Cochrane Database of Systematic Reviews

\title{
Structured telephone support or non-invasive telemonitoring for patients with heart failure (Review)
}

Inglis SC, Clark RA, Dierckx R, Prieto-Merino D, Cleland JGF

Inglis SC, Clark RA, Dierckx R, Prieto-Merino D, Cleland JGF.

Structured telephone support or non-invasive telemonitoring for patients with heart failure.

Cochrane Database of Systematic Reviews 2015, Issue 10. Art. No.: CD007228.

DOI: 10.1002/14651858.CD007228.pub3.

www.cochranelibrary.com 
TABLE OF CONTENTS

HEADER 1

ABSTRACT

PLAIN LANGUAGE SUMMARY

SUMMARY OF FINDINGS

BACKGROUND

OBJECTIVES

METHODS

RESULTS

Figure 1.

Figure 2.

Figure 3.

Figure 4.

Figure 5.

Figure 6.

Figure 7.

Figure 8.

Figure 9.

Figure 10.

Figure 11.

Figure 12.

Figure 13.

Figure 14

DISCUSSION

AUTHORS' CONCLUSIONS

ACKNOWLEDGEMENTS

REFERENCES

CHARACTERISTICS OF STUDIES

DATA AND ANALYSES

Analysis 1.1. Comparison 1 Impact of structured telephone support and telemonitoring in heart failure on all-cause mortality, Outcome 1 All-cause mortality: STS vs UC.

Analysis 1.2. Comparison 1 Impact of structured telephone support and telemonitoring in heart failure on all-cause mortality, Outcome 2 All-cause mortality: TM vs UC.

Analysis 1.3. Comparison $1 \mathrm{Impact}$ of structured telephone support and telemonitoring in heart failure on all-cause mortality, Outcome 3 Subgroup technology: all-cause mortality STS vs UC.

Analysis 1.4. Comparison 1 Impact of structured telephone support and telemonitoring in heart failure on all-cause mortality, Outcome 4 Subgroup technology: all-cause mortality Complex TM vs UC.

Analysis 1.5. Comparison $1 \mathrm{Impact}$ of structured telephone support and telemonitoring in heart failure on all-cause mortality, Outcome 5 Subgroup technology: all-cause mortality Videophone vs UC.

Analysis 1.6. Comparison $1 \mathrm{Impact}$ of structured telephone support and telemonitoring in heart failure on all-cause mortality, Outcome 6 Subgroup technology: all-cause mortality IVR vs UC.

Analysis 1.7. Comparison $1 \mathrm{Impact}$ of structured telephone support and telemonitoring in heart failure on all-cause mortality, Outcome 7 Subgroup technology: all-cause mortality Mobile/PDA vs UC.

Analysis 1.8. Comparison 1 Impact of structured telephone support and telemonitoring in heart failure on all-cause mortality, Outcome 8 Subgroup TM intensity: all-cause mortality Office hours vs UC.

Analysis 1.9. Comparison $1 \mathrm{Impact}$ of structured telephone support and telemonitoring in heart failure on all-cause mortality, Outcome 9 Subgroup TM intensity: all-cause mortality $24 / 7$ or 7 days/week vs UC.

Analysis 1.10. Comparison $1 \mathrm{Impact}$ of structured telephone support and telemonitoring in heart failure on all-cause mortality, Outcome 10 Subgroup Publication year: all-cause mortality STS vs UC $<2000$.

Analysis 1.11. Comparison $1 \mathrm{Impact}$ of structured telephone support and telemonitoring in heart failure on all-cause mortality, Outcome 11 Subgroup Publication year: all-cause mortality STS vS UC 2000 - 2007.

Analysis 1.12. Comparison 1 Impact of structured telephone support and telemonitoring in heart failure on all-cause mortality, Outcome 12 Subgroup Publication year: all-cause mortality STS vs UC $\geq 2008$. 
Analysis 1.13. Comparison 1 Impact of structured telephone support and telemonitoring in heart failure on all-cause mortality, Outcome 13 Subgroup Publication year: all-cause mortality TM vs UC 2000 - 2007.

Analysis 1.14. Comparison $1 \mathrm{Impact}$ of structured telephone support and telemonitoring in heart failure on all-cause mortality, Outcome 14 Subgroup Publication year: all-cause mortality TM vs UC $\geq 2008$.

Analysis 1.15. Comparison 1 Impact of structured telephone support and telemonitoring in heart failure on all-cause mortality, Outcome 15 Subgroup Participant age: all-cause mortality: STS vS UC $<70$ years of age.

Analysis 1.16. Comparison 1 Impact of structured telephone support and telemonitoring in heart failure on all-cause mortality, Outcome 16 Subgroup Participant age: all-cause mortality STS vs UC $\geq 70$ years of age.

Analysis 1.17. Comparison 1 Impact of structured telephone support and telemonitoring in heart failure on all-cause mortality, Outcome 17 Subgroup Participant age: all-cause mortality: TM vs UC $<70$ years of age.

Analysis 1.18. Comparison 1 Impact of structured telephone support and telemonitoring in heart failure on all-cause mortality, Outcome 18 Subgroup Participant age: all-cause mortality TM vs UC $\geq 70$ years of age.

Analysis 1.19. Comparison $1 \mathrm{Impact}$ of structured telephone support and telemonitoring in heart failure on all-cause mortality, Outcome 19 Subgroup STS focus: all-cause mortality STS (clinical support) vs UC.

Analysis 1.20. Comparison $1 \mathrm{Impact}$ of structured telephone support and telemonitoring in heart failure on all-cause mortality, Outcome 20 Subgroup STS focus: all-cause mortality STS (education) vs UC.

Analysis 1.21. Comparison $1 \mathrm{Impact}$ of structured telephone support and telemonitoring in heart failure on all-cause mortality, Outcome 21 Sensitivity analysis follow-up period (> 6 months), all-cause mortality: STS vs UC.

Analysis 1.22. Comparison 1 Impact of structured telephone support and telemonitoring in heart failure on all-cause mortality, Outcome 22 Sensitivity analysis follow-up period (> 6 months), all-cause mortality: TM vs UC.

Analysis 2.1. Comparison 2 Impact of structured telephone or telemonitoring in heart failure on risk of all-cause hospitalisation, Outcome 1 All-cause hospitalisation: STS vs UC.

Analysis 2.2. Comparison 2 Impact of structured telephone or telemonitoring in heart failure on risk of all-cause hospitalisation, Outcome 2 All-cause hospitalisation: TM vs UC.

Analysis 2.3. Comparison 2 Impact of structured telephone or telemonitoring in heart failure on risk of all-cause hospitalisation, Outcome 3 Subgroup technology: all-cause hospitalisation STS vs UC.

Analysis 2.4. Comparison 2 Impact of structured telephone or telemonitoring in heart failure on risk of all-cause hospitalisation, Outcome 4 Subgroup technology: all-cause hospitalisation Mobile/PDA vs UC.

Analysis 2.5. Comparison $2 \mathrm{Impact}$ of structured telephone or telemonitoring in heart failure on risk of all-cause hospitalisation, Outcome 5 Subgroup technology: all-cause hospitalisation Videophone vs UC.

Analysis 2.6. Comparison 2 Impact of structured telephone or telemonitoring in heart failure on risk of all-cause hospitalisation, Outcome 6 Subgroup technology: all-cause hospitalisation IVR vs UC.

Analysis 2.7. Comparison 2 Impact of structured telephone or telemonitoring in heart failure on risk of all-cause hospitalisation, Outcome 7 Subgroup technology: all-cause hospitalisation Complex TM vs UC.

Analysis 2.8. Comparison 2 Impact of structured telephone or telemonitoring in heart failure on risk of all-cause hospitalisation, Outcome 8 Subgroup TM intensity: all-cause hospitalisation Office hours vs UC.

Analysis 2.9. Comparison 2 Impact of structured telephone or telemonitoring in heart failure on risk of all-cause hospitalisation, Outcome 9 Subgroup TM intensity: all-cause hospitalisation $24 / 7$ or 7 days vs UC.

Analysis 2.10. Comparison 2 Impact of structured telephone or telemonitoring in heart failure on risk of all-cause hospitalisation, Outcome 10 Subgroup Publication year: all-cause hospitalisation STS vs UC $<2000$.

Analysis 2.11. Comparison 2 Impact of structured telephone or telemonitoring in heart failure on risk of all-cause hospitalisation, Outcome 11 Subgroup Publication year: all-cause hospitalisation STS vs UC $2000-2007$.

Analysis 2.12. Comparison 2 Impact of structured telephone or telemonitoring in heart failure on risk of all-cause hospitalisation, Outcome 12 Subgroup Publication year: all-cause hospitalisation STS vs UC $\geq 2008$.

Analysis 2.13. Comparison 2 Impact of structured telephone or telemonitoring in heart failure on risk of all-cause hospitalisation, Outcome 13 Subgroup Publication year: all-cause hospitalisation TM vs UC 2000 - 2007.

Analysis 2.14. Comparison 2 Impact of structured telephone or telemonitoring in heart failure on risk of all-cause hospitalisation, Outcome 14 Subgroup Publication year: all-cause hospitalisation TM vs UC $\geq 2008$.

Analysis 2.15. Comparison 2 Impact of structured telephone or telemonitoring in heart failure on risk of all-cause hospitalisation, Outcome 15 Subgroup Participant age: all-cause hospitalisation STS vs UC $\geq 70$ years of age.

Analysis 2.16. Comparison 2 Impact of structured telephone or telemonitoring in heart failure on risk of all-cause hospitalisation, Outcome 16 Subgroup Participant age: all-cause hospitalisation: STS vs UC < 70 years of age.

Analysis 2.17. Comparison 2 Impact of structured telephone or telemonitoring in heart failure on risk of all-cause hospitalisation, Outcome 17 Subgroup Participant age: all-cause hospitalisation TM vs UC $\geq 70$ years of age.

Analysis 2.18. Comparison 2 Impact of structured telephone or telemonitoring in heart failure on risk of all-cause hospitalisation, Outcome 18 Subgroup Participant age: all-cause hospitalisation: TM vs UC < 70 years of age. 
Analysis 2.19. Comparison 2 Impact of structured telephone or telemonitoring in heart failure on risk of all-cause hospitalisation, Outcome 19 Subgroup STS focus: all-cause hospitalisation: STS (clinical support) vs UC.

Analysis 2.20. Comparison 2 Impact of structured telephone or telemonitoring in heart failure on risk of all-cause hospitalisation, Outcome 20 Subgroup STS focus: all-cause hospitalisation: STS (education) vs UC.

Analysis 2.21. Comparison 2 Impact of structured telephone or telemonitoring in heart failure on risk of all-cause hospitalisation, Outcome 21 Sensitivity analysis follow-up period (> 6 months), all-cause hospitalisation: STS vs UC.

Analysis 2.22. Comparison 2 Impact of structured telephone or telemonitoring in heart failure on risk of all-cause hospitalisation, Outcome 22 Sensitivity analysis follow-up period (> 6 months), all-cause hospitalisation: TM vs UC.

Analysis 3.1. Comparison $3 \mathrm{Impact}$ of structured telephone support or telemonitoring in heart failure on risk of heart failurerelated hospitalisation, Outcome 1 CHF-related hospitalisation: STS vs UC.

Analysis 3.2. Comparison 3 Impact of structured telephone support or telemonitoring in heart failure on risk of heart failurerelated hospitalisation, Outcome 2 CHF-related hospitalisation: TM vs UC.

Analysis 3.3. Comparison 3 Impact of structured telephone support or telemonitoring in heart failure on risk of heart failurerelated hospitalisation, Outcome 3 Subgroup technology: CHF-related hospitalisation STS vs UC.

Analysis 3.4. Comparison 3 Impact of structured telephone support or telemonitoring in heart failure on risk of heart failurerelated hospitalisation, Outcome 4 Subgroup technology: CHF-related hospitalisation Mobile/PDA vs UC.

Analysis 3.5. Comparison 3 Impact of structured telephone support or telemonitoring in heart failure on risk of heart failurerelated hospitalisation, Outcome 5 Subgroup technology CHF-related hospitalisation Complex TM vs UC.

Analysis 3.6. Comparison 3 Impact of structured telephone support or telemonitoring in heart failure on risk of heart failurerelated hospitalisation, Outcome 6 Subgroup technology: CHF-related hospitalisation IVR vs UC.

Analysis 3.7. Comparison 3 Impact of structured telephone support or telemonitoring in heart failure on risk of heart failurerelated hospitalisation, Outcome 7 Subgroup TM intensity: CHF-related hospitalisation Office hours vs UC.

Analysis 3.8. Comparison 3 Impact of structured telephone support or telemonitoring in heart failure on risk of heart failurerelated hospitalisation, Outcome 8 Subgroup TM intensity: CHF-related hospitalisation 24/7 or 7 days vs UC.

Analysis 3.9. Comparison 3 Impact of structured telephone support or telemonitoring in heart failure on risk of heart failurerelated hospitalisation, Outcome 9 Subgroup Publication year: CHF-related hospitalisation STS vS UC $<2000$.

Analysis 3.10. Comparison 3 Impact of structured telephone support or telemonitoring in heart failure on risk of heart failurerelated hospitalisation, Outcome 10 Subgroup Publication year: CHF-related hospitalisation STS vs UC 2000 - 2007.

Analysis 3.11. Comparison $3 \mathrm{Impact}$ of structured telephone support or telemonitoring in heart failure on risk of heart failurerelated hospitalisation, Outcome 11 Subgroup Publication year: CHF-related hospitalisation STS vs UC $\geq 2008$.

Analysis 3.12. Comparison $3 \mathrm{Impact}$ of structured telephone support or telemonitoring in heart failure on risk of heart failurerelated hospitalisation, Outcome 12 Subgroup Publication year: CHF-related hospitalisation TM vs UC $2000-2007$.

Analysis 3.13. Comparison 3 Impact of structured telephone support or telemonitoring in heart failure on risk of heart failurerelated hospitalisation, Outcome 13 Subgroup Publication year: CHF-related hospitalisation TM vs UC $\geq 2008$.

Analysis 3.14. Comparison 3 Impact of structured telephone support or telemonitoring in heart failure on risk of heart failurerelated hospitalisation, Outcome 14 Subgroup Participant age: CHF-related hospitalisation: STS vs UC $<70$ years of age. ....... Analysis 3.15. Comparison 3 Impact of structured telephone support or telemonitoring in heart failure on risk of heart failurerelated hospitalisation, Outcome 15 Subgroup Participant age: CHF-related hospitalisation: STS vS UC $\geq 70$ years of age. ....... Analysis 3.16. Comparison $3 \mathrm{Impact}$ of structured telephone support or telemonitoring in heart failure on risk of heart failurerelated hospitalisation, Outcome 16 Subgroup Participant age: CHF-related hospitalisation: TM vs UC < 70 years of age. ......... Analysis 3.17. Comparison $3 \mathrm{Impact}$ of structured telephone support or telemonitoring in heart failure on risk of heart failurerelated hospitalisation, Outcome 17 Subgroup Participant age: CHF-related hospitalisation: TM vs UC $\geq 70$ years of age. ........ Analysis 3.18. Comparison 3 Impact of structured telephone support or telemonitoring in heart failure on risk of heart failurerelated hospitalisation, Outcome 18 Subgroup STS focus: CHF-related hospitalisation: STS (clinical support) vs UC.

Analysis 3.19. Comparison 3 Impact of structured telephone support or telemonitoring in heart failure on risk of heart failurerelated hospitalisation, Outcome 19 Subgroup STS focus: CHF-related hospitalisation: STS (education) vs UC.

Analysis 3.20. Comparison 3 Impact of structured telephone support or telemonitoring in heart failure on risk of heart failurerelated hospitalisation, Outcome 20 Sensitivity analysis follow-up period (> 6 months), CHF-related hospitalisation: STS vs UC.

Analysis 3.21. Comparison 3 Impact of structured telephone support or telemonitoring in heart failure on risk of heart failurerelated hospitalisation, Outcome 21 Sensitivity analysis follow-up period (> 6 months), CHF-related hospitalisation: TM vs UC.

ADDITIONAL TABLES 
[Intervention Review]

\title{
Structured telephone support or non-invasive telemonitoring for patients with heart failure
}

\author{
Sally C Inglis 1 , Robyn A Clark², Riet Dierckx³ , David Prieto-Merino4 ${ }^{4}$, John GF Cleland 5 \\ 1Centre for Cardiovascular and Chronic Care, Faculty of Health, University of Technology Sydney, Sydney, Australia. 2 School of Nursing \\ and Midwifery, The Flinders University of South Australia, Adelaide, Australia. ${ }^{3}$ Cardiovascular Center, OLV Hospital, Aalst, Belgium. \\ ${ }^{4}$ Department of Non-communicable Disease Epidemiology, London School of Hygiene \& Tropical Medicine, London, UK. ${ }^{5} \mathrm{National}$ Heart \\ and Lung Institute, Imperial College London, London, UK
}

Contact address: Sally C Inglis, Centre for Cardiovascular and Chronic Care, Faculty of Health, University of Technology Sydney, Sydney, Australia.sally.inglis@uts.edu.au.

Editorial group: Cochrane Heart Group.

Publication status and date: New search for studies and content updated (conclusions changed), published in Issue 10, 2015.

Citation: Inglis SC, Clark RA, Dierckx R, Prieto-Merino D, Cleland JGF. Structured telephone support or non-invasive telemonitoring for patients with heart failure. Cochrane Database of Systematic Reviews 2015, Issue 10. Art. No.: CD007228. DOI: 10.1002/14651858.CD007228.pub3.

Copyright ( 2015 The Cochrane Collaboration. Published by John Wiley \& Sons, Ltd.

\section{A B S T R A C T}

\section{Background}

Specialised disease management programmes for heart failure aim to improve care, clinical outcomes and/or reduce healthcare utilisation. Since the last version of this review in 2010, several new trials of structured telephone support and non-invasive home telemonitoring have been published which have raised questions about their effectiveness.

\section{Objectives}

To review randomised controlled trials (RCTs) of structured telephone support or non-invasive home telemonitoring compared to standard practice for people with heart failure, in order to quantify the effects of these interventions over and above usual care.

\section{Search methods}

We updated the searches of the Cochrane Central Register of Controlled Trials (CENTRAL), Database of Abstracts of Reviews of Effects (DARE), Health Technology AsseFssment Database (HTA) on the Cochrane Library; MEDLINE (OVID), EMBASE (OVID), CINAHL (EBSCO), Science Citation Index Expanded (SCI-EXPANDED), Conference Proceedings Citation Index- Science (CPCI-S) on Web of Science (Thomson Reuters), AMED, Proquest Theses and Dissertations, IEEE Xplore and TROVE in January 2015. We handsearched bibliographies of relevant studies and systematic reviews and abstract conference proceedings. We applied no language limits.

\section{Selection criteria}

We included only peer-reviewed, published RCTs comparing structured telephone support or non-invasive home telemonitoring to usual care of people with chronic heart failure. The intervention or usual care could not include protocol-driven home visits or more intensive than usual (typically four to six weeks) clinic follow-up.

\section{Data collection and analysis}

We present data as risk ratios (RRs) with 95\% confidence intervals (Cls). Primary outcomes included all-cause mortality, all-cause and heart failure-related hospitalisations, which we analysed using a fixed-effect model. Other outcomes included length of stay, healthrelated quality of life, heart failure knowledge and self care, acceptability and cost; we described and tabulated these. We performed meta-regression to assess homogeneity (the null hypothesis) in each subgroup analysis and to see if the effect of the intervention varied according to some quantitative variable (such as year of publication or median age). 


\section{Main results}

We include 41 studies of either structured telephone support or non-invasive home telemonitoring for people with heart failure, of which 17 were new and 24 had been included in the previous Cochrane review. In the current review, 25 studies evaluated structured telephone support (eight new studies, plus one study previously included but classified as telemonitoring; total of 9332 participants), 18 evaluated telemonitoring (nine new studies; total of 3860 participants). Two of the included studies trialled both structured telephone support and telemonitoring compared to usual care, therefore 43 comparisons are evident.

Non-invasive telemonitoring reduced all-cause mortality (RR 0.80,95\% Cl 0.68 to $0.94 ;$ participants $=3740 ;$ studies $=17 ; I^{2}=24 \%$, GRADE: moderate-quality evidence) and heart failure-related hospitalisations (RR $0.71,95 \% \mathrm{Cl} 0.60$ to $0.83 ;$ participants $=2148 ;$ studies $=8 ; I^{2}=20 \%$, GRADE: moderate-quality evidence). Structured telephone support reduced all-cause mortality (RR $0.87,95 \% \mathrm{Cl} 0.77$ to 0.98 ; participants $=9222$; studies $=22 ; \mathrm{I}^{2}=0 \%$, GRADE: moderate-quality evidence) and heart failure-related hospitalisations (RR $0.85,95 \% \mathrm{Cl} 0.77$ to 0.93 ; participants $=7030 ;$ studies $=16 ; I^{2}=27 \%$, GRADE: moderate-quality evidence) .

Neither structured telephone support nor telemonitoring demonstrated effectiveness in reducing the risk of all-cause hospitalisations (structured telephone support: RR $0.95,95 \% \mathrm{Cl} 0.90$ to 1.00 ; participants $=7216$; studies $=16 ; I^{2}=47 \%$, GRADE: very low-quality evidence; non-invasive telemonitoring: RR $0.95,95 \% \mathrm{Cl} 0.89$ to 1.01 ; participants $=3332$; studies $=13 ; \mathrm{I}^{2}=71 \%$, GRADE: very low-quality evidence).

Seven structured telephone support studies reported length of stay, with one reporting a significant reduction in length of stay in hospital. Nine telemonitoring studies reported length of stay outcome, with one study reporting a significant reduction in the length of stay with the intervention. One telemonitoring study reported a large difference in the total number of hospitalisations for more than three days, but this was not an analysis of length of stay per hospitalisation. Nine of 11 structured telephone support studies and five of 11 telemonitoring studies reported significant improvements in health-related quality of life. Nine structured telephone support studies and six telemonitoring studies reported costs of the intervention or cost effectiveness. Three structured telephone support studies and one telemonitoring study reported a decrease in costs and two telemonitoring studies reported increases in cost, due both to the cost of the intervention and to increased medical management. Adherence was rated between $55.1 \%$ and $98.5 \%$ for those structured telephone support and telemonitoring studies which reported this outcome. Participant acceptance of the intervention was reported in the range of $76 \%$ to $97 \%$ for studies which evaluated this outcome. Seven of nine studies that measured these outcomes reported significant improvements in heart failure knowledge and self-care behaviours.

\section{Authors' conclusions}

For people with heart failure, structured telephone support and non-invasive home telemonitoring reduce the risk of all-cause mortality and heart failure-related hospitalisations; these interventions also demonstrated improvements in health-related quality of life and heart failure knowledge and self-care behaviours. Studies also demonstrated participant satisfaction with the majority of the interventions which assessed this outcome.

\section{PLAIN LANGUAGE SUMMARY}

\section{Structured telephone support and non-invasive telemonitoring in the management of people with heart failure}

\section{Review question}

We reviewed the evidence about the effect of structured telephone support and non-invasive telemonitoring in the management of people with heart failure. We found 41 studies. Two of the included studies trialled both structured telephone support and telemonitoring compared to usual care, therefore 43 comparisons are evident. The evidence is current to January 2015.

\section{Background}

In the context of limited health funding and a rapidly expanding population of older people, it is increasingly difficult for healthcare systems to provide high-quality care to those with heart failure. Multidisciplinary specialist heart failure clinics are available only to a minority of people and do not have the capacity for frequent patient review. Patients may be unwilling or unable to make frequent clinic attendance due to cost, difficulty with transport or disability and frailty. Structured telephone support and telemonitoring can provide specialised heart failure care to a large number of people with limited access to healthcare services.

\section{Study characteristics}

We include 41 full-text peer-reviewed studies of either structured telephone support or home telemonitoring in this review. Twenty-five studies evaluate structured telephone support (eight new studies, plus one previously included study now classified as telemonitoring; total of 9332 participants), 18 evaluated telemonitoring (nine new studies; total of 3860 participants) and two studies evaluated both interventions (included in listed counts).

\section{Key results}


This review demonstrates that supporting people with heart failure at home using information technology can reduce the rates of death and heart failure-related hospitalisation. It can improve people's quality of life and knowledge about heart failure and self care. Most patients, even those who are elderly, learn to use the technology easily and are satisfied with these interventions.

\section{Quality of the evidence}

We assessed the quality of the evidence for the primary outcomes in this review (all-cause mortality, all-cause hospitalisation and heart failure-related hospitalisation) according to GRADE criteria. We rated it from very low (all-cause hospitalisations) to moderate (all-cause mortality and heart failure-related hospitalisations). 


\section{SUMMARY OF FINDINGS}

Summary of findings for the main comparison. Summary of findings - structured telephone support or telemonitoring versus usual care - all-cause mortality

Impact of structured telephone support and telemonitoring in heart failure on all-cause mortality2

Patient or population: people with heart failure

Setting:

Intervention: structured telephone support or telemonitoring

Comparison: usual care

\begin{tabular}{|c|c|c|c|c|c|c|}
\hline \multirow[t]{2}{*}{ Outcomes } & \multicolumn{2}{|c|}{ Anticipated absolute effects ${ }^{\star}(95 \% \mathrm{Cl})$} & \multirow{2}{*}{$\begin{array}{l}\text { Relative effect } \\
(95 \% \mathrm{Cl})\end{array}$} & \multirow{2}{*}{$\begin{array}{l}\text { № of partici- } \\
\text { pants } \\
\text { (studies) }\end{array}$} & \multirow{2}{*}{$\begin{array}{l}\text { Quality of the } \\
\text { evidence } \\
\text { (GRADE) }\end{array}$} & \multirow[t]{2}{*}{ Comments } \\
\hline & Risk with usual care & $\begin{array}{l}\text { Risk with Impact of structured tele- } \\
\text { phone support and telemonitoring in } \\
\text { CHF on all-cause mortality }\end{array}$ & & & & \\
\hline \multirow{4}{*}{$\begin{array}{l}\text { All-cause mortality: } \\
\text { Structured telephone } \\
\text { support versus usual } \\
\text { care }\end{array}$} & \multicolumn{2}{|l|}{ Study population } & \multirow{4}{*}{$\begin{array}{l}\text { RR } 0.87 \\
\text { (0.77 to } 0.98 \text { ) }\end{array}$} & \multirow{4}{*}{$\begin{array}{l}9222 \\
\text { (22 RCTs) }\end{array}$} & \multirow{4}{*}{$\begin{array}{l}\oplus \oplus \oplus \\
\text { MODERATE } 1\end{array}$} & \\
\hline & 116 per 1000 & $\begin{array}{l}101 \text { per } 1000 \\
\text { (89 to } 113 \text { ) }\end{array}$ & & & & \\
\hline & \multicolumn{2}{|c|}{ Moderate risk population } & & & & \\
\hline & 109 per 1000 & $\begin{array}{l}94 \text { per } 1000 \\
(84 \text { to } 106)\end{array}$ & & & & \\
\hline \multirow{4}{*}{$\begin{array}{l}\text { All-cause mortality: } \\
\text { Telemonitoring versus } \\
\text { usual care }\end{array}$} & \multicolumn{2}{|l|}{ Study population } & \multirow{4}{*}{$\begin{array}{l}\text { RR } 0.80 \\
\text { (0.68 to } 0.94 \text { ) }\end{array}$} & \multirow{4}{*}{$\begin{array}{l}3740 \\
(17 \mathrm{RCTs})\end{array}$} & \multirow{4}{*}{$\begin{array}{l}\oplus \oplus \oplus \\
\text { MODERATE } 1\end{array}$} & \\
\hline & 145 per 1000 & $\begin{array}{l}116 \text { per } 1000 \\
\text { (99 to } 136)\end{array}$ & & & & \\
\hline & \multicolumn{2}{|c|}{ Moderate risk population } & & & & \\
\hline & 155 per 1000 & $\begin{array}{l}124 \text { per } 1000 \\
\text { (105 to } 145)\end{array}$ & & & & \\
\hline
\end{tabular}

${ }^{\star}$ The risk in the intervention group (and its $95 \%$ confidence interval) is based on the assumed risk in the comparison group and the relative effect of the intervention (and its $95 \% \mathrm{Cl}$ ).

Cl: Confidence interval; RR: Risk ratio.

GRADE Working Group grades of evidence

High quality: We are very confident that the true effect lies close to that of the estimate of the effect 


\begin{tabular}{|c|c|c|c|c|c|c|}
\hline \multicolumn{7}{|c|}{$\begin{array}{l}\text { Moderate quality: We are moderately confident in the effect estimate: The true effect is likely to be close to the estimate of the effect, but there is a po } \\
\text { stantially different } \\
\text { Low quality: Our confidence in the effect estimate is limited: The true effect may be substantially different from the estimate of the effect } \\
\text { Very low quality: We have very little confidence in the effect estimate: The true effect is likely to be substantially different from the estimate of effect }\end{array}$} \\
\hline \multicolumn{7}{|c|}{$\begin{array}{l}\text { 1Publication bias strongly suspected. } \\
2 \text { Length of follow-up ranged from } 3 \text { months to } 18 \text { months for structured telephone support studies and } 3 \text { months to } 26 \text { months for telemonitoring studies. } \\
\text { Summary of findings } 2 \text {. Summary of findings - structured telephone support or telemonitoring versus usual care - all-cause hospitalisation }\end{array}$} \\
\hline \multicolumn{7}{|c|}{ Impact of structured telephone or telemonitoring in heart failure on risk of all-cause hospitalisation 4} \\
\hline \multicolumn{7}{|c|}{$\begin{array}{l}\text { Patient or population: people with heart failure } \\
\text { Setting: } \\
\text { Intervention: structured telephone or telemonitoring } \\
\text { Comparison: usual care }\end{array}$} \\
\hline \multirow[t]{2}{*}{ Outcomes } & \multicolumn{2}{|c|}{ Anticipated absolute effects ${ }^{\star}(95 \% \mathrm{Cl})$} & \multirow{2}{*}{$\begin{array}{l}\text { Relative effect } \\
(95 \% \mathrm{Cl})\end{array}$} & \multirow{2}{*}{$\begin{array}{l}\text { No of partici- } \\
\text { pants } \\
\text { (studies) }\end{array}$} & \multirow{2}{*}{$\begin{array}{l}\text { Quality of the } \\
\text { evidence } \\
\text { (GRADE) }\end{array}$} & \multirow[t]{2}{*}{ Comments } \\
\hline & Risk with usual care & $\begin{array}{l}\text { Risk with Impact of structured telephone } \\
\text { or telemonitoring in CHF on risk of all- } \\
\text { cause hospitalisation }\end{array}$ & & & & \\
\hline \multirow{4}{*}{$\begin{array}{l}\text { All-cause hospitalisa- } \\
\text { tion: Structured tele- } \\
\text { phone support verus } \\
\text { usual care }\end{array}$} & Study population & & \multirow{4}{*}{$\begin{array}{l}\text { RR } 0.95 \\
\text { (0.90 to } 1.00)\end{array}$} & \multirow{4}{*}{$\begin{array}{l}7216 \\
\text { (16 RCTs) }\end{array}$} & \multirow{4}{*}{$\begin{array}{l}\text { OOO } \\
\text { VERY LOW 1, 2, } 3\end{array}$} & \\
\hline & 422 per 1000 & $\begin{array}{l}401 \text { per } 1000 \\
\text { (380 to } 422 \text { ) }\end{array}$ & & & & \\
\hline & \multicolumn{2}{|c|}{ Moderate risk population } & & & & \\
\hline & 432 per 1000 & $\begin{array}{l}411 \text { per } 1000 \\
\text { (389 to } 432 \text { ) }\end{array}$ & & & & \\
\hline \multirow{4}{*}{$\begin{array}{l}\text { All-cause hospitalisa- } \\
\text { tion: Telemonitoring } \\
\text { versus usual care }\end{array}$} & Study population & & \multirow{4}{*}{$\begin{array}{l}\text { RR } 0.95 \\
\text { (0.89 to } 1.01)\end{array}$} & \multirow{4}{*}{$\begin{array}{l}3332 \\
\text { (13 RCTs) }\end{array}$} & \multirow{4}{*}{$\begin{array}{l}\bigcirc \bigcirc \bigcirc \\
\text { VERY LOW 1, 2, } 3\end{array}$} & \\
\hline & 517 per 1000 & $\begin{array}{l}491 \text { per } 1000 \\
(460 \text { to } 522)\end{array}$ & & & & \\
\hline & \multicolumn{2}{|c|}{ Moderate risk population } & & & & \\
\hline & 541 per 1000 & $\begin{array}{l}514 \text { per } 1000 \\
\text { ( } 482 \text { to } 547)\end{array}$ & & & & \\
\hline
\end{tabular}




\begin{tabular}{|c|c|c|c|c|c|c|}
\hline \multicolumn{7}{|c|}{$\begin{array}{l}{ }^{\star} \text { The risk in the intervention group (and its } 95 \% \text { confidence interval) is based on the assumed risk in the comparison group and the relative effect of the intervention (and } \\
\text { its } 95 \% \mathrm{Cl} \text { ). } \\
\text { Cl: Confidence interval; RR: Risk ratio. }\end{array}$} \\
\hline \multicolumn{7}{|c|}{$\begin{array}{l}\text { GRADE Working Group grades of evidence } \\
\text { High quality: We are very confident that the true effect lies close to that of the estimate of the effect } \\
\text { Moderate quality: We are moderately confident in the effect estimate: The true effect is likely to be close to the estimate of the effect, but there is a possibility that it is sub- } \\
\text { stantially different } \\
\text { Low quality: Our confidence in the effect estimate is limited: The true effect may be substantially different from the estimate of the effect } \\
\text { Very low quality: We have very little confidence in the effect estimate: The true effect is likely to be substantially different from the estimate of effect }\end{array}$} \\
\hline \multicolumn{7}{|c|}{$\begin{array}{l}\text { 1Evidence of serious inconsistency in this outcome, due to moderate to substantial heterogeneity (structured telephone support } I^{2}=47 \% \text {; telemonitoring } I^{2}=71 \% \text { ) } \\
\text { 2Evidence of serious imprecision in this outcome, due to wide confidence intervals around the effect measure for individual studies. } \\
\text { 3Publication bias strongly suspected. } \\
4 \text { Length of follow-up ranged from } 3 \text { months to } 16 \text { months for structured telephone support studies and from } 3 \text { months to } 802 \text { days for telemonitoring studies. }\end{array}$} \\
\hline \multicolumn{7}{|c|}{ Impact of structured telephone support or telemonitoring in heart failure on risk of heart failure-related hospitalisation 2} \\
\hline \multicolumn{7}{|c|}{$\begin{array}{l}\text { Patient or population: people with heart failure } \\
\text { Setting: } \\
\text { Intervention: structured telephone support or telemonitoring } n \\
\text { Comparison: usual care }\end{array}$} \\
\hline \multirow[t]{2}{*}{ Outcomes } & \multicolumn{2}{|c|}{ Anticipated absolute effects* $(95 \% \mathrm{Cl})$} & \multirow{2}{*}{$\begin{array}{l}\text { Relative effect } \\
(95 \% \mathrm{CI})\end{array}$} & \multirow{2}{*}{$\begin{array}{l}\text { No of partici- } \\
\text { pants } \\
\text { (studies) }\end{array}$} & \multirow{2}{*}{$\begin{array}{l}\text { Quality of the } \\
\text { evidence } \\
\text { (GRADE) }\end{array}$} & Comments \\
\hline & Risk with usual care & $\begin{array}{l}\text { Risk with Impact of structured telephone } \\
\text { support or telemonitoring in CHF on risk } \\
\text { of CHF-related hospitalisation }\end{array}$ & & & & \\
\hline \multirow{4}{*}{$\begin{array}{l}\text { CHF-related hospitali- } \\
\text { sation: Structured tele- } \\
\text { phone support versus } \\
\text { usual care }\end{array}$} & Study population & & \multirow{4}{*}{$\begin{array}{l}\text { RR } 0.85 \\
\text { (0.77 to } 0.93 \text { ) }\end{array}$} & \multirow{4}{*}{$\begin{array}{l}7030 \\
(16 \mathrm{RCTs})\end{array}$} & \multirow{4}{*}{\multicolumn{2}{|c|}{$\begin{array}{l}\oplus \oplus \oplus \\
\text { MODERATE } 1\end{array}$}} \\
\hline & 214 per 1000 & $\begin{array}{l}182 \text { per } 1000 \\
(165 \text { to } 199)\end{array}$ & & & & \\
\hline & \multicolumn{2}{|c|}{ Moderate risk population } & & & & \\
\hline & 179 per 1000 & $\begin{array}{l}152 \text { per } 1000 \\
\text { (138 to } 167)\end{array}$ & & & & \\
\hline
\end{tabular}




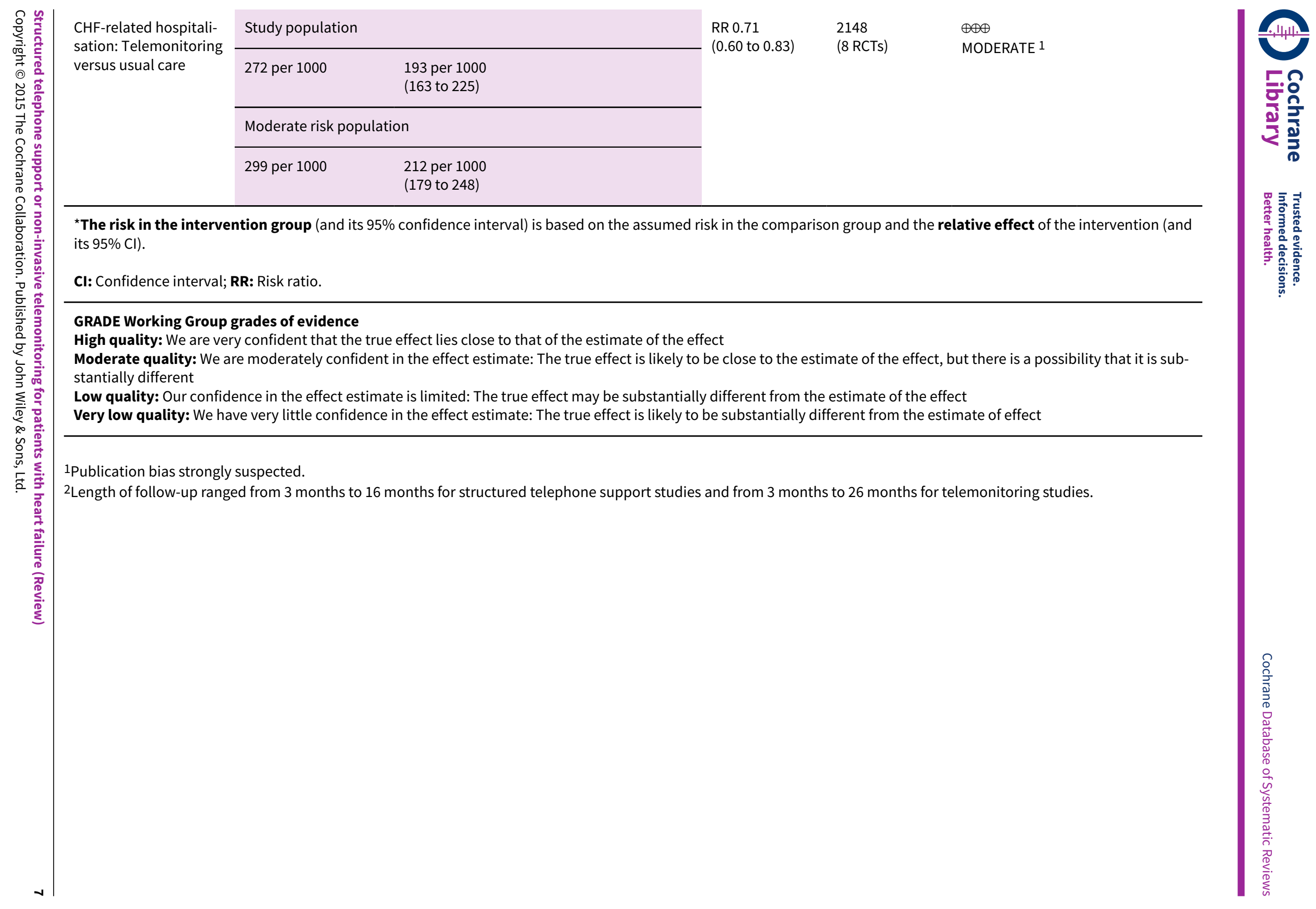




\section{B A C K G R O U N D}

A 2010 Cochrane review of structured telephone support or home telemonitoring for people with heart failure (HF) concluded that structured telephone support and telemonitoring were effective in reducing the risk of all-cause mortality and heart failure relatedhospitalisations (Inglis 2010). Since then, doubt has been cast on the efficacy of these interventions, due to publication of several neutral studies (Anker 2011; Chaudhry 2010 (Tele-HF); Koehler 2011 (TIM-HF)). Current international heart failure guidelines have so far not recommended widespread implementation of remote monitoring due to these conflicting trial results (National Heart Foundation of Australia 2011; Mant 2011; McMurray 2012; Yancy 2013) and have called for more research before recommending these interventions Ponikowski 2014. This review sought to update the previous version by identifying and classifying all peerreviewed trials of structured telephone support or non-invasive home telemonitoring published since the Inglis 2010 review.

\section{Description of the condition}

Heart failure (HF) is a complex, debilitating syndrome due to cardiac dysfunction that impairs the ability of the ventricle to fill with or eject blood. As a result, typical symptoms such as dyspnoea and fatigue occur at rest or with reduced physical effort. Heart failure often results from damage to the myocardium for which the aetiology differs according to the population studied. In highincome countries, hypertension and coronary heart disease are the most important causes of heart failure (Cleland 2011; Gerber 2015; Maggioni 2013; Yusuf 2014). Hypertension is a risk factor for coronary disease, which is the most common direct cause for heart failure with reduced ejection fraction (HFrEF). Hypertension, often associated with atrial fibrillation, is the predominant cause of heart failure with preserved ejection fraction (HFpEF). In low- to middle-income countries, the syndrome is also often the result of longstanding hypertension but cardiomyopathy and rheumatic heart disease are also common. However, the incidence of atherosclerotic disease is increasing in low- to middle-income countries (Callender 2014b). Heart failure exerts a substantial burden on healthcare systems, due to the high consumption of human resources caused predominantly by repeated and lengthy admissions to hospital (Dunlay 2014).

Ageing of populations will drive up the prevalence of heart failure internationally, making it increasingly difficult to maintain the quality of care. Switching resources from crisis management (by hospitalising people) to health maintenance (through structured telephone support or home telemonitoring) may be an affordable method to maintain and improve the quality of care for heart failure.

Pharmacological treatments, including angiotensin-converting enzyme (ACE) inhibitors, angiotensin receptor blockers, angiotensin-receptor neprilysin inhibitors beta-blockers, aldosterone antagonists and ivabradine, and devices such as implantable defibrillators and cardiac resynchronisation therapy, can improve symptoms and prolong survival in those with heart failure with reduced left ventricular ejection fraction (LVEF) (McMurray 2012). In people who are stable, mildly symptomatic (NYHA I - II) and optimally treated, annual mortality is $1.6 \%$ (Linde 2008), but it rises up to $12 \%$ for those with moderate to severe symptoms (Cleland 2006a; Kjekshus 2007). Several projection models predict an increase in the prevalence of $\mathrm{HF}$ and the number of $\mathrm{HF}$ hospitalisations by more than $20 \%$ in the next 20 years (Heidenreich 2013; Stewart 2013).

Fewer RCTs of medicines or devices have been conducted in people with heart failure with preserved LVEF (HFpEF) and none have been conclusively positive (Cleland 2014b). RCTs in participants with stable HFpEF suggest an annual mortality of $5 \%$ to $13 \%$ (Cleland 2006b; MAGGIC 2012; Zile 2002) and a similar rate of hospitalisations for worsening heart failure (Massie 2008).

For acute heart failure, no new treatments have been identified in the last 40 years and much of clinical practice is opinion- rather than evidence-based (Mebazaa 2015). In RCTs, six-month mortality ranges from $11 \%$ to $24 \%$, depending on age (Metra 2015).

Most trials of telemonitoring have focused on people with a recent history of heart failure hospitalisation and have shown a high mortality in those assigned to usual care, which is consistent with the above.

The worse outcome of participants in epidemiological studies compared to RCTs (Jhund 2009; Levy 2002) may reflect the strict inclusion/exclusion criteria of randomised controlled trials, with selection of better-educated participants with fewer comorbidities and a lower likelihood that older and sicker people will agree to be researched (Cleland 2007; Jhund 2009; Lenzen 2005). Also, participants in trials are likely to be much more closely monitored, and this may have a favourable influence on prognosis, consistent with the evidence from trials of telemonitoring. However, perhaps the most important reason why people in surveys have an outcome similar to those in trials of acute heart failure is because that is the point at which most people in surveys of heart failure are enrolled (Cleland 2001). Surveys suggest that about $20 \%$ of participants will die in the first year of new-onset heart failure (Harjola 2010; Jhund 2009; Levy 2002). The mortality one year after an acute exacerbation of chronic heart failure is about 30\% (Harjola 2010), but much worse in older people. Using National Audit data for England and Wales, which captures an even broader population admitted with heart failure, inpatient mortality ranges from less than $5 \%$ in those aged under 65 years to almost $15 \%$ in those aged over 84 years. Rates of death and readmission in the following weeks are also high; 30-day mortality after discharge ranges from under $10 \%$ in people aged less than 65 years to more than $25 \%$ in those aged over 84 years, whilst 30-day rates for readmission regardless of age run at about $15 \%$, although only one-third are primarily due to heart failure (Cleland 2003; Cleland 2011). In contrast to clinical trials, in epidemiological studies LVEF does not appear to be a major determinant of prognosis, possibly because people with heart failure with preserved ejection fraction are often older and have more comorbidities (Bhatia 2006; Cleland 2007; Cleland 2011; Owan 2006).

\section{Description of the intervention}

The effectiveness of multidisciplinary approaches to managing people with heart failure has been demonstrated by meta-analyses (Holland 2005; McAlister 2004) and specialist heart failure disease management programmes are recommended in best practice guidelines (Mant 2011; McMurray 2012; National Heart Foundation of Australia 2011; Yancy 2013).To date, trials of specialist, multidisciplinary heart failure management programmes have tested multifaceted approaches (multidisciplinary input, home/ clinic visits, telephone support). As a consequence, it has been 
difficult to identify the incremental benefits of the components of each intervention (McAlister 2004; Yu 2006). Nevertheless, it is clear that within most populations access to these programmes is limited as a result of barriers related to funding or accessibility (Clark 2005; Jaarsma 2006).

To meet the needs of heart failure populations who have difficulty accessing multidisciplinary heart failure disease management programmes, alternative models of care have been proposed and tested. These alternative models typically involve information communication technology and may include self monitoring and education delivered via standard telephone or more advanced telemonitoring technology (e.g. electronic transfer of physiological data), electrocardiograph (ECG), blood pressure (BP), weight, pulse oximetry, respiratory rate and medicine administration (Clark 2007a; Inglis 2010).

In this review, we classified programmes a priori as being 'structured telephone support' if the monitoring or selfcare management or both were delivered using simple telephone technology (data may have been collected and stored by a computer), and 'telemonitoring' if there was digital/broadband/ satellite/wireless or blue-tooth transmission of physiological and other non-invasive data. This review focuses only on non-invasive remote monitoring of people with heart failure. We consider that there is significant heterogeneity in monitoring of people using non-invasive and invasive technologies, in particular monitoring using implanted therapeutic devices such as defibrillators; this will form a part of future reviews.

We consider that structured telephone support and telemonitoring are two similar but distinctly different interventions, and as such we have reported outcomes for each intervention separately rather than as either telemonitoring or structured telephone support. It should be noted that in the context of this review the term 'remote monitoring' refers to the use of these technologies (structured telephone support or telemonitoring) outside of a heart-failure specialist centre of care, and not necessarily remote in the geographical sense. Most studies have been conducted in urban or semi-rural populations in regions with high population densities. In all studies of structured telephone support, having access to a touch-tone telephone was an essential inclusion criterion.

\section{How the intervention might work}

Some studies have demonstrated a beneficial effect of remote monitoring on mortality and morbidity in people with heart failure. The mechanisms by which improvements in outcomes with these interventions is achieved are not clear, but may include enhanced self care by improving the person's knowledge and adherence through education and monitoring, higher prescription rates of guideline-based medication by clinicians, or earlier intervention when heart failure worsens. It might also be that recipients find the presence of remote monitoring comforting and that it reduces their anxiety and need for formal contact with healthcare providers. A common finding is that participants in the active arm of studies showing a benefit from telemonitoring are more likely to be on higher doses of disease-modifying agents, which probably mediates benefit (Abraham 2011; Antonicelli 2010; Cleland 2005 (Struct Tele) (TENS-HMS)). This makes sense; simply monitoring a person will not modify the course of the disease unless action results.
Structured telephone support and home telemonitoring are more likely to be effective when delivery of care by conventional means is deficient. Trials that select participants carefully and ensure that treatment is optimal prior to randomisation may be less likely to show a benefit from intervention. Thus, trials that randomly assign individual participants to different strategies may place structured telephone support and home telemonitoring at a disadvantage, since care is likely to improve in those assigned to conventional care.

\section{Why it is important to do this review}

In this era of rapidly advancing and wider community access and adaptation to information technology, new trials of remote monitoring interventions have been continually commissioned and published. Results from earlier systematic reviews and metaanalyses (Clark 2007a; Inglis 2010; Klersy 2009; Louis 2003; McAlister 2004) supported the beneficial effect of non-invasive home telemonitoring or structured telephone support or both within heart failure disease management programmes on mortality and hospitalisation. In the 2010 Cochrane review, participants assigned to telemonitoring had lower all-cause mortality and heart failure-related hospitalisations, while structured telephone support mainly reduced heart failure-related hospitalisations (Inglis 2010). However several large trials of remote monitoring interventions have since been published, and some have shown a lack of clinical benefit. Also, one criticism of the previous Cochrane review (Inglis 2010) was that it accepted the study by Kielblock 2007 as an RCT while others considered it to be a cohort study (Anker 2011). A more recent systematic review and meta-analysis included some (but not all) of the newer trials and excluded the Kielblock study, and again provided support for remote monitoring interventions in reducing all-cause mortality. However, only trials that focused on people with a recent discharge diagnosis of heart failure were included (Pandor 2013b).

Remote monitoring interventions have often been more effective in reducing all-cause mortality rather than heart failure-related hospitalisations, and some trials have even reported an increase in the number of hospitalisations (Cleland 2005 (Struct Tele) (TENSHMS); Cleland 2005 (Telemon) (TENS-HMS); Steventon 2012a; Steventon 2013a). This may reflect the effect of overcautious, risk-averse health professionals increasing participant anxiety. Alternatively, appropriate expert review and timely hospitalisation can be life-saving and improved survival means that more people are alive and at risk of hospitalisation. Given the conflicting evidence on this topic and the interests of the European Society of Cardiology and the American Heart Association heart failure guideline committees, an updated systematic review incorporating recent results is appropriate at this time.

\section{O B JECTIVES}

To review randomised controlled trials (RCTs) of structured telephone support or non-invasive home telemonitoring compared to standard practice for people with heart failure, in order to quantify the effects of these interventions over and above usual care.

This is an update of previous versions (Clark 2007a; Inglis 2010). 


\section{METHODS}

\section{Criteria for considering studies for this review}

\section{Types of studies}

We include randomised controlled trials (RCTs) comparing heart failure management delivered via structured telephone support or non-invasive home telemonitoring with usual post-discharge care for people with heart failure living within the community. We include only RCTs that have been published in full in the peerreviewed literature. We excluded any studies that did not report data for any of our outcomes of interest in an extractable format (we contacted authors of each primary study in an attempt to obtain data).

\section{Types of participants}

Adults (aged 18 years and over) of either sex, any age or ethnic group, with a definitive diagnosis of heart failure. Participants may have been recently discharged from an acute-care setting (including emergency departments and acute assessment units) to home (including a relative's home but excluding nursing homes or convalescence homes) or they may have been recruited to a study while managed in the community setting. We excluded studies dealing with general cardiac disorders rather than specifically with heart failure.

\section{Types of interventions}

Structured telephone support or non-invasive telemonitoring interventions needed to be scheduled, as opposed to offering telephone follow-up on an 'as needed' basis. The intervention must have been initiated by a healthcare professional (medical, nursing, social work, pharmacist) and delivered to people with heart failure living in the community as the only aftercare intervention, without protocol-driven home visits or intensified clinic follow-up. The intervention had to be targeted at the person and intended to address their concerns and problems, not those of caregivers. The participant must not have been visited at home by a specialised heart failure healthcare professional or study personnel for the purpose of education or clinical assessment other than as an initiation visit to set up equipment. 'Usual care' consisted of standard post-discharge care without intensified attendance at cardiology clinics or clinic-based heart failure disease management programme, or home visiting as described above. We excluded studies if there was any previous exposure to telemonitoring or structured telephone support for the usual care or intervention arms prior to the start of the study.

\section{Types of outcome measures}

\section{Primary outcomes}

- All-cause mortality (total number of deaths at the end of study follow-up in each arm of the study)

- All-cause hospitalisations (calculated as the proportion of participants readmitted to hospital at least once during the period of follow-up)

- Heart failure-related hospitalisations (calculated as the proportion of participants readmitted to hospital at least once during the period of follow-up due to heart failure)

\section{Secondary outcomes}

- Length of stay (number of days for hospitalisations)
- Health-related quality of life as assessed by validated questionnaires

- Healthcare costs and cost effectiveness (reduction in healthcare costs, cost of care)

- Adherence to the intervention

- Participant acceptance of the intervention

- Heart failure knowledge and self care as assessed by validated questionnaires

\section{Search methods for identification of studies}

This review updates a previously published Cochrane review which examined the period 2006 to November 2008 (Inglis 2010); that in turn had updated a previously published review examining the period January 1966 to May 2006 (Clark 2007a).

For this update, we searched all databases from 2008 until January 2015 , without any language restrictions. If we found an abstract or thesis reporting an eligible RCT in the searches, then we included the study as long as a full-text peer-reviewed publication was available (online or in print) by 1 June 2015 .

\section{Electronic searches}

We searched the following databases on 12 January 2015:

1. Cochrane Central Register of Controlled Trials (CENTRAL) (Issue 12 of 12, 2014, Cochrane Library);

2. Database of Abstracts of Reviews of Effects (DARE) (Issue 4 of 4 , 2014, Cochrane Library);

3. Health Technology Assessment Database (HTA) (Issue 4 of 4 , 2014, Cochrane Library),

4. MEDLINE, MEDLINE In-Process \& Other Non-Indexed Citations, MEDLINE Daily and OLDMEDLINE (OVID, 1946 to 12 January 2015);

5. EMBASE (OVID, 1980 to 2015 week 2);

6. CINAHL Plus with Full text (EBSCO, 1937 to 12 January 2015);

7. Science Citation Index Expanded (SCI-EXPANDED, 1970 to 12 January 2015) - and Conference Proceedings Citation IndexScience (CPCl-S, 1990 to 12 January 2015) on Web of Science (Thomson Reuters);

8. AMED (to 31 December 2014).

The search strategies are listed in Appendix 1 (for this version of the review) and Appendix 2 (for the 2010 version of the review (Inglis 2010)).

\section{Searching other resources}

We also searched the following resources ("heart failure" and tele* / "cardiac failure" and tele $\left.{ }^{\star}\right)$ :

1. IEEE Xplore (2008 to 31 December 2014);

2. TROVE (2008 to 31 December 2014);

3. Proquest Digital Dissertations (2008 to 31 December 2014).

We handsearched bibliographies of identified studies and published systematic reviews relevant to this topic area. We also handsearched abstracts and conference proceedings from the following international conferences for relevant studies:

1. European Society of Cardiology Congress (2009, 2010, 2011, 2012, 2013, 2014); 
2. American College of Cardiology Congress (2009, 2010, 2011, 2012, 2013, 2014);

3. American Heart Association $(2009,2010,2011,2012,2013,2014)$;

4. World Congress of Cardiology (2010, 2012, 2014);

5. Heart Failure Society of America (2009, 2010, 2011, 2012, 2013, 2014);

6. European Society of Cardiology Heart Failure Congress (2009, 2010, 2011, 2012, 2013, 2014);

7. European Society of Cardiology Annual Spring Meeting of Cardiovascular Nursing (2009, 2010, 2011, 2012, 2013, 2014).

\section{Data collection and analysis}

Two review authors (from $\mathrm{SCl}, \mathrm{RAC}$ or $\mathrm{RD}$ ) checked all identified abstracts and results from database searches for relevance to the review topic. If the reference appeared to be relevant, we obtained a full copy of the reference for detailed review, to determine inclusion/exclusion of the study.

\section{Selection of studies}

Two review authors ( $\mathrm{SCl}$ and $\mathrm{RAC}$ ) independently reviewed the results of each search according to exclusion and inclusion criteria. We excluded studies if home visits were performed as part of the intervention or by the clinical staff involved in the intervention, or if there were clinic visits (more than usual care) offered to participants in the intervention or control groups. A third review author (JGFC) adjudicated in cases of disagreement between the first two review authors.

\section{Data extraction and management}

Two review authors (RD and $\mathrm{SA}$ ) abstracted the data from the included studies in a blinded manner, and a third review author (either $\mathrm{SCl}$ or RAC) checked all extracted data.

\section{Assessment of risk of bias in included studies}

Two review authors (RD and SA) independently assessed risks of bias for each study, and a third review author ( $\mathrm{SCl}$ or RAC) checked them, using the Cochrane tool for assessing risk of bias (Higgins 2011).

\section{Measures of treatment effect}

Data were dichotomous and the statistical method used for analysis was a fixed-effect analysis model (Deeks 2011). We calculated risk ratios (RRs) and 95\% confidence intervals (Cls) for all-cause mortality, and proportions of participants with at least one allcause and heart failure-related hospitalisation. We conducted all analyses on an intention-to-treat (ITT) basis, i.e. all participants and their outcomes analysed in the groups to which they were allocated, regardless of whether they received the treatment or whether or not they were measured for the outcome.

We tabulated and described all measured outcomes such as quality of life, using tools such as validated questionnaires, or length of stay.

\section{Dealing with missing data}

In the absence of appropriate details regarding the participants, intervention, usual care or outcomes assessed for a study potentially eligible for inclusion, we contacted authors via email to request further details. We contacted authors of studies which we identified as a published abstract, to request a copy of a full-text peer-reviewed publication for the study if one was available. We sent authors a follow-up email if we received no response.

\section{Assessment of heterogeneity}

We explored statistical heterogeneity in each outcome of interest using the $\mathrm{Chi}^{2}$ test and $\mathrm{I}^{2}$ statistic, as recommended in the Cochrane Handbook (Deeks 2011).

\section{Assessment of reporting biases}

We assessed reporting bias through funnel plots and visual assessment.

\section{Data synthesis}

Owing to differences in participant populations, programme characteristics, and length of follow-up, we performed all metaanalyses using a fixed-effect model.

\section{Summary of Findings}

We assessed the primary outcomes examined in the review using GRADEPro methodology (Schünemann 2011) to create 'Summary of findings' tables. We performed quality assessment and rated using the measures of very low, low, moderate or high. We assessed only primary outcomes, as these were the most consistently reported outcomes across the included studies.

\section{Subgroup analysis and investigation of heterogeneity}

We conducted several subgroup analyses to investigate heterogeneity. These were based on previously published post hoc subgroup analyses from the data included in the 2010 version of this review (Inglis 2014), and in Conway 2014.

Each of the subgroup analyses and their methodology are detailed below:

\section{Technology}

We categorised included studies according to the following technology types: a) telephone calls; b) videophone; c) interactive voice response (IVR) involving the manual input of data using a telephone keypad in response to questions from a computerised interactive voice response system and computerassisted telephone interviewing (CATI); d) complex/clinical telemonitoring, involving automatic transmission of physiological data, such as weight, heart rate and rhythm, oxygen saturations and blood pressure, from the measuring device to a central server via telephonic, satellite or broadband capabilities for interpretation by the healthcare team (Conway 2014).

\section{Telemonitoring intensity}

We categorised included studies of non-invasive telemonitoring according to the level of data monitoring and response, adapted from a categorisation proposed by Anker 2011. Categories included: a) office hours (typically Monday to Friday, $9-5$ pm); b) 24 hours per day/seven days per week.

\section{Publication year}

We categorised included studies according to the year that a fulltext peer-reviewed publication for that study was published: a) 
pre-2000; b) 2000 - 2007; c) since 2008. These nominal periods were based on time points of change to heart failure care.

- Pre-2000: era before contemporary pharmacological prescribing (beta-blockers, ACE inhibitors);

- 2000 - 2007: development of several important heart failure treatments (pharmacological and devices) influencing the standard of 'usual care';

- 2008 onwards: revised heart failure guidelines.

\section{Mean/median age of study participants}

We categorised included studies according to the mean or median age of the study participants. Categories included: a) $<70$ years of age; $b$ ) $\geq 70$ years of age (Inglis 2014).

\section{Focus of structured telephone support studies}

We categorised included studies of structured telephone support according to the focus of the intervention. Categories included: a) clinical monitoring of heart failure signs and symptoms with clinical support provided (clinical support); b) self-management education.

\section{Meta-regression}

In each subgroup analysis we performed a heterogeneity test to investigate whether there was enough evidence that the treatment effect differed between subgroups. We estimated the $Q$ statistics that follows a Chi ${ }^{2}$ distribution and produced a $\mathrm{P}$ value using the command 'metabin' from the package 'meta' in R.

To investigate whether the intervention effect depended on variables that were originally recorded as a continuous scale (year of publication and median age), we conducted linear metaregressions between the logarithm of the RR in the trials and each variable. We performed the meta-regression assuming a fixedeffect model and using the command 'rma.uni' in the package 'metareg' in R.

\section{Sensitivity analysis}

We performed a sensitivity analysis for each of the primary outcomes, to assess the impact of length of follow-up on outcomes. We excluded studies with a follow-up period of six months or less from these sensitivity analyses.

\section{RES U LT S}

\section{Description of studies}

\section{Results of the search}

We identified 17 new studies for inclusion in the review.

Searching the databases and search engines retrieved a total of 5051 new results (Figure 1): 


\section{Figure 1. Study flow diagram for update}

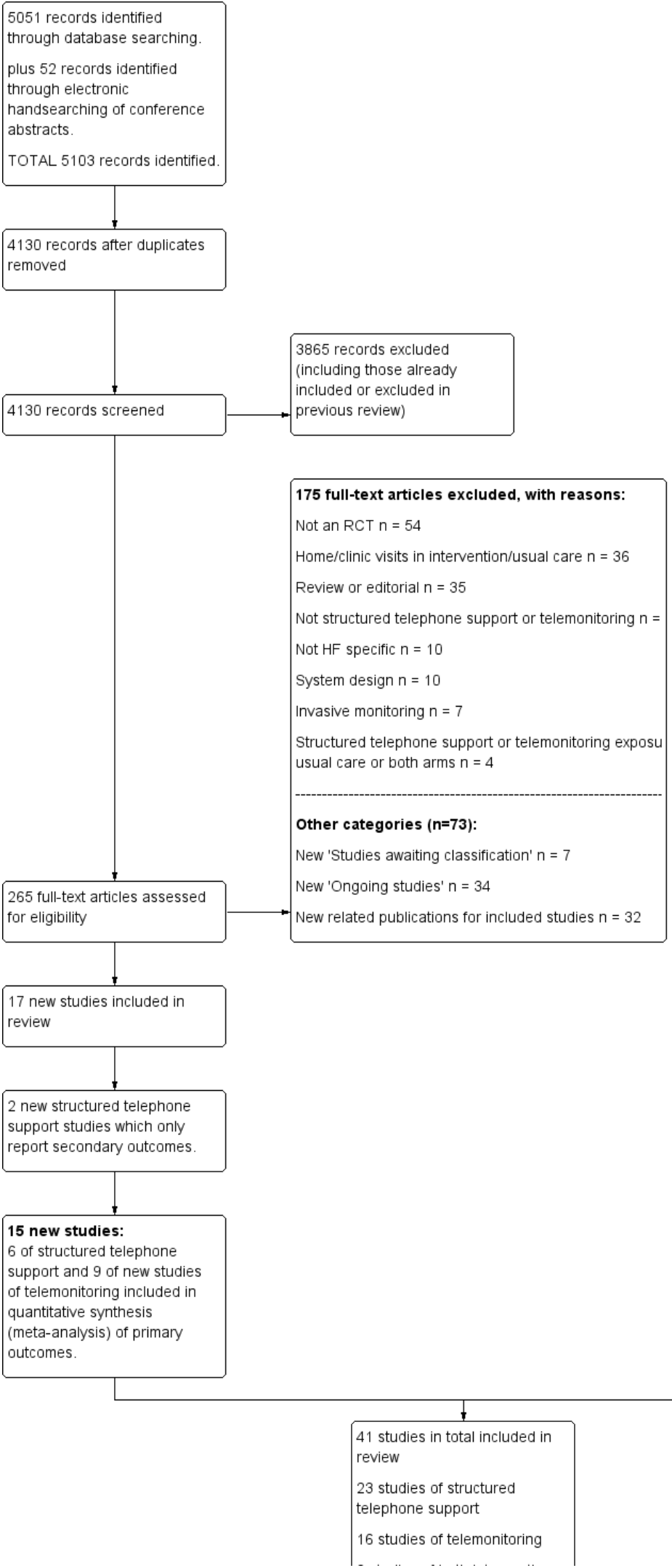

\begin{tabular}{|c|c|}
\hline $\begin{array}{l}30 \text { studies already included in } \\
\text { review }\end{array}$ & \multirow{2}{*}{$\begin{array}{l}\text { Kielblock } 2007 \text { excluded, as } \\
\text { considered a cohort study. } \\
\text { Zugck } 2010 \text { HiTel moved to } \\
\text { 'Ongoing studies', as abstracts } \\
\text { no longer included in review } \\
\text { and no full-text peer-reviewed } \\
\text { publication available }\end{array}$} \\
\hline & \\
\hline $\begin{array}{l}24 \text { studies already included in } \\
\text { the review carried into the } \\
\text { updated review. } \\
\text { [does not include } 4 \text { studies } \\
\text { included in the } 2010 \text { review as } \\
\text { abstracts only which are now } \\
\text { available as full peer-reviewed } \\
\text { publications and detailed in the } \\
\text { search results of this study } \\
\text { flow diagram] }\end{array}$ & $\begin{array}{l}\text { Four studies included in the } \\
2010 \text { review as abstract only, } \\
\text { where a full peer-reviewed } \\
\text { publication is now available: } \\
\text { Angermann } 2012 \text { (INH); Blum } \\
2014 \text { (MCCD); Krum 2013 } \\
\text { (CHAT); Villani } 2014 \\
\text { (ICAROS). Abstract references } \\
\text { for these studies are listed as } \\
\text { related publications for these } \\
\text { included studies. }\end{array}$ \\
\hline
\end{tabular}


Figure 1. (Continued)

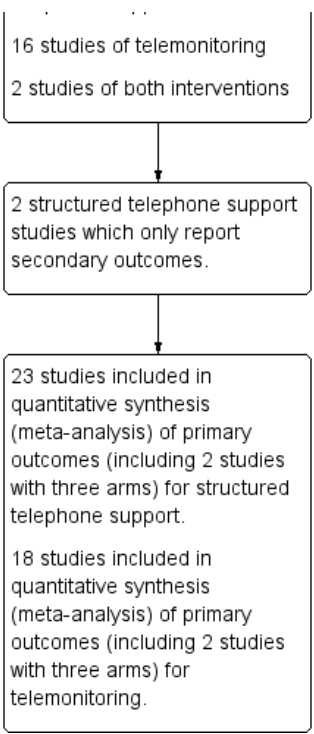

- CENTRAL $n=548$

- DARE $n=62$

- $\operatorname{HTA} n=19$

- MEDLINE, MEDLINE In-Process \& Other Non-Indexed Citations, MEDLINE Daily and OLDMEDLINE (OVID) $n=731$

- EMBASE (OVID) $n=936$

- Science Citation Index Expanded (SCI-EXPANDED) and Conference Proceedings Citation Index-Science (CPCI-S) $n=881$

- CINAHL Plus with Full text (EBSCO) $n=306$

- AMED $n=21$

- IEEE Xplore $n=1524$

- TROVE $n=0$

- Proquest Digital Dissertations $n=23$

We excluded 973 references as duplicates.

Handsearching of conference abstracts identified 52 references.

Overall, we identified 265 studies as potentially relevant, and we obtained full-text copies for assessment according to our inclusion and exclusion criteria.

We have identified 34 new studies which are currently ongoing, which includes those identified as conference abstracts but for which a full-text peer-reviewed publication was not available by the census date (Characteristics of ongoing studies).
We have identified seven new references as awaiting classification (Characteristics of studies awaiting classification), as we have contacted the study authors but have not received a reply to assist with determining the study eligibility, with several remaining in this category from the 2010 version of this review (Dunlap 2006 (HearTI); Kulshreshtha 2010; Levine 2006 (Mind My Heart); Yakushin 2006).

We have now excluded one study (Kielblock 2007) which was included in the previous version of this review, since, based on debate and discussion in the literature (Anker 2011) since the publication of the previous version of this review, we consider it not to be a randomised controlled trial.

\section{Included studies}

We include 41 full-text peer-reviewed studies of either structured telephone support or home telemonitoring. Two included studies trialled both structured telephone support and telemonitoring compared to usual care, therefore 43 comparisons are evident. These include 24 studies published as full-text peer-reviewed publications included in the 2010 Cochrane review (Inglis 2010); (Figure 2 and Characteristics of included studies). Twenty-five studies evaluated structured telephone support (eight new studies, plus one included study previously classified as telemonitoring; total of 9332 participants), 18 evaluated telemonitoring (nine new studies; total of 3860 participants) and two studies evaluated both interventions (included in listed counts). 
Figure 2. Study flowchart.

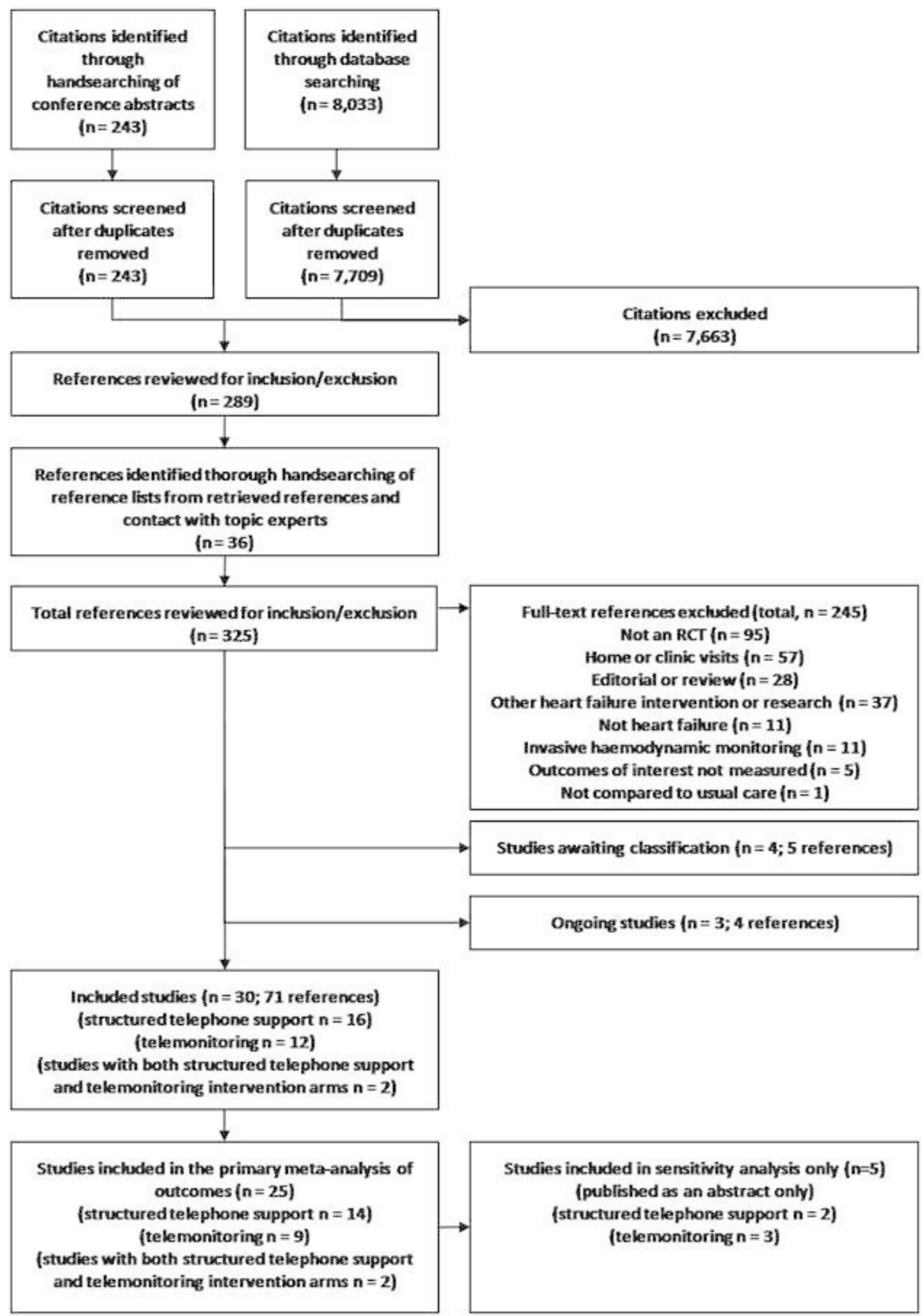


We have excluded one telemonitoring study (Kielblock 2007) included in the previous version of this review (Inglis 2010). Another study (Capomolla 2004) which used IVR technology and was previously included as a telemonitoring study in the previous version of this review we now classify as structured telephone support.

We have added 17 new studies to the review for this update. These include eight new studies of structured telephone support (Angermann 2012 (INH); Baker 2011; Bento 2009; Brandon 2009; Chaudhry 2010 (Tele-HF); Domingues 2011; Krum 2013 (CHAT); Zamanzadeh 2013) and nine new studies of non-invasive home telemonitoring (Biannic 2012 (SEDIC); Blum 2014 (MCCD); Dendale 2012 (TEMA-HF1); Koehler 2011 (TIM-HF); Lyngå 2012 (WISH); Scherr 2009 (MOBITEL); Seto 2012; Villani 2014 (ICAROS); Vuorinen 2014).

Studies with multiple intervention arms included in this review are: Cleland 2005 (Struct Tele) (TENS-HMS); Cleland 2005 (Telemon) (TENS-HMS); Mortara 2009 (Struct Tele) (HHH); Mortara 2009 (Telemon) $(\mathrm{HHH})$. We have separated out the data from these studies into our two interventions of interest (structured telephone support and telemonitoring). Structured telephone support (Mortara 2009 (Struct Tele) (HHH)) data are from the "Strategy 2" study arm, and telemonitoring data (Mortara 2009 (Telemon) $(\mathrm{HHH}))$ are from the "Strategy 3" study arm. (Wakefield 2008) includes two intervention arms, one using standard telephone equipment and the other a videophone; for the purposes of our analyses, we combined these two intervention arms and classed them as structured telephone support.

Two new structured telephone support studies (Brandon 2009; Zamanzadeh 2013) did not examine the main outcomes of interest (all-cause mortality, all-cause hospitalisations and heart failurerelated hospitalisations) but did include data on some secondary outcomes.

Of the newly-included studies, four had been included in the 2010 review as conference abstracts and have now been included in this version of the review as they are now available as full-text peerreviewed publications. These are: Angermann 2012 (INH); Blum 2014 (MCCD); Krum 2013 (CHAT); Villani 2014 (ICAROS). Two studies identified in the 2010 version of this review as ongoing (Chaudhry 2010 (Tele-HF); Koehler 2011 (TIM-HF)) and one study awaiting assessment in 2010 (Scherr 2009 (MOBITEL)) are now included in this version of the review.

For the structured telephone support studies ( $\mathrm{n}=25$ studies, including two three-armed studies):

- Trials ranged in size from small (34 participants in Barth 2001) to large (1653 participants in Chaudhry 2010 (Tele-HF));

- Mean/median age of participants ranged from 45 years (Ramachandran 2007) to 75 years (Barth 2001);

- Mean percentage of male participants was $63 \%$, which ranged from $45 \%$ to $99 \%$. Only five out of the 25 included studies recruited more women than men (Barth 2001; Brandon 2009; DeWalt 2006; Riegel 2002; Riegel 2006);

- $56 \%(n=14)$ of the studies reviewed originated from the USA; other countries were Australia (1), Argentina (1), Brazil (2), Canada (1), Germany (1), India (1), Iran (1), Italy (1) and two studies which were involved several European countries (Germany, Netherlands, UK, Poland, Italy).
- Ethnic groups: Riegel 2006 examined the effect of structured telephone support on a Hispanic population;

- $66 \%$ of studies had a length of follow-up less than six months;

- Most studies included participants with symptomatic heart failure, NYHA Class II - III.

For non-invasive home telemonitoring studies ( $\mathrm{n}=18$, including two three-armed studies):

- Trials ranged in size from small (20 participants in De Lusignan 2001) to large (710 participants in Koehler 2011 (TIM-HF));

- Mean/median age of participants ranged from 55 years of age in Seto 2012 to 78 years of age in Antonicelli 2008;

- Mean percentage of male participants was $72 \%$, and ranged from $35 \%$ to $85 \%$. Only one of the 18 included studies recruited more women than men (Soran 2008);

- Studies were undertaken in a variety of countries: Italy (3), USA (3), Canada (2), Austria (1), Belgium (1), Finland (1), France (1), Germany (1), Sweden (1), The Netherlands (1), UK (1) and two studies involved several European countries (Germany, The Netherlands and the UK; UK, Poland and Italy)

- Minority populations: Soran 2008 included older minorities (elderly women and non-white men);

- $38 \%$ of studies had a length of follow-up less than six months;

- Most studies included participants with symptomatic heart failure, NYHA Class III.

Structured telephone support studies included in the primary meta-analysis for the primary outcomes of interest funded by industry/health insurers (reported in publications):

1. Cleland 2005 (Struct Tele) (TENS-HMS) - Phillips.

2. DeWalt 2006 - Pfizer Inc.

3. Domingues 2011 - FIPE and CNPq.

4. GESICA 2005 (DIAL) - Roche, Boehringer Ingelheim, Bago. Pharmacia, Novartis, Merck Sharp \& Dohme.

5. Krum 2013 (CHAT) - National Health and Medical Research Council (NHMRC) and National Heart Foundation of Australia and Medical Benefits Fund.

6. Laramee 2003 - Novartis Pharmaceuticals.

7. Riegel 2002 - Pfizer Inc.

8. Tsuyuki 2004 - Park Davis Canada (Pfizer Canada).

Non-invasive telemonitoring studies included in the primary metaanalysis of the primary outcomes of interest funded by industry (reported in publications):

1. Balk 2008 - Achmea, Philips; provision of the MOTIVA system.

2. Cleland 2005 (Telemon) (TENS-HMS) - Philips.

3. De Lusignan 2001 - Nexan Telemed Ltd, Cambridge.

4. Dendale 2012 (TEMA-HF1) - Belgium Government Health Insurance Institute, Leo Pharma.

5. Goldberg 2003 (WHARF) - Alere Medical, Incorporated.

6. Koehler 2011 (TIM-HF) - German Federal Ministry of Economics and Technology, Robert Bosch Healthcare $\mathrm{GmbH}$, InterComponentWare AG, Aipermon GmbH \& Co KG.

7. Scherr 2009 (MOBITEL) - Novartis Pharma Austria, Roche Pharma Australia Mobilkom Austria.

8. Woodend 2008 - Merck-Frost Canada. 


\section{Excluded studies}

We excluded the majority of studies for the following reasons (Characteristics of excluded studies):

- Not an RCT: $\mathrm{n}=54$

- Involved home or intensive clinic visits: $\mathrm{n}=36$

- Review or editorial: $\mathrm{n}=35$

- Not structured telephone support or telemonitoring: $n=19$

- Not heart failure-specific: $n=10$

- System design: $\mathrm{n}=10$

- Invasive telemonitoring: $\mathrm{n}=7$

- Structured telephone support or telemonitoring exposure in usual care or both arms: $n=4$

\section{Risk of bias in included studies}

Overall the heterogeneity ranged from "might not be important" to "may represent substantial heterogeneity" according to the rough guide provided by Deeks 2011:

- $0 \%$ to $40 \%$ : might not be important;
- $30 \%$ to $60 \%$ : may represent moderate heterogeneity;

- $50 \%$ to $90 \%$ : may represent substantial heterogeneity;

- $75 \%$ to $100 \%$ : considerable heterogeneity.

- All-cause mortality:

* Structured telephone support vs usual care: Heterogeneity: $\mathrm{Chi}^{2}=13.56, \mathrm{df}=20(\mathrm{P}=0.85) ; \mathrm{I}^{2}=0 \%$

* Telemonitoring vs usual care: Heterogeneity: $\mathrm{Chi}^{2}=19.70, \mathrm{df}$ $=15(P=0.18) ; I^{2}=24 \%$

- All-cause hospitalisation:

* Structured telephone support vs usual care: Heterogeneity: $\mathrm{Chi}^{2}=28.51, \mathrm{df}=15(\mathrm{P}=0.02) ; \mathrm{I}^{2}=47 \%$

* Telemonitoring vs usual care: Heterogeneity: $\mathrm{Chi}^{2}=41.72, \mathrm{df}$ $=12(P<0.0001) ; I^{2}=71 \%$

- Heart failure-related hospitalisation:

* Structured telephone support vs usual care: Heterogeneity: $\mathrm{Chi}^{2}=19.20, \mathrm{df}=14(\mathrm{P}=0.16) ; \mathrm{I}^{2}=27 \%$

* Telemonitoring vs usual care: Heterogeneity: $\mathrm{Chi}^{2}=8.71, \mathrm{df}=$ $7(P=0.27) ; I^{2}=20 \%$

Analysis of the distribution in the funnel plots (Figure 3; Figure 4; Figure 5; Figure 6; Figure 7; Figure 8) demonstrates a strong publication bias in the studies selected for this review.

Figure 3. Funnel plot of comparison: 1 Impact of structured telephone support and telemonitoring in CHF on allcause mortality, outcome: 1.1 All-cause mortality: STS vs UC.

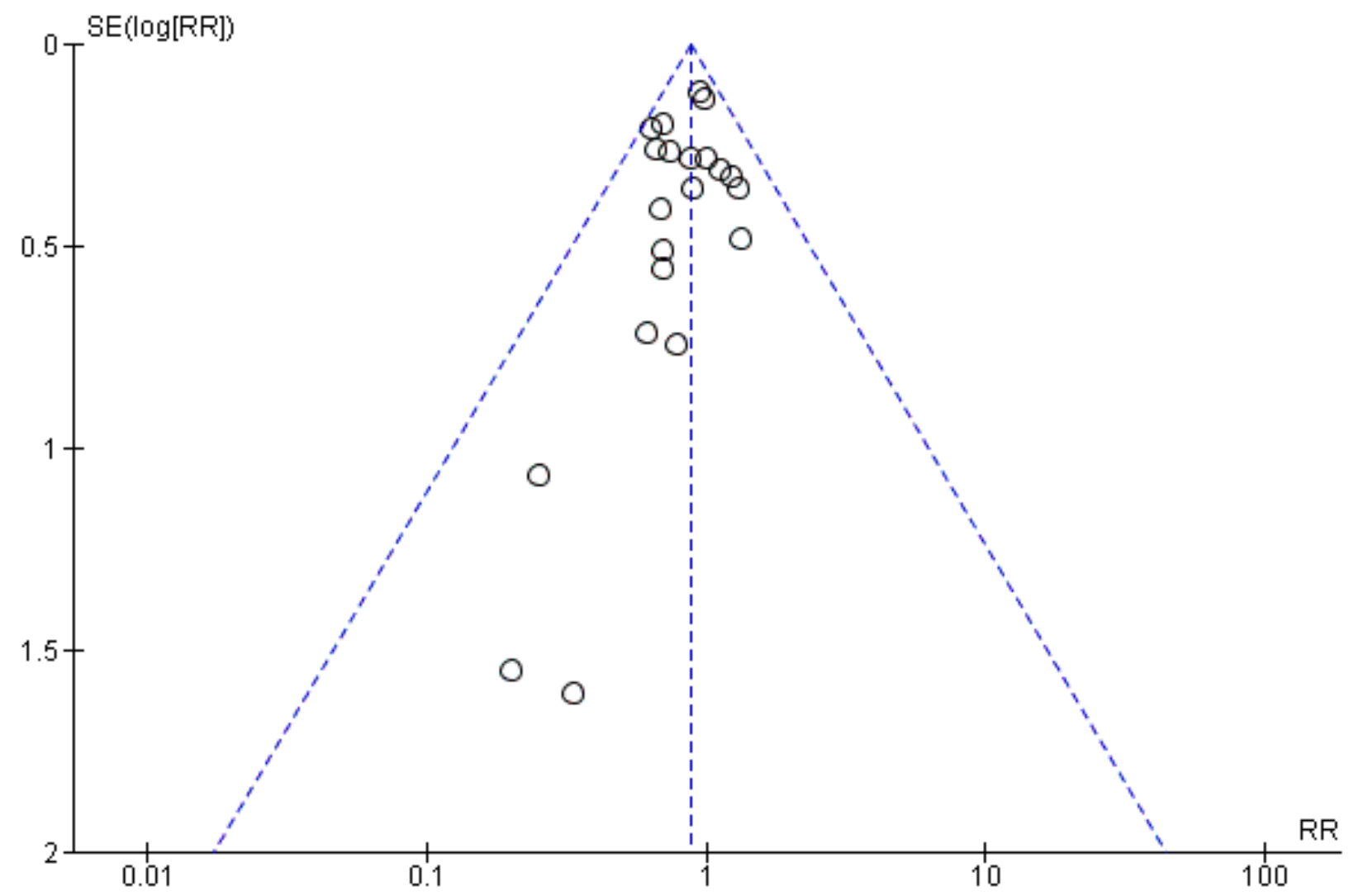

Structured telephone support or non-invasive telemonitoring for patients with heart failure (Review) 
Figure 4. Funnel plot of comparison: 1 Impact of structured telephone support and telemonitoring in CHF on allcause mortality, outcome: 1.2 All-cause mortality: TM vs UC.

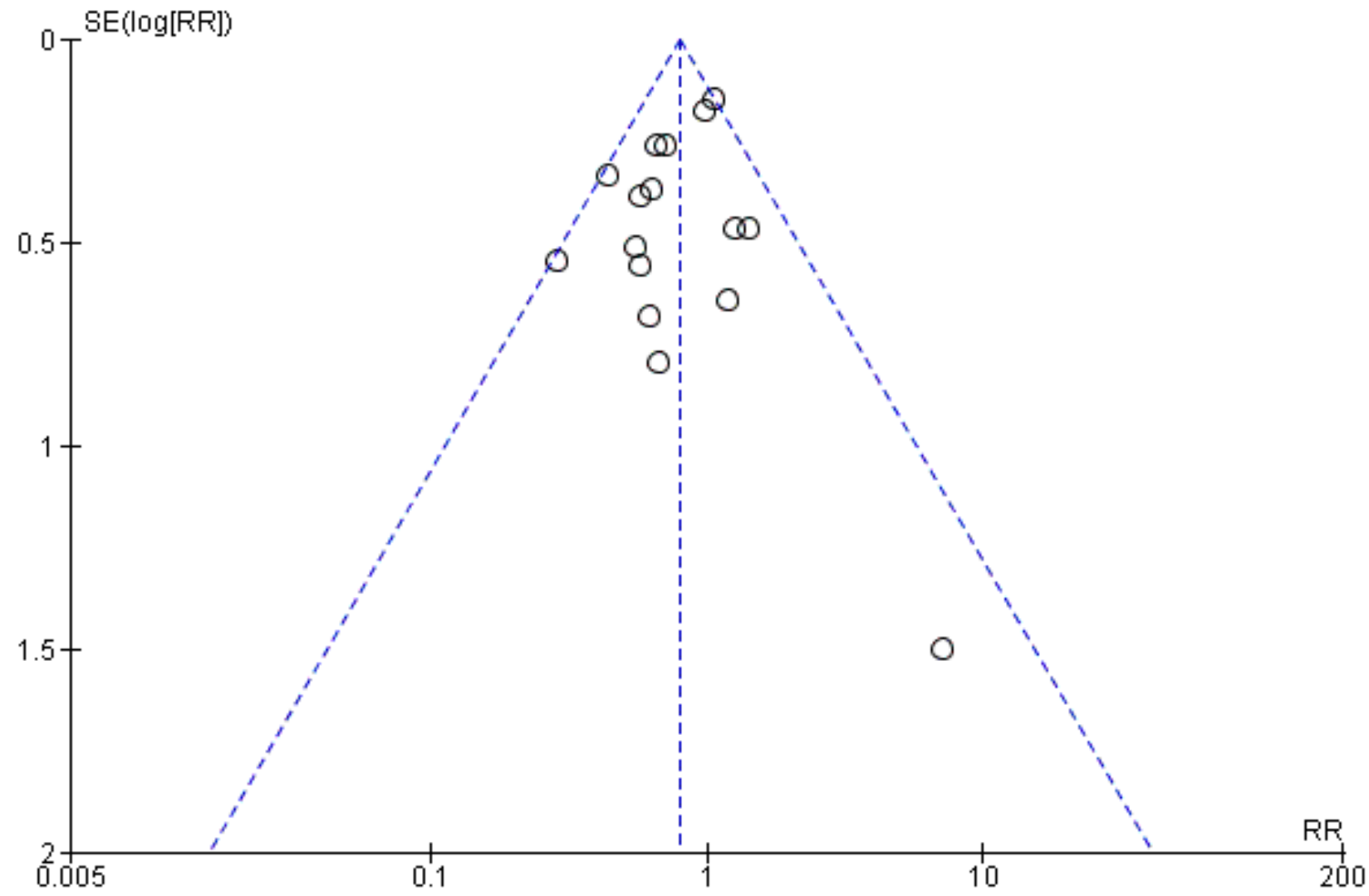


Figure 5. Funnel plot of comparison: 2 Impact of structured telephone or telemonitoring in CHF on risk of all-cause hospitalisation, outcome: 2.1 All-cause hospitalisation: STS vs UC.

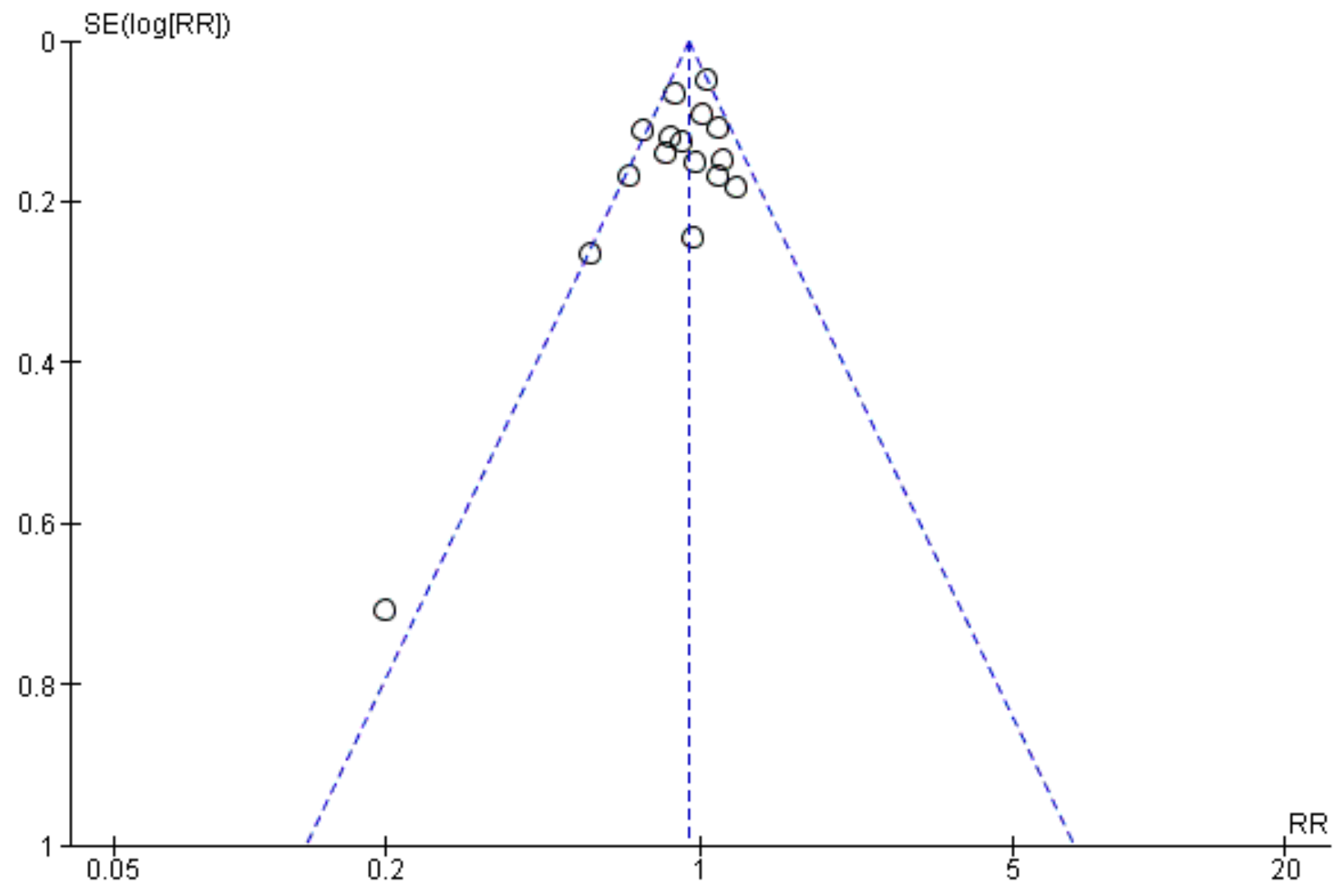


Figure 6. Funnel plot of comparison: 2 Impact of structured telephone or telemonitoring in CHF on risk of all-cause hospitalisation, outcome: 2.2 All-cause hospitalisation: TM vs UC.

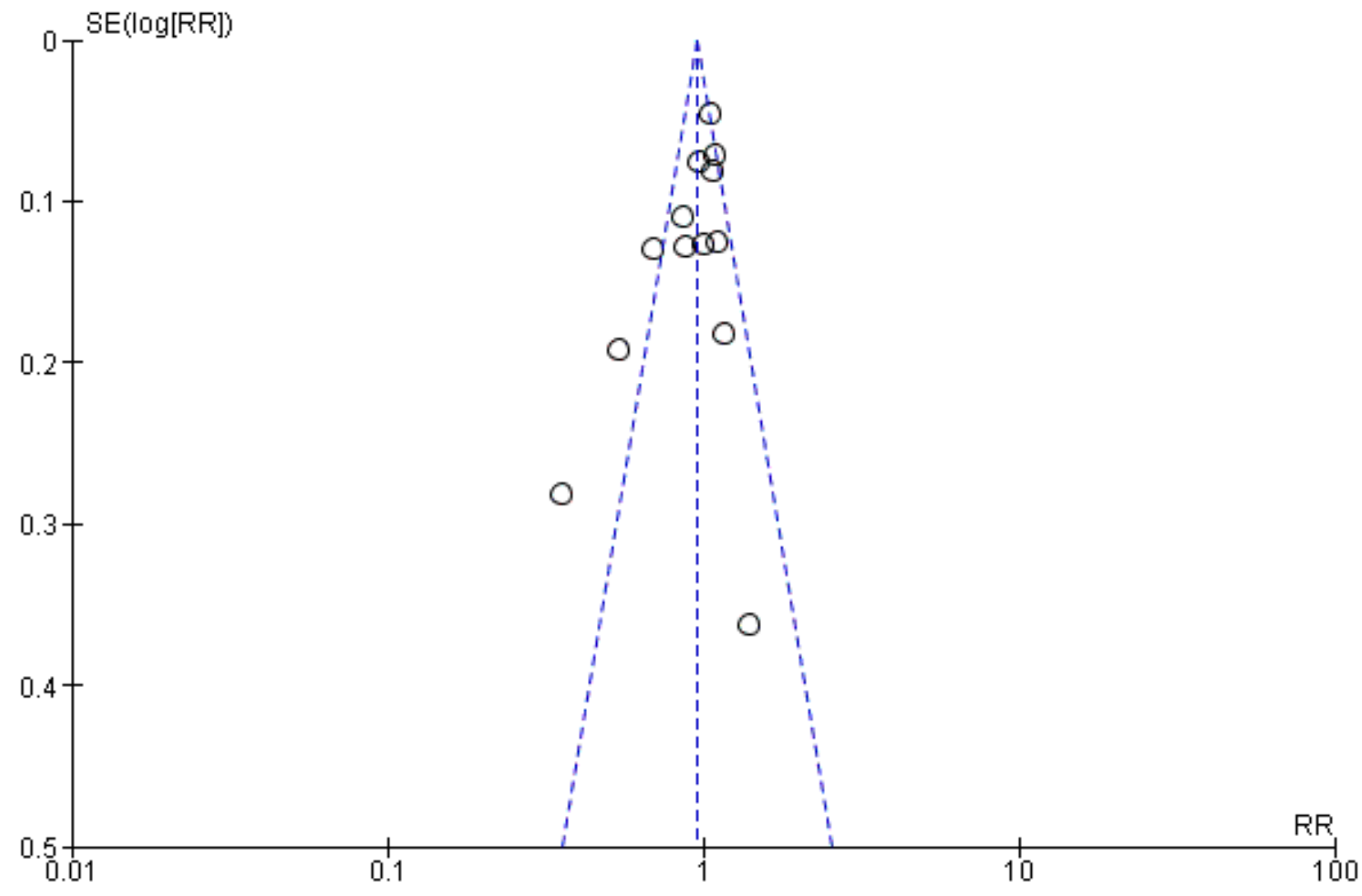


Figure 7. Funnel plot of comparison: 3 Impact of structured telephone support or telemonitoring in CHF on risk of CHF-related hospitalisation, outcome: 3.1 CHF-related hospitalisation: STS vs UC.

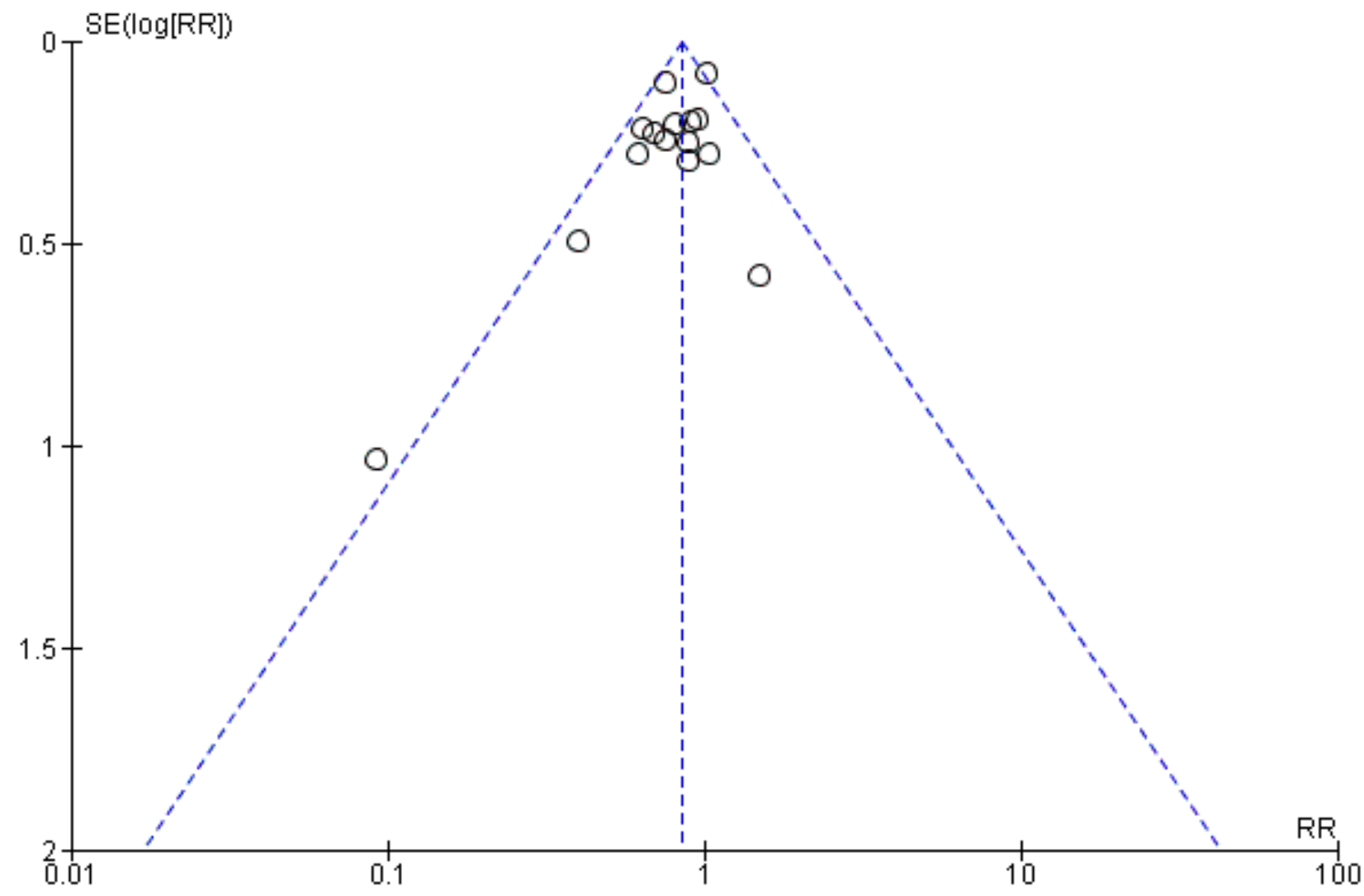


Figure 8. Funnel plot of comparison: 3 Impact of structured telephone support or telemonitoring in CHF on risk of CHF-related hospitalisation, outcome: 3.2 CHF-related hospitalisation: TM vs UC.

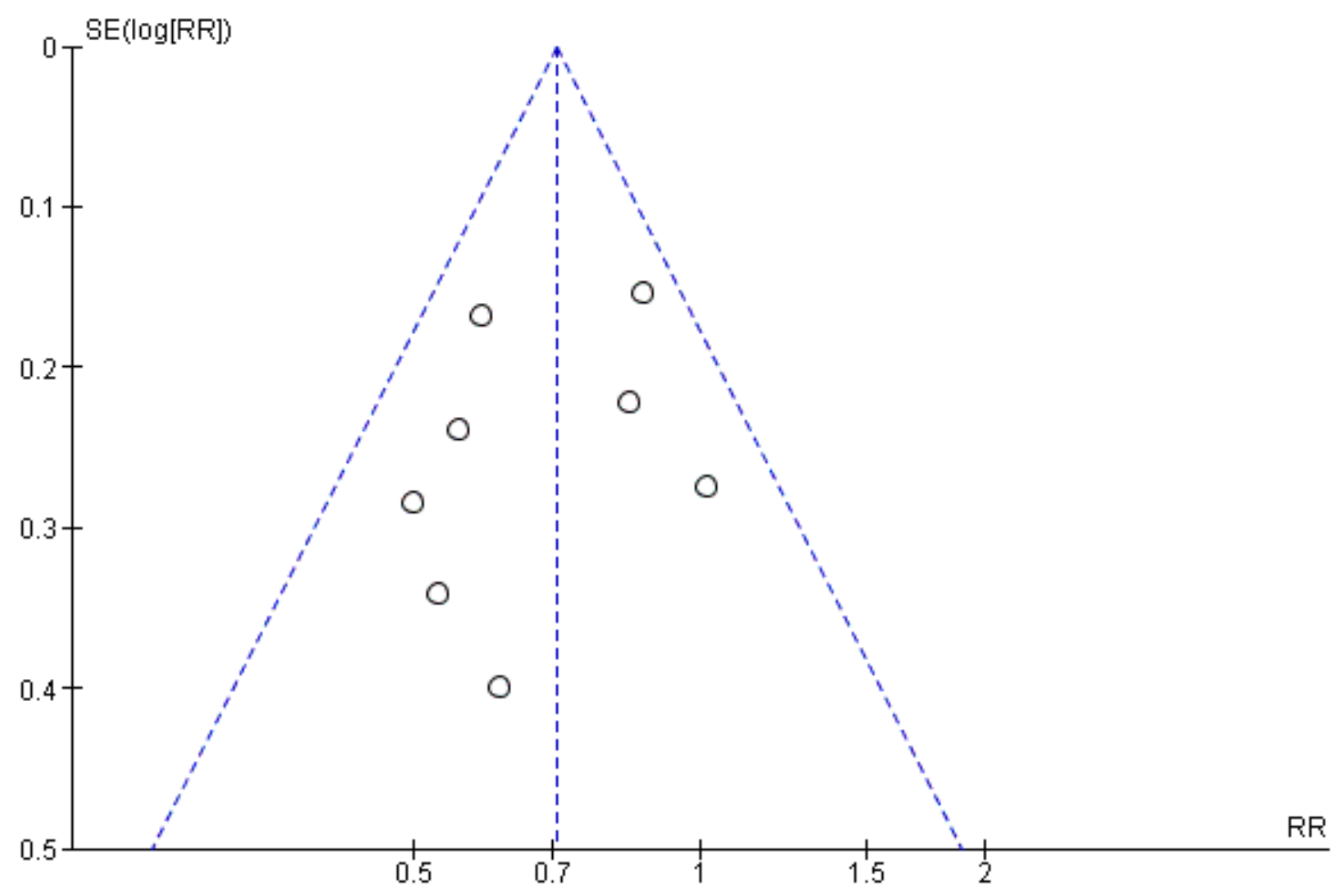

\section{Allocation}

included in this review. Many studies did not report the method of

We assessed random sequence generation as either low risk of bias random sequence generation Figure 9; Figure 10. ( $50 \%$ of all studies) or unclear for more than $95 \%$ of all studies

Figure 9. Risk of bias graph: review authors' judgements about each methodological quality item presented as percentages across all included studies.

Random sequence generation (selection bias)

Allocation concealment (selection bias)

Blinding of outcome assessment (detection bias)

Incomplete outcome data (attrition bias)

Selective reporting (reporting bias)
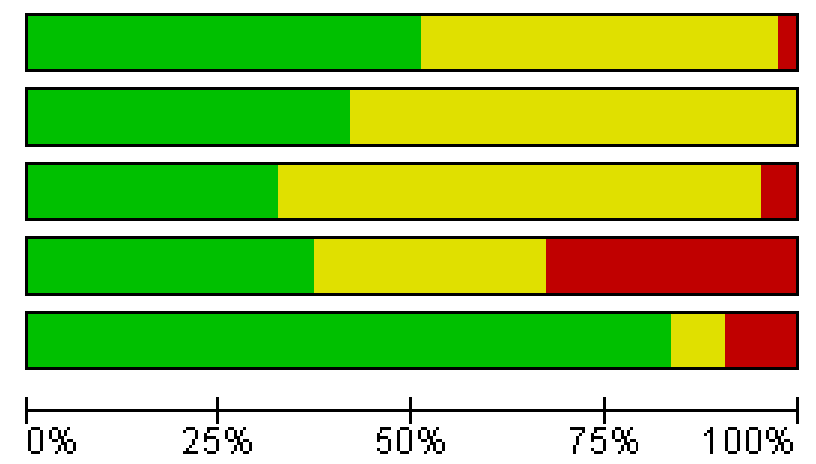

Low risk of bias

Unclear risk of bias

High risk of bias 
Figure 10. Risk of bias summary: review authors' judgements about each methodological quality item for each included study.

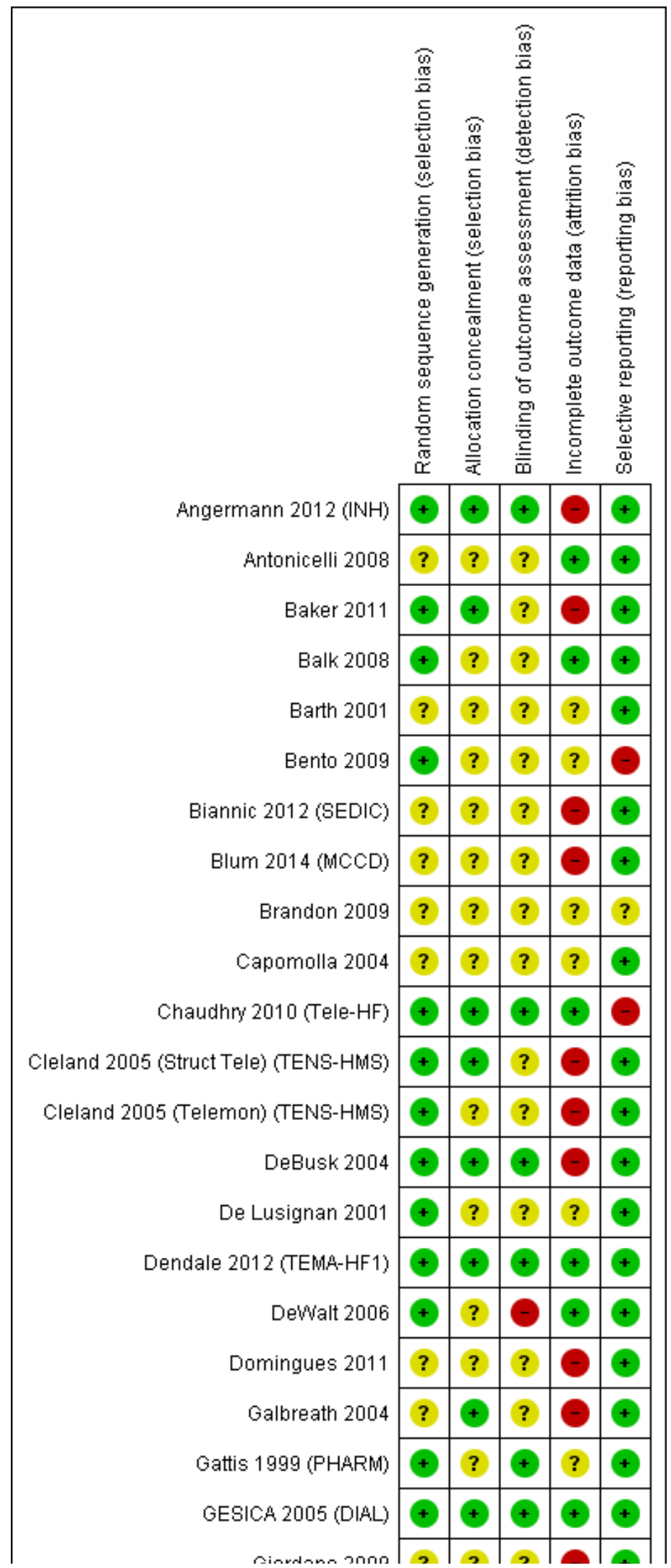


Figure 10. (Continued)

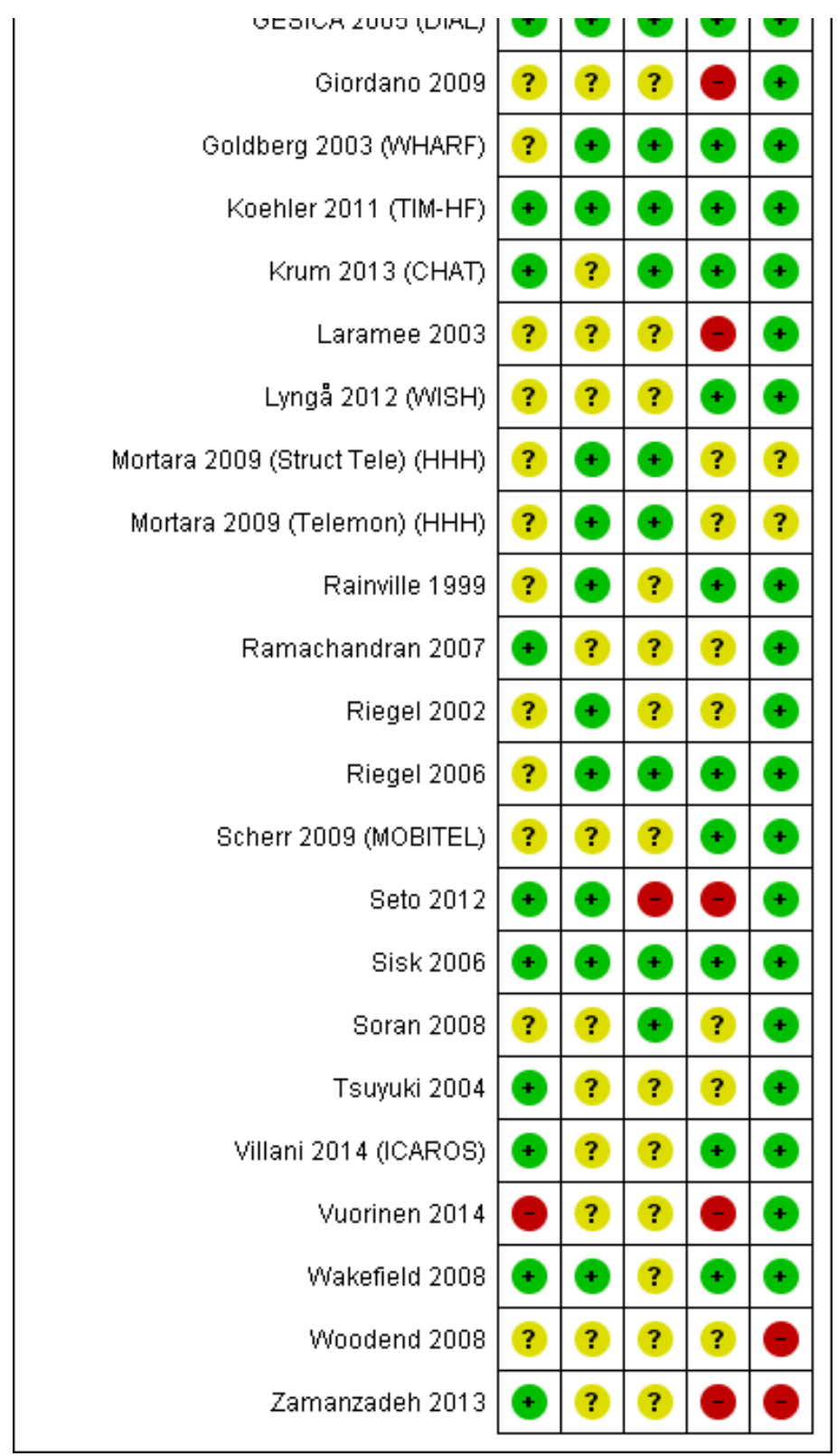

Allocation concealment was not clearly reported for more than $50 \%$ of all studies included in the review. We rated those that did report allocation concealment at low risk.

\section{Blinding}

We do not consider that blinding of participants and study personnel delivery was possible for these types of interventions. We assessed blinding of outcome assessment, and rated the majority of included studies at unclear risk of detection bias, due to the absence of details in the publications for outcome assessment (Figure 9; Figure 10).

\section{Incomplete outcome data}

Several studies reported losses to follow-up without detailing how or whether outcome data for these studies were included in an intention-to-treat analysis (Figure 9; Figure 10).

\section{Selective reporting}

We assessed more than $75 \%$ of all studies included in this review to be at low risk for selective reporting, with a small number assessed as being at high risk (Figure 9; Figure 10).

\section{Effects of interventions}

See: Summary of findings for the main comparison Summary of findings - structured telephone support or telemonitoring versus usual care - all-cause mortality; Summary of findings 
2 Summary of findings - structured telephone support or telemonitoring versus usual care - all-cause hospitalisation; Summary of findings 3 Summary of findings - structured telephone support or telemonitoring versus usual care - heart failure-related hospitalisation

\section{All-cause mortality}

All-cause mortality was available for 22 peer-reviewed studies comparing structured telephone support with usual care and for 17 studies comparing telemonitoring with usual care. Both structured telephone support ( $\mathrm{RR} 0.87,95 \% \mathrm{Cl} 0.77$ to 0.98 ; participants $=9222$; studies $=22 ; I^{2}=0 \%$; Analysis 1.1 ) and non-invasive telemonitoring (RR $0.80,95 \% \mathrm{Cl} 0.68$ to 0.94 ; participants $=3740$; studies $=17 ; \mathrm{I}^{2}=$ 24\%; Analysis 1.2) reduced all-cause mortality in people with heart failure.

GRADE assessment rated all-cause mortality evidence included in the review as of moderate quality, limited by a strong suspicion publication bias (Summary of findings for the main comparison).

Subgroup analyses designed to explore heterogeneity in the included studies found the following:

\section{Technology}

Heterogeneity tests between groups were not significant $(P=0.34)$ and therefore it is difficult to conclude that the apparent differences amongst technologies on all-cause mortality are true.

Effective technologies for reducing the risk of all-cause mortality in people with heart failure were telephone (RR $0.81,95 \% \mathrm{Cl} 0.71$ to 0.93 ; participants $=6629$; studies $=17 ; I^{2}=0 \%$; Analysis 1.3 ) and complex telemonitoring (RR $0.81,95 \% \mathrm{Cl} 0.68$ to 0.96 ; participants $=2885$; studies $=12 ; I^{2}=31 \%$; Analysis 1.4 ).

Technology categories that did not individually demonstrate statistically significant effects on all-cause mortality in people with heart failure included videophone (RR $1.14,95 \% \mathrm{Cl} 0.65$ to 1.99; participants $=269$; studies $=2 ; I^{2}=0 \%$; Analysis 1.5), IVR (RR 1.01, $95 \% \mathrm{Cl} 0.80$ to 1.28 ; participants $=2445$; studies $=4 ; I^{2}=0 \%$; Analysis 1.6), and mobile telephone or person digital assistant (PDA) (RR $0.71,95 \% \mathrm{Cl} 0.46$ to 1.11 ; participants $=734$; studies $=4 ; I^{2}=25 \%$; Analysis 1.7).

\section{Telemonitoring intensity}

A heterogeneity test did not show strong statistical evidence of differences between the two groups $(P=0.21)$ according to the intensity of telemonitoring.

When we categorised telemonitoring studies according to data monitoring intensity, only the first subgroup with monitoring in conventional office hours showed statistically significant evidence of an effect (RR 0.69, 95\% Cl 0.52 to 0.92; participants $=1548$; studies $=10 ; 1^{2}=0 \%$; Analysis 1.8), while the subgroup of seven days per week/24 hours per day showed no significant effect: (RR 0.86, 95\% $\mathrm{Cl} 0.71$ to 1.04 ; participants $=2192$; studies $=7 ; I^{2}=44 \%$; Analysis 1.9).

\section{Publication year}

A test came close to identifying significant heterogeneity between the two subgroups $(P=0.0760)$. The linear meta-regression coefficient for year of publication for telemonitoring studies was
$1.0477(P=0.0463)$, providing further support for the view that more recent studies have shown a smaller impact on mortality.

For structured telephone support studies, full-text peer-reviewed publications prior to 2000 reported a reduction in all-cause mortality in people with heart failure, but was not statistically significant. This could be due to the few studies and cases. (RR 0.45, $95 \% \mathrm{Cl} 0.14$ to 1.40; participants $=219$; studies $=2 ; I^{2}=0 \%$; Analysis 1.10). For studies published between 2000 and 2007 the effect was smaller, but the larger sample size made it (just) statistically significant (RR 0.86, 95\% Cl 0.74 to 0.99; participants $=5668$; studies $=13 ;\left.\right|^{2}=0 \%$; Analysis 1.11). Studies published after 2007 showed an even smaller effect and were clearly statistically non-significant (RR $0.91,95 \% \mathrm{Cl} 0.75$ to 1.10 ; participants $=3335$; studies $=7$; $1^{2}=0 \%$; Analysis 1.12). Heterogeneity tests between these three subgroups were non-significant $(P=0.47)$ and a meta-regression failed to identify a significant linear trend (slope $=1.008, P=0.67$ ).

For non-invasive telemonitoring studies, full-text peer-reviewed publications during the period 2000 to 2007 reported a reduction in all-cause mortality in people with heart failure (RR $0.58,95 \%$ $\mathrm{Cl} 0.39$ to 0.86 ; participants $=553$; studies $=3 ; \mathrm{I}^{2}=0 \%$; Analysis 1.13), but for those published after 2008 the effect did not have strong statistically significant evidence (RR $0.85,95 \% \mathrm{Cl} 0.72$ to 1.02 ; participants $=3187$; studies $=14 ; I^{2}=21 \%$; Analysis 1.14$)$. We did not identify any telemonitoring study published prior to 2000 for inclusion.

\section{Mean/median age of participants}

Again, a test failed to identify heterogeneity $(P=0.4534)$ and the linear regression coefficient for median age of participants was $1.0010(P=0.9405)$.

When we categorised structured telephone support studies according to the age of the participants, the benefit of structured telephone support for all-cause mortality was similar for studies where the mean/median age of participants was less than 70 years (RR $0.88,95 \% \mathrm{Cl} 0.77$ to 1.01 ; participants $=6158$; studies $=13 ; \mathrm{I}^{2}$ $=0 \%$; Analysis 1.15) and for those aged 70 years or older (RR 0.84 , $95 \% \mathrm{Cl} 0.67$ to 1.04 ; participants $=3064$; studies $=9 ; I^{2}=0 \%$; Analysis 1.16).

Tests for heterogeneity were not significant $(P=0.69)$.The linear meta-regression coefficient for age of participants for structured telephone support studies was $0.9886(P=0.3871)$ supporting the view that age is not a major determinant of the effect of the intervention.

For non-invasive telemonitoring studies, the effect on mortality was similar to the above, with studies where the mean/median age of participants was less than 70 years (RR $0.84,95 \% \mathrm{Cl} 0.68$ to 1.04 ; participants $=2493$; studies $=9 ; I^{2}=31 \%$; Analysis 1.17 ), and for those aged 70 or over (RR $0.74,95 \% \mathrm{Cl} 0.59$ to 0.94 ; participants $=$ 1247 ; studies $=8 ;\left.\right|^{2}=30 \%$; Analysis 1.18$)$.

\section{Focus of structured telephone support studies}

The heterogeneity test was non-significant $(P=0.8921)$.

Structured telephone support which focused on monitoring of signs and symptoms of heart failure and provided clinical support reduced all-cause mortality ( $\mathrm{RR} 0.87,95 \% \mathrm{Cl} 0.77$ to 0.98 ; participants $=8094 ;$ studies $=18 ; I^{2}=0 \%$; Analysis 1.19$)$, to a similar 
extent as those that focused on self-management education (RR $0.90,95 \% \mathrm{Cl} 0.55$ to 1.45 ; participants $=1128$; studies $=4 ; I^{2}=0 \%$; Analysis 1.20).

Sensitivity analyses, performed to explore the influence of length of follow-up greater than six months on all-cause mortality removed the statistically significant effect of structured telephone support (RR $0.88,95 \% \mathrm{Cl} 0.75$ to $1.02 ;$ participants $=4818$; studies $=11$; $\mathrm{I}^{2}=0 \%$; Analysis 1.21) and telemonitoring (RR $0.89,95 \% \mathrm{Cl} 0.74$ to 1.06; participants = 2580; studies $=10 ; I^{2}=0 \%$; Analysis 1.22), but reduced heterogeneity for telemonitoring studies. However, meta-regression tests revealed no heterogeneity according to study duration.

\section{All-cause hospitalisation}

All-cause hospitalisation data were available for 16 studies comparing structured telephone support with usual care and 13 studies comparing telemonitoring with usual care. Both structured telephone support (RR $0.95,95 \% \mathrm{Cl} 0.90$ to 1.00 ; participants $=7216$; studies $=16 ; I^{2}=47 \%$; Analysis 2.1 ), and non-invasive telemonitoring $(\mathrm{RR} 0.95,95 \% \mathrm{Cl} 0.89$ to 1.01 ; participants = 3332; studies $=13 ; 1^{2}=71 \%$; Analysis 2.2 ), showed an average reduction in all-cause hospitalisation of people with heart failure but statistically significant evidence was not strong in either study and heterogeneity between trial effects was important.

GRADE assessment rated the evidence for all-cause hospitalisation as very low, due to serious inconsistency and serious imprecision in this outcome, and a strong suspicion of publication bias (Summary of findings 2).

Figure 11.
Subgroup analyses designed to explore heterogeneity in the included studies found the following:

\section{Technology}

The heterogeneity test between subgroups was not significant $(P=0.25)$, suggesting that observed effect differences between subgroups may be due to the play of chance.

Effective technologies for reducing the risk of all-cause hospitalisation in people with heart failure was telephone (RR $0.93,95 \% \mathrm{Cl} 0.86$ to 0.99 ; participants $=4756$; studies $=12 ; \mathrm{I}^{2}$ $=51 \%$; Analysis 2.3) and mobile phone/PDA (RR $0.76,95 \% \mathrm{Cl}$ 0.60 to 0.97 ; participants $=560 ;$ studies $=2 ; 1^{2}=70 \%$; Analysis 2.4). Technology categories that did not individually demonstrate statistically significant reductions in all-cause hospitalisation in people with heart failure included videophone (RR 0.91, 95\% $\mathrm{Cl} 0.80$ to 1.04; participants $=269$; studies $=2 ; 1^{2}=92 \%$; Analysis 2.5), IVR (RR $0.99,95 \% \mathrm{Cl} 0.91$ to 1.08 ; participants $=2312$; studies $=3 ; I^{2}=76 \%$; Analysis 2.6) and complex telemonitoring (RR $0.97,95 \% \mathrm{Cl} 0.90$ to 1.04; participants $=2651$; studies $=10 ; I^{2}=69 \%$; Analysis 2.7). But again, heterogeneity between studies was important in all these analyses.

\section{Telemonitoring intensity}

The heterogeneity test identified differences between the two subgroups $(P=0.0125)$ (Figure 11$)$.

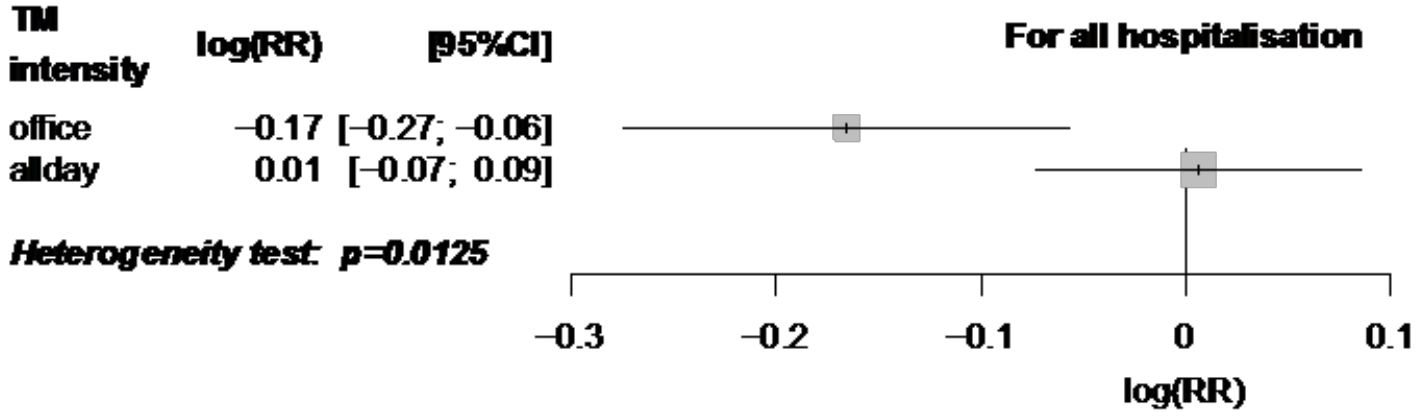

When we categorised telemonitoring studies according to data monitoring intensity (office hours or seven days per week/24 hours per day) only those which operated during standard office hours reduced the risk of all-cause hospitalisation in people with heart failure (RR $0.85,95 \% \mathrm{Cl} 0.76$ to 0.94 ; participants = 1140 ; studies $=6 ; I^{2}=76 \%$; Analysis 2.8$)$, whereas studies that operated telemonitoring seven days per week or 24 hours per day did not (RR $1.01,95 \% \mathrm{Cl} 0.93$ to 1.09 ; participants $=2192$; studies $=7 ; \mathrm{I}^{2}=50 \%$; Analysis 2.9).

\section{Publication year}

A test did not identify heterogeneity $(P=0.9269)$ and the linear meta-regression coefficient for year of publication was $1.0000(\mathrm{P}=$ 0.9992).

For structured telephone support studies, only one study (Gattis 1999 (PHARM)) was published as a full-text peer-reviewed report prior to 2000 , and demonstrated a statistically significant effect on reducing the risk of all-cause hospitalisation for people with heart failure (RR $0.57,95 \% \mathrm{Cl} 0.34$ to 0.96 ; participants = 181 ; studies $=1$; Analysis 2.10). Structured telephone support studies published during the period 2000 to 2007 (RR $0.93,95 \% \mathrm{Cl} 0.86$ 
to 1.01 ; participants $=3700$; studies $=8 ; \mathrm{I}^{2}=0 \%$; Analysis 2.11 ) and from 2008 onwards (RR $0.98,95 \% \mathrm{Cl} 0.91$ to 1.06; participants $=3335$; studies $=7 ; \mathrm{I}^{2}=68 \%$; Analysis 2.12 ) did not show strong evidence of a reduced risk. However, there was no strong evidence of heterogeneity between the three subgroups $(P=0.1031)$ and the linear meta-regression coefficient for year of publication was also non-significant (slope $=1.0078, \mathrm{P}=0.3453)$.

For non-invasive telemonitoring studies, we found no statistically significant reduction in all-cause hospitalisation for the period 2000 to 2007: (RR 0.94, $95 \% \mathrm{Cl} 0.79$ to 1.12; participants $=533$; studies $=$ $2 ; I^{2}=0 \%$; Analysis 2.13) or for the period from 2008 onward: (RR $0.95,95 \% \mathrm{Cl} 0.89$ to 1.02 ; participants $=2799$; studies $=11 ; \mathrm{I}^{2}=76 \%$; Analysis 2.14).

\section{Mean/median age of participants}

Heterogeneity tests $(P=0.1990)$ and linear meta-regression coefficient for age (slope $=0.9983 ; \mathrm{P}=0.7271$ ) did not provide statistically significant evidence of heterogeneity of effect.

The borderline effect of STS on all-cause hospitalisation was similar for trials with a median/mean participant age above 70 (RR 0.94 $95 \% \mathrm{Cl} 0.85$ to 1.04; participants = 1923; studies $=6 ; \mathrm{I}^{2}=36 \%$; Analysis 2.15), or below 70 years of age (RR $0.95,95 \% \mathrm{Cl} 0.89$ to 1.01 ; participants $=5293$; studies $=10 ; I^{2}=56 \%$; Analysis 2.16). Neither heterogeneity tests $(P=0.88)$ nor linear meta-regression coefficient for age (slope $=0.9933 ; P=0.2369$ ) identified significant heterogeneity amongst studies.

Non-invasive telemonitoring studies with a mean/median participant age above 70 years demonstrated a marginally significant reduction in the risk of all-cause hospitalisation for people with heart failure (RR $0.90,95 \% \mathrm{Cl} 0.82$ to 0.99 ; participants $=1147$; studies $=6 ; 1^{2}=80 \%$; Analysis 2.17 ) that we did not observe for studies with a mean/median age of participants below 70 years of age (RR $0.98,95 \% \mathrm{Cl} 0.90$ to 1.07; participants $=2185$; studies $=7$; $\mathrm{I}^{2}=57 \%$; Analysis 2.18).

\section{Focus of structured telephone support studies}

Heterogeneity tests did not find important heterogeneity $(\mathrm{P}=$ 0.3052).

Neither structured telephone support which focused on monitoring of signs and symptoms of heart failure and providing clinical support (RR 0.94, $95 \% \mathrm{Cl} 0.89$ to 1.00; participants $=6820$; studies $=14 ; I^{2}=52 \%$; Analysis 2.19), nor those that focused on selfmanagement education (RR $1.08,95 \% \mathrm{Cl} 0.84$ to 1.38 ; participants $=396$; studies $=2 ; 1^{2}=0 \%$; Analysis 2.20 ) showed statistically significant reductions in all-cause hospitalisation.

Sensitivity analyses, performed to explore the influence of length of follow-up greater than six months showed slightly better results and less heterogeneity for STS (RR $0.89,95 \% \mathrm{Cl} 0.82$ to 0.96 ; participants $=3451$; studies $=7 ; 1^{2}=37 \%$; Analysis 2.21 ), but had no influence on the results of home telemonitoring (RR $0.94,95 \% \mathrm{Cl}$ 0.87 to $1.01 ;$ participants $=2387 ;$ studies $=8 ;\left.\right|^{2}=78 \%$; Analysis 2.22 ).

\section{Heart failure-related hospitalisation}

Data on heart failure-related hospitalisations were available for 16 studies comparing structured telephone support with usual care and eight studies comparing non-invasive telemonitoring with usual care.

Both structured telephone support (RR $0.85,95 \% \mathrm{Cl} 0.77$ to 0.93 ; participants $=7030$; studies $=16 ; \mathrm{I}^{2}=27 \%$; Analysis 3.1 ) and noninvasive telemonitoring (RR $0.71,95 \% \mathrm{Cl} 0.60$ to 0.83 ; participants $=2148$; studies $=8 ; I^{2}=20 \%$; Analysis 3.2 ) showed a statistically significant reduction in heart failure-related hospitalisations.

GRADE assessment rated the evidence for heart failure-related hospitalisation evidence as moderate, due to a strong suspicion publication bias.(Summary of findings 3 ).

Subgroup analyses designed to explore heterogeneity in the included studies found the following:

\section{Technology}

A heterogeneity test $(P=0.0029)$ strongly suggested that there are differences in effect for this outcome amongst these technologies (Figure 12).

\section{Figure 12.}

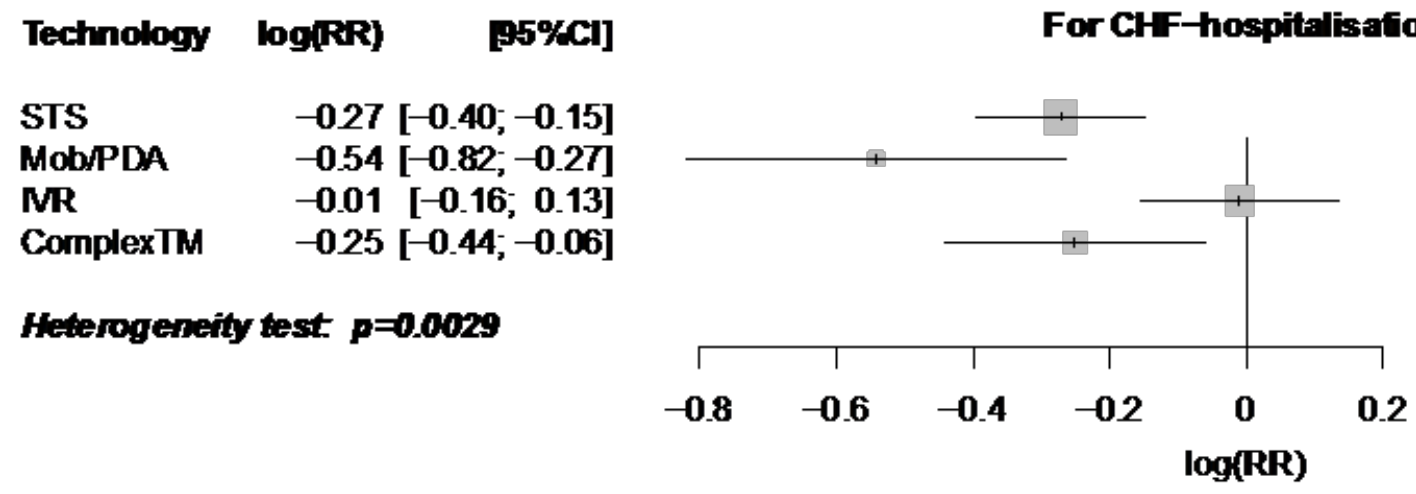


Effective technologies for reducing the risk of heart failure-related hospitalisation included telephone (RR $0.76,95 \% \mathrm{Cl} 0.67$ to 0.86 ; participants $=4718$; studies $=13 ; \mathrm{I}^{2}=2 \%$; Analysis 3.3 ), mobile phone/PDA (RR $0.58,95 \% \mathrm{Cl} 0.44$ to 0.77 ; participants $=674$; studies $=3 ; I^{2}=0 \%$; Analysis 3.4) and complex non-invasive telemonitoring (RR 0.78, 95\% Cl 0.64 to 0.94; participants $=1474$; studies $=5 ; I^{2}=$ 33\%; Analysis 3.5).

IVR was the only technology which did not demonstrate a benefit for reducing the risk of heart failure-related hospitalisation (RR $0.99,95 \% \mathrm{Cl} 0.86$ to 1.14 ; participants $=2312$; studies $=3 ; \mathrm{I}^{2}=0 \%$; Analysis 3.6).

\section{Telemonitoring intensity}

The heterogeneity test did not find evidence of differences in the effect $(P=0.9907)$.

When we categorised telemonitoring studies according to data monitoring intensity (office hours or seven days per week/24 hours per day), we found significant reductions in heart failurerelated hospitalisation (office hours: RR $0.71,95 \% \mathrm{Cl} 0.56$ to 0.89 ; participants $=858$; studies $=5 ; I^{2}=20 \%$; Analysis 3.7; seven days per week/24 hours per day: RR $0.71,95 \% \mathrm{Cl} 0.57$ to 0.87 ; participants = 1290; studies = 3; $\left.\right|^{2}=46 \%$; Analysis 3.8).

\section{Publication year}

The heterogeneity tests did not demonstrate heterogeneity ( $P$ $=0.3978$ ) and the linear meta-regression coefficient for year of publication was non-significant $(0.9694 ; \mathrm{P}=0.3721)$.

For structured telephone support studies, the two studies published prior to 2000 demonstrated a large reduction in the risk of heart failure-related hospitalisations (RR $0.24,95 \% \mathrm{Cl} 0.10$ to 0.58 ; participants $=219$; studies $=2 ; I^{2}=48 \%$; Analysis 3.9 ). The effect size was smaller with more recent publications: 2000 to $2007 \mathrm{RR} 0.78,95 \% \mathrm{Cl} 0.69$ to 0.89 ; participants $=3784$; studies $=10 ; I^{2}=0 \%$; Analysis 3.10; 2008 onwards RR $0.96,95 \% \mathrm{Cl} 0.84$ to 1.11 ; participants $=3027$; studies $=4 ; \mathrm{I}^{2}=0 \%$; Analysis 3.11 . The heterogeneity test strongly suggested differences between these three groups $(P=0.0019)$ and the linear meta-regression coefficient for year of publication was $1.0360(P=0.0177)$, indicating a reduction in benefit for more recent publications for this outcome (Figure 13; Figure 14).

\section{Figure 13.}

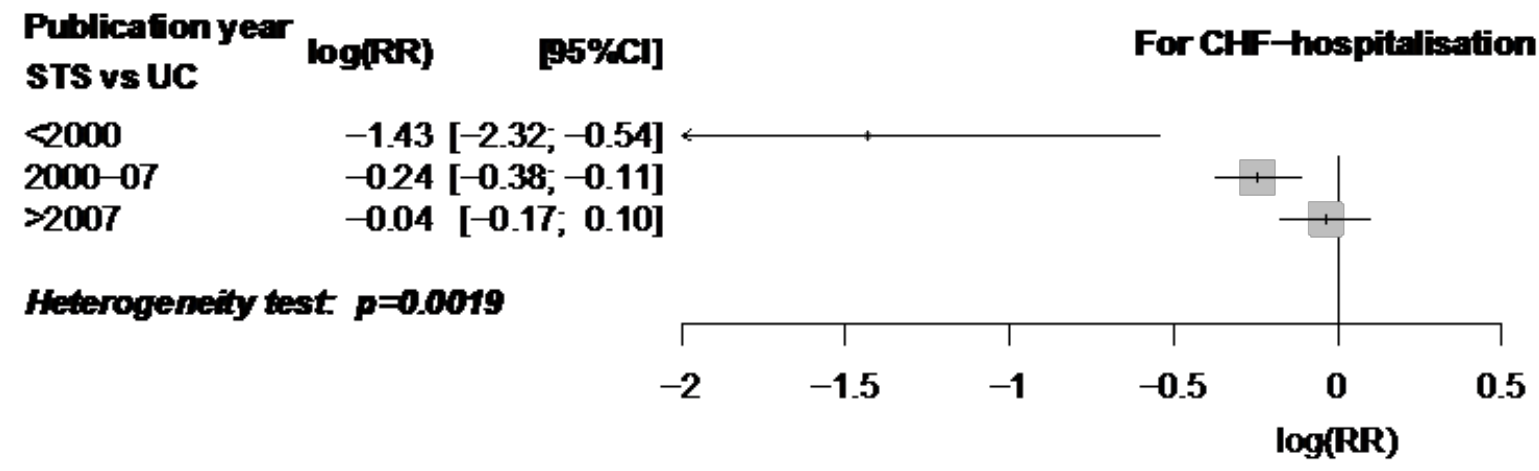


Figure 14.

\section{Meta-regression using a fixed effect model for CHF-related hospitalisations (STS vs UC) (Dot size is proportional to study precision)}

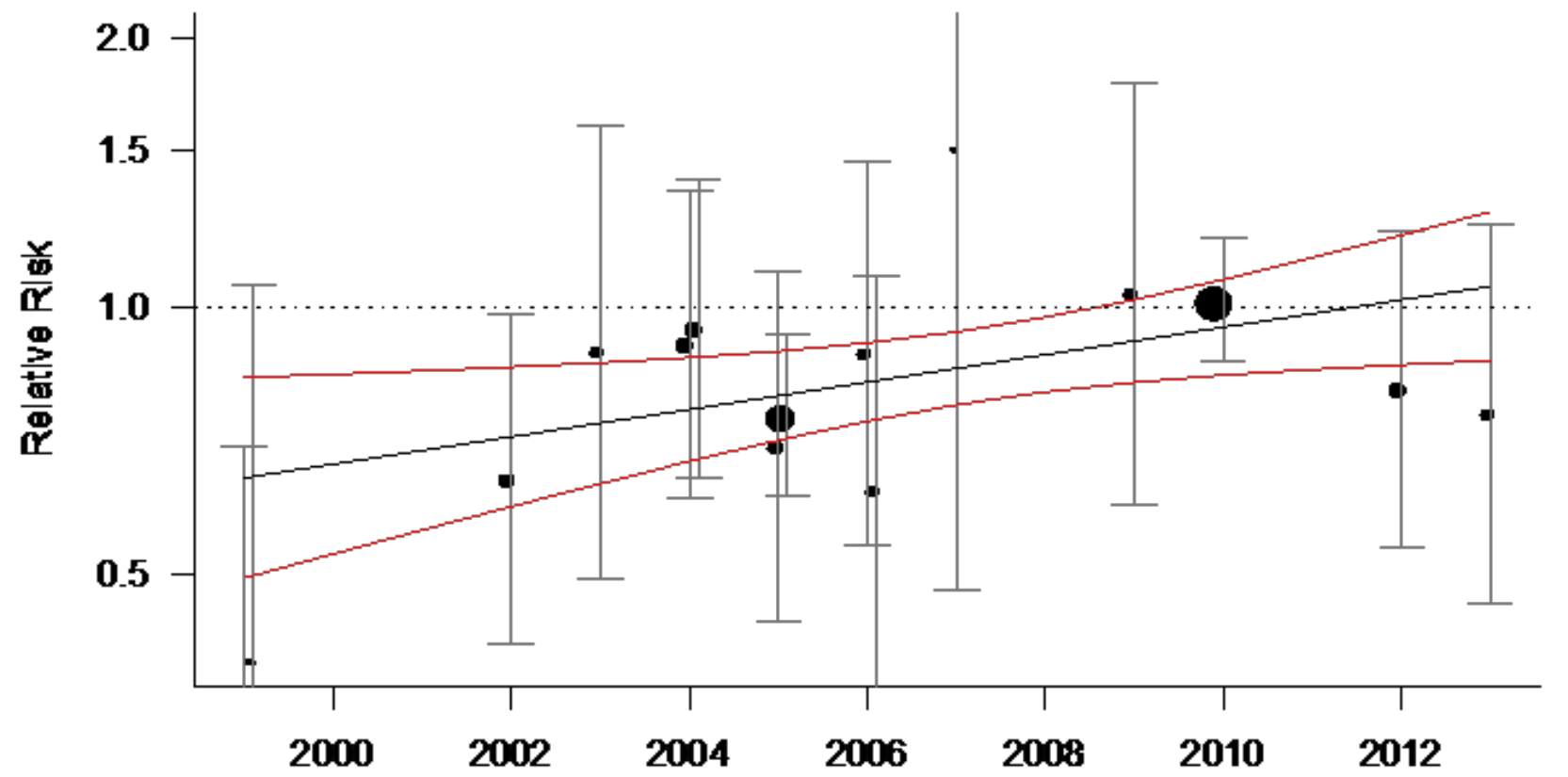

Year of publication

Only one non-invasive telemonitoring study reporting heart failurerelated hospitalisations was published during the period 2000 to 2007 (Cleland 2005 (Telemon) (TENS-HMS)), which did not demonstrate a significant benefit for reducing the risk of heart failure-related hospitalisations in people with heart failure (RR 0.84, $95 \% \mathrm{Cl} 0.55$ to 1.30; participants = 253; studies = 1; Analysis 3.12). Telemonitoring studies published from 2008 onwards did detect a significant reduction in heart failure-related hospitalisations (RR $0.69,95 \% \mathrm{Cl} 0.58$ to 0.82 ; participants $=1895$; studies $=7 ; I^{2}=24 \%$; Analysis 3.13).

\section{Mean/median age of participants}

A test did not identify heterogeneity between the three groups $(P=0.0952)$ and the linear meta-regression coefficient for age of participants was $0.9915(P=0.4784)$.

Structured telephone support reduced heart failure-related hospitalisations in studies where the mean/median age of participants was below 70 years (RR $0.86,95 \% \mathrm{Cl} 0.77$ to 0.96 ; participants $=5035$; studies $=8 ; I^{2}=51 \%$; Analysis 3.14) and for those where the mean/median age was 70 years and above (RR $0.81,95 \% \mathrm{Cl} 0.67$ to 0.96 ; participants $=1995 ;$ studies $=8 ; 1^{2}=$ $0 \%$; Analysis 3.15). A test did not find evidence of heterogeneity $(P=0.5349)$. The linear meta-regression coefficient for median age of participants was $0.9821(P=0.0562)$. This result does not provide strong evidence that studies with an older median age for participant have slightly larger effects.
There were similar findings for non-invasive telemonitoring studies where the mean/median age of participants was below 70 years (RR $0.75,95 \% \mathrm{Cl} 0.63$ to 0.89 ; participants $=1898$; studies $=6 ; I^{2}=14 \%$; Analysis 3.16), and for those where the mean/median age was 70 years and above (RR $0.53,95 \% \mathrm{Cl} 0.37$ to 0.76 ; participants $=250$; studies $=2 ;\left.\right|^{2}=0 \%$; Analysis 3.17).

\section{Focus of structured telephone support studies}

A test did not demonstrate heterogeneity ( $\mathrm{P} 0.5607)$.

Structured telephone support which focused on monitoring of signs and symptoms of heart failure and provided clinical support reduced heart failure-related hospitalisations (RR 0.84, 95\% Cl 0.76 to 0.93 ; participants $=6754$; studies $=15 ; \mathrm{I}^{2}=31 \%$; Analysis 3.18 ). There was only one included structured telephone support study (Tsuyuki 2004) which focused on self-management education and the effect on heart failure-related hospitalisations, and did not show a significant effect (RR $0.95,95 \% \mathrm{Cl} 0.64$ to 1.39 ; participants = 276; studies $=1$; Analysis 3.19).

The effect was somewhat greater for studies of structured telephone support with a follow-up greater than six months (RR $0.76,95 \% \mathrm{Cl} 0.66$ to 0.88 ; participants $=3341$; studies $=7 ; I^{2}=0 \%$; Analysis 3.20), but restricting the analysis to longer duration of follow-up in studies of non-invasive telemonitoring, did not seem to affect the results (RR $0.78,95 \% \mathrm{Cl} 0.65$ to 0.94 ; participants $=1684$; studies $=4 ; I^{2}=31 \%$; Analysis 3.21 ). 


\section{Length of stay}

Of the 25 studies reporting on structured telephone support versus usual care, seven reported length-of-stay data (Chaudhry 2010 (Tele-HF); Galbreath 2004; Laramee 2003; Riegel 2002; Riegel 2006; Tsuyuki 2004; Wakefield 2008). Only Tsuyuki 2004 reported a statistically significant reduction in length of stay in hospital. Nine telemonitoring studies reported length of stay (Balk 2008; Blum 2014 (MCCD); Dendale 2012 (TEMA-HF1); Koehler 2011 (TIM-HF); Lyngå 2012 (WISH); Scherr 2009 (MOBITEL); Soran 2008; Vuorinen 2014; Wakefield 2008). Only Scherr 2009 (MOBITEL) reported a significant reduction in the length of stay with the intervention. One telemonitoring study (Villani 2014 (ICAROS)) reported a large difference in the total number of hospitalisations for longer than three days, but this was not an analysis of length of stay per hospitalisation. Studies which assessed both telemonitoring and structured telephone support (Cleland 2005 (Struct Tele) (TENSHMS); Cleland 2005 (Telemon) (TENS-HMS); Mortara 2009 (Struct Tele) (HHH); Mortara 2009 (Telemon) (HHH)) reported no significant difference in length of stay for hospital admissions between groups (Table 1).

\section{Health-related quality of life}

Health-related quality of life (HRQoL) was a secondary outcome for 22 of the 41 included studies (Table 2). Several different psychometric tools were used for evaluation (Chronic Heart Failure Symptomatology Questionnaire (CHFSQ); Minnesota Living with Heart Failure Questionnaire (MLWHFQ); Kansas City Cardiomyopathy Questionnaire (KCCQ); Short Form 12 Item (SF-12); Short Form 36 Item (SF-36); Health Distress Score (HDS); Improving Chronic Illness Care Evaluation (ICICE); and Heart Failure Symptom Scale (HFSS)). Eleven structured telephone support studies measured HRQoL, of which nine (Angermann 2012 (INH); Baker 2011; Barth 2001; Brandon 2009; Galbreath 2004; Smith 2005; GESICA 2005 (DIAL); Ramachandran 2007; Sisk 2006; Hebert 2008; Wakefield 2008) demonstrated significant improvements in component scores or overall HRQoL measures, and two studies (DeWalt 2006; Riegel 2006) did not. Of the 11 telemonitoring studies that measured HRQoL, five (45\%) (Antonicelli 2008; Blum 2014 (MCCD); Koehler 2011 (TIM-HF); Seto 2012; Woodend 2008) reported statistically significant improvements in HRQoL outcomes. Studies which assessed both telemonitoring and structured telephone support (Cleland 2005 (Struct Tele) (TENS-HMS); Cleland 2005 (Telemon) (TENS-HMS); Mortara 2009 (Struct Tele) (HHH); Mortara 2009 (Telemon) (HHH)) have not reported HRQoL outcomes.

\section{Healthcare costs and cost effectiveness}

Fifteen studies presented detailed cost analysis (cost of the intervention or cost effectiveness) for these two types of technologies (structured telephone support (Barth 2001; Galbreath 2004 - Smith 2008; Laramee 2003; Ramachandran 2007; Riegel 2002; Riegel 2006; Sisk 2006 - Hebert 2008; Tsuyuki 2004; Wakefield 2008) and telemonitoring (Balk 2008; Blum 2014 (MCCD); Dendale 2012 (TEMA-HF1); Giordano 2009; Soran 2008 - Soran 2010; Villani 2014 (ICAROS)). Costs varied according to the intensity and technologies used in the intervention. Studies which reported reduction in the cost of care per admission or overall cost reduction due to fewer hospitalisations reported cost savings ranging between 14\% (Laramee 2003) and 86\% (Wakefield 2008). Three structured telephone support studies (Riegel 2002; Tsuyuki 2004; Wakefield 2008) and one telemonitoring study (Giordano 2009) reported a decrease in costs. Two telemonitoring studies (Balk 2008; Villani
2014 (ICAROS)) reported increases in cost, due both to the cost of the intervention and to increased medical management (Table 3).

\section{Adherence to the intervention}

Adherance (compliance) was between $55.1 \%$ and $65.8 \%$ for structured telephone support (Chaudhry 2010 (Tele-HF); Krum 2013 (CHAT) - Clark 2007b) and from $75 \%$ to $98.5 \%$ for telemonitoring (Capomolla 2004; Cleland 2005 (Struct Tele) (TENS-HMS); Cleland 2005 (Telemon) (TENS-HMS); De Lusignan 2001; Goldberg 2003 (WHARF); Mortara 2009 (Struct Tele) (HHH); Mortara 2009 (Telemon) (HHH), Soran 2008) (Table 4).

\section{Acceptability (satisfaction and usability)}

Acceptance (satisfaction and usability) of people receiving health care via technology was rated between $76 \%$ and $97 \%$ (Balk 2008; Cleland 2005 (Struct Tele) (TENS-HMS); Cleland 2005 (Telemon) (TENS-HMS); Krum 2013 (CHAT) - Clark 2007b; Vuorinen 2014; Woodend 2008).

The only two videophone studies in this review both had low satisfaction (De Lusignan 2001) and statistically non-significant satisfaction ratings (Wakefield 2008) (Table 5).

\section{Heart failure knowledge and self care}

Six structured telephone support studies (Baker 2011; Brandon 2009; DeWalt 2006; Domingues 2011; Wakefield 2008; Zamanzadeh 2013) and one telemonitoring study (Balk 2008) evaluated the effects of the intervention on participants' knowledge of their heart failure. All studies except Wakefield 2008 demonstrated significant improvements in heart failure knowledge, although Wakefield 2008 did report an improvement in medication knowledge. Four structured telephone support (Baker 2011; GESICA 2005 (DIAL); DeWalt 2006; Zamanzadeh 2013) and one telemonitoring study (Seto 2012) reported significant improvements in self-care (Table 6).

\section{DISCUSSION}

\section{Summary of main results}

This review summarises data from 41 trials covering 12,947 participants. Two of the included studies trialled both structured telephone support and telemonitoring compared to usual care, therefore 43 comparisons are evident. This review demonstrates that structured telephone support and non-invasive home telemonitoring programmes for people with heart failure living in the community reduce all-cause mortality by $13 \%(95 \% \mathrm{Cl} .0 .77$ to 0.98$)$ and $20 \%(95 \% \mathrm{Cl} 0.68$ to 0.94$)$ respectively and heart failure-related hospitalisation by $15 \%(95 \% \mathrm{Cl} 0.77$ to 0.93$)$ and $29 \%(95 \% \mathrm{Cl} 0.60$ to 0.83$)$ respectively relative to usual care, but do not provide evidence of an important effect on allcause hospitalisations. These results confirm those reported in previous Cochrane reviews (Clark 2007a; Inglis 2010), and place in context recent studies with a neutral outcome that have not materially changed the point estimates for mortality. However, the reduction in all-cause hospitalisation for people with heart failure reported in the previous version of this review (Inglis 2010), was not sustained in this updated analysis. Several studies reported significant improvements in health-related quality of life, as well as heart failure knowledge and self care. There is also evidence to support some reduction in healthcare costs, although this was less consistent across studies. 
The first RCTs of structured telephone support (compared to usual care, without home or clinic visits) were published in 1999 (Gattis 1999 (PHARM); Rainville 1999) and the first study of non-invasive telemonitoring was published in 2001 (De Lusignan 2001). In the past 15 or more years, information technology has progressed rapidly alongside the need and demand for chronic disease management for conditions such as heart failure. The first version of this review (Clark 2007a) included just nine studies of structured telephone support and four studies of non-invasive telemonitoring. The first Cochrane review in 2010 (Inglis 2010) included 14 studies of structured telephone support and nine studies of non-invasive telemonitoring. The current review now includes 25 studies of structured telephone support and 18 studies of non-invasive telemonitoring.

Although we found a reduction in the proportion of participants with a heart failure-related hospitalisation, we did not identify a consistent effect of structured telephone support or telemonitoring on length of stay for such admissions. Length of stay was inconsistently reported, thus preventing meta-analysis of this outcome. It is reasonable to suppose that while remote monitoring interventions would prevent episodes of hospitalisation through early detection and management of clinical deterioration, in more serious episodes of decompensation, hospitalisation would still be necessary and it cannot be expected that communitybased interventions such as structured telephone support or telemonitoring would affect the care administered in hospital.

Remote monitoring might be most useful in reducing the risk of death if it is implemented when patients are unstable or newly diagnosed, and over a relatively brief period when they need support and education. We therefore performed sensitivity analyses to investigate length of follow-up on outcomes. Restricting the analyses only to studies with more than six months follow-up failed to demonstrate a significant effect of either intervention on all-cause mortality. However, this might reflect the smaller number of trials and participants rather than a true lack of effect.

For all-cause mortality there was little heterogeneity amongst prespecified subgroups, although we noted a trend of borderline significance for a smaller effect of home telemonitoring in more recent studies. Although some technologies appeared inferior, such as the videophone and IVR, tests for heterogeneity were unable to confirm differences. Meta-regression tests did not identify heterogeneity $(P=0.34)$ and it is therefore inappropriate to conclude that apparent differences are well founded.

Neither age group, not the intensity of home telemonitoring, nor the nature of the structured telephone support proffered had an influence on the effect of interventions.

For reducing the risks of all-cause hospitalisations, we found no difference for non-invasive telemonitoring, while for structured telephone support the effect became barely statistically significant. When we limited the studies to those with more than six months follow-up the effect did not change for the telemonitoring intervention, but was improved (and reached statistical significance) for the structured telephone support intervention.

Both interventions (structured telephone support and non-invasive telemonitoring) showed a consistent and clear reduction in the risk of heart failure-related hospitalisations. When we used only studies with more than six months of follow-up, the first effect improved and the second remained basically unchanged.

We undertook several subgroup analyses to explore heterogeneity across the included studies. Using data from the previous version of this review (Inglis 2010), two of these subgroup analyses have been published (Conway 2014; Inglis 2014). We used heterogeneity tests to explore differences across the prespecified subgroups and meta-regressions to test whether the intervention effect could depend on the median age of participants or the year of publication of the trial. We found little conclusive evidence of heterogeneity, although in some cases this may have reflected the paucity of data. These analyses indicate that the effect of the intervention might change depending on the technologies involved, the intensity of the telemonitoring regimen and also on the year of publication. For all-cause mortality, only telephone and complex telemonitoring studies demonstrated a significant benefit, with mobile/PDA and IVR shown to be ineffective. For both structured telephone support studies and telemonitoring, studies published over the period 2000 to 2007 were effective in reducing the risk of all-cause mortality, but with this effect absent for more recent studies. Structured telephone support studies focusing on clinical support demonstrated a significant benefit in reducing the risk of all-cause mortality, whereas those which focused on self-management education did not. For heart failure-related hospitalisations, telephone studies, mobile phone/PDA studies and complex telemonitoring studies were effective in reducing this risk, while IVR interventions were ineffective. For structured telephone support studies, the reduction in the risk of heart failure-related hospitalisations reduced by year of publication. Assessment of this effect was limited for telemonitoring studies, as only one study reporting heart failure-related hospitalisations was published prior to 2008. The differences between the mean/median age of participants was most notable for the outcome of heart failure-related hospitalisations for telemonitoring studies, where studies with a mean/median age of participants of 70 years or older demonstrated a $43 \%$ reduction in the risk of this outcome (relative to a $25 \%$ reduction for those with a mean/median age below 70 years).

Of the three outcomes included in our primary meta-analysis, we found the greatest heterogeneity in all-cause hospitalisations (structured telephone support $\mathrm{I}^{2}=47 \%$ and telemonitoring studies $\left.1^{2}=71 \%\right)$. Two telemonitoring studies with small numbers of participants (Antonicelli 2008; Biannic 2012 (SEDIC)) reported a substantially lower RR for hospitalisation for all-causes (RRs of 0.36 and 0.54 respectively). These two studies account for a large part of the heterogeneity observed for this outcome, as between the other studies the heterogeneity is much lower $\left(I^{2}=40 \%\right)$. The methodological reasons for this difference in reported outcomes for these two studies are unclear, but may relate to the intervention and the clinical management of these participants. There are no obvious differences in the study participants or study methodology that we can identify to account for this difference.

This review demonstrated good evidence for cost effectiveness for structured telephone support. The expense of telemonitoring has not been shown to have a cost benefit. Strong evidence is also emerging for the effect of structured telephone support and telemonitoring for improving health-related quality of life, heart failure knowledge and self-care behaviours. 
Adherence was good in most studies reporting this outcome, and acceptance was strong among the participants in the studies reviewed. However, it appears that not all technologies are favoured by patients exposed to video and IVR. After a decade and a half of research in this area, what is indisputable from the satisfaction and quality-of-life evidence was that overall participants liked these interventions (structured telephone support and telemonitoring). This is demonstrated by levels of adherence, acceptance, knowledge, self-care behaviours and health-related quality of life. As described in the Background, the majority of people with heart failure do not have access to specialist services. Structured telephone support and telemonitoring can bridge this gap.

People who use non-invasive home telemonitoring are generally very positive about their experience. Structured telephone support and non-invasive home telemonitoring may provide greater benefits when targeting people requiring education about their disease and optimisation of therapy tailored to their needs. Home telemonitoring provides the person with the possibility of taking much more informed responsibility for their own care. Integrating structured telephone support and home telemonitoring into services may increase their benefits. It may be possible to deliver benefits similar to those of structured telephone support and home telemonitoring by intensification of conventional clinic- or community-based interventions. However, structured telephone support or home telemonitoring or both might prove more cost-effective than intensified conventional care. The possibility of additive or synergistic benefit from enhanced conventional services and structured telephone support and home telemonitoring should not be discounted. For geographically isolated people, remote management may be the only feasible means of delivering advice, and providing monitoring and support. The evidence supports the use of these interventions.

\section{Overall completeness and applicability of evidence}

Like any systematic review and meta-analysis, our findings are only as good as the studies which met our inclusion criteria. Future updates of this review will incorporate new data along with the findings of studies which are currently underway but not yet completed, or only available as a conference abstract or awaiting classification. We were unable to stratify results according to age, functional class or sex, as outcomes were not reported in a manner that allowed us to extract these subgroup-specific data. We were unable to consider 'patient-years' as the denominator for our metaanalysis in order to adjust for the differing lengths of follow-up of the included studies, as these data were very rarely reported.

Correctly identifying all of the outcomes available for each included study was challenging because of multiple publications arising from some of the included studies. Many studies published hospitalisation and mortality findings in one paper, cost findings in another, and quality of life, acceptability and adherence in another. Often these multiple publications were published with a different order or list of authors and the study was not always clearly identified from the title of the paper or the abstract. Future publications arising from RCTs of structured telephone support and telemonitoring should be published in a manner which permits easy identification of multiple publications.

Krumholz 2006 has outlined a taxonomy for disease management which encourages authors to describe their study intervention under eight domains (patient population; intervention recipient; intervention content; delivery personnel; method of communication; intensity and complexity; environment; and clinical outcomes). We found that studies inconsistently reported these details, which in turn did not allow us to explore differences in these domains as potential explanations for observed heterogeneity between studies. Many of the included studies were published prior to publication of the taxonomy. Similarly, Clark 2009 has called for a more specific evidence base to support the development of effective programmes for different populations, and for future reviews to pool data by sex or age; however, we were unable to do so in this review as very few of the included studies presented outcomes in a manner that permitted us to extract these data. Most studies recruited more men than women. We were also unable to pool outcomes based on age, other than by using mean/median age of the study participants. An individual patient data meta-analysis would be the best method to stratify outcomes according to important demographic and clinical variables, such as sex, age, cardiac function and comorbidities.

A major limitation to the studies conducted so far is that the research study is conducted in parallel with the existing service to patients who are willing to participate in research and by staff that are usually supernumerary to the service that delivers routine clinical care. This division between research study and clinical service is likely to make home monitoring less efficient and less effective. Moreover, the lack of a service means that health professionals will struggle to get experience and training in conducting home monitoring. Ideally, studies should integrate home monitoring into the routine service. However, until home monitoring, and in particular telemonitoring, is adopted as a service, this is exceedingly difficult.

The nature of the control group should be considered with care when interpreting clinical trials of home telemonitoring. Intensification of more conventional methods of delivering care, such as more home or clinic visits, can deliver results similar to those of home telemonitoring. This may account for the neutral outcome observed in some studies (Cleland 2009; Dar 2009 (HOME-HF); Chaudhry 2010 (Tele-HF); Koehler 2011 (TIM-HF)). However, employing health professionals is by far the largest part of healthcare costs. Technology that can make staff more efficient and effective may well be cost-neutral or cost-saving.

Participants from socio-economically disadvantaged groups may have been excluded if they did not have access to a touchtone telephone. In the case of telemonitoring the information communication technology equipment and monitoring devices were provided by the project, regardless of socioeconomic status.

\section{Quality of the evidence}

We assessed the primary outcomes examined in the review using GRADEPro methodology (Schünemann 2011) to create 'Summary of findings' tables. Quality assessment ranged from very low (allcause hospitalisation) to moderate (all-cause mortality and heart failure-related hospitalisation). We downgraded all-cause mortality and heart failure-related hospitalisation evidence by one point for strong suspicions of publication bias. We downgraded all-cause hospitalisation evidence by three points because of evidence of serious inconsistency and imprecision in this outcome, as well as a strong suspicion of publication bias. 
The strong suspicion of publication bias may in some part be due to the inclusion of only full-text peer-reviewed publications. However we stand by our decision to exclude studies not yet published as a full-text peer-reviewed publication and have discussed this methodological aspect below in the strengths and weaknesses of this review.

\section{Potential biases in the review process}

Our review has adhered to Cochrane methodology, and all review authors and personnel have at all times tried to avoid or minimise any biases in the review process. The introduction of several subgroup analyses in this version of the review was initiated in order to investigate potential heterogeneity across the included studies. We classified studies into these categories adhering to clear definitions and processes; however, minimal details of interventions and participants reported in some studies may lead to some minor inaccuracies in these classifications. Of most concern is that categorisation of studies according to year of publication does not take into account that for a small number of studies there may be a substantial time lag between the start of study recruitment and full-text peer-reviewed publication in a journal.

Publication bias has been noted as an issue in evidence presented in the review. It may be that the exclusion of studies not yet published as a full peer-reviewed publication has given rise to some of this publication bias. The review captures eight studies currently awaiting classification and 25 currently ongoing studies. Some of these currently ongoing studies have not yet reported any study findings and only a study protocol was available, for others, interim results have been presented in the form of conference abstracts, with very little detail provided to clarify the intervention or usual care. Our decision to exclude studies not published as a full peer-reviewed publication was based on research indicating inconsistency between trial findings presented as conference abstracts to those reported in a full, peer-reviewed publication (Toma 2006). Another factor which was important in the decision to exclude studies only available as a conference abstract was the lack of detail provided regarding the intervention and usual care, thereby not providing confidence as to the classification of the study in terms of inclusion/exclusion in the review. Authors of studies available only as a conference abstract or study protocol were contacted in order to identify a full peer-reviewed publication for the study. A response was not received from several studies which are classified as ongoing or awaiting classification despite multiple attempts to contact authors.

\section{Agreements and disagreements with other studies or reviews}

This review is novel in that we sought to delineate the benefits of one form of heart failure disease management on patient outcomes while controlling for other disease management interventions which may confound the benefits of structured telephone support and non-invasive home telemonitoring. It is important to consider the benefits that these specific interventions can deliver, as there are some circumstances where such interventions may be the only option for providing specialised heart failure management. Multidisciplinary heart failure management programmes, designed to improve patient outcomes through structured follow-up with patient education, optimisation of treatment and psychosocial support, are advocated worldwide as a fundamental component to the delivery of care. However, because of conflicting trial results, national and international guidelines have so far not recommended widespread implementation of remote monitoring. The latest European Society of Cardiology Heart Failure Guidelines (McMurray 2012) state that the effect of invasive (implantable devices) and non-invasive telemonitoring, and structured telephone support remain unclear, with insufficient evidence to support a guideline recommendation. Similarly the 2013 ACCF/AHA Guidelines for the Management of Heart Failure (Yancy 2013) acknowledged the problem of the mixed quality of evidence for specific components of heart failure clinical management interventions, such as home-based care, disease management, and remote telemonitoring programmes. The National Institute for Health and Clinical Excellence (NICE) guidelines in the United Kingdom (Mant 2011) summarise that telemonitoring reduces mortality and hospitalisation for any reason but does not seem to improve quality of life or decrease heart failure-related hospitalisations. For this reason again the guideline did not include a recommendation for telemonitoring.

Several systematic reviews and meta-analyses have been published on this topic (Clarke 2011; Kotb 2015; Pandor 2013b; Polisena 2010). Although superficially the recently published reviews appear similar, there are important differences in inclusion criteria from our review, and particularly the inclusion of home visits or invasive haemodynamic monitoring in their definitions of 'remote monitoring'. Our review focuses solely on non-invasive monitoring and structured telephone support, and as such we believe is more relevant for healthcare service planning in resource-poor environments where access to invasive monitoring or specially-trained staff to conduct home visits is not an option.

Differences in the inclusion criteria of the recently published meta-analyses on this topic impair the possibility of directly comparing our findings with the findings of previous metaanalyses. However, a critical review of these other reports with our findings highlights the uniqueness and importance of our findings. The previous version of this review included 30 randomised controlled trials ( 25 peer-reviewed publications and 5 abstracts) comparing telemonitoring or structured telephone support to usual care. Other recently published systematic reviews and metaanalyses have included fewer studies, used different selection criteria, and some have included cohort studies and/or did not systematically exclude intensified follow-up such as home visits (Clarke 2011; Kotb 2015; Pandor 2013b; Polisena 2010). Nonetheless, overall findings have been consistent in confirming that telemonitoring reduces all-cause mortality, especially in those situations where the quality of usual care is inferior. However, the effect on all-cause or heart failure-related hospitalisations was more variable.

\section{Strengths and weaknesses of this review}

The major strength and advantage of our review is the quantification of the benefit of structured telephone support and non-invasive telemonitoring in the absence of home visits or intensified clinic follow-up, which identifies for clinicians and healthcare service planners the value of each of the 'building blocks' in contemporary heart failure disease management programmes, allowing them to be better customised to needs. Another strength of our systematic review and meta-analysis is that we have considered and synthesised evidence on many important aspects of heart failure (mortality, hospitalisations, length of stay, 
quality of life, heart failure knowledge and self care, acceptability, and cost).

Weaknesses of this review are due to inadequate reporting by some studies, which has precluded classification of risks of bias as either low or high risk, leading to many studies across the categories being rated as at unclear risk.

Although we have only included studies which were published as a full-text peer-reviewed publication, we consider that this is a strength of this review. We do, however, acknowledge that this has resulted in some studies which are near to reporting final results not being included in this update. Studies which are yet to report final results are listed as Studies awaiting classification, and those which are still ongoing are listed as Ongoing studies.

\section{AUTHORS' CONCLUSIONS}

\section{Implications for practice}

Compared to usual conventional care, implementation of structured telephone support and non-invasive home telemonitoring reduces mortality and heart failure-related hospitalisations, improves quality of life, heart failure knowledge and self-care behaviours and therefore should be considered evidence-based strategies to improve the quality of care and outcomes for people with heart failure.

As the equipment for these interventions was in most cases provided as part of the study, the implications for purchase, installation and maintenance of such equipment for use of telemonitoring in particular in the real world needs to be considered as these may be barriers to the use of telemonitoring.

\section{Implications for research}

- Patient preference and engagement is a key factor in successful delivery of structured telephone support and non-invasive home telemonitoring services and should be a greater focus for future research. Patient approval for these services appears to be high.

- Structured telephone support and non-invasive home telemonitoring are a heterogeneous group of interventions. Further RCTs investigating the benefits of these interventions would be valuable, but should take into account many factors including the population to be studied and the intended interventions for both the active and control groups. Structured telephone support and non-invasive home telemonitoring may not be superior to intensive management by conventional means, and this should be taken into account when planning studies. Monitoring alone is unlikely to change outcome; actions as a consequence of monitoring may.

- However, further studies in geographically isolated communities could be considered unnecessary, as there may be no satisfactory alternative to structured telephone support and non-invasive home telemonitoring, and the data in support of this approach are substantial.

- More consideration should be given to the potential advantages of cluster-RCTs when evaluating service models, including structured telephone support and non-invasive home telemonitoring. This allows structured telephone support and non-invasive home telemonitoring to be evaluated alongside other disease management strategies and the 'best' multimodal strategy identified.

- Although there is a plethora of evidence in well-resourced, high-income countries, consideration should be given to more studies undertaken in low- and middle-income nations where the burden of heart failure may increase in the future (Callender 2014b).

- Standard design and reporting criteria for studies of structured telephone support and non-invasive home telemonitoring should be developed. The published taxonomy (Krumholz 2006) should be developed for this purpose.

- Investigators are encouraged to share data. This will enable individual patient data meta-analysis that can provide insights into overall effect and in subgroups.

- Future research into 'remote monitoring' of people with heart failure should compare the value of remote monitoring using a variety of different non-invasive technologies as well as implanted devices.

- The development of closed-loop systems that integrate physiological measurements into decision-support tools that allow the patient greater participation in their own disease management is an exciting area requiring more research. Patient motivation as well as education may be important.

- More work is required on business models to identify sustainable, cost-effective services, particularly telemonitoring. High capital acquisition costs with low running costs argue for long-term monitoring of individuals. However, renting equipment argues for monitoring over shorter periods of high risk with intensive monitoring and education. Business models will help define how the clinical community use the technology.

- Stratified implementation of technology should be investigated, according to the severity of illness on the trajectory of heart failure.

- The potential for remote monitoring in end-of-life care for heart failure should be investigated.

- Publications relating to studies of these interventions should clearly identify to which study they relate and where outcomes are reported across multiple publications, all publications for a study should detail all of the outcomes and then indicate which are reported in each publication.

\section{ACKNOWLEDGEMENTS}

Sally C Inglis is a Cardiovascular Life Sciences Fellow, supported by the New South Wales Cardiovascular Research Network which is supported by the Heart Foundation of Australia and the NSW Office for Health and Medical Research (CR 11S 6226). During the previous version of this review she was supported by a Sidney Sax Overseas Post-Doctoral Research Fellow supported by the National Health and Medical Research Council of Australia (NHMRC) and National Heart Foundation of Australia (NHMRC Grant ID 472 699).Robyn A Clark has no personal financial support to report. During the previous version of this review Robyn A Clark was a Post-Doctoral Research Fellow supported by National Health and Medical Research Council of Australia (NHMRC) and a Research SA Fellowship (NHMRC Grant ID 570 141).

John GF Cleland is a National Institute of Health Research (United Kingdom) Senior Investigator for which he receives grant funding. 
We wish to thank Ms Sabine Allida for her contribution to this review in the role of author support.

Our team wishes to thank all of the personnel at the Cochrane Heart Review Group, in particular, Mrs Nicole Martin, Managing Editor and Professor Juan Pablo Casas, Co-ordinating Editor, for their ongoing support of our review and team.

We wish to thank Dr Daniel Sabater-Hernández for assistance with Spanish translation.

We wish to thank and acknowledge the following study authors who were very generous in sharing further details and data with us: Angermann, Domingues, Blum, Scherr, Seto, Vourinen.

\section{Support for authors of previous versions of this review:}

Finlay A McAlister received salary support from the Alberta Heritage Foundation for Medical Research Health Scholar Program and the University of Alberta/Merck Frost/Aventis Chair in Patient Health Management.

Simon Stewart was supported by the National Health and Medical Research Council of Australia (NHMRC) (NHMRC Grant ID 472 658).

\section{Acknowledgement of contributions of authors of previous versions of this review}

Finlay A McAlister (author on 2010 version of this review and 2007 version published in BMJ): Responsible for checking data extraction, assisting with analyses and interpretation of data (providing a methodological and clinical view), revising the review critically for important intellectual content and final approval of the version to be published. Performed previous work that was the foundation of this study.

Jocasta Ball (author on 2010 version of this review): Responsible to retrieving studies for review, for drafting of the review and final approval of the version to be published.
Christian Lewinter (author on 2010 version of this review): Responsible for handsearching of conference abstracts, retrieving studies for review and final approval of the version to be published.

Damien Cullington (author on 2010 version of this review): Responsible for handsearching of conference abstracts, retrieving studies for review and final approval of the version to be published.

Simon Stewart (author on 2010 version of this review and 2007 version published in $\mathrm{BMJ}$ ): Responsible for interpretation of data (providing a clinical view) and final approval of the version to be published. Performed previous work that was the foundation of this study.

\section{Acknowledgements for support of previous versions of this review:}

Our team wishes to make special acknowledgement to the contribution of the University of Glasgow's Medical Science Librarian Dr Helen Marlborough, the University of South Australia's Health Sciences Librarian, Ms Margaret Goedhart and the Cochrane Heart Group Trials Search Co-ordinator, Ms Margaret Burke. Dr Marlborough's, Ms Goedhart's and Ms Burke's knowledge and skill in navigating current bibliographies and electronic sources of knowledge was essential to ensuring quality and rigor of the search strategies. Our team also wishes to thank Ms Stefanie Nagendirarajah from Preventative Health, Baker IDI Heart and Diabetes Institute for her assistance with retrieving studies. We would like to also thank Monika, Horst and Erika Winterstein and Ms Andrea Horsky for their assistance with German translations.

Our team also wishes to acknowledge the following study authors who were very generous in sharing further details and data with us, some of which is unpublished, in order to include the most upto-date data in our meta-analysis: K Blum; DA DeWalt; M Blasius and P Brocki (B. Kielblock); WA Gattis; LR Goldberg; A Laramee; A Mortarra; G Parati; B Riegel; RT Tsuyuki; BJ Wakefield; A Woodend; C Zugck. 


\section{R E F E R E N C E S}

\section{References to studies included in this review}

Angermann 2012 (INH) \{published data only\}

Angermann CE, Störk S, Gelbrich G, Faller H, Jahns R, Frantz S, et al. Abstract 2709: a prospective randomised trial comparing the efficacy of a standardised supraregionally transferable program for monitoring and education of patients with systolic heart failure with usual care: the Interdisciplinary Network for Heart failure (INH) study. Circulation 2007;116(11):601.

* Angermann CE, Störk S, Gelbrich G, Faller H, Jahns R, Frantz S, et al. Competence Network Heart Failure. Mode of action and effects of standardized collaborative disease management on mortality and morbidity in patients with systolic heart failure: the Interdisciplinary Network for Heart Failure (INH) study. Circulation: Heart Failure 2012;5(1):25-35.

Berliner D, Stoerk S, Gelbrich G, Jahns R, Frantz S, Jany B, et al. Predeterminants of mortality and rehospitalisation at 180 days in the interdisciplinary network for heart failure (INH) study. European Heart Journal 2009;30:717.

Güder G, Gelbrich G, Brenner S, Morbach C, Berliner D, Deubner N, et al. Prescription frequencies, dosing and clinical effects of heart failure drugs in the randomised INH-study. European Heart Journal 2012;33:43.

Güder G, Störk S, Gelbrich G, Brenner S, Deubner N, Morbach C, et al. Nurse-coordinated collaborative disease management improves the quality of guideline-recommended heart failure therapy, patient-reported outcomes, and left ventricular remodelling. European Journal of Heart Failure 2015;17(4):442.

Güder G, Störk S, Gelbrich G, Brenner S, Morbach C, Berliner D, et al. Heart failure medication in the extended randomized INH study: Clinical outcomes according to prescription frequency and dosing of guideline-recommended drugs. Journal of the American College of Cardiology 2013;1:E764.

\section{Antonicelli 2008 \{published data only\}}

Antonicelli R, Mazzanti I, Abbatecola AM, Parati G. Impact of home patient telemonitoring on use of beta-blockers in congestive heart failure. Drugs \& Aging 2010;27(10):801-5. [Antonicelli 2010]

* Antonicelli R, Testarmata P, Spazzafumo L, Gagliardi C, Bilo G, Valentini $\mathrm{M}$, et al. Impact of telemonitoring at home on the management of elderly patients with congestive heart failure. Journal of Telemedicine and Telecare 2008;14(6):300-5.

\section{Baker 2011 \{published data only\}}

Baker DW, DeWalt DA, Schillinger D, Hawk V, Ruo B, BibbinsDomingo K, et al. Randomized trial of intensive telephone education and counseling to overcome disparities in knowledge, self-care behaviors and symptom severity for patients with heart failure and low health literacy. Circulation 2010;122:2.

* Baker DW, DeWalt DA, Schillinger D, Hawk V, Ruo B, BibbinsDomingo $\mathrm{K}$, et al. The effect of progressive, reinforcing telephone education and counseling versus brief education intervention on knowledge, self-care behaviors and heart failure symptoms. Journal of Cardiac Failure 2011;17(10):789-96.

Balk 2008 \{published data only\}

* Balk AHMM, Davidse W, Van Dommelen P, Klaassen E, Caliskan K, Van der Burg P, et al. Tele-guidance of chronic heart failure patients enhances knowledge about the disease. European Journal of Heart Failure 2008;10(11):1136-42.

Balk AHMM, Leenders CM, Davidse W, Westerteicher C, Van Montfort G. 275 personalised tele-guidance of heart failure patients: effects of the MOTIVA interactive health care platform on hospital admissions, quality of life, knowledge of disease and self-care: a pilot study. European Journal of Heart Failure Supplement 2007;6(Suppl):56.

Van Montfort APWP, Van der Helm MHJ. Telemonitoring of patients with chronic heart failure. Disease Management and Health Outcomes 2006;14(Supplement 1):33-5.

\section{Barth 2001 \{published data only\}}

Barth V. A nurse-managed discharge program for congestive heart failure patients: outcomes and costs. Home Health Care Management and Practice 2001;13(6):436-43.

\section{Bento 2009 \{published data only\}}

Bento VF, Brofman PR. Impact of the nursing consultation on the frequency of hospitalizations in patients with heart failure in Curitiba, Parana State. Arquivos Brasileiros de Cardiologia 2009;92(6):454-60, 473-9, 490-6.

\section{Biannic 2012 (SEDIC) \{published data only\}}

* Biannic C, Coutance G, Calus J, Belin A, Loiselet P, Michel L, et al. Educational home follow-up by telemedicine in cases of cardiac insufficiency. Randomised, multicentric study from the Basse-Normandie region. Preliminary results. European Research in Telemedicine 2012;1:40-8.

Coutance G, Belin A, Biannic C, Parienti JJ, Loiselet P, Michel $L$, et al. Educative telemedicine reduces the severity of rehospitalizations for acute heart failure in an elderly population: Post-hoc analysis of SEDIC study. European Journal of Heart Failure 2013;12:S144.

Sabatier R, Coutance G, Belin A, Biannic C, Loiselet P, Pradere G, et al. Three months educational remote telemonitoring in elderly patients with heart failure reduces hospitalizations for acute heart failure at one year: A randomized trial. European Journal of Heart Failure 2013;12:S313.

\section{Blum 2014 (MCCD) \{published data only\}}

Blum K, Gottlieb SS. Morbidity and mortality benefits of reliable instrumental support. Journal of Cardiac Failure 2007;13(6 Suppl. 2):S164.

* Blum K, Gottlieb SS. The effect of a randomised trial of home telemonitoring on medical costs, 30-day readmissions, mortality, and health-related quality of life in a cohort of community-dwelling heart failure patients. Journal of Cardiac Failure 2014;20(7):513-21. 
Blum K, Janowick F, Gottlieb SS. One year changes in quality of life for heart failure patients in a home telemonitoring program. Journal of Cardiac Failure 2006;12(6 Suppl):S122.

Gottlieb S, Blum K. Coordinated care, telemonitoring, and the therapeutic relationship: heart failure management in the United States. Disease Management and Health Outcomes 2006;14(Suppl 1):29-31.

Nahm ES, Blum K, Scharf B, Friedmann E, Thomas S, Jones D, et al. Exploration of patients' readiness for an eHealth management program for chronic heart failure: a preliminary study. Journal of Cardiovascular Nursing 2008;23(6):463-71.

\section{Brandon 2009 \{published data only\}}

Brandon A, Ellison KJ, Schuessler J, Lazenby R. The effects of a clinical nurse specialist--led, telephone-based intervention on hospital readmissions, quality of life, and self-care behaviors of heart failure patients. Clinical Nurse Specialist 2009;23:99-100.

* Brandon AF, Schuessler JB, Ellison KJ, Lazenby RB. The effects of an advanced practice nurse led telephone intervention on outcomes of patients with heart failure. Applied Nursing Research 2009;22(4):e1-7.

\section{Capomolla 2004 \{published data only\}}

Capomolla S, Pinna G, La Rovere MT, Maestri R, Ceresa M, Ferrari $\mathrm{M}$, et al. Heart failure case disease management program: a pilot study of home telemonitoring versus usual care. European Heart Journal 2004;6(Suppl F):F91-8.

\section{Chaudhry 2010 (Tele-HF) \{published data only\}}

Bikdeli B, Wayda B, Bao HK, Ross JS, Xu X, Chaudhry SI, et al. Place of residence and outcomes of patients with heart failure analysis from the telemonitoring to improve heart failure outcomes trial. Circulation. Cardiovascular Quality and Outcomes 2014;7(5):749-U169.

Chaudhry SI, Barton B, Mattera J, Spertus J, Krumholz HM. Randomized trial of telemonitoring to improve heart failure outcomes (Tele-HF): study design. Journal of Cardiac Failure 2007;13(9):709-14.

* Chaudhry SI, Mattera JA, Curtis JP, Spertus JA, Herrin J, Lin Z, et al. Telemonitoring in patients with heart failure. New England Journal of Medicine 2010;363(24):2301-9.

Chaudhry SI, Mattera JA, Curtis JP, Spertus JA, Herrin J, Lin Z, et al. Telemonitoring to improve outcomes after heart failure hospitalization: a randomized controlled trial. Circulation 2010;122:2223.

Jayaram N, Krumholz HM, Chaudhry SI, Mattera J, Tang F, Spertus JA. Impact of telemonitoring on health status in patients with heart failure. Circulation. Cardiovascular Quality of Care Outcomes 2013;6:A27.

\section{Cleland 2005 (Struct Tele) (TENS-HMS) \{published data only\}}

Cleland JG. The trans-European network - home care management system (TEN-HMS) study: an investigation of the effect of telemedicine on outcomes in Europe. Disease Management and Health Outcomes 2006;14 (Suppl 1):23-8.
* Cleland JGF, Louis AA, Rigby AS, Janssen U, Balk AHMM, for the Trans-European Network-Home-Care Management System (TENS-HMS) Study. Noninvasive home telemonitoring for patients with heart failure at high risk of recurrent admission and death. Journal of the American College of Cardiology 2005;45(10):1654-64.

Goode K, Zhang J, Rigby AS, Atkin P, Cullington D, Balk AH, et al. Home-telemonitoring or nurse telephone support improves short-term outcomes in patients at greatest risk of death or hospitalisation compared to usual care: Evidence from the TEN-HMS Study. European Journal of Heart Failure, Supplement 2009;8:ii195.

Louis AA, Balk A, Janssens U, Westerteicher C, Cleland JG. Patient acceptance and satisfaction of home telemonitoring in the management of heart failure: TENS-HMS. Journal of the American College of Cardiology 2002;19:537A.

Robinson S, Stroetmann K, Stroetmann V. Tele-homecare for chronically-ill patients: improved outcomes and new developments. The Journal on Information Technology in Healthcare 2004;2(4):251-62.

Zhang J, Goode K, Cuddihy P, Cleland JG. Predicting hospitalisation due to worsening heart failure using daily weight measurement: an analysis of the Trans-EuropeanNetwrok-Home-Care Management System (TEM-HMS) Study. European Journal of Heart Failure 2009;11(4):420-7.

\section{Cleland 2005 (Telemon) (TENS-HMS) \{published data only\}}

Cleland JG. The trans-European network - home care management system (TEN-HMS) study: an investigation of the effect of telemedicine on outcomes in Europe. Disease Management and Health Outcomes 2006;14(Suppl 1):23-8.

* Cleland JGF, Louis AA, Rigby AS, Janssen U, Balk AHMM, for the Trans-European Network-Home-Care Management System (TENS-HMS) Study. Noninvasive home telemonitoring for patients with heart failure at high risk of recurrent admission and death. Journal of the American College of Cardiology 2005;45(10):1654-64.

Goode K, Zhang J, Rigby AS, Atkin P, Cullington D, Balk AH, et al. Home-telemonitoring or nurse telephone support improves short-term outcomes in patients at greatest risk of death or hospitalisation compared to usual care: Evidence from the TEN-HMS Study. European Journal of Heart Failure, Supplement 2009;8:ii195.

Louis AA, Balk A, Janssens U, Westerteicher C, Cleland JG. Patient acceptance and satisfaction of home telemonitoring in the management of heart failure: TENS-HMS. Journal of the American College of Cardiology 2002;19:537A.

Robinson S, Stroetmann K, Stroetmann V. Tele-homecare for chronically-ill patients: improved outcomes and new developments. The Journal on Information Technology in Healthcare 2004;2(4):251-62.

Zhang J, Goode K, Cuddihy P, Cleland JG. Predicting hospitalisation due to worsening heart failure using daily weight measurement: an analysis of the Trans-European- 
Netwrok-Home-Care Management System (TEM-HMS) Study. European Journal of Heart Failure 2009;11(4):420-7.

\section{DeBusk 2004 \{published data only\}}

DeBusk RF, Miller NH, Parker KM, Bandura A, Kraemer HC, Cher DJ, et al. Care management for low-risk patients with heart failure: a randomised, controlled trial. Annals of Internal Medicine 2004;141(8):606-13.

\section{De Lusignan 2001 \{published data only\}}

De Lusignan S. A controlled pilot study in the use of telemedicine in the community on the management of heart failure: a report of the first three months. In: Nerlich M, Kretschmer R editor(s). The Impact of Telemedicine on Health Care Management. IOS, 1999.

* De Lusignan S, Wells S, Johnson P, Meredith K, Leatham E. Compliance and effectiveness of 1 year's home telemonitoring: the report of a pilot study of patients with chronic heart failure. European Journal of Heart Failure 2001;3(6):723-30.

\section{Dendale 2012 (TEMA-HF1) \{published data only\}}

* Dendale P, De Keulenaer G, Troisfontaines P, Weytjens C, Mullens W, Elegeert I, et al. Effect of a telemonitoring-facilitated collaboration between general practitioner and heart failure clinic on mortality and rehospitalization rates in severe heart failure: the TEMA-HF 1 (TElemonitoring in the MAnagement of Heart Failure) study. European Journal of Heart Failure 2012;14(3):333-40

Willekens K, Dendale P, De Keulenaer G, Troisfontaines P, Weytjens C, Mullens W, et al. Effect of a telemonitoringfacilitated collaboration between general practitioner and heart failure clinic on mortality and rehospitalization rates in severe heart failure. Acta Cardiologica 2011;66 (1):107.

\section{DeWalt 2006 \{published data only\}}

DeWalt DA, Malone RM, Bryant ME, Kosnar MC, Corr KE, Rothman RL, et al. A heart failure self-management program for patients of all literacy levels: a randomised, controlled trial. BMC Health Services Research 2006;13(6):30.

\section{Domingues 2011 \{published data only\}}

Domingues FB, Clausell N, Aliti GB, Dominguez DR, Rabelo ER. Education and telephone monitoring by nurses of patients with heart failure: randomized clinical trial. Arquivos Brasileiros de Cardiologia 2011;96(3):233-9.

\section{Galbreath 2004 \{published data only\}}

Correction to: Long-term healthcare and cost outcomes of disease management in a large, randomized, community-based population with heart failure. Circulation 2006; Vol. 113, issue 3:e48

* Galbreath AD, Krasuski RA, Smith B, Stajduhar KC, Kwan MD, Ellis $\mathrm{R}$, et al. Long-term healthcare and cost outcomes of disease management in a large, randomised, community-based population with heart failure. Circulation 2004;110(23):3518-26.

Smith B, Forkner E, Zaslow B, Krasuski RA, Stajduhar K, Kwan M, et al. Disease management produces limited quality-oflife improvements in patients with congestive heart failure: evidence from a randomised trial in community-dwelling patients. American Journal of Managed Care 2005;11(11):701-3.

Smith B, Hughes-Cromwick PF, Forkner E, Galbreath AD. Costeffectiveness of telephonic disease management in heart failure. American Journal of Managed Care 2008;14(2):106-15.

Gattis 1999 (PHARM) \{published data only\}

Gattis WA, Hasselblad V, Whellan DJ, O'Connor CM. Reduction in heart failure events by the addition of a clinical pharmacist to the heart failure management team. Archives of Internal Medicine 1999;159(16):1939-45.

\section{GESICA 2005 (DIAL) \{published data only\}}

Ferrante D, Varini S, Macchia A, Soifer S, Badra R, Nul D, et al. Long-term results after a telephone intervention in chronic heart failure: DIAL (Randomized Trial of Phone Intervention in Chronic Heart Failure) follow-up. Journal of the American College of Cardiology 2010;56(5):372-8.

\section{* GESICA Investigators. Randomised trial of telephone intervention in chronic heart failure: DIAL trial. BMJ 2005;331(7514):425.}

Grancelli H, Varini S, Ferrante D, Schwartzman R, Zambrano C, Soifer S, et al. GESICA Investigators. Randomized trial of telephone intervention in chronic heart failure (DIAL): study design and preliminary observations. Journal of Cardiac Failure 2003;9(3):172-9.

Grancelli HO. Disease management programs in heart failure: findings of the DIAL study. Revista Espanola de Cardiologia 2007;60(Suppl 3):15-22

\section{Giordano 2009 \{published data only\}}

* Giordano A, Scalvini S, Zanelli E, Corrà U, Longobardi GL, Ricci VA, et al. Multicentre randomised trial on home-based telemanagement to prevent hospital readmission of patients with chronic heart failure. International Journal of Cardiology 2009;131(2):192-9.

Scalvini S, Zanelli E, Corrà U, Ricci VA, Longobardi GL, Giordano A. 243 multicenter randomised trial on home based tele-management to prevent hospital readmission of patients with chronic heart failure. European Journal of Heart Failure 2008;7(Suppl 1):57.

Scalvini S, Zanelli E, Volterrani M, Martinelli G, Baratti D, Buscaya $\mathrm{O}$, et al. A pilot study of nurse-led home-based telecardiology for patients with chronic heart failure. Journal of Telemedicine and Telecare 2004;10(2):113-7.

Goldberg 2003 (WHARF) \{published data only\}

* Goldberg LR, Piette JD, Walsh MN, Frank TA, Jaski BE, Smith AL, et al. Randomized trial of a daily electronic home monitoring system in patients with advanced heart failure: the Weight Monitoring in Heart Failure (WHARF) trial. American Heart Journal 2003;146(4):705-12.

Jones NA, Frankel DS, Piette JD, Goldberg LR. Abstract 3277: withdrawal of a technology-based daily weight monitoring system in patients with advanced heart failure eliminates mortality benefit. Circulation 2007;116:II 737-8. 
Koehler 2011 (TIM-HF) \{published data only\}

Koehler F, Winkler S, Schieber M, Sechtem U, Stangl K, Böhm M, et al. Telemedical Interventional Monitoring in Heart Failure (TIM-HF), a randomized, controlled intervention trial investigating the impact of telemedicine on mortality in ambulatory patients with chronic heart failure. Circulation 2010;122:2224.

Koehler F, Winkler S, Schieber M, Sechtem U, Stangl K, Böhm M, et al. Telemedicine in heart failure: pre-specified and exploratory subgroup analyses from the TIM-HF trial. International Journal of Cardiology 2012;161(3):143-50.

Koehler F, Winkler S, Schieber M, Sechtem U, Stangl K, Böhm M, et al. TIM-HF Investigators. Telemedical Interventional Monitoring in Heart Failure (TIM-HF), a randomized, controlled intervention trial investigating the impact of telemedicine on mortality in ambulatory patients with heart failure: study design. European Journal of Heart Failure 2010;12(12):1354-62.

* Koehler F, Winkler S, Schieber M, Sechtem U, Stangl K, Böhm M, et al. Telemedical Interventional Monitoring in Heart Failure Investigators. Impact of remote telemedical management on mortality and hospitalisations in ambulatory patients with chronic heart failure: the Telemedical Interventional Monitoring in Heart Failure Study. Circulation 2011;123(17):1873-80.

Prescher S, Deckwart O, Winkler S, Koehler K, Honold M, Koehler F. Telemedical care: feasibility and perception of the patients and physicians: a survey-based acceptance analysis of the Telemedical Interventional Monitoring in Heart Failure (TIMHF) trial. European Journal of Preventive Cardiology 2013;20(2 Suppl):18-24.

\section{Krum 2013 (CHAT) \{published data only\}}

Clark RA, Yallop JJ, Piterman L, Croucher J, Tonkin A, Stewart S, et al. Adherence, adaptation and acceptance of elderly chronic heart failure patients to receiving healthcare via telephone-monitoring. European Journal of Heart Failure 2007;9(11):1104-11.

Driscoll A, Liew D, Tonkin A, Krum H. Is a telephone support program for rural and remote heart failure patients costeffective?. Global Heart 2014;1:e23.

* Krum H, Forbes A, Yallop J, Driscoll A, Croucher J, Chan B, et al. Telephone support to rural and remote patients with heart failure: The chronic heart failure assessment by telephone (CHAT) study. Cardiovascular Therapeutics 2013;31(4):230-7.

Tonkin A, Yallop J, Driscoll A, Forbes A, Croucher J, Chan B, et al. Does telephone support of the rural and remote patients with heart failure improve clinical outcomes?: results of the Chronic Heart Failure Assistance by Telephone (CHAT) study. Heart Lung Circulation 2009;18S(Suppl 1):S105.

Yallop J, Chan B, Piterman P, Tonkin A, Forbes A, Davidson PM, et al. The Chronic Heart-failure Assistance by Telephone (CHAT) study: a new model of care to support aging rural patients with chronic disease. Asian Pacific Family Medicine 2006;5:2.
Yallop J, Clark RA, Chan B, Croucher J, Wilson A, Sellar B, et al. CHAT-a study of a nurse-led system of care. Australian Nursing Journal 2006;14(4):19.

\section{Laramee 2003 \{published data only\}}

Laramee AS, Levinsky SK, Sargent J, Ross R, Callas P. Case management in a heterogenous congestive heart failure population: a randomised controlled trial. Archives of Internal Medicine 2003;163(7):809-17.

\section{Lyngå 2012 (WISH) \{published data only\}}

Lyngå P, Fridlund B, Langius-Eklof A, Bohm K. Patients experiences of telemonitoring and transmission of body weight. European Journal of Cardiovascular Nursing 2013;12:S49-50.

Lyngå P, Fridlund B, Langius-Eklof A, Bohm K. Perceptions of transmission of body weight and telemonitoring in patients with heart failure?. International Journal of Qualitative Studies on Health and Well-being 2013;8:21524.

\footnotetext{
* Lyngå P, Persson H, Hägg-Martinell A, Hägglund E, Hagerman I, Langius-Eklof A, et al. Weight monitoring in patients with severe heart failure (WISH). A randomized controlled trial. European Journal of Heart Failure 2012;14(4):438-44.
}

\section{Mortara 2009 (Struct Tele) (HHH) \{published and unpublished data\}}

Capomolla S, Pinna G, Maestri R, Ferrari M, Ceresa M. The telemonitoring service [Il servizio di telemonitoraggoio]. Monaldi Archives for Chest Disease 2005;64(2):135-6.

Mortara A, Pinna GD, Capomolla S, Johnson P, La Rovere MT, Ponikowski P, et al. P2160 a new telemonitoring system in heart failure: preliminary data from the multi-country randomized study HHH (Home or Hospital in Heart Failure). European Heart Journal 2006;27(Suppl 1):S345.

Mortara A, Pinna GD, Capomolla S, Johnson P, La Rovere MT, Ponikowski P, et al. P358 a multi-country randomised trial of the role of a new telemonitoring system in CHF: the HHH Study (Home or Hospital in Heart Failure): preliminary data. European Heart Journal 2005;26(Suppl 1):20.

Mortara A, Pinna GD, Capomolla S, Maestri R, Johnson P, La Rovere MT, et al. Rehabilitation and models of domiciliary care of patients with chronic heart failure: preliminary results of the $\mathrm{HHH}$ study [Riabilitazione e modelli di gestione domiciliare del paziente con scompenso cardiaco cronico: risultati preliminari dello studio HHH]. Monaldi Archives for Chest Disease 2005;64(2):143-5.

* Mortara A, Pinna GD, Johnson P, Maestri R, Capomolla S, LaRovere MT, et al. Home telemonitoring in heart failure patients: the HHH study (Home of Hospital in Heart Failure). European Journal of Heart Failure 2009;11(3):312-8.

Pinna GD. Home telemonitoring of respiratory activity and heart rate variability in chronic heart failure patients: the challenge of the home or hospital in heart failure project. Computers in Cardiology 2003;30:197-200. 
Pinna GD, Maestri R, Andrews D, Witkowski T, Capomolla S, Scanferlato JL, et al. Home telemonitoring of vital signs and cardiorespiratory signals in heart failure patients: system architecture and feasibility of the $\mathrm{HHH}$ model. International Journal of Cardiology 2007;120(3):371-9.

Pinna GD, Maestri R, Capomolla S, La Rovere MT, Andrews D, Johnson P, et al. Home telemonitoring of vital signs and cardiorespiratory signals in chronic heart failure patients. Conference Proceedings of the IEEE EMBS Asian-Pacific Conference on Biomedical Engineering. 2003:34-35. [DOI: 10.1109/APBME.2003.1302570]

Pinna GD, Maestri R, Capomolla S. Domiciliary telemonitoring of vital and cardiorespiratory signs of the $\mathrm{HHH}$ project [Telemonitoraggio domestico di segni vitali e cardiorespiratori: la sfida del progetto $\mathrm{HHH}$. Monaldi Archives for Chest Disease 2005;64(2):145-7.

Pinna GD, Maestri R, Gobbi E, Capomolla S, Campana C, Emdin M, et al. Long-term monitoring of sleep apnea at home in heart failure patients: preliminary results from the $\mathrm{HHH}$ study. Conference Proceedings of the 26th Annual International Conference of the IEEE Engineering in Medicine and Biology Society. 2004; Vol. 5:3874-7.

Pinna GD, Maestri R, Roma M, Scanferlato JL, Giordano A, Comazzi F. Home telemonitoring of chronic heart failure patients: novel system architecture of the home or hospital study. Computers in Cardiology 2003;30:105-8.

Mortara 2009 (Telemon) (HHH) \{published and unpublished data\} Capomolla S, Pinna G, Maestri R, Ferrari M, Ceresa M. The telemonitoring service [Il servizio di telemonitoraggoio]. Monaldi Archives for Chest Disease 2005;64(2):135-6.

Mortara A, Pinna GD, Capomolla S, Johnson P, La Rovere MT, Ponikowski P, et al. P2160 a new telemonitoring system in heart failure: preliminary data from the multi-country randomized study HHH (Home or Hospital in Heart Failure). European Heart Journal 2006;27(Suppl 1):S345.

Mortara A, Pinna GD, Capomolla S, Johnson P, La Rovere MT, Ponikowski P, et al. P358 a multi-country randomised trial of the role of a new telemonitoring system in CHF: the HHH Study (Home or Hospital in Heart Failure): preliminary data. European Heart Journal 2005;26(Suppl 1):20.

Mortara A, Pinna GD, Capomolla S, Maestri R, Johnson P, La Rovere MT, et al. Rehabilitation and models of domiciliary care of patients with chronic heart failure: preliminary results of the HHH study [Riabilitazione e modelli di gestione domiciliare del paziente con scompenso cardiaco cronico: risultati preliminari dello studio HHH]. Monaldi Archives for Chest Disease 2005;64(2):143-5.

* Mortara A, Pinna GD, Johnson P, Maestri R, Capomolla S, LaRovere MT, et al. Home telemonitoring in heart failure patients: the HHH study (Home of Hospital in Heart Failure). European Journal of Heart Failure 2009;11(3):312-8.

Pinna GD. Home telemonitoring of respiratory activity and heart rate variability in chronic heart failure patients: the challenge of the home or hospital in heart failure project. Computers in Cardiology 2003;30:197-200.

Pinna GD, Maestri R, Andrews D, Witkowski T, Capomolla S, Scanferlato JL, et al. Home telemonitoring of vital signs and cardiorespiratory signals in heart failure patients: system architecture and feasibility of the $\mathrm{HHH}$ model. International Journal of Cardiology 2007;120(3):371-9.

Pinna GD, Maestri R, Capomolla S. Domiciliary telemonitoring of vital and cardiorespiratory signs of the $\mathrm{HHH}$ project [Telemonitoraggio domestico di segni vitali e cardiorespiratori: la sfida del progetto $\mathrm{HHH}$ ]. Monaldi Archives for Chest Disease 2005;64(2):145-7.

Pinna GD, Maestri R, Capomolla S, La Rovere MT, Andrews D, Johnson $\mathrm{P}$, et al. Home telemonitoring of vital signs and cardiorespiratory signals in chronic heart failure patients. Conference Proceedings of the IEEE EMBS Asian-Pacific Conference on Biomedical Engineering. 2003:34-35. [DOI: 10.1109/APBME.2003.1302570]

Pinna GD, Maestri R, Gobbi E, Capomolla S, Campana C, Emdin M, et al. Long-term monitoring of sleep apnea at home in heart failure patients: preliminary results from the $\mathrm{HHH}$ study. Conference Proceedings of the 26th Annual International Conference of the IEEE Engineering in Medicine and Biology Society. 2004; Vol. 5:3874-7.

Pinna GD, Maestri R, Roma M, Scanferlato JL, Giordano A, Comazzi F. Home telemonitoring of chronic heart failure patients: novel system architecture of the home or hospital study. Computers in Cardiology 2003;30:105-8.

\section{Rainville 1999 \{published data only\}}

Rainville EC. Impact of pharmacist intervention on hospital readmissions for heart failure. American Journal of HealthSystem Pharmacy 1999;56(13):1339-42.

\section{Ramachandran 2007 \{published data only\}}

Ramachandran K, Husain N, Maikhuri R, Seth S, Vij A, Kumar M, et al. Impact of a comprehensive telephone-based disease management programme on quality-of-life in patients with heart failure. The National Medical Journal of India 2007;20(2):67-73.

\section{Riegel 2002 \{published data only\}}

Riegel B, Carlson B, Kopp Z, LePetri B, Glaser D, Unger A. Effect of a standardized nurse case-management telephone intervention on resource use in patients with chronic heart failure. Archives of Internal Medicine 2002;162(6):705-12.

\section{Riegel 2006 \{published data only\}}

Riegel B. Standardized telephonic case management in a Hispanic heart failure population: an effective intervention. Disease Management and Health Outcomes 2002;10(4):241-9.

* Riegel B, Carlson B, Glaser D, Romero T. Randomized controlled trial of telephone case management in Hispanics of Mexican origin with heart failure. Journal of Cardiac Failure 2006;12(3):211-9. 
Scherr 2009 (MOBITEL) \{published data only\}

Fruhwald FM, Scherr D, Kastner P, Kollmann A, Schreier G. Telemonitoring using mobile phones reduces the event rate after recent acute heart failure. Results of the MOBIle TELemonitoring in heart failure patients study (MOBITEL). European Heart Journal 2009;30:911-2.

* Scherr D, Kastner P, Kollmann A, Hallas A, Auer J, Krappinger $\mathrm{H}$, et al and the MOBITEL investigators. Effect of home-based telemonitoring using mobile phone technology on the outcome of heart failure patients after an episode of acute decompensation: randomised controlled trial. Journal of Medical Internet Research 2009;11(3):e34.

Scherr D, Kollman A, Hallas A, Krappinger H, Auer J, Kastner P, et al. Telemonitoring for heart failure patients following acute decompensation: first results on influences of the system on functional status and heart failure therapy. European Heart Journal. 2005.

\section{Seto 2012 \{published data only\}}

Seto E, Leonard KJ, Cafazzo JA, Barnsely J, Masino C, Ross HJ. Mobile phone-based telemonitoring for heart failure management: a randomised controlled trial. Journal of Medical Internet Research 2012;14(1):e31.

\section{Sisk 2006 \{published data only\}}

Hebert PL, Sisk JE, Wang JJ, Tuzzio L, Casablanca JM, Chassin MR, et al. Cost-effectiveness of nurse-led disease management for heart failure in an ethnically diverse urban community. Annals of Internal Medicine 2008;149(8):540-8.

Sisk J, Hebert P, Horowitz C, McLaughlin MA, Wang J, Tuzzio L. Effectiveness and cost-effectiveness of nurse-management to improve heart-failure care in minority communities.

Proceedings of the First Annual Meeting of the Health Technology Assessment International (HTAi) 2004;1:111.

* Sisk JE, Hebert PL, Horowitz CR, McLaughlin MA, Wang JJ, Chassin MR. Effects of nurse management on the quality of heart failure care in minority communities: a randomised trial. Annals of Internal Medicine 2006;145(4):273-83.

\section{Soran 2008 \{published data only\}}

Soran O, Piña IL, Lamas GA, Kelsey SF, Selzer F, Pilotte J, et al. Randomized clinical trial of the clinical effects of enhanced heart failure monitoring using a computer-based telephonic monitoring system in older minorities and women. Journal of Cardiac Failure 2007;13(9):793.

Soran OZ, Feldman AM, Piña IL, Lamas GA, Kelsey SF, Selzer F, et al. Cost of medical services in older patients with heart failure: those receiving enhanced monitoring using a computer-based telephonic monitoring system compared with those in usual care: the heart failure home care trial. Journal of Cardiac Failure 2010;16(11):859-66.

* Soran OZ, Piña IL, Lamas GA, Kelsey SF, Selzer F, Pilotte J, et al. A randomised clinical trial of the clinical effects of enhanced heart failure monitoring using a computer-based telephonic monitoring system in older minorities and women. Journal of Cardiac Failure 2008;114(9):711-7.
Soran OZ, Piña IL, Lamas GA, Kelsey SF, Selzer F, Pilotte J, et al. Impact of a sophisticated computer based telephonic heart failure monitoring system on quality of life in patients with heart failure (Abstract \#1012-151). Journal of the American College of Cardiology 2008;51:A236-70.

\section{Tsuyuki 2004 \{published data only\}}

Tsuyuki RT, Fradette M, Johnson JA, Bungard TJ, Eurich DT, Ashton $\mathrm{T}$, et al. A multicenter disease management program for hospitalised patients with heart failure. Journal of Cardiac Failure 2004;10(6):473-80.

\section{Villani 2014 (ICAROS) \{published data only\}}

* Villani A, Malfatto G, Compare A, Della Rosa F, Bellardita L, Branzi G, et al. Clinical and psychological telemontoring and telecare of high risk heart failure patients. Journal of Telemedicine and Telecare 2014;20(8):468-75.

Villani A, Malfatto G, Della Rosa F, Branzi G, Boarin S, Borghi C, et al. Disease management for heart failure patients: role of wireless technologies for telemedicine: the ICAROS project. Giornale Italiano di Cardiologia 2007;8(2):107-17.

\section{Vuorinen 2014 \{published data only\}}

Vuorinen AL, Leppänen J, Kaijanranta H, Kuliju M, Heliö T, Van Gils M, Lähteenmäki J. Use of home telemonitoring to support multidisciplinary care of heart failure patients in Finland: randomised controlled trial. Journal of Medical Internet Research 2014;16(12):e282.

\section{Wakefield 2008 \{published data only\}}

Wakefield BJ, Bylund CL, Holman JE, Ray A, Scherubel M, Kienzle MG, et al. Nurse and patient communication profiles in a home-based telehealth intervention for heart failure management. Patient Education and Counseling 2008;71(2):285-92.

Wakefield BJ, Holman JE, Ray A, Scherubel M, Burns TL, Kienzle MG, et al. Outcomes of a home telehealth intervention for patients with heart failure. Journal of Telemedicine and Telecare 2009;15(1):46-50.

* Wakefield BJ, Ward MM, Holman JE, Ray A, Scherubel M, Burns TL, et al. Evaluation of home telehealth following hospitalizations for heart failure: a randomised trial. Telemedicine Journal and E-Health 2008;4(8):753-61.

Woodend 2008 \{published and unpublished data\}

Woodend A, Sherrard H, Fraser M, Stuewe L, Haddad H, Cheung T, et al. Getting connected: tele-home care for patients with heart disease. Canadian Journal of Health Economics 2003;52(2):19-28.

* Woodend KA, Sherrard H, Fraser M, Stuewe L, Cheung T, Struthers $\mathrm{C}$. Telehome monitoring in patients with cardiac disease who are at high risk of readmission. Heart and Lung 2008;37(1):36-45.

\section{Zamanzadeh 2013 \{published data only\}}

Zamanzadeh V, Valizadeh L, Howard A F, Jamshidi F. A supportive-educational intervention for heart failure patients 
in Iran: the effect on self-care behaviours. Nursing Research and Practice 2013;2013:492729.

\section{References to studies excluded from this review}

\section{Abu-Awwad 2012 \{published data only\}}

Abu-Awwad R, Alkhatib Y, Bukannan A, Bandak G, El Atrache M, Pflaum J, et al. Telemonitoring in patients with heart failure: $A$ single-center experience. Journal of General Internal Medicine 2012;27:S313.

\section{Akosah 2005 \{published data only\}}

Akosah KO, Schaper AM, Haus LM, Mathiason MA, Barnhart SI, McHugh VL. Improving outcomes in heart failure in the community: long-term survival benefit of a diseasemanagement program. Chest 2005;127(6):2042-8.

\section{Albanese 2001 \{published data only\}}

Albanese MC, Bulfoni A, Rossi P, Gregori D, Badano LP, Gremese E, et al. The SCOOP II trial in heart failure. Italian Heart Journal Supplement 2001;2(4):390-5.

\section{Albert 2007 \{published data only\}}

Albert NM, Buchsbaum R, Li J. Randomized study of the effect of video education on heart failure healthcare utilization, symptoms, and self-care behaviours. Patient Education and Counseling 2007;69(1-3):129-39.

Aliti 2007 \{published data only\}

Aliti GB, Rabelo ER, Domingues FB, Clausell N. Educational settings in the management of patients with heart failure. Revista Latino-Americana de Enfermagem 2007;15:344-9.

\section{Al Khateeb 2012 \{published data only\}}

Al Khateeb M. Telephone intervention in chronic heart failure: Quality project. Journal of the Saudi Heart Association 2012;24 (4):289.

\section{Alluhaidan 2015 \{published data only\}}

Alluhaidan A, Lee E, Alnosayan N, Chatterjee S, HoustonFeenstra L, Dysinger W, et al. Designing Patient-Centered mHealth Technology Intervention to Reduce Hospital Readmission for Heart-Failure Patients. System Sciences. 2015:2886-95.

\section{Alnosayan 2014 \{published data only\}}

Alnosayan N, Lee E, Alluhaidan A, Chatterjee S, HoustonFeenstra L, Kagoda M, et al. MyHeart: An intelligent mHealth home monitoring system supporting heart failure self-care. eHealth Networking, Applications and Services (Healthcom). 2014:311-6.

\section{Anderson 2005 \{published data only\}}

Anderson C, Deepak BV, Amoateng-Adjepong Y, Zarich S. Benefits of comprehensive inpatient education and discharge planning combined with outpatient support in elderly patients with congestive heart failure. Congestive Heart Failure 2005;11(6):315-21.

\section{Andrikopoulou 2014 \{published data only\}}

Andrikopoulou E, Abbate K, Whellan D J. Conceptual model for heart failure disease management. Canadian Journal of Cardiology 2014;30:304-11.

Ansinelli 2013 \{published data only\}

Ansinelli DJ. The Effectiveness of a Structured Telephone Support Program for Rural Patients with Heart Failure. http:// wvuscholar.wvu.edu:8881/R/?func=dbin-jump-full\&object_ $i d=28040$ (accessed 20 July 2015). West Virginia University, 2013.

\section{Archelrod 2014 \{published data only\}}

Archelrod D. Policy expectations and reality of telemedicine - a critical analysis of health care outcomes, costs and acceptance for congestive heart failure. Journal of Telemedicine and Telecare 2014;20(4):192-200.

\section{Artinian 2003 \{published data only\}}

Artinian NT, Harden JK, Kronenberg MW, Vander Wal JS, Daher E, Stephens Q, et al. Pilot study of a web-based compliance monitoring. Heart and Lung 2003;32(4):226-33.

Artinian 2006 \{published data only\}

Artinian NT, Nordstrom CK, Flack JM, Washington OG, Jen KLC. 3307: Nurse-managed telemonitoring is associated with improved blood pressure at 12-months follow-up in African Americans. Circulation 2006;114:II_702-b.

\section{Arya 2008 \{published data only\}}

Arya A, Block M, Kautzner J, Lewalter T, Mortel H, Sack S, et al. Influence of home monitoring on the clinical status of heart failure patients: design and rationale of the IN-TIME study. European Journal of Heart Failure 2008;10(11):1143-8.

\section{Austin 2012 \{published data only\}}

Austin LS, Landis C. An interactive technology self-management solution. Journal of Nursing Administration 2012;42:442-6.

\section{Baden 2007a \{published data only\}}

Baden D, Fleck J, Klingelberg M, Waehner M, Zugck CH, Korb H. P3939: Telemonitoring by congestive heart failure: early detection of pending cardiac decompensation and its predictive values of blood pressure and weight instability. European Heart Journal 2007;28(Suppl 1):650.

\section{Baden 2007b \{published data only\}}

Baden D, Fleck J, Klingelberg M, Waehner M, Zugck CH, Korb H. 289: Telemonitoring by congestive heart failure: predictive value of blood pressure and weight instability in the early detection of pending cardial decompensation. European Journal of Heart Failure 2007;6(Suppl 1):61-2.

\section{Baer 1999 \{published data only\}}

Baer CA, Di Salvo TG, Cail MI, Noyes D, Kvedar JC. Electronic home monitoring of congestive heart failure patients: design and feasibility. Congestive Heart Failure 1999;5:105-13.

\section{Bakhshi 2011 \{published data only\}}

Bakhshi S, Xin Li, Semenov N, Apodaca-Madrid J, Mahoor MH, Newman KE, et al. Congestive heart failure home monitoring 
pilot study in urban Denver. Conf Proc IEEE Eng Med Biol Soc. 2011:3150-3.

\section{Baldauf 2008 \{published data only\}}

Baldauf RD, Williams CM, Natasha C, Menon S, Chung E. Establishing the telephonic connection: easing the transition from hospital to home through experienced nursing. Journal of Cardiac Failure 2008;14(6):S110.

\section{Baldonado 2013 \{published data only\}}

Baldonado A, Rodriguez L, Renfro D, Sheridan SB, McElrath M, Chardos J. A home telehealth heart failure management program for veterans through care transitions. Dimensions of Critical Care Nursing 2013;32:162-5.

\section{Barber 1999 \{published data only\}}

Barber ML. Community-focused nurse case management by telephone for patients with congestive heart failure. eScholarship Repository - Electronic Thesis and Dissertation Collection, Texas Tech University 1999.

\section{Bekelman 2013 \{published data only\}}

Bekelman DB, Plomondon ME, Sullivan MD, Nelson K, Hattler B, McBryde $C$, et al. Patient-centered disease management (PCDM) for heart failure: Study protocol for a randomised controlled trial. BMC Cardiovascular Disorders 2013;13:49.

\section{Benatar 2003 \{published data only\}}

Benatar D, Bondmass M, Ghitelam J, Avitall B. Outcomes of chronic heart failure. Archives of Internal Medicine 2003;163(10):347-52.

\section{Ben Gal 2013 \{published data only\}}

Ben Gal T, Amir O, Rappaport D, Rekant K, Adamson PB, Anker S, et al. A pilot study on the feasibility of heart failure medical management based on daily monitoring of pulmonary subclinical congestion with ReDS technology in outpatient set up. European Journal of Heart Failure 2013;12:S75.

\section{Bennett 2006 \{published data only\}}

Bennett SJ, Litzelman DK, Wright A, Perkins SM, Wu J, Meyer L, et al. The PUMP-UP tailored computerized program for heart failure care. Nursing Outlook 2006;54(1):39-45.

\section{Bennett Milburna 2014 \{published data only\}}

Bennett Milburna A, Hewittb M, Griffinc P, Savelsberghd M. The value of remote monitoring systems for treatment of chronic disease. IIE Transactions on Healthcare Systems Engineering 2014;4(2):65-79.

\section{Berkley 2010 \{published data only\}}

Berkley R, Bauer S, Rowland C. How telehealth can increase the effectiveness of chronic heart failure management. Nursing Times 2010;106:14-5.

\section{Blue 2001 \{published data only\}}

Blue L, Lang E, McMurray JJ, DAvie AP, McDonagh TA, Murdoch DR, et al. Randomised controlled trial of specialist nurse intervention in heart failure. BMJ 2001;323(7315):715-8.
Bocchi 2007 (REMADHE) \{published data only\}

Bocchi EA, Cruz F, Guimarães G, Brandão SM, Costa P, Ferreira SMA, et al. Late effects of a disease program management on mortality, hospitalisation, and quality of life in heart failure patients followed by cardiologists with experience in heart failure- a randomised and prospective trial (Abstract $\# 1022-143)$. Journal of the American College of Cardiology 2007;49:42A-98A.

\section{Bocchi 2013 \{published data only\}}

Bocchi EA, Cruz F, Brandao S, Guimaraes G, Bacal F, Issa VS, et al. Cost-effectiveness of long-term disease management program in heart failure: Results from the REMADHE trial. European Journal of Heart Failure 2013;12:S122-3.

\section{Bohacik 2014 \{published data only\}}

Bohacik J, Kambhampati C, Davis DN, Cleland JGF. Use of cumulative information estimations for risk assessment of heart failure patients. FUZZ IEEE. 2014:1402-7.

\section{Bohmer 2011 \{published data only\}}

Bohmer A, Wieser M, Weywar I, Frauendorfer H, Kronik G. The "Kremser model": Successful disease management programme for patients with chronic heart failure. [German] Das "Kremser modell": Erfolgreiches disease-management zur betreuung von patienten mit herzinsuffizienz. Journal fur Kardiologie 2011;18:299-306.

\section{Bolz 2005 \{published data only\}}

Bolz A, Braecklein M, Moor C, Gmelin M. The technical possibilities in telemonitoring of physiological parameters [Technische Möglichkeiten des Telemonitorings physiologischer Parameter]. Herzschrittmachertherapie und Elektrophysiologie 2005;16(3):134-42.

\section{Bondmass 1999 \{published data only\}}

Bondmass M, Bolger N, Castro G, Avitall B. The effect of physiological home monitoring and tele-management on chronic heart failure outcomes. International Journal of Asthma Allergery and Immunology 1999;3:online.

\section{Bondmass 2002 \{published data only\}}

Bondmass MD. Thesis: Outcomes of home management methods for chronic heart failure. University of Illinois at Chicago Health Sciences Centre 2002.

\section{Bondmass 2007 \{published data only\}}

Bondmass MD. Improving outcomes for African Americans with chronic heart failure: a comparison of two home care management delivery methods. Home Health Care Management \& Practice 2007;20(1):8-20.

\section{Boriani 2013 \{published data only\}}

Boriani G, Da Costa A, Ricci RP, Quesada A, Favale S, lacopino S, et al. The MOnitoring Resynchronization dEvices and CARdiac patiEnts (MORE-CARE) randomized controlled trial: phase 1 results on dynamics of early intervention with remote monitoring. Journal of Medical Internet Research 2013;15(8):e167. 
Bourge 2008 (COMPASS-HF) \{published data only\}

Bourge RC, Abraham WT, Adamson PB, Aaron MF, Aranda JM Jr, Magalski A, et al. Randomized controlled trial of an implantable continuous hemodynamic monitor in patients with advanced heart failure: the COMPASS-HF study. Journal of the American College of Cardiology 2008;51(11):1073-9.

\section{Bowles 2007 \{published data only\}}

Bowles $\mathrm{KH}$, Baugh AC. Applying research evidence to optimise tele home care. Journal of Cardiovascular Nursing 2007;22(1):5-15.

\section{Bowles 2008 \{published data only\}}

Bowles K, Horowitz D. The advantages and disadvantages of heart failure disease management when randomly assigned to be delivered by telehealth or telephone. Journal of Cardiac Failure 2008;14(6):6S.

\section{Bowles 2009 \{published data only\}}

Bowles KH, Holland DE, Horowitz DA. A comparison of inperson home care, home care with telephone contact and home care with telemonitoring for disease management. Journal of Telemedicine and Telecare 2009;15(7):344-50.

\section{Bowles 2011 \{published data only\}}

Bowles KH, Hanlon AL, Glick HA, Naylor MD, O'Connor M, Riegel $B$, et al. Clinical effectiveness, access to, and satisfaction with care using a telehomecare substitution intervention: a randomized controlled trial. International Journal of Telemedicine and Applications 2011.

\section{Brennan 2006 \{published data only\}}

Brennan PF, Burke L, Casper G, Sebern M, Krause C, Kossman S, et al. Creating technology-enhanced practice: a universityhome care-corporate alliance. Consumer-Centered Computer Supported Care for Healthy People 2006;122:644-8.

\section{Brennan 2010 \{published data only\}}

Brennan PF, Casper GR, Burke LJ, Johnson K, Brown R, Valdez RS, et al. Technology-enhanced practice for patients with chronic cardiac disease: Home implementation and evaluation. Heart \& Lung 2010;39(6 Suppl):S34-46.

\section{Browning 2011 \{published data only\}}

Browning SV, Clark RC, Poff R, Todd D. Telehealth monitoring: a smart investment for home care patients with heart failure? Home Healthcare Nurse 2011;29:368-74.

\section{Brownsell 2006a \{published data only\}}

Brownsell S, Blackburn S, Aldred H, Hawley M. Two tele-care examples from the UK: lifestyle reassurance and chronic heart failure. The 10th World Multi-Conference on Systematics, Cybernetics and Informatics. Orlando, Florida, July 16-19 2006; Vol. IV:136-41.

\section{Brownsell 2006b \{published data only\}}

Brownsell S, Aldred H, Hawley MS. Telemonitoring chronic heart failure: interim findings from a pilot study in South Yorkshire. British Journal of Healthcare Computing and Information Management 2006;23(8):14-8.

\section{Brownsell 2008 \{published data only\}}

Brownsell S, Aldred H, Young T, Hawley MS. Reforming health care through information and communication technologies. Journal of Care Service Management 2008;2(3):286-300.

\section{Brunetti 2014 \{published data only\}}

Brunetti ND, Dellegrottaglie G, Di Giuseppe G, Di Biase M. Remote tele-medicine cardiologist support for care manager nursing of chronic cardiovascular disease: preliminary report. International Journal of Cardiology 2014;176:552-6.

\section{Byrnes 2012 \{published data only\}}

Byrnes JM, Goldstein S, Venator B, Pollicino C, Ng SK, Veroff D, et al. The impact of population-based disease management services for selected chronic conditions: the Costs to Australian Private Insurance - Coaching Health (CAPICHe) study protocol. BMC Public Health 2012;12:6.

\section{Caldwell 2005 \{published data only\}}

Caldwell MA, Peters KJ, Dracup KA. A simplified education program improves knowledge, self-care behavior, and disease severity in heart failure patients in rural settings. American Heart Journal 2005;150(5):983.e7-e12.

\section{Call 2010 \{published data only\}}

Call C, Hartwig M, Pope S, Hedrick M, Bacher S, Jennison S. Opportunity for improvement: heart failure readmissions 30 days. 2010 AAHFN 6th Annual Meeting, June 24-26, 2010. Heart \& Lung 2010;39:368.

\section{Calò 2013 \{published data only\}}

Calò L, Gargaro A, De Ruvo E, Palozzi G, Sciarra L, Rebecchi M, et al. Economic impact of remote monitoring on ordinary followup of implantable cardioverter defibrillators as compared with conventional in-hospital visits. A single-center prospective and randomized study. Journal of Interventional Cardiac Electrophysiology 2013;37(1):69-78.

\section{Calvin 2008 \{published data only\}}

Calvin JE, Powell L, Richardson D, Janssen I. Impact of selfmanagement training on high-risk patients with heart failure: results from the Heart Failure Adherence and Retention Trial (HART). Abstract \#63. Circulation 2008;117(21):e423.

\section{Capomolla 2002 \{published data only\}}

Capomolla S, Febo O, Ceresa M, Caporotondi A, Guazzotti G, La Rovere MT, et al. Cost/utility ratio in chronic heart failure: comparison between heart failure management program delivered by day-hospital and usual care. Journal of the American College of Cardiology 2002;40(7):1259-66.

\section{Chen 2010 \{published data only\}}

Chen YH, Ho YL, Huang HC, Wu HW, Lee CY, Hsu TP, et al. Assessment of the Clinical Outcomes and Cost-effectiveness of the Management of Systolic Heart Failure in Chinese Patients Using a Home-based Intervention. Journal of International Medical Research 2010;38:242-52. 
Chen 2014 \{published data only\}

Chen L, Ho TW, Lai F, Ho YL, Hsu TP. Evaluating the requirements of patients with heart diseases in a telehealthcare service. IEEE. 2014:1-4.

\section{Cherry 2000 \{published data only\}}

Cherry JC, Colliflower SJ, Tsiperfal A. Meeting the challenges of case management with remote patient monitoring technology. Lippincotts Case Mangagement 2000;5:191-8.

Chetney 2003 \{published data only\}

Chetney R. Home care that doesn't miss a beat. Home Healthcare Nurse 2003;21:680-6.

\section{Chetney 2008 \{published data only\}}

Chetney R. Using telehealth to avoid urgent care and hospitalisation. Home Health Care Management and Practice 2008;20(2):154-60.

\section{Clappers 2006 \{published data only\}}

Clappers N, Verheugt FWA. Hotline sessions of the 28th European Congress of Cardiology/World Congress of Cardiology 2006. European Heart Journal 2006;27(23):2896-9.

Clark 2008 \{published data only\}

Clark AM, Reid ME, Morrison CE, Capewell S, Murdoch DL, McMurray JJ. The complex nature of informal care in homebased heart failure management. Journal of Advanced Nursing 2008;61(4):373-83.

\section{Clarke 2005 \{published data only\}}

Clarke M, Jones R. A telemonitoring architecture to support chronic disease management and acute episode monitoring. Proceedings of the Annual International Conference of the Engineering in Medicine and Biology Society 2005;4:3711-3.

\section{Cleland 2012 \{published data only\}}

Cleland JGF, Atkin P, Slottje K, Domingo M, Mabote T, Navarro $X$, et al. The HeartCycle trial of a 3rd generation home telemonitoring system for patients with severe heart failure. European Journal of Heart Failure, Supplement 2012;11:S85-6.

\section{Cleland 2014a \{published data only\}}

Cleland JGF, Frankenstein L, Slottje K, Dierckx R, Whitehead C, Domingo $\mathrm{M}$, et al. Home telemonitoring, algorithm-assisted, diuretic-minimization in patients with heart failure stabilised after recent hospitalization. A report from theHeartCycle Programme. European Journal of Heart Failure 2014;16:s2.

\section{Cline 1998 \{published data only\}}

Cline CM, Israelsson BY, Willenheimer RB, Broms K, Erhardt LR. Cost effective management programme for heart failure reduces hospitalisation. Heart 1998;80(5):442-6.

\section{Cole 2006 \{published data only\}}

Cole SA, Farber NC, Weiner JS, Sulfaro M, Katzelnick DJ, Blader JC. Double-disease management or one care manager for two chronic conditions: pilot feasibility study of nurse telephonic disease management for depression and congestive heart failure. Disease Management 2006;9(5):266-76.

\section{Coll 2011 \{published data only\}}

Coll J, Sanjoaquin AC, Lopez M, Romero D, Pinilla R, Ochoa P. Does a Preventive Telemonitoring Service improve the quality of life of frail elderly? Preliminary results of the European project Dreaming. European Geriatric Medicine 2011;2:S128-9.

Conway 2014 \{published data only\}

Conway A, Inglis SC, Clark RA. Effective Technologies for Noninvasive Remote Monitoring in Heart Failure. Telemedicine and E-Health 2014;20:531-8.

\section{Copeland 2010 \{published data only\}}

Copeland LA, Berg GD, Johnson DM, Bauer RL. An intervention for VA patients with congestive heart failure. American Journal of Managed Care 2010;16(3):158-65.

\section{Cordisco 1999 \{published data only\}}

Cordisco ME, Beniamonivitz A, Hammond K, Mancini D. Use of telemonitoring to decrease the rate of hospitalisation in patients with severe congestive heart failure. American Journal of Cardiology 1999;84:860-2.

\section{Courtney 2009 \{published data only\}}

Courtney M, Edwards H, Chang A, Parker A, Finlayson K, Hamilton K. Fewer emergency readmissions and better quality of life for older adults at risk of hospital readmission: a randomised controlled trial to determine the effectiveness of a 24-week exercise and telephone follow-up program. Journal of the American Geriatrics Society 2009;57(3):395-402.

Cross 1999 \{published data only\}

Cross M. A scale that talks back. Health Data Management 1999;7:76-8.

\section{Crundall-Goode 2014 \{published data only\}}

Crundall-Goode A, Goode KM. Using telehealth for heart failure: Barriers, pitfalls and nursing service models. British Journal of Cardiac Nursing 2014;9:396-406.

\section{Cruz 2010 \{published data only\}}

Cruz F, Issa VS, Ayub-Ferreira SM Chizzola PR, Souza Germano EC, Moreira Luiz FP, et al. Effect of a sequential education and monitoring programme on quality-of-life components in heart failure. European Journal of Heart Failure 2010;12(9):1009-15.

\section{Dalmiani 2001 \{published data only\}}

Dalmiani S, Passino C, Macerata A, Emdin M. An informative system for chronic heart failure patients follow-up (CHeF). Computers in Cardiology. 23-26 September 2001:569-72.

Dang 2006 \{published data only\}

Dang S, Ma F, Nedd N, Aguilar EJ, Roos BA. Differential resource utilisation benefits with Internet -based care coordination in elderly veterans with chronic disease associated with high resource utilization. Telemedicine Journal and e-Health 2006;12:14-23 


\section{Dansky 2008a \{published data only\}}

Dansky KH, Vasey J, Bowles K. Impact of telehealth on clinical outcomes in patients with heart failure. Clinical Nursing Research 2008;17(3):182-99.

\section{Dansky 2008b \{published data only\}}

Dansky KH, Vasey J, Bowles K. Use of telehealth by older adults to manage heart failure. Research in Gerontological Nursing 2008;1(1):25-32.

\section{Dansky 2009 \{published data only\}}

Dansky K, Vasey J. Managing heart failure patients after formal homecare. Telemedicine Journal \& E-Health 2009;15(10):983-91.

\section{Dar 2009 (HOME-HF) \{published data only\}}

* Dar O, Riley J, Chapman C, Dubrey SW, Morris S, Rosen SD, et al. A randomised trial of home telemonitoring in atypical elderly heart failure population in northwest London: results of the Home-HF study. European Journal of Heart Failure 2009;11(3):319-25.

Dar OA, Riley J, Chapman C, Dubrey SW, Morris S, Rosen S, et al. The Home Heart Failure Study (HOME-HF): A randomised controlled trial of home telemonitoring of heart failure patients at high risk of readmission and death (Abstract \#197). Heart 2008;94:A106-7.

Dar OA, Riley JA, Chapman C, Dubrey S, Rosen S, Morris S, et al. 2341 Telemonitoring in an elderly, urban, multi-ethnic population: results of a UK multi-centre randomised controlled trial. The Home Heart Failure (HOME-HF) study. European Heart Journal 2008;29:p381.

Riley J, Cowie MR. Telemonitoring in heart failure. Heart 2009;95(23):1964-8.

Riley J, Dar O, Gabe J, Cowie MR. 1400 Telemonitoring in heart failure: changing roles and challenging relationships. The HOME-HF study. European Journal of Cardiovascular Nursing 2008;7(Suppl 1):S58.

Riley JP, Dar OA, Chapman C, Dubrey SW, Rosen SD, Roughton M, et al. 626 The HOME-HF Study: A multi-centre randomised controlled trial of home telemonitoring in a general heart failure population of elderly patients with co-morbidity. European Journal of Heart Failure 2008;7(Suppl 1):152-3.

Deckwart 2011 \{published data only\}

Deckwart O, Fiss G, Laurisch A, Winkler S, Koehler F. P166 Analysis of the content of consultations between patients and nursing staff in the course of telemedical care for chronic heart failure. European Journal of Cardiovascular Nursing 2011;10:S29.

\section{Dedier 2008 \{published data only\}}

Dedier J, Migneault J, Heeren T, Friedman R. 4430: A culturallyadapted automated telecommunications system improves medication adherence and blood pressure control in urban African Americans. Circulation 2008;118:S888.
Deepak 2008 \{published data only\}

Deepak SM, Waywell CA, Khan WM, Coppinger T, Borg A, Harper LB, et al. 1036-152 Home uptitration of beta-blockers by heart failure clinic patients with telephone advice: a British Heart experience. Journal of the American College of Cardiology 2006;47(4 Suppl A):270A.

De Feo 2002 \{published data only\}

De Feo S, Opasich C. To educate or to communicate: a nonpharmacological treatment for the patient with heart failure? [Educare e comunicare: terapienon farmacologiche per il pazientecon scompenso cardiaco cronico?]. Monaldi Archives for Chest Disease 2002;58(1):52-3.

Delaney 2013 \{published data only\}

Delaney C, Apostolidis B, Bartos S, Morrison H, Smith Li, Fortinsky R. A randomized trial of telemonitoring and selfcare education in heart failure patients following home care discharge. Home Health Care Management \& Practice 2013;25:187-95.

\section{Del Sindaco 2007 \{published data only\}}

Del Sindaco D, Pulignano G, Minardi G, Apostoli A, Guerrieri L, Rotoloni M, et al. Two-year outcome of a prospective, controlled study of a disease management programme for elderly patients with heart failure. Journal of Cardiovascular Medicine 2007;8(5):324-9.

\section{Demarzo 2006 \{published data only\}}

Demarzo AP, Calvin JE. Using impedance cardiography as a decision aid for diagnosing and managing heart failure. Progress in Cardiovascular Nursing 2006;21(2):114.

De Vries 2011a \{published data only\}

de Vries AE, de Jong RM, van der Wal MH, Jaarsma T, van Dijk RB, Hillege HL. The value of INnovative ICT guided disease management combined with Telemonitoring in OUtpatient clinics for Chronic Heart failure patients. Design and methodology of the IN TOUCH study: a multicenter randomised trial. BMC Health Services Research 2011;11:167.

De Vries 2011b \{published data only\}

De Vries AE, Jaarsma T, Van Der Wal MHW, De Jong RM, Van Dijk RB, Hillege HL. State of the art of telemonitoring in Dutch heart failure clinics. European Journal of Cardiovascular Nursing 2011;11:S72.

\section{Dickens 2012 \{published data only\}}

Dickens C, Vuckovic K, Nehmer M, DiDomenico R, Kerbow D, Stamos $\mathrm{T}$, et al. Improving the heart failure readmission rate at an urban medical center. Western Journal of Nursing Research 2012;34:1066-7.

\section{Dickerson 2011 \{published data only\}}

Dickerson A, Alexander C, Bucher G, Holt J. Christiana care visiting nurse association telehealth program: A work in progress. Heart \& Lung 2011;40:373-4.

Dimmick 2003 \{published data only\}

Dimmick SL, Burgiss SG, Robbin S, Black D, Jarnagin B, Andres M. Outcomes of an integrated telehealth network 
demonstration project. Telemedicine and Journal of E-Health 2003;9:13-23.

\section{Dollard 2004 \{published data only\}}

Dollard J, Smith J, Thompson DR, Stewart S. Broadening the reach of cardiac rehabilitation to rural and remote Australia. European Journal of Cardiovascular Nursing 2004;3(1):27-42.

\section{Dougherty 2005 \{published data only\}}

Dougherty CM, Thompson EA, Lewis FM. Long-term outcomes of a telephone intervention after an ICD. Pacing and Clinical Electrophysiology 2005;28(11):1157-67.

\section{Doughty 2002 \{published data only\}}

Doughty RN, Wright SP, Pearl A, Walsh HJ, Muncaster S, Whalley GA, et al. Randomized, controlled trial of integrated heart failure management: the Auckland Heart Failure Management Study. European Heart Journal 2002;23(2):139-46.

Downey 2001 \{published data only\}

Downey C. Disease management uses web to net savings. Managed Care Magazine http://www.managedcaremag.com/ archives/0107/0107.online_dm.html 2001 (accessed 16 June 2010).

\section{Dracup 2012 \{published data only\}}

Dracup K, Moser D, Pelter M, Nesbitt T, Southard J, Paul S M, et al. A focused heart failure self-care intervention reduces cardiac deaths in rural patients. Circulation 2012; Vol. 126: A10687.

\section{Ducharme 2005 \{published data only\}}

Ducharme A, Doyon O, White M, Rouleau JL, Brophy JM. Impact of care at a multidisciplinary congestive heart failure clinic: a randomised trial. Canadian Medical Association Journal 2005;173(1):40-5.

\section{Duffy 2005 \{published data only\}}

Duffy JR, Hoskins LM, Dudley-Brown S. Development and testing of a caring-based intervention for older adults with heart failure. Journal of Cardiovascular Nursing 2005;20(5):325-33.

\section{Duffy 2008 \{published data only\}}

Duffy JR, Hoskins LM. Research challenges and lessons learned from a heart failure tele-homecare study. Home Healthcare Nurse 2008;26(1):58-65.

\section{Duffy 2010 \{published data only\}}

Duffy JR, Hoskins LM, Dudley-Brown S. Improving outcomes for older adults with heart failure: a randomized trial using a theory-guided nursing intervention. Journal of Nursing Care Quality 2010;25(1):56-64.

\section{Dunagan 2005 \{published data only\}}

Dunagan C, Littenberg B, Ewald GA, Jones CA, Emery VB, Waterman BM, et al. Randomised trial of a nurse-administered, telephone-based diseased management program for patients with heart failure. Journal of Cardiac Failure 2005;11(5):358-65.

\section{Dunn 2006 \{published data only\}}

Dunn P, Gambetta M, Nelson D, Herron B, Arena R. Impact of a heart failure disease management program on hospitalizations. Journal of Cardiac Failure 2006;12(6):S125.

\section{Dunn 2007 \{published data only\}}

Dunn P, Gambetta M, Nelson D, Herron B, Arena R. Reduction of B-type natriuretic peptide using tele-management in patients with heart failure. Journal of Cardiac Failure 2007;13(6):S187.

Dwinger 2013 \{published data only\}

Dwinger S, Dirmaier J, Herbarth L, Konig HH, Eckardt M, Kriston L, et al. Telephone-based health coaching for chronically ill patients: Study protocol for a randomized controlled trial. Trials [Electronic Resource] 2013; Vol. 14:337.

\section{Ekman 1998 \{published data only\}}

Ekman I, Andersson B, Ehnfors M, Matejka G, Persson B, Fagerberg B. Feasibility of a nurse-monitored, outpatient-care programme for elderly patients and moderate-to server, chronic heart failure. European Heart Journal 1998;19(8):1254-60.

Ellery 2006 \{published data only\}

Ellery S, Pakrashi T, Paul V, Sack S. Predicting mortality and rehospitalization in heart failure patients with home monitoring: the Home CARE pilot study. Clinical Research in Cardiology 2006;95(Suppl 3):iii29-35.

\section{Enemuoh 2013 \{published data only\}}

Enemuoh Chiamaka J. Implementation of telehealth for rural patients with congestive heart failure: Evaluation of healthcare access and outcome. College of St. Scholastica 2013; Vol. D.N.P.:101 p.

\section{Enjuanes 2013a \{published data only\}}

Enjuanes Grau C, Comin Colet J, Verdu Rotellar JM, Gonzalez RG, Linas AA, Ruiz RP, et al. Evaluation of the efficacy and costs impact of a telemonitoring and teleintervention trough videoconference program for patients with chronic heart failure: A randomized controlled trial. European Heart Journal 2013;34:941.

Erich 2013a \{published data only\}

Erich J. MIH Approach Proves Positive for Chicago CHF Trial. EMS World 2013;42:32-7.

\section{Erich 2013b \{published data only\}}

Erich J. Chicago MIH Effort Targets CHF Patients. EMS World 2013;42:18-20.

\section{Esposito 2008 \{published data only\}}

Esposito D, Brown R, Chen A, Schore J, Shapiro R. Impacts of a disease management program for dually eligible beneficiaries. Health Care Financing Review 2008;30(1):27-45.

\section{Evangelista 2004 \{published data only\}}

Evangelista LS, Stromberg A, Westlake C, Ter-Galstanyan A, Anderson N, Dracup K. Developing a web-based education and counselling program for heart failure patients. Progress in Cardiovascular Nursing 2006;21(4):196-201. 
Fairbrother 2014 \{published data only\}

Fairbrother P, Ure J, Hanley J, McCloughan L, Denvir M, Sheikh A, et al. Telemonitoring for chronic heart failure: the views of patients and healthcare professionals - a qualitative study. Journal of Clinical Nursing 2014;23:132-44.

Falces 2008 \{published data only\}

Falces C, López-Cabezas C, Andrea R, Arnau A, Ylla M, Sadurní J. [An educative intervention to improve treatment compliance and to prevent readmissions of elderly patients with heart failure]. Medicina Clinica 2008;131(12):452-6.

\section{Fan 2010 \{published data only\}}

Fan HH, Shi HY, Jin W, Zhu YJ, Huang DN, Yan YW, et al. Effects of integrated disease management program on the outcome of patients with heart failure. Zhonghua Xin Xue Guan Bing Za Zhi (Chinese Journal of Cardiology) 2010;38(7):592-6.

Feldman 2004 \{published data only\}

Feldman PH, Peng TR, Murtaugh CM, Kelleher C, Donelson SM, McCann ME, et al. A randomised intervention to improve heart failure outcomes in community-based home health care. Home Health Care Services Quarterly 2004;23(1):1-23.

Feldman 2005 \{published data only\}

Feldman PH, Murtaugh CM, Pezzin LE, McDonald MV, Peng TR. Just-in-time evidence-based e-mail "reminders" in home health care: impact on patient outcomes. Health Services Research 2005;40(3):865-85.

\section{Ferguson 2010 \{published data only\}}

Ferguson G, Quinn J, Horwitz C, Swift M, Allen J, Galescu L. Towards a Personal Health Management Assistant. Journal of Biomedical Informatics 2010;43:S13-6.

\section{Finkelstein 2004 \{published data only\}}

Finkelstein SM, Speedie SM, Demiris G, Veen M, Lundgren JM, Potthoff S. Telehomecare: quality, perception, satisfaction. Telemedicine Journal and E-Health 2004;10(2):122-8.

\section{Finkelstein 2006 \{published data only\}}

Finkelstein SM, Speedie SM, Potthoff S. Home telehealth improves clinical outcomes at lower cost for home healthcare. Telemedicine Journal and E-Health 2006;12(2):128-36.

\section{Finkelstein 2010a \{published data only\}}

Finkelstein J, Cha E, Dennison C R. Exploring feasibility of home telemanagement in African Americans with congestive heart failure. MEDINFO 2010: Proceedings of the 13th World Congress on Medical Informatics, Part 1. Studies in Health Technology \& Informatics 2010;160:535-9.

\section{Finkelstein 2010b \{published data only\}}

Finkelstein J, Dennison CR. A Pilot Study of Home Automated Telemanagement (HAT) System in African Americans with Congestive Heart Failure. eHealth, Telemedicine, and Social Medicine, 2010 ETELEMED '10 Second International Conference. 2010:90-4.

\section{Finkelstein 2011 \{published data only\}}

Finkelstein J, Wood J. Implementing home telemanagement of congestive heart failure using Xbox gaming platform. 33rd Annual International Conference of the IEEE EMBS. 2011:3158-63.

\section{Florea 2011 \{published data only\}}

Florea VG, Anand IS. Clinical trial report: Reevaluating telemonitoring in heart failure. Current Heart Failure Reports 2011;8:84-6.

\section{Foley 2008 \{published data only\}}

Foley S. Heart Failure study: nurse-led management proves disheartening. American Journal of Nursing 2008;108(5):19-20.

Fragrasso 2007 \{published data only\}

Fragasso G, Cuko A, Spoladore R, Montano C, Palloshi A, Silipigni $C$, et al. Validation of remote cardiopulmonary examination in patients with heart failure with a videophonebased system. Journal of Cardiac Failure 2007;13(4):281-6.

Friedberg 2008 \{published data only\}

Friedberg MW. Nurse-led counselling had no effect on heart failure outcomes. Journal of Clinical Outcomes Management 2008;15(4):170-1.

\section{Fursse 2008 \{published data only\}}

Fursse J, Clarke M, Jones R, Khemka S, Findlay G. Early experience in using telemonitoring for the management of chronic disease in primary care. Journal of Telemedicine and Telecare 2008;14(3):122-4

\section{Gambetta 2007 \{published data only\}}

Gambetta M, Dunn P, Nelson D, Herron B, Arean R. Impact of the implementation of tele-management on a disease management program in an elderly heart failure cohort. Progress in Cardiovascular Nursing 2007;22(FALL):196-200.

Gámez-López 2012 \{published data only\}

Gámez-López AL, Bonilla-Palomas JL, Anguita-Sánchez M, Castillo-Domínguez JC, Arizón del Prado JM, Suárez de Lezo J. [Effects of three different disease management programs on outcomes in patients hospitalized with heart failure: a randomized trial]. Medicina Clinica 2012;138(5):192-8.

\section{Garcia 2009 \{published data only\}}

Garcia Lizana F, Yanes Lopez V. Information and communications technologies in CHF management programmes. Organizational challenges and evidence status (Structured abstract). Agencia de Evaluacion de Tecnologias Sanitarias (AETS) 2009.

\section{Gellis 2012 \{published data only\}}

Gellis ZD, Kenaley B, McGinty J, Bardelli E, Davitt J, Ten Have T. Outcomes of a telehealth intervention for homebound older adults with heart or chronic respiratory failure: a randomized controlled trial. Gerontologist 2012;52:541-52.

\section{Gellis 2014 \{published data only\}}

Gellis ZD, Kenaley BL, Have TT. Integrated Telehealth Care for Chronic Illness and Depression in Geriatric Home Care Patients: 
The Integrated Telehealth Education and Activation of Mood (ITEAM) Study [2014]. Journal of the American Geriatrics Society 2014;62(5):889-95.

\section{Giamouzis 2012 \{published data only\}}

Giamouzis G, Mastrogiannis D, Koutrakis K, Karayannis G, Parisis C, Rountas C, et al. Telemonitoring in chronic heart failure: a systematic review. Cardiology Research \& Practice 2012;2012:410820.

\section{Giordano 2013 \{published data only\}}

Giordano A, Scalvini S, Paganoni AM, Baraldo S, Frigerio M, Vittori C, et al. Home-Based Telesurveillance Program in Chronic Heart Failure: Effects on Clinical Status and Implications for 1Year Prognosis. Telemedicine and E-Health 2013;19:605-12.

\section{Goldstein 2014 \{published data only\}}

Goldstein CM, Gathright EC, Dolansky MA, Gunstad J, Sterns A, Redle JD, et al. Randomized controlled feasibility trial of two telemedicine medication reminder systems for older adults with heart failure. Journal of Telemedicine and Telecare 2014;20(6):293-9.

\section{Grancelli 2007 \{published data only\}}

Grancelli HO, Ferrante DC. Telephone interventions for disease management in heart failure. BMJ 2007;334:910-1.

\section{Granger 2013 \{published data only\}}

Granger BB, Bosworth H, Hernandez A, Reed S, Ekman I. Results of the chronic heart failure intervention to improve medication adherence (CHIME) study: A randomized self-management intervention in high risk non-adherent patients. Circulation 2013;128:A18245.

\section{Graves 2013 \{published data only\}}

Graves BA, Ford CD, Mooney KD. Telehealth technologies for heart failure disease management in rural areas: an integrative review. Online Journal of Rural Nursing \& Health Care 2013;13:56-83.

\section{Gregory 2006 (SPAN-CHF) \{published data only\}}

Gregory D, Kimmelstiel C, Perry K, Parikh A, Konstam V, Konstam MA. Hospital cost effect of a heart failure disease management program: The Specialized Primary and Networked Care in Heart Failure (SPAN-CHF) trial. American Heart Journal 2006;151(5):1013-8.

\section{Grustam 2014 \{published data only\}}

Grustam AS, Severens JL, van Nijnatten J, Koymans R, Vrijhoef HJM. Cost-effectiveness of telehealth interventions for chronic heart failure patients: a literature review. International Journal of Technology Assessment in Health Care 2014;30:59-68.

\section{Gund 2008 \{published data only\}}

Gund A, Ekman I, Lindecrantz K, Sjoqvist BA, Staaf EL, Thorneskold N. Design evaluation of a home-based telecare system for chronic heart failure patients. Proceedings of the 2008 IEEE Engineering in Medicine and Biology Society Conference 2008:5851-4.

\section{Gurne 2012 \{published data only\}}

Gurne O, Conraads V, Missault L, Mullens W, Vachierys JL, Van Mieghem W, et al. A critical review on telemonitoring in heart failure. Acta Cardiologica 2012;67:439-44.

\section{Hall 2010 \{published data only\}}

Hall P, Morris M. Improving heart failure in home care with chronic disease management and telemonitoring. Home Healthcare Nurse 2010;28:606-19.

Hanssen 2007 \{published data only\}

Hanssen TA, Nordrehaug JE, Eide GE, Hanestad BR. Can telephone follow-up after discharge improve lifestyle factors after a myocardial infarction? A randomised controlled trial. European Journal of Cardiovascular Nursing 2007;6(Suppl 1):S43-S44.

\section{Harkness 2006 \{published data only\}}

Harkness K. A telephone intervention reduced combined all cause mortality or admission for worsening heart failure in chronic heart failure. Evidence Based Nursing 2006;9(2):55.

\section{Harrison 2002 \{published data only\}}

Harrison MB, Browne GB, Roberts J, Tugwell P, Gafni A, Graham ID. Quality of life of individuals with heart failure: a randomised trial of the effectiveness of two models of hospitalto-home transition. Medical Care 2002;40(4):271-82.

\section{Hart-Wright 2006 \{published data only\}}

Hart-Wright J, Patrick S, He H, Green J, Rierson M, Teal G, et al. Effectiveness of a nurse-led telephonic support program for heart failure patients. Progress in Cardiovascular Nursing 2006;21(2):111.

Hayes, 2008 \{published data only\}

Hayes, Inc. Remote monitoring of patients with congestive heart failure (Structured abstract). HAYES, Inc 2008.

Hayes, 2011 \{published data only\}

Hayes, Inc. Mobile cardiac outpatient telemetry (MCOT) (CardioNet ambulatory ECG monitor; CardioNet Inc.) for home monitoring of cardiac patients (Structured abstract). HAYES, Inc 2011.

\section{Heidenreich 1999 \{published data only\}}

Heidenreich PA, Ruggerio CM, Massie BM. Effect of a home monitoring system on hospitalisation and resource use for patients with heart failure. American Heart Journal 1999;138:633-40.

\section{Heisler 2007 \{published data only\}}

Heisler M, Halasyamani L, Resnicow K, Neaton M, Shanahan J, Brown S, et al. "I am not alone": the feasibility and acceptability of interactive voice response-facilitated telephone peer support among older adults with heart failure. Congestive Heart Failure 2007;13:149-57.

\section{Helms 2007 \{published data only\}}

Helms TM, Pelleter JT, Ronneberger DL. Telemetric care of patients suffering from chronic heart failure with special reference to the telemetric care and education program 
"Telemedizin fürs Herz" ("Telemedicine for the heart"). Herz 2007;32(8):623-9.

\section{Helms 2010 \{published data only\}}

Helms TM, Pelleter J, Kroettinger A, Budych K, Thoden E, Sohn S, et al. Advantages and restrictions of telemonitoring in chronic heart failure - experiences and results from the integrated care concept telemedicine for the heart. European Heart Journal 2010;31((Abstract Suppl)):P1443. 226-227.

Hennrikus 2012 \{published data only\}

Hennrikus E. Communication between the primary care physician and the hospitalist at the time of patient admission. Journal of Clinical Outcomes Management 2012;19:453-9.

\section{Hindricks 2014 \{published data only\}}

Hindricks G, Taborsky M, Glikson M, Heinrich U, Schumacher B, Katz A, et al. IN-TIME Study Group. Implant-based multiparameter telemonitoring of patients with heart failure (IN-TIME): a randomised controlled trial. Lancet 2014;384(9943):583-90.

\section{Hinterbuchner 2010 \{published data only\}}

Hinterbuchner L, Fritsch MF. CardioMobile home care for congestive heart failure. European Journal of Cardiovascular Nursing 2010;9:S30.

\section{Ho 2007 \{published data only\}}

Ho YL, Hsu TP, Chen CP, Lee CY, Lin YH, Hsu RB, et al. Improved cost-effectiveness for management of chronic heart failure by combined home-based intervention with clinical nursing specialists. Journal of the Formosan Medical Association 2007;106(4):313-9.

\section{Hoban 2013 \{published data only\}}

Hoban MB, Fedor M, Reeder S, Chernick M. The effect of telemonitoring at home on quality of life and self-care behaviors of patients with heart failure. Home Healthcare Nurse 2013;31(7):368-77.

\section{Holland 2014 \{published data only\}}

Holland DE, Vanderboom CE, Ingram CJ, Dose AM, Borkenhagen LS, Skadahl P, et al. The feasibility of using technology to enhance the transition of palliative care for rural patients. Computers, Informatics, Nursing: CIN 2014;32(6):257-66.

\section{Holly 2011 \{published data only\}}

Holly C. Structured telephone support or telemonitoring programmes for patients with chronic heart failure. International Journal of Evidence-Based Healthcare 2011;9:274-5.

\section{Holst 2007 \{published data only\}}

Holst M, Willen Heimer R, Mårtensson J, Lindholm M, Strömberg A. Telephone follow-up of self-care behaviour after a single session education of patients with heart failure in primary health care. European Journal of Cardiovascular Nursing 2007;6(2):153-9.
Hoover 2007 \{published data only\}

Hoover CA, Simones J, Wilson R, Greenlee K, Schimnich M, Lilemoen J, et al. Abstract 2235: promoting self-management in heart failure patients through education and telemonitoring. Circulation 2007;116:II_486.

\section{Hoover 2009 \{published data only\}}

Hoover C. The difference in outcomes for the use of telemonitoring on elderly home care patients with a primary or secondary diagnosis of heart failure. Heart \& Lung 2009;38:262.

Howlett 2011 \{published data only\}

Howlett JG, Marr D, Palmer K, O'Neill BJ, Rajda M. A comparison of primary care, home-based telemonitoring, telemonitoring with video, or specialist directed heart failure clinic care for patients with high risk heart failure. Circulation 2011;124:A17680.

\section{Hudson 2005 \{published data only\}}

Hudson LR, Hamar B, Orr P, Johnson JH, Neftzger A, Chung RS, et al. Remote physiological monitoring: clinical, financial and behavioural outcomes in a heart failure population. Disease Management 2005;5:372-81.

\section{Huynh 2006 \{published data only\}} Huynh BC, Rovner A, Rich MW. Long-term survival in elderly patients hospitalized for heart failure. Archives of Internal Medicine 2006;166:1892-8.

\section{Jaarsma (COACH Study) \{published data only\}}

Jaarsma T, Van Veldhuisen DJ, Van der Wal MHL. NHF$\mathrm{COACH}$ multicenter trial in the Netherlands: searching for underlying potentially beneficial mechanisms in nurse led heart failure management. Progress in Cardiovascular Nursing 2002;17(2):96-8.

Jaarsma T, Van der Wal MHL, Hogenhuis J, Lesman I, Luttik MLA, Veeger NJGM, et al. Design and methodology of the $\mathrm{COACH}$ study: a multicenter randomised Coordinating study evaluating Outcomes of Advising and Counselling in Heart failure. European Journal of Heart Failure 2004;6(2):227-33.

* Jaarsma T, Van der Wal MHL, Lesman-Leegte I, Luttik ML, Hogenhuis J, Veeger $\mathrm{NJ}$, et al. Effect of moderate or intensive disease management program on outcome in patients with heart failure: Coordinating study evaluating Outcomes of Advising and Counseling in Heart failure (COACH). Archives of Internal Medicine 2008;168(3):316-24.

\section{Jaarsma 1999 \{published data only\}}

Jaarsma T. Nurse led, multidisciplinary intervention in chronic heart failure. Heart 1999;81:676.

\section{Jenkins 2001 \{published data only\}}

Jenkins RL, McSweeney M. Assessing elderly patients with congestive heart failure via in-home interactive telecommunication. Journal of Gerontological Nursing 2001;27(1):21-7. 


\section{Jerant 2001 \{published data only\}}

Jerant AF, Azari R, Nesbit TS. Reducing the cost of frequent hospital admissions for congestive heart failure: a randomized trial of a home telecare intervention. Medical Care 2001;39(11):1234-45.

\section{Jerant 2003 \{published data only\}}

Jerant AF, Azari R, Martinez C, Nesbitt TS. A randomised trial of tele-nursing to reduce hospitalizations for heart failure: patientcentred outcomes and nursing indicators. Home Health Care Services Quarterly 2003;22(1):1-20.

\section{Johnston 2000 \{published data only\}}

Johnston B, Wheeler L, Deuser J, Sousa KH. Outcomes of the Kaiser Permanente Tele-Home Health Research Project. Archives of Family Medicine 2000;9(1):40-5.

\section{Jolly 2007 \{published data only\}}

Jolly K, Tayor RS, Lip GYH, Greenfield SM, Davies MK, Davies RC, et al. Home-based exercise rehabilitation in addition to specialist heart failure nurse care: design, rationale and recruitment to the Birmingham Rehabilitation Uptake Maximisation study for patients with congestive heart failure (BRUM-CHF): a randomised controlled trial. BMC Cardiovascular Disorders 2007;7:9.

\section{Jones 2002 \{published data only\}}

Jones JF, Brennan PF. Telehealth interventions to improve clinical nursing of elders. In: Archbold PG, Stewart BJ, Lyons KS editor(s). Annual Review of Nursing Research. New York: Springer, 2002:293-322.

\section{Jones 2014 \{published data only\}}

Jones A, Hedges-Chou J, Bates J, Loyola M, Lear S A, JarvisSelinger S. Home Telehealth for Chronic Disease Management: Selected Findings of a Narrative Synthesis. Telemedicine and EHealth 2014;20:346-80.

\section{Juan 2011 \{published data only\}}

Juan C, Sanjoaquın AC, Lopez M, Romeroa D, Pinilla R, Ochoa P. Presentation of a frail elderly telemonitoring service lessons learned in the Barbarstro health care area. European Geriatric Medicine 2011;2(1):S24-206.

\section{Karlsson 2005 \{published data only\}}

Karlsson MR, Edner M, Henrisksson P, Mejhert M, Persson H, Grut $\mathrm{M}$, et al. A nurse-based management program in heart failure patients affects females and persons with cognitive dysfunction most. Patient Education and Counseling 2005;58(2):146-53.

\section{Kashem 2007 \{published data only\}}

Kashem A, Droogan MT, Santamore WP, Wald JW, Bove AA. Managing heart failure care using an internetbased telemedicine system. Journal of Cardiac Failure 2008;14(2):121-6.

Kashem A, Droogan MT, Santamore WP, Wald JW, Marble JF, Cross RC, et al. Web-based internet telemedicine management of patients with heart failure. Telemedicine and e-Health 2006;12(4):439-47.
* Kashem A, Santamore WP, Cross RC, Homko CJ, Zamora L, Berger PT, et al. 1018-192 Preliminary outcome of web-based telemedicine clinical trial for disease management: comparison of 4-versus 8-months follow-up visits. Journal of the American College of Cardiology 2007;49(9 Suppl A):285A-286A.

Santamore WP, Homko C, Marble J, Wald J, Bove AA. Improving heart failure care by using a telemedicine system. Conference Proceedings of the Annual International Conference of the IEEE Engineering in Medicine and Biology Society 2004;4:3076-9.

\section{Kasper 2002 \{published data only\}}

Kasper EK, Gerstenblith G, Hefter G, Van Anden E, Brinker JA, Terrin $\mathrm{M}$, et al. A randomised trial of the efficacy of multidisciplinary care in heart failure outpatients at high risk of hospital readmission. Journal of the American College of Cardiology 2002;39(3):471-80.

\section{Kastner 2010 \{published data only\}}

Kastner P, Morak J, Modre R, Kollmann A, Ebner C, Fruhwald F, et al. Innovative telemonitoring system for cardiology: from science to routine operation. Applied Clinical Informatics 2010;1:165-76.

\section{Khoury 2008 \{published data only\}}

Khoury DS, Naware M, Siou J, Mathuria NS, Wang J, Shih HT, et al. Abstract 820: usefulness of monitoring in congestive heart failure by multiple bioelectric impedance vectors. 2008 Circulation;118:S_618.

\section{Kielblock 2007 \{published and unpublished data\}}

Kielblock B, Frye Ch, Kottmair S, Hudler T, Siegmund-Schultze E, Middeke M. Impact of telemetric management on overall treatment costs and mortality rate among patients with chronic heart failure [Einfluss einer telemedizinisch unterstützten Betreuung auf Gesamtbehandlungskosten und Mortalität bei chronischer Herzinsuffizienz]. Deutsche Medizinische Wochenschrift 2007;132(9):417-22.

\section{Kimmelsteil 2004 \{published data only\}}

Kimmelstiel C, Levine D, Perry K, Patel AR, Sadaniantz A, Gorham N, et al. Randomized, controlled evaluation of shortand long-term benefits of heart failure disease management within a diverse provider network: the SPAN-CHF trial. Circulation 2004;110(11):1450-5.

\section{Kirschner 2006 \{published data only\}}

Kirschner L, Cram N. The benefits of a tele-home health initiative on an elderly population. Journal of Clinical Psychopharmacology 2006; January/March:40-5.

\section{Kitsiou 2013 \{published data only\}}

Kitsiou S, Paré G, Jaana M. Systematic reviews and metaanalyses of home telemonitoring interventions for patients with chronic diseases: a critical assessment of their methodological quality. Journal of Medical Internet Research 2013;15:e150.

Kleet 2013 \{published data only\}

Kleet AC, Aron MA, Henry A, Fearon-Clarke J, Giordano JI, Roman $\mathrm{L}$, et al. Leveraging novel technologies: Risk 
stratification and care coordination. Journal of Cardiac Failure 2013;1:S82-3.

\section{Kline 2006 \{published data only\}}

Kline S. Telehealth and care coordination improves outcomes for veterans with heart failure. Progress in Cardiovascular Nursing 2006;Spring:111.

\section{Koehler 2006 \{published data only\}}

Koehler R, Anker SD. Noninvasive home telemonitoring:the Trans-European Network-Home-Care Management System. Journal of the American College of Cardiology 2006;48(4):850-1.

Koelling 2005 \{published data only\}

Koelling TM, Johnson ML, Cody RJ, Aaronson KD. Discharge education improves clinical outcomes in patients with chronic heart failure. Circulation 2005;111(2):179-85.

\section{Konstam 2012 \{published data only\}}

Konstam MA. Home monitoring should be the central element in an effective program of heart failure disease management. Circulation 2012;125:820-7.

\section{Kottmair 2005 \{published data only\}}

Kottmair S, Frye C, Ziegenhagen DJ. Germany's disease management program: improving outcomes in congestive heart failure. Health Care Financing Review 2005;27(1):79-87.

Koutkias 2003 \{published data only\}

Koutkias VG, Chouvarda I, Maglaveras N. Multi agent system architecture for heart failure management in a home care environment. Computers in Cardiology 2003;30:383-6.

\section{Kraal 2014 \{published data only\}}

Kraal JJ, Peek N, Van den Akker-Van Marle ME, Kemps HMC. Effects of home-based training with telemonitoring guidance in low to moderate risk patients entering cardiac rehabilitation: short-term results of the FIT@Home study. European Journal of Preventive Cardiology 2014;21:26-31.

\section{Kropf 2014 \{published data only\}}

Kropf M, Modre-Osprian R, Hayn D, Fruhwald F, Schreier G. Telemonitoring in heart failure patients with clinical decision support to optimize medication doses based on guidelines. Engineering in Medicine and Biology Society. 2014:3168-71.

\section{Krumholz 2002 \{published data only\}}

Krumholz HM, Amatruda J, Smith GL, Mattera JA, Roumanis SA, Radford MJ, et al. Randomised trial of an education and support intervention to prevent readmission of patients with heart failure. Journal of the American College of Cardiology 2002;39(1):84-9.

\section{Krumholz 2011 \{published data only\}}

Krumholz HM. Telemonitoring did not reduce readmissions or mortality in patients recently hospitalized for heart failure. Annals of Internal Medicine 2011;154:JC3-8.

\section{Kurtz 2011 \{published data only\}}

Kurtz B, Lemercier M, Pouchin SC, Benmokhtar E, Vallet C, Cribier A, et al. Automated home telephone self-monitoring reduces hospitalization in patients with advanced heart failure. Journal of Telemedicine and Telecare 2011;17:298-302.

Kutzleb 2006 \{published data only\}

Kutzleb J, Reiner D. The impact of nurse-directed patient education on quality of life and functional capacity in people with heart failure. Journal of the American Academy of Nurse Practitioners 2006;18(3):116-23.

Kwok 2008 \{published data only\}

Kwok T, Lee J, Woo J, Lee DTF, Griffith S. A randomised controlled trial of a community nurse-supported hospital discharge programme in older patients with chronic heart failure. Journal of Clinical Nursing 2008;17(1):109-17.

\section{LaFramboise 2003 \{published data only\}}

LaFramboise LM, Todero CM, Zimmerman L, Agrawal S. Comparison of Health Buddy with traditional approaches to heart failure management. Family Community Health 2003;4:275-88

\section{Lagido 2014 \{published data only\}}

Lagido RB, Lobo J, Leite S, Sousa C, Ferreira L, Silva-Cardoso J. Using the smartphone camera to monitor heart rate and rhythm in heart failure patients. Biomedical and Health Informatics. 2014:556-9.

\section{Lavenberg 2012 \{published data only\}}

Lavenberg J G, Williams K, Behta M. Reducing heart failure readmissions (Structured abstract). Health technology Assessment Database. Center for Evidence-based Practice (CEP), 2012.

\section{Lehmann 2006 \{published data only\}}

Lehmann CA, Mintz N, Giacini JM. Impact of telehealth on healthcare utilization by congestive heart failure patients. Disease Management and Health Outcomes 2006;14(3):163-9.

Lemay 2013 \{published data only\}

Lemay G, Azad N, Struthers C. Utilization of home telemonitoring in patients 75 years of age and over with complex heart failure. Journal Of Telemedicine And Telecare 2013; Vol. 19:18-22.

\section{Leventhal 2011 \{published data only\}}

Leventhal ME, Denhaerynck K, Brunner-La Rocca HP, Burnand B, Conca-Zeller A, Bernasconi AT, et al. Swiss Interdisciplinary Management Programme for Heart Failure (SWIM-HF): a randomised controlled trial study of an outpatient interprofessional management programme for heart failure patients in Switzerland. Swiss Medical Weekly 2011;141:w13171.

\section{Lind 2013 \{published data only\}}

Lind L, Karlsson D. Digital pen-based telemonitoring of elderly heart failure patients. MEDINFO 2013. Studies in Health Technology \& Informatics 2013;192:1062.

Lucas 2007 \{published data only\}

Lucas CMHB, Cleuren GVJ. P622 telephone support by heart failure nurses has a significant impact on readmission rate and survival. European Heart Journal 2007;28(Suppl 1):85. 
Machingo 2003 \{published data only\}

Machingo KA, Woods LA, Parish L. CHF outcomes following implementation of telephone follow-up monitoring. Journal of Cardiac Failure 2003;9(5 Suppl 1):S82.

\section{Maddukuri 2006 \{published data only\}}

Maddukuri P, Woods P, Joseph J, Aragam J, McIntyre K, Sharma GVRK. Abstract 2953: Non-invasive haemodynamic assessment by Vericor reveals heart failure management by clinical assessment alone is sub-optimal in ambulatory patients and leads to increased heart failure hospitalisations. Circulation 2006;114:II_621.

\section{Madigan 2008 \{published data only\}}

Madigan EA. People with heart failure and home health care resource use and outcomes. Journal of Nursing and Healthcare of Chronic Illness in association with the Journal of Clinical Nursing 2008;17(7b):253-9.

\section{Madigan 2013 \{published data only\}}

Madigan E, Schmotzer BJ, Struk CJ, DiCarlo CM, Kikano G, Piña IL, et al. Home health care with telemonitoring improves health status for older adults with heart failure. Home Health Care Services Quarterly 2013;32(1):57-74.

\section{Maglaveras 2002 \{published data only\}}

Maglaveras N, Gogou G, Chouvarda I, Koutkias V, Lekka I, Adamidis $\mathrm{D}$, et al. Using contact centres in tele-management and home care of congestive heart failure patients: the CHS experience. Computers in Cardiology 2002;29:281-4.

\section{Maglaveras 2003 \{published data only\}}

Maglaveras N, Lekka I, Chouvarda I, Adamidis D, Karvounis H, Louridas $\mathrm{G}$, et al. Congestive heart failure management in a home-care system through the CHS contact centre. Computers in Cardiology 2003;30:189-92.

\section{Maglaveras 2006 \{published data only\}}

Maglaveras N, Maglavera S, Lekka I, Chouvarda I, Kaimakamis V, Kilintzis V, et al. Quality home telemedicine services for chronic cardiac disease patients through the INTERLIFE platform. Computers in Cardiology 2006;33:245-8.

\section{Mair 2007 \{published data only\}}

Mair FS. Does remote monitoring improve outcome in patients with chronic heart failure?. Nature Clinical Practice Cardiovascular Medicine 2007;4(10):588-9.

\section{Makaya 2008 \{published data only\}}

Makaya M, Tsutsui H, Hamaguchi S, Kingugawa S, Yokota T, Goto D, et al. Beta-blocker use at discharge in patients hospitalized for heart failure is associated with improved survival: an analysis from JCARE-CARD. Journal of Cardiac Failure 2008;14(7 Suppl):S167.

\section{Mansfield 2006 \{published data only\}}

Mansfield W. Thesis: A feasibility study examining telecardiology in New Hampshire: telemonitoring in patients with congestive heart failure. Dartmouth College 2006.

\section{Marangelli 2007 \{published data only\}}

Marangelli V, Giorgio A, Sorgente L, Lepera ME, Di Summa, Perri AG, et al. 4522 High quality heart and lung ascultation using a web-based mobile home-telecare equipment for heart failure monitoring. European Heart Journal 2007;28(Suppl 1):788.

\section{Maric 2010 \{published data only\}}

Maric B, Kaan A, Araki Y, Ignaszewski A, Lear S A. The Use of the Internet to Remotely Monitor Patients with Heart Failure. Telemedicine Journal and E-Health 2010;16:26-33.

\section{Martensson 2005 \{published data only\}}

Mårtensson J, Strömberg A, Dahlström U, Karlsson JE, Fridlund B. Patients with heart failure in primary health care: effects of a nurse-led intervention on health-related quality of life and depression. European Journal of Heart Failure 2005;7(3):393-403.

\section{Martín-Lesende 2011 \{published data only\}}

Martín-Lesende I, Orruño E, Cairo C, Bilbao A, Asua J, Romo MI, et al. Assessment of a primary care-based telemonitoring intervention for home care patients with heart failure and chronic lung disease. The TELBIL study. BMC Health Services Research 2011;11:56.

\section{Martín-Lesende 2013 \{published data only\}}

Martín-Lesende I, Orruño E, Bilbao A, Vergara I, Cairo MC, Bayon JC, et al. Impact of telemonitoring home care patients with heart failure or chronic lung disease from primary care on healthcare resource use (the TELBIL study randomised controlled trial). BMC Health Services Research 2013;13:118.

\section{Masterson Creber 2014 \{published data only\}}

Masterson Creber RM, Patey M, Riegel B. Motivational interviewing tailored intervention to promote heart failure self-care (MITI-HF): primary study results. Circulation 2014;130:A19245.

\section{Mau 2006 \{published data only\}}

Mau J, Kolk M, Pelon J, Frauenheim W, Johnson D, Culina J. Nurse-directed home-based heart failure management program decreases death/readmission rates and increases dietary and medication compliance. Progress in Cardiovascular Nursing 2006;21(2):112.

\section{McCauley 2006 \{published data only\}}

McCauley KM, Bixby MB, Naylor MD. Advanced practice nurse strategies to improve outcomes and reduce cost in elders with heart failure. Disease Management 2006;9(5):302-10.

\section{McCoy 2007 \{published data only\}}

McCoy ML, Davidhizar R, Gillum DR. AcCorrelational pilot study of home health nurse management of heart failure patients and hospital readmissions. Home Health Care Management \& Practice 2007;19(5):392-6.

McDonald 2002 \{published data only\}

McDonald K, Ledwidge M, Cahill J, Quigley P, Maurer B, Travers $\mathrm{B}$, et al. Heart failure management: multidisciplinary 
care has intrinsic benefit above the optimizations of medical care. Journal of Cardiac Failure 2002;8(3):142-8.

McEntee 2010 \{published data only\}

McEntee M L, Johnson B J, Dennison C R, Finkelstein J. Establishing feasibility of a telemanagement system to facilitate self care among African American heart failure patients... 2010 AAHFN 6th Annual Meeting, June 24-26, 2010. Heart \& Lung 2010;39:370-1.

\section{McKinstry 2014 \{published data only\}}

McKinstry Brian. Telemonitoring: the future for managing long-term conditions?. International Journal of Therapy \& Rehabilitation 2014;21:407.

\section{McManus 2004 \{published data only\}}

McManus SG. A telehealth program to reduce readmission rates among heart failure patients: one agency's experience. Home Mealth Care Management \& Practice 2004;16(4):250-4

\section{Mendoza 2002 \{published data only\}}

Mendoza GG. In-home wireless monitoring of physiological data for heart failure patients. Engineering in medicine and biology society. 24th Annual conference of the IEEE 2002;3:1849-50.

\section{Meriggi 2009 \{published data only\}}

Meriggi P, Rizzo F, Faini A, Chiarugi F, Karatzanis I, Zacharioudakis $\mathrm{G}$, et al. A new simple multimodal platform for home monitoring of cardiac patients through textile technology. Computers in Cardiology. 2009:93-6.

\section{Metten 2011 \{published data only\}}

Metten L, Zueca F, Haver Y, Neukirch B, Rauchhaus M. 22 Effects of intensified care for heart failure patients by telemonitoring. European Journal of Cardiovascular Nursing 2011;10:S26.

\section{Mistiaen 2006 \{published data only\}}

Mistiaen P, Poot E. Telephone follow-up, initiated by a hospital-based health professional, for post discharge problems in patients discharged from hospital to home. Cochrane Database of Systematic Reviews 2006, Issue 4. [DOI: 10.1002/14651858.CD004510.pub3]

\section{Mitchell 2011 \{published data only\}}

Mitchell M D, Norris A, Umscheid C A. Telemedicine for patients with congestive heart failure (Structured abstract). Health Technology Assessment Database. Center for Evidence-based Practice (CEP), 2011.

\section{Mitchell 2014 \{published data only\}}

Mitchell JS, McCormick J, Trumbo N, Horne K, Workman J, Lovgren M, et al. Using 30 day remote telemedicine to support heart failure patients following discharge. Heart \& Lung 2014;43:382.

\section{Moore 2013 \{published data only\}}

Moore C, Sanchez M, Mueller D, Reed M, Heithoff K. A program to assist transitions from hospital to home. Journal of Cardiac Failure 2013;1):S85.
Morales-Ascencio 2008 \{published data only\}

Morales-Asencio JM, Gonzalo-Jiménez E, Martin-Santos FJ, Morilla-Herrera JC M Celdráan-Mañas M, Millán Carrasco A, et al. Effectiveness of a nurse-led case management home care model in primary health care: a quasi-experimental, controlled, multi-centre study. BMC Health Services Research 2008;8:193.

Morcillo 2005 \{published data only\}

Morcillo C, Valderas JM, Aguado O, Delás J, Sort D, Pujadas R, et al. Evaluation of a home-based intervention in heart failure patients: results of a randomized study [Evaluación de una intervención domiciliaria en pacientes con insuficiencia cardíaca. Resultados de un estudio aleatorizado]. Revista Espaňola de Cardiologia 2005;58(6):618-25.

\section{Morgan 2014 \{published data only\}}

Morgan JM, Dimitrov BD, Gill J, Kitt S, Andre NG, McComb JM, et al. Rationale and study design of the REM-HF study: Remote management of heart failure using implanted devices and formalized follow-up procedures. European Journal of Heart Failure 2014; Vol. 16, issue 9:1039-45.

\section{Morguet 2006 \{published data only\}}

Morguet AJ. Impact of home-based monitoring on the care of patients with congestive heart failure. Home Health Care Management and Practice 2006:107.

\section{Morguet 2007a \{published data only\}}

Morguet AJ, Kuehnelt P, Kallel A, Jaster M, Schultheiss HP. 4521 Telemedical care and monitoring to reduce morbidity in patients with NYHA class II and III heart failure. European Heart Journal 2007;28(Suppl 1):788.

\section{Morguet 2007b \{published data only\}}

Morguet AJ, Kuehnelt P, Kallel A, Jaster M, Schultheiss HP. 295 Staged tele-medical service for patients with mild-to-moderate congestive heart failure. European Journal of Heart Failure Supplement 2007;6:63.

\section{Morguet 2008 \{published data only\}}

Morguet AJ, Kühnelt P, Kallel A, Jaster M, Schultheiss HP. Impact of tele medical care and monitoring on morbidity in mild to moderate chronic heart failure. Cardiology 2008;111(2):134-9.

\section{Mueller 2002 \{published data only\}}

Mueller TM, Vuckovic KM, Knox DA, Williams RE. Telemanagement of heart failure: a diuretic treatment algorithm for advanced practice nurses. Heart Lung 2002;31:340-7.

\section{Muller 2013 \{published data only\}}

Muller A, Goette A, Perings C, Nägele H, Konorza T, Spitzer W, et al. Potential role of telemedical service centers in managing remote monitoring data transmitted daily by cardiac implantable electronic devices: results of the early detection of cardiovascular events in device patients with heart failure (detecT-Pilot) study. Telemedicine Journal and E-Health 2013;19(6):460-6. 
Murtaugh 2005 \{published data only\}

Murtaugh CM, Pezzin LE, McDonald MV, Feldman PH, Peng TR. Just-in-time evidence-based e-mail "reminders" in home health care: impact on nurse practices. Health Services Research 2005;40(3):849-64.

\section{Myers 2006 \{published data only\}}

Myers S, Grant RW, Lugn NE, Holbert B, Kvedar JC. Impact of home-based monitoring on the care of patients with congestive heart failure. Home Health Care Managment \& Practice 2006;18(6):444-51.

\section{Nanevicz 2000 \{published data only\}}

Nanevicz T, Zipkin D, Ennis S, Modin G. The feasibility of a telecommunications service in support of outpatients congestive heart failure in a diverse patient population. Congestive Heart Failure 2008;6:140-5.

\section{Naylor 1999 \{published data only\}}

Naylor MD, McCauley KM. The effects of a discharge planning and home follow-up intervention on elders hospitalized with common medical and surgical cardiac conditions. Journal of Cardiovascular Nursing 1999;14(1):44-54.

\section{Naylor 2004 \{published data only\}}

Naylor MD, Brooten DA, Campbell RL, Maislin G, McCauley KM, Sanford Schwartz J. Transitional care of older adults hospitalized with heart failure: a randomized, controlled trial. Journal of the American Geriatrics Society 2004;52(5):675-84.

\section{Nguyen 2007 \{published data only\}}

Nguyen V, Ducharme A, White M, Racine N, O'Meara E, Zhang B, et al. Lack of long-term benefits of a 6-month heart failure disease management program. Journal of Cardiac Failure 2007;13(4):287-93.

\section{Nobel 2003 \{published data only\}}

Nobel JJ, Norman GK. Emerging information management technologies and the future of disease management. Disease Management 2003;6:219-31.

\section{Noel 2004 \{published data only\}}

Noel HC, Vogel DC, Erdos JJ, Cornwall D, Levin F. Home telehealth reduces healthcare costs. Telemedicine Journal and e-Health 2004;10(2):170-83.

\section{Nohria 2007 \{published data only\}}

Nohria A, Warner Stevenson L, Bourge R, Israeli D, Kueffer F, Zile M. Frequency and impact of hypervolemic states during monitored heart failure management. Abstract \#047. Journal of Cardiac Failure 2007;13(6 Suppl 2):S88.

\section{Nucifora 2006 \{published data only\}}

Nucifora G, Albanese MC, De Biaggio P, Caliandro D, Gregori D, Goss P, et al. Lack of improvement of clinical outcomes by a low-cost, hospital-based heart failure management programme. Journal of Cardiovascular Medicine 2006;7(8):614-22.

\section{O'Reilly 1999 \{published data only\}}

O'Reilly M. Is Internet-based disease management on the way?. Candian Medical Association Journal 1999;160:1039.

Oddone 1999 \{published data only\}

Oddone EZ, Weinberger M, Giobbie-Hurder A, Landsman P Henderson W. Enhanced access to primary care for patients with congestive heart failure: Veterans Affairs Cooperative Study Group on Primary Care and Hospital Readmission. Efficient Clinical Practice 1999;2:201-9.

\section{Odeh 2013 \{published data only\}}

Odeh B, Kayyali R, Nabhani S, Philip N, Wallace C, Wigmore B, et al. Impact of telehealth on patients' outcomes-A patients' perceptions evaluation study. International Journal of Pharmacy Practice 2013;21:104-5.

\section{Oeff 2005a \{published data only\}}

Oeff M, Kotsch P, Gösswald A, Wolf U. Telemonitoring of patients with heart failure using extended vital sign recording [Überwachung multipler Herzkreislaufparameter mittels Telemonitoring bei Patienten mit chronischer Herzinsuffizienz]. Herzschrittmachertherapie und Elektrophysiologie 2005;16(3):150-8.

\section{Oeff 2005b \{published data only\}}

Oeff M, Kotsch P, Gösswald A, Wolf U. Telemonitoing of multiple parameters in chronic heart failure achieves impressive success in clinical management. European Heart Journal 2005;26:360.

\section{Ojeda 2005 \{published data only\}}

Ojeda S, Anguita M, Delgado M, Atienza F, Rus C, Granados AL, et al. Short- and long-term results of a programme for the prevention of readmissions and mortality in patients with heart failure: are effects maintained after stopping the programme?. European Journal of Heart Failure 2005;7(5):921-6.

\section{Oliveira 2013 \{published data only\}}

Oliveira M, Fernandes M, Primo J, Reis H, Nicola P, Los investigadores do Estudo PORTLink. Remote versus face-toface monitoring for implantable cardiac devices: Rationale and design of the PORTLink (PORTuguese research on telemonitoring with CareLink). [Portuguese, English] Monitorizacao a distancia versus seguimento convencional presencial em portadores de dispositivos cardiacos implantados: Racional e desenho do estudo PORTLink (PORTuguese research on telemonitoring with CareLink). Revista Portuguesa de Cardiologia 2013;32(12):957-64.

\section{Opasich 2005 \{published data only\}}

Opasich G. Educating and communicating: non-pharmacologic treatment for patients with chronic heart failure? [Educare e comunicare: terapie non farmacologiche per il paziente con scompenso cardiaco cronico?]. Mondaldi Archives for Chest Disease 2002;58(1):S1-S3.

\section{Page 2012 \{published data only\}}

Page RL, Kao DP, Macaulay D, Birnbaum HG, Jarvis J, Desai U, et al. Abstract 14767: Integrated Telehealth and Care Management Program Reduces All Cause Mortality in Medicare Beneficiaries with Heart Failure. Circulation 2012;126:A14767. 


\section{Pandor 2013a \{published data only\}}

Pandor A, Thokala P, Gomersall T, Baalbaki H, Stevens J W, Wang J, et al. Home telemonitoring or structured telephone support programmes after recent discharge in patients with heart failure: systematic review and economic evaluation. Health Technology Assessment 2013;17:1-+.

\section{Pascual 2011 \{published data only\}}

Pascual CR, Galan EP, Guerrero JL, Colino RM, Soler PA, Calvo $\mathrm{MH}$, et al. Rationale and methods of the multicenter randomised trial of a heart failure management programme among geriatric patients (HF-Geriatrics). BMC Public Health 2011;11:627.

\section{Pasqualini 2006 \{published data only\}}

Pasqualini MF, Monopoli DM, Mazzucco R, Negrelli M, Pozzetti D, Sandrini R, et al. P876 Home management of old patients with chronic heart diseases: role of tele-cardiolography and tele consultancy. European Heart Journal 2006;27(Suppl 1):140.

Patja 2012 \{published data only\}

Patja K, Absetz P, Auvinen A, Tokola K, Kytö J, Oksman E, et al. Health coaching by telephony to support self-care in chronic diseases: clinical outcomes from The TERVA randomized controlled trial. BMC Health Services Research 2012;12:147.

\section{Peikes 2009 \{published data only\}}

Peikes D, Chen A, Schore J, Brown R. Effects of care coordination on hospitalization, quality of care, and health care expenditures among Medicare beneficiaries: 15 randomized trials. Journal of the American Medical Association 2009;301:603-18.

\section{Philbin 2000 \{published data only\}}

Philbin EF, Rocco TA, Lindenmuth NW, Ulrich K, McCall M, Jenkins $\mathrm{PL}$. The results of a randomized trial of a quality improvement intervention in the care of patients with heart failure. American Journal of Medicine 2000;109:443-9.

\section{Phillips 2008 \{published data only\}}

Phillips JL, Davidson PM, Newton PJ, DiGiacomo M. Supporting patients and their caregivers after-hours at the end of life: the role of telephone support. Journal of Pain and Symptom Management 2008;36(1):11-21.

\section{Picard 2008 \{published data only\}}

Picard F, Bordachar P, Dos Santos P. Remote monitoring of heart failure [Suivi a distance des patients en insuffisance cardiaque]. Medecine Therapeutique - Cardio 2008;4(2):104-11.

\section{Piepoli 2006 \{published data only\}}

Piepoli MF, Villani GQ, Aschieri D, Bennati S, Groppi F, Pisati MS, et al. Multidisciplinary and multi-setting team management programme in heart failure patients affects hospitalisation and costing. International Journal of Cardiology 2006;111:377-85.

\section{Pinto 2014 \{published data only\}}

Pinto JM, Coppola JF, Stanford M, Gaur C, Gaur D. Educational effects of telehealth implementation on older adults with socioeconomic disparities. Systems, Applications and Technology Conference. 2014:1-6.

\section{Piorkowski 2006 \{published data only\}}

Piorkowski C. Abstract 3513: Homemonitoring in MADIT II patients: a prospective randomised multi-centre comparison against a standard follow-up (REFORM Trial). Circulation 2006;114:II_749.

\section{Piotrowicz 2012 \{published data only\}}

Piotrowicz E, Jasionowska A, Banaszak-Bednarczyk M, Gwilkowska J, Piotrowicz R. ECG telemonitoring during homebased cardiac rehabilitation in heart failure patients. Journal of Telemedicine \& Telecare 2012;18:193-7.

Pugh 2001 \{published data only\}

Pugh L, Havens D, Xie S, Robinson J, Blaha C. Case management for elderly persons with heart failure:the quality of life and cost outcome. MEDSURG Nursing 2001;10:71-8.

Purcell 2014 \{published data only\}

Purcell R, McInnes S, Halcomb E J. Telemonitoring can assist in managing cardiovascular disease in primary care: a systematic review of systematic reviews. BMC family practice 2014;15:43.

Quinn 2006 \{published data only\}

Quinn C. Low-technology heart failure care in home health: improving patient outcomes. Home Healthcare Nurse 2006:24:533-40.

\section{Quinn 2008 \{published data only\}}

Quinn JR, Tucker R, Chen L, Horwitz C, Ferguson G. 321 Heart failure patients' use of computer technology for self-care management. Journal of Cardiac Failure 2008;14(6 Suppl Aug):S98.

\section{Rabelo 2007 \{published data only\}}

Aliti GB, Rabelo ER, Domingues FB, Clausell N. Educational settings in the management of patients with heart failure. Revista Latino-Americana de Enfermagem 2007;15:344-9.

Raborn 2012 \{published data only\}

Raborn N. Preventing avoidable heart failure readmissions: the University Hospital experience. Journal of Clinical Outcomes Management 2012;19:460-4.

Rahimpour 2008 \{published data only\}

Rahimpour M, Lovell NH, Celler BG, McCormick J. Patients' perceptions of a home telecare system. International Journal of Medical Informatics 2008;77:486-98.

Ramaekers 2009 \{published data only\}

Ramaekers BL, Janssen-Boyne JJ, Gorgels AP, Vrijhoef HJ. Adherence among telemonitored patients with heart failure to pharmacological and nonpharmacological recommendations. Telemedicine Journal and E-Health 2009;15(6):517-24.

Raman 2008 \{published data only\}

Raman G, DeVine D, Lau J. Non-pharmacological interventions for post-discharge care in heart failure (Structured abstract). Agency for Healthcare Research and Quality 2008:1. 
Reble 2006 \{published data only\}

Reble C, Jensen M, Schneider K, Koots S, Crisman T. Impact of heart failure tele-management program on patient outcomes. Progress in Cardiovascular Nursing 2006;21(2):113.

\section{Repoley 2006 \{published data only\}}

Repoley JL, Dukes-Graves D, Gohn DC. Monitoring intrathoracic impedence intensifies heart failure management. Progress in Cardiovascular Nursing 2006;Spring:109.

Rich 2002 \{published data only\}

Rich MW. Management of heart failure in the elderly. Heart Failure Reviews 2002;7:89-97.

\section{Ross 2004 \{published data only\}}

Ross SE, Moore LA, Earnest MA, Wittevrongel L, Lin CT. Providing a web-based online medical record with electronic communication capabilities to patients with congestive heart failure: randomised trial. Journal of Medical Internet Research 2004;6(2):e12.

\section{Roth 2005 \{published data only\}}

Roth A, Gadot R, Kalter E. Tele-cardiology for patients with chronic heart failure: The 'SHL' experience in Israel and Germany. Studies in Health Technology Information 2005;114:235-7.

\section{Roth 2006 \{published data only\}}

Roth A, Rogowski O, Yanay Y, Kehati M, Malov N, Golovner M. Teleconsultation for cardiac patients: a comparison between nurses and physicians: the SHL experience in Israel. Telemedicine and e-Health 2006;5:528-35.

\section{Rozenman 2007 \{published data only\}}

Rozenman Y. Abstract 3278: Noninvasive home monitoring of pulmonary artery pressure by RemonCHF device. First multicentre experience. Circulation 2007;116:II_738.

\section{Saxon 2007 \{published data only\}}

Saxon LA, Boehmer JP, Neuman S, Mullin CM. Remote active monitoring in patients with heart failure (RAPID-RF): design and rationale. Journal of Cardiac Failure 2007;13(4):241-6.

\section{Scalvini 2004 \{published data only\}}

Scalvini S, Zanelli E, Volterrani M, Martinelli G, Baratti D, Buscaya $\mathrm{O}$, et al. A pilot study of nurse-led home-based telecardiology for patients with chronic heart failure. Journal of Telemedicine and Telecare 2004;10(2):113-7.

\section{Scalvini 2005a \{published data only\}}

Scalvini S, Capomolla S, Zanelli E, Benigno M, Domenighini D, Paletta L, et al. Effect of home-based tele-cardiology on chronic heart failure: costs and outcomes. Journal of Telemedicine and Telecare 2005;11(Suppl 1):16-8.

\section{Scalvini 2005b \{published data only\}}

Scalvini S, Martinelli G, Baratti D, Domenighini D, Paletta L, Zanelli E, Giordano A. Telecardiology: one-lead electrocardiogram monitoring and nurse triage in chronic heart failure. Journal of Telemedicine and Telecare 2005;11(Suppl 1):18-20.

\section{Scalvini 2006 \{published data only\}}

Scalvini S, Zanelli E, Paletta L, Benigno M, Domeneghini D, De Giuli F, et al. Chronic heart Failure home-based management with a tele-cardiology system: a comparison between patients followed by general practitioners and by a cardiology department. Journal of Telemedicine and Telecare 2006;12(Suppl 1):46-48

\section{Scherr 2006 \{published data only\}}

Scherr D, Zweiker R, Kollman A, Kastener P, Schreir G, Fruhwald FM. Mobile phone-based surveillance of cardiac patients at home. Journal of Telemedicine and Telecare 2006;12(5):255-61.

\section{Schmidt 2008 \{published data only\}}

Schmidt S, Sheikzadeh S, Beil B, Patten M, Stettin J. Acceptance of telemonitoring to enhance medication compliance in patients with chronic heart failure. Telemedicine and e-Health 2008;14(5):426-33.

Schneider 2004 \{published data only\}

Schneider NM. Managing congestive heart failure using home health. Home Healthcare Nurse 2004;22:719-22.

\section{Schofield 2005 \{published data only\}}

Schofield R, Kline SE, Schmalfuss CM, Carver HM, Arnada JM Jr, Pauly DF, et al. Early outcomes of a care coordination-enhanced telephone care program for elderly veterans with chronic heart failure. Telemedicine Journal and E-Health 2005;11:20-7.

\section{Schofield 2008 \{published data only\}}

Schofield R, Scott L, Hassan M, Kline S, Marshall E, Schmalfuss $C$, et al. Telehealth management of heart failure improves cardiac remodeling. Journal of Cardiac Failure 2008;14(6 Suppl August):S114-S115.

\section{Schwamm 2014 \{published data only\}}

Schwamm L H. Telehealth: Seven Strategies To Successfully Implement Disruptive Technology And Transform Health Care. Health Affairs 2014;33:200-6.

\section{Schwarz 2008 \{published data only\}}

Schwarz KA, Mion LC, Hudock D, Litman G. Telemonitoring of heart failure patients and their caregivers: a pilot randomised controlled trial. Progress in Cardiovascular Nursing 2008;23(1):18-26.

\section{Scott 2004 \{published data only\}}

Scott LD, Setter-Kline K, Britton AS. The effects of nursing interventions to enhance mental health and quality of life among individuals with heart failure. Applied Nursing Research 2004;17(4):248-56

\section{Seibert 2008a \{published data only\}}

Seibert PS, Whitmore TA, Patterson C, Parker PD, Otto C, Basom J, et al. Telemedicine facilitates CHF home health care for those with systolic dysfunction. International Journal of Telemedicine and Applications 2008;2008:Article ID 235031, 7 pages. [DOI: 10.1155/2008/235031] 
Sen 2014 \{published data only\}

Sen AP, Sewell TB, Riley EB, Stearman B, Bellamy SL, Hu MF, et al. Financial Incentives for Home-Based Health Monitoring: A Randomized Controlled Trial. Journal of General Internal Medicine 2014;29(5):770-7.

\section{Serxner 1998 \{published data only\}}

Serxner S, Miyaji M, Jeffords J. Congestive heart failure disease management study: a patient education intervention. Congestive Heart Failure 1998;4(3):23-8.

\section{Shah 2007 \{published data only\}}

Shah MR, Connor CM, Nohria A, Whellan DJ, Xue Z, Hasselblad V, et al. 338 Telephone heart failure disease management: getting something for nothing. Journal of Cardiac Failure 2007;13(6, Suppl 2):S172.

\section{Shah 2008 \{published data only\}}

Shah MR, Whellen DJ, Peterson ED, Nohria A, Hasselblad V, Xue $Z$, et al. Delivering heart failure disease management in 3 tertiary care centres: key clinical components and venues of care. American Heart Journal 2008;155(4):764.e1-5.

\section{Shearer 2007 \{published data only\}}

Shearer NB, Cisar N, Greenberg EA. A telephone-delivered empowerment intervention with patients diagnosed with heart failure. Heart and Lung 2007;36(3):159-69.

\section{Simpson 2006 \{published data only\}}

Simpson L, Idelchik G, Delgado R, Gregoric I, Loyalka P, Kar B. Abstract 2365: Overall experience with the tandem heart at the Texas Heart Institute. Circulation 2006;114:II 485.

\section{Slater 2006 \{published data only\}}

Slater SG, Neander L, Carey G. Measuring quality of life outcomes through the use of home telehealth: using a case study model in a terminal heart failure patient. Home Health Care Management \& Practice 2006;18(4):333-5.

\section{Slater 2008 \{published data only\}}

Slater MR, Phillips DM, Woodard EK. Cost-effective care a phone call away: a nurse managed telephonic program for patients with chronic heart failure. Nursing Economics 2008;26(1):41-4.

\section{Smart 2005 \{published data only\}}

Smart N, Haluska B, Jeffriess L, Marwick TH. Predictors of a sustained response to exercise training in patients with chronic heart failure: a telemonitoring study. American Heart Journal 2005;150:1240-7.

\section{Smeulders 2006 \{published data only\}}

Smeulders ESTF, Van Haastregt JCM, Van Hoef EFM, Van Eijk JTM, Kempen GIJM. Evaluation of a self-management programme for congestive heart failure patients: design of a randomised controlled trial. BMC Health Services Research 2006;6:91.

\section{Smolis-Bak 2012 \{published data only\}}

Smolis-Bak E, Dabrowski R, Chwyczko T, Piotrowicz E, Kowalik I, Kazimierska B. Hospital training and its continuation at home with central telemonitoring significantly improves exercise capacity and echocardiographic parameters in heart failure patients after CRT implantation. Circulation 2012;125(19):e18.

Sonntag 2009 \{published data only\}

Sonntag SM, Sohn H, Klauss V, Ziegler M, H Moehlmann, Seifel $M$, et al. The disease management/telemonitoring programme CORDIVA for patients with heart failure reduces hospitalizations and costs in both rural and urban areas. European Heart Journal 2009.

Sousa 2014 \{published data only\}

Sousa C, Leite S, Lagido R, Ferreira L, Silva-Cardoso J, Maciel M J. Telemonitoring in heart failure: A state-of-the-art review. Revista Portuguesa De Cardiologia 2014;33:229-39.

Spaeder 2006 \{published data only\}

Spaeder J, Najjar SS, Gerstenblith G, Hefter G, Kern L, Palmer JG, et al. Rapid titration of carvedilol in patients with congestive heart failure: a randomised trial of automated telemedicine versus frequent outpatient clinic visits. American Heart Journal 2006;151(4):844 e1-10.

\section{Sprenger 2007 \{published data only\}}

Sprenger C, Kotsch P, Oeff M. 450 Telemonitoring in patients with chronic heart failure - usage of telemonitoring for the outpatient care. European Journal of Heart Failure Supplements 2007;6(Suppl):95-6.

\section{Stampehl 2012 \{published data only\}}

Stampehl MR, Call CF, Pope SM, Hartwig M, Jennison S. Rural heart failure management by telemedicine: lessons learned. Journal of Cardiac Failure 2012;18:S98.

\section{Steckler 2008 \{published data only\}}

Steckler AE, Wassif H, Wagner J, Jaenicke C, Rector T, Anand IS. 1033-154: Long distance titration of heart failure medications by telephone calls. Journal of the American College of Cardiology 2008;51:A268.

\section{Steventon 2013 \{published data only\}}

Steventon A, Tunkel S, Blunt I, Bardsley M. Effect of telephone health coaching (Birmingham OwnHealth) on hospital use and associated costs: cohort study with matched controls. BMJ 2013;347:f4585.

\section{Stoerk 2013 \{published data only\}}

Stoerk S, Gietzen F, Kerber S, Gebhard B, Holzendorf V, Brenner S, et al. Effectiveness of HeartNetCare-HF, a heart failure disease management program, in the real-world setting. European Journal of Heart Failure 2013;12:S147.

\section{Stone 2009 \{published data only\}}

Stone PW. Nurse-led heart failure management improved quality of life and was cost-effective.. Evidence Based Nursing 2009;2012(2):59.

\section{Stone 2012 \{published data only\}}

Stone S. Evaluation of a nurse-led telephone intervention with symptomatic heart failure patients. West Virginia University 2012; Vol. D.N.P.:82 p. 
Stork 2009 \{published data only\}

Stork S, Faller H, Schowalter M, Ertl G, Angermann C E. Evidence-based disease management in patients with heart failure (HeartNetCare-HF Wurzburg). [German] Evidenzbasiertes Krankheitsmanagement bei Herzinsuffizienz (HeartNetCare-HF Wurzburg). Deutsche medizinische Wochenschrift (1946) 2009;134:773-6.

\section{Stromberg 2003 \{published data only\}}

Strömberg A, Mårtensson J, Fridlund B, Leving LA, Karlssond JE, Dahlström U. Nurse-led heart failure clinics improve survival and self-care behaviour in patients with heart failure: results from a prospective, randomised trial. European Heart Journal 2003;24(11):1014-23.

\section{Stromberg 2006 \{published data only\}}

Strömberg A, Dahlström U, Fridlund B. Computer-based education for patients with chronic heart failure. A randomised, controlled, multicentre trial of the effects on knowledge, compliance and quality of life. Patient Education and Counseling 2006;64:128-35.

\section{Stut 2011 \{published data only\}}

Stut W, Deighan C, Armitage W, Clark M, Jaarsma T. 18 A tailored on-line education and coaching programme to increase compliance of heart failure patients. European Journal of Cardiovascular Nursing 2011;10:S21.

\section{Sullivan 2006 \{published data only\}}

Sullivan A, Cleary R, Hales S, Pryde I, Baker V, Davidson P, et al. 442: Interventions by heart failure nurse specialists: potential for reducing hospital admissions. Journal of Cardiac Failure 2006;12(6 Suppl August):S133.

\section{Takagawa 2013 \{published data only\}}

Takagawa J, Asanoi H. Telemonitoring for chronic heart failure using fully-automated and real-time biosignal processing system. Journal of Cardiac Failure 2013;19:S125.

\section{Takahashi 2010 \{published data only\}}

Takahashi PY, Hanson GJ, Pecina JL, Stroebel RJ, Chaudhry R, Shah ND, et al. A randomized controlled trial of telemonitoring in older adults with multiple chronic conditions: the Tele-ERA study. BMC Health Services Research 2010;10:7.

\section{Takahashi 2012 \{published data only\}}

Takahashi PY, Pecina JL, Upatising B, Chaudhry R, Shah ND, Van Houten $\mathrm{H}$, et al. A randomized controlled trial of telemonitoring in older adults with multiple health issues to prevent hospitalizations and emergency department visits. Archives of Internal Medicine 2012;172(10):773-9.

\section{TEHAF Study \{published data only\}}

Boyne J, Van Asselt ADI, Gorgels APM, Steuten LMG, Kragten J, De Weerd GD, et al. Cost-effectiveness analysis of telemonitoring versus usual care in patients with heart failure (the TEHAF-study). European Heart Journal Suppl 1 2013;34:626.

Boyne J, Vrijhoef H, Crijns H, Nieman F, Deweerd G, Kragten J, et al. Tailored Telemonitoring in patients with heart failure: results from a multicentre randomized controlled trial (the TEHAFstudy). International Journal of Integrated Care 2012;12:1.

Boyne J, Vrijhoef HJ, Nieman FH, De Wit R, Kragten J, De Weerd GJ, et al. Telemonitoring in patients with heart failure: Results from a multicenter randomized controlled trial (the TEHAFstudy). Journal of the American College of Cardiology 2011;1:E389.

Boyne J, Vrijhoef HJM, Spreeuwenberg M, Kragten J, Deweerd G, Gorgels APM. The effects of telemonitoring on heart failure patients' knowledge, self-care, self-efficacy and adherence: A randomized controlled trial. European Heart Journal 2012;33:166.

Boyne J, Vrijhoef HJM, de Wit R, Kragten J, Platteel P, Heerlen AMC, et al. Telemonitoring in patients with heart failure: a feasibility study (TEHAF). European Journal of Cardiovascular Nursing 2008;7(Suppl 1):S20-1.

Boyne JJ, Vrijhoef HJ, Crijns HJ, De Weerd G, Kragten J, Gorgels AP, et al. Tailored telemonitoring in patients with heart failure: results of a multicentre randomized controlled trial. European Journal of Heart Failure 2012;14:791-801.

Boyne JJ, Vrijhoef HJM, Spreeuwenberg M, Weerd GD, Kragten J, Gorgels APM. The effects of telemonitoring on heart failure patients' knowledge, self-care, self-efficacy and adherence: a randomized controlled trial [European Journal of Cardiovascular Nursing]. European Journal of Cardiovascular Nursing 2013; Vol. 13, issue 3:243-52.

Boyne JJJ, Ramaekers B, De Wit R, Gorgels APM, Vrijhoef HJM. Adherence to medication and non-pharmacological recommendations in telemonitored heart failure patients. European Journal of Heart Failure, Supplement 2009;8:ii564.

Boyne JJJ, Ramaekers B, De Wit R, Gorgels APM, Vrijhoef HJM. Patients telemonitored for heart failure: Adherence to medication and non-pharmacological recommendations. European Journal of Cardiovascular Nursing 2010;9:S30.

Boyne JJJ, Ramaekers B, De Wit R, Gorgels APM, Vrijhoef HJM. Patients telemonitoring for heart failure: adherence to medication and non-pharmacological recommendations. European Journal of Heart Failure 2010; Vol. 9:S30.

Boyne JJJ, Spreeuwenberg MD, Gorgels APM, Vrijhoef HJM. Effects of telemonitoring on heart failure patients' quality of life and depression scores: a randomised controlled trial. eTELEMED 2014 : The Sixth International Conference on eHealth, Telemedicine, and Social Medicine. 2014:35-43.

Boyne JJJ, Van Asselt ADI, Gorgels APM, Steuten LMG, De Weerd G, Kragten J, et al. Cost-effectiveness analysis of telemonitoring versus usual care in patients with heart failure: the TEHAF-study. Journal of Telemedicine and Telecare 2013;19:242-8.

Boyne JJJ, Vrijhoef HJM, Nieman FHN, De Wit R, De Weerd GJ, Kragten J, et al. Tailored telemonitoring in patients with heart failure: The TEHAF-study. European Journal of Heart Failure, Supplement 2011;10:S2. 
Boyne JJJ, Vrijhoef HJM, Wit RD, Gorgels APM. Telemonitoring in patients with heart failure, the TEHAF study: Study protocol of an ongoing prospective randomised trial. International Journal of Nursing Studies 2011;48:94-9.

Boyne JJJ, Vrijhoef HJVM, Spreeuwenberg M, Weerd GD, Kragten J, Gorgels APM on behalf of the TEHAF investigators. Effects of tailored telemonitoring on heart failure patients' knowledge, self-care, self-efficacy and adherence: A randomized controlled trial. Journal of Cardiovascular Nursing 2014;13:243-52.

\section{Terschuren 2007 \{published data only\}}

Terschüren C, Fendrich K, van den Berg N, Hoffmann W. Implementing telemonitoring in the daily routine of a GP practice in a rural setting in northern Germany. Journal of Telemedicine and Telecare 2007;13:197-201.

Thokala 2013a \{published data only\}

Thokala P, Baalbaki H, Brennan A. Telemonitoring after discharge from hospital with heart failure - Cost-effectiveness modelling of alternative service designs. Value in Health 2013;16 (7):A530.

\section{Thokala 2013b \{published data only\}}

Thokala P, Brennan A, Baalbaki H. Cost-effectiveness modelling of telemonitoring after discharge from hospital with heart failure. Value in Health 2013;16 (3):A290.

\section{Thompson 2005 \{published data only\}}

Thompson DR, Roebuck A, Stewart S. Effects of a nurse-led, clinic and home-based intervention on recurrent hospital use in chronic heart failure. European Journal of Heart Failure 2005;7(3):377-84.

\section{Thompson 2008 \{published data only\}}

Thompson DR. Telehome monitoring reduced readmissions and improved quality of life in heart failure or angina. Evidenced Based Nursing 2008;11(3):86.

\section{Tompkins 2010 \{published data only\}}

Tompkins C, Orwat J. A randomized trial of telemonitoring heart failure patients. Journal of Healthcare Management 2010;55(5):312-22; Discussion 322-3.

\section{Tramarin 2005 \{published data only\}}

Tramarin R. La telecardiology come strumento di integrazione tra ospedale e territorio. Monaldi Archives for Chest Disease 2005;64:1134-50.

\section{Tran 2008 \{published data only\}}

Tran K, Polisena J, Coyle D, Coyle K, Kluge E H, Cimon K, et al. Home telehealth for chronic disease management (Structured abstract). Database of Abstracts of Reviews of Effects. Title to be Checked, 2008:1.

\section{Trudel 2007 \{published data only\}}

Trudel M, Cafazzo JA, Hamill M, Igharas W, Tallevi K, Picton P, et al. A mobile phone based remote patient monitoring system for chronic disease management. Studies in Health Technology and Informatics 2007;129(Part 1):167-71.

\section{Tsuji 2013 \{published data only\}}

Tsuji M, Akematsu Y. Long-term effect of telecare on patients with chronic diseases. Computing in Cardiology Conference. 2013:261-4

\section{Umeda 2014 \{published data only\}}

Umeda A, Inoue T, Takahashi T, Wakamatsu H. Telemonitoring of patients with implantable cardiac devices to manage heart failure: an evaluation of tablet-PC-based nursing intervention program. Open Journal of Nursing 2014;4:237-50.

\section{Vaccaro 2001 \{published data only\}}

Vaccaro J, Cherry J, Harper A, O'Connell M. Utilzation reduction, cost savings and return on investment for the pacificare chronic heart failure program 'taking charge of your heart health'. Disease Management 2001;4:131-42.

Valle 2004 \{published data only\}

Valle R, Carbonieri E, Tenderini P, Zanella C, De Cian F, Ginocchio G, et al. Proposed protocol for the ambulatory management of patients discharged with heart failure diagnosis: collaborative project Venice-HF. Italian Heart Journal Supplement 2004;5(4):282-91.

Vallina 2008 \{published data only\} Vallina $\mathrm{H}$, Effken JA. Identifying moderators of telemonitoring outcomes in heart failure patients. Communicating Nursing Research 2008;41:326.

Vallina 2010 \{published data only\} Vallina H, Effken J. Telemonitoring and heart failure outcomes. Communicating Nursing Research 2010;43:433.

van den Bussche 2004 \{published data only\} van den Bussche $\mathrm{H}$, Steinberg B, von Brandis S, Sperber S, Zimmermann T. Effectiveness of an outpatient disease management programme for chronic heart insufficiency patients [Nutzen eines ambulanten Disease-ManagementProgramms für Patienten mit chronischer Herzinsuffizienz]. Gesundheitswesen 2004;66(10):656-60.

\section{Varon 2014 \{published data only\}}

Varon C, Alao M, Minter J, Stapleton M, Thomson S, Jaecques S, et al. Effect of telehealth on self-care behavior of heart failure patients. Proc. of the 41st Annual Computing in Cardiology. 2014:1-4.

\section{Vasoncelos 2013 \{published data only\}}

Vasoncelos G, Carmargo MEJ, Oliveira LR. Prevention of heart failure with telemedicine. European Journal of Heart Failure 2013:S257.

VA Technology Assessment \{published data only\}

Physiologic telemonitoring in CHF. VA Technology Assessment Program. Short Report 2001, issue 5:1-11.

\section{Villalba 2006a \{published data only\}}

Villalba E, Ottaviano M, Arredondo MT, Martinez A, Guillen S. Wearable monitoring system for heart failure assessment in a mobile environment. Computers in Cardiology 2006;33:237-40. 
Villalba 2006b \{published data only\}

Villalba E, Ottaviano M, Arredondo MT, Martinez A, Guille S. A new solution for a heart failure monitoring system based on wearable and information technologies. International workshop on wearable and implantable body sensor networks 2006, issue 3-5 April:4.

\section{Vrijhoef 2007 \{published data only\}}

Vrijhoef HJM, Janssen-Boyne, Engering G, Kragten JA, De Weerd GJ, Frederix M, et al. 741 The Health Buddy: telemonitoring system for patients with heart failure. European Journal of Heart Failure Supplement 2006, issue 5 Suppl:174.

Waldman 2008 \{published data only\}

Waldmann A, Katalinic A, Schwabb B, Richardt G, Sheikhzadeh A, Raspe H. The TeleGuard trial of additional telemedicine care in CAD patients: 2 morbidity and mortality after 12 months. Journal of Telemedicine and Telecare 2008;14(1):22-6.

\section{Walsh 2005 \{published data only\}}

Walsh M, Coleman JR. Trials and tribulations: a small pilot telehealth home care program for Medicare patients. Geriatric Nursing 2005;26(6):343-6.

\section{Wang 2010 \{published data only\}}

Wang H, Xiao C, Li Junwei, Wang J. The Research of Transtelephonic Electrocardiogram Monitoring Between. Circulation 2010;122(2):e120.

\section{Wang 2012 \{published data only\}}

Wang HY, Wang JY, Yang L, Xiao CS. Ambulatory monitoring of four physiology parameters to the chronic patients in community by long range wireless transmission technology. Circulation 2012;125(19):e896.

\section{Waywell 2007 \{published data only\}}

Waywell C, Coppinger T. An audit of telephone call nurse-led intervention for chronic heart failure patients in a heart failure clinic. Abstract \#1388. European Journal of Cardiovascular Nursing 2007;6(Suppl 1):s49.

\section{Weintraub 2005 \{published data only\}}

Weintraub AR, Kimmelstiel C, Levine D, Venesy D, Levin A, Lorell $\mathrm{B}$, et al. A multicenter randomised controlled comparison of telephonic disease management vs. automated home monitoring in patients recently hospitalised with heart failure: Span-CHF II Trial. Journal of Cardiac Failure 2005;11(9):647-744.

\section{West 2013 \{published data only\}}

West P. Enhancing patient care across borders. Health Management Technology 2013;34:13.

\section{West-Frasier 2008 \{published and unpublished data\}}

West-Frasier J. The impact of telemonitoring on self-efficacy, emotional well-being, and clinical outcomes in patients with chronic obstructive pulmonary disease or heart failure (PhD Thesis). Western Michigan University 2008.

\section{Westlake 2007 \{published data only\}}

Westlake C, Evangelista LS, Strömberg A, Ter-Galstanyan A, Vazirani S, Dracup K. Evaluation of a web-based education and counseling pilot program for older heart failure patients. Progress in Cardiovascular Nursing 2007;22(1):20-6.

Wheeler 2006 \{published data only\}

Wheeler EC, Waterhouse JK. Telephone interventions by nursing students: improving outcomes for heart failure patients in the community. Journal of Community Health Nursing 2006;23(3):137-46.

\section{Whitten 2007 \{published data only\}}

Whitten P, Mickus M. Home telecare for COPD/CHF patients: outcomes and perceptions. Journal of Telemedicine and Telecare 2007;13:69-73.

\section{Wierzchowiecki 2006a \{published data only\}}

Wierzchowiecki M, Poprawski K, Nowicka A, Kandziora M, Piatkowska A, Jankowiak $M$, et al. A new programme of multidisciplinary care for patients with heart failure in Poznan: One-year follow-up. Kardiologia Polska 2006;64(10):1063-70.

\section{Wierzchowiecki 2006b \{published data only\}}

Wierzchowiecki M, Poprawski K, Nowicka A, Kandziora M, Piatkowska A, Janlowiak M, et al. New multidisciplinary heart failure care program (six-month preliminary observation) [Nowy wielodyscyplinarny program opieki nad chorymi z niewydolnoscia serca (szsciomiesieczna obserwacja wstepna)]. Polski Merkuriusz Lekarski 2006;126:511-5.

\section{Willyard 2006 \{published data only\}}

Willyard DA. The use of automated tele-management system for depression screening in a chronic illness care management program. Progress in Cardiovascular Nursing 2006;Spring:116.

\section{Wong 2005 \{published data only\}}

Wong KW, Wong FKY, Chan MF. Effects of nurse-initiated telephone follow-up on self-efficacy among patients with chronic obstructive pulmonary disease. Journal of Advanced Nursing 2005;49(2):210-2.

\section{Wongpiriyayothar 2008 \{published data only\}}

Wongpiriyayothar A, Pothiban L, Liehr P, Senaratana W, Sucumvang K. Effects of home-based care program on symptom alleviation and well-being among persons with chronic heart failure. Thai Journal of Nursing Research 2008;12(1):25-39.

\section{Woodside 2011 \{published data only\}}

Woodside PA, Schofield LC, Holman C. 3. Use of telehealth to reduce heart failure readmissions in a rural community. Heart \& Lung 2011;40:371-2.

\section{Wright 2003 \{published data only\}}

Wright SP, Walsh H, Ingley KM, Muncaster SA, Gamble GD, Pearl A, et al. Uptake of self-management strategies in a heart failure management programme. European Journal of Heart Failure 2003;5(3):371-80. 


\section{Wu 2006 \{published data only\}}

Delgado D, Costigan J, Wu R, Ross HJ. An interactive site for the management of patients with congestive heart failure. Canadian Journal of Cardiology 2003;19(12):1381-5.

* Wu R, Delgado D, Costigan J, Maclver J. Pilot study of an internet patient-physician communication tool for heart failure disease management. World Hospital Health Services 2006;42(3):32-8.

Wu RC, Delgado D, Costigan J, Msclver J, Ross H. Pilot study of an internet based patient-physician communication tool for heart failure disease management. Journal of Medical and Internet Research 2005;7(1):e8.

\section{Xiao 2010 \{published data only\}}

Xiao C, Wang H, Wang J. The benefit of establishing transtelephonic electrocardiogram monitoring between urban and rural patients. Circulation 2010;122(2):e120.

\section{Zaphiriou 2006 \{published data only\}}

Zaphiriou A, Mulligan K, Hagrave P, Patterson D, Cowie M, Newman S, et al. 303 Improved outcomes following hospitalisation in patients with a new diagnosis of heart failure: results from a randomised controlled trial of a novel, nurse-led self-management intervention. Heart 2006;92:A119.

\section{Zentner 2007 \{published data only\}}

Zentner D, Joshi S, Price P, Ryan M, Kurzel A, Treacher B, Grigg L. 35 Multidisciplinary Community Care for Congestive Cardiac Failure Decreases Hospital Admissions in an Elderly Patient Population. Heart, Lung and Circulation 2007;16:S15.

\section{Zugck 2006 \{published data only\}}

Zugck C, Frankenstein L, Nelles M, Remppis A, Baden D, Waehner M, et al. P2298 Prove of concept - clinical and economical effectiveness of telemonitoring in chronic heart failure (CHF). European Heart Journal 2006;27(Suppl 1):379.

\section{References to studies awaiting assessment}

\section{Dunlap 2006 (HearT-I) \{published data only\}}

Dunlap ME. HSR\&D Study CHI 99-074: Randomized trial of a telephone intervention in heart failure patients. www.hsrd.research.va.gov/research/abstracts.cfm?Project_ ID=4115075 2006 .

\section{Kulshreshtha 2010 \{published data only\}}

* Kulshreshtha A, Kvedar J C, Goyal A, Halpern E F, Watson A J. Use of remote monitoring to improve outcomes in patients with heart failure: a pilot trial. International Journal of Telemedicine and Applications 2010;2010:870959.

Kulshreshtha A, Nieves R, Kvedar J, Watson A. Using information technology to improve outcomes in patients with heart failure: the value of remote monitoring. Circulation 2008;117(21):e414.

Kulshreshtha A, Nieves R, Kvedar JC, Watson AJ. Remote monitoring program may improve outcomes for heart failure patients (Abstract \#4356). Circulation 2008;118:S_872.
Levine 2006 (Mind My Heart) \{published data only\}

Levine BA, McAlinden E, Hu TM-J, Fang FM, Alaoui A, Angelus P, et al. Home monitoring of congestive heart failure patients.

Distributed Diagnosis and Home Healthcare, 2006. 2006:33-6.

\section{Suh 2010 \{published data only\}}

Suh M, Evangelista LS, Chen V, Hong WS, Macbeth J, Nahapetian A, et al. WANDA B: Weight and activity with blood Pressure monitoring system for heart failure patients. IEEE Transactions on Information Technology in Biomedicine 2010;Jun 14:1-6.

\section{Wade 2011 \{published data only\}}

Wade MJ, Desai AS, Spettell CM, Snyder AD, McGowanStackewicz V, Kummer PJ, et al. Telemonitoring with case management for seniors with heart failure. American Journal of Managed Care 2011;17(3):e71-9.

\section{Wongpiriyayothar 2011 \{published data only\}}

Wongpiriyayothar A, Piamjariyakul U, Williams P D. Outcomes of a telephone intervention in heart failure. Clinical and Translational Science 2010;3 (2):S28.

* Wongpiriyayothar A, Piamjariyakul U, Williams PD. Effects of patient teaching, educational materials, and coaching using telephone on dyspnea and physical functioning among persons with heart failure. Applied Nursing Research 2011;24(4):e59-66.

\section{Wootton 2010 \{published data only\}}

Wootton R, Gramotnev H, Hailey D. A randomized controlled trial of telephone-supported care coordination in patients with congestive heart failure. Journal of Telemedicine and Telecare 2009;15(4):182-6.

* Wootton R, Gramotnev H, Hailey D. Telephone-supported care coordination in an Australian veterans population: a randomized controlled trial. Journal of Telemedicine and Telecare 2010;16(2):57-62.

\section{Yakushin 2006 \{published data only\}}

Yakushin SS, Nikulina NN. 593 Two-year prognosis in chronic heart failure patients undergone therapeutic education and out-patient observation. European Journal of Heart Failure 2006;5(Supplement):136.

\section{References to ongoing studies}

\section{Andrei 2011 \{published data only\}}

Andrei CL, Sinescu CJ, Ianula RM, Popa Al, Chioncel VP, Mischie AN, et al. Can be the home care of the heart failure patients a better economic alternative?. European Journal of Heart Failure 2011;10(Suppl):S29.

\section{Black 2014 \{published data only\}}

Black JT, Romano PS, Sadeghi B, Auerbach AD, Ganiats TG, Greenfield S, et al. BEAT-HF Research Group. A remote monitoring and telephone nurse coaching intervention to reduce readmissions among patients with heart failure: study protocol for the Better Effectiveness After Transition - Heart Failure (BEAT-HF) randomized controlled trial. Trials 2014;15:124. 
Boxer 2010 \{published data only\}

Boxer R S, Burant C J, DiCarlo C M, Struk C, Madigan E, Pina I $\mathrm{L}$. Home telemonitoring improves health status in the posthospitalization period for patients with heart failure. Journal of Cardiac Failure 2010;1:S91.

\section{Cavusoglu 2013 Hit-Point \{published data only\}}

* Çavuşoğlu Y, Zoghi M, Eren M, Bozçalı E, Kozdağ G, Şentürk T. Does Cardiologist Lead Enhanced Heart Failure Education and Follow-up Program Affect Cardiovascular Mortality Rate?: HitPoint. Circulation 2013;128:Abstract 17804.

Çavuşoğlu Y, Zoghi M, Eren M, Bozçalı E, Kozdağ G, Şentürk T. Post-discharge heart failure monitorization program in turkey: Hit-point. Journal of the American College of Cardiology 2013;62:C74.

\section{De Vries 2011 IN TOUCH \{published data only\}}

* De Vries AE, De Jong RM, Van der Wal MH, Jaarsma T, Van Dijk RB, Hillege HL. The value of INnovative ICT guided disease management combined with Telemonitoring in OUtpatient clinics for Chronic Heart failure patients. Design and methodology of the IN TOUCH study: a multicenter randomised trial. BMC Health Services Research 2011;11:167.

Kraai IH, Otten RM, De Vries AE, De Jong RM, Van Dijk RB, Van Veldhuisen DJ, et al. 56 Poster: Moderated Innovative ICT guided disease management and Telemonitoring in OUt patient clinics for Chronic Heart failure patients (In Touch study design): a disease management study with patient oriented outcomes. European Journal of Cardiovascular Nursing 2010;9:S9.

\section{Dwinger 2013a \{published data only\}}

Dwinger S, Dirmaier J, Herbarth L, König HH, Eckardt M, Kriston L, et al. Telephone-based health coaching for chronically ill patients: Study protocol for a randomized controlled trial. Trials 2013; Vol. 14:337.

\section{Enjuanes 2013b \{published data only\}}

Enjuanes Grau C, Comin Colet J, Verdu Rotellar JM, Gonzalez Robledo G, Linas Alonso A, Ruiz Rodriguez P, et al. Evaluation of the efficacy and costs impact of a telemonitoring and teleintervention trough videoconference program for patients with chronic heart failure: a randomized controlled trial. European Heart Journal 2013; Vol. 34.

\section{Gupta 2013 \{published data only\}}

Gupta J, Fletcher T, Larcombe T, Gupta T. Telemonitoring as bridge to discharge in advanced heart failure. European Journal of Heart Failure 2013;12:S296.

\section{Janssen 2010 \{published data only\}}

Janssen DJ, Becker R, Zucca F, Wendisch T, Pethig K. Long termcost effectiveness analysis of a telemedicine programme for patients with chronic heart failure. spo.escardio.org/eslides/ view.aspx?eevtid=40\&fp=P1441 2010 (accessed 15th October 2015).

\section{Kalowes 2012 \{published data only\}}

Kalowes P, Peters C, Catipon K, Hawkins D, Long P, Tin E, et al. Effect of telephone intervention on heart failure self care: randomized clinical trial. Communicating Nursing Research 2012;45:522.

Karanam 2012 \{published data only\}

Karanam C, Dayanand S, Dang S, Cobian S, Gomez-Marin O, Mallon S, et al. Outcomes from a mobile-phone study for heart failure in an ethnically diverse County Hospital. Journal of the American Geriatrics Society 2012;60:S221.

\section{Kessing 2011 Telemed-HF \{published data only\}}

Kessing D, Denollet J, Widdershoven J, Kupper N. Investigating a TELEmedicine solution to improve MEDication adherence in chronic Heart Failure (TELEMED-HF): study protocol for a randomized controlled trial. Trials 2011;12:227.

Kotooka 2013 Homes-HF \{published data only\}

* Kotooka N, Asaka M, Nagatomo D, Node K. Home telemonitoring for Japanese patients with chronic heart failure. Journal of Cardiac Failure 2013;19:S125.

Kotooka N, Asaka M, Sato Y, Kinugasa Y, Nochioka K, Mizuno A, et al. Home telemonitoring study for Japanese patients with heart failure (HOMES-HF): protocol for a multicentre randomised controlled trial. BMJ Open 2013; Vol. 3.

\section{Lambrinou 2013 MEETTinCy \{published data only\}}

Lambrinou E, Kalogirou F, Protopapas A, Papathanassoglou E, Barberis V, Sourtzi P, et al. Management of patients with heart failure using education or education \& yelephone or telephone in Cyprus (MEETTinCy) trial. Preliminary results. European Journal of Heart Failure 2013;12:S220.

\section{Mareev 2010 CHANCE-AND \{published data only\}}

Mareev V, Daniely MD, Yu B, CHANCE-AND investigators. Congestive Heart failure: A multidisciplinary Nonpharmacological approach for Changing in $\mathrm{rE}$-hospitalisation and prognosis in patients with concomitant Anxiety aNd Depression (CHANCE-AND). Journal of Cardiac Failure 2010;16(11):913.

\section{McCall 2011 \{published data only\}}

McCall N, Cromwell J. Results of the Medicare Health Support disease-management pilot program. New England Journal of Medicine 2011;365(18):1704-12.

\section{Mizukawa 2014 \{published data only\}}

Mizukawa M, Moriyama M, Naka M, Tomiyama M, Kobayashi S, Kitagawa T, et al. Telemonitoring in patients with chronic heart failure: multicenter randomized trial in Japan (pilot study). Journal of Cardiac Failure 2014;20(10S):S144.

\section{Moye 2012 \{published data only\}}

Moye P M, Douglass P, Owen PS, Pounds T. A hospital's effort to lower the readmission of patients with heart failure (HELP-HF): a pilot study. Pharmacotherapy 2012;32(10):e306.

Papadopoulou 2010 \{published data only\}

* Papadopoulou E. Cardiac failure patients telephone intervention program by specialized trained nurse and the impact on the occurrence of patient readmissions and cardiac mortality rate. Heart Surgery Forum 2010;13:S41. 
Papadopoulou E, Mavrogeni S, Kolovou G, Sida V, Cokkinos D V. Phone call evaluation in end stage heart failure One year follow-up. European Journal of Heart Failure 2009;8(Suppl):ii354.

\section{Persson 2011 \{published data only\}}

Persson H, Lofsjogard J, Melin M, Jaraj D, Fjellner A, Straat E. Results of a randomized clinical study evaluating nurse-driven telemedicine interventions for high risk chronic heart failure patients with frequent rehospitalizations. European Heart Journal 2011;32:292.

\section{Ritchie 2012 E-coach \{published data only\}}

Ritchie C, Richman J, Sobko H, Bodner E, Phillips B, Houston T. The E-coach transition support computer telephony implementation study: protocol of a randomized trial. Contemporary Clinical Trials 2012;33(6):1172-9.

\section{Voon 2013 \{published data only\}}

Voon V, Ledwidge M T, Edwards N, Lawlor L, Travers B, Kelly D, et al. Telemonitoring in heart failure-is there a need to target highrisk populations and optimize background standards of care?. European Journal of Heart Failure 2013;12:S297.

\section{Whole Systems Demonstrator \{published data only\}}

* Bardsley M, Steventon A, Doll H. Impact of telehealth on general practice contacts: findings from the whole systems demonstrator cluster randomised trial. BMC Health Services Research 2013;13:395.

Bower P, Cartwright M, Hirani S P, Barlow J, Hendy J, Knapp $M$, et al. A comprehensive evaluation of the impact of telemonitoring in patients with long-term conditions and social care needs: protocol for the whole systems demonstrator cluster randomised trial. BMC Health Services Research 2011;11:12.

Cartwright M, Hirani SP, Rixon L, Beynon M, Doll H, Bower P, et al. Effect of telehealth on quality of life and psychological outcomes over 12 months (Whole Systems Demonstrator telehealth questionnaire study): nested study of patient reported outcomes in a pragmatic, cluster randomised controlled trial. BMJ 2013;346:f653.

Henderson C, Knapp M, Fernandez J L, Beecham J, Hirani S P, Cartwright $\mathrm{M}$, et al. Cost effectiveness of telehealth for patients with long term conditions (Whole Systems Demonstrator telehealth questionnaire study): nested economic evaluation in a pragmatic, cluster randomised controlled trial. [Erratum appears in BMJ. 2013;346:j2065]. BMJ 2013;346:f1035.

Sanders C, Rogers A, Bowen R, Bower P, Hirani S, Cartwright M, et al. Exploring barriers to participation and adoption of telehealth and telecare within the Whole System Demonstrator trial: a qualitative study. BMC Health Services Research 2012;12:220.

Steventon A, Bardsley M, Billings J, Dixon J, Doll H, Hirani S, et al. Effect of telehealth on use of secondary care and mortality: findings from the Whole System Demonstrator cluster randomised trial. BMJ 2012;344:e3874.

\section{Zhang 2009 \{published data only\}}

Zhang H, Shen Y, Zhang Z, Feng F, Zhao W, Yin Y, et al. Twentyfour hours nurse telephone service improves clinical outcomes in patients with chronic heart failure. Journal of the American College of Cardiology 2009:A368.

\section{Zugck 2010 HiTel \{published data only\}}

* Zugck C, Cebola R, Frankenstein L, Nelles M, Taeger T, Pribe R, et al. Telemedicine reduces hospitalisation rates in patients with chronic heart failure-results of the randomized HiTel trial. European Journal of Heart Failure 2010;9(Suppl):S170.

Zugck C, Frankenstein L, Nelles M, Froehlich H, Schellberg D, Cebola R, et al. 52 Telemedicine reduces hospitalisation rates in patients with chronic heart failure: results of the randomised HiTel trial. European Journal of Heart Failure 2008;7(Suppl 1):9.

Zugck C, Nelles M, Frankenstein L, Schultz C, Helms T, Korb H, et al. Telemonitoring in chronic heart failure patients: which diagnostic finding prevents hospital readmission? [Telemedizinisches Monitoring bei herzinsuffizienten Patienten: welche Befundkonstellation verhindert die stationäre Wiedereinweisung?]. Herzschrittmachertherapie und Elektrophysiologie 2005;16(3):176-82.

\section{Additional references}

\section{Abraham 2011}

Abraham WT, Adamson PB, Bourge RC, Aaron MF, Costanzo MR, Stevenson LW, et al. Wireless pulmonary artery haemodynamic monitoring in chronic heart failure: a randomised controlled trial. Lancet 2011;377(9766):658-66.

\section{Anker 2011}

Anker SD, Koehler F, Abraham WT. Telemedicine and remote management of patients with heart failure. Lancet 2011;378(9792):731-9.

\section{Antonicelli 2010}

Antonicelli R, Mazzanti I, Abbatecola AM, Parati G. Impact of home patient telemonitoring on use of beta-blockers in congestive heart failure. Drugs \& Aging 2010;27(10):801-5.

\section{Bhatia 2006}

Bhatia RS, Tu JV, Lee DS, Austin PC, Fang J, Haouzi A, et al. Outcome of heart failure with preserved ejection fraction in a population-based study. New England Journal of Medicine 2006;355(3):260-9.

\section{Callender 2014b}

Callender T, Woodward M, Roth G, Farzadfar F, Lemarie JC, Cicquel S, et al. Heart Failure Care in Low- and Middle-Income Countries: A Systematic Review and Meta-Analysis. PLoS Medicine 2014;12(11):e1001699.

\section{Clark 2005}

Clark RA, McLennan S, Eckert K, Dawson A, Wilkinson D, Stewart S. Chronic heart failure beyond city limits. Rural and Remote Health 2005;5(4):443. 


\section{Clark 2007b}

Clark RA, Yallop JJ, Piterman L, Croucher J, Tonkin AM, Stewart S, et al. Adherence, adaptation and acceptance of elderly chronic heart failure participants to receiving healthcare by telemonitoring. European Journal of Heart Failure 2007;9(11):1104-11.

\section{Clark 2009}

Clark AM, Savard LA, Thompson DR. What is the strength of evidence for heart failure disease-management programs?. Journal of the American College of Cardiology 2009;54(5):397-401.

\section{Clarke 2011}

Clarke M, Shah A, Sharma U. Systematic review of studies on telemonitoring of patients with congestive heart failure: a metaanalysis. Journal of Telemedicine and Telecare 2011;17(1):7-14.

\section{Cleland 2001}

Cleland JGF, Khand A, Clark AC. The heart failure epidemic: exactly how big is it?. European Heart Journal 2001;22(8):623-6.

\section{Cleland 2003}

Cleland JG, Swedberg K, Follath F, Komajda M, Cohen-Solal A, Aguilar JC, et al. The EuroHeart Failure survey programme-a survey on the quality of care among patients with heart failure in Europe. Part 1: patient characteristics and diagnosis. European Heart Journal 2003;24(5):442-63.

\section{Cleland 2006a}

Cleland JG, Daubert JC, Erdmann E, Freemantle N, Gras D, Kappenberger $\mathrm{L}$, et al. Longer-term effects of cardiac resynchronization therapy on mortality in heart failure [the CArdiac REsynchronization-Heart Failure (CARE-HF) trial extension phase]. European Heart Journal 2006; Vol. 27, issue $16: 1928-32$

\section{Cleland 2006b}

Cleland JG, Tendera M, Adamus J, Freemantle N, Polonski L, Taylor J. The perindopril in elderly people with chronic heart failure (PEP-CHF) study. European Heart Journal 2006; Vol. 27, issue 19:2338-45.

\section{Cleland 2007}

Cleland JGF, Tendera M, Taylor J. Prognosis in heart failure with a normal ejection fraction. New England Journal of Medicine 2007;357(8):829-30.

\section{Cleland 2009}

Cleland JGF, Lewinter C, Goode KM. Telemonitoring for heart failure: the only feasible option for good universal care?. European Journal of Heart Failure 2009;11(3):227-8.

\section{Cleland 2011}

Cleland JG, McDonagh T, Rigby AS, Yassin A, Whittaker T, Dargie HJ, National Heart Failure Audit Team for England and Wales. The national heart failure audit for England and Wales 2008-2009. Heart 2011;97(11):876-86.

\section{Cleland 2014b}

Cleland JG, Pellicori P, Dierckx R. Clinical trials in patients with heart failure and preserved left ventricular ejection fraction. Heart Failure Clinics 2014; Vol. 10, issue 3:511-23.

\section{Deeks 2011}

Deeks JJ, Higgins JPT, Altman DG (editors). Chapter 9: Analysing data and undertaking meta-analyses. in: Higgins JPT, Green $\mathrm{S}$ (editors). Cochrane Handbook for Systematic Reviews of Interventions Version 5.1.0 [updated March 2011]. The Cochrane Collaboration, 2011. Available from www.cochranehandbook.org.

\section{Dunlay 2014}

Dunlay SM, Roger VL. Understanding the epidemic of heart failure: past, present, and future. Current Heart Failure Reports 2014;11(4):404-15.

\section{Gerber 2015}

Gerber Y, Weston SA, Redfield MM, Chamberlain AM, Manemann SM, Jiang R, et al. A contemporary appraisal of the heart failure epidemic in Olmsted County, Minnesota, 2000 to 2010. JAMA Internal Medicine 2015;175(6):996-1004.

\section{Harjola 2010}

Harjola VP, Follath F, Nieminen MS, Brutsaert D, Dickstein K, Drexler $\mathrm{H}$, et al. Characteristics, outcomes, and predictors of mortality at 3 months and 1 year in patients hospitalised for acute heart failure. European Journal of Heart Failure 2010;12(Mar 3):239-48.

\section{Hebert 2008}

Hebert PL, Sisk JE, Wang JJ, Tuzzio L, Casablanca JM, Chassin MR, et al. Cost-effectiveness of nurse-led disease management for heart failure in an ethnically diverse urban community. Annals of Internal Medicine 2008;149(8):540-8.

\section{Heidenreich 2013}

Heidenreich PA, Albert NM, Allen LA, Bluemke DA, Butler J, Fonarow J, et al. Forecasting the impact of heart failure in the United States: a policy statement from the American Heart Association. Circulation Heart Failure 2013;6(3):606-19.

\section{Higgins 2011}

Higgins JPT, Altman DG, Gøtzsche PC, Jüni P, Moher D, Oxman AD, et al. Cochrane Bias Methods Group, Cochrane Statistical Methods Group. The Cochrane Collaboration's tool for assessing risk of bias in randomised trials. $B M J$ 2011;343:d5928.

\section{Holland 2005}

Holland R, Battersby J, Harvey I, Lenaghan E, Smith J, Hay, L. Systematic review of multidisciplinary interventions in heart failure. Heart 2005; Vol. 91, issue 7:899-906.

\section{Jaarsma 2006}

Jaarsma T, Strömberg A, De Geest S, Fridlund B, Heikkila J, Mårtensson $\mathrm{J}$, et al. Heart failure management programmes in Europe. European Journal of Cardiovascular Nursing 2006;5(3):197-205. 


\section{Jhund 2009}

Jhund PS, Maclntyre K, Simpson CR, Lewsey JD, Stewart S, Redpath A, et al. Long-term trends in first hospitalisation for heart failure and subsequent survival between 1986 and 2003: a population study of 5.1 million people. Circulation 2009;119(4):515-23.

\section{Kjekshus 2007}

Kjekshus J, Apetrei E, Barrios V, Böhm M, Cleland JG, Cornel JH, et al. Rosuvastatin in older patients with systolic heart failure. New England Journal of Medicine 2007;357(22):2248-61.

\section{Klersy 2009}

Klersy C, De Silvestri A, Gabutti G, Regoli F, Auricchio A. Metaanalysis of remote monitoring of heart failure patients. Journal of the American College of Cardiology 2009;54(18):1683-94.

\section{Kotb 2015}

Kotb A, Cameron C, Hsieh S, Wells G. Comparative effectiveness of different forms of telemedicine for individuals with heart failure (HF): a systematic review and network meta-analysis. PLoS One 2015;10(2):e0118681.

\section{Krumholz 2006}

Krumholz HM, Currie PM, Riegel B, Phillips CO, Peterson ED, Smith R, et al. A taxonomy of disease management: a scientific statement from the American Heart Association Disease Management Taxonomy Writing Group. Circulation 2006;114(13):1432-45.

\section{Lenzen 2005}

Lenzen MJ, Boersma E, Reimer WJ, Balk AH, Komajda M, Swedburg K, et al. Under-utilization of evidence-based drug treatment in patients with heart failure is only partially explained by dissimilarity to patients enrolled in landmark trials. European Heart Journal 2005;Sep 23(24):2706-13.

\section{Levy 2002}

Levy D, Kenchaiah S, Larson MG, Benjamin EJ, Kupka MJ, Ho KK, et al. Long-term trends in the incidence of and survival with heart failure. New England Journal of Medicine 2002;347(18):1397-402.

\section{Linde 2008}

Linde C, Abraham WT, Gold MR, St John Sutton M, Ghio S, Daubert C. Randomized trial of cardiac resynchronization in mildly symptomatic heart failure patients and in asymptomatic patients with left ventricular dysfunction and previous heart failure symptoms. Journal of the American College of Cardiology 2008;52(23):1834-43.

\section{Louis 2003}

Louis AA, Turner T, Gretton M, Baksh A, Cleland JGF. A systematic review of telemonitoring for the management of heart failure. European Journal of Heart Failure 2003;5(5):585-90.

\section{MAGGIC 2012}

Meta-analysis Global Group in Chronic Heart Failure (MAGGIC). The survival of patients with heart failure with preserved or reduced left ventricular ejection fraction: an individual patient data meta-analysis. European Heart Journal 2012; Vol. 33, issue 14:1750-7.

\section{Maggioni 2013}

Maggioni AP, Dahlström U, Filippatos G, Chioncel O, Crespo Leiro M, et al. EURObservational Research Programme: regional differences and 1-year follow-up results of the Heart Failure Pilot Survey (ESC-HF Pilot). European Journal of Heart Failure 2013;15(7):808-17.

\section{Mant 2011}

Mant J, Al-Mohammad A, Swain S, Laramee P. Management of chronic heart failure in adults: synopsis of the National Institute For Health and Clinical Excellence guideline. Annals of Internal Medicine 2011;155(4):252-9.

\section{Massie 2008}

Massie BM, Carson PE, McMurray JJ, Komajda M, McKelvie R, Zile MR, et al. Irbesartan in patients with heart failure and preserved ejection fraction. New England Journal of Medicine 2008;359(23):2456-67.

\section{McAlister 2004}

McAlister FA, Stewart S, Ferrua J, McMurray JJV. Multidisciplinary strategies for the management of heart failure patients at high risk for admission: a systematic review of randomised trials. Journal of the American College of Cardiology 2004;44(4):810-9.

\section{McMurray 2012}

McMurray JJ, Adamopoulos S, Anker SD, Auricchio A, Bohm M, Dickstein $\mathrm{K}$, et al. ESC guidelines for the diagnosis and treatment of acute and chronic heart failure 2012: The Task Force for the Diagnosis and Treatment of Acute and Chronic Heart Failure 2012 of the European Society of Cardiology. Developed in collaboration with the Heart Failure Association (HFA) of the ESC. European Journal of Heart Failure 2012; Vol. 14 , issue 8:803-69.

\section{Mebazaa 2015}

Mebazaa A, Yilmaz MB, Levy P, Ponikowski P, Peacock WF, Laribi $S$, et al. Recommendations on pre-hospital \& early hospital management of acute heart failure: a consensus paper from the Heart Failure Association of the European Society of Cardiology, the European Society of Emergency Medicine and the Society of Academic Emergency Medicine. European Journal of Heart Failure 2015; Vol. 17, issue 6:544-58.

\section{Metra 2015}

Metra M, Mentz RJ, Chiswell K, Bloomfield DM, Cleland JG, Cotter G, et al. Acute heart failure in elderly patients: worse outcomes and differential utility of standard prognostic variables. Insights from the PROTECT trial. European Journal of Heart Failure 2015; Vol. 17, issue 1:109-18.

\section{National Heart Foundation of Australia 2011}

National Heart Foundation of Australia and the Cardiac Society of Australia and New Zealand (Chronic Heart Failure Guidelines Expert Writing Panel). Guidelines for the prevention, detection and management of chronic heart failure in Australia. Updated October 2011. National Heart Foundation of Australia and the 
Cardiac Society of Australia and New Zealand, 2011. [ISBN 978-1-921748-71-4]

\section{Owan 2006}

Owan TE, Hodge DO, Herges RM, Jacobsen SJ, Roger VL, Redfield MM. Trends in prevalence and outcome of heart failure with preserved ejection fraction. New England Journal of Medicine 2006; Vol. 355, issue 3:251-9.

\section{Pandor 2013b}

Pandor A, Gomersall T, Stevens JW, Wang J, Al-Mohammad A, Bakhai $A$, et al. Remote monitoring after recent hospital discharge in patients with heart failure: a systematic review and network meta-analysis. Heart 2013;99(23):1717-26.

\section{Polisena 2010}

Polisena J, Tran K, Cimon K, Hutton B, McGill S, Palmer K, et al. Home telemonitoring for congestive heart failure: a systematic review and meta-analysis. Journal of Telemedicine and Telecare 2010;16(2):68-76.

\section{Ponikowski 2014}

Ponikowski P, Anker SD, AlHabib KF, Cowie MR, Force TL, Hu S, et al. Heart Failure: preventing disease and death worldwide. European Society of Cardiology 2014.

\section{Schünemann 2011}

Schünemann HJ, Oxman AD, Higgins JPT, Vist GV, Glasziou P, Guyatt GH, Cochrane Applicability and Recommendations Methods Group and the Cochrane Statistical Methods Group. Chapter 11: Presenting results and 'Summary of findings' tables. In: Higgins JPT, Green S (editors). Cochrane Handbook for Systematic Reviews of Interventions Version 5.1.0 [updated March 2011]. The Cochrane Collaboration, 2011. Available from www.cochrane-handbook.org.

\section{Smith 2005}

Smith B, Forkner E, Zaslow B, Krasuski RA, Stajduhar K, Kwan M, et al. Disease management produces limited quality-oflife improvements in patients with congestive heart failure: evidence from a randomised trial in community- dwelling patients. American Journal of Managed Care 2005;11:701-3.

\section{Smith 2008}

Smith B, Hughes-Cromwick PF, Forkner E, Galbreath AD. Costeffectiveness of telephonic disease management in heart failure. American Journal of Managed Care 2008;14(2):106-15.

\section{Soran 2010}

Soran OZ, Feldman AM, Piña IL, Lamas GA, Kelsey SF, Selzer F, et al. Cost of medical services in older patients with heart failure: those receiving enhanced monitoring using a computer-based telephonic monitoring system compared with those in usual care: the heart failure home care trial. Journal of Cardiac Failure 2010;16(11):859-66.

\section{Steventon 2012a}

Steventon A, Bardsley M, Billings J, Dixon J, Doll H, Hirani S, et al. Effect of telehealth on use of secondary care and mortality: findings from the Whole System Demonstrator cluster randomised trial. BMJ 2012; Vol. 344:e3874.

\section{Steventon 2013a}

Steventon A, Tunkel S, Blunt I, Bardsley M. Effect of telephone health coaching (Birmingham OwnHealth) on hospital use and associated costs: cohort study with matched controls. British Medical Journal 2013;347:f4585.

\section{Stewart 2013}

Stewart S, MacIntyre K, Capewell S, McMurray JJ. Heart failure and the aging population: an increasing burden in the 21st century?. Heart 2003;89(1):49-53.

\section{Toma 2006}

M Toma, FA McAlister, L Bialy, D Adams, B Vandermeer, PW Armstrong. JAMA. 295:1281-1287, 2006. Transition from meeting abstract to full-length journal article for randomized controlled trials.. Journal of the American Medical Association 2006;295:1281-1287,.

\section{Yancy 2013}

Yancy CW, Jessup M, Bozkurt B, Butler J, Casey DE, Drazner MH, et al. 2013 ACCF/AHA Guideline for the Management of Heart failure: Executive Summary: A Report of the American College of Cardiology Foundation/American Heart Association Task Force on Practice Guidelines. Circulation 2013;128(16):1810-52.

\section{Yu 2006}

Yu D, Thompson DR, Lee D. Disease management programmes for older people with heart failure: crucial characteristics which improve post-discharge outcomes. European Heart Journal 2006;27(5):596-612.

\section{Yusuf 2014}

Yusuf S, Rangarajan S, Koon T, Islam S, Li W, Liu L, et al. PURE Investigators. Cardiovascular Risk and Events in 17 Low-, Middle-, and High-Income Countries. New England Journal of Medicine 2014;371(9):818-27.

\section{Zile 2002}

Zile MR, Brutsaert DL. New concepts in diastolic dysfunction and diastolic heart failure: Part I: diagnosis, prognosis, and measurements of diastolic function. Circulation 2002; Vol. 105, issue 11:1387-93.

\section{References to other published versions of this review \\ Clark 2007a}

Clark RA, Inglis SC, McAlister FA, Cleland JGF, Stewart S. Telemonitoring or structured telephone support programmes for patients with chronic heart failure: systematic review and meta-analysis. BMJ 2007;334(7600):942-7.

\section{Conway 2014}

Conway A, Inglis SC, Clark RA. Effective technologies for noninvasive remote monitoring in heart failure. Telemedicine Journal and e-Health 2014;20(6):531-8.

\section{Inglis $\mathbf{2 0 1 0}$}

Inglis SC, Clark RA, McAlister FA, Ball J, Lewinter C, Cullington D, et al. Structured telephone support or telemonitoring programmes for patients with chronic heart failure. 
Cochrane Database of Systematic Reviews 2010, Issue 8. [DOI: 10.1002/14651858.CD007228.pub2]

\section{Inglis 2011a}

Inglis SC, Clark RA, McAlister FA, Stewart S, Cleland JG. Telemedicine and remote management of heart failure (Letter to the Editor). Lancet 2011;378:e9.

\section{Inglis 2011b}

Inglis SC, Clark RA, Cleland JGF. Telemonitoring in patients with heart failure (Letter to the Editor). New England Journal of Medicine 2011;364(11):1078-80.

\section{CHARACTERISTICS OF STUDIES}

Characteristics of included studies [ordered by study ID]

\section{Inglis 2014}

Inglis SC, Conway A, Cleland JGF, Clark RA. Is age a factor in the success or failure of remote monitoring in heart failure? Telemonitoring and structured telephone support in elderly heart failure patients. European Journal of Cardiovascular Nursing 2015;14(3):248-55.

* Indicates the major publication for the study

\section{Angermann 2012 (INH)}

Methods Open, randomised, 2-armed, parallel-group, multicentre trial.

2 groups: nurse co-ordinated disease management programme (HeartNetCare-HF, HNC) or usual care (UC).

Participants

715 people with heart failure $\geq 18$ years of age hospitalised with signs and symptoms of decompensated heart failure (dyspnoea at rest/minimal exercise plus at least 1 the following: raised jugular venous pressure, peripheral oedema, third heart sound or pulmonary congestion on either clinical examination or radiography) and LVEF $\leq 40 \%$.

Mean age 68.6 years.

$71 \%$ of participants were men.

$2 \%$ NYHA class I, 58\% NYHA class II, 36\% NYHA class III, 4\% NYHA class IV, mean LVEF 30\%

HNC: $n=352$, mean age 67.7 years, $71 \%$ men, 3\% NYHA class I, 54\% NYHA class II, 40\% NYHA class III, $3 \%$ NYHA class IV, mean LVEF $30 \%$.

UC: $\mathrm{n}=363$, mean age 69.4 years, $71 \%$ men, 2\% NYHA class I, 62\% NYHA class II, 31\% NYHA class III, 5\% NYHA class IV, mean LVEF $30 \%$.

9 hospitals in Germany.

Electronic scale and BP at participant's home.

Intervention included: 1) in-hospital face-to-face education; 2) telephone-based structured monitoring using 19-item questionnaire (assessing indicators of worsening HF, other cardiac symptoms, medication, health care utilisation, state of mood and general health and well-being; 3) up titration of HF medication in co-operation with GPs; 4) needs-adjusted specialist care, which nurses co-ordinated with participant's physician.

All nurses received supervision by cardiologist (weekly) and a psychologist (bimonthly), and had unrestricted access to their supervisor for questions.

Professionals involved: skilled nurses, general practitioners and cardiologist.

Frequency of intervention: weekly during the first month, and then individualised according to NYHA class at discharge (weekly or fortnightly for NYHA III - IV, monthly for NYHA I - II) and participant's needs.

Outcomes

Follow-up: 180 days

Primary endpoint was time to all-cause rehospitalisation (combined endpoints). 
Angermann 2012 (INH) (Continued)

Secondary endpoints were cardiovascular and all-cause death or hospitalisation separately, time to, number and duration of readmissions, number of days alive and not hospitalised, changes in NYHA class, HF medication, cardiac function and quality of life.

Funding source German Ministry of Education and Research, German Competence Network Heart Failure, Comprehen sive Heart Failure Centre Wurzburg, University of Wurzburg Cardiovascular Centre.

Comparison Group(s) Usual care: fixed appointment with GP or cardiologist within 7 - 14 days after discharge. No restrictions were placed on outpatient care.

Professionals involved: skilled nurses, general practitioners and cardiologist.

Notes

New in 2015 review - included as abstract in sensitivity analysis in previous version of review (Inglis 2010).

\section{Risk of bias}

\section{Bias Authors' judgement Support for judgement}

Random sequence genera- Low risk tion (selection bias)

\section{Allocation concealment Low risk}

(selection bias)

Blinding of outcome as- Low risk sessment (detection bias)

All outcomes
Quote: "Central computer-generated block random assignment was used (strata: age ( $>70$ vs. $<70$ years), sex and type of outpatient care (cardiologist vs. GP)".

Quote: "Patients were randomly assigned 1:1 to either HNC or UC, using sealed envelopes".

Quote: "An independent committee adjudicated the end points (see online-only Data Supplement Appendix), blinded to treatment assignment".
Incomplete outcome data High risk
(attrition bias)

All outcomes
Quote: "More patients withdrew consent in HNC; life status at 180 days was ascertained in dropouts and reported as uncensored survival data".

22 UC (6\%) and 45 HNC (12\%) participants withdrew consent. No participants were lost to follow-up.

Comment: There were imbalances between dropouts in the 2 groups. (Uncensored) survival was assessed but unsure about other endpoints.

All prespecified endpoints have been addressed (except for total duration of readmissions; duration of first and second hospitalisation has been reported).

\begin{tabular}{|c|c|c|}
\hline $\begin{array}{l}\text { Selective reporting (re- } \\
\text { porting bias) }\end{array}$ & Low risk & $\begin{array}{l}\text { All prespecified endpoints have been addressed (except for total duration of } \\
\text { readmissions; duration of first and second hospitalisation has been reported). }\end{array}$ \\
\hline
\end{tabular}

Antonicelli 2008

\begin{tabular}{|c|c|}
\hline Methods & Randomised controlled trial; intervention arm and control (usual care) arm. \\
\hline \multirow[t]{4}{*}{ Participants } & $\begin{array}{l}57 \text { people hospitalised for worsening symptoms and signs of CHF with NYHA class II - IV, evidence of } \\
\text { pulmonary congestions on chest } x \text {-ray and EF }<40 \% \text {. People with NYHA class II - III with an EF }>40 \% \\
\text { and diastolic LV dysfunction were also included. }\end{array}$ \\
\hline & Mean age 78 years. \\
\hline & $61 \%$ of participants were men. \\
\hline & Italy \\
\hline
\end{tabular}

Interventions

Telemonitoring 
Antonicelli 2008 (Continued)

Participants randomised to home telemonitoring-based care were contacted by telephone at least once a week to collect information on symptoms and treatment adherence as well as BP, HR, weight and $24 \mathrm{~h}$ urine output on the previous day. A weekly ECG transmission was also obtained. Participants were then evaluated and their regimen altered when necessary based on these data. Additionally, clinic visits were performed when required based on the data collected or telephone interviews.

Outcomes Combined rate of mortality and hospitalisation, these rates considered individually, quality of life.

12-month follow-up.

\begin{tabular}{ll}
\hline Funding source & Italian Ministry of Health \\
\hline Comparison Group(s) & $\begin{array}{l}\text { Usual care involved receiving stand care based on routinely scheduled clinic visits (every 4 months) } \\
\text { performed by a team specialised in CHF patient management. These participants were also contacted } \\
\text { monthly by telephone to collect data on new hospital admissions, complications and death. Additional } \\
\text { clinic visits were performed whenever required when clinical status altered. }\end{array}$ \\
\hline Notes & Included previous version of review (Inglis 2010). \\
\hline
\end{tabular}

\section{Risk of bias}

\begin{tabular}{lll}
\hline Bias & Authors' judgement & Support for judgement \\
\hline $\begin{array}{l}\text { Random sequence genera- } \\
\text { tion (selection bias) }\end{array}$ & Unclear risk & Method of randomisation not stated. \\
\hline $\begin{array}{l}\text { Allocation concealment } \\
\text { (selection bias) }\end{array}$ & Unclear risk & Not detailed. \\
\hline $\begin{array}{l}\text { Blinding of outcome as- } \\
\text { sessment (detection bias) } \\
\text { All outcomes }\end{array}$ & Unclear risk & Not detailed. \\
\hline $\begin{array}{l}\text { Incomplete outcome data } \\
\text { (attrition bias) } \\
\begin{array}{l}\text { All outcomes } \\
\text { Selective reporting (re- }\end{array}\end{array}$ & Low risk \\
\begin{tabular}{l} 
porting bias) \\
\hline
\end{tabular}
\end{tabular}

\section{Baker 2011}

\begin{tabular}{|c|c|}
\hline Methods & Randomised controlled trial; intervention arm and control (usual care) arm. \\
\hline \multirow[t]{5}{*}{ Participants } & $\begin{array}{l}605 \text { people with heart failure from general and internal medicine and cardiology clinics at } 4 \text { different } \\
\text { sites. }\end{array}$ \\
\hline & $\begin{array}{l}\text { Inclusion criteria: diagnosis of heart failure, NYHA Class II - IV symptoms in the past } 6 \text { months, current } \\
\text { use of a loop diuretic, fluency in English or Spanish and adequate cognitive function (based on Mi- } \\
\text { ni-Cog screening tool). }\end{array}$ \\
\hline & Mean age 60.7 years. \\
\hline & $52 \%$ of participants were men. \\
\hline & USA \\
\hline
\end{tabular}


Baker 2011 (Continued)

Intensive education and self-care training which was based on social cognitive theory and adult learning theory. This included specific instruction using daily weights to guide diuretic self adjustment and included an individualised plan developed with the participant's clinician. Over 4 weeks, participants were scheduled to receive 5 - 8 phone calls from the study educator to reinforce education and to guide the participant towards improved self-care skills. Each call lasted about 10 minutes. The calls focused on reviewing the content of the initial education session, assessing the participant's knowledge and behaviour and providing additional information and encouragement.

\begin{tabular}{ll} 
Outcomes & $\begin{array}{l}30 \text { days - Heart failure-related quality of life and heart failure knowledge was assessed using the Im- } \\
\text { proving Chronic Illness Care Evaulation Heart Failure Symptom Scale } \\
\text { All-cause hospitalisation and mortality at } 6 \text { and } 12 \text { months. }\end{array}$ \\
\hline Funding source & Not reported. \\
\hline Comparison Group(s) & $\begin{array}{l}\text { Control was a brief educational intervention (BEI), duration of } 40 \text { minutes which covered daily self as- } \\
\text { sessment and action planning in case of exacerbation, salt avoidance, exercise and medication adher- } \\
\text { ence. Participants received an educational manual and a new digital scale for weighing themselves. All } \\
\text { participants in both study arms received this BEl, after which participants were randomised to the con- } \\
\text { trol arm where they continued to receive usual care, or the intervention. }\end{array}$
\end{tabular}

Notes New in 2015 review.

\section{Risk of bias}

\begin{tabular}{lll}
\hline Bias & Authors' judgement & Support for judgement \\
\hline $\begin{array}{l}\text { Random sequence genera- } \\
\text { tion (selection bias) }\end{array}$ & Low risk & $\begin{array}{l}\text { Quote: "Allocation to study group is done with concealed, stratified, block ran- } \\
\text { domization by the statistical team at UNC." }\end{array}$ \\
\hline $\begin{array}{l}\text { Allocation concealment } \\
\text { (selection bias) }\end{array}$ & Low risk & $\begin{array}{l}\text { Quote: "Randomization assignments are placed in sets of opaque envelopes } \\
\text { and distributed to the health educators at each site. After literacy status is de- } \\
\text { termined and the BEl is delivered, the health educator opens the opaque enve- } \\
\text { lope and learns the intervention status of the patient" }\end{array}$
\end{tabular}

Blinding of outcome as- Unclear risk sessment (detection bias) All outcomes
Quote: "Patients were called by the UNC Survey Research Unit (SRU) on day 30 of the study (with day 1 defined as the day of the initial in-person educational session) and a blinded interview conducted."

Incomplete outcome data High risk

(attrition bias)

Quote: "During the 30-day study period...72 (11.7\%) patients did not complete

All outcomes the 1-month assessment call (41 in the BEI group and 31 in the TTG group), leaving 259 patients in the BEI group and 272 in the TTG group".

Selective reporting (re- Low risk porting bias) No evidence of selective outcome reporting.

\section{Balk 2008}

\begin{tabular}{ll}
\hline Methods & Randomised controlled trial; intervention arm and control (usual care) arm. \\
\hline Participants & 214 patients with CHF and NYHA class I - IV. \\
& Mean age 66 years. \\
$70 \%$ of participants were men. \\
The Netherlands. \\
\hline
\end{tabular}


Balk 2008 (Continued)

Telemonitoring.
Participants in the Intervention group were provided a MOTIVA system (TV-channel providing educa-
tional material, reminders of medication, health-related surveys and motivational messages to encour-
age the prescribed lifestyle regimen) in addition to scheduled cardiologist appointments. A subgroup
of intervention participants also received automated BP and weight devices that automatically com-
municated readings via the telephone (those who had been hospitalised in the prior year for HF). Par-
ticipaent guidance followed a personalised plan.

288 days - mean follow-up.
Outcomes
All-cause hospital days per year, days alive and out of hospital, quality of life, knowledge of disease, self
care.

Funding source Study proposed and funded by the healthcare insurance company Achmea.

Philips- provision of the MOTIVA system.

Comparison Group(s) Control participants were followed by their cardiologists and HF nurses according to standard local practice.

All participants recorded all contacts with healthcare professionals and hospital admissions.

\begin{tabular}{|c|c|c|}
\hline Notes & \multicolumn{2}{|c|}{ Included in previous version of review (Inglis 2010). } \\
\hline \multicolumn{3}{|l|}{ Risk of bias } \\
\hline Bias & Authors' judgement & Support for judgement \\
\hline $\begin{array}{l}\text { Random sequence genera- } \\
\text { tion (selection bias) }\end{array}$ & Low risk & $\begin{array}{l}\text { Quote: "Randomisation was performed in a 1:1 ratio, in randomly permuted } \\
\text { blocks of } 30 \text { per participating centre. Randomisation was independently per- } \\
\text { formed...via a special Web-based application". }\end{array}$ \\
\hline $\begin{array}{l}\text { Allocation concealment } \\
\text { (selection bias) }\end{array}$ & Unclear risk & Not detailed. \\
\hline $\begin{array}{l}\text { Blinding of outcome as- } \\
\text { sessment (detection bias) } \\
\text { All outcomes }\end{array}$ & Unclear risk & Not detailed. \\
\hline $\begin{array}{l}\text { Incomplete outcome data } \\
\text { (attrition bias) } \\
\text { All outcomes }\end{array}$ & Low risk & No evidence of incomplete outcome data. \\
\hline $\begin{array}{l}\text { Selective reporting (re- } \\
\text { porting bias) }\end{array}$ & Low risk & No evidence of selective outcome reporting. \\
\hline
\end{tabular}

Barth 2001

\begin{tabular}{ll}
\hline Methods & Randomised controlled trial; intervention arm and control (usual care) arm. \\
\hline Participants & 34 people discharged from acute care to home with primary diagnosis of CHF. \\
& Mean age 75 years. \\
& $47 \%$ of participants were men.
\end{tabular}


Barth 2001 (Continued)

USA

Interventions Structured telephone support.

Structured nurse-managed telephonic post-discharge programme involving predischarge education plus post-discharge telephone follow-up. Structured interaction at 72 hours, 144 hours, and then fortnightly.

\begin{tabular}{|c|c|c|}
\hline Outcomes & \multicolumn{2}{|c|}{$\begin{array}{l}\text { Mortality, rehospitalisation, physician and emergency department visits, quality of life, cost of the in- } \\
\text { tervention. }\end{array}$} \\
\hline Funding source & \multicolumn{2}{|l|}{ Not reported. } \\
\hline Comparison Group(s) & \multicolumn{2}{|c|}{$\begin{array}{l}\text { The control group received routine discharge teaching at the time of discharge as per hospital proce- } \\
\text { dure. Participants were contacted at } 2 \text { months for collection of data. }\end{array}$} \\
\hline Notes & \multicolumn{2}{|c|}{ Included in previous version of review (Inglis 2010) } \\
\hline \multicolumn{3}{|l|}{ Risk of bias } \\
\hline Bias & Authors' judgement & Support for judgement \\
\hline $\begin{array}{l}\text { Random sequence genera- } \\
\text { tion (selection bias) }\end{array}$ & Unclear risk & Method of randomisation not stated. \\
\hline $\begin{array}{l}\text { Allocation concealment } \\
\text { (selection bias) }\end{array}$ & Unclear risk & Not detailed. \\
\hline $\begin{array}{l}\text { Blinding of outcome as- } \\
\text { sessment (detection bias) } \\
\text { All outcomes }\end{array}$ & Unclear risk & Not detailed. \\
\hline $\begin{array}{l}\text { Incomplete outcome data } \\
\text { (attrition bias) } \\
\text { All outcomes }\end{array}$ & Unclear risk & Not detailed. \\
\hline $\begin{array}{l}\text { Selective reporting (re- } \\
\text { porting bias) }\end{array}$ & Low risk & Not evident. \\
\hline
\end{tabular}

Bento 2009

Methods Randomised controlled trial. Single-blinded

2 groups: intervention group (nursing consultation and telephone monitoring of educational nature every 15 days) versus usual care (with monthly telephone monitoring of epidemiological and administrative nature).

Participants

40 participants with a diagnosis of heart failure and NYHA class I - IV, treated at a heart failure outpatient clinic with telephone access.

Mean age (intervention 54 years; control 61 years, $P=0.046$ )

$70 \%$ of participants were men.

IG: $\mathrm{n}=20$, mean age 54.3 years, 65\% men, 5\% NYHA I, 65\% NYHA II, 30\% NYHA III, 0\% NYHA IV 
Bento 2009 (Continued)

CG: $n=20$, mean age 60.7 years, 75\% men, 5\% NYHA I, 50\% NYHA II, 45\% NYHA III, 0\% NYHA IV

Brazil

Structured telephone support
Conventional medical assistance (not otherwise specified), nursing consultation (fortnightly or month-
ly depending on participants' needs) and telephone monitoring every 15 days (education, recording
hospitalisations and emergency treatments). Recomendations on pharmacological treatment, water
intake, sodium intake, BP control, body weight control.
Duration: 6 months
Professionals involved: nurses

\begin{tabular}{ll}
\hline Outcomes & $\begin{array}{l}\text { Follow-up: } 6 \text { months. } \\
\text { All-cause hospitalisations. }\end{array}$ \\
\hline Funding source & No funding received. \\
\hline Comparison Group(s) & $\begin{array}{l}\text { Conventional medical assistance and monthly telephone monitoring of administrative and epidemio- } \\
\text { logical nature, with no education. The phone calls were aimed at recording hospitalisations and emer- } \\
\text { gency treatments. }\end{array}$ \\
\hline Professionals involved: not specified
\end{tabular}

\section{Risk of bias}

\begin{tabular}{lll}
\hline Bias & Authors' judgement & Support for judgement \\
\hline $\begin{array}{l}\text { Random sequence genera- } \\
\text { tion (selection bias) }\end{array}$ & Low risk & $\begin{array}{l}\text { Quote: "The groups were created by simple random allocation (drawing } \\
\text { lots)...". }\end{array}$ \\
\hline $\begin{array}{l}\text { Allocation concealment } \\
\text { (selection bias) }\end{array}$ & Unclear risk & Comment: not reported. \\
\hline $\begin{array}{l}\text { Blinding of outcome as- } \\
\text { sessment (detection bias) } \\
\text { All outcomes }\end{array}$ & Unclear risk & Comment: not reported. \\
\hline $\begin{array}{l}\text { Incomplete outcome data } \\
\text { (attrition bias) } \\
\text { All outcomes }\end{array}$ & Unclear risk & Comment: No dropouts reported. \\
\hline $\begin{array}{l}\text { Selective reporting (re- } \\
\text { porting bias) }\end{array}$ & High risk & $\begin{array}{l}\text { Comment: Some hospitalisations in the CG might have been underreported } \\
\text { due to the fact that some emergency services do not record these cases. }\end{array}$ \\
\hline
\end{tabular}

\section{Biannic 2012 (SEDIC)}

\begin{tabular}{ll}
\hline Methods & Randomised controlled trial. \\
& 2 groups - classic follow-up group and telemedicine group. \\
\hline Participants & Telemonitoring \\
\hline
\end{tabular}


Biannic 2012 (SEDIC) (Continued)

Elderly and well-treated population suffering from severe heart failure. Participants older than 65 years of age, recently hospitalised for $\mathrm{HF}(\mathrm{EF}<45 \%)$.

73 participants (99 randomised); 35 in control group (UC) and 38 in the TM group.

TM: mean age 76; 79\% men, mean LVEF 31.9\%, NYHA II 45.9\%, NYHA III 54\%.

UC: mean age $77.9 ; 77 \%$ men, mean LVEF 31.1\%, NYHA II 35.3\%, NYHA III 64.7\%

France

Interventions

TM group: TM during 3 months, after which participants all received usual care up until 1 year.

TM: intensity 3 times per week; variables: symptoms, weight and BP

Outcomes $\quad$ Follow-up 3 months (preliminary results; main trial will report on follow up of 1 year)

Primary outcome: number of days in hospital for acute cardiac reasons:

\begin{tabular}{|c|c|}
\hline Funding source & No funding \\
\hline Comparison Group(s) & $\begin{array}{l}\text { Author correspondence: "After an initial consultation with a specialized nurse, all eligible patients par- } \\
\text { ticipated in therapeutic education sessions, which focused on disease knowledge and dietetic advice } \\
\text { and were part of the French I-Care project endorsed by the French Society of Cardiology (14). All inves- } \\
\text { tigators were asked to treat patients in accordance with the current guidelines for the management } \\
\text { of HF, irrespective of the group assignment. Automated sphygmomanometers were given to all pa- } \\
\text { tients. Patients assigned to the SOC group were treated in the same manner as those assigned to the } \\
\text { ETM group (including standard therapeutic education), and all subjects were instructed to contact their } \\
\text { physician for HF symptom worsening and weight increase." }\end{array}$ \\
\hline
\end{tabular}

physician for HF symptom worsening and weight increase."

Notes New in 2015 review.

\section{Risk of bias}

\begin{tabular}{lll}
\hline Bias & Authors' judgement & Support for judgement \\
\hline $\begin{array}{l}\text { Random sequence genera- } \\
\text { tion (selection bias) }\end{array}$ & Unclear risk & $\begin{array}{l}\text { Quote: "Randomized occurred centrally by the Unit of Biostatistics and Clinical } \\
\text { Research" in Caen." }\end{array}$ \\
\hline $\begin{array}{l}\text { Allocation concealment } \\
\text { (selection bias) }\end{array}$ & Unclear risk & No information provided. \\
\hline
\end{tabular}

Blinding of outcome as- Unclear risk No information provided.

sessment (detection bias)

All outcomes

$\begin{array}{ll}\begin{array}{l}\text { Incomplete outcome data } \\ \text { (attrition bias) }\end{array} & \text { High risk } \\ \text { All outcomes } & 99 \text { participants were randomized, } 11 \text { withdrew consent after inclusion or re- } \\ & \text { fused further follow-up, } 3 \text { were excluded because of breach of protocol. } 4 \text { nev- } \\ & \text { er used the TM system and in } 1 \text { participant it was never installed (because of } \\ \text { prolonged hospitalisation). } 73 \text { participants completed the 3-month follow-up. } & \text { Conflict in the report: "34 patients in the telemonitoring arm produced at least } \\ 1 \text { alert" (Page 43) is in conflict with the number of } 38 \text { patients reported to be } & \text { included in the telemonitoring group. }\end{array}$

$\begin{aligned} & \text { Selective reporting (re- Low risk } \\ & \text { porting bias) }\end{aligned}$


Blum 2014 (MCCD)

\begin{tabular}{|c|c|}
\hline Methods & Randomised controlled trial; intervention arm and control (usual care) arm. \\
\hline \multirow[t]{4}{*}{ Participants } & $\begin{array}{l}204 \text { people with heart failure. Inclusion criteria: hospital admission in the past year; able to provide in- } \\
\text { formed consent; access to a telephone; systolic or diastolic dysfunction; and enrolled in Medicare Part } \\
\text { A and B. }\end{array}$ \\
\hline & Mean age 72 years. \\
\hline & $71 \%$ of participants were men. \\
\hline & USA \\
\hline \multirow[t]{3}{*}{ Interventions } & Telemonitoring. \\
\hline & $\begin{array}{l}\text { All participants were given written material about heart failure and self-management activities such } \\
\text { as daily weights, medication administration, signs and symptoms of worsening heart failure, and were } \\
\text { given an opportunity to ask questions or seek clarification as the handout was discussed. }\end{array}$ \\
\hline & $\begin{array}{l}\text { Intervention participants were instructed to use the scale, BP cuff/HR monitor and the heart rhythm } \\
\text { strip monitor at the same time each day. The transmitted data were then compared to individually as- } \\
\text { signed parameters based on the participant's admission and subsequent evaluations. Readings out- } \\
\text { side these parameters were flagged for the nurse practitioner (NP) who did the monitoring. This NP, } \\
\text { who had extensive experience in the management of people with heart failure contacted the partic- } \\
\text { ipant to gather more information and, if appropriate, adjusted medications, usually diuretics. There } \\
\text { were no specific protocols as to the management decisions, and decisions were based on the NP's ex- } \\
\text { perience or consultation with the participant's cardiologist, or both. If no flags were noted over the pe- } \\
\text { riod of } 1 \text { month, the participants were called just to maintain contact, provide encouragement and an- } \\
\text { swer any questions they might have. }\end{array}$ \\
\hline
\end{tabular}

Outcomes Mean follow-up $802 \pm 430$ days.

All-cause mortality, hospitalisations (as provided by the authors). Quality of life using SF-36 and Minnesota Living with Heart Failure Questionnaire at 12 months.

\begin{tabular}{ll} 
Funding source & Center for Medicare and Medicaid Services (CMS). \\
\hline Comparison Group(s) & $\begin{array}{l}\text { The usual-care group was not contacted again until time to schedule the 6-month follow-up appoint- } \\
\text { ment. }\end{array}$
\end{tabular}
ment.

Notes $\quad$ New in 2015 review - included as abstract in sensitivity analysis in previous version of review (Inglis
2010).

\section{Risk of bias}

\begin{tabular}{lll}
\hline Bias & Authors' judgement & Support for judgement \\
\hline $\begin{array}{l}\text { Random sequence genera- } \\
\text { tion (selection bias) }\end{array}$ & Unclear risk & $\begin{array}{l}\text { Quote: "The subject's identification information was entered into the Mathe- } \\
\text { matica Policy Research (MPR) randomisation web site and the designation of } \\
\text { participant (tele monitored group) or control (usual care) was returned"; au- } \\
\text { thor correspondence. }\end{array}$ \\
\hline $\begin{array}{l}\text { Allocation concealment } \\
\text { (selection bias) }\end{array}$ & Unclear risk & Not detailed. \\
\hline $\begin{array}{l}\text { Blinding of outcome as- } \\
\text { sessment (detection bias) } \\
\text { All outcomes }\end{array}$ & Unclear risk & Not detailed. \\
\hline $\begin{array}{l}\text { Incomplete outcome data } \\
\text { (attrition bias) }\end{array}$ & High risk & $\begin{array}{l}\text { Quote: "Two subjects declined to continue after signing consent and only par- } \\
\text { tial or no data were collected on them, one in the usual care group and one in }\end{array}$ \\
\hline
\end{tabular}


Blum 2014 (MCCD) (Continued)

All outcomes the monitored group. Therefore, complete baseline data was collected on 202 subjects. One subject completed all of the first visit data and then declined to accept the monitoring equipment when it was delivered. These subjects were eliminated from the data analysis leaving 201 subjects; 100 in the usual care group and 101 in the monitored group"; author correspondence.

$\begin{aligned} & \text { Selective reporting (re- } \quad \text { Low risk } \\ & \text { porting bias) }\end{aligned}$
porting bias)

$\begin{array}{ll}\text { Methods } & \text { Randomised controlled trial using pretest, post-test design. } \\ & 2 \text { study arms, a control (usual care) and a intervention (advanced-practice nurse-led telephone inter- } \\ \text { vention). }\end{array}$
vention).

Participants People with heart failure under the care of 1 cardiologist. Inclusion criteria included living with heart failure for more than 6 months, capable of self care, telephone access.

Mean age of participants was 60 years.

$45 \%$ men.

USA.

Structured telephone support.
Advanced Practice Nurse-led intervention was delivered by the same APN throughout the study. The
frequency of the APN-led intervention was weekly for 2 weeks and every 2 weeks for the following 10
weeks. There were 7 telephone appointments for each participant in the intervention group. The APN-
led intervention included education about the pathophysiology of HF, a low sodium diet, smoking ces-
sation, flu/pneumonia vaccination, when to call the physician with symptoms of exacerbation and
medication adherence.

\begin{tabular}{ll}
\hline Outcomes & Length of follow-up 3 months. \\
HF-related hospital admissions; QoL and self-care behaviours.
\end{tabular}

\begin{tabular}{ll}
\hline Funding source & Not reported. \\
\hline Comparison Group(s) & $\begin{array}{l}\text { Participants randomised to the control group received usual care from the cardiologist clinic. This in- } \\
\text { cluded education by the physician or a registered nurse about exercise recommendations, low sodium } \\
\text { intake, medications, and when to call the physician with increased swelling or shortness of breath. }\end{array}$ \\
\hline
\end{tabular}

Notes HF-hospitalisation data presented only as ANOVA output and unable to be included in meta-analysis. Authors contacted, but no response received.

New in 2015 review.

\section{Risk of bias}

\begin{tabular}{lll}
\hline Bias & Authors' judgement & Support for judgement \\
\hline $\begin{array}{l}\text { Random sequence genera- } \\
\text { tion (selection bias) }\end{array}$ & Unclear risk & $\begin{array}{l}\text { Not detailed: "...participants were randomly assigned to the intervention or } \\
\text { usual care group". }\end{array}$ \\
\hline $\begin{array}{l}\text { Allocation concealment } \\
\text { (selection bias) }\end{array}$ & Unclear risk & Not detailed. \\
\hline \hline
\end{tabular}


Brandon 2009 (Continued)

Blinding of outcome as- Unclear risk Not detailed. sessment (detection bias)

All outcomes

\begin{tabular}{lll}
$\begin{array}{l}\text { Incomplete outcome data } \\
\text { (attrition bias) } \\
\text { All outcomes }\end{array}$ & Unclear risk & Not detailed. \\
\hline $\begin{array}{l}\text { Selective reporting (re- } \\
\text { porting bias) }\end{array}$ & Unclear risk & $\begin{array}{l}\text { HF-hospitalisation data presented only as ANOVA output and unable to be in- } \\
\text { cluded in meta-analysis. Authors contacted, but no response received. }\end{array}$ \\
\hline
\end{tabular}

\section{Capomolla 2004}

\begin{tabular}{ll}
\hline Methods & Randomised controlled trial; intervention arm and control (usual care) arm. \\
\hline Participants & 133 people discharged from specialist CHF unit to home. \\
& Mean age 57 years. \\
& $88 \%$ of participants were men. \\
Italy
\end{tabular}

$\begin{array}{ll}\text { Interventions } & \text { Structured telephone support. } \\ & \begin{array}{l}\text { Daily communication of vital signs (including weight, systolic BP, HR) and symptoms with review by } \\ \text { nurses and physicians. Access to medical staff via phone was available as needed . }\end{array}\end{array}$

Outcomes Mortality, re hospitalisation, emergency department visits, compliance with intervention.

12 month follow-up.

\begin{tabular}{ll}
\hline Funding source & Ministero della Salute \\
\hline Comparison Group(s) & $\begin{array}{l}\text { Usual care consisted of a referral to the participant's primary care physician or cardiology department } \\
\text { at discharge. Post-discharge care was governed by the care provider. }\end{array}$ \\
\hline Notes & Included in previous version of this review (Inglis 2010). \\
\hline
\end{tabular}

\section{Risk of bias}

\begin{tabular}{lll}
\hline Bias & Authors' judgement & Support for judgement \\
\hline $\begin{array}{l}\text { Random sequence genera- } \\
\text { tion (selection bias) }\end{array}$ & Unclear risk & Method of randomisation not stated. \\
\hline $\begin{array}{l}\text { Allocation concealment } \\
\text { (selection bias) }\end{array}$ & Unclear risk & Not detailed. \\
\hline $\begin{array}{l}\text { Blinding of outcome as- } \\
\text { sessment (detection bias) } \\
\text { All outcomes }\end{array}$ & Unclear risk & Not detailed. \\
\hline $\begin{array}{l}\text { Incomplete outcome data } \\
\text { (attrition bias) } \\
\text { All outcomes }\end{array}$ & Unclear risk & Not detailed. \\
\hline
\end{tabular}


Capomolla 2004 (Continued)

Selective reporting (re- Low risk No evidence of selective outcome reporting. porting bias)

\begin{tabular}{ll}
\hline Methods & Randomised controlled trial. \\
\hline Participants & $\begin{array}{l}1653 \text { people who had recently been hospitalised for heart failure enrolled from } 2006 \text { through } 2009 \text { at } 33 \\
\text { cardiology practices across the USA. } \\
\text { Median age of the participants was } 61 \text { years. } \\
58 \% \text { of participants were men. } \\
\text { USA. }\end{array}$
\end{tabular}

Interventions Structured telephone support.

All study participants received educational materials developed by the Heart Failure Society of America, and if needed, a weighing scale.

Participants in the intervention group were also provided with detailed instructions and a demonstration by site co-ordinators of how to use the system, as well as a touch-tone telephone, if needed. The intervention was performed using a commercial system, Tel-Assurance (Pharos Innovations).

The intervention group was instructed to make daily, toll-free calls to the system. During each call, participants, via an interactive voice response system, heard a series of questions about general health and heart-failure symptoms, and entered responses using the telephone keypad. Validated depression screening questions were included monthly. Information from the system was downloaded daily to a secure Internet site and was reviewed every weekday (except on holidays) by site co-ordinators. All questions had predetermined responses that triggered "variances" to flag clinicians' attention. The protocol required the sites to contact any participant whose response generated variances and document their management of the variances.

Clinicians were instructed to treat participants in accordance with national guidelines for the management of heart failure.

Outcomes

Primary endpoint was a composite of readmission for any reason or death from any cause within 180 days after enrolment. Prespecified secondary endpoints included hospitalisation for any reason or death from any cause, hospitalisation for heart failure, number of days in the hospital, number of hospitalisations for any cause, and times to the primary endpoint and its components.

Funding source National Heart, Lung, and Blood Institute.

Comparison Group(s) All study participants received educational materials developed by the Heart Failure Society of America, and if needed, a weighing scale.

Clinicians were instructed to treat participants in accordance with national guidelines for the management of heart failure.

Notes New in 2015 review.

\section{Risk of bias}

Bias Authors' judgement Support for judgement


Chaudhry 2010 (Tele-HF) (Continued)

Random sequence genera- Low risk tion (selection bias)
Quote: "Patients were randomly assigned to receive usual care or undergo telemonitoring, according to a sequence of computer-generated random numbers, with stratification on the basis of the study site".

\section{Allocation concealment Low risk} (selection bias)
Quote: "All patients provided written informed consent before randomization".

"Randomization is centralized and performed by telephone. Randomization is stratified by study site, and force randomized within each study site in blocks of 20 (10 intervention, 10 control), to ensure a balance across study arms within each site. The randomization sequence is developed by the coordinating center using a computer random-number generator. The sequence is unknown to the attending cardiologists and nurses. The study nurses call the coordinating center when they enroll a new patient; the coordinating center personnel then assigns the new patient to intervention or control group according to the randomization list for that study site".

\begin{tabular}{|c|c|c|}
\hline $\begin{array}{l}\text { Blinding of outcome as- } \\
\text { sessment (detection bias) } \\
\text { All outcomes }\end{array}$ & Low risk & $\begin{array}{l}\text { Quote: "A committee of physicians, all of whom were unaware of the treat- } \\
\text { ment-group assignments, adjudicated each potential readmission to ensure } \\
\text { that the event qualified as a readmission (and not another clinical encounter } \\
\text { such as an emergency department visit) and to determine the primary cause of } \\
\text { the readmission". }\end{array}$ \\
\hline
\end{tabular}

Incomplete outcome data Low risk

(attrition bias)

Quote: "A total of $21 \%$ of the study patients did not complete the final tele-

All outcomes phone interview at 6 months. This rate is not surprising, given the severity of illness in the study population. Missing data for these patients should have had minimal influence on our assessment of hospitalization and vital status, which were verified through medical-record review and electronic databases."

Selective reporting (re- High risk porting bias)

Comment: The study protocol is available and the primary endpoint has been adequately reported. However, following secondary endpoints are missing: number of office visits with the clinician receiving information from the telemonitoring system; cost of inpatient and outpatient medical care; health status; participants' satisfaction with care; and participants' reported confidence in their self management of HF.

\section{Cleland 2005 (Struct Tele) (TENS-HMS)}

\begin{tabular}{ll} 
Methods & Randomised controlled trial; multiple intervention arms and control (usual care) arm. \\
& 3-armed study with both telephone and telemonitoring. \\
\hline Participants & 426 people with a recent admission for heart failure and LVEF < 40\%. \\
& Mean age 67 years. \\
& $77 \%$ of participants were men. \\
& Germany, Netherlands, UK. \\
& Structured telephone support; telemonitoring. \\
& Participants assigned to the nurse telephone support arm received a telephone call each month by a \\
heart failure specialist nurse to assess their symptoms and current medications. & \\
Participants assigned to telemonitoring received the nurse telephone support and had their weight, BP \\
and ECG monitored twice daily.
\end{tabular}


Cleland 2005 (Struct Tele) (TENS-HMS) (Continued)

Outcomes

Mortality, rehospitalisation, compliance with intervention.

240-day and 450-day follow-up.

Funding source

Quote: "The study was funded jointly by the European Union's Trans European Network (TEN) Telecom programme, which provided most of the financial support for clinical investigators, data collection, and analysis, and by Philips Medical Systems, which provided information technology systems, telemonitoring solutions, and support engineers and contributed to investigator-site staff costs".

Comparison Group(s) Usual care consisted of a management plan forwarded to the participant's primary care physician, who was asked to implement it. If the practice involved nurse titration of drugs this was allowed. Participants were assessed at a research clinic every four months; contact with the clinic was discouraged between clinic visits.

Notes Included in previous review (Inglis 2010).

Results included in meta-analysis are from 240-day follow-up.

\section{Risk of bias}

Bias Authors' judgement Support for judgement

Random sequence genera- Low risk Random permuted blocks - correspondence from author. tion (selection bias)

\begin{tabular}{|c|c|c|}
\hline $\begin{array}{l}\text { Allocation concealment } \\
\text { (selection bias) }\end{array}$ & Low risk & $\begin{array}{l}\text { After consent and collection of baseline data an independent statistical centre } \\
\text { was contacted - correspondence from author. }\end{array}$ \\
\hline
\end{tabular}

\begin{tabular}{lll}
\hline Blinding of outcome as- & Unclear risk & $\begin{array}{l}\text { Quote: "Investigators were asked to classify hospitalizations as due to heart } \\
\text { fessment (detection bias) }\end{array}$ \\
$\begin{array}{l}\text { All outcomes } \\
\text { sudden, due to circulatory failure, or due to other causes." }\end{array}$
\end{tabular}

\begin{tabular}{|c|c|c|}
\hline \multirow{2}{*}{$\begin{array}{l}\text { Incomplete outcome data } \\
\text { (attrition bias) } \\
\text { All outcomes }\end{array}$} & High risk & $\begin{array}{l}\text { Quote: "...Four were lost to follow-up and } 12 \text { declined to comply with regular } \\
\text { telemonitoring". }\end{array}$ \\
\hline & & Quote: "Analyses were conducted by intention-to-treat". \\
\hline
\end{tabular}

Selective reporting (re- Low risk Selective outcome reporting not evident.
porting bias)

\section{Cleland 2005 (Telemon) (TENS-HMS)}

\begin{tabular}{ll} 
Methods & Randomised controlled trial; multiple intervention arms and control (usual care) arm. \\
& 3-armed study with both telephone and telemonitoring. \\
\hline Participants & 426 people with a recent admission for heart failure and LVEF $<40 \%$. \\
& Mean age 67 years. \\
& $77 \%$ of participants were men. \\
& Germany, Netherlands, UK. \\
& Structured telephone support; telemonitoring. \\
Interventions & Participants assigned to the nurse telephone support arm received a telephone call each month by a \\
heart failure specialist nurse to assess their symptoms and current medications.
\end{tabular}


Cleland 2005 (Telemon) (TENS-HMS) (Continued)

Participants assigned to telemonitoring received the nurse telephone support and had their weight, BP and ECG monitored twice daily.

\begin{tabular}{ll}
\hline Outcomes & Mortality, rehospitalisation, compliance with intervention. \\
& 240-day and 450-day follow-up. \\
\hline Funding source & $\begin{array}{l}\text { Quote: "The study was funded jointly by the European Union's Trans European Network (TEN) Telecom } \\
\text { programme, which provided most of the financial support for clinical investigators, data collection, } \\
\text { and analysis, and by Philips Medical Systems, which provided information technology systems, tele- } \\
\text { monitoring solutions, and support engineers and contributed to investigator-site staff costs". }\end{array}$ \\
\hline Comparison Group(s) & $\begin{array}{l}\text { Usual care consisted of a management plan forwarded to the participant's primary care physician, who } \\
\text { was asked to implement it. If the practice involved nurse titration of drugs this was allowed. Partici- } \\
\text { pants were assessed at a research clinic every 4 months; contact with the clinic was discouraged be- } \\
\text { tween clinic visits. }\end{array}$ \\
\hline Notes & $\begin{array}{l}\text { Included in previous review (Inglis 2010). } \\
\text { Results included in meta-analysis are from 240-day follow-up. }\end{array}$ \\
\hline
\end{tabular}

\section{Risk of bias}

\begin{tabular}{|c|c|c|}
\hline Bias & Authors' judgement & Support for judgement \\
\hline $\begin{array}{l}\text { Random sequence genera- } \\
\text { tion (selection bias) }\end{array}$ & Low risk & Random permuted blocks; correspondence from author. \\
\hline $\begin{array}{l}\text { Allocation concealment } \\
\text { (selection bias) }\end{array}$ & Unclear risk & $\begin{array}{l}\text { After consent and collection of baseline data an independent statistical centre } \\
\text { was contacted; correspondence from author. }\end{array}$ \\
\hline $\begin{array}{l}\text { Blinding of outcome as- } \\
\text { sessment (detection bias) } \\
\text { All outcomes }\end{array}$ & Unclear risk & $\begin{array}{l}\text { Quote: "Investigators were asked to classify hospitalizations as due to heart } \\
\text { failure, other cardiovascular, or noncardiovascular. Deaths were classified as } \\
\text { sudden, due to circulatory failure, or due to other causes." }\end{array}$ \\
\hline \multirow{2}{*}{$\begin{array}{l}\text { Incomplete outcome data } \\
\text { (attrition bias) } \\
\text { All outcomes }\end{array}$} & High risk & $\begin{array}{l}\text { Quote: "...Four were lost to follow-up and } 12 \text { declined to comply with regular } \\
\text { telemonitoring". }\end{array}$ \\
\hline & & Quote: "Analyses were conducted by intention-to-treat". \\
\hline $\begin{array}{l}\text { Selective reporting (re- } \\
\text { porting bias) }\end{array}$ & Low risk & Selective outcome reporting not evident. \\
\hline
\end{tabular}

\section{De Lusignan 2001}

\begin{tabular}{|c|c|}
\hline Methods & Randomised controlled trial; intervention arm and control (usual care) arm. \\
\hline \multirow[t]{4}{*}{ Participants } & $\begin{array}{l}20 \text { people with heart failure confirmed by cardiologist, identified from the database of an academic } \\
\text { general practice. }\end{array}$ \\
\hline & Mean age 75 years. \\
\hline & Number or proportion of men and women not specified. \\
\hline & UK. \\
\hline
\end{tabular}


De Lusignan 2001 (Continued)

Telemonitoring of vital signs (pulse, BP, weight) and clinical status daily assessed daily by nurses along with video consultations with a nurse weekly for 3 months, fortnightly for 3 months, then monthly.

\section{Outcomes}

Mortality, compliance with intervention and medication, participant satisfaction, quality of life.

12 month follow-up. $\begin{array}{ll}\text { Funding source } & \text { Hewlett Packard now Agilent. MSD Pharmaceuticals, Herts who funded the assessments of the con- } \\ \text { trols. }\end{array}$

\begin{tabular}{ll}
\hline Comparison Group(s) & $\begin{array}{l}\text { Usual care consisted of standard general practice treatment; in addition they had their pulse, BP and } \\
\text { weight measured quarterly. They were evaluated in the same manner as the intervention group. }\end{array}$
\end{tabular}

Notes Included in previous version of review (Inglis 2010).

\section{Risk of bias}

\begin{tabular}{|c|c|c|}
\hline Bias & Authors' judgement & Support for judgement \\
\hline $\begin{array}{l}\text { Random sequence genera- } \\
\text { tion (selection bias) }\end{array}$ & Low risk & $\begin{array}{l}\text { Quote: "The first } 20 \text { patients identified by random table allocation } 10 \text { to the } \\
\text { telemedicine and } 10 \text { to the control group...". }\end{array}$ \\
\hline $\begin{array}{l}\text { Allocation concealment } \\
\text { (selection bias) }\end{array}$ & Unclear risk & Not detailed. \\
\hline $\begin{array}{l}\text { Blinding of outcome as- } \\
\text { sessment (detection bias) } \\
\text { All outcomes }\end{array}$ & Unclear risk & Not detailed. \\
\hline $\begin{array}{l}\text { Incomplete outcome data } \\
\text { (attrition bias) } \\
\text { All outcomes }\end{array}$ & Unclear risk & Not detailed. \\
\hline $\begin{array}{l}\text { Selective reporting (re- } \\
\text { porting bias) }\end{array}$ & Low risk & No evidence of selective outcome reporting. \\
\hline
\end{tabular}

DeBusk 2004

\begin{tabular}{ll} 
Methods & Randomised controlled trial; intervention arm and control (usual care) arm. \\
\hline Participants & 462 people hospitalised with a provisional diagnosis of CHF from Kaiser Permanente. \\
& Mean age 72 years. \\
& $51 \%$ of participants were men. \\
& USA. \\
\hline Interventions & Structured telephone support. \\
& $\begin{array}{l}\text { Standardised telephonic physician-directed nurse-managed case management, involving CHF lifestyle } \\
\text { education and medication management. Participants contacted weekly for } 6 \text { weeks, biweekly for } 8 \\
\text { weeks and then monthly and bimonthly. }\end{array}$
\end{tabular}

Outcomes

Mortality, rehospitalisation, emergency and outpatient department visits, prescription of recommended pharmacotherapy. 
DeBusk 2004 (Continued)

12 months follow-up.

\begin{tabular}{ll} 
Funding source & National Heart, Lung, and Blood Institute. \\
\hline Comparison Group(s) & $\begin{array}{l}\text { Usual care not clearly defined, but was provided by the participating Kaiser Permanente medical cen- } \\
\text { tres, appeared to involve a high frequency of all of kinds of follow-up clinic visits (13 in } 12 \text { months fol- } \\
\text { lowing hospitalisation). }\end{array}$
\end{tabular}

Notes Included in previous version of review (Inglis 2010).

\section{Risk of bias}

\begin{tabular}{|c|c|c|}
\hline Bias & Authors' judgement & Support for judgement \\
\hline $\begin{array}{l}\text { Random sequence genera- } \\
\text { tion (selection bias) }\end{array}$ & Low risk & $\begin{array}{l}\text { Quote: "Equal numbers of patients were allocated to the } 2 \text { groups in each } \\
\text { medical center by using the Efron procedure". }\end{array}$ \\
\hline $\begin{array}{l}\text { Allocation concealment } \\
\text { (selection bias) }\end{array}$ & Low risk & $\begin{array}{l}\text { Quote: "Research staff who were not associated with delivering the interven- } \\
\text { tion randomly assigned patients to treatment conditions by using sealed as- } \\
\text { signments." }\end{array}$ \\
\hline $\begin{array}{l}\text { Blinding of outcome as- } \\
\text { sessment (detection bias) } \\
\text { All outcomes }\end{array}$ & Low risk & $\begin{array}{l}\text { Quote: "Research staff who were not associated with, and were blinded to, the } \\
\text { intervention conditions measured health outcomes at } 12 \text { months." }\end{array}$ \\
\hline $\begin{array}{l}\text { Incomplete outcome data } \\
\text { (attrition bias) } \\
\text { All outcomes }\end{array}$ & High risk & $\begin{array}{l}\text { Quote: "During the first year of follow-up, } 23 \text { patients (3\%) dropped out of the } \\
\text { trial ( } 8 \text { in the treatment group and } 15 \text { in the usual care group)" } \\
\text { The analysis was by intention-to-treat. }\end{array}$ \\
\hline $\begin{array}{l}\text { Selective reporting (re- } \\
\text { porting bias) }\end{array}$ & Low risk & No evidence of selective reporting. \\
\hline
\end{tabular}

$\begin{array}{ll}\text { Methods } & \text { Multicentre RCT (all centres located in Belgium). } \\ & \text { Follow-up: } 6 \text { months } \\ & 2 \text { arms: Telemonitoring (TM) versus usual care. }\end{array}$

Participants

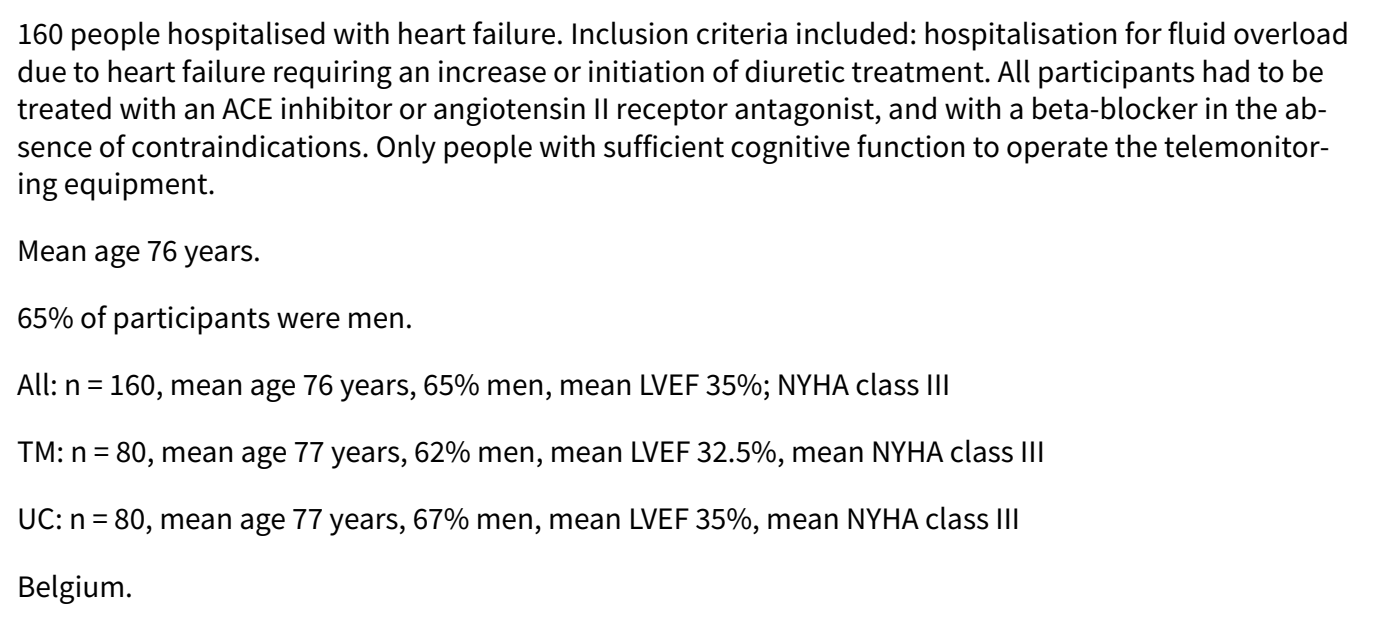


Dendale 2012 (TEMA-HF1) (Continued)

Interventions Telemonitoring.

Daily measurement of weight, BP and HR for 6 months. Participants were seen at the HF clinic 2 weeks after discharge, and at 3 and 6 months (but were allowed to visit the clinic sooner or more frequently if necessary).

Professionals involved: GP, heart failure clinic (HF nurse and cardiologist).

Primary endpoint was all-cause mortality.
Secondary endpoints were days lost until death, hospitalisation or dialysis and number of hospitalisa-
tions and cost of hospitalisations.
6 month follow-up.

Funding source The Belgian Government Health Insurance Institute; Leo Pharma

\begin{tabular}{ll}
\hline Comparison Group(s) & $\begin{array}{l}\text { All participants were seen at the HF clinic } 2 \text { weeks after discharge. No intervention by the study nurse } \\
\text { or HF clinic was done thereafter. }\end{array}$
\end{tabular}
or HF clinic was done thereafter.

Professionals involved: GP (who could refer participants to their cardiologist if needed).

\begin{tabular}{|c|c|c|}
\hline Notes & New in 2015 review. & \\
\hline \multicolumn{3}{|l|}{ Risk of bias } \\
\hline Bias & Authors' judgement & Support for judgement \\
\hline $\begin{array}{l}\text { Random sequence genera- } \\
\text { tion (selection bias) }\end{array}$ & Low risk & $\begin{array}{l}\text { Quote: "Next, patients were block randomised by sealed envelopes to } 6 \\
\text { months of intense follow-up facilitated by TM or usual care". }\end{array}$ \\
\hline $\begin{array}{l}\text { Allocation concealment } \\
\text { (selection bias) }\end{array}$ & Low risk & Comment: Method of allocation concealment was sealed envelopes. \\
\hline $\begin{array}{l}\text { Blinding of outcome as- } \\
\text { sessment (detection bias) } \\
\text { All outcomes }\end{array}$ & Low risk & $\begin{array}{l}\text { Quote: "To decrease the risk of bias in an unblinded study, the data were col- } \\
\text { lected by a data manager not involved in patient care, and not stationed in } \\
\text { one of the participating hospitals. The statistical analysis was done by a sta- } \\
\text { tistician not involved in patient care. The reason for hospitalization was deter- } \\
\text { mined based on the discharge letter and adjudicated after the end of the study } \\
\text { in a blinded way, and the primary endpoint was all-cause mortality, which } \\
\text { avoided the difficulty of determining the cause of death." }\end{array}$ \\
\hline $\begin{array}{l}\text { Incomplete outcome data } \\
\text { (attrition bias) } \\
\text { All outcomes }\end{array}$ & Low risk & $\begin{array}{l}\text { Quote: "Four patients dropped out prematurely because of lack of motivation } \\
\text { (timing and assigned group not specified). Data from these subjects were in- } \\
\text { cluded in the analysis (intention to treat)". }\end{array}$ \\
\hline $\begin{array}{l}\text { Selective reporting (re- } \\
\text { porting bias) }\end{array}$ & Low risk & $\begin{array}{l}\text { Comment: The study protocol is not available but the results paper reports on } \\
\text { all prespecified outcomes. }\end{array}$ \\
\hline
\end{tabular}

\section{DeWalt 2006}

\begin{tabular}{ll}
\hline Methods & Randomised controlled trial; intervention arm and control (usual care) arm. \\
\hline Participants & $\begin{array}{l}127 \text { people with confirmed HF, NYHA class II - IV symptoms within the last } 3 \text { months and currently tak- } \\
\text { ing furosemide from the University of North Carolina (UNC) General Internal Medicine Practice. } \\
\text { Mean age } 62.5 \text { years. }\end{array}$
\end{tabular}


DeWalt 2006 (Continued)

$47 \%$ of participants were men.

USA.

\begin{tabular}{ll}
\hline Interventions & Structured telephone support. \\
& Intervention participants received self-care education, picture-based educational materials with verbal \\
explanation, a digital scale and scheduled follow-up phone calls (days $3,7,14,21,28,56)$ and monthly \\
during months $3-6$ for reinforcement of education and revision of individualised care plan.
\end{tabular}

\begin{tabular}{ll}
\hline Outcomes & $\begin{array}{l}\text { Mortality, all-cause rehospitalisation, HF-related quality of life, HF self efficacy, HF knowledge, reported } \\
\text { weight monitoring (self-management behaviour). } \\
12 \text { month follow-up. }\end{array}$ \\
\hline Funding source & $\begin{array}{l}\text { Pfizer Health Literacy Initiative, the Robert Wood Johnson Clinical Scholars Program, the University of } \\
\text { North Carolina Program on Health Outcomes, and the National Institute of Nursing Research, NIH. }\end{array}$ \\
\hline Comparison Group(s) & $\begin{array}{l}\text { Control group participants received a general heart failure education pamphlet and usual care from } \\
\text { their primary physician (not specified). Data collection occurred at } 6 \text { and } 12 \text { months via in-person inter- } \\
\text { view and medical record review. }\end{array}$ \\
\hline Notes & Included in previous version of review (Inglis 2010).
\end{tabular}

\section{Risk of bias}

\begin{tabular}{lll}
\hline Bias & Authors' judgement & Support for judgement \\
\hline $\begin{array}{l}\text { Random sequence genera- } \\
\text { tion (selection bias) }\end{array}$ & Low risk & $\begin{array}{l}\text { Quote: "..randomised patients by concealed allocation based on a random } \\
\text { number generator" }\end{array}$ \\
\hline $\begin{array}{l}\text { Allocation concealment } \\
\text { (selection bias) }\end{array}$ & Unclear risk & $\begin{array}{l}\text { Comment: Unclear if randomisation performed before or after consent provid- } \\
\text { ed. }\end{array}$ \\
\hline $\begin{array}{l}\text { Blinding of outcome as- } \\
\text { sessment (detection bias) }\end{array}$ & High risk & $\begin{array}{l}\text { Quote: "We assessed outcomes at } 6 \text { and } 12 \text { months through in-person inter- } \\
\text { views and review of the medical record. To be sensitive to low literacy, all in- } \\
\text { terviews were conducted verbally by a trained research assistant. If patients } \\
\text { were unable to come to clinic for the interview, it was conducted by phone. } \\
\text { The research assistant was not blinded to the patient's study group." }\end{array}$ \\
\hline $\begin{array}{l}\text { Incomplete outcome data } \\
\text { (attrition bias) }\end{array}$ & Low risk & $\begin{array}{l}\text { Quote: "Patients who did not return any phone calls and did not return for fol- } \\
\text { low-up assessment did not have outcome data for analysis. Patients who with- } \\
\text { drew from the study were censored at the time of withdrawal; any data col- } \\
\text { lected prior to withdrawal were included in the analysis" p5. }\end{array}$ \\
$\begin{array}{l}\text { "Of those randomised to the control group, 1 never returned after the first vis- } \\
\text { it, 1 withdrew during the study and } 4 \text { died during the study. Follow-up was } \\
\text { completed for all of the remaining participants (98\%)" }\end{array}$ \\
\hline $\begin{array}{l}\text { Selective reporting (re- } \\
\text { porting bias) }\end{array}$ & Low risk & \begin{tabular}{l} 
No evidence of selective reporting. \\
\hline
\end{tabular} \\
\hline
\end{tabular}

Domingues 2011

Methods Randomised controlled trial.


Domingues 2011 (Continued)

2 groups: intervention group (IG): education during hospitalisation followed by telephone monitoring after discharge, and control group (CG): in-hospital education only.

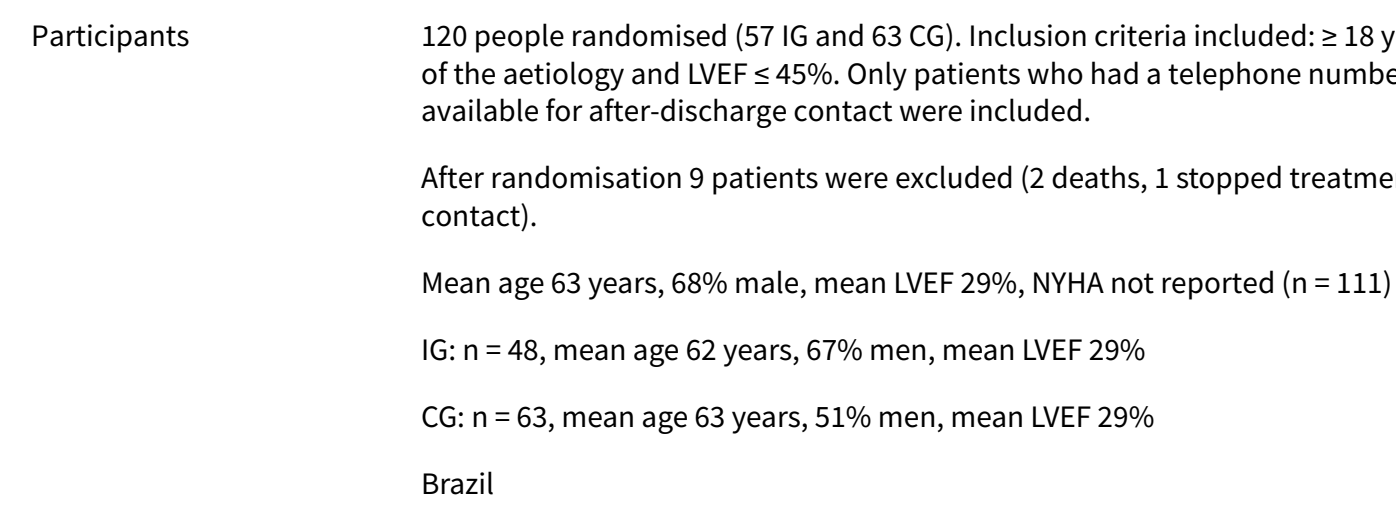

Interventions Education in hospital (3 - 5 visits).

Systematic telephone contact (study nurse) for a 3-month period.

1 telephone contact per week during the 1st month, followed by 1 every 15 days in the 2 nd and 3rd month.

\begin{tabular}{ll}
\hline Outcomes & Follow-up: 3 months \\
& $\begin{array}{l}\text { Primary outcome was HF awareness and self-care knowledge scores. } \\
\text { Secondary outcomes were frequency of visits to the emergency room, rehospitalisations and death at } \\
\text { the end of the } 3 \text { month follow-up. }\end{array}$ \\
\hline Funding source & $\begin{array}{l}\text { FIPE (Fundação Instituto de Pasquisas Econŏmicas) and CNPq (Conselho Nacional de Desenvolvimento } \\
\text { Cientico e Tecnologico) }\end{array}$ \\
\hline Comparison Group(s) & $\begin{array}{l}\text { Education in hospital (3 - } 5 \text { visits). } \\
\text { Follow-up of the participant at the return appointment to the outpatient clinic without any telephone } \\
\text { contact in between. }\end{array}$ \\
\hline Notes & New in 2015 review. \\
\hline
\end{tabular}

\section{Risk of bias}

Bias Authors' judgement Support for judgement

Random sequence genera- Unclear risk tion (selection bias)

Quote: "After discharge from the hospital, patients were randomised to receive systematic telephone contacts for a 3-month investigational period (intervention group- IG) or to receive the usual care."

Comment: Method of randomisation not specified.

Allocation concealment $\quad$ Unclear risk $\quad$ Comment: Not detailed.
(selection bias)

Blinding of outcome as- Unclear risk Comment: Not detailed.
sessment (detection bias)

All outcomes

$\begin{array}{ll}\begin{array}{l}\text { Incomplete outcome data } \\ \text { (attrition bias) }\end{array} & \text { High risk } \\ \text { All outcomes } & \text { Quote: "After randomisation, } 9 \text { patients were excluded from the IG ( } \mathrm{n}=2 \\ \text { deaths, } n=1 \text { quit treatment, } n=6 \text { could not contact). At the end of the study, }\end{array}$


Comment: Data for 9 participants excluded after randomisation were not included in the analysis.

Selective reporting (re- Low risk Comment: All prespecified outcomes have been addressed.
porting bias)

\section{Galbreath 2004}

\begin{tabular}{|c|c|}
\hline Methods & Randomised controlled trial; intervention arm and control (usual care) arm. \\
\hline \multirow[t]{4}{*}{ Participants } & $\begin{array}{l}1069 \text { people with symptoms of CHF and documented systolic (mean EF 35\%) or diastolic dysfunction } \\
\text { (echo confirmed). }\end{array}$ \\
\hline & Mean age 71 years. \\
\hline & $71 \%$ of participants were men. \\
\hline & USA. \\
\hline
\end{tabular}

Interventions Structured telephone support.

All intervention participants received bathroom scales and were assigned a disease manager who administered the disease management programme telephonically. Initial call frequency was weekly then transitioned to monthly for the duration of the study. Call frequency could be adjusted for acuity or need. After each call a call summary was faxed to the participant's primary care provider.

An additional randomisation was performed within the intervention arm, with some participants provided with in-home technology (BP monitor, pulse oximeter). These measurements were reported by the participant to the disease manager, but the data were not forwarded to the primary care provider. These participants also wore activity monitors at regular intervals and had 6-monthly measurement of thoracic bioimpedance cardiac output; these data were not forwarded to the primary care physician.

The authors state: "because data derived from the technology were not used in clinical management, we combined results from the two treatment groups for the purposes of this analysis."

Traditional-care participants were managed as usual by their physicians.

Outcomes All-cause mortality, 6-minute walk performance, functional therapeutic class improvement, total healthcare costs. Improvement in ejection fraction and medication adherence were assessed in a subgroup.

18-month follow-up.

Funding source
by Department of Defense, US Army Medical Research Acquisition Activity. Clinical space was provided
Neighborhood Clinic, and Hill Country Medical Associates.

\begin{tabular}{ll}
\hline Comparison Group(s) & Traditional-care participants were managed as usual by their physicians. \\
\hline Notes & Included in previous version of review (Inglis 2010). \\
\hline Risk of bias & \\
\hline Bias & Authors' judgement Support for judgement \\
\hline
\end{tabular}


Galbreath 2004 (Continued)

Random sequence genera- Unclear risk Quote: "Participants were randomised in a 2:1 ratio between the treatment tion (selection bias) and control groups".

Method of randomisation not detailed.

$\begin{aligned} & \text { Allocation concealment } \\ & \text { (selection bias) }\end{aligned}$ Low risk Randomisation performed after informed consent obtained.

Blinding of outcome as- Unclear risk sessment (detection bias) All outcomes
Quote: "To assess economic and utilization outcomes, we thoroughly reviewed patient medical records. Records covering the period of a patient's enrollment in the trial were requested from both PCPs and specialty physicians. Records of inpatient and outpatient encounters and emergency department visits were culled from patient self-reported data, reviews of electronic hospital records, and documents received from physician and clinic charts. Reviews were performed by study staff, consisting of physicians, nurses, and ancillary health providers."

Some evidence of attrition of study participants but actual numbers not presented.

Incomplete outcome data High risk
(attrition bias)

All outcomes

Selective reporting (re- Low risk No evidence of selective reporting.
porting bias)

\section{Gattis 1999 (PHARM)}

\begin{tabular}{|c|c|}
\hline Methods & Randomised controlled trial; intervention arm and control (usual care) arm. \\
\hline \multirow[t]{4}{*}{ Participants } & 181 people with heart failure being evaluated in cardiology clinic. \\
\hline & Mean age 67 years. \\
\hline & $68 \%$ of participants were men. \\
\hline & USA. \\
\hline \multirow[t]{2}{*}{ Interventions } & Structured telephone support. \\
\hline & $\begin{array}{l}\text { Clinical pharmacist-led medication review and patient education. Regularly-scheduled telephone con- } \\
\text { tact (at 2, } 12 \text { and } 24 \text { weeks) to detect clinical deterioration early. }\end{array}$ \\
\hline \multirow[t]{2}{*}{ Outcomes } & Mortality, rehospitalisation, medication prescription. \\
\hline & 6-month follow-up. \\
\hline Funding source & $\begin{array}{l}\text { American Society of Health-System Pharmacists Research and Education Foundation, Bethesda, Md } \\
\text { and the Duke Clinical Research Institute. }\end{array}$ \\
\hline Comparison Group(s) & $\begin{array}{l}\text { The control group received usual care which did not include the pharmacist providing recommenda- } \\
\text { tions regarding drug therapy to the attending physician or providing education to the participant. Par- } \\
\text { ticipant assessment and education were provided by the attending physician and/or physician assis- } \\
\text { tant or nurse practitioner. The participant was contacted by the pharmacist via telephone to identify } \\
\text { clinical events. }\end{array}$ \\
\hline Notes & Included in previous version of review (Inglis 2010). \\
\hline
\end{tabular}

\section{Risk of bias}

Structured telephone support or non-invasive telemonitoring for patients with heart failure (Review)

Copyright @ 2015 The Cochrane Collaboration. Published by John Wiley \& Sons, Ltd. 
Gattis 1999 (PHARM) (Continued)

\begin{tabular}{lll} 
Bias & Authors' judgement & Support for judgement \\
\hline $\begin{array}{l}\text { Random sequence genera- } \\
\text { tion (selection bias) }\end{array}$ & Low risk & $\begin{array}{l}\text { Quote: "...patients were randomised according to a computer-generated ran- } \\
\text { domisation scheme..". }\end{array}$ \\
\hline $\begin{array}{l}\text { Allocation concealment } \\
\text { (selection bias) }\end{array}$ & Unclear risk & Not detailed. \\
\hline $\begin{array}{l}\text { Blinding of outcome as- } \\
\begin{array}{l}\text { sessment (detection bias) } \\
\text { All outcomes }\end{array}\end{array}$ & Low risk & Quote: "...clinical events were adjudicated by a blinded end point committee". \\
\hline $\begin{array}{l}\text { Incomplete outcome data } \\
\text { (attrition bias) } \\
\text { All outcomes }\end{array}$ & Unclear risk & Not detailed. \\
\hline $\begin{array}{l}\text { Selective reporting (re- } \\
\text { porting bias) }\end{array}$ & Low risk & No evidence of selective reporting. \\
\hline
\end{tabular}

GESICA 2005 (DIAL)

\begin{tabular}{ll} 
Methods & Randomised controlled trial; intervention arm and control (usual care) arm. \\
\hline Participants & 1518 outpatients with stable CHF. \\
& Mean age 65 years. \\
& $71 \%$ of participants were men. \\
& Argentina.
\end{tabular}

Structured telephone support.
$\begin{aligned} & \text { Nurses trained in the management of people with CHF performed structured telephone follow-up } \\ & \text { based on adherence to diet and treatment, monitoring of symptoms, control of fluid retention and dai- } \\ & \text { ly physical activity. Participants were contacted } 4 \text { times in the first fortnight and then as needed. }\end{aligned}$

\begin{tabular}{ll}
\hline Outcomes & Mortality, rehospitalisation, quality of life. \\
& Mean 16-month follow-up. \\
\hline Funding source & $\begin{array}{l}\text { GESICA (Grupo de Estudio de Sobrevida en la Insuficiencia Cardíaca en la Argentina) Foundation with } \\
\text { unrestricted collaboration from the following local companies in Argentina: Roche, Boehringer Ingel- } \\
\text { heim, Bagó, Pharmacia, Novartis, and Merck Sharp and Dohme. }\end{array}$
\end{tabular}

\begin{tabular}{ll}
\hline Comparison Group(s) & $\begin{array}{l}\text { Participants in the control group were followed by their attending cardiologists and received care simi- } \\
\text { lar to the intervention group. }\end{array}$ \\
\hline Notes & Included in previous version of review (Inglis 2010). \\
\hline
\end{tabular}

\section{Risk of bias}

\begin{tabular}{lll}
\hline Bias & Authors' judgement & Support for judgement \\
\hline $\begin{array}{l}\text { Random sequence genera- } \\
\text { tion (selection bias) }\end{array}$ & Low risk & $\begin{array}{l}\text { Quote: "We then used concealed randomisation lists to do permuted block } \\
\text { randomisation stratified by attending cardiologist ". }\end{array}$ \\
\hline
\end{tabular}


GESICA 2005 (DIAL) (Continued)

\begin{tabular}{|c|c|c|}
\hline $\begin{array}{l}\text { Allocation concealment } \\
\text { (selection bias) }\end{array}$ & Low risk & $\begin{array}{l}\text { After provision of consent, patient's cardiologist contacted study centre (BMJ } \\
\text { comment). }\end{array}$ \\
\hline
\end{tabular}

\begin{tabular}{|c|c|c|}
\hline $\begin{array}{l}\text { Blinding of outcome as- } \\
\text { sessment (detection bias) }\end{array}$ & Low risk & $\begin{array}{l}\text { Quote: "The clinical events committee, which was blinded to the patients' } \\
\text { treatment group assignment, adjudicated all outcomes." }\end{array}$ \\
\hline
\end{tabular}

All outcomes

\begin{tabular}{ll}
\hline $\begin{array}{l}\text { Incomplete outcome data } \\
\text { (attrition bias) }\end{array}$ & Low risk \\
All outcomes & Quote: "Follow-up was completed in $1511(99.5 \%)$ randomised patients". \\
\hline
\end{tabular}

Selective reporting (re- Low risk No evidence of selective reporting.
porting bias)

\section{Giordano 2009}

\begin{tabular}{|c|c|}
\hline Methods & Randomised controlled trial; intervention arm and control (usual care) arm. \\
\hline \multirow[t]{4}{*}{ Participants } & $\begin{array}{l}460 \text { people with confirmed } \mathrm{CHF} \text { with } \mathrm{LVEF}<40 \% \text { and at least } 1 \text { hospitalisation for acute } \mathrm{HF} \text { in the prior } \\
\text { year. }\end{array}$ \\
\hline & Mean age 57 years. \\
\hline & $85 \%$ of participants were men. \\
\hline & Italy. \\
\hline
\end{tabular}

Telemonitoring.
Home-Based Telemanagement (HBT) participants received a 1-lead trace portable device that trans-
ferred results via telephone where a nurse was available for interactive teleconsultation. Scheduled
standardised telemonitoring appointments were performed every week to15 days depending on HF
severity discussing symptomology, medications, self care and, if required, the transmission of the ECG
trace.

Outcomes Unplanned cardiovascular hospital readmissions, hospitalisation for HF, haemodynamic instability
episode occurrence, cardiovascular mortality.

12-month follow-up.

\begin{tabular}{|c|c|c|}
\hline Funding source & \multicolumn{2}{|c|}{ National Ministry of Health. } \\
\hline Comparison Group(s) & \multicolumn{2}{|c|}{$\begin{array}{l}\text { Usual care consisted of participants being referred to their primary care physician (PCP) and cardiolo- } \\
\text { gist for clinical management. These participants attended a 2-weeks post-discharge PCP appointment } \\
\text { and a structured follow-up outpatient cardiologist appointment at } 12 \text { months. }\end{array}$} \\
\hline Notes & \multicolumn{2}{|c|}{ Included in previous version of review (Inglis 2010). } \\
\hline \multicolumn{3}{|l|}{ Risk of bias } \\
\hline Bias & Authors' judgement & Support for judgement \\
\hline $\begin{array}{l}\text { Random sequence genera- } \\
\text { tion (selection bias) }\end{array}$ & Unclear risk & $\begin{array}{l}\text { Quote: "Random permuted blocks for each center were used to allocate pa- } \\
\text { tients to treatment groups". }\end{array}$ \\
\hline
\end{tabular}


Giordano 2009 (Continued)

Allocation concealment $\quad$ Unclear risk Not detailed.
(selection bias)

\begin{tabular}{lll}
\hline $\begin{array}{l}\text { Blinding of outcome as- } \\
\text { sessment (detection bias) }\end{array}$ & Unclear risk & $\begin{array}{l}\text { Quote: "The data and the cause of readmission were obtained from the GP and } \\
\text { confirmed by hospital records. Episodes of clinical instability were confirmed } \\
\text { All outcomes }\end{array}$ \\
$\begin{array}{l}\text { by the GP. Cardiovascular deaths were ascertained through the GP or hospital } \\
\text { records." }\end{array}$
\end{tabular}

\begin{tabular}{|c|c|c|}
\hline $\begin{array}{l}\text { Incomplete outcome data } \\
\text { (attrition bias) } \\
\text { All outcomes }\end{array}$ & High risk & $\begin{array}{l}\text { Quote: "...one patient in UC group and four in HBT group were lost to fol- } \\
\text { low-up" p196. } \\
\text { Quote: "Analyses were conducted according to the intention-to treat ap- } \\
\text { proach". }\end{array}$ \\
\hline
\end{tabular}

\begin{tabular}{l}
\hline $\begin{array}{l}\text { Selective reporting (re- Low risk } \\
\text { porting bias) }\end{array}$ \\
\hline
\end{tabular}

Goldberg 2003 (WHARF)

\begin{tabular}{|c|c|}
\hline Methods & Randomised controlled trial; intervention arm and control (usual care) arm. \\
\hline \multirow[t]{4}{*}{ Participants } & 280 people hospitalised with NYHA Class III - IV, with a LVEF $<35 \%$. \\
\hline & Mean age 59 years. \\
\hline & $68 \%$ of participants were men. \\
\hline & USA. \\
\hline \multirow[t]{2}{*}{ Interventions } & Telemonitoring. \\
\hline & $\begin{array}{l}\text { Daily transmission of weight and symptoms using a customised monitor, data was reviewed daily by } \\
\text { nurses and concerns reported to the physician. }\end{array}$ \\
\hline \multirow[t]{2}{*}{ Outcomes } & $\begin{array}{l}\text { Mortality, rehospitalisation, emergency department visits, quality of life, participant satisfaction, com- } \\
\text { pliance with intervention. }\end{array}$ \\
\hline & Mean 6-month follow-up. \\
\hline Funding source & Alere Medical, Incorporated. \\
\hline Comparison Group(s) & $\begin{array}{l}\text { Participants in the control group were instructed to contact their physician for weight increases of } \\
\text { more than a prespecified amount or if their symptoms of heart failure worsened. They had a weight log } \\
\text { to bring to visits. Follow-up visits, other than study visits were at the discretion of the treating physi- } \\
\text { cian. Telephone contacts were permitted at the discretion of the treating physician or nurse. }\end{array}$ \\
\hline Notes & Included in previous version of review (Inglis 2010). \\
\hline
\end{tabular}

\section{Risk of bias}

\begin{tabular}{lll}
\hline Bias & Authors' judgement & Support for judgement \\
\hline $\begin{array}{l}\text { Random sequence genera- } \\
\text { tion (selection bias) }\end{array}$ & Unclear risk & Comment: method of randomisation not detailed. \\
\hline $\begin{array}{l}\text { Allocation concealment } \\
\text { (selection bias) }\end{array}$ & Low risk & $\begin{array}{l}\text { Quote: "After informed consent was obtained and screening laboratory evalu- } \\
\text { ations were completed, patients were randomized". }\end{array}$ \\
\hline \hline
\end{tabular}


Goldberg 2003 (WHARF) (Continued)

Blinding of outcome as- Low risk sessment (detection bias)

All outcomes
Quote: "To insure that all hospitalizations, emergency room visits, and deaths were identified, all patients were contacted by telephone on a monthly basis by a non medical surveyor (blinded to patient treatment group randomization), located outside of the enrollment sites and Alere monitoring center. Records were obtained for each of these events, including those occurring outside of the participating health systems."

$\begin{array}{ll}\begin{array}{l}\text { Incomplete outcome data } \\ \text { (attrition bias) }\end{array} & \text { Quote: "During the study, } 32 \text { patients either refused follow-up data collection } \\ \text { All outcomes } & \text { or were lost to follow-up. Seven patients received cardiac transplantation and } \\ & \text { were censored on the day of transplant. Excluding deaths, there was no differ- } \\ & \text { ence between groups in the percentage of patients who failed to complete six } \\ & \text { months of follow-up". }\end{array}$

Selective reporting (re- Low risk Comment: some nominated outcomes (satisfaction) were not reported. porting bias)

Koehler 2011 (TIM-HF)

\begin{tabular}{|c|c|}
\hline Methods & Open, randomised, parallel-group, prospective multicentre clinical trial. \\
\hline \multirow[t]{4}{*}{ Participants } & $\begin{array}{l}\text { A total of } 710 \text { people ( } 354 \text { patients RTM group and } 356 \text { to the usual care group) with chronic HF who } \\
\text { had signed informed consent were eligible to participate if they were at least } 18 \text { years of age, were in } \\
\text { NYHA class II or III, and had a LVEF of } 35 \% \text {. }\end{array}$ \\
\hline & Mean age 66.9 years \\
\hline & $80 \%$ men \\
\hline & Germany \\
\hline Interventions & $\begin{array}{l}\text { Quote: "The telemonitoring system used in the TIM-HF trial is based on a wireless Bluetooth system } \\
\text { with a personal digital assistant (PDA) as the central structural element. The only prerequisite for this } \\
\text { system to function once installed is the availability of a mobile phone network connection. Three mea- } \\
\text { suring devices are integrated into the system, namely one to collect electrocardiogram (ECG) measure- } \\
\text { ments, one to collect BP measurements, and one to collect body weight. Each device is equipped with } \\
\text { a Bluetooth chip and connected to the PDA. The patient performs the daily self-assessment of health } \\
\text { status by using the PDA interface. A subgroup of patients in the intervention group performed a 6-min } \\
\text { walk test using a telemedical accelerometer once a month starting } 3 \text { months after randomization." }\end{array}$ \\
\hline
\end{tabular}

Outcomes

Quote: "The primary endpoint is all-cause mortality. The first secondary endpoint is a composite of the combined rate of cardiovascular death and hospitalization for worsening heart failure. For this composite endpoint, patients will be followed for all hospitalizations for heart failure until death or the end of follow-up thus enabling the possibility to report event rates for each event. Other secondary endpoints include:

- days lost due to death or heart failure hospitalization,

- cardiovascular mortality,

- rate of cardiovascular hospitalization at 6,12 and 24 months

- rate of hospitalization for Heart Failure at 6,12 and 24 months

- hospitalization for any reason,

- cardiovascular hospitalization,

- hospitalization for heart failure,

- duration of all hospitalizations for heart failure,

- NYHA functional class at 12 months and 24 months adjusted for baseline,

- SF-36 physical functioning score at 12 months and 24 months adjusted for baseline, and

- PHQ-9 depression score, 12 months and 24 months adjusted for baseline. 
Koehler 2011 (TIM-HF) (Continued)

As for the first secondary endpoint, patients will be followed for all events until death or the end of follow-up."

Quote: "Power Calculation: The sample size calculation was based on the assumption of a mortality rate of $27 \%$ in the control group and $17 \%$ in the intervention group. This corresponds to a relative risk (RR) of 0.63 , which is comparable to the effect of remote patient management reported by Cleland et al (2005) ( $R R=0.65)$ and more conservative than the findings of Goldberg et al (2003) (RR=0.44)."

Funding source

Quote: "The technology development as well as the clinical trial was funded in a public-private partnership through a research grant of the German Federal Ministry of Economics and Technology (01MG531) and by the following companies: Robert Bosch Healthcare GmbH, Waiblingen, Germany; InterComponentWare AG, Walldorf, Germany; and Aipermon GmbH \& Co KG, Munich, Germany."

Comparison Group(s)

Quote: "Patients randomized to the usual care group were followed by their treating physician who was instructed to ensure that the patients were optimally treated for their heart failure in accordance with the current standards and guidelines for treatment of patients with CHF."

Notes New in 2015 review.

\section{Risk of bias}

\begin{tabular}{|c|c|c|}
\hline Bias & Authors' judgement & Support for judgement \\
\hline $\begin{array}{l}\text { Random sequence genera- } \\
\text { tion (selection bias) }\end{array}$ & Low risk & $\begin{array}{l}\text { Quote: "Patients were then assigned to one of the two treatment arms by a } \\
\text { central computerized randomization system. In order to achieve a balance of } \\
\text { potential risk factors in the treatment arms, Pocock's minimization algorithm5 } \\
\text { (with } 20 \% \text { residual randomness) was used, with the following variables: NYHA } \\
\text { class II or III, hospitalization for heart failure within } 2 \text { years prior to randomiza- } \\
\text { tion, implanted defibrillator, region (Berlin-Brandenburg or Baden-Württem- } \\
\text { berg), age group (,60 or } 60-70 \text { or . } 70 \text { years), known diabetes mellitus, known } \\
\text { cerebrovascular disease, living alone or with partner, gender, presence of CRT, } \\
\text { use of statins, and use of aldosterone receptor antagonists." }\end{array}$ \\
\hline
\end{tabular}

\begin{tabular}{|c|c|c|}
\hline $\begin{array}{l}\text { Allocation concealment } \\
\text { (selection bias) }\end{array}$ & Low risk & Comment: Investigators were unaware of the randomisation sequence. \\
\hline $\begin{array}{l}\text { Blinding of outcome as- } \\
\text { sessment (detection bias) } \\
\text { All outcomes }\end{array}$ & Low risk & $\begin{array}{l}\text { Quote: "A Clinical Endpoint Committee (CEC), blinded to treatment allocation, } \\
\text { will classify all deaths and hospitalizations using pre-defined criteria as de- } \\
\text { tailed in the CEC charter". }\end{array}$ \\
\hline
\end{tabular}

\begin{tabular}{lll}
\hline $\begin{array}{l}\text { Incomplete outcome data } \\
\text { (attrition bias) }\end{array}$ & Low risk & $\begin{array}{l}\text { Quote: "The essential features of the planned statistical analyses are as fol- } \\
\text { lows: two analysis populations will be distinguished-the full analysis set (FAS) } \\
\text { and the per protocol analysis population." }\end{array}$ \\
\hline $\begin{array}{l}\text { Selective reporting (re- } \\
\text { porting bias) }\end{array}$ & Low risk & $\begin{array}{l}\text { Comment: Not all data for changes in NYHA class, PHQ-9 and SF-36 are report- } \\
\text { ed (but percentages at } 12 \text { and } 24 \text { months are reported) }\end{array}$
\end{tabular}

Krum 2013 (CHAT)

\begin{tabular}{ll} 
Methods & $\begin{array}{l}\text { Cluster-randomised controlled trial. Computer-generated random sequence. } \\
\text { GP practices were the unit of randomisation. GPs were not blinded to allocation group before recruiting } \\
\text { and consenting participants. }\end{array}$ \\
\hline Participants & $\begin{array}{l}405 \text { people with a recent hospital discharge due to a primary diagnosis of heart failure with an EF of } \\
40 \% \text { and in NYHA class II - IV were randomised to either usual care or usual care plus telephone moni- } \\
\text { toring performed at least once per month. }\end{array}$
\end{tabular}


Krum 2013 (CHAT) (Continued)

Mean age 73 years.

$61 \%$ of participants were men.

Australia.

Structured telephone support.
Nurse-led telephone monitoring using the Telewatch System (Baltimore).
Participant responded to computer-generated CHF self-monitoring questions by pressing the numbers
on the touch-phone key pad.
Nurse survey incoming calls daily and responded to preset variations to participant's parameters.

Outcomes The primary endpoint was the change in Packer clinical composite score. HRQOL, BNP. Secondary endpoints included: all-cause death and all-cause hospitalisation as well as heart failure-related death and heart failure-specific hospitalisation.

Participants were assessed by a blinded reviewer at baseline and then after 6 and 12 months.

12 months follow-up.

\begin{tabular}{ll}
\hline Funding source & National Health and Medical Research Council, National Heart Foundation of Australia, and Medical \\
Benefits Fund.
\end{tabular}

\begin{tabular}{ll}
\hline Comparison Group(s) & Usual care discharge follow-up with GP and copy of guidelines. \\
\hline Notes & $\begin{array}{l}\text { New in } 2015 \text { review; included as abstract in sensitivity analysis in previous version of review (Inglis } \\
\text { 2010). }\end{array}$
\end{tabular}

\section{Risk of bias}

\begin{tabular}{lll}
\hline Bias & Authors' judgement & Support for judgement \\
\hline $\begin{array}{l}\text { Random sequence genera- } \\
\text { tion (selection bias) }\end{array}$ & Low risk & Computer-generated random sequence. \\
\hline $\begin{array}{l}\text { Allocation concealment } \\
\text { (selection bias) }\end{array}$ & Unclear risk & $\begin{array}{l}\text { Quote: "The study involved cluster randomization at the level of the general } \\
\text { practitioner (1:1, usual care, usual care plus intervention, stratified by rural, re- } \\
\text { mote and outer metropolitan area [RRMA] classification). This was to minimize } \\
\text { contamination across the two interventions to which patients were random- } \\
\text { ized." }\end{array}$ \\
\hline
\end{tabular}

Blinding of outcome as- Low risk sessment (detection bias)

All outcomes
Quote: "All patients regardless of treatment allocation were followed up by an independent reviewer, blinded to treatment allocation, and asked to complete a telephone survey at baseline and at 6 and 12 months. The survey included questions relating to quality of life (Minnesota Living with Heart Failure questionnaire), NYHA class, global health assessment, EQ-5D EuroQOL, and questions regarding utilization of health services."

Quote: "All hospitalization data were adjudicated by three cardiologists (blinded to randomization allocation) to determine whether the hospitalizations were related to heart failure." 
Krum 2013 (CHAT) (Continued)

Selective reporting (re- Low risk No evidence of selective outcome reporting. porting bias)

Laramee 2003

Methods Randomised controlled trial; intervention arm and control (usual care) arm.

Participants 287 people admitted to hospital with primary or secondary diagnosis of CHF: LVSD $<40 \%$ or radiological evidence of pulmonary oedema.

Mean age 71 years.

$54 \%$ of participants were men.

USA.

Interventions Structured telephone support.

Telephonic case management performed by $1 \mathrm{CHF}$ nurse case manager, involving 4 major components: early discharge planning, participant and family $\mathrm{CHF}$ education, promotion of optimal CHF medications and 12 weeks of telephone follow-up.

\begin{tabular}{ll}
\hline Outcomes & Mortality, rehospitalisation, inpatient and outpatient costs, medication prescription and adherence. \\
& 3-month follow-up. \\
\hline Funding source & Novartis Pharmaceuticals. \\
\hline Comparison Group(s) & $\begin{array}{l}\text { Usual care consisted of standard care typical of a tertiary-care hospital. It included inpatient social } \\
\text { service evaluation (25\%), dietary consultation (15\%), physiotherapy/occupational therapy (17\%) and } \\
\text { medication and CHF education by nurses. Post-discharge was conducted by the participant's own local } \\
\text { physician, 44\% received some home-care services. }\end{array}$ \\
\hline Notes & Included in previous version of this review (Inglis 2010).
\end{tabular}

\section{Risk of bias}

\begin{tabular}{|c|c|c|}
\hline Bias & Authors' judgement & Support for judgement \\
\hline $\begin{array}{l}\text { Random sequence genera- } \\
\text { tion (selection bias) }\end{array}$ & Unclear risk & $\begin{array}{l}\text { Quote: "After simple randomisation of the first } 42 \text { patients resulted in large } \\
\text { amounts of patients being assigned to one group or the other, patients were } \\
\text { randomised in blocks of } 8 \text { to endure an even group allocation across time". }\end{array}$ \\
\hline $\begin{array}{l}\text { Allocation concealment } \\
\text { (selection bias) }\end{array}$ & Unclear risk & Not detailed. \\
\hline $\begin{array}{l}\text { Blinding of outcome as- } \\
\text { sessment (detection bias) } \\
\text { All outcomes }\end{array}$ & Unclear risk & Not detailed. \\
\hline $\begin{array}{l}\text { Incomplete outcome data } \\
\text { (attrition bias) } \\
\text { All outcomes }\end{array}$ & High risk & $\begin{array}{l}\text { Quote: "Patients who withdrew, died or were otherwise lost before } 90 \text { days of } \\
\text { follow-up were censored on the day of early attrition". }\end{array}$ \\
\hline $\begin{array}{l}\text { Selective reporting (re- } \\
\text { porting bias) }\end{array}$ & Low risk & One secondary outcome not reported: number of days until first readmission. \\
\hline
\end{tabular}


Lyngå 2012 (WISH)

Methods A multicentre, randomised controlled trial (RCT) involving 6 hospitals in Sweden.

Participants

Quote: "A total of 344 patients were randomized in the study and, of them, 319 were included in the final ITT analysis. Twenty-five patients were, after being randomized, found not to be satisfy the inclusion criteria due to reasons such as having an LVEF .50\%, myocardial infarction within 2 months before inclusion, not being hospitalized before enrolment, or moved to a nursing home just after randomization. These patients were excluded from further analysis."

$75 \%$ of participants were men.

Mean age 73.9 years

Sweden

Interventions Telemonitoring.

Quote: "Patients randomized to the IG were given an electronic scale (Zenicor Medical Systems AB ) to install in their homes. A few patients required help to install the electronic scale. The scale could be placed anywhere in the patients' home and, after weighing, a wireless signal was sent from the scale to a modem plugged into the patient's telephone. The weight was then automatically transmitted via the telephone network to a central internet-based data server system (Zenicor Medical Systems AB). Hence, the weight could be checked from any computer with internet access. The Zenicor system produces an alarm if patients show a weight gain of . $2 \mathrm{~kg}$ from the target weight (body weight at discharge from hospital) and also if there is an upward trend with a weight increase of . $2 \mathrm{~kg}$ in 3 days."

Outcomes Quote: "Primary endpoint, i.e. cardiac re-hospitalization. Information on hospitalization, cardiac and other, and death was collected from the medical register at the local health authorities."

Quote: "Based on previous research, we assumed a re-admission rate due to a cardiac cause of $40 \%$ in the control group (CG) 3 and $25 \%$ in the intervention group (IG). To detect the difference between the groups, with a power of $80 \%$ and an alpha-value of 0.05 , a sample size of 152 patients in each group was needed."

12 month follow-up.

Funding source The Swedish Governmental Agency for Innovation Systems (VINNOVA); the Swedish Heart and Lung foundation.

Comparison Group(s) Quote: "The patients in the CG were informed to contact the HF clinic on a special telephone in the case of a weight gain of $.2 \mathrm{~kg}$ in 3 days."

Notes New in 2015 review.

\section{Risk of bias}

\begin{tabular}{lll}
\hline Bias & Authors' judgement & Support for judgement \\
\hline $\begin{array}{l}\text { Random sequence genera- } \\
\text { tion (selection bias) }\end{array}$ & Unclear risk & $\begin{array}{l}\text { Comment: method of randomisation not detailed. } \\
\text { Quote: "Patients were then randomized to either the CG or the IG, and both } \\
\text { groups were recommended to weigh themselves daily after emptying their } \\
\text { bladder but before having breakfast." }\end{array}$ \\
\hline
\end{tabular}

\begin{tabular}{lll}
\hline $\begin{array}{l}\text { Allocation concealment } \\
\text { (selection bias) }\end{array}$ & Unclear risk detailed.
\end{tabular}

\begin{tabular}{lll}
\hline Blinding of outcome as- & Unclear risk & Not detailed. \\
sessment (detection bias) &
\end{tabular}


Lyngå 2012 (WISH) (Continued)

All outcomes

\section{Incomplete outcome data Low risk} (attrition bias)

All outcomes
Quote: "The study was based on intention to treat (ITT) except that randomized patients not fulfilling the inclusion criteria were excluded from analysis. Baseline characteristics are presented by descriptive statistics."

Quote: "One obvious limitation in the present study is that 25 patients were randomized before all inclusion criteria were fully checked (e.g., having an LVEF $>50 \%$, myocardial infarction within 2 months before inclusion, not being hospitalized before enrolment, or moved to a nursing home just after randomization). However, these patients were equally distributed between the groups. Also the number of all eligible patients is unknown".

Selective reporting (re- Low risk No evidence of selective outcome reporting.

\section{Mortara 2009 (Struct Tele) (HHH)}

\begin{tabular}{ll}
\hline Methods & Randomised controlled trial; multiple intervention arms and control (usual care) arm. \\
& Study arms include structured telephone support and telemonitoring. \\
\hline Participants & 461 people with heart failure NYHA class II - IV and LVEF $\leq 40 \%$. \\
& Mean age 60 years. \\
& $85 \%$ of participants were men. \\
UK, Poland, Italy.
\end{tabular}

Interventions

Structured telephone support; telemonitoring.

Strategy 2 is classed as structured telephone support.

Strategy 3 is classed as telemonitoring.

Strategy 2 received monthly supportive telephone contacts from a study nurse to check on their clinical status and transmitted their vital signs and other data including details of changes in weight, BP and symptoms weekly by telephone. These participants also performed monthly $24 \mathrm{~h}$ cardiorespiratory recordings which were not made available to the clinical team.

Strategy 3 carried out the same measurements as strategy 2 participants, but the monthly $24 \mathrm{~h}$ cardiorespiratory recordings were made available for clinical management.

\begin{tabular}{ll} 
Outcomes & $\begin{array}{l}\text { Mortality and hospitalisation due to HF, all-cause mortality, all-cause hospitalisation, bed-days occu- } \\
\text { pancy (due to cardiovascular cause). } \\
\text { Mean } 11.6 \text { month follow-up. }\end{array}$ \\
\hline Funding source & HHH was supported by E.C. grant (Action line 10.1 'Public Health, contract no. QLGA-CT-2001-02424). \\
\hline Comparison Group(s) & Usual care was only described as usual outpatient care. \\
\hline Notes & Authors provided additional unpublished data. \\
& Included in previous version of this review (Inglis 2010).
\end{tabular}

\section{Risk of bias}


Mortara 2009 (Struct Tele) (HHH) (Continued)

\begin{tabular}{lll} 
Bias & Authors' judgement & Support for judgement \\
\hline $\begin{array}{l}\text { Random sequence genera- } \\
\text { tion (selection bias) }\end{array}$ & Unclear risk & $\begin{array}{l}\text { Quote: "The randomisation list was generated by the coordinating centre with } \\
\text { separate blocks held in each country". }\end{array}$ \\
\hline $\begin{array}{l}\text { Allocation concealment } \\
\text { (selection bias) }\end{array}$ & Low risk & $\begin{array}{l}\text { Quote: "The individual patient allocation was to be revealed only after the pa- } \\
\text { tient identifiers (name, surname and the date of birth) had been received at } \\
\text { the national randomisation centre". }\end{array}$ \\
\hline $\begin{array}{l}\text { Blinding of outcome as- } \\
\text { sessment (detection bias) } \\
\text { All outcomes }\end{array}$ & Low risk & $\begin{array}{l}\text { Quote: "All endpoints were adjudicated by an independent, blinded, Endpoint } \\
\text { Committee." }\end{array}$ \\
\hline $\begin{array}{l}\text { Incomplete outcome data } \\
\text { (attrition bias) } \\
\text { All outcomes }\end{array}$ & Unclear risk & $\begin{array}{l}\text { Quote: "...18 patients dropped out of the study...". } \\
\text { No statement asserting that analyses were performed as intention-to-treat. }\end{array}$ \\
\hline $\begin{array}{l}\text { Selective reporting (re- } \\
\text { porting bias) }\end{array}$ & Unclear risk & $\begin{array}{l}\text { All-cause mortality listed as a secondary outcome but not reported in publica- } \\
\text { tion according to study group. Author contacted for this information. }\end{array}$ \\
& $\begin{array}{l}\text { Bed days occupancy for all cardiovascular causes listed as secondary out- } \\
\text { come. Not reported, unless "all-causes" is actually all "cardiovascular causes". }\end{array}$
\end{tabular}

\section{Mortara 2009 (Telemon) (HHH)}

\begin{tabular}{ll} 
Methods & Randomised controlled trial; multiple intervention arms and control (usual care) arm. \\
& Study arms include structured telephone support and telemonitoring. \\
\hline Participants & 461 people with heart failure with NYHA class II - IV and LVEF $\leq 40 \%$. \\
& Mean age 60 years. \\
& $85 \%$ of participants were men. \\
UK, Poland, Italy.
\end{tabular}

Interventions Structured telephone support; telemonitoring.

Strategy 2 is classed as structured telephone support.

Strategy 3 is classed as telemonitoring.

Strategy 2 received monthly supportive telephone contacts from a study nurse to check on their clinical status and transmitted their vital signs and other data including details of changes in weight, BP and symptoms weekly by telephone. These participants also performed monthly $24 \mathrm{~h}$ cardiorespiratory recordings which were not made available to the clinical team.

Strategy 3 carried out the same measurements as strategy 2 participants, but the monthly $24 \mathrm{~h}$ cardiorespiratory recordings were made available for clinical management.

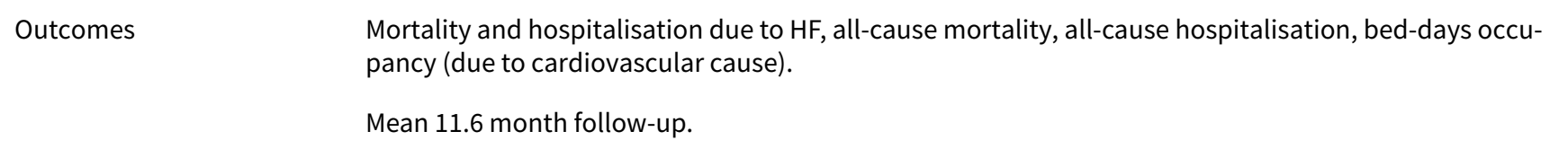


Mortara 2009 (Telemon) (HHH) (Continued)

Comparison Group(s) Usual care was only described as usual outpatient care.

\begin{tabular}{ll}
\hline Notes & Authors provided additional unpublished data. \\
Included in previous version of this review (Inglis 2010).
\end{tabular}

\section{Risk of bias}

\begin{tabular}{lll}
\hline Bias & Authors' judgement & Support for judgement \\
\hline $\begin{array}{l}\text { Random sequence genera- } \\
\text { tion (selection bias) }\end{array}$ & Unclear risk & $\begin{array}{l}\text { Quote: "The randomisation list was generated by the coordinating centre with } \\
\text { separate blocks held in each country". }\end{array}$ \\
\hline $\begin{array}{l}\text { Allocation concealment } \\
\text { (selection bias) }\end{array}$ & Low risk & $\begin{array}{l}\text { Quote: "The individual patient allocation was to be revealed only after the pa- } \\
\text { tient identifiers (name, surname and the date of birth) had been received at } \\
\text { the national randomisation centre". }\end{array}$ \\
\hline $\begin{array}{l}\text { Blinding of outcome as- } \\
\text { sessment (detection bias) }\end{array}$ & Low risk & $\begin{array}{l}\text { Quote: "All endpoints were adjudicated by an independent, blinded, Endpoint } \\
\text { Committee." }\end{array}$ \\
\hline $\begin{array}{l}\text { Incomplete outcome data } \\
\text { (attrition bias) } \\
\text { All outcomes }\end{array}$ & Unclear risk & $\begin{array}{l}\text { "...18 patients dropped out of the study...". } \\
\text { No statement asserting that analyses were performed as intention-to-treat. }\end{array}$ \\
\hline $\begin{array}{l}\text { Selective reporting (re- } \\
\text { porting bias) }\end{array}$ & Unclear risk & $\begin{array}{l}\text { All-cause mortality listed as a secondary outcome but not reported in publica- } \\
\text { tion according to study group. Author contacted for this information. }\end{array}$ \\
& $\begin{array}{l}\text { Bed days occupancy for all cardiovascular causes listed as secondary out- } \\
\text { come. Not reported, unless "all-causes" is actually all "cardiovascular causes". }\end{array}$ \\
\hline
\end{tabular}

Rainville 1999

\begin{tabular}{ll}
\hline Methods & Randomised controlled trial; intervention arm and control (usual care) arm. \\
\hline Participants & 38 people aged $\geq 50$ years discharged from hospital with heart failure. \\
& Mean age 70 years. \\
& $50 \%$ of participants were men. \\
& USA. \\
\hline Interventions & Structured telephone support. \\
& $\begin{array}{l}\text { Usual care plus a pharmacist-led medication review, patient education, medication management prior } \\
\text { to discharge and at day } 3, \text { day } 7,30 \text { days, } 90 \text { days and } 12 \text { months via telephone. }\end{array}$
\end{tabular}

Outcomes

Mortality, rehospitalisation, functional assessment score. NYHA Functional Class.

12-month follow-up.

\begin{tabular}{ll}
\hline Funding source & Not reported. \\
\hline Comparison Group(s) & $\begin{array}{l}\text { Usual care consisted of routine care and preparation for discharge including written prescriptions, } \\
\text { physician discharge instructions and a nurse review of diet, treatment plans and medications. The }\end{array}$
\end{tabular}


Rainville 1999 (Continued)

nurses provided the participant with computer-generated drug information sheets. Participants were contacted by a pharmacist at 30 days, 90 days and 12 months to determine readmissions.

Notes Included in previous version of this review (Inglis 2010).

\section{Risk of bias}

\begin{tabular}{lll}
\hline Bias & Authors' judgement & Support for judgement \\
\hline $\begin{array}{l}\text { Random sequence genera- } \\
\text { tion (selection bias) }\end{array}$ & Unclear risk & Method of randomisation not detailed. \\
\hline $\begin{array}{l}\text { Allocation concealment } \\
\text { (selection bias) }\end{array}$ & Low risk & $\begin{array}{l}\text { Quote: "Qualified patients were randomly assigned to a control group or an in- } \\
\text { tervention group, with the patients, nurses, and physicians blinded to the ran- } \\
\text { domisation results". }\end{array}$ \\
\hline
\end{tabular}

Blinding of outcome as- Unclear risk Not detailed.

sessment (detection bias)

All outcomes

Incomplete outcome data Low risk

(attrition bias)

38 participants randomised; 2 participants in intervention group and 1 in con-

All outcomes trol group were excluded during the initial hospitalisation because test results showed normal LVF, long-term dialysis was initiated or because the participant was moving out of state after DC. 1 control participant was lost to follow-up within the first 30 days after discharge and was excluded from the analysis.

Final sample included 34 participants equally divided between the 2 groups.

Selective reporting (re- Low risk Selective outcome reporting not evident.
porting bias)

Ramachandran 2007

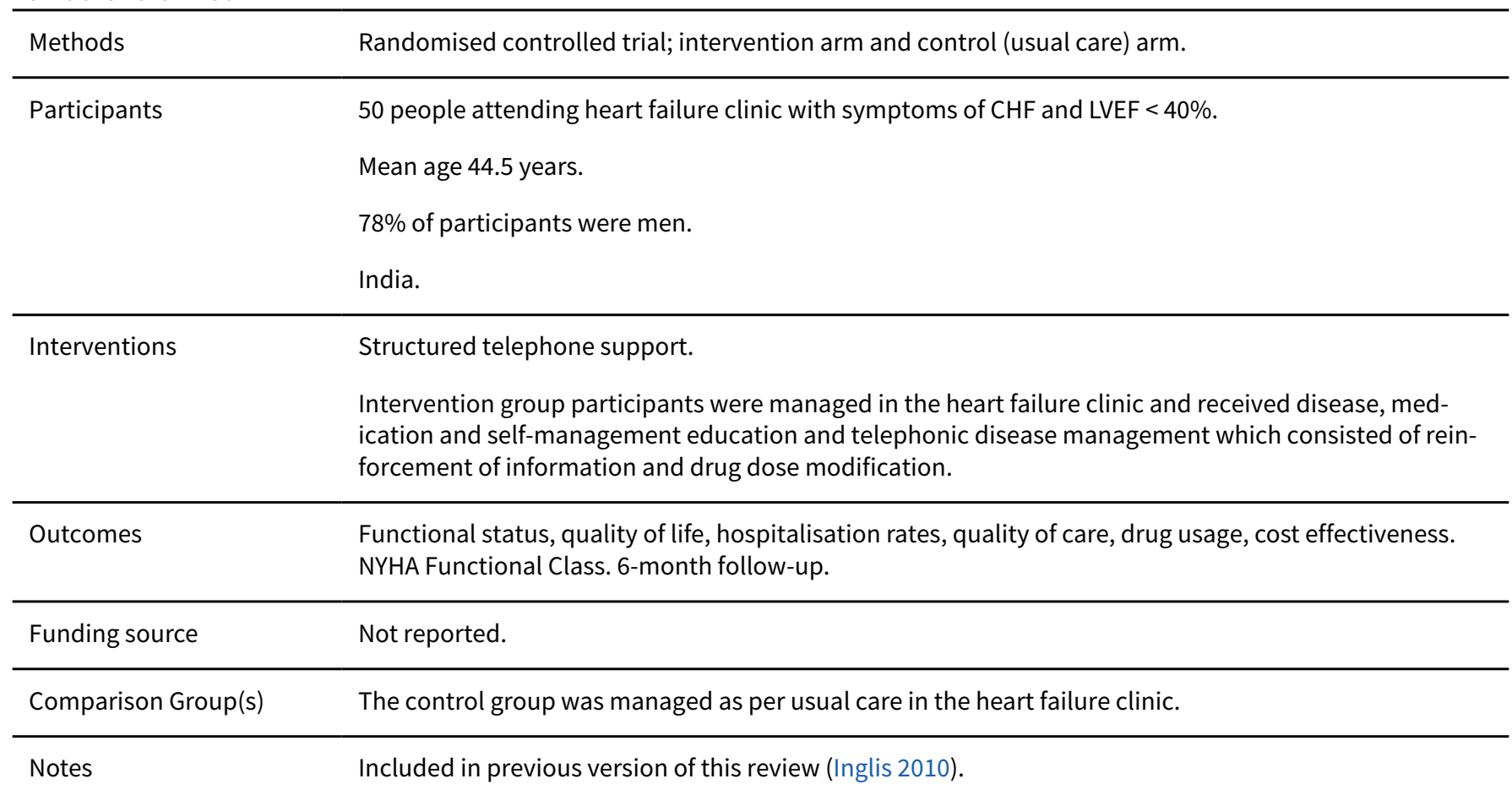


Ramachandran 2007 (Continued)

Mortality not reported. No response from authors for further detail.

\section{Risk of bias}

Bias Authors' judgement Support for judgement

Random sequence genera- Low risk Quote: "An investigator, unaware of the patients' demographic and clinical tion (selection bias) profile, using a computer-generated list, initiated randomisation".

$\begin{aligned} & \text { Allocation concealment } \\ & \text { (selection bias) }\end{aligned}$
Unclear risk $\quad$ Not detailed.

Blinding of outcome as- Unclear risk Not detailed.

sessment (detection bias)

All outcomes

\begin{tabular}{lll}
$\begin{array}{l}\text { Incomplete outcome data } \\
\text { (attrition bias) }\end{array}$ & Unclear risk & Not detailed. \\
All outcomes & & \\
\hline $\begin{array}{l}\text { Selective reporting (re- } \\
\text { porting bias) }\end{array}$ & Low risk & No evidence of selective outcome reporting
\end{tabular}

\section{Riegel 2002}

\begin{tabular}{|c|c|}
\hline Methods & Cluster randomised controlled trial; intervention arm and control (usual care) arm. \\
\hline \multirow[t]{4}{*}{ Participants } & 358 people discharged from hospital with heart failure. \\
\hline & Mean age 74 years. \\
\hline & $49 \%$ of participants were men. \\
\hline & USA. \\
\hline \multirow[t]{2}{*}{ Interventions } & Structured telephone support. \\
\hline & $\begin{array}{l}\text { Telephonic case management by a registered nurse using decision support software, involving patient } \\
\text { education and counselling and liaison with primary care physician. Participants were telephoned with- } \\
\text { in } 5 \text { days of discharge and thereafter at a frequency guided by the software and case manager (mean } 17 \\
\text { calls). }\end{array}$ \\
\hline \multirow[t]{2}{*}{ Outcomes } & $\begin{array}{l}\text { Mortality, rehospitalisation, physician and emergency department visits, inpatient costs, participant } \\
\text { satisfaction. }\end{array}$ \\
\hline & 6-month follow-up. \\
\hline Funding source & Pfizer. \\
\hline Comparison Group(s) & $\begin{array}{l}\text { Usual care was not standardised, and no formal telephonic case management was in existence at these } \\
\text { institutions. These participants presumably received some education regarding HF management prior } \\
\text { to hospital discharge. }\end{array}$ \\
\hline Notes & Included in previous version of this review (Inglis 2010). \\
\hline
\end{tabular}

\section{Risk of bias}


Riegel 2002 (Continued)

\begin{tabular}{lll} 
Bias & Authors' judgement & Support for judgement \\
\hline $\begin{array}{l}\text { Random sequence genera- } \\
\text { tion (selection bias) }\end{array}$ & Unclear risk & $\begin{array}{l}\text { Method of randomisation not stated. (Physicians were the unit of randomisa- } \\
\text { tion). }\end{array}$ \\
\hline $\begin{array}{l}\text { Allocation concealment } \\
\text { (selection bias) }\end{array}$ & Low risk & $\begin{array}{l}\text { Quote: "Physicians were not informed of the group to which they were as- } \\
\text { signed". }\end{array}$ \\
\hline $\begin{array}{l}\text { Blinding of outcome as- } \\
\text { sessment (detection bias) } \\
\text { All outcomes }\end{array}$ & Unclear risk & Not detailed. \\
\hline $\begin{array}{l}\text { Incomplete outcome data } \\
\text { (attrition bias) } \\
\text { All outcomes }\end{array}$ & Unclear risk & Not detailed. \\
\hline $\begin{array}{l}\text { Selective reporting (re- } \\
\text { porting bias) }\end{array}$ & Low risk & Selective outcome reporting not evident. \\
\hline
\end{tabular}

Riegel 2006

Methods Randomised controlled trial; intervention arm and control (usual care) arm.

Participants 135 hospitalised Hispanic people with CHF.
Mean age 72 years.
$46 \%$ of participants were men.
USA.
Structured telephone support.
Education, monitoring and guidance by bilingual-bicultural Mexican-American registered nurses via
telephone case management standardised using decision support software. Participants were con-
tacted on average within 5 days of discharge and thereafter at a frequency guided by the software and
nurse case manager over a 6-month period (mean 13.5 calls to participants and 8.4 additional calls to
families). Printed educational material was provided monthly and upon request in the relevant lan-
guage.

Outcomes

Mortality, re hospitalisation, cost of care, self-reported health-related quality of life and depression.

6-month follow-up.

\begin{tabular}{ll}
\hline Funding source & American Heart Association. \\
\hline Comparison Group(s) & $\begin{array}{l}\text { Usual care was not standardised and no formal disease management programme existed at these in- } \\
\text { stitutions. The standard of usual care was that participants were educated regarding HF management } \\
\text { before discharge, assuming that the nurse spoke the participant's language or someone bilingual was } \\
\text { available to translate. In reality, only a small proportion of staff were bilingual . }\end{array}$
\end{tabular}

Notes Included in previous version of review (Inglis 2010).

\section{Risk of bias}

Bias Authors' judgement Support for judgement


Riegel 2006 (Continued)

Random sequence genera- Unclear risk tion (selection bias)
Quote: "After the baseline data were collected, the nurse case manager opened a sealed envelope with the random assignment. These envelopes had been prepared by the project director and attached to the numbered data collection forms, to be opened in sequence".

Method of randomisation not detailed.

\begin{tabular}{lll}
\hline $\begin{array}{l}\text { Allocation concealment } \\
\text { (selection bias) }\end{array}$ & Low risk & See above. \\
\hline $\begin{array}{l}\text { Blinding of outcome as- } \\
\text { sessment (detection bias) } \\
\text { All outcomes }\end{array}$ & Low risk & $\begin{array}{l}\text { Quote: "We were unable to strictly blind staff about which patients were in the } \\
\text { intervention group, but a research assistant uninvolved with the clinical care } \\
\text { collected all follow-up data." }\end{array}$
\end{tabular}

Incomplete outcome data Low risk (attrition bias)

All outcomes
For intervention arm, only 69 participants were included in analysis as 1 outlier excluded from analysis.

Quote: "One outlier was removed from the data set before analyses began because he spent three months in the hospital while his family debated taking him off life support".

Selective reporting (re- Low risk Selective outcome reporting not evident.

porting bias)

\section{Scherr 2009 (MOBITEL)}

$\begin{array}{ll}\text { Methods } & \text { A prospective, randomised, open-label study. } \\ & \text { Recruitment started on October 1, } 2003 \text { - April 29, } 2008 .\end{array}$

\section{Participants}

Quote: "Inclusion criteria: acute worsening of heart failure (acute cardiac decompensation) with hospital admission lasting $>24$ hours within the last 4 weeks, treatment according to the guidelines of the European Society of Cardiology (ESC) with an angiotensin converting enzyme (ACE) inhibitor or an angiotensin receptor blocker (ARB), diuretic, and beta-blocker (except in cases with documented intolerance to beta-blockers). Initially, patients older than 18 years and younger than 75 years were eligible; the latter was amended to 80 years after 4 months of recruitment. For the definition of CHF, we adopted the ESC guidelines".

Austria.

Interventions Telemonitoring

Quote: "Tele group patients were asked to measure vital parameters (blood pressure, heart rate, body weight) on a daily basis at the same time, preferably in the morning after emptying the bladder and before dressing and taking medication. Thereafter, patients were advised to enter these values as well as their dosage of heart failure medication into the mobile phone's Internet browser and send them to the monitoring center provided by the Austrian Institute of Technology (AIT) - Information Management \& eHealth, Graz. Study physicians had access to a secure website providing both numerical and graphical depiction of data for each patient."

Outcomes

Quote: "The combined primary endpoint of this study was cardiovascular mortality or re-hospitalization for worsening heart failure. Besides evaluation of patients' functional status according to the NYHA classification and length of stay during re-hospitalizations, further secondary endpoints focused on technical parameters: system availability, cumulative transmissions, and transmissions per patient."

Quote: "For statistical planning, we assumed that patients in the control arm would show an event rate of $30 \%$ over 6 months. For the telemedicine arm, we expected a $50 \%$ reduction of the event rate. To 
Scherr 2009 (MOBITEL) (Continued)

show a statistically significant difference at an error of .05 with a power of $80 \%$, a sample size of 240 subjects was calculated."

6 month follow-up.

Funding source

Quote: "This study was partly funded by restricted research grants from Novartis Pharma Austria, Roche Pharma Austria, and Mobilkom Austria."

Quote: "Baseline demographics and medication were recorded for all patients, and an appointment for the 6-month follow-up was made. There was no planned interaction between study site and patients in the control group within the follow-up period of 6 months."

Notes New in 2015 review.

\section{Risk of bias}

\begin{tabular}{lll}
\hline Bias & Authors' judgement & Support for judgement \\
\hline $\begin{array}{l}\text { Random sequence genera- } \\
\text { tion (selection bias) }\end{array}$ & Unclear risk & Comment: method of randomisation not detailed. \\
& $\begin{array}{l}\text { Quote: "Patients were allocated randomly to pharmacological treatment (con- } \\
\text { trol group) or pharmacological treatment plus telemedical surveillance (tele } \\
\text { group). The adaptive randomization procedure was stratified by patient age, } \\
\text { New York Heart Association (NYHA) class, gender, and study center." }\end{array}$ \\
\end{tabular}

\begin{tabular}{ll}
\hline $\begin{array}{l}\text { Allocation concealment } \\
\text { (selection bias) }\end{array}$ & Unclear risk \\
\hline
\end{tabular}

Blinding of outcome as- Unclear risk Not detailed

sessment (detection bias)

All outcomes

\begin{tabular}{ll}
\hline $\begin{array}{l}\text { Incomplete outcome data } \\
\text { (attrition bias) }\end{array}$ & Low risk \\
All outcomes & $\begin{array}{l}\text { Comment: Baseline demographics data not presented for intention-to-treat } \\
\text { sample. }\end{array}$
\end{tabular}

Selective reporting (re- Low risk Selective outcome reporting not evident.
porting bias)

\section{Seto 2012}

\begin{tabular}{|c|c|}
\hline Methods & $\begin{array}{l}\text { Quote: "The primary intent of the trial was to pilot the telemonitoring system in order to determine the } \\
\text { impact of the system on self-care and clinical management." }\end{array}$ \\
\hline \multirow[t]{4}{*}{ Participants } & $\begin{array}{l}\text { Quote: "Eligible participants were ambulatory patients diagnosed with heart failure. Other eligibility } \\
\text { criteria included } 18 \text { years of age or older, ability to speak and read in English, not on the heart trans- } \\
\text { plantation list, an expected survival of greater than one year, and a left ventricular ejection fraction } \\
\text { (LVEF) less than } 40 \% . "\end{array}$ \\
\hline & Mean age $55.1 \mathrm{SD}(13.7)$ years \\
\hline & $82 \%$ male \\
\hline & Canada \\
\hline
\end{tabular}

Interventions Telemonitoring


Seto 2012 (Continued)

Quote: "The participants in the telemonitoring group received the telemonitoring system in addition to standard care. They were asked to use the telemonitoring system for 6 months to take daily morning weight and blood pressure readings as well as weekly single-lead electrocardiograms (ECGs) if provided with an ECG recorder. They were also asked to answer daily morning symptom questions on a mobile phone. Only the 17 patients who did not have an implantable cardioverter defibrillator (ICD) were provided with an ECG recorder because the recorder was not certified for use with ICDs. Patients were also told to report their symptoms through the mobile phone if they did not feel well during the day. The patients in the telemonitoring group were given an individual training session on how to use the system during the recruitment session, and were provided with technical support by telephone throughout the study. The daily measurements took about 5 minutes each morning."

Outcomes Quote: "The primary outcomes of this study included a surrogate for heart failure prognosis, specifically brain natriuretic peptide (BNP), self-care as measured by the SCHFI, and quality of life as measured by the MLHFQ. Hospital readmissions, number of nights in hospital, and mortality were secondary outcome measures because the study was underpowered to detect differences between groups for these metrics. Other secondary outcome measures included number of emergency department visits and number of Heart Function Clinic visits. In addition, LVEF, NYHA class, medication prescriptions, and blood test results (specifically creatinine, sodium, potassium, hemoglobin, and urate values) were also subsequently analyzed."

Quote: "A sample size calculation was based on the Self-Care of Heart Failure Index (SCHFI), using a population standard deviation of 20 and an effect size of 10 (effect size represents a clinically significant change of more than half a standard deviation) as determined in previous studies (alpha $=0.05$, power $=0.8)[7,8]$. We calculated the required sample size per group to be 34 , and recruited 50 participants for the intervention group and 50 for the control group to compensate for the patients estimated as lost to follow-up, including due to mortality, over the six-month trial."

6 month follow-up.

Funding source

Quote: "Funding for this work was in part provided by the Toronto General Hospital Foundation and the Natural Sciences and Engineering Research Council of Canada Strategic Research Network Grant entitled Healthcare Support through Information Technology Enhancements (hSITE)."

Comparison Group(s)

Quote: "The standard care group received standard care at the UHN Heart Function Clinic, which includes visiting the clinic between once every 2 weeks to once every 3 to 6 months, depending on the severity of the patient's heart failure condition and the need for optimizing their medication. Standard care also includes heart failure education during preliminary visits at the Heart Function Clinic and the ability to telephone the clinic as necessary. Participants in the standard care group were not contacted again regarding the study until the end of the trial."

Notes New in 2015 review.

\section{Risk of bias}

Bias Authors' judgement Support for judgement
tion (selection bias)
Random sequence genera- Low risk

Quote: "The 100 participants were randomized into the telemonitoring (TM) group and standard care (SC) group using stratified four-block randomization. Stratification was based on NYHA classification (NYHA class II-III and NYHA class IV). There were no participants in NYHA class I. An online computer-generated randomization tool, Research Randomizer was used to determine the order of participants in the telemonitoring and standard care groups."

\begin{tabular}{|c|c|c|}
\hline $\begin{array}{l}\text { Allocation concealment } \\
\text { (selection bias) }\end{array}$ & Low risk & $\begin{array}{l}\text { Quote: "The study coordinatorwas blinded to which group the patient would } \\
\text { be assigned until each patient consented to participate in the trial." }\end{array}$ \\
\hline
\end{tabular}

Quote from author contact: "We had to rely on self-reported indications of hospitalization and tried to confirm with charts because it was possible that patients would be admitted to hospital outside of the clinical setting where 


Incomplete outcome data High risk
(attrition bias)

All outcomes
Quote from author contact: "We had to rely on self-reported indications of hospitalization and tried to confirm with charts because it was possible that patients would be admitted to hospital outside of the clinical setting where the study took place. Therefore, the denominators are smaller than the number of participants."

Selective reporting (re- Low risk Selective outcome reporting not evident.
porting bias)

\begin{tabular}{ll}
\hline Sisk 2006 & Randomised controlled trial; intervention arm and control (usual care) arm. \\
\hline Methods & 406 non-Hispanic and Hispanic people with documented systolic dysfunction. \\
\hline Participants & Mean age 59 years. \\
& $54 \%$ of participants were men. \\
& USA. \\
& Structured telephone support. \\
& $\begin{array}{l}\text { An in-person appointment was arranged for each intervention participant, which included symptom } \\
\text { and disease education and referral to additional patient services (if required). Follow-up telephone } \\
\text { calls consisted of participant assessment, recording of admission information reinforcement of self } \\
\text { monitoring and administration of a food-frequency questionnaire (at 2, 4, 8, } 12 \text { and } 24 \text { weeks and a re- } \\
\text { port sent to participants). Intervention nurses co-ordinated flow of information between participant } \\
\text { and clinician and arranged medication adjustment and required examinations. }\end{array}$
\end{tabular}

Outcomes

Mortality, hospitalisations, functional status (including quality of life). Cost.

12-month follow-up.

\begin{tabular}{ll}
\hline Funding source & The Agency for Healthcare Research and Quality. \\
\hline Comparison Group(s) & $\begin{array}{l}\text { Usual care participants received guidelines for managing systolic dysfunction, but no other care infor- } \\
\text { mation was specified. }\end{array}$ \\
\hline Notes & Included in previous version of this review (Inglis 2010). \\
\hline
\end{tabular}

\section{Risk of bias}

\begin{tabular}{lll}
\hline Bias & Authors' judgement & Support for judgement \\
\hline $\begin{array}{l}\text { Random sequence genera- } \\
\text { tion (selection bias) }\end{array}$ & Low risk & $\begin{array}{l}\text { Quote: "The project's statistician used a computer-generated, random-num- } \\
\text { ber sequence without blocking or stratification to centrally determine ran- } \\
\text { domizations assignments and concealed treatment group assignments in } \\
\text { sealed, opaque envelopes". }\end{array}$ \\
\hline $\begin{array}{l}\text { Allocation concealment } \\
\text { (selection bias) }\end{array}$ & Low risk & See above. \\
\hline $\begin{array}{l}\text { Blinding of outcome as- } \\
\text { sessment (detection bias) }\end{array}$ & Low risk & $\begin{array}{l}\text { Quote: "To measure hospitalizations, we used billing data from the 4 partici- } \\
\text { pating hospitals. At quarterly telephone surveys, interviewers who were blind- }\end{array}$ \\
\hline
\end{tabular}

Structured telephone support or non-invasive telemonitoring for patients with heart failure (Review) 
Sisk 2006 (Continued)

All outcomes ed to treatment assignment asked patients about hospitalizations at nonparticipating hospitals; however, we present the analysis of billing data because they measure hospitalizations independent of possibly socially acceptable responses or survey non response of the patients."

Incomplete outcome data Low risk No losses to follow-up in the first 12 months of follow-up.

(attrition bias)

All outcomes

Selective reporting (re- Low risk Selective outcome reporting not evident.
porting bias)

\section{Soran 2008}

Methods Randomised controlled trial; intervention arm and control (usual care) arm.

Participants

315 people with HF diagnosis secondary to systolic dysfunction (LVEF $\leq 40 \%)$.

Mean age 76 years.

$35 \%$ of participants were men.

USA.

\section{Telemonitoring.}

Participants randomised to the Heart Failure Monitoring System (HFMS) cohort received a disease management programme using telecommunication equipment including an electronic scale and individualised symptom response system linked to a database staffed by nurses. Participants weighed themselves and answered questions related to their heart failure. Participants were contacted if any changes were observed in symptoms or weight.
Treatment failure (cardiovascular mortality or rehospitalisation for HF within 6 months), length of hospital stay, 6-month all-cause hospitalisation, 6-month heart failure hospitalisation, number of emergency room visits, Medicare expenditure, total participant costs, quality of life.

6-month follow-up.
Funding source Centers for Medicare \& Medicaid Services Baltimore, Maryland.

Comparison Group(s) Participants allocated to standard heart failure care (SC) received enhanced patient education, education to clinicians and follow-up. They were provided with a digital home scale to weigh themselves daily and educational materials related to worsening of $\mathrm{HF}$ and were asked to record heart failure symptoms.

All participants were telephoned 30 days and 3 months post-randomisation for blinded clinical data collection (vital signs, hospital visits, quality of life questionnaires). Included in previous version of this review (Inglis 2010).

\section{Risk of bias}

\begin{tabular}{lll}
\hline Bias & Authors' judgement & Support for judgement \\
\hline $\begin{array}{l}\text { Random sequence genera- } \\
\text { tion (selection bias) }\end{array}$ & Unclear risk & Quote: "...patients were randomised in a 1:1 ratio..".
\end{tabular}


Soran 2008 (Continued)

Method of randomisation not detailed.

\begin{tabular}{lll}
\hline $\begin{array}{l}\text { Allocation concealment } \\
\text { (selection bias) }\end{array}$ & Unclear risk & Not detailed. \\
\hline $\begin{array}{l}\text { Blinding of outcome as- } \\
\begin{array}{l}\text { sessment (detection bias) } \\
\text { All outcomes }\end{array}\end{array}$ & Low risk & $\begin{array}{l}\text { Quote: "The HFHC Trial was a multicenter, randomized controlled clinical trial } \\
\text { with blinded end point evaluation...". }\end{array}$ \\
\hline $\begin{array}{l}\text { Incomplete outcome data } \\
\text { (attrition bias) }\end{array}$ & Unclear risk & $\begin{array}{l}\text { Quote: "Eight patients refused to be re contacted after randomisation and } \\
\text { were considered lost to follow-up". }\end{array}$ \\
$\begin{array}{l}\text { ll outcomes } \\
\begin{array}{l}\text { Selective reporting (re- } \\
\text { porting bias) }\end{array}\end{array}$ & Low risk & Quote: "The intention-to-treat principle was used to compare HFMS to SC". \\
\hline
\end{tabular}

\section{Tsuyuki 2004}

\begin{tabular}{ll}
\hline Methods & Randomised controlled trial; intervention arm and control (usual care) arm. \\
\hline Participants & 276 people discharged from hospital with heart failure. \\
& Mean age 72 years. \\
$58 \%$ of participants were men. \\
Canada.
\end{tabular}

Interventions Structured telephone support.

Early discharge planning with provision of adherence aids, patient education, regularly scheduled telephone contact with local research co-ordinator at 2 and 4 weeks then monthly thereafter for 6 months. Recommendations to see primary care physician if not on target dose ACE inhibitor or deterioration.

\begin{tabular}{|c|c|}
\hline \multirow[t]{2}{*}{ Outcomes } & $\begin{array}{l}\text { Mortality, rehospitalisation, medication adherence, physician and emergency department visits, cost } \\
\text { analysis. }\end{array}$ \\
\hline & 6-month follow-up. \\
\hline
\end{tabular}

\begin{tabular}{ll}
\hline Funding source & Parke Davis Canada (now Pfizer Canada) and the University of Alberta Hospital Foundation. \\
\hline Comparison Group(s) & $\begin{array}{l}\text { Participants assigned to usual care received a general heart disease pamphlet before discharge, but no } \\
\text { formal counselling beyond what was routine at the hospital. Participants were contacted monthly for } 6 \\
\text { months to ascertain clinical events. }\end{array}$
\end{tabular}

Notes Included in previous version of this review (Inglis 2010).

\section{Risk of bias}

\begin{tabular}{lll}
\hline Bias & Authors' judgement & Support for judgement \\
\hline $\begin{array}{l}\text { Random sequence genera- } \\
\text { tion (selection bias) }\end{array}$ & Low risk & $\begin{array}{l}\text { Quote: "Randomization was conducted by a computer-generated sequence } \\
\text { using block randomisation (block size of 4), stratified by study site (hospital)". }\end{array}$ \\
\hline $\begin{array}{l}\text { Allocation concealment } \\
\text { (selection bias) }\end{array}$ & Unclear risk & Quote: "...patients were randomised via a telephone call to the project office". \\
\hline
\end{tabular}


Tsuyuki 2004 (Continued)

Blinding of outcome as- Unclear risk Quote: "...the primary outcome was medication adherence, as measured by sessment (detection bias) pharmacy records."

All outcomes

Quote: "Clinical events, the secondary outcome, were recorded by patient report and through examination of hospital records".

Incomplete outcome data Unclear risk Intervention: early withdrawal $\mathrm{n}=5$; lost to follow-up $\mathrm{n}=3$.
(attrition bias)

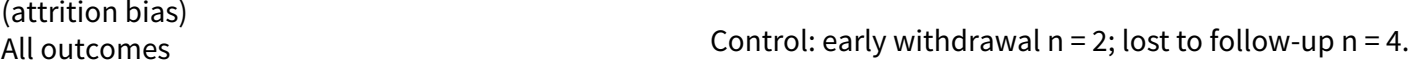

Selective reporting (re- Low risk Selective outcome reporting not evident.
porting bias)

Villani 2014 (ICAROS)

Methods Quote: "A randomized, parallel open study was conducted on patients with chronic heart failure leaving hospital after being treated for clinical instability."

Power Calculation: No sample size calculation was detailed.

Quote: "...80 agreed to participate and were randomized to the two groups (40 in each Inclusion criteria
(1) NYHA class III/IV during hospital stay (2) Left ventricular systolic dysfunction (ejection fraction $40 \%$ )
(3) High risk of early re-hospitalization at discharge (at least two of): age $>70$ years, $>2$ hospitalizations
for heart failure in the last 6 months, $>1$ co-pathologies (diabetes, COPD, cerebrovascular disease, renal
failure)".
Mean age 72 (SD 3) years.
$73.7 \%$ men.
Italy.
Telemonitoring.
Quote: "Integrated Management group, patients and their caregivers had specific training in the use of
the dedicated PDA described above. Each day, the PDA acted as a reminder of the correct timing for the
pills. At a predefined time patients were asked to send their body weight, blood pressure and heart rate
data via the PDA. In some cases patients were asked to monitor their diuresis. Each month, a psycho-
logical assessment was performed through the PDA software about anxiety (STAI-6; Spielberger's State
Trait Anxiety Inventory, depression (PHQ-9; Patient Health Questionnaire)18 and perceived well being
InGWBI; Perception of General Well-Being Inventory)."

Outcomes Quote: "Cardiovascular death or hospitalization for heart failure lasting more than 3 days were considered major adverse effects. We considered the following as minor adverse effects: (1) any hospital stay of less than three days, including Emergency Department visits; (2) any unplanned investigation and/or blood testing; (3) any unplanned clinical visit (4) cost."

12 month follow-up

\begin{tabular}{ll}
\hline Funding source & The research received a grant from the Italian Ministry of Research and Public Instruction (FIRB RB- \\
NE01KYE4 2003).
\end{tabular}
NE01KYE4 2003).

\begin{tabular}{ll} 
Comparison Group(s) & $\begin{array}{l}\text { Quote: "In the Usual Care group, patients were discharged with appointments for follow-up every th } \\
\text { months at our Heart Failure clinic, in accordance with guidelines for fragile patients." }\end{array}$ \\
\hline Notes & $\begin{array}{l}\text { New in } 2015 \text { review - included as abstract in sensitivity analysis in previous version of review (Inglis } \\
2010) .\end{array}$
\end{tabular}


Villani 2014 (ICAROS) (Continued)

Risk of bias

\begin{tabular}{|c|c|c|}
\hline Bias & Authors' judgement & Support for judgement \\
\hline $\begin{array}{l}\text { Random sequence genera- } \\
\text { tion (selection bias) }\end{array}$ & Low risk & $\begin{array}{l}\text { Quote: "Patients were randomized } 1: 1 \text { either to an Integrated Management } \\
\text { group or to a Usual Care group, using a computerised random number genera- } \\
\text { tor." }\end{array}$ \\
\hline $\begin{array}{l}\text { Allocation concealment } \\
\text { (selection bias) }\end{array}$ & Unclear risk & Not detailed. \\
\hline $\begin{array}{l}\text { Blinding of outcome as- } \\
\text { sessment (detection bias) } \\
\text { All outcomes }\end{array}$ & Unclear risk & Not detailed. \\
\hline $\begin{array}{l}\text { Incomplete outcome data } \\
\text { (attrition bias) } \\
\text { All outcomes }\end{array}$ & Low risk & Not evident. \\
\hline $\begin{array}{l}\text { Selective reporting (re- } \\
\text { porting bias) }\end{array}$ & Low risk & No evidence of selective outcome reporting. \\
\hline
\end{tabular}

Vuorinen 2014

$\begin{array}{ll}\text { Methods } & \text { Quote: "Two-arm randomized controlled trial conducted at the Cardiology Outpatient Clinic of HUCH in } \\ \text { 2010-2012." }\end{array}$
Participants 94 people were randomised. 1 from each pair was randomly assigned to receive the usual care, and the other was assigned to the telemonitoring group (47 per group)
Quote: "The inclusion criteria were (1) diagnosis of systolic heart failure, (2) age of 18-90 years, (3) NYHA class $\geq 2$ (an interview-based classification by the New York Heart Association concerning limitations to physical activity), (4) left ventricular ejection fraction $\leq 35 \%$ as measured during hospital visits, (5) need for a regular check-up visit, and (6) time from the last visit of less than 6 months."
$83 \%$ of participants were men.
Mean age 58 years.
Finland

Interventions Telemonitoring.

Quote: "Patients regularly reported their most important health parameters to the nurse using a mobile phone app. At the beginning of the study, the patients were given a home-care package including a weight scale, a blood pressure meter, a mobile phone, and self-care instructions. The patients were advised to carry out and report the measurements together with the assessment of symptoms once a week."

Outcomes

Quote: "The primary outcome was the number of HF-related hospital days during the follow-up. The data were obtained from the electronic health record system of $\mathrm{HUCH}$. Secondary outcomes included clinical outcomes, use of health care resources, and user experience. death from any cause, heart transplant operation or listing for transplant operation, left ventricular ejection fraction (LVEF, \%) measured by echocardiography, plasma concentration of $\mathrm{N}$-terminal of the prohormone brain natriuretic peptide (NT-proBNP, $\mathrm{ng} / \mathrm{l})$, creatinine $(\mu \mathrm{mol} / \mathrm{l})$, sodium $(\mathrm{mmol} / \mathrm{l})$, and potassium $(\mathrm{mmol} / \mathrm{l})$. Self-care behavior was measured using the European Heart Failure Self-Care Behaviour Scale (EHFSBS). Patients' acceptance and experience towards home telemonitoring were evaluated using a questionnaire delivered to patients in the telemonitoring group at the end-point visit." 
Vuorinen 2014 (Continued)

Quote: "The study was designed to have a power of $90 \%$, an alpha level of .05, and an effect size of 0.5 determined as the expected difference of $3 \mathrm{HF}$-related hospital days between the study groups (SD 6). A t test was used as a calculation framework. With these parameters, we calculated that 44 patients per treatment arm needed to be recruited".

6 month follow-up.

Funding source

Quote: "The Finnish Funding Agency for Technology and Innovation and VTT Technical Research Centre of Finland are acknowledged for funding the study."

Comparison Group(s)

Quote: "A multidisciplinary care approach including patient guidance and support for self-care has been adopted at the clinic. In the care of these HF patients, the cardiac team plays a central role in monitoring and interpreting patient symptoms, optimizing medication, and providing education."

Notes New in 2015 review.

\title{
Risk of bias
}

\begin{tabular}{lll}
\hline Bias & Authors' judgement & Support for judgement \\
\hline $\begin{array}{l}\text { Random sequence genera- } \\
\text { tion (selection bias) }\end{array}$ & High risk & $\begin{array}{l}\text { Quote: "Matched pair design was used in the randomization. The eligible pa- } \\
\text { tients, who were similar in left ventricular ejection fraction, NYHA classifica- } \\
\text { tion, age, and gender, respectively, were matched in pairs. One was random- } \\
\text { ized to the control group and the other to the intervention group." }\end{array}$ \\
& $\begin{array}{l}\text { The first } 30 \text { intervention participants and } 29 \text { control participants started step- } \\
\text { wise from November } 2010 \text { to February } 2011 . \text { After the first } 59 \text { participants had } \\
\text { finished their follow-up, the second group (17 intervention participants and } \\
\text { 18 control participants) started in May to August 2011. The nominal follow-up } \\
\text { time was } 6 \text { months. The study was completed in February 2012. }\end{array}$
\end{tabular}

Allocation concealment Unclear risk Not detailed.

(selection bias)

Blinding of outcome as- Unclear risk Not detailed.

sessment (detection bias)

All outcomes

$\begin{array}{ll}\begin{array}{l}\text { Incomplete outcome data } \\ \begin{array}{l}\text { (attrition bias) } \\ \text { All outcomes }\end{array}\end{array} & \begin{array}{l}\text { Quote: "There was one dropout in the intervention group. The patient with- } \\ \text { drew from the study shortly after the beginning, and no endpoint measure- } \\ \text { ments were available. The patient was excluded in the endpoint analyses." }\end{array}\end{array}$

Selective reporting (re- Low risk No evidence of selective outcome reporting.

porting bias)

\section{Wakefield 2008}

$\begin{array}{ll}\text { Methods } & \text { Randomised controlled trial; intervention arm and control (usual care) arm. } \\ & \text { Telephone and videophone intervention arms were combined and classed as structured telephone } \\ \text { support for this review. }\end{array}$

Participants

\author{
148 people hospitalised for HF exacerbation. \\ Mean age 69 years. \\ $99 \%$ of participants were men.
}


Wakefield 2008 (Continued)

USA.

Interventions Structured telephone support.

Participants allocated to the intervention group were allocated to 1 of 2 interventions: telephone follow-up or videophone follow-up. Intervention participants were contacted by a nurse 3 times in the first week then weekly for 11 weeks. Symptoms and the participant's discharge plan were reviewed and reinforced as well as referrals made if required. Additionally, the intervention nurses employed behaviour skill training strategies to maximise self management, self monitoring and self efficacy.

Outcomes Mortality, readmissions, hospital days, time to first readmission, urgent care clinic visits, quality of life, intervention dose and technical issues.

12-month follow-up.

Department of Veterans Affairs, Veterans Health Administration, Health Services Research and Devel-
opment (VA HSR\&D) Service (\#NRI 99-345), a VA HSR\&D Career Development Award to Dr. Wakefield, the
VA HSR\&D Center for Research in the Implementation of Innovative Strategies in Practice (CRIISP) at
the lowa City VA Medical Center, lowa City, IA, and by the Harry S. Truman Memorial Veterans Hospital.

Comparison Group(s) Usual care was not specified except to state that "subjects contacted their primary care nurse case manager by telephone if needed".

Notes Included in previous version of this review (Inglis 2010).

\section{Risk of bias}

\begin{tabular}{|c|c|c|}
\hline Bias & Authors' judgement & Support for judgement \\
\hline $\begin{array}{l}\text { Random sequence genera- } \\
\text { tion (selection bias) }\end{array}$ & Low risk & $\begin{array}{l}\text { Quote: "The project coordinator prepared sealed envelopes containing group } \\
\text { assignments in blocks of } 24 \text { ". }\end{array}$ \\
\hline $\begin{array}{l}\text { Allocation concealment } \\
\text { (selection bias) }\end{array}$ & Low risk & $\begin{array}{l}\text { Quote: "Following informed consent and baseline data collection, study nurs- } \\
\text { es opened the envelope to assign subjects to one of three treatment condi- } \\
\text { tions: usual care, telephone follow-up, or videophone follow-up". }\end{array}$ \\
\hline $\begin{array}{l}\text { Blinding of outcome as- } \\
\text { sessment (detection bias) } \\
\text { All outcomes }\end{array}$ & Unclear risk & Not detailed. \\
\hline $\begin{array}{l}\text { Incomplete outcome data } \\
\text { (attrition bias) } \\
\text { All outcomes }\end{array}$ & Low risk & $\begin{array}{l}\text { Qoute: "At } 3 \text { months, } 85 \%(n=126) \text { completed follow-up; at } 6 \text { months, } 74 \% \text { ( } n= \\
\text { 109) completed follow-up". } \\
\text { Quote: "All data analyses were conducted using an intent-to-treat approach". }\end{array}$ \\
\hline $\begin{array}{l}\text { Selective reporting (re- } \\
\text { porting bias) }\end{array}$ & Low risk & Selective outcome reporting not evident. \\
\hline
\end{tabular}

Woodend 2008

\begin{tabular}{ll}
\hline Methods & Randomised controlled trial; intervention arm and control (usual care) arm. \\
\hline Participants & 121 people with symptomatic heart failure (NYHA Class II or greater). \\
& Mean age 68 years. \\
& $74 \%$ of participants were men.
\end{tabular}


Woodend 2008 (Continued)

Canada.

Interventions Telemonitoring.

Daily transmission of weight and periodic transmission of ECG and BP. Weekly video conferences by tele-home care nurse. Video conferences more frequent in first few weeks and tapered over the 3 months.

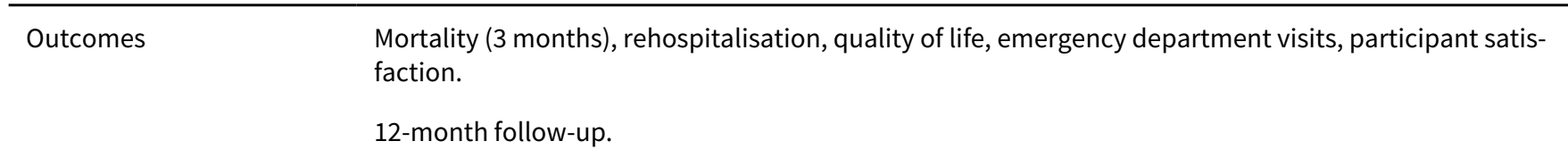

Funding source

The Richard Ivey Foundation, The Change Foundation and an unrestricted educational grant from Merck-Frosst Canada.

\begin{tabular}{ll}
\hline Comparison Group(s) & Usual care was not described. \\
\hline Notes & Included in the previous version of this review (Inglis 2010). \\
\hline
\end{tabular}

\section{Risk of bias}

\begin{tabular}{lll}
\hline Bias & Authors' judgement & Support for judgement \\
\hline $\begin{array}{l}\text { Random sequence genera- } \\
\text { tion (selection bias) }\end{array}$ & Unclear risk & Method of randomisation not detailed. \\
\hline $\begin{array}{l}\text { Allocation concealment } \\
\text { (selection bias) }\end{array}$ & Unclear risk & Not detailed. \\
\hline $\begin{array}{l}\text { Blinding of outcome as- } \\
\text { sessment (detection bias) } \\
\text { All outcomes }\end{array}$ & Unclear risk & Not detailed. \\
\hline $\begin{array}{l}\text { Incomplete outcome data } \\
\text { (attrition bias) } \\
\text { All outcomes }\end{array}$ & Unclear risk & Not detailed. \\
\hline $\begin{array}{l}\text { Selective reporting (re- } \\
\text { porting bias) }\end{array}$ & High risk & \\
\hline
\end{tabular}

Zamanzadeh 2013

\begin{tabular}{|c|c|}
\hline Methods & Prospective, randomised trial of a supportive-educational intervention. \\
\hline \multirow[t]{4}{*}{ Participants } & $\begin{array}{l}\text { Quote: "Participants who were included were of } 18 \text { years age and older, diagnosed with New York Heart } \\
\text { Association class III or IV HF, had an ejection fraction less than } 40 \% \text {, agreed to predischarge education } \\
\text { and follow-up care, and would be available by phone after discharge." }\end{array}$ \\
\hline & Mean age $65.82 \pm 9.87$ years \\
\hline & $57.9 \%$ men. \\
\hline & Iran \\
\hline Interventions & $\begin{array}{l}\text { Quote: "HF participants randomized to the intervention group received a two-part intervention aimed } \\
\text { at improving self-care behaviours. The first phase consisted of a one-hour, nurse-led, in-person HF ed- }\end{array}$ \\
\hline
\end{tabular}


ucation session that was customized by the nurse according to the participant's level of education. An individualized education booklet was reviewed with literate patients, while for illiterate patients this booklet was reviewed with the participant as well as a family member.

The second phase of the intervention included postdischarge telephone followup. The objective of this phase was to reiterate and review information covered during the initial education session and improve the participant's ability to cope with the disease, as well as enhance self-care behaviours. The first followup telephone call was made by a nurse two days after hospital discharge to verify participant information and determine the next date of contact. The nurse then contacted the participant by phone every two weeks for 3 months. During these phone calls the nurse asked the participant whether they were experiencing any signs or symptoms that would suggest worsening HF.The nurse also reviewed the recommended self-care behaviours and provided support in the form of advice and encouragement when deemed necessary. These follow-up telephone calls typically lasted 15 minutes."

Self-care (SCHFI)
Quote: "A sample size of 80 ( 40 individuals in the intervention group and 40 in the control group)was
deemed sufficient based on a preliminary analysis of self-care scores of $5 \mathrm{HF}$ patients. The following pa-
rameters guided the present study; the optimal self-care behaviour score in the study was 70 , the mean
and standard deviation of self-care behaviours scores were estimated (Mean $=25, \mathrm{SD}=6.15)$, alpha $=$
0.05 and power $=0.9$ were chosen, and no attrition during followup was anticipated".

\begin{tabular}{ll}
\hline Funding source & Nil disclosed. \\
\hline Comparison Group(s) & $\begin{array}{l}\text { Quote: "Participants who were randomized to the control group received usual care provided by the } \\
\text { hospital and attending physician (nonsystematic and informal teaching)." }\end{array}$ \\
\hline Notes & New in 2015 review. \\
\hline
\end{tabular}

\section{Risk of bias}

\begin{tabular}{lll}
\hline Bias & Authors' judgement & Support for judgement \\
\hline $\begin{array}{l}\text { Random sequence genera- } \\
\text { tion (selection bias) }\end{array}$ & Low risk & $\begin{array}{l}\text { Quote:"The participants were randomized into the control and experimental } \\
\text { groups using random number software". }\end{array}$ \\
\hline
\end{tabular}

\begin{tabular}{|c|c|c|}
\hline $\begin{array}{l}\text { Allocation concealment } \\
\text { (selection bias) }\end{array}$ & Unclear risk & Not detailed. \\
\hline $\begin{array}{l}\text { Blinding of outcome as- } \\
\text { sessment (detection bias) } \\
\text { All outcomes }\end{array}$ & Unclear risk & Not detailed. \\
\hline $\begin{array}{l}\text { Incomplete outcome data } \\
\text { (attrition bias) } \\
\text { All outcomes }\end{array}$ & High risk & $\begin{array}{l}\text { Comment: } 2 \text { participants from the intervention group lost to follow-up in inter- } \\
\text { vention arm, analyses not performed as intention-to-treat. }\end{array}$ \\
\hline $\begin{array}{l}\text { Selective reporting (re- } \\
\text { porting bias) }\end{array}$ & High risk & Comment: No evidence of selective outcome reporting. \\
\hline
\end{tabular}

ACE: angiotensin-converting enzyme

BNP: brain natriuretic peptide

CHF: congestive heart failure

CNPq: Conselho Nacional de Desenvolvimento Cientico e Tecnologico

ECG: electrocardiograph

EF: ejection fraction

FIPE: Fundação Instituto de Pasquisas Econŏmicas

HF: heart failure

HR: heart rate 
LVEF: left ventricular ejection fraction

LVSD: left ventricular systolic dysfunction

NYHA: New York Heart Association

SD: standard deviation

Characteristics of excluded studies [ordered by study ID]

\begin{tabular}{|c|c|}
\hline Study & Reason for exclusion \\
\hline Abu-Awwad 2012 & System design paper. \\
\hline Akosah 2005 & Contra to protocol: intervention included frequent clinic visits. \\
\hline Al Khateeb 2012 & Contra to protocol: not an RCT. \\
\hline Albanese 2001 & $\begin{array}{l}\text { Contra to protocol: invasive impedence monitoring (SCOOP II Trial Evaluating CRT/ICD/Impedence } \\
\text { Monitoring). }\end{array}$ \\
\hline Albert 2007 & Contra to protocol: intervention was an education video. \\
\hline Aliti 2007 & Discussion paper. \\
\hline Alluhaidan 2015 & System design paper. \\
\hline Alnosayan 2014 & Review paper. \\
\hline Anderson 2005 & Contra to protocol: intervention was a heart failure clinic. \\
\hline Andrikopoulou 2014 & Review paper. \\
\hline Ansinelli 2013 & Contra to protocol: not an RCT. \\
\hline Archelrod 2014 & Review paper. \\
\hline Artinian 2003 & Contra to protocol: web-based intervention, not an RCT. \\
\hline Artinian 2006 & Contra to protocol: intervention specific for hypertension, not heart failure. \\
\hline Arya 2008 & Contra to protocol: invasive haemodynamic monitoring. \\
\hline Austin 2012 & Contra to protocol: not an RCT. \\
\hline Baden 2007a & Contra to protocol: not an RCT. \\
\hline Baden 2007b & Contra to protocol: not an RCT. \\
\hline Baer 1999 & $\begin{array}{l}\text { Asessment of correlation between electronic patient measurements and manual nurse measure- } \\
\text { ments. }\end{array}$ \\
\hline Bakhshi 2011 & Contra to protocol: not an RCT. \\
\hline Baldauf 2008 & Contra to protocol: not an RCT. \\
\hline Baldonado 2013 & Review paper. \\
\hline Barber 1999 & Contra to protocol: not an RCT, quasi experimental design. \\
\hline
\end{tabular}




\begin{tabular}{|c|c|}
\hline Study & Reason for exclusion \\
\hline Bekelman 2013 & Contra to protocol: telemonitoring was offered to usual care patients. \\
\hline Ben Gal 2013 & Contra to protocol: not an RCT. \\
\hline Benatar 2003 & Contra to protocol: comparison was between telemonitoring and home visits (not usual care). \\
\hline Bennett 2006 & Contra to protocol: intervention was a computer-based intervention. \\
\hline Bennett Milburna 2014 & Review paper. \\
\hline Berkley 2010 & Review paper. \\
\hline Blue 2001 & Contra to protocol: intervention included home visits. \\
\hline Bocchi 2007 (REMADHE) & $\begin{array}{l}\text { Contra to protocol: intervention involved intensive group education sessions and face-to-face indi- } \\
\text { vidual/group communication. }\end{array}$ \\
\hline Bocchi 2013 & $\begin{array}{l}\text { Contra to protocol: intervention involved intensive group education sessions and face-to-face indi- } \\
\text { vidual/group communication. }\end{array}$ \\
\hline Bohacik 2014 & Contra to protocol: invasive monitoring. \\
\hline Bohmer 2011 & Contra to protocol: intensive clinic follow-up in intervention (monthly). \\
\hline Bolz 2005 & Review paper. \\
\hline Bondmass 1999 & Contra to protocol: not an RCT. \\
\hline Bondmass 2002 & Contra to protocol: not an RCT. \\
\hline Bondmass 2007 & Contra to protocol: not an RCT. \\
\hline Boriani 2013 & Home visits. \\
\hline Bourge 2008 (COMPASS-HF) & Contra to protocol: invasive implantable haemodynamic monitoring. \\
\hline Bowles 2007 & Reivew paper. \\
\hline Bowles 2008 & Systematic review. \\
\hline Bowles 2009 & Contra to protocol: home visits in intervention and usual care arms. \\
\hline Bowles 2011 & Home visits. \\
\hline Brennan 2006 & Contra to protocol: not an RCT. \\
\hline Brennan 2010 & Contra to protocol: not HF patients. \\
\hline Browning 2011 & Contra to protocol: not an RCT. \\
\hline Brownsell 2006a & Author contacted: primary and secondary outcomes for this review were not measured. \\
\hline Brownsell 2006b & Author contacted: primary and secondary outcomes for this review were not measured. \\
\hline Brownsell 2008 & Author contacted: primary and secondary outcomes for this review were not measured. \\
\hline
\end{tabular}




\begin{tabular}{|c|c|}
\hline Study & Reason for exclusion \\
\hline Brunetti 2014 & Contra to protocol: not an RCT. \\
\hline Byrnes 2012 & Contra to protocol: not HF patients. \\
\hline Caldwell 2005 & Contra to protocol: education session with one follow-up telephone call. \\
\hline Call 2010 & Contra to protocol: not an RCT. \\
\hline Calvin 2008 & $\begin{array}{l}\text { Contra to protocol: participants received } 18 \text { education sessions aimed to develop self-manage- } \\
\text { ment skills. The intervention did not include telemonitoring or structured telephone support. }\end{array}$ \\
\hline Calò 2013 & Contra to protocol: invasive monitoring. \\
\hline Capomolla 2002 & Contra to protocol: intervention was a day hospital. \\
\hline Chen 2010 & Not an RCT \\
\hline Chen 2014 & Contra to protocol: not an RCT. \\
\hline Cherry 2000 & Review article. \\
\hline Chetney 2003 & Contra to protocol: not an RCT. \\
\hline Chetney 2008 & Contra to protocol: not an RCT. \\
\hline Clappers 2006 & Review of abstracts. \\
\hline Clark 2008 & Interviews with carers of patients with heart failure regarding their experiences. \\
\hline Clarke 2005 & Conference discussion paper. \\
\hline Cleland 2012 & Not an RCT. \\
\hline Cleland 2014a & Not an RCT. \\
\hline Cline 1998 & $\begin{array}{l}\text { Contra to protocol: Intervention group received education on heart failure and self management, } \\
\text { with follow-up at an outpatient clinic. }\end{array}$ \\
\hline Cole 2006 & Contra to protocol: not an RCT. \\
\hline Coll 2011 & Not heart failure-specific. \\
\hline Conway 2014 & Review paper. \\
\hline Copeland 2010 & Home visits. \\
\hline Cordisco 1999 & Contra to protocol: not an RCT. \\
\hline Courtney 2009 & Contra to protocol: intervention was an exercise programme. \\
\hline Cross 1999 & Contra to protocol: not an RCT. \\
\hline Crundall-Goode 2014 & Review paper. \\
\hline Cruz 2010 & Contra to protocol: intensive face to face education sessions. \\
\hline
\end{tabular}




\begin{tabular}{|c|c|}
\hline Study & Reason for exclusion \\
\hline Dalmiani 2001 & Contra to protocol: not an RCT. \\
\hline Dang 2006 & Contra to protocol: not an RCT. \\
\hline Dansky 2008a & Contra to protocol: intervention included home visits. \\
\hline Dansky 2008b & Contra to protocol: not an RCT. \\
\hline Dansky 2009 & Contra to protocol: both groups had telemonitoring exposure. Home visits. \\
\hline Dar 2009 (HOME-HF) & Contra to protocol: both study groups received a home visit from study nurse. \\
\hline De Feo 2002 & Contra to protocol: not an RCT. \\
\hline De Vries 2011a & Contra to protocol: not an RCT. \\
\hline De Vries 2011b & Not an RCT. \\
\hline Deckwart 2011 & Not an RCT. \\
\hline Dedier 2008 & Contra to protocol: intervention specific for hypertension, not heart failure. \\
\hline Deepak 2008 & Contra to protocol: not an RCT. \\
\hline Del Sindaco 2007 & Contra to protocol: intervention included clinic and home visits. \\
\hline Delaney 2013 & Previous exposure to telemonitoring. \\
\hline Demarzo 2006 & Contra to protocol: invasive haemodynamic monitoring. \\
\hline Dickens 2012 & Contra to protocol: not an RCT. \\
\hline Dickerson 2011 & Contra to protocol: not an RCT. \\
\hline Dimmick 2003 & Contra to protocol: not an RCT. \\
\hline Dollard 2004 & Review paper. \\
\hline Dougherty 2005 & Contra to protocol: invasive monitoring. \\
\hline Doughty 2002 & Contra to protocol: intervention included regular clinic visits. \\
\hline Downey 2001 & Contra to protocol: not an RCT. \\
\hline Dracup 2012 & $\begin{array}{l}\text { Contra to protocol: face-to-face education and telephone counselling for intervention and control } \\
\text { groups. }\end{array}$ \\
\hline Ducharme 2005 & Contra to protocol: intervention was an outpatient clinic. \\
\hline Duffy 2005 & Contra to protocol: intervention included home visits. \\
\hline Duffy 2008 & Discussion paper. \\
\hline Duffy 2010 & Contra to protocol: home visits. \\
\hline
\end{tabular}




\begin{tabular}{|c|c|}
\hline Study & Reason for exclusion \\
\hline Dunagan 2005 & Contra to protocol: intervention included home visits. \\
\hline Dunn 2006 & Contra to protocol: not an RCT, intervention included clinic visits. \\
\hline Dunn 2007 & Contra to protocol: not an RCT, intervention included clinic visits. \\
\hline Dwinger 2013 & Contra to protocol: education intervention (no monitoring data). \\
\hline Ekman 1998 & Contra to protocol: intervention was a nurse-led outpatient clinic and telephone follow-up. \\
\hline Ellery 2006 & Contra to protocol: intervention was invasive monitoring. \\
\hline Enemuoh 2013 & Contra to protocol: not an RCT. \\
\hline Enjuanes 2013a & Contra to protocol: usual care is a HF disease management program. \\
\hline Erich 2013a & Contra to protocol: not an RCT. \\
\hline Erich 2013b & Contra to protocol: not an RCT. \\
\hline Esposito 2008 & Not heart failure-specific. \\
\hline Evangelista 2004 & Contra to protocol: web-based education and counselling for patients with heart failure. \\
\hline Fairbrother 2014 & Contra to protocol: not an RCT. \\
\hline Falces 2008 & Not structured telephone support or telemonitoring. \\
\hline Fan 2010 & Not an RCT. \\
\hline Feldman 2004 & Contra to protocol: intervention was email-communication to nurses. \\
\hline Feldman 2005 & Contra to protocol: intervention was email-communication to nurses. \\
\hline Ferguson 2010 & Contra to protocol: not an RCT. \\
\hline Finkelstein 2004 & Contra to protocol: intervention included home visits. \\
\hline Finkelstein 2006 & Contra to protocol: intervention included home visits. \\
\hline Finkelstein 2010a & System design paper. \\
\hline Finkelstein 2010b & System design paper. \\
\hline Finkelstein 2011 & System design paper. \\
\hline Florea 2011 & Editorial. \\
\hline Foley 2008 & Contra to protocol: not an RCT. \\
\hline Fragrasso 2007 & Not an intervention for management of heart failure, validation of remote clinical examination. \\
\hline Friedberg 2008 & Review of COACH study. \\
\hline Fursse 2008 & Contra to protocol: not an RCT. \\
\hline
\end{tabular}




\begin{tabular}{|c|c|}
\hline Study & Reason for exclusion \\
\hline Gambetta 2007 & Contra to protocol: not an RCT. \\
\hline Garcia 2009 & Review paper. \\
\hline Gellis 2012 & Contra to protocol: home visits. \\
\hline Gellis 2014 & Not structured telephone support or telemonitoring. \\
\hline Giamouzis 2012 & Review paper. \\
\hline Giordano 2013 & Contra to protocol: not an RCT. \\
\hline Goldstein 2014 & Contra to protocol: medication adherence only. \\
\hline Grancelli 2007 & Editorial for previous version of this review. \\
\hline Granger 2013 & Not structured telephone support or telemonitoring. \\
\hline Graves 2013 & Review paper. \\
\hline Gregory 2006 (SPAN-CHF) & Contra to protocol: intervention included home visits. \\
\hline Grustam 2014 & Review paper. \\
\hline Gund 2008 & Contra to protocol: not an RCT. \\
\hline Gurne 2012 & Review paper. \\
\hline Gámez-López 2012 & Contra to protocol: home visits in intervention. \\
\hline Hall 2010 & Review paper. \\
\hline Hanssen 2007 & $\begin{array}{l}\text { Contra to protocol: intervention was telephone follow-up of patients following a myocardial infarc- } \\
\text { tion. }\end{array}$ \\
\hline Harkness 2006 & Review of DIAL Trial. \\
\hline Harrison 2002 & Contra to protocol: intervention included home visits. \\
\hline Hart-Wright 2006 & Contra to protocol: not an RCT. \\
\hline Hayes, 2008 & Contra to protocol: health technology assessment. \\
\hline Hayes, 2011 & Contra to protocol: health technology assessment. \\
\hline Heidenreich 1999 & Contra to protocol: not an RCT. \\
\hline Heisler 2007 & Contra to protocol: not an RCT. \\
\hline Helms 2007 & Discussion / review paper. \\
\hline Helms 2010 & Contra to protocol: not an RCT. \\
\hline Hennrikus 2012 & Contra to protocol: not an RCT. \\
\hline
\end{tabular}




\begin{tabular}{|c|c|}
\hline Study & Reason for exclusion \\
\hline Hindricks 2014 & Contra to protocol: invasive monitoring. \\
\hline Hinterbuchner 2010 & Contra to protocol: not an RCT. \\
\hline Ho 2007 & Contra to protocol: intervention included home visits. \\
\hline Hoban 2013 & Contra to protocol: home visits. \\
\hline Holland 2014 & Contra to protocol: not telemonitoring or telephone support. \\
\hline Holly 2011 & Review paper. \\
\hline Holst 2007 & $\begin{array}{l}\text { Contra to protocol: not structured telephone support or telemonitoring, telephone follow-up fol- } \\
\text { lowing an education intervention. }\end{array}$ \\
\hline Hoover 2007 & Contra to protocol: not an RCT. \\
\hline Hoover 2009 & Contra to protocol: not an RCT. \\
\hline Howlett 2011 & Contra to protocol: usual care in a HF clinic. \\
\hline Hudson 2005 & Contra to protocol: not an RCT. \\
\hline Huynh 2006 & $\begin{array}{l}\text { Contra to protocol: intensive education session; not structured telephone support or telemonitor- } \\
\text { ing. }\end{array}$ \\
\hline Jaarsma (COACH Study) & Contra to protocol: intervention included clinic and home visits. \\
\hline Jaarsma 1999 & Review paper. \\
\hline Jenkins 2001 & Contra to protocol: intervention included home visits. \\
\hline Jerant 2001 & Contra to protocol: intervention included home visits. \\
\hline Jerant 2003 & Contra to protocol: intervention included home visits. \\
\hline Johnston 2000 & Intervention not specific to heart failure patients. \\
\hline Jolly 2007 & Home-based exercise intervention. \\
\hline Jones 2002 & Review paper. \\
\hline Jones 2014 & Review paper. \\
\hline Juan 2011 & Not heart failure-specific. \\
\hline Karlsson 2005 & Contra to protocol: intervention was an outpatient clinic. \\
\hline Kashem 2007 & Contra to protocol: web-based intervention. \\
\hline Kasper 2002 & Contra to protocol: intervention included home visits. \\
\hline Kastner 2010 & Contra to protocol: not an RCT. \\
\hline Khoury 2008 & Contra to protocol: invasive haemodynamic monitoring. \\
\hline
\end{tabular}




\begin{tabular}{|c|c|}
\hline Study & Reason for exclusion \\
\hline Kielblock 2007 & Not an RCT. \\
\hline Kimmelsteil 2004 & Contra to protocol: intervention included home visits. \\
\hline Kirschner 2006 & Discussion paper. \\
\hline Kitsiou 2013 & Review paper. \\
\hline Kleet 2013 & Contra to protocol: not an RCT. \\
\hline Kline 2006 & Contra to protocol: not an RCT. \\
\hline Koehler 2006 & Review of TEN-HMS study. \\
\hline Koelling 2005 & Contra to protocol: intervention was a face-to-face education session. \\
\hline Konstam 2012 & Review paper. \\
\hline Kottmair 2005 & Discussion paper. \\
\hline Koutkias 2003 & Contra to protocol: not an RCT. \\
\hline Kraal 2014 & Contra to protocol: cardiac rehabilitation. \\
\hline Kropf 2014 & Contra to protocol: not an RCT. \\
\hline Krumholz 2002 & Contra to protocol: frequent clinic and home visits. \\
\hline Krumholz 2011 & Editorial \\
\hline Kurtz 2011 & Contra to protocol: not an RCT. \\
\hline Kutzleb 2006 & Contra to protocol: not an RCT. \\
\hline Kwok 2008 & Contra to protocol: intervention included home visits. \\
\hline LaFramboise 2003 & Contra to protocol: not an RCT. \\
\hline Lagido 2014 & System design paper. \\
\hline Lavenberg 2012 & Contra to protocol: health technology assessment. \\
\hline Lehmann 2006 & Contra to protocol: not an RCT. \\
\hline Lemay 2013 & Chart review paper. \\
\hline Leventhal 2011 & Contra to protocol: home visit in the intervention. \\
\hline Lind 2013 & System design paper. \\
\hline Lucas 2007 & Contra to protocol: not an RCT. \\
\hline Machingo 2003 & Contra to protocol: not an RCT. \\
\hline Maddukuri 2006 & Contra to protocol: not an RCT. \\
\hline
\end{tabular}




\begin{tabular}{|c|c|}
\hline Study & Reason for exclusion \\
\hline Madigan 2008 & Contra to protocol: not an RCT. \\
\hline Madigan 2013 & Contra to protocol: home visits. \\
\hline Maglaveras 2002 & Contra to protocol:Not RCT \\
\hline Maglaveras 2003 & Contra to protocol: not an RCT \\
\hline Maglaveras 2006 & Contra to protocol: not an RCT \\
\hline Mair 2007 & Review paper. \\
\hline Makaya 2008 & Contra to protocol: intervention included home visits. \\
\hline Mansfield 2006 & Contra to protocol: not an RCT. \\
\hline Marangelli 2007 & Contra to protocol: not an RCT. \\
\hline Maric 2010 & Contra to protocol: not an RCT. \\
\hline Martensson 2005 & Contra to protocol: intervention included home visits. \\
\hline Martín-Lesende 2011 & Contra to protocol: home visits when required. \\
\hline Martín-Lesende 2013 & Contra to protocol: home visits when required. \\
\hline Masterson Creber 2014 & Home visits. \\
\hline Mau 2006 & Contra to protocol: intervention included home visits. \\
\hline McCauley 2006 & Contra to protocol: intervention included home visits. \\
\hline McCoy 2007 & Contra to protocol: intervention included home visits. \\
\hline McDonald 2002 & Contra to protocol: frequent clinic visits with unstructured telephone follow-up. \\
\hline McEntee 2010 & Contra to protocol: not an RCT. \\
\hline McKinstry 2014 & Editorial. \\
\hline McManus 2004 & Contra to protocol: not an RCT. \\
\hline Mendoza 2002 & Contra to protocol: not an RCT. \\
\hline Meriggi 2009 & System development paper. \\
\hline Metten 2011 & Contra to protocol: not an RCT. \\
\hline Mistiaen 2006 & Review paper. \\
\hline Mitchell 2011 & Contra to protocol: health technology assessment. \\
\hline Mitchell 2014 & Contra to protocol: not an RCT. \\
\hline Moore 2013 & Not an RCT. \\
\hline
\end{tabular}




\begin{tabular}{|c|c|}
\hline Study & Reason for exclusion \\
\hline Morales-Ascencio 2008 & Contra to protocol: not an RCT. \\
\hline Morcillo 2005 & Intervention was a single, home-based educational intervention. \\
\hline Morgan 2014 & Contra to protocol: invasive monitoring. \\
\hline Morguet 2006 & Contra to protocol: not an RCT. \\
\hline Morguet 2007a & Contra to protocol: not an RCT. \\
\hline Morguet 2007b & Contra to protocol: not an RCT. \\
\hline Morguet 2008 & Contra to protocol: not RCT. \\
\hline Mueller 2002 & Contra to protocol: not an RCT. \\
\hline Muller 2013 & Contra to protocol: invasive monitoring. \\
\hline Murtaugh 2005 & Contra to protocol: intervention was email-communication to nurses. \\
\hline Myers 2006 & Contra to protocol: not an RCT. \\
\hline Nanevicz 2000 & Contra to protocol: not an RCT. \\
\hline Naylor 1999 & Contra to protocol: intervention included home visits. \\
\hline Naylor 2004 & Contra to protocol: intervention included home visits. \\
\hline Nguyen 2007 & Contra to protocol: not an RCT. \\
\hline Nobel 2003 & Contra to protocol: not an RCT. \\
\hline Noel 2004 & Contra to protocol: intervention not specific to patients with heart failure. \\
\hline Nohria 2007 & Contra to protocol: intervention was invasive haemodynamic monitoring. \\
\hline Nucifora 2006 & $\begin{array}{l}\text { Contra to protocol: intervention was not structured telephone support (a telephone number was } \\
\text { available for participants to talk to a nurse). }\end{array}$ \\
\hline O'Reilly 1999 & Contra to protocol: not an RCT. \\
\hline Oddone 1999 & Contra to protocol: not an RCT. \\
\hline Odeh 2013 & Contra to protocol: not an RCT. \\
\hline Oeff 2005a & Contra to protocol: not an RCT. \\
\hline Oeff 2005b & Discussion paper. \\
\hline Ojeda 2005 & $\begin{array}{l}\text { Contra to protocol: intervention included clinic visits. A telephone number was made available to } \\
\text { participants to contact clinic staff. }\end{array}$ \\
\hline Oliveira 2013 & Contra to protocol: invasive monitoring. \\
\hline Opasich 2005 & Review paper. \\
\hline
\end{tabular}




\begin{tabular}{|c|c|}
\hline Study & Reason for exclusion \\
\hline Page 2012 & Contra to protocol: not an RCT. \\
\hline Pandor 2013a & Review paper. \\
\hline Pascual 2011 & Contra to protocol: education, not monitoring, intensive clinic visits. \\
\hline Pasqualini 2006 & Contra to protocol: not an RCT. \\
\hline Patja 2012 & Contra to protocol: education, not monitoring (outcomes of interest not assessed). \\
\hline Peikes 2009 & Review paper. \\
\hline Philbin 2000 & Report on a quality improvement intervention. \\
\hline Phillips 2008 & Report of a 24-hour telephone support programme for patients and caregivers at the end of life. \\
\hline Picard 2008 & Review paper. \\
\hline Piepoli 2006 & Contra to protocol: not an RCT. \\
\hline Pinto 2014 & Contra to protocol: not an RCT. \\
\hline Piorkowski 2006 & Contra to protocol: invasive haemodynamic monitoring. \\
\hline Piotrowicz 2012 & Contra to protocol: cardiac rehabilitation, not an RCT. \\
\hline Pugh 2001 & Contra to protocol: nurse visits were part of the intervention. \\
\hline Purcell 2014 & Review paper. \\
\hline Quinn 2006 & Contra to protocol: not an RCT. \\
\hline Quinn 2008 & Contra to protocol: not an RCT. \\
\hline Rabelo 2007 & Contra to protocol: not an RCT. \\
\hline Raborn 2012 & Contra to protocol: not an RCT. \\
\hline Rahimpour 2008 & Contra to protocol: not an RCT. \\
\hline Ramaekers 2009 & Contra to protocol: home visits in usual care. \\
\hline Raman 2008 & Review paper. \\
\hline Reble 2006 & Contra to protocol: not an RCT. \\
\hline Repoley 2006 & Contra to protocol: not an RCT. \\
\hline Rich 2002 & Review paper. \\
\hline Ross 2004 & Comparison of interactive internet electronic record. \\
\hline Roth 2005 & Contra to protocol: not an RCT, not specific to heart failure. \\
\hline Roth 2006 & Contra to protocol: not an RCT, not specific to heart failure. \\
\hline
\end{tabular}




\begin{tabular}{|c|c|}
\hline Study & Reason for exclusion \\
\hline Rozenman 2007 & Contra to protocol: invasive haemodynamic monitoring. \\
\hline Saxon 2007 & Contra to protocol: invasive haemodynamic monitoring. \\
\hline Scalvini 2004 & Contra to protocol: not an RCT. \\
\hline Scalvini 2005a & Contra to protocol: not an RCT. \\
\hline Scalvini 2005b & Contra to protocol: not an RCT. \\
\hline Scalvini 2006 & Contra to protocol: GP monitoring vs home based monitoring. \\
\hline Scherr 2006 & Contra to protocol:intervention not specific for heart failure patients. \\
\hline Schmidt 2008 & Medication box which monitored medication adherence. \\
\hline Schneider 2004 & Contra to protocol: not an RCT. \\
\hline Schofield 2005 & Contra to protocol: not an RCT. \\
\hline Schofield 2008 & Contra to protocol: not an RCT. \\
\hline Schwamm 2014 & Review paper. \\
\hline Schwarz 2008 & Contra to protocol: intervention involved caregivers as well as the person with heart failure. \\
\hline Scott 2004 & Contra to protocol: not an RCT. \\
\hline Seibert 2008a & Contra to protocol: not an RCT. \\
\hline Sen 2014 & Contra to protocol: diabetes not HF \\
\hline Serxner 1998 & Contra to protocol: not an RCT. \\
\hline Shah 2007 & Discussion paper. \\
\hline Shah 2008 & Contra to protocol: intervention based in clinic. \\
\hline Shearer 2007 & Author contacted: primary and secondary outcomes for this review were not measured. \\
\hline Simpson 2006 & Heart transplant technology. \\
\hline Slater 2006 & Contra to protocol: not an RCT. \\
\hline Slater 2008 & Contra to protocol: not an RCT. \\
\hline Smart 2005 & Contra to protocol: not an RCT. \\
\hline Smeulders 2006 & Contra to protocol: intervention based in clinic. \\
\hline Smolis-Bak 2012 & Contra to protocol: not an RCT. \\
\hline Sonntag 2009 & Contra to protocol: not an RCT. \\
\hline Sousa 2014 & Review paper. \\
\hline
\end{tabular}




\begin{tabular}{|c|c|}
\hline Study & Reason for exclusion \\
\hline Spaeder 2006 & Contra to protocol: very frequent clinic visits. \\
\hline Sprenger 2007 & Contra to protocol: not an RCT. \\
\hline Stampehl 2012 & Contra to protocol: not an RCT. \\
\hline Steckler 2008 & Contra to protocol: not an RCT. \\
\hline Steventon 2013 & Contra to protocol: not an RCT. \\
\hline Stoerk 2013 & Contra to protocol: not an RCT. \\
\hline Stone 2009 & Editorial. \\
\hline Stone 2012 & Contra to protocol: not an RCT. \\
\hline Stork 2009 & Review paper. \\
\hline Stromberg 2003 & Contra to protocol: intervention based in clinic. \\
\hline Stromberg 2006 & Contra to protocol: intervention based in clinic. \\
\hline Stut 2011 & System development paper. \\
\hline Sullivan 2006 & Contra to protocol: not an RCT. \\
\hline Takagawa 2013 & System development paper. \\
\hline Takahashi 2010 & Not heart failure-specific. \\
\hline Takahashi 2012 & Not heart failure-specific. \\
\hline TEHAF Study & Contra to protocol: not an RCT, pre- and post-test study design. \\
\hline Terschuren 2007 & Contra to protocol: not an RCT. \\
\hline Thokala 2013a & Cost-effectiveness modelling. \\
\hline Thokala 2013b & Not an RCT. \\
\hline Thompson 2005 & Contra to protocol: intervention included home and clinic visits. \\
\hline Thompson 2008 & Review of Woodend 2003. \\
\hline Tompkins 2010 & Home visits. \\
\hline Tramarin 2005 & $\begin{array}{l}\text { Collection of abstracts, not relating to structured telephone support or telemonitoring in heart fail- } \\
\text { ure. }\end{array}$ \\
\hline Tran 2008 & Review paper. \\
\hline Trudel 2007 & $\begin{array}{l}\text { Study included participants with diabetes and hypertension. Intervention not specific to heart fail- } \\
\text { ure. }\end{array}$ \\
\hline Tsuji 2013 & Contra to protocol: not an RCT. \\
\hline
\end{tabular}




\begin{tabular}{|c|c|}
\hline Study & Reason for exclusion \\
\hline Umeda 2014 & Contra to protocol: invasive monitoring. \\
\hline VA Technology Assessment & Report on telemonitoring technologies. \\
\hline Vaccaro 2001 & Contra to protocol: not an RCT. Compared 638 matched controls. \\
\hline Valle 2004 & $\begin{array}{l}\text { Contra to protocol: intervention consisted of education for participant and family, prescribed diet } \\
\text { and guideline-based pharmacotherapy and did not include structured telephone support or tele- } \\
\text { monitoring. }\end{array}$ \\
\hline Vallina 2008 & Contra to protocol: not an RCT. \\
\hline Vallina 2010 & Contra to protocol: not an RCT. \\
\hline van den Bussche 2004 & Contra to protocol: not an RCT, observation study. \\
\hline Varon 2014 & Contra to protocol: not an RCT. \\
\hline Vasoncelos 2013 & Contra to protocol: not an RCT. \\
\hline Villalba 2006a & Contra to protocol: not an RCT. \\
\hline Villalba 2006b & Contra to protocol: not an RCT. \\
\hline Vrijhoef 2007 & Contra to protocol: not an RCT. \\
\hline Waldman 2008 & Included participants with coronary artery disease, intervention not specific to heart failure. \\
\hline Walsh 2005 & Contra to protocol: not an RCT. \\
\hline Wang 2010 & Contra to protocol: not an RCT. \\
\hline Wang 2012 & Not heart failure. \\
\hline Waywell 2007 & Contra to protocol: not an RCT. \\
\hline Weintraub 2005 & Contra to protocol: intervention included home-visits. \\
\hline West 2013 & Editorial paper. \\
\hline West-Frasier 2008 & Contra to protocol: home visits by cardiac nurses to both groups (communication from author). \\
\hline Westlake 2007 & Contra to protocol: intervention was web-based. \\
\hline Wheeler 2006 & Contra to protocol: intervention included home visits. \\
\hline Whitten 2007 & Review. \\
\hline Wierzchowiecki 2006a & Contra to protocol: intervention included home visits. \\
\hline Wierzchowiecki 2006b & Contra to protocol: intervention included home visits. \\
\hline Willyard 2006 & Contra to protocol: not an RCT. \\
\hline Wong 2005 & Intervention for people with chronic obstructive pulmonary disease. \\
\hline
\end{tabular}




\begin{tabular}{ll}
\hline Study & Reason for exclusion \\
\hline Wongpiriyayothar 2008 & Contra to protocol: intervention included home visits. \\
\hline Woodside 2011 & Contra to protocol: not an RCT. \\
\hline Wright 2003 & $\begin{array}{l}\text { Contra to protocol: intervention consisted of symptom diary, attended three education session and } \\
\text { clinic visits. }\end{array}$ \\
\hline Wu 2006 & Comparison of internet-based technology. \\
\hline Xiao 2010 & Contra to protocol: not telemonitoring or telephone support. \\
\hline Zaphiriou 2006 & Contra to protocol: intervention included a home visit. \\
\hline Zentner 2007 & Contra to protocol: not an RCT. \\
\hline Zugck 2006 & Contra to protocol: not an RCT. \\
\hline
\end{tabular}

\section{Characteristics of studies awaiting assessment [ordered by study ID]}

Dunlap 2006 (HearT-I)

\begin{tabular}{ll}
\hline Methods & Randomised controlled trial; Intervention versus usual care. \\
\hline Participants & 455 participants to date (NYHA Class II or greater). \\
& Mean age no data. \\
\% of participants were male - no data. & USA. \\
\hline Interventions & $\begin{array}{l}\text { Structured Telephone Support } \\
\text { cess to education modules; 3) Computer-initiated phone calls with a series of question regarding } \\
\text { weight and symptoms. }\end{array}$ \\
\hline Outcomes & $\begin{array}{l}\text { All-cause hospitalisation; unscheduled outpatient visits. } \\
\text { KCCQ; satisfaction; adherence to medications; knowledge of self care and heart failure }\end{array}$ \\
\hline 12-month follow-up.
\end{tabular}

\section{Kulshreshtha 2010}

\begin{tabular}{ll}
\hline Methods & Randomised controlled trial. \\
\hline Participants & 150 eligible participants from Massachusetts General Hospital. \\
& USA. \\
\hline
\end{tabular}


Kulshreshtha 2010 (Continued)

Interventions "Participants transmitted daily vital signs data and weight to a nurse who coordinated care with a physician. Timely interventions and teaching were offered over the course of the 6 month study."

Outcomes

"All cause readmission, HF related admission mortality ER Visits and Length of Stay".

Notes $\quad$ Authors contacted to clarify study methods, but no response received.

Levine 2006 (Mind My Heart)

\begin{tabular}{ll}
\hline Methods & Randomised controlled trial; intervention arm and control (usual care) arm. \\
\hline Participants & 234 participants. \\
& Mean age not reported. \\
& $\%$ of participants were male not reported. \\
& USA. \\
\hline Interventions & Telemonitoring. \\
& Intervention group transmitted to monitoring centre via modem vital signs, BP SpO. \\
\hline Outcomes & Usual care no further contact with project staff. \\
\hline Notes & Technology use and Satisfaction Survey \\
\hline
\end{tabular}

Suh 2010

Methods "The WANDA B. wireless health technology leverages sensor technology and wireless communication to monitor heart failure patient activity and to provide tailored guidance. Patients who have cardiovascular system disorders can measure their weight, blood pressure, activity levels, and other vital signs in a real-time automated fashion. The system was developed in conjunction with the UCLA Nursing School and the UCLA Wireless Health Institute for use on actual patients. It is currently in use with real patients in a clinical trial."

\section{Participants}

Interventions

\section{Outcomes}

Notes $\quad$ Authors contacted requesting details of clinical trial, no details provided.

Wade 2011

\begin{tabular}{ll}
\hline Methods & Randomised clinical trial. \\
\hline Participants & High-risk people with heart failure.
\end{tabular}


Wade 2011 (Continued)

Interventions Telehealth system with case management (THCM) versus case management (CM) alone.

\begin{tabular}{ll}
\hline Outcomes & Composite of all-cause hospitalisation, ED visit, or death. \\
\hline Notes & Unable to locate valid author contact details. \\
\hline
\end{tabular}

Wongpiriyayothar 2011

Methods 2-group pretest-post-test experimental research design with random assignment to groups.

\begin{tabular}{ll}
\hline Participants & 22 people with heart failure. \\
\hline Interventions & Coaching using telephone programme. \\
& $\begin{array}{l}\text { "The experimental group received the coaching by telephone program (CTP) from the researcher } \\
\text { who is a cardiac nurse practitioner; the control group received the usual care from the hospital } \\
\text { (routine hospital or clinic assessment and education)." }\end{array}$ \\
\hline Outcomes & Self management of heart failure symptoms, dyspnoea, physical functioning. \\
\hline Notes & Authors contacted, no response received. \\
\hline
\end{tabular}

Wootton 2010

\begin{tabular}{ll}
\hline Methods & Randomised controlled trial. \\
\hline Participants & People with heart failure. \\
\hline Interventions & $\begin{array}{l}\text { "An evaluation was undertaken on the effectiveness and efficiency of care coordination as a means } \\
\text { of delivering health services to Australian veterans with a diagnosis of congestive heart failure." }\end{array}$ \\
\hline Outcomes & Authors contacted, no response received. \\
\hline Notes &
\end{tabular}

\section{Yakushin 2006}

\begin{tabular}{ll}
\hline Methods & Randomised controlled trial; intervention arm and control (usual care) arm. \\
\hline Participants & 78 participants \\
& Mean age 56 years. \\
& $\%$ of participants were male not reported. \\
& Russian Federation. \\
\hline
\end{tabular}

Interventions

Unable to determine intervention from abstract.

Some telephone follow-up.

Usual care not described. 
Yakushin 2006 (Continued)

Outcomes Hospitalisations and cardiovascular death

Notes Unable to contact authors.

BP: blood pressure

KCCQ: Kansas City cardiomyopathy questionnaire

$\mathrm{SpO}_{2}$ : Pulse oximetry

Characteristics of ongoing studies [ordered by study ID]

Andrei 2011

Trial name or title

\begin{tabular}{ll}
\hline Methods & Randomised controlled trial. \\
\hline Participants & $\begin{array}{l}\text { 45 people that have been followed } 1 \text { year. All these participants had established diagnosis of chron- } \\
\text { ic congestive heart failure. }\end{array}$
\end{tabular}

\begin{tabular}{ll}
\hline Interventions & "Two groups: group 1 - patients with worsening CCFH hospitalized for treatment and monitories \\
and group 2 - patients with worsening chronic congestive heart failure treated and monitories at \\
home."
\end{tabular}

Outcomes Cost

\section{Starting date}

Contact information

Notes Published as conference abstract.

Unable to locate contact details for authors.

\section{Black 2014}

Trial name or title

\begin{tabular}{ll}
\hline Methods & Multicentre, randomised controlled trial. \\
\hline Participants & $\begin{array}{l}\text { "1,500 patients aged } 50 \text { years and older will be enrolled during a hospitalization for treatment of } \\
\text { heart failure." }\end{array}$ \\
\hline Interventions & "Patients in the intervention group will receive intensive patient education using the 'teach-back' \\
& $\begin{array}{l}\text { method and receive instruction in using the telemonitoring equipment. Following hospital dis- } \\
\text { charge, they will receive a series of nine scheduled health coaching telephone calls over } 6 \text { months } \\
\text { from nurses located in a centralized call center. The nurses also will call patients and patients' } \\
\text { physicians in response to alerts generated by the telemonitoring system, based on predetermined } \\
\text { parameters." }\end{array}$ \\
\hline Outcomes & "The primary outcome is readmission for any cause within 180 days. Secondary outcomes include \\
30-day readmission, mortality, hospital days, emergency department (ED) visits, hospital cost, and \\
health-related quality of life."
\end{tabular}

\section{Starting date}


Black 2014 (Continued)

Contact information

Notes Study protocol only.

Boxer 2010

Trial name or title

Methods Randomised controlled trial.

\section{Participants}

Interventions

\section{Outcomes}

\section{Starting date}

Contact information

\begin{tabular}{ll}
\hline Notes & Published as conference abstract. \\
& Authors emailed, no response received.
\end{tabular}

\section{Cavusoglu 2013 Hit-Point}

\begin{tabular}{ll}
\hline Trial name or title & Hit-Point \\
\hline Methods & Multicentre, randomised, controlled trial. \\
\hline Participants & $\begin{array}{l}\text { "Patients who carried the diagnosis of HF secondary to systolic dysfunction, had been hospitalized } \\
\text { for HF within six months of randomization, and had symptoms despite optimal medical therapy." }\end{array}$ \\
\hline Interventions & $\begin{array}{l}\text { "Enhanced HF education with a } 6 \text { month phone follow-up program (EHFP) vs routine care (RC). Ed- } \\
\text { ucation included information on the adherence to treatment, symptoms recognition, diet and flu- } \\
\text { id intake, weight monitoring, activity, exercise training and when to contact cardiologist. Patients } \\
\text { were contacted by phone at 1, 3 and } 6 \text { month." }\end{array}$ \\
\hline Outcomes & "The primary study endpoint was cardiovascular death." \\
\hline Starting date & Conference abstract, authors contacted, full-text peer-reviewed publication not yet available. \\
\hline Nontact information & \\
\hline
\end{tabular}

\section{De Vries 2011 IN TOUCH}

Trial name or title IN-TOUCH


De Vries 2011 IN TOUCH (Continued)

\begin{tabular}{ll} 
Methods & Randomised controlled trial. \\
\hline Participants & 220 participants will be included after worsening of CHF. \\
\hline Interventions & Telemonitoring added to ICT-guided disease management.
\end{tabular}

\section{Outcomes}

"The primary endpoint of this study is a composite score of: 1 : death from any cause during the follow-up of the study, 2: first readmission for HF and 3: change in quality of life compared to baseline, assessed by the Minnesota Living with Heart failure Questionnaire."

\begin{tabular}{ll}
\hline Starting date & 2009 \\
\hline Contact information & \\
\hline Notes & Study protocol only. \\
\hline
\end{tabular}

\section{Dwinger 2013a}

Trial name or title

\begin{tabular}{ll}
\hline Methods & Prospective randomised controlled trial. \\
\hline Participants & Telephone-based health coaching with usual care. \\
\hline Interventions & $\begin{array}{l}\text { "Patients are selected based on one of the following chronic conditions: diabetes, coronary artery } \\
\text { disease, asthma, hypertension, heart failure, COPD, chronic depression or schizophrenia". }\end{array}$ \\
\hline Outcomes & \\
\hline Starting date & Study protocol only. \\
\hline Notes &
\end{tabular}

\section{Enjuanes 2013b}

Trial name or title

\begin{tabular}{ll}
\hline Methods & Prospective, randomised, controlled trial. \\
\hline Participants & \\
\hline Interventions & "Strategies of high risk HF patients: HFP (heart failure programme) or HFP+T heart failure pro- \\
& $\begin{array}{l}\text { gramme based on telemedicine). Telemedicine management consisted in daily telemonitoring of } \\
\text { weight, blood pressure, heart rate and symptoms that were transmitted to a central station con- } \\
\text { trolled by HF nurses. Teleintervention was carried out replacing the physical appointments by } \\
\text { videoconference, promoting self-care and performing therapeutic optimization." }\end{array}$ \\
\hline Outcomes & "The determination of direct costs was performed using cost accounting methodology. Primary \\
endpoint: non-fatal HF events by requiring hospital attention at 6 months of inclusion (decompen- \\
sations requiring parenteral treatment)."
\end{tabular}


Enjuanes 2013b (Continued)

Starting date

\section{Contact information}

Notes

Conference abstract only, authors contacted, full-text peer-reviewed publication not yet available.

Gupta 2013

Trial name or title

\begin{tabular}{ll}
\hline Methods & Randomised controlled trial. \\
\hline Participants & 40 participants with advanced heart failure. \\
\hline Interventions & $\begin{array}{l}\text { "Usual in-hospital care versus telemonitoring-facilitated discharge. Interventions made on the ba- } \\
\text { sis of telemonitoring data were limited to telephonically communicated advice to adjust medica- } \\
\text { tion and to arrange domiciliary blood samples to monitor renal function and electrolytes." }\end{array}$
\end{tabular}

\section{Outcomes}

Starting date

Contact information

\begin{tabular}{ll}
\hline Notes & Published as conference abstract. \\
& Unable to locate contact details for authors. \\
\hline
\end{tabular}

\section{Janssen 2010}

Trial name or title

\begin{tabular}{ll}
\hline Methods & Controlled trial \\
\hline Participants & People with heart failure. \\
\hline Interventions & Telemonitoring. \\
\hline Outcomes & \\
\hline Starting date & \\
\hline Contact information & Published as conference abstract. \\
\hline Notes & Unable to locate contact details for authors. \\
\hline
\end{tabular}


Kalowes 2012

Trial name or title

\begin{tabular}{ll}
\hline Methods & Multi-site randomised, controlled trial (RCT) \\
\hline Participants & "Recently hospitalized HF patients". \\
\hline Interventions & $\begin{array}{l}\text { "post-discharge, telephone intervention" compared to "usual care group receiving outpatient care } \\
\text { by their primary care physician." }\end{array}$ \\
\hline Outcomes & $\begin{array}{l}\text { "...self-care/adherence...incidence of all cause mortality or hospital readmissions (30-day /over- } \\
\text { all)". "A secondary aim is to assess the impact of the intervention on patient self-care/adherence by } \\
\text { examining the effect on QoL and the role of multiple variables (socioeconomic, HC setting/system } \\
\text { related, condition related, treatment and patient related)". }\end{array}$
\end{tabular}

Starting date

Contact information

Notes

Published as conference abstract.

Authors emailed, no response received.

Karanam 2012

Trial name or title

\begin{tabular}{ll}
\hline Methods & Unclear. \\
\hline Participants & 61 participants. \\
\hline Interventions & $\begin{array}{l}\text { "Mobile phone based case management program for patients with Heart Failure (HF) to monitor, } \\
\text { educate, and improve self-care efficacy." }\end{array}$ \\
\hline Outcomes & "Knowledge, behavior, self care efficacy, and quality of life(QoL) at baseline and 12 weeks." \\
\hline Starting date & Published as conference abstract. \\
\hline Contact information & Unable to locate contact details for authors. \\
\hline Notes & \\
\hline
\end{tabular}

Kessing 2011 Telemed-HF

\begin{tabular}{ll}
\hline Trial name or title & TELEMED-HF \\
\hline Methods & Randomised controlled trial. \\
\hline Participants & People with chronic, systolic HF presenting to the outpatient clinic. \\
\hline Interventions & Medication support device for 6 months in addition to usual care. \\
\hline
\end{tabular}


Kessing 2011 Telemed-HF (Continued)

Outcomes

"The efficacy and cost-efficiency of an electronic medication adherence support system in improving and monitoring HF patients' medication adherence; (2) the effect of medication adherence on hospitalizations and health care consumption; as well as on (3) clinical characteristics, and Quality of Life (QoL); and (4) clinical, sociodemographic, and psychological determinants of medication adherence."

\section{Starting date}

Contact information

Notes Study protocol.

\section{Kotooka 2013 Homes-HF}

Trial name or title

\begin{tabular}{ll}
\hline Methods & Multicentre RCT (Japan). \\
& 2 groups: Telemonitoring versus usual care. \\
& Follow-up: 12 months. \\
\hline Participants & n $=420$ participants planned. \\
\hline Interventions & Peight and blood pressure measurements to be performed at least once daily for 12 months. \\
& responsible for acting on the information." \\
\hline Outcomes & "Primary endpoints are all-cause death or HF hospitalisation. Secondary endpoints are all-cause \\
death, cardiac death, all-cause rehospitalisation, CV rehospitalisation, HF rehospitalisation, wors- \\
ening of symptoms, cost of care, LVEF, NTpro-BNP, high sensitive CRP, PTX3, high sensitive tropo- \\
nine, high molecular weight adiponectin, changes in MMSE score, GSES, MLWHF score, PHQ-9 score \\
and adherence to medications."
\end{tabular}

\section{Starting date}

Contact information

Notes Study protocol only.

\section{Lambrinou 2013 MEETTinCy}

\begin{tabular}{ll}
\hline Trial name or title & MEETTinCy. \\
\hline Methods & Randomised controlled trial, 4 arms. \\
\hline Participants & People hospitalised with HF, NYHA I - IV, were recruited from 4 general hospitals in Cyprus. \\
\hline Interventions & $\begin{array}{l}\text { "Patients were randomly assigned to receive pre-discharge education or telephone support for } 3 \\
\text { months after discharge or both, while patients in the control group received the usual care." }\end{array}$ \\
\hline
\end{tabular}


Lambrinou 2013 MEETTinCy (Continued)

Outcomes

"Measures of self-care (using the European Heart Failure Selfcare Behavior Scale) and QoL (using the Minnesota Living in Heart Failure Questionnaire) were collected at baseline and 3 months."

\section{Starting date}

Contact information

Notes

Published as conference abstract.

Authors emailed, no response received.

\section{Mareev 2010 CHANCE-AND}

\begin{tabular}{ll}
\hline Trial name or title & CHANCE-AND \\
\hline Methods & "The aim of this trial was to determine whether a special multidisciplinary, non-pharmacological, \\
& intervention (including pre-discharge patient education and active follow-up with regular bi-lateral \\
& telephone contact) could reduce prevalence of anxiety and depression and morbidity and mortali- \\
ty of patients with heart failure depending on severity of concomitant anxiety and depression."
\end{tabular}

\section{Participants}

Interventions

\section{Outcomes}

\section{Starting date}

\section{Contact information}

\section{McCall 2011}

\begin{tabular}{ll}
\hline Trial name or title & Medicare Health Support Pilot Program \\
\hline Methods & $\begin{array}{l}\text { Randomised study of } 8 \text { commercial programmes for disease management that used nurse-based } \\
\text { call centres. }\end{array}$ \\
\hline Participants & People with heart failure, diabetes, or both to the intervention or to usual care (control). \\
\hline Interventions & $\begin{array}{l}\text { "Quality of clinical care, acute care utilization, and Medicare expenditures for Medicare fee-for-ser- } \\
\text { vice beneficiaries." }\end{array}$ \\
\hline Outcomes & \\
\hline Starting date & Authors emailed to clarify methods, no response received. \\
\hline Notes & \\
\hline
\end{tabular}


Mizukawa 2014

Trial name or title

\begin{tabular}{ll}
\hline Methods & Multicentre randomised controlled trial. \\
\hline Participants & People with heart failure. \\
\hline Interventions & "Three groups: A) TM group with a device of noninvasive blood pressure, heart rate, body weight \\
measurements that automatically send data to the monitor center. Nurses gave patients tele-con- \\
sultation when the data were out of the optimal values. Patients also received a DM program to \\
gain self-management skills. B) DM group with the DM program. Patients write the value of blood \\
pressure, heart rate, and body weight to monitor their conditions. C) UC group with standard self- \\
management education once from a nurse, and the patients visited the physicians as usual."
\end{tabular}

Outcomes Primary outcome was hospital readmissions for heart failure.

Starting date January 2013 and April 2014

Contact information

Notes

Conference abstract, authors contacted, full-text peer-reviewed publication not yet available.

\section{Moye 2012}

Trial name or title

\begin{tabular}{ll}
\hline Methods & Randomised controlled trial. \\
\hline Participants & Primary or secondary diagnosis of heart failure. \\
\hline Interventions & $\begin{array}{l}\text { "Pharmacist-lead education intervention program. The control group received standard of care. } \\
\text { The intervention included one-on-one medication/disease management discharge counseling } \\
\text { from a pharmacist, patients were given individualized information regarding their disease state, } \\
\text { patients were called on days } 14 \text { and } 28 \text { post discharge." }\end{array}$
\end{tabular}

Outcomes Hospital re-admissions

Starting date August 1, 2011

Contact information

Notes Published as a conference abstract.

Authors emailed, no response received.

\section{Papadopoulou 2010}

\section{Trial name or title}

\begin{tabular}{ll}
\hline Methods & Randomised controlled trial. \\
\hline Participants & 100 outpatients with stable chronic HF (NYHA III and IV) and optimal drug treatment. \\
\hline \hline
\end{tabular}


Papadopoulou 2010 (Continued)

Interventions $\quad$ "Telephone intervention (a liaison nurse telephoned within seven days of hospital discharge and then at least weekly for one year).vs. usual care."

Outcomes Mortality, hospitalisations.

\section{Starting date}

Contact information

Notes Published as a conference abstract.

Unable to locate author contact details.

\section{Persson 2011}

Trial name or title

\begin{tabular}{ll}
\hline Methods & Randomised controlled clinical study. \\
\hline Participants & $\mathrm{n}=139$ \\
\hline Interventions & $\begin{array}{l}\text { "Patients were invited to meet with a specially trained nurse/care manager for a one-hour struc- } \\
\text { tured interview investigating possible causes of frequent rehospitalizations. Gaps within the pa- } \\
\text { tients' care were identified and specific actions to alleviate the patient's situation (e.g. establishing } \\
\text { a primary care contact, booking a specialist or a heart failure nurse appointment, securing home } \\
\text { care) were completed. Thereafter regular medical telephone support (structured and planned } \\
\text { phone calls) by the same nurse was offered to the patients with the aim to coach and support pa- } \\
\text { tients." }\end{array}$ \\
\hline Outcomes & $\begin{array}{l}\text { Quality of life and hospitalisations. } \\
\text { Starting date }\end{array}$ \\
\hline Contact information & Published as a conference abstract. \\
\hline Notes & Authors emailed, no response received. \\
\hline
\end{tabular}

\section{Ritchie 2012 E-coach}

\begin{tabular}{ll}
\hline Trial name or title & E-Coach \\
\hline Methods & Randomised controlled trial. \\
\hline Participants & People with congestive heart failure and chronic obstructive pulmonary disease. \\
\hline Interventions & $\begin{array}{l}\text { "Interactive voice response (IVR)-enhanced care transition intervention that monitors patients } \\
\text { at home using their personal phone." }\end{array}$ \\
\hline
\end{tabular}

\section{Outcomes}

\section{Starting date}


Ritchie 2012 E-coach (Continued)

Contact information

\begin{tabular}{ll}
\hline Notes & Study protocol. \\
& Authors emailed, no response received. \\
\hline
\end{tabular}

\section{Voon 2013}

Trial name or title

\begin{tabular}{ll}
\hline Methods & Unclear. \\
\hline Participants & "79 consecutive patients with HF deemed high-risk (hospital admission for acute decompensated \\
HF/IV diuretic outpatient treatment within 6 months, euvolemic BNP.300pg/mL, non-compliance \\
to therapy)."
\end{tabular}

\begin{tabular}{ll}
\hline Interventions & "TM was defined as daily body weight (BW) remote home monitoring using Bluetooth-enabled \\
& weighing scale via mobile phone software to a remote web server. DMP was defined as $24-$ hour \\
outpatient access via phone contact with adhoc review. In addition, structured physician review at & 2,6 and 12 weeks post-discharge and concurrent weekly phone-contacts by nurse, was available."
\end{tabular}

\begin{tabular}{ll}
\hline Outcomes & "Patients were monitored for clinical deterioration (CD), defined as evidence of HF decompensa- \\
tion requiring diuretic therapy or hospitalization as per guidelines."
\end{tabular}

\section{Starting date}

Contact information

\begin{tabular}{ll}
\hline Notes & Published as a conference abstract. \\
& Authors contacted, no response received. \\
\hline
\end{tabular}

\section{Whole Systems Demonstrator}

\begin{tabular}{ll}
\hline Trial name or title & Whole System Demonstrator \\
\hline Methods & Cluster-randomised trial \\
\hline Participants & 79 general practices in 3 areas of England \\
\hline Interventions & $\begin{array}{l}\text { "Telehealth or usual care to eligible patients. Telehealth included remote exchange of vitals signs } \\
\text { and symptoms data between patients and healthcare professionals as part of the continuing man- } \\
\text { agement of patients. Usual care reflected the range of services otherwise available in the sites, ex- } \\
\text { cluding telehealth". }\end{array}$ \\
\hline
\end{tabular}

\section{Outcomes}

\section{Starting date}

\section{Contact information}

Notes

Authors contacted to assess if intervention tailored for people with heart failure and if data available for them. Authors did not respond. 
Zhang 2009

Trial name or title

\begin{tabular}{ll}
\hline Methods & Randomised study \\
\hline Participants & 181 participants with heart failure \\
\hline Interventions & $\begin{array}{l}\text { "Nurse } 24 \text { hours telephone services group (24h-telephone, } \mathrm{n}=91 \text { ) and non-24 hours telephone ser- } \\
\text { vices (non-24h-telephone, } \mathrm{n}=90) \text { group. Telephone services must tell patients how to do next step } \\
\text { when patients } \\
\text { have any questions." }\end{array}$ \\
\hline Outcomes & $\begin{array}{l}\text { "The primary end point of the study was the total number of hospital readmission and dead in the } \\
\text { one year follow-up period". }\end{array}$ \\
\hline Starting date & \begin{tabular}{l} 
Conference abstract, unable to locate author contact details. \\
\hline Notes
\end{tabular}
\end{tabular}

\section{Zugck $2010 \mathrm{HiTel}$}

\begin{tabular}{|c|c|}
\hline Trial name or title & HiTel \\
\hline Methods & Randomised controlled trial; intervention arm and control (usual care) arm. \\
\hline \multirow[t]{4}{*}{ Participants } & $\begin{array}{l}88 \text { participants recruited from hospital, mean LVEF was } 24 \pm 7 \% \text {. Inclusion criteria NYHA II - IV on } \\
\text { optimum therapy and telephone at home. }\end{array}$ \\
\hline & Mean age 58.1 years. \\
\hline & $82 \%$ of participants were men. \\
\hline & Germany. \\
\hline \multirow[t]{3}{*}{ Interventions } & Telemonitoring. \\
\hline & $\begin{array}{l}\text { Intervention group transmitted to monitoring centre via modem vital signs, } \mathrm{BP} \mathrm{SpO}_{2} \text { and received } \\
\text { lifestyle and medication education. NYHA III and IV transmitted weekly and NYHA II monthly. Med- } \\
\text { ical advice was available } 24 / 7 \text {. }\end{array}$ \\
\hline & Usual care not described. \\
\hline \multirow[t]{2}{*}{ Outcomes } & All-cause hospitalisation. \\
\hline & 12-month follow-up. \\
\hline \multicolumn{2}{|l|}{ Starting date } \\
\hline \multicolumn{2}{|l|}{ Contact information } \\
\hline Notes & \\
\hline
\end{tabular}

BP: blood pressure 
$\mathrm{SpO}_{2}$ : pulse oximetry

\section{DATA AND ANALYSES}

\section{Comparison 1. Impact of structured telephone support and telemonitoring in heart failure on all-cause mortality}

\begin{tabular}{|c|c|c|c|c|}
\hline Outcome or subgroup title & No. of studies & $\begin{array}{l}\text { No. of partici- } \\
\text { pants }\end{array}$ & Statistical method & Effect size \\
\hline 1 All-cause mortality: STS vs UC & 22 & 9222 & $\begin{array}{l}\text { Risk Ratio (M-H, Fixed, } \\
95 \% \mathrm{Cl})\end{array}$ & $0.87[0.77,0.98]$ \\
\hline 2 All-cause mortality: TM vs UC & 17 & 3740 & $\begin{array}{l}\text { Risk Ratio }(\mathrm{M}-\mathrm{H}, \text { Fixed, } \\
95 \% \mathrm{Cl})\end{array}$ & $0.80[0.68,0.94]$ \\
\hline $\begin{array}{l}3 \text { Subgroup technology: all-cause mortality } \\
\text { STS vs UC }\end{array}$ & 17 & 6629 & $\begin{array}{l}\text { Risk Ratio (M-H, Fixed, } \\
95 \% \mathrm{Cl})\end{array}$ & $0.81[0.71,0.93]$ \\
\hline $\begin{array}{l}4 \text { Subgroup technology: all-cause mortality } \\
\text { Complex TM vs UC }\end{array}$ & 12 & 2885 & $\begin{array}{l}\text { Risk Ratio }(\mathrm{M}-\mathrm{H}, \text { Fixed, } \\
95 \% \mathrm{Cl})\end{array}$ & $0.81[0.68,0.96]$ \\
\hline $\begin{array}{l}5 \text { Subgroup technology: all-cause mortality } \\
\text { Videophone vs UC }\end{array}$ & 2 & 269 & $\begin{array}{l}\text { Risk Ratio }(\mathrm{M}-\mathrm{H}, \text { Fixed, } \\
95 \% \mathrm{Cl})\end{array}$ & $1.14[0.65,1.99]$ \\
\hline $\begin{array}{l}6 \text { Subgroup technology: all-cause mortality } \\
\text { IVR vs UC }\end{array}$ & 4 & 2445 & $\begin{array}{l}\text { Risk Ratio (M-H, Fixed, } \\
95 \% \mathrm{Cl})\end{array}$ & $1.01[0.80,1.28]$ \\
\hline $\begin{array}{l}7 \text { Subgroup technology: all-cause mortality } \\
\text { Mobile/PDA vs UC }\end{array}$ & 4 & 734 & $\begin{array}{l}\text { Risk Ratio (M-H, Fixed, } \\
95 \% \mathrm{Cl})\end{array}$ & $0.71[0.46,1.11]$ \\
\hline $\begin{array}{l}8 \text { Subgroup TM intensity: all-cause mortality } \\
\text { Office hours vs UC }\end{array}$ & 10 & 1548 & $\begin{array}{l}\text { Risk Ratio }(\mathrm{M}-\mathrm{H}, \text { Fixed, } \\
95 \% \mathrm{Cl})\end{array}$ & $0.69[0.52,0.92]$ \\
\hline $\begin{array}{l}9 \text { Subgroup TM intensity: all-cause mortality } \\
24 / 7 \text { or } 7 \text { days/week vs UC }\end{array}$ & 7 & 2192 & $\begin{array}{l}\text { Risk Ratio (M-H, Fixed, } \\
95 \% \mathrm{Cl})\end{array}$ & $0.86[0.71,1.04]$ \\
\hline $\begin{array}{l}10 \text { Subgroup Publication year: all-cause } \\
\text { mortality STS vs UC }<2000\end{array}$ & 2 & 219 & $\begin{array}{l}\text { Risk Ratio (M-H, Fixed, } \\
95 \% \mathrm{Cl})\end{array}$ & $0.45[0.14,1.40]$ \\
\hline $\begin{array}{l}11 \text { Subgroup Publication year: all-cause } \\
\text { mortality STS vs UC } 2000-2007\end{array}$ & 13 & 5668 & $\begin{array}{l}\text { Risk Ratio (M-H, Fixed, } \\
95 \% \mathrm{Cl})\end{array}$ & $0.86[0.74,0.99]$ \\
\hline $\begin{array}{l}12 \text { Subgroup Publication year: all-cause } \\
\text { mortality STS vs UC } \geq 2008\end{array}$ & 7 & 3335 & $\begin{array}{l}\text { Risk Ratio (M-H, Fixed, } \\
95 \% \mathrm{Cl})\end{array}$ & $0.91[0.75,1.10]$ \\
\hline $\begin{array}{l}13 \text { Subgroup Publication year: all-cause } \\
\text { mortality TM vs UC } 2000 \text { - } 2007\end{array}$ & 3 & 553 & $\begin{array}{l}\text { Risk Ratio (M-H, Fixed, } \\
95 \% \mathrm{Cl})\end{array}$ & $0.58[0.39,0.86]$ \\
\hline $\begin{array}{l}14 \text { Subgroup Publication year: all-cause } \\
\text { mortality TM vs UC } \geq 2008\end{array}$ & 14 & 3187 & $\begin{array}{l}\text { Risk Ratio (M-H, Fixed, } \\
95 \% \mathrm{Cl})\end{array}$ & $0.85[0.72,1.02]$ \\
\hline $\begin{array}{l}15 \text { Subgroup Participant age: all-cause mor- } \\
\text { tality: STS vs UC }<70 \text { years of age }\end{array}$ & 13 & 6158 & $\begin{array}{l}\text { Risk Ratio }(\mathrm{M}-\mathrm{H}, \text { Fixed, } \\
95 \% \mathrm{Cl})\end{array}$ & $0.88[0.77,1.01]$ \\
\hline $\begin{array}{l}16 \text { Subgroup Participant age: all-cause mor- } \\
\text { tality STS vs UC } \geq 70 \text { years of age }\end{array}$ & 9 & 3064 & $\begin{array}{l}\text { Risk Ratio (M-H, Fixed, } \\
95 \% \mathrm{Cl})\end{array}$ & $0.84[0.67,1.04]$ \\
\hline
\end{tabular}




\begin{tabular}{|c|c|c|c|c|}
\hline Outcome or subgroup title & No. of studies & $\begin{array}{l}\text { No. of partici- } \\
\text { pants }\end{array}$ & Statistical method & Effect size \\
\hline $\begin{array}{l}17 \text { Subgroup Participant age: all-cause mor- } \\
\text { tality: TM vs UC }<70 \text { years of age }\end{array}$ & 9 & 2493 & $\begin{array}{l}\text { Risk Ratio (M-H, Fixed, } \\
95 \% \mathrm{Cl})\end{array}$ & $0.84[0.68,1.04]$ \\
\hline $\begin{array}{l}18 \text { Subgroup Participant age: all-cause mor- } \\
\text { tality TM vs UC } \geq 70 \text { years of age }\end{array}$ & 8 & 1247 & $\begin{array}{l}\text { Risk Ratio (M-H, Fixed, } \\
95 \% \mathrm{Cl})\end{array}$ & $0.74[0.59,0.94]$ \\
\hline $\begin{array}{l}19 \text { Subgroup STS focus: all-cause mortality } \\
\text { STS (clinical support) vs UC }\end{array}$ & 18 & 8094 & $\begin{array}{l}\text { Risk Ratio (M-H, Fixed, } \\
95 \% \mathrm{Cl})\end{array}$ & $0.87[0.77,0.98]$ \\
\hline $\begin{array}{l}20 \text { Subgroup STS focus: all-cause mortality } \\
\text { STS (education) vs UC }\end{array}$ & 4 & 1128 & $\begin{array}{l}\text { Risk Ratio (M-H, Fixed, } \\
95 \% \mathrm{Cl})\end{array}$ & $0.90[0.55,1.45]$ \\
\hline $\begin{array}{l}21 \text { Sensitivity analysis follow-up period (> } 6 \\
\text { months), all-cause mortality: STS vs UC }\end{array}$ & 11 & 4818 & $\begin{array}{l}\text { Risk Ratio (M-H, Fixed, } \\
95 \% \mathrm{Cl})\end{array}$ & $0.88[0.75,1.02]$ \\
\hline $\begin{array}{l}22 \text { Sensitivity analysis follow-up period (> } 6 \\
\text { months), all-cause mortality: TM vs UC }\end{array}$ & 10 & 2580 & $\begin{array}{l}\text { Risk Ratio (M-H, Fixed, } \\
95 \% \mathrm{Cl})\end{array}$ & $0.89[0.74,1.06]$ \\
\hline
\end{tabular}

\section{Analysis 1.1. Comparison 1 Impact of structured telephone support and telemonitoring in heart failure on all-cause mortality, Outcome 1 All-cause mortality: STS vs UC.}

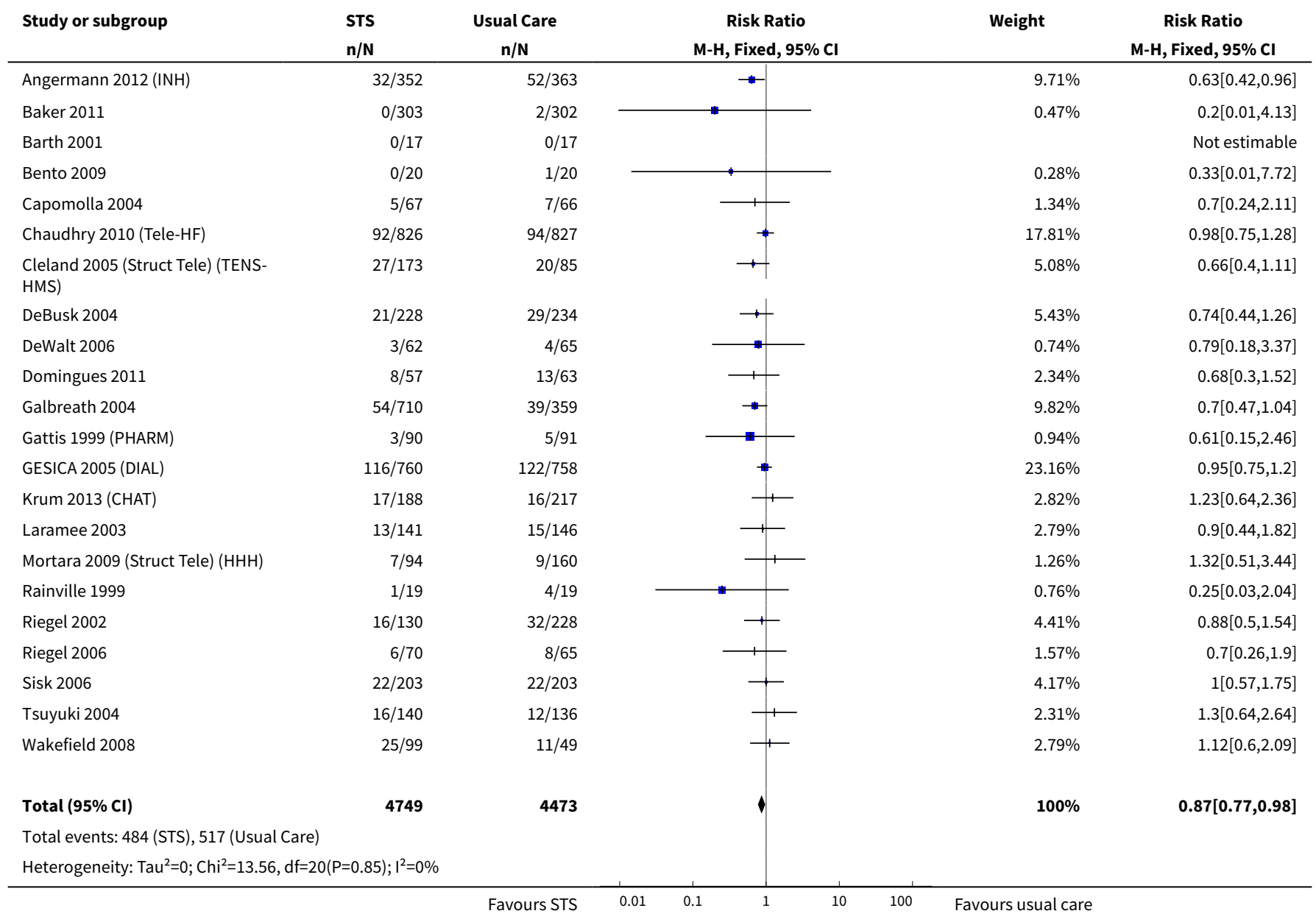




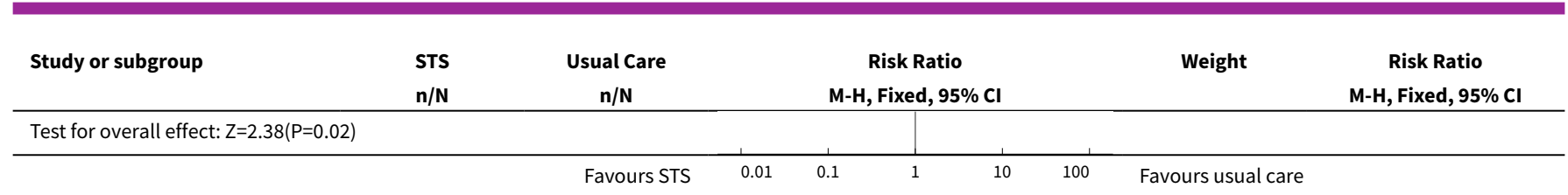

Analysis 1.2. Comparison 1 Impact of structured telephone support and telemonitoring in heart failure on all-cause mortality, Outcome 2 All-cause mortality: TM vs UC.

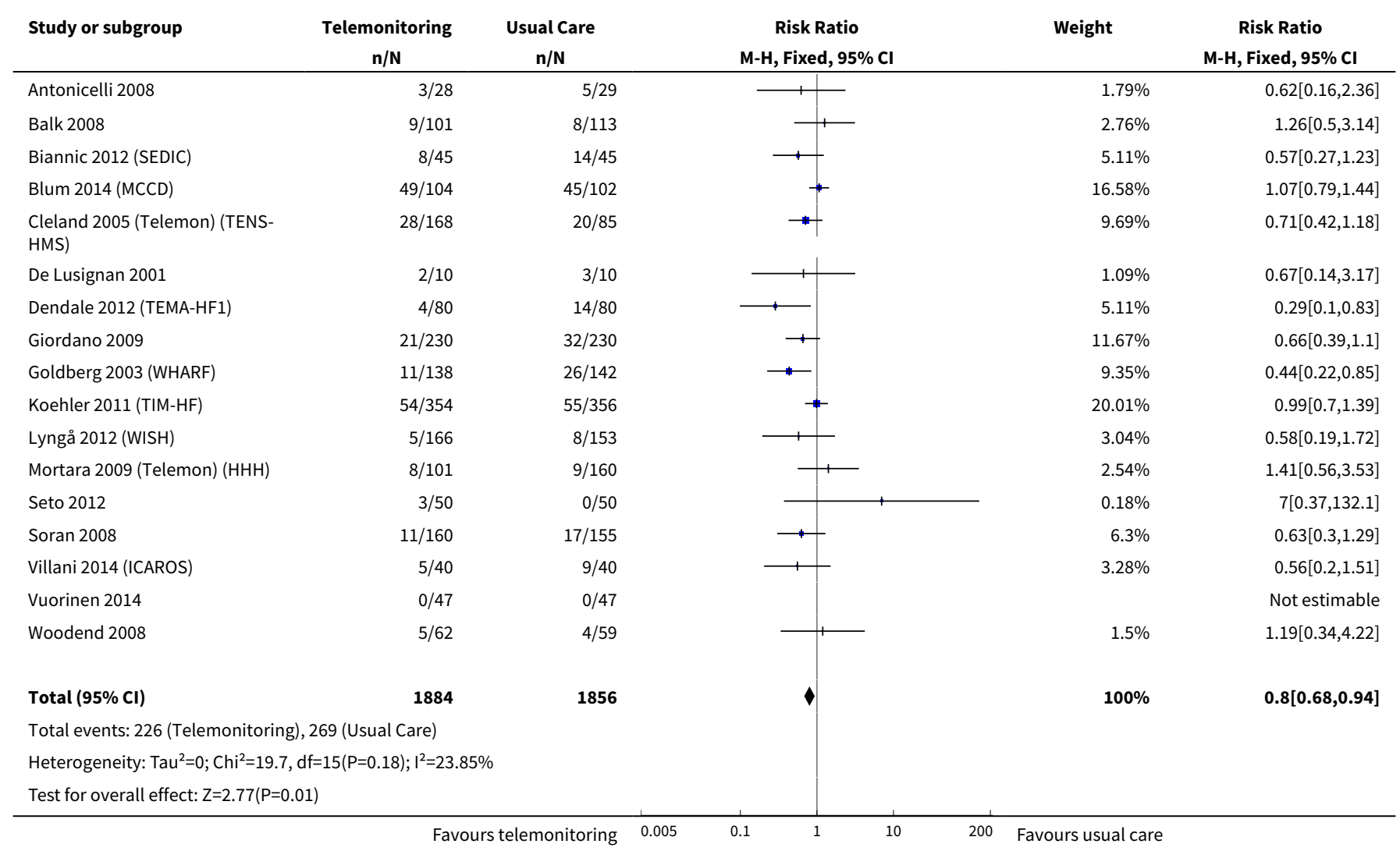

Analysis 1.3. Comparison 1 Impact of structured telephone support and telemonitoring in heart failure on all-cause mortality, Outcome 3 Subgroup technology: all-cause mortality STS vs UC.

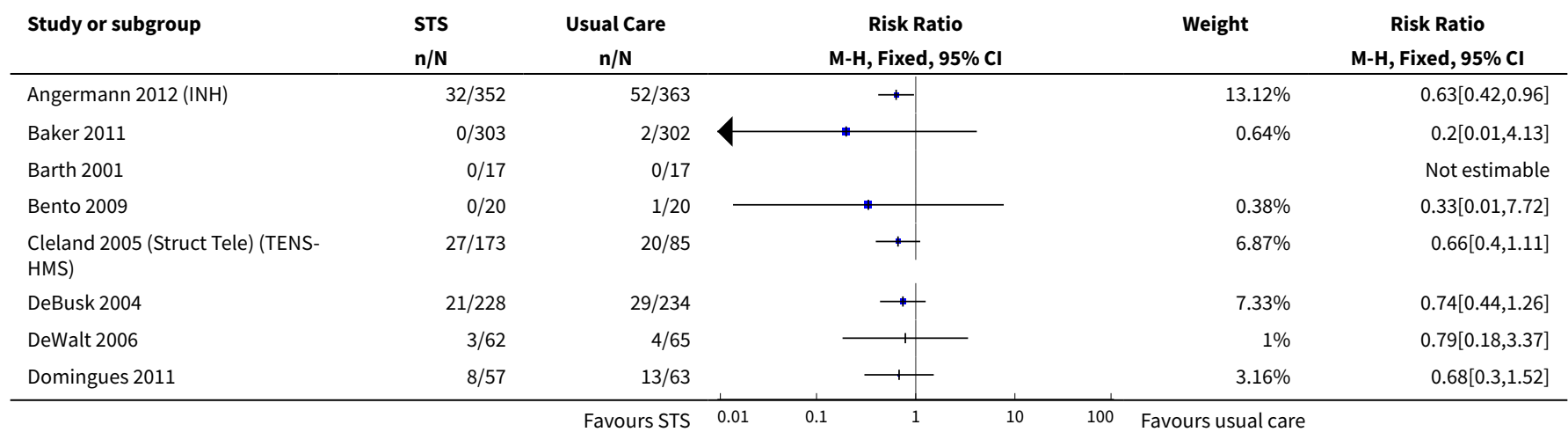




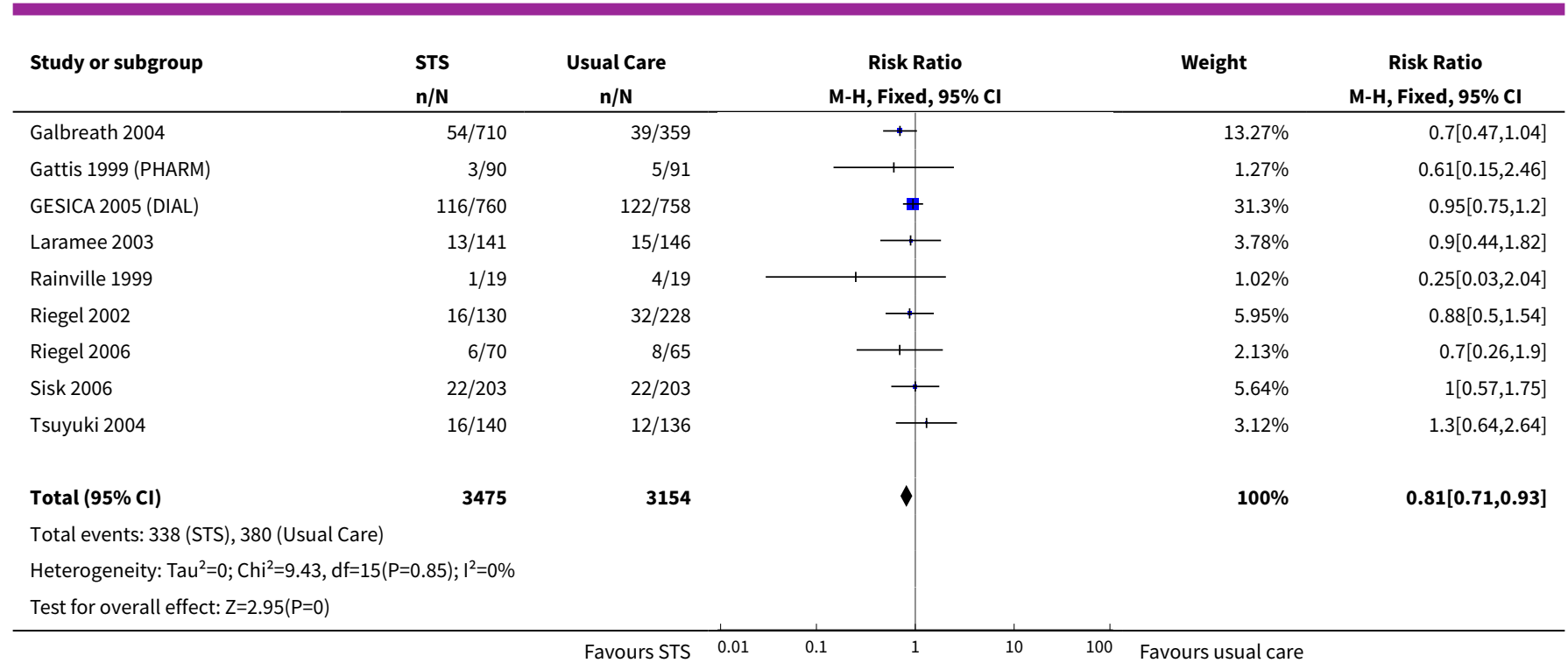

Analysis 1.4. Comparison 1 Impact of structured telephone support and telemonitoring in heart failure on all-cause mortality, Outcome 4 Subgroup technology: all-cause mortality Complex TM vs UC.

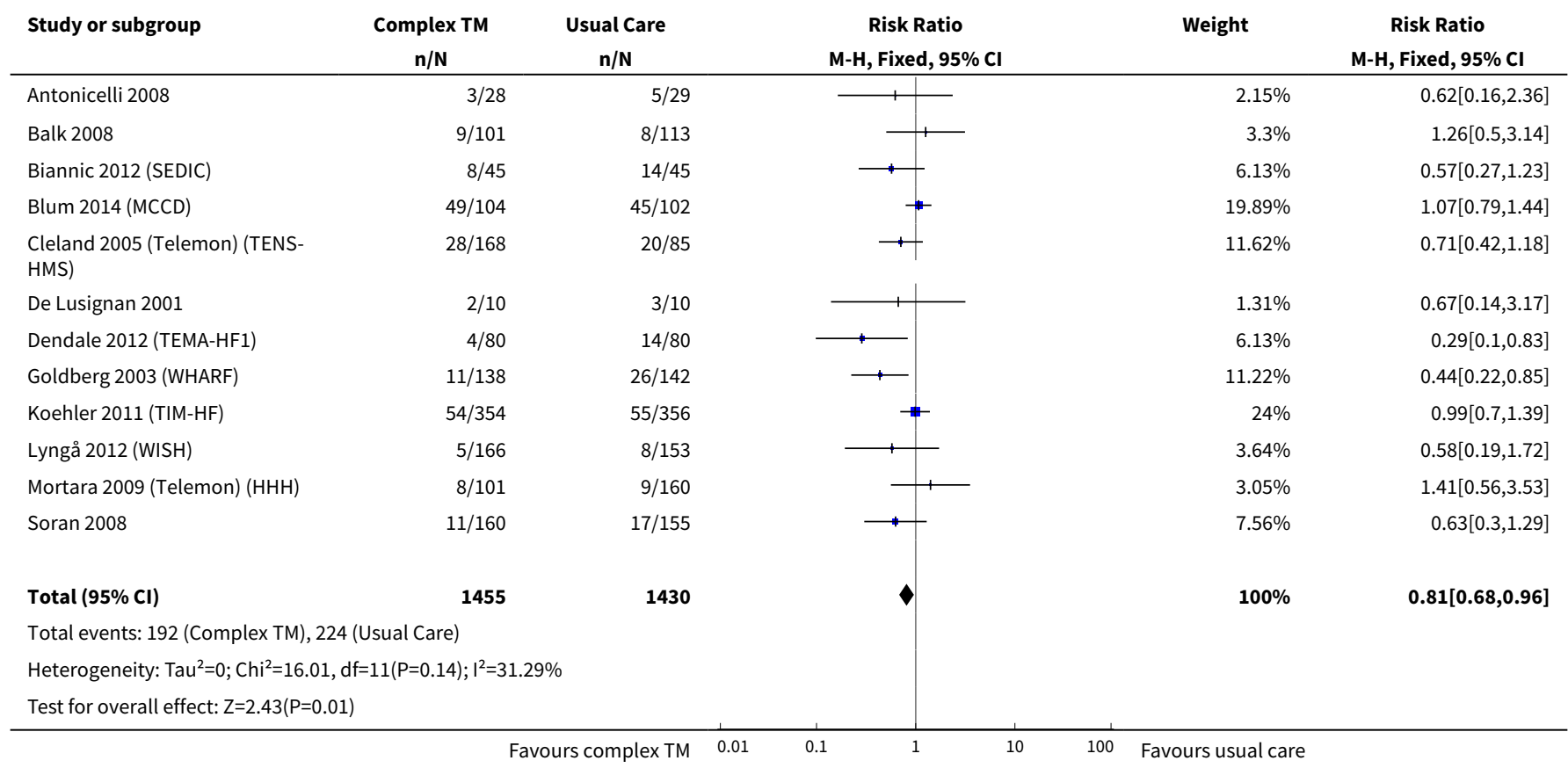

Analysis 1.5. Comparison 1 Impact of structured telephone support and telemonitoring in heart failure on all-cause mortality, Outcome 5 Subgroup technology: all-cause mortality Videophone vs UC.

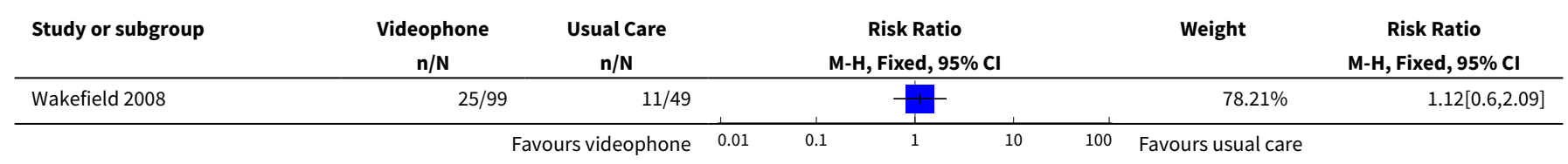




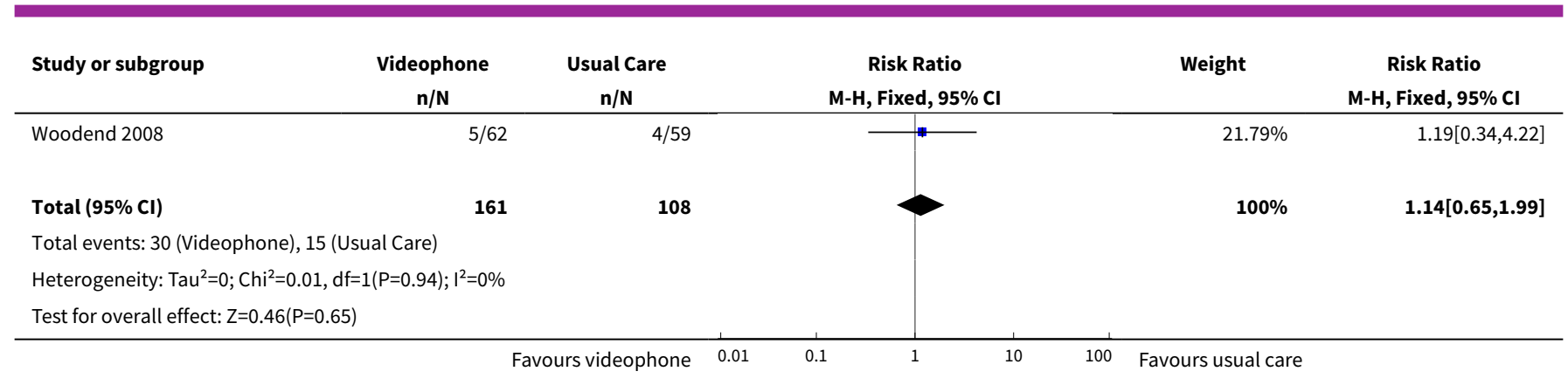

Analysis 1.6. Comparison 1 Impact of structured telephone support and telemonitoring in heart failure on all-cause mortality, Outcome 6 Subgroup technology: all-cause mortality IVR vs UC.

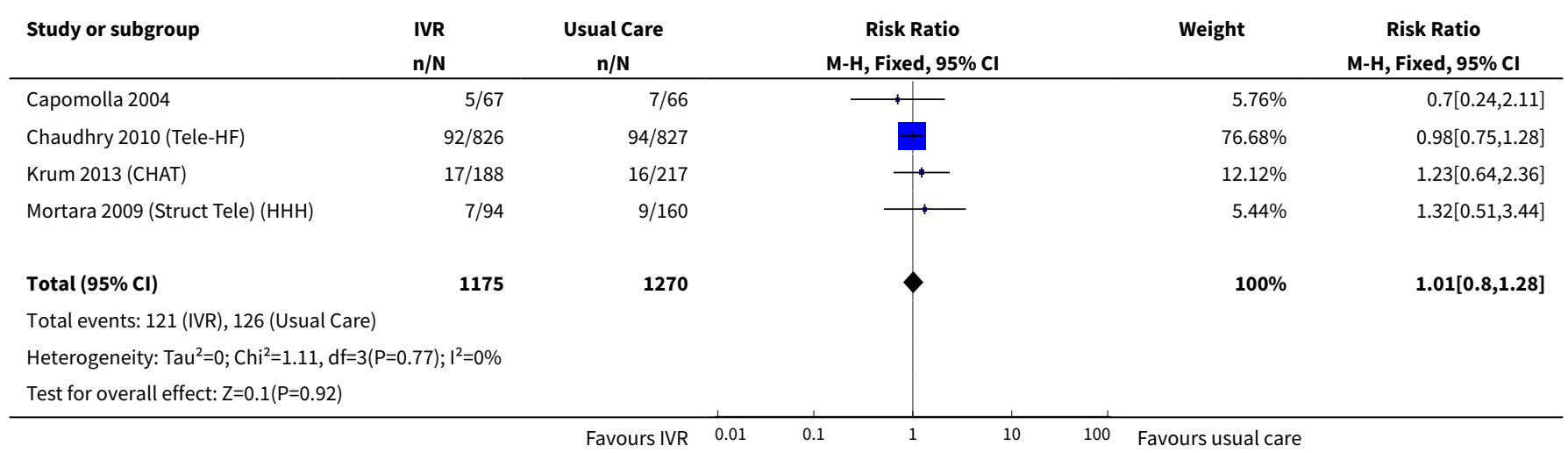

Analysis 1.7. Comparison 1 Impact of structured telephone support and telemonitoring in heart failure on all-cause mortality, Outcome 7 Subgroup technology: all-cause mortality Mobile/PDA vs UC.

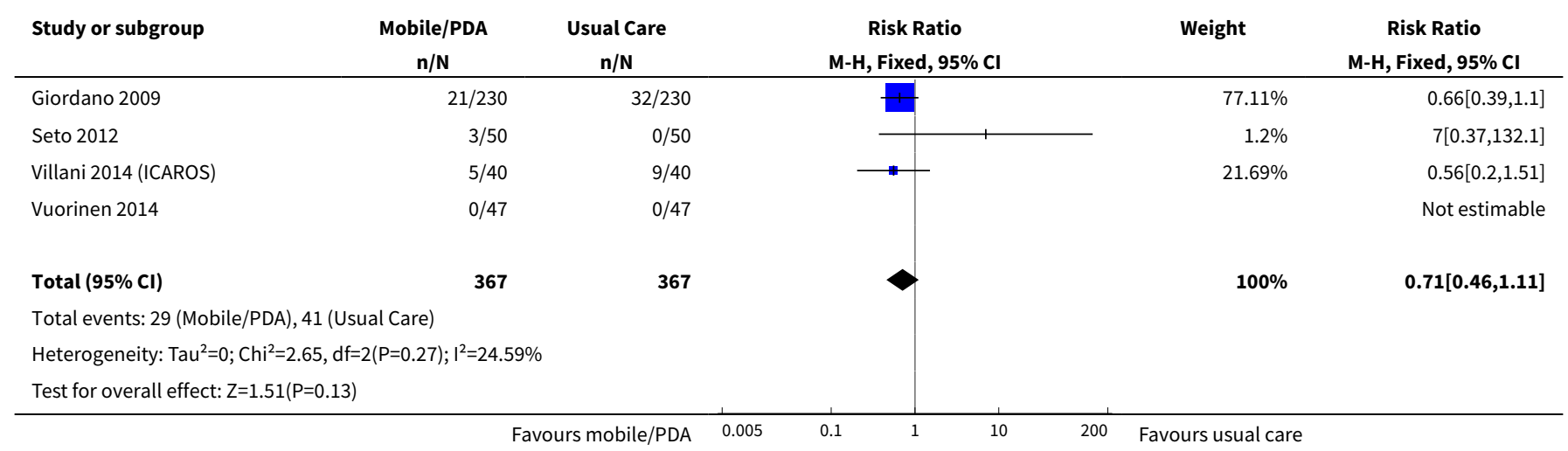


Analysis 1.8. Comparison 1 Impact of structured telephone support and telemonitoring in heart failure on all-cause mortality, Outcome 8 Subgroup TM intensity: all-cause mortality Office hours vs UC.

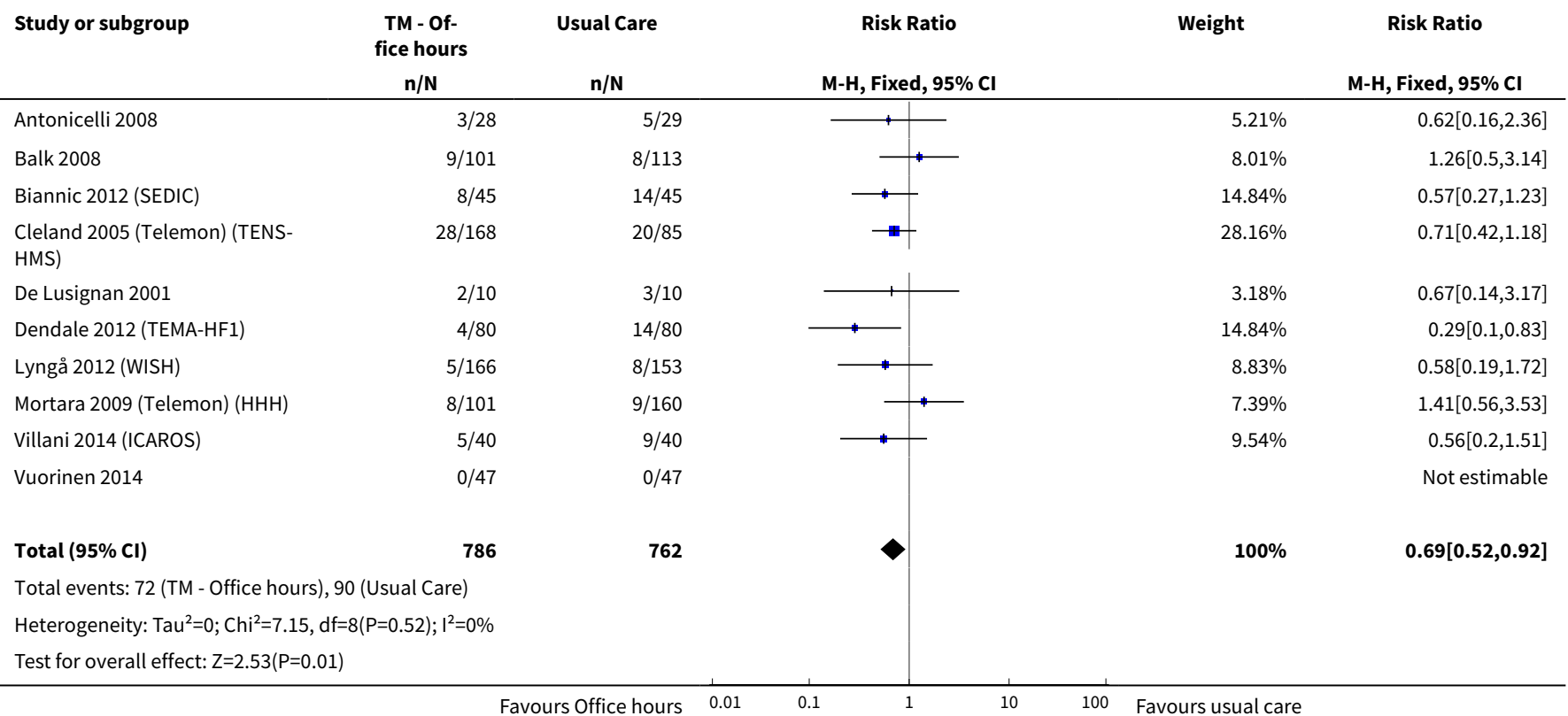

Analysis 1.9. Comparison 1 Impact of structured telephone support and telemonitoring in heart failure on all-cause mortality, Outcome 9 Subgroup TM intensity: all-cause mortality 24/7 or 7 days/week vs UC.

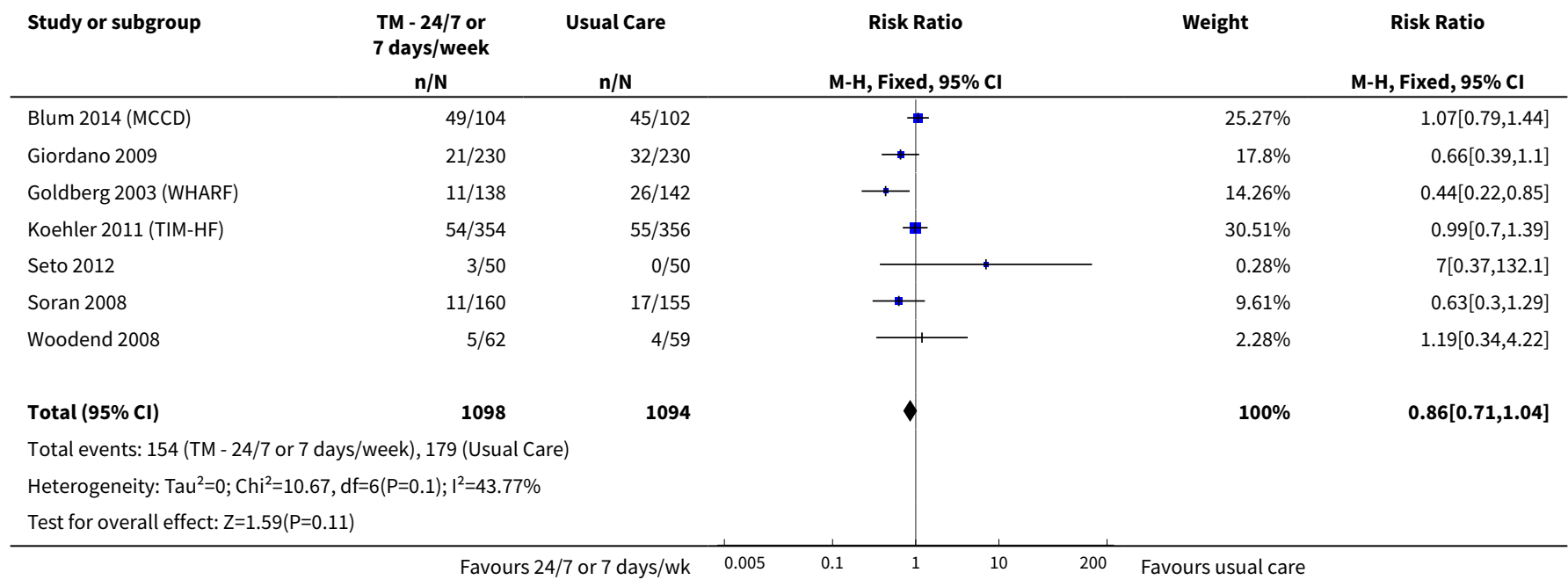

\section{Analysis 1.10. Comparison 1 Impact of structured telephone support and telemonitoring in heart failure on all-cause mortality, Outcome 10 Subgroup Publication year: all-cause mortality STS vs UC $<2000$.}

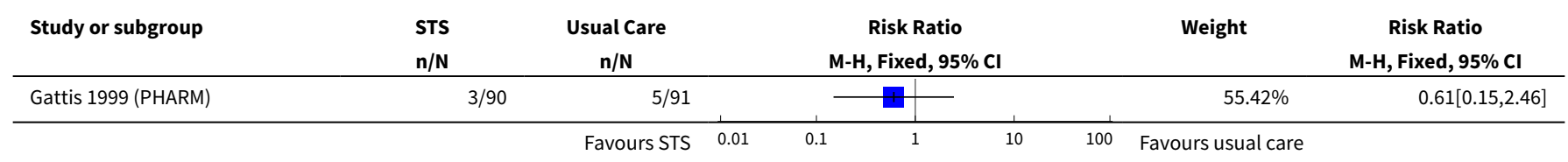




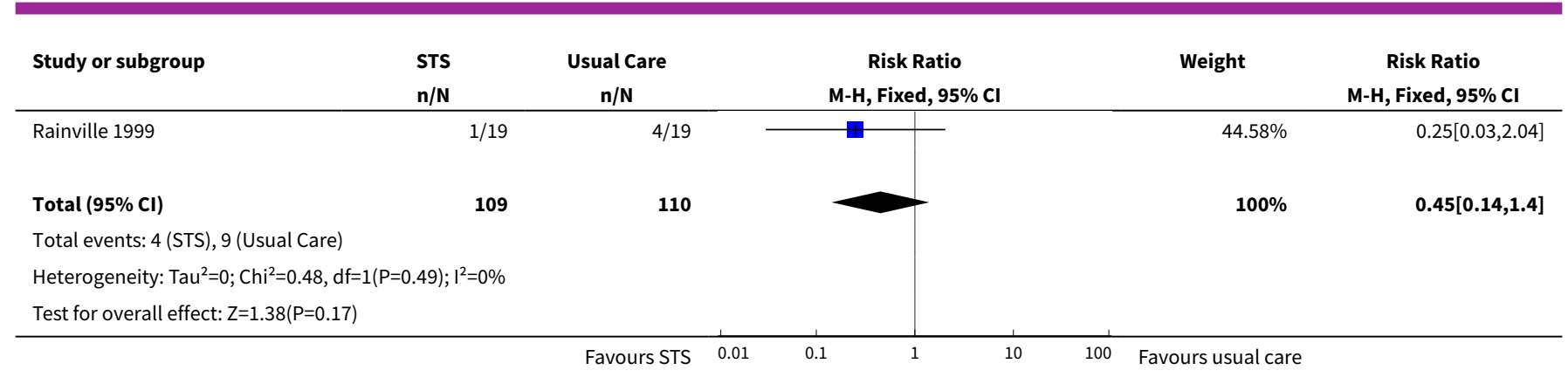
Analysis 1.11. Comparison 1 Impact of structured telephone support and telemonitoring in heart failure
on all-cause mortality, Outcome 11 Subgroup Publication year: all-cause mortality STS vs UC $2000-2007$.

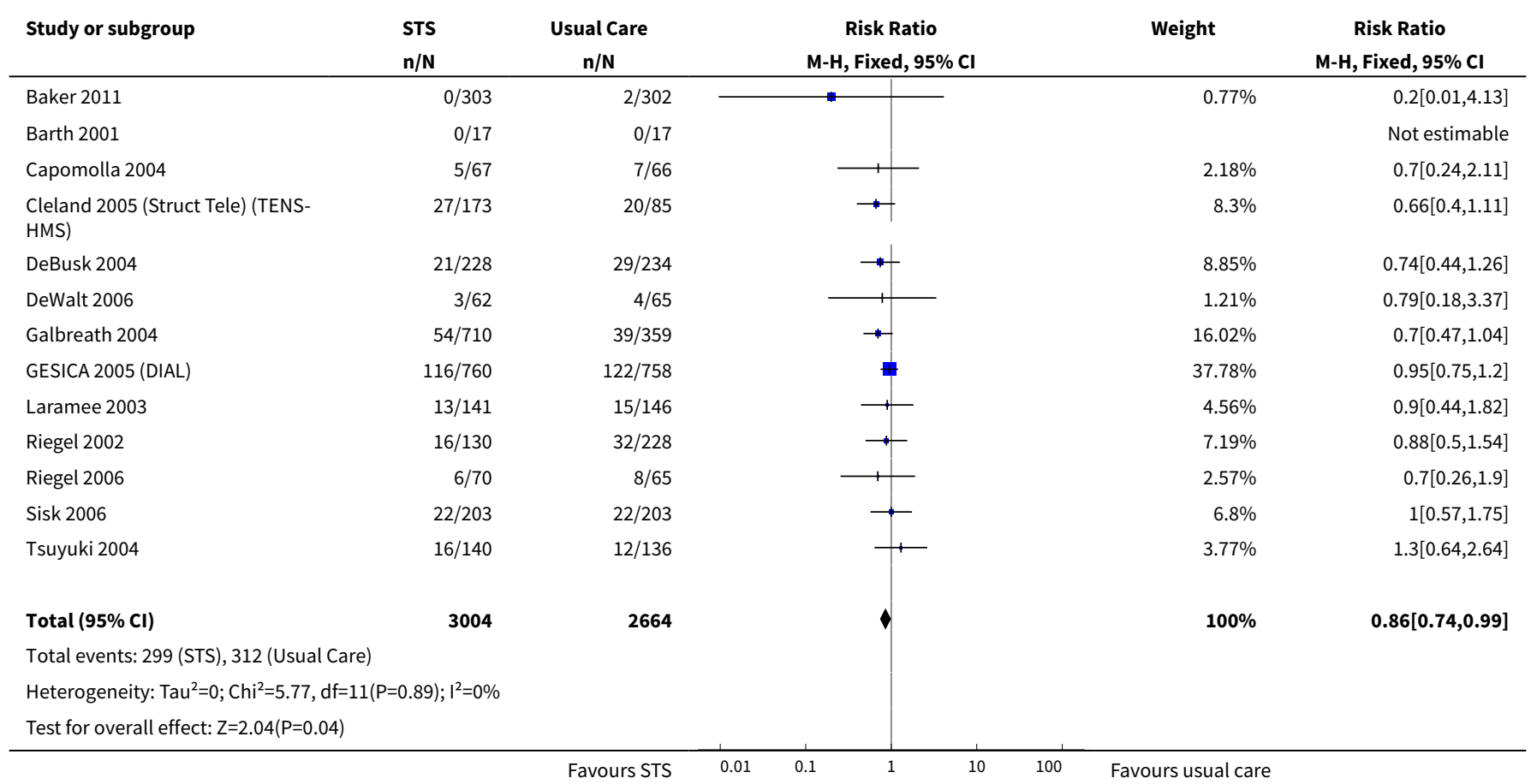

\section{Analysis 1.12. Comparison $1 \mathrm{Impact}$ of structured telephone support and telemonitoring in heart failure on all-cause mortality, Outcome 12 Subgroup Publication year: all-cause mortality STS vs UC $\geq 2008$.}

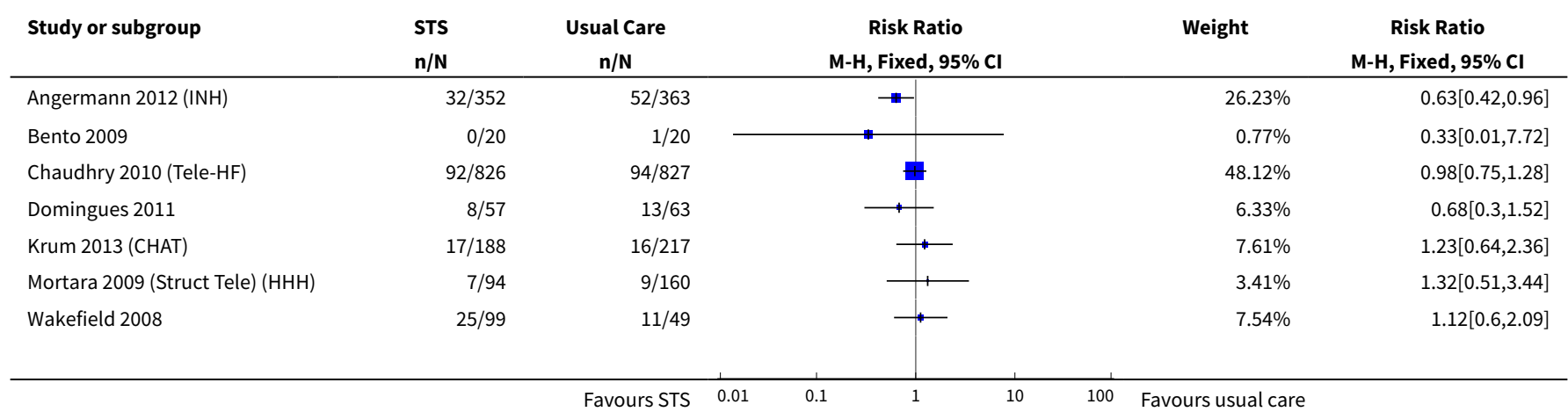




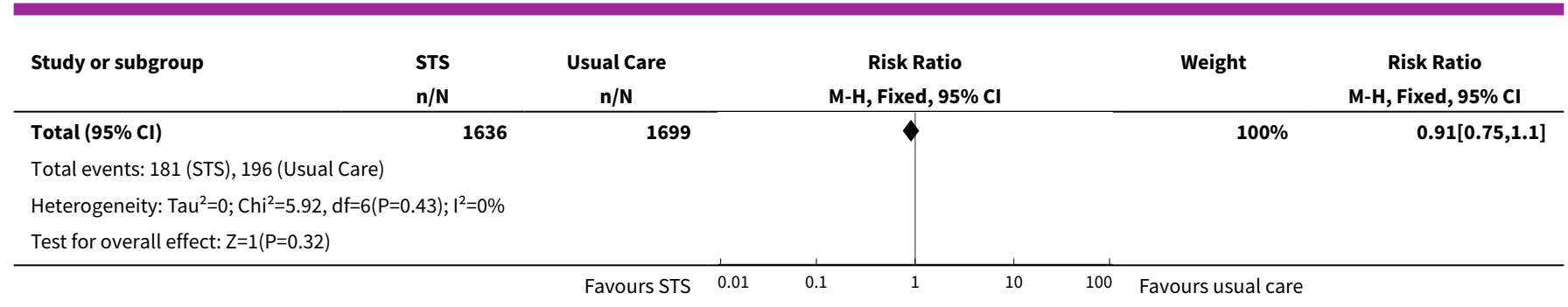

Analysis 1.13. Comparison 1 Impact of structured telephone support and telemonitoring in heart failure on all-cause mortality, Outcome 13 Subgroup Publication year: all-cause mortality TM vs UC 2000 - 2007.

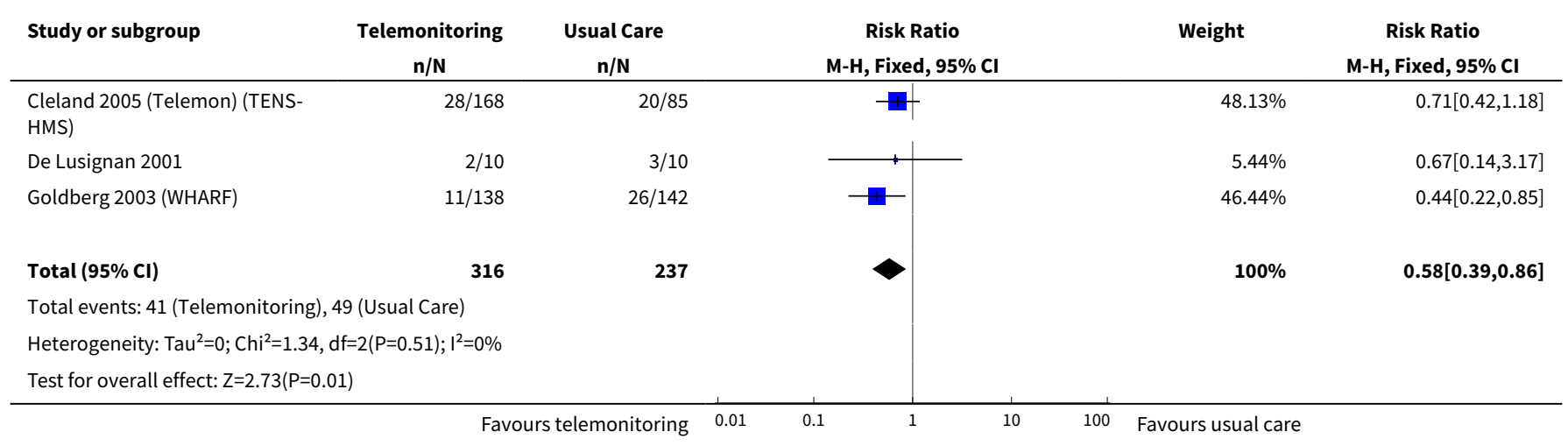

\section{Analysis 1.14. Comparison 1 Impact of structured telephone support and telemonitoring in heart failure on all-cause mortality, Outcome 14 Subgroup Publication year: all-cause mortality TM vs UC $\geq 2008$.}

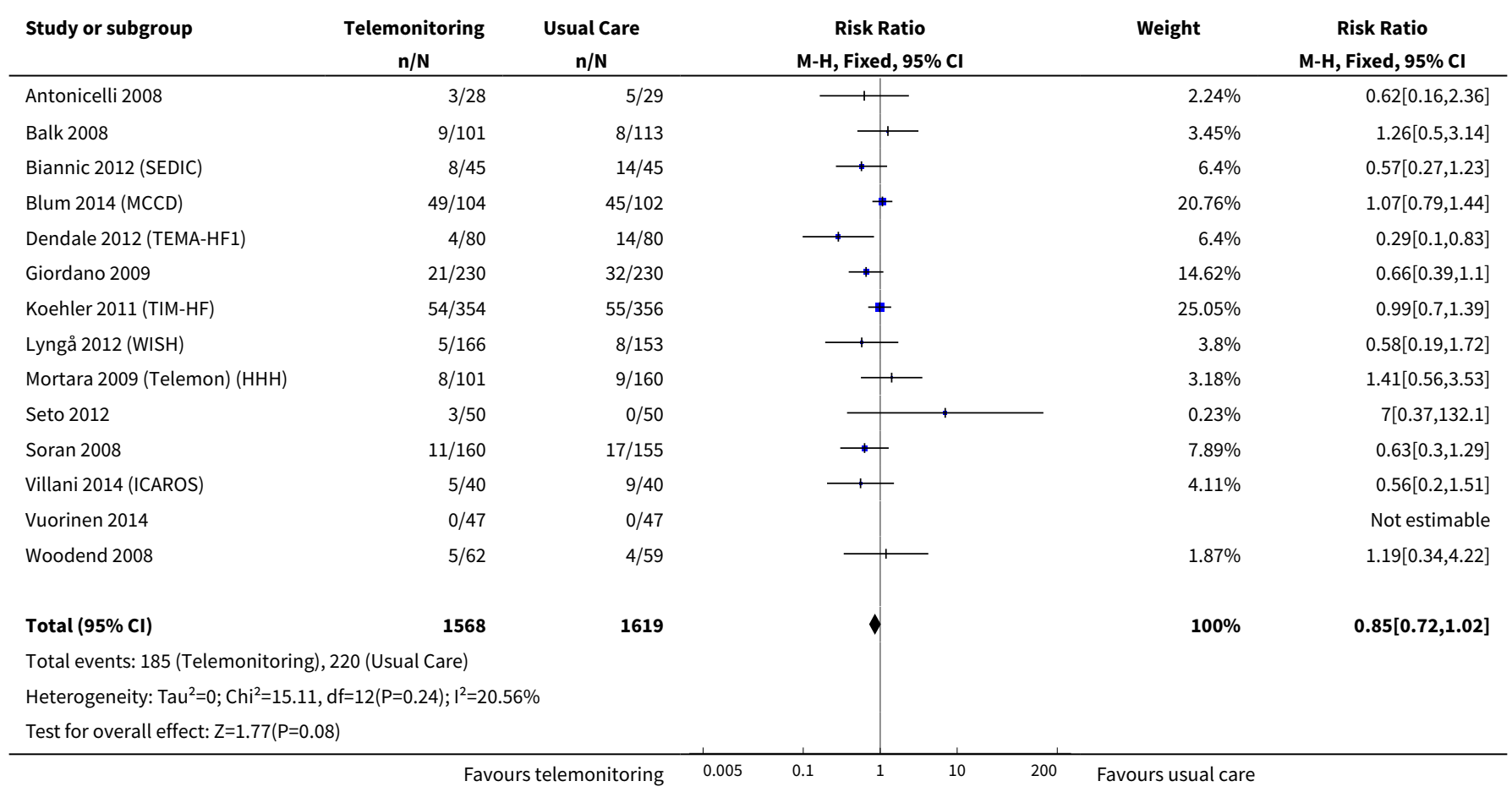


Analysis 1.15. Comparison 1 Impact of structured telephone support and telemonitoring in heart failure on all-cause mortality, Outcome 15 Subgroup Participant age: all-cause mortality: STS vs UC $<70$ years of age.

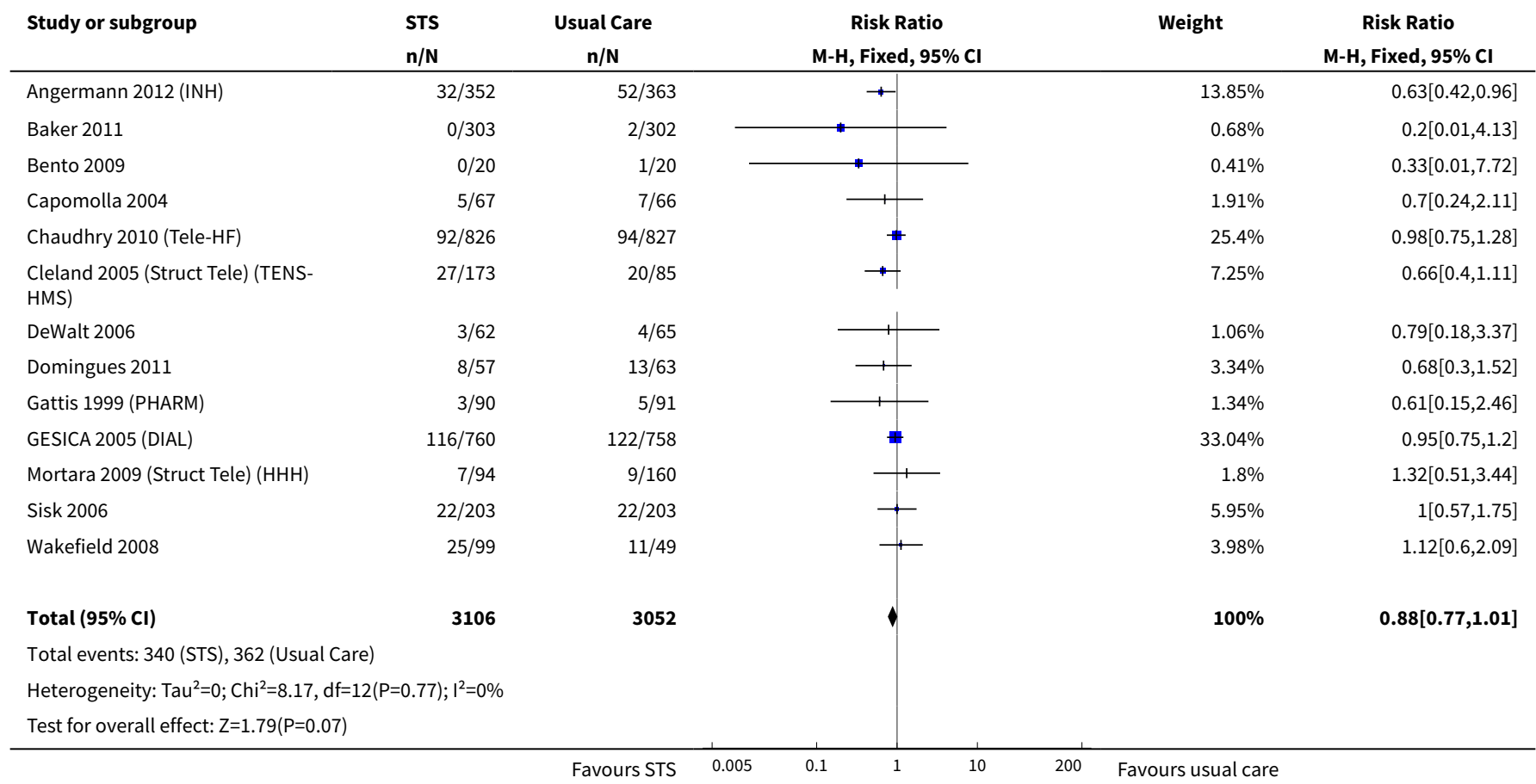

Analysis 1.16. Comparison 1 Impact of structured telephone support and telemonitoring in heart failure on all-cause mortality, Outcome 16 Subgroup Participant age: all-cause mortality STS vs UC $\geq 70$ years of age.

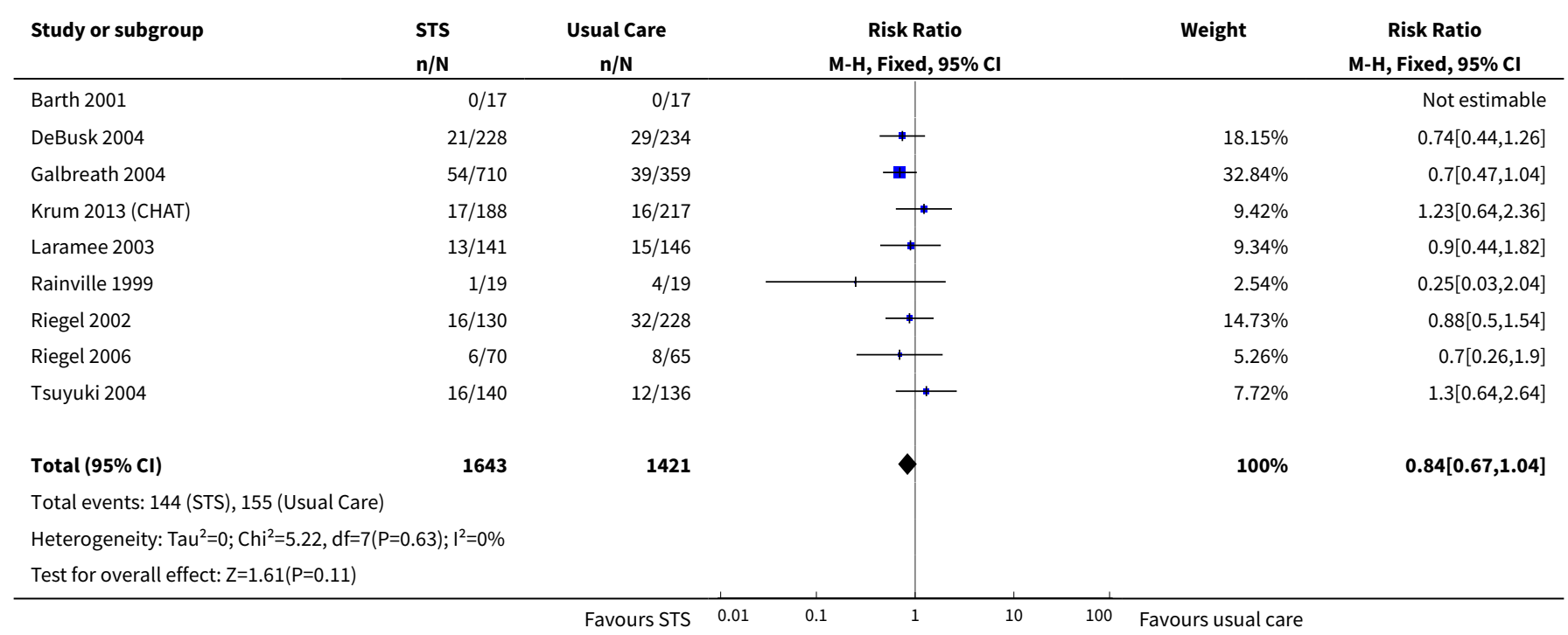


Analysis 1.17. Comparison 1 Impact of structured telephone support and telemonitoring in heart failure on all-cause mortality, Outcome 17 Subgroup Participant age: all-cause mortality: TM vs UC $<70$ years of age.

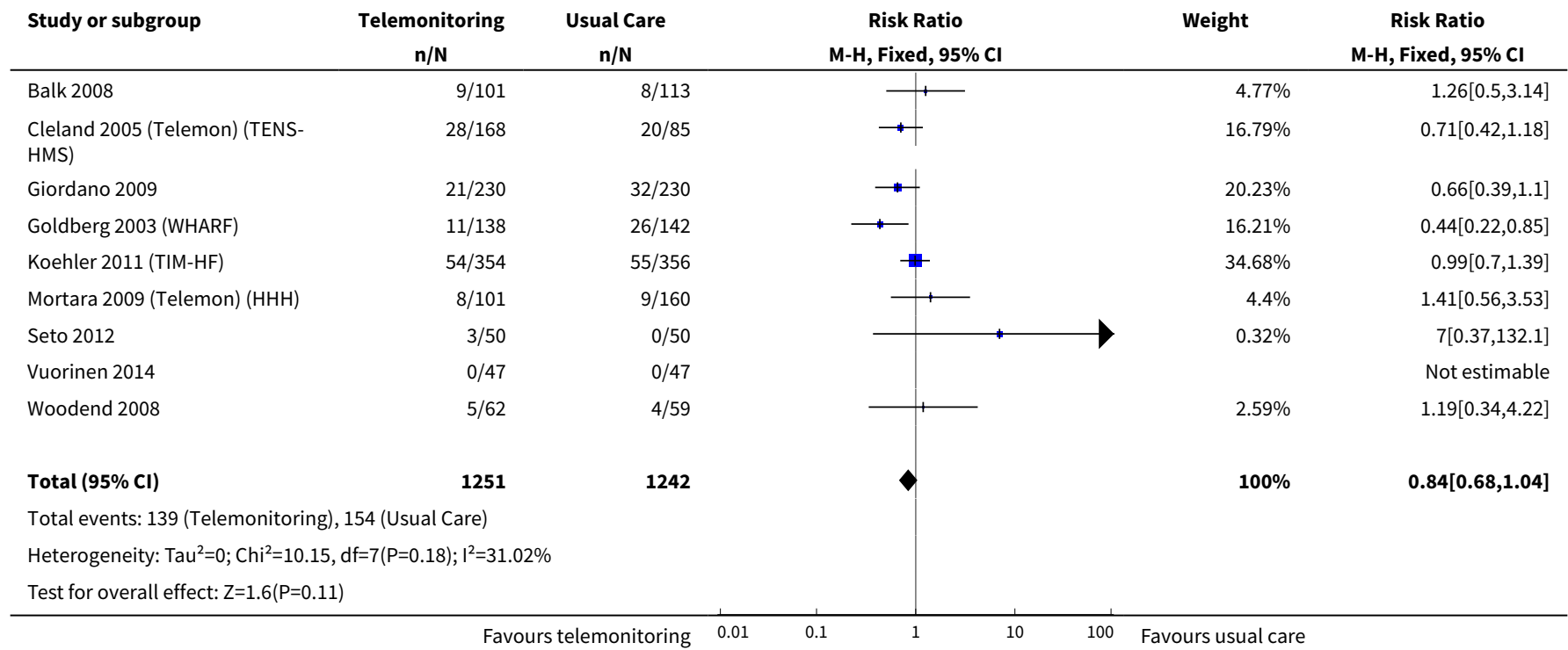

Analysis 1.18. Comparison 1 Impact of structured telephone support and telemonitoring in heart failure on all-cause mortality, Outcome 18 Subgroup Participant age: all-cause mortality TM vs UC $\geq 70$ years of age.

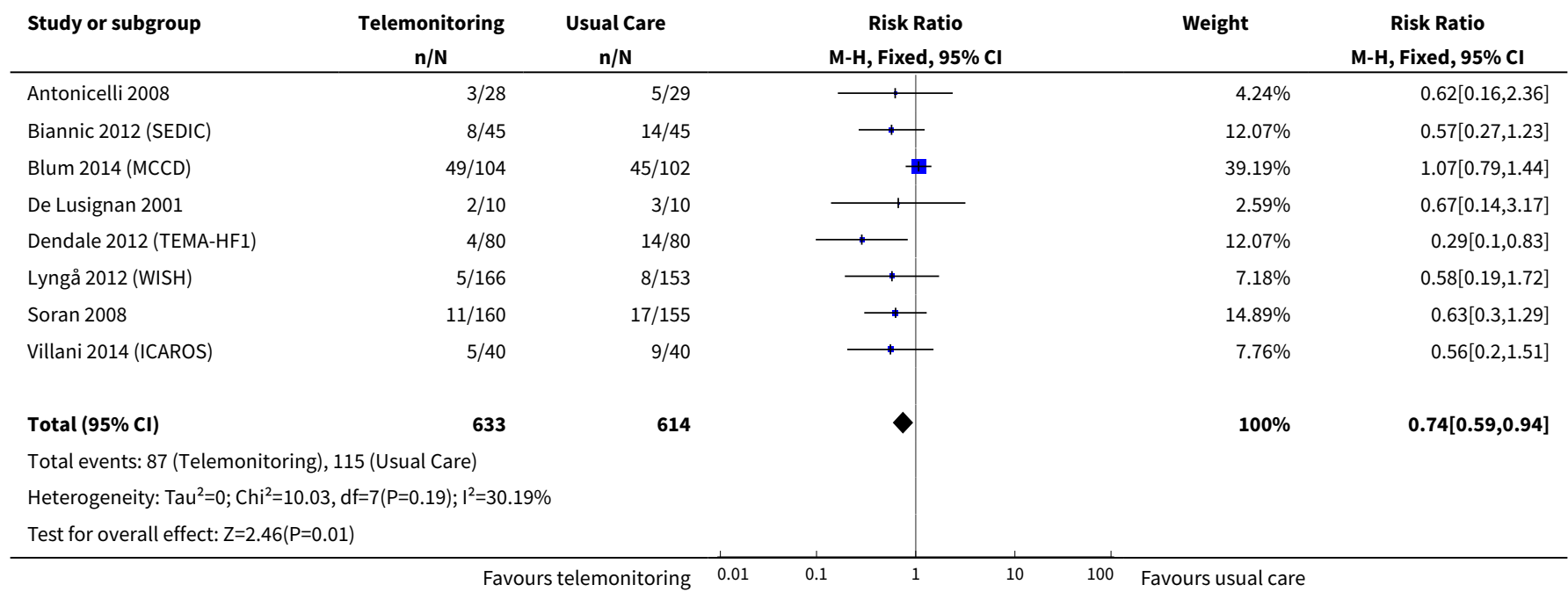

Analysis 1.19. Comparison 1 Impact of structured telephone support and telemonitoring in heart failure on all-cause mortality, Outcome 19 Subgroup STS focus: all-cause mortality STS (clinical support) vs UC.

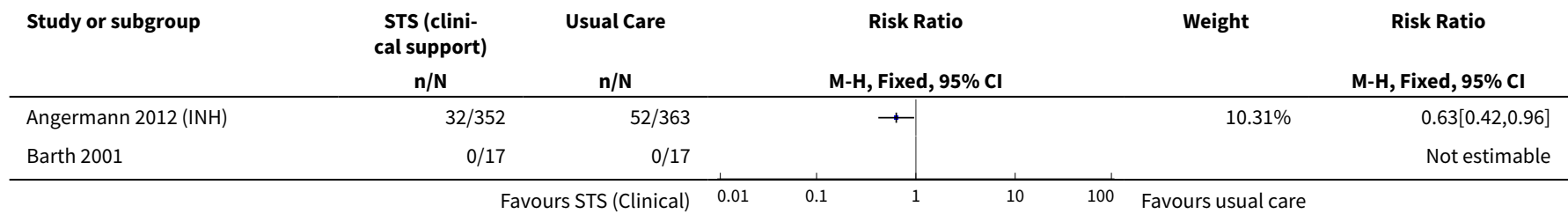




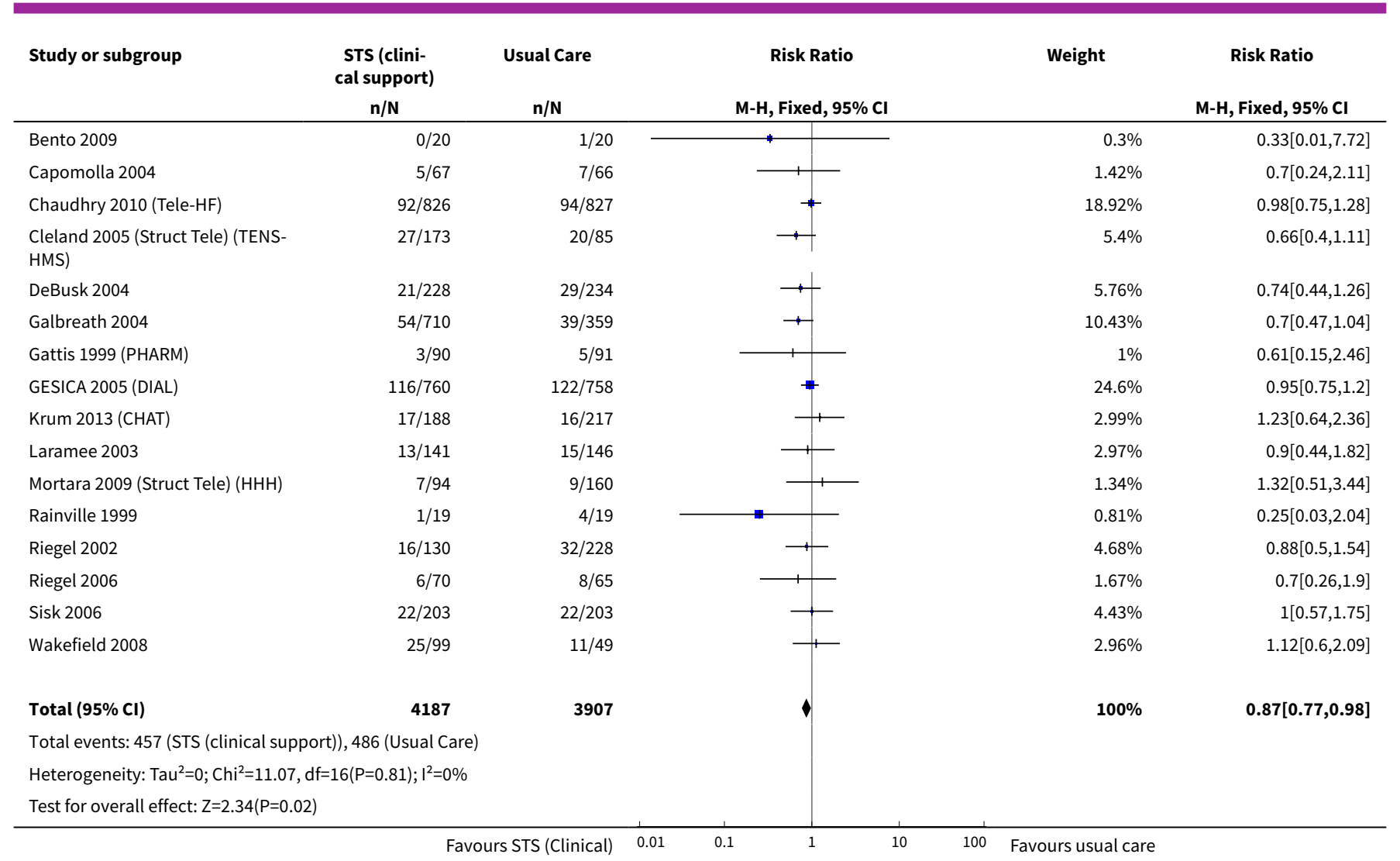
Analysis 1.20. Comparison 1 Impact of structured telephone support and telemonitoring in heart failure
on all-cause mortality, Outcome 20 Subgroup STS focus: all-cause mortality STS (education) vs UC.

\begin{tabular}{|c|c|c|c|c|c|}
\hline Study or subgroup & $\begin{array}{c}\text { STS (education) } \\
\mathrm{n} / \mathrm{N}\end{array}$ & $\begin{array}{c}\text { Usual Care } \\
n / N\end{array}$ & $\begin{array}{c}\text { Risk Ratio } \\
\text { M-H, Fixed, 95\% CI }\end{array}$ & Weight & $\begin{array}{c}\text { Risk Ratio } \\
\text { M-H, Fixed, } 95 \% \mathrm{Cl}\end{array}$ \\
\hline Baker 2011 & $0 / 303$ & $2 / 302$ & \begin{tabular}{l|l}
. & \\
\end{tabular} & $8.1 \%$ & $0.2[0.01,4.13]$ \\
\hline DeWalt 2006 & $3 / 62$ & $4 / 65$ & $\rightarrow$ & $12.63 \%$ & $0.79[0.18,3.37]$ \\
\hline Domingues 2011 & $8 / 57$ & $13 / 63$ & $\longrightarrow$ & $39.92 \%$ & $0.68[0.3,1.52]$ \\
\hline Tsuyuki 2004 & $16 / 140$ & $12 / 136$ & - & $39.36 \%$ & $1.3[0.64,2.64]$ \\
\hline Total $(95 \% \mathrm{Cl})$ & 562 & 566 & & $100 \%$ & $0.9[0.55,1.45]$ \\
\hline \multicolumn{6}{|c|}{ Total events: 27 (STS (education)), 31 (Usual Care) } \\
\hline \multicolumn{6}{|c|}{ Heterogeneity: $\operatorname{Tau}^{2}=0 ; \mathrm{Chi}^{2}=2.46, \mathrm{df}=3(\mathrm{P}=0.48) ; \mathrm{I}^{2}=0 \%$} \\
\hline Test for overall effect & & & & & \\
\hline
\end{tabular}

Analysis 1.21. Comparison 1 Impact of structured telephone support and telemonitoring in heart failure on allcause mortality, Outcome 21 Sensitivity analysis follow-up period (> 6 months), all-cause mortality: STS vs UC.

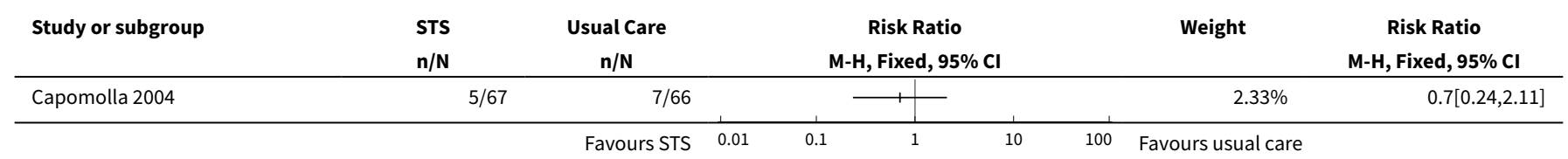




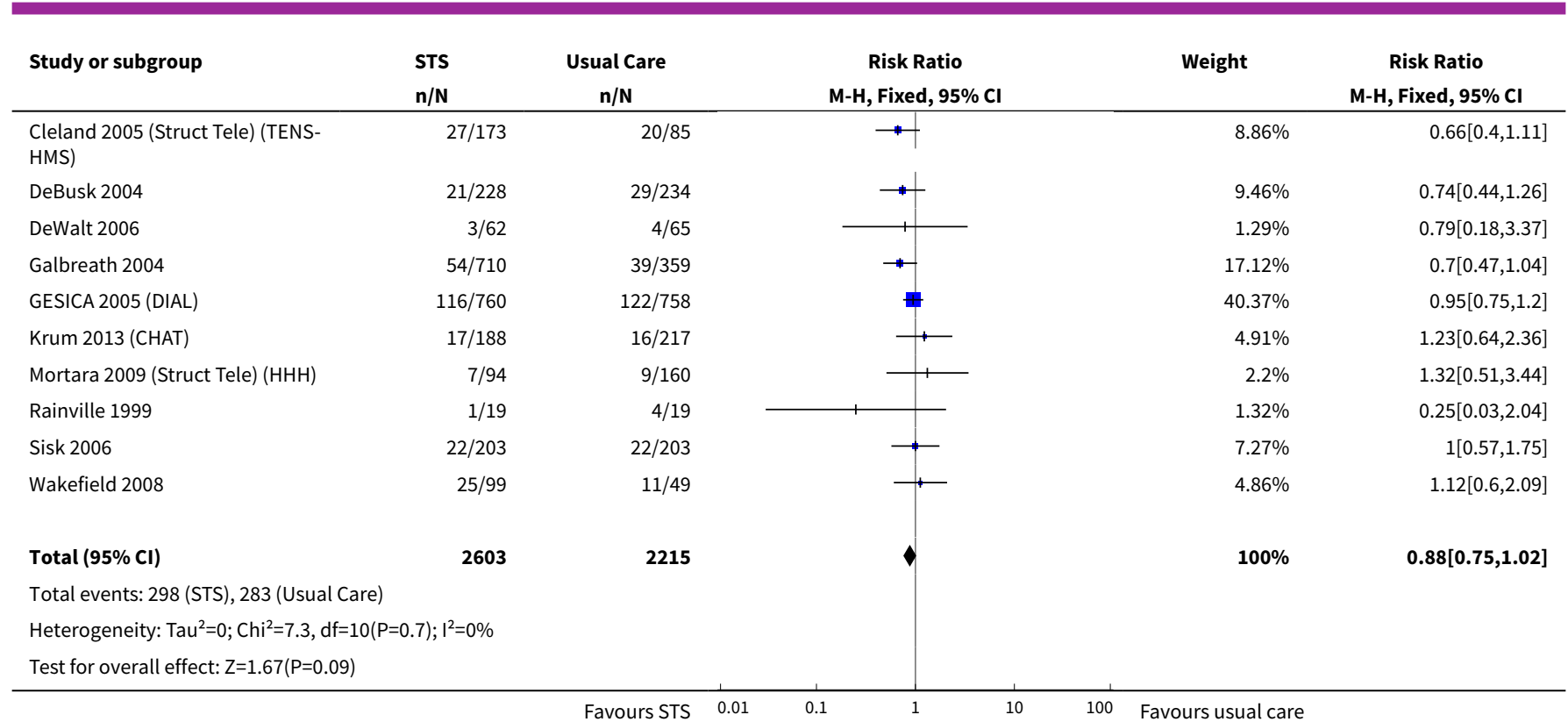

Analysis 1.22. Comparison 1 Impact of structured telephone support and telemonitoring in heart failure on allcause mortality, Outcome 22 Sensitivity analysis follow-up period (> 6 months), all-cause mortality: TM vs UC.

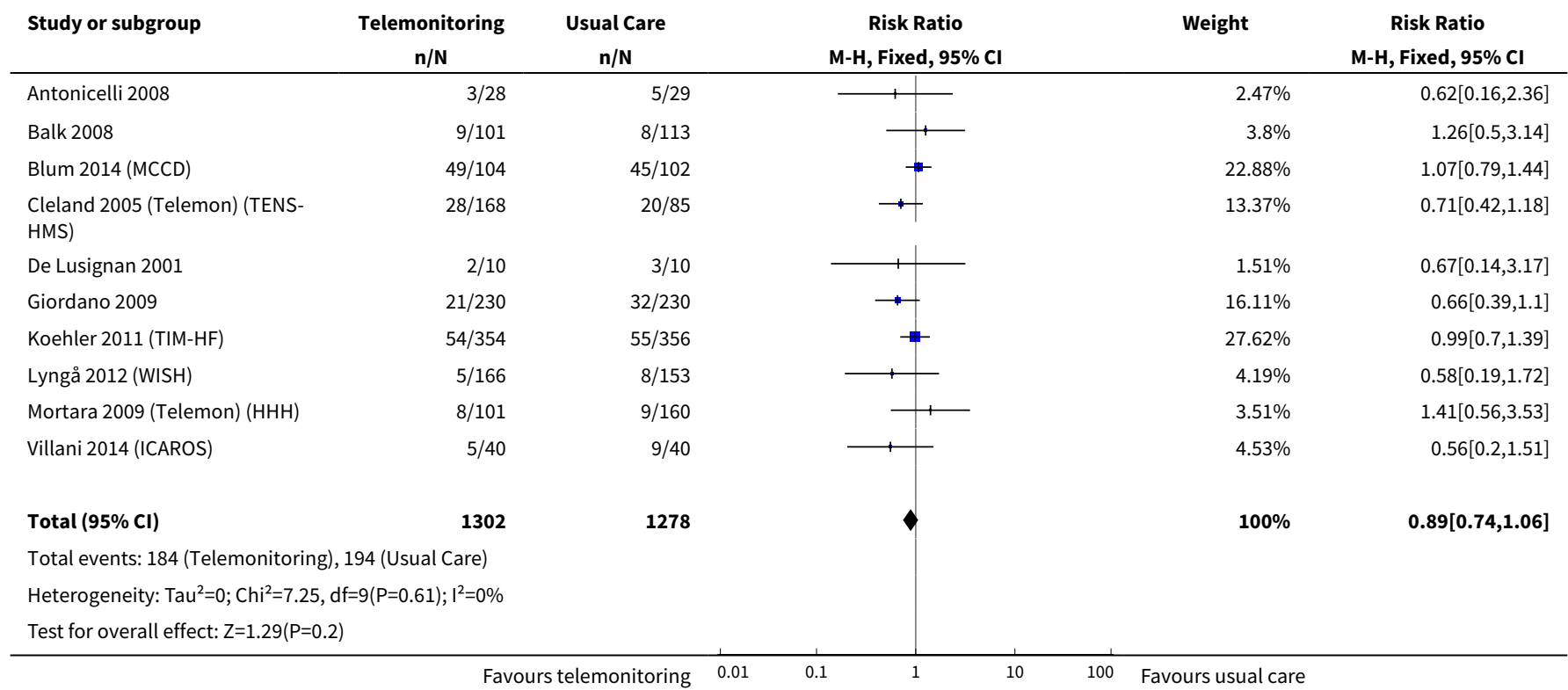

\section{Comparison 2. Impact of structured telephone or telemonitoring in heart failure on risk of all-cause hospitalisation}

\begin{tabular}{lllll}
\hline Outcome or subgroup title & No. of studies & $\begin{array}{l}\text { No. of partici- } \\
\text { pants }\end{array}$ & Statistical method & Effect size \\
\hline 1 All-cause hospitalisation: STS vs UC & 16 & 7216 & $\begin{array}{l}\text { Risk Ratio (M-H, Fixed, } \\
95 \% \text { Cl) }\end{array}$ & $0.95[0.90,1.00]$ \\
\hline
\end{tabular}




\begin{tabular}{|c|c|c|c|c|}
\hline Outcome or subgroup title & No. of studies & $\begin{array}{l}\text { No. of partici- } \\
\text { pants }\end{array}$ & Statistical method & Effect size \\
\hline $\begin{array}{l}3 \text { Subgroup technology: all-cause hospitali- } \\
\text { sation STS vs UC }\end{array}$ & 12 & 4756 & $\begin{array}{l}\text { Risk Ratio (M-H, Fixed, } \\
95 \% \mathrm{Cl})\end{array}$ & $0.93[0.86,0.99]$ \\
\hline $\begin{array}{l}4 \text { Subgroup technology: all-cause hospitali- } \\
\text { sation Mobile/PDA vs UC }\end{array}$ & 2 & 560 & $\begin{array}{l}\text { Risk Ratio (M-H, Fixed, } \\
95 \% \mathrm{Cl})\end{array}$ & $0.76[0.60,0.97]$ \\
\hline $\begin{array}{l}5 \text { Subgroup technology: all-cause hospitali- } \\
\text { sation Videophone vs UC }\end{array}$ & 2 & 269 & $\begin{array}{l}\text { Risk Ratio (M-H, Fixed, } \\
95 \% \mathrm{Cl})\end{array}$ & $0.91[0.80,1.04]$ \\
\hline $\begin{array}{l}6 \text { Subgroup technology: all-cause hospitali- } \\
\text { sation IVR vs UC }\end{array}$ & 3 & 2312 & $\begin{array}{l}\text { Risk Ratio (M-H, Fixed, } \\
95 \% \mathrm{Cl})\end{array}$ & $0.99[0.91,1.08]$ \\
\hline $\begin{array}{l}7 \text { Subgroup technology: all-cause hospitali- } \\
\text { sation Complex TM vs UC }\end{array}$ & 10 & 2651 & $\begin{array}{l}\text { Risk Ratio (M-H, Fixed, } \\
95 \% \mathrm{Cl})\end{array}$ & $0.97[0.90,1.04]$ \\
\hline $\begin{array}{l}8 \text { Subgroup TM intensity: all-cause hospitali- } \\
\text { sation Office hours vs UC }\end{array}$ & 6 & 1140 & $\begin{array}{l}\text { Risk Ratio (M-H, Fixed, } \\
95 \% \mathrm{Cl})\end{array}$ & $0.85[0.76,0.94]$ \\
\hline $\begin{array}{l}9 \text { Subgroup TM intensity: all-cause hospitali- } \\
\text { sation } 24 / 7 \text { or } 7 \text { days vs UC }\end{array}$ & 7 & 2192 & $\begin{array}{l}\text { Risk Ratio (M-H, Fixed, } \\
95 \% \mathrm{Cl})\end{array}$ & $1.01[0.93,1.09]$ \\
\hline $\begin{array}{l}10 \text { Subgroup Publication year: all-cause hos- } \\
\text { pitalisation STS vs UC }<2000\end{array}$ & 1 & & $\begin{array}{l}\text { Risk Ratio (M-H, Fixed, } \\
95 \% \mathrm{Cl})\end{array}$ & $\begin{array}{l}\text { Totals not select- } \\
\text { ed }\end{array}$ \\
\hline $\begin{array}{l}12 \text { Subgroup Publication year: all-cause hos- } \\
\text { pitalisation STS vs UC } \geq 2008\end{array}$ & 7 & 3335 & $\begin{array}{l}\text { Risk Ratio (M-H, Fixed, } \\
95 \% \mathrm{Cl})\end{array}$ & $0.98[0.91,1.06]$ \\
\hline $\begin{array}{l}13 \text { Subgroup Publication year: all-cause hos- } \\
\text { pitalisation TM vs UC } 2000 \text { - } 2007\end{array}$ & 2 & 533 & $\begin{array}{l}\text { Risk Ratio (M-H, Fixed, } \\
95 \% \mathrm{Cl})\end{array}$ & $0.94[0.79,1.12]$ \\
\hline $\begin{array}{l}14 \text { Subgroup Publication year: all-cause hos- } \\
\text { pitalisation TM vs UC } \geq 2008\end{array}$ & 11 & 2799 & $\begin{array}{l}\text { Risk Ratio (M-H, Fixed, } \\
95 \% \mathrm{Cl})\end{array}$ & $0.95[0.89,1.02]$ \\
\hline $\begin{array}{l}15 \text { Subgroup Participant age: all-cause hos- } \\
\text { pitalisation STS vs UC } \geq 70 \text { years of age }\end{array}$ & 6 & 1923 & $\begin{array}{l}\text { Risk Ratio (M-H, Fixed, } \\
95 \% \mathrm{Cl})\end{array}$ & $0.94[0.85,1.04]$ \\
\hline $\begin{array}{l}16 \text { Subgroup Participant age: all-cause hos- } \\
\text { pitalisation: STS vs UC < } 70 \text { years of age }\end{array}$ & 10 & 5293 & $\begin{array}{l}\text { Risk Ratio (M-H, Fixed, } \\
95 \% \mathrm{Cl})\end{array}$ & $0.95[0.89,1.01]$ \\
\hline $\begin{array}{l}17 \text { Subgroup Participant age: all-cause hos- } \\
\text { pitalisation TM vs UC } \geq 70 \text { years of age }\end{array}$ & 6 & 1147 & $\begin{array}{l}\text { Risk Ratio (M-H, Fixed, } \\
95 \% \mathrm{Cl})\end{array}$ & $0.90[0.82,0.99]$ \\
\hline $\begin{array}{l}18 \text { Subgroup Participant age: all-cause hos- } \\
\text { pitalisation: TM vs UC < } 70 \text { years of age }\end{array}$ & 7 & 2185 & $\begin{array}{l}\text { Risk Ratio (M-H, Fixed, } \\
95 \% \mathrm{Cl})\end{array}$ & $0.98[0.90,1.07]$ \\
\hline $\begin{array}{l}19 \text { Subgroup STS focus: all-cause hospitali- } \\
\text { sation: STS (clinical support) vs UC }\end{array}$ & 14 & 6820 & $\begin{array}{l}\text { Risk Ratio (M-H, Fixed, } \\
95 \% \mathrm{Cl})\end{array}$ & $0.94[0.89,1.00]$ \\
\hline
\end{tabular}




\begin{tabular}{lllll}
\hline Outcome or subgroup title & No. of studies & $\begin{array}{l}\text { No. of partici- } \\
\text { pants }\end{array}$ & Statistical method & Effect size \\
\hline $\begin{array}{l}\text { 20 Subgroup STS focus: all-cause hospitali- } \\
\text { sation: STS (education) vs UC }\end{array}$ & 2 & 396 & $\begin{array}{l}\text { Risk Ratio (M-H, Fixed, } \\
95 \% \mathrm{Cl})\end{array}$ & $1.08[0.84,1.38]$ \\
\hline $\begin{array}{l}21 \text { Sensitivity analysis follow-up period (> } \\
\begin{array}{l}\text { months), all-cause hospitalisation: STS vs } \\
\text { UC }\end{array}\end{array}$ & 7 & 3451 & Risk Ratio (M-H, Fixed, & $0.89[0.82,0.96]$ \\
\hline $\begin{array}{l}22 \text { Sensitivity analysis follow-up period (> 6 } \\
\text { months), all-cause hospitalisation: TM vs UC }\end{array}$ & 8 & & $95 \%$ Cl) & \\
\hline
\end{tabular}

Analysis 2.1. Comparison 2 Impact of structured telephone or telemonitoring in heart failure on risk of all-cause hospitalisation, Outcome 1 All-cause hospitalisation: STS vs UC.

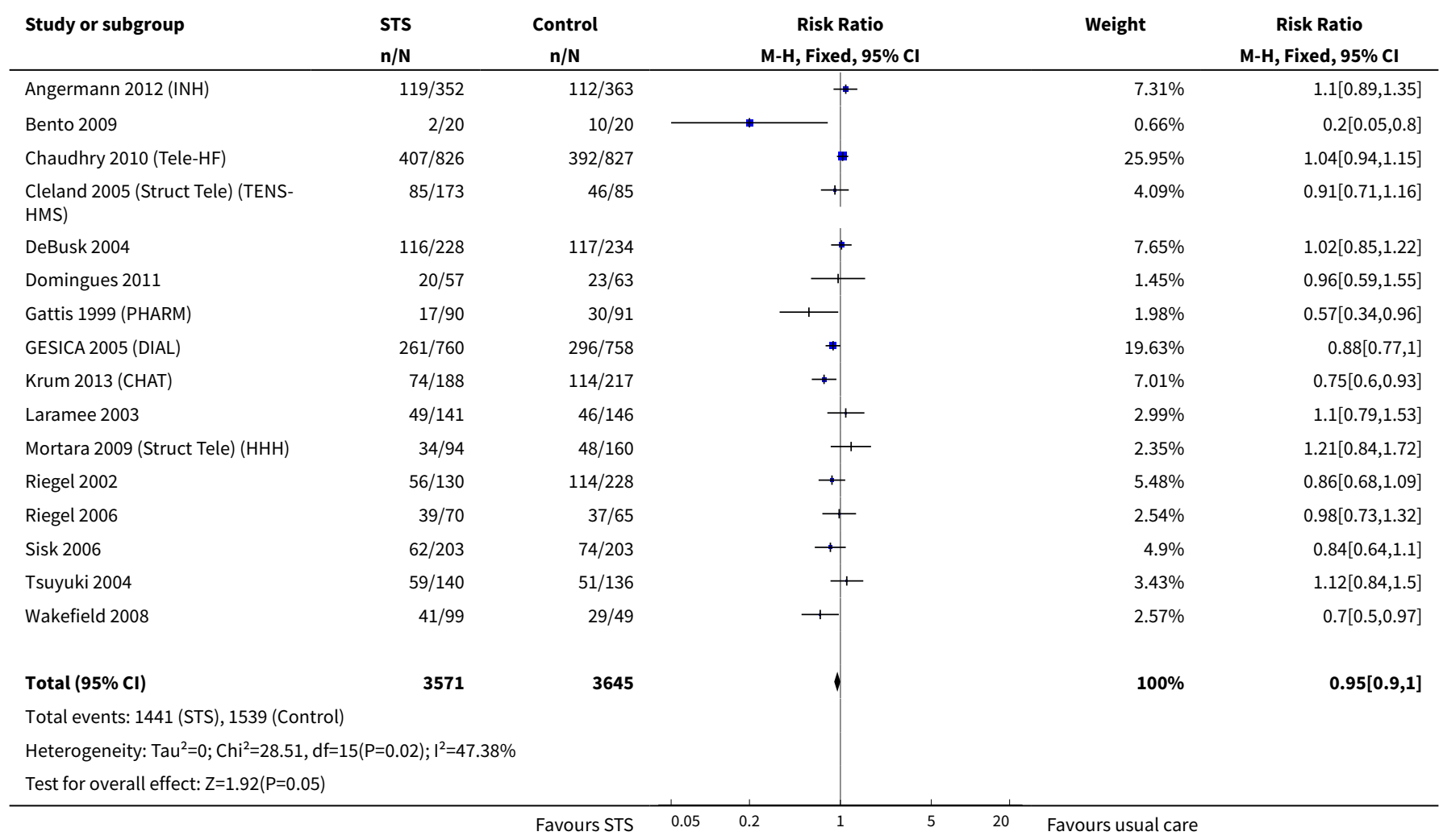

Analysis 2.2. Comparison 2 Impact of structured telephone or telemonitoring in heart failure on risk of all-cause hospitalisation, Outcome 2 All-cause hospitalisation: TM vs UC.

\begin{tabular}{|c|c|c|c|c|c|}
\hline Study or subgroup & $\begin{array}{c}\text { Telemonitoring } \\
\mathrm{n} / \mathrm{N}\end{array}$ & $\begin{array}{c}\text { Control } \\
n / N\end{array}$ & $\begin{array}{r}\text { Risk } \\
\text { M-H, Fix } \\
\end{array}$ & Weight & $\begin{array}{c}\text { Risk Ratio } \\
\text { M-H, Fixed, 95\% CI }\end{array}$ \\
\hline Antonicelli 2008 & $9 / 28$ & $26 / 29$ & 千 & $2.97 \%$ & $0.36[0.21,0.62]$ \\
\hline Biannic 2012 (SEDIC) & $19 / 45$ & $35 / 45$ & + & $4.07 \%$ & $0.54[0.37,0.79]$ \\
\hline Blum 2014 (MCCD) & $80 / 104$ & $74 / 102$ & & $8.69 \%$ & $1.06[0.9,1.24]$ \\
\hline
\end{tabular}




\begin{tabular}{|c|c|c|c|c|c|}
\hline Study or subgroup & $\begin{array}{c}\text { Telemonitoring } \\
\mathbf{n} / \mathbf{N}\end{array}$ & $\begin{array}{c}\text { Control } \\
n / N\end{array}$ & $\begin{array}{c}\text { Risk Ratio } \\
\text { M-H, Fixed, 95\% Cl }\end{array}$ & Weight & $\begin{array}{c}\text { Risk Ratio } \\
\text { M-H, Fixed, 95\% Cl }\end{array}$ \\
\hline $\begin{array}{l}\text { Cleland } 2005 \text { (Telemon) (TENS- } \\
\text { HMS) }\end{array}$ & $80 / 168$ & $46 / 85$ & $\rightarrow+$ & $7.1 \%$ & $0.88[0.68,1.13]$ \\
\hline Dendale 2012 (TEMA-HF1) & $64 / 80$ & $66 / 80$ & + & $7.68 \%$ & $0.97[0.84,1.13]$ \\
\hline Giordano 2009 & $67 / 230$ & $96 / 230$ & + & $11.16 \%$ & $0.7[0.54,0.9]$ \\
\hline Goldberg 2003 (WHARF) & $65 / 138$ & $67 / 142$ & + & $7.68 \%$ & $1[0.78,1.28]$ \\
\hline Koehler 2011 (TIM-HF) & $192 / 354$ & $179 / 356$ & * & $20.76 \%$ & $1.08[0.94,1.24]$ \\
\hline Lyngå 2012 (WISH) & $79 / 166$ & $84 / 153$ & + & $10.17 \%$ & $0.87[0.7,1.07]$ \\
\hline Mortara 2009 (Telemon) (HHH) & $35 / 101$ & $48 / 160$ & + & $4.32 \%$ & $1.16[0.81,1.65]$ \\
\hline Seto 2012 & $14 / 50$ & $10 / 50$ & 1 & $1.16 \%$ & $1.4[0.69,2.85]$ \\
\hline Soran 2008 & $75 / 160$ & $66 / 155$ & + & $7.8 \%$ & $1.1[0.86,1.41]$ \\
\hline Woodend 2008 & $60 / 62$ & $54 / 59$ & * & $6.44 \%$ & $1.06[0.97,1.16]$ \\
\hline Total $(95 \% \mathrm{Cl})$ & 1686 & 1646 & 1 & $100 \%$ & $0.95[0.89,1.01]$ \\
\hline \multicolumn{6}{|c|}{ Total events: 839 (Telemonitoring), 851 (Control) } \\
\hline \multicolumn{6}{|c|}{ Heterogeneity: $\operatorname{Tau}^{2}=0 ; \mathrm{Chi}^{2}=41.72, \mathrm{df}=12(\mathrm{P}<0.0001) ; \mathrm{I}^{2}=71.24 \%$} \\
\hline Test for overall effect: $\mathrm{Z}=1.61(\mathrm{P}=\mathrm{C}$ & & & & & \\
\hline
\end{tabular}

\section{Analysis 2.3. Comparison 2 Impact of structured telephone or telemonitoring in heart failure on risk of all-cause hospitalisation, Outcome 3 Subgroup technology: all-cause hospitalisation STS vs UC.}

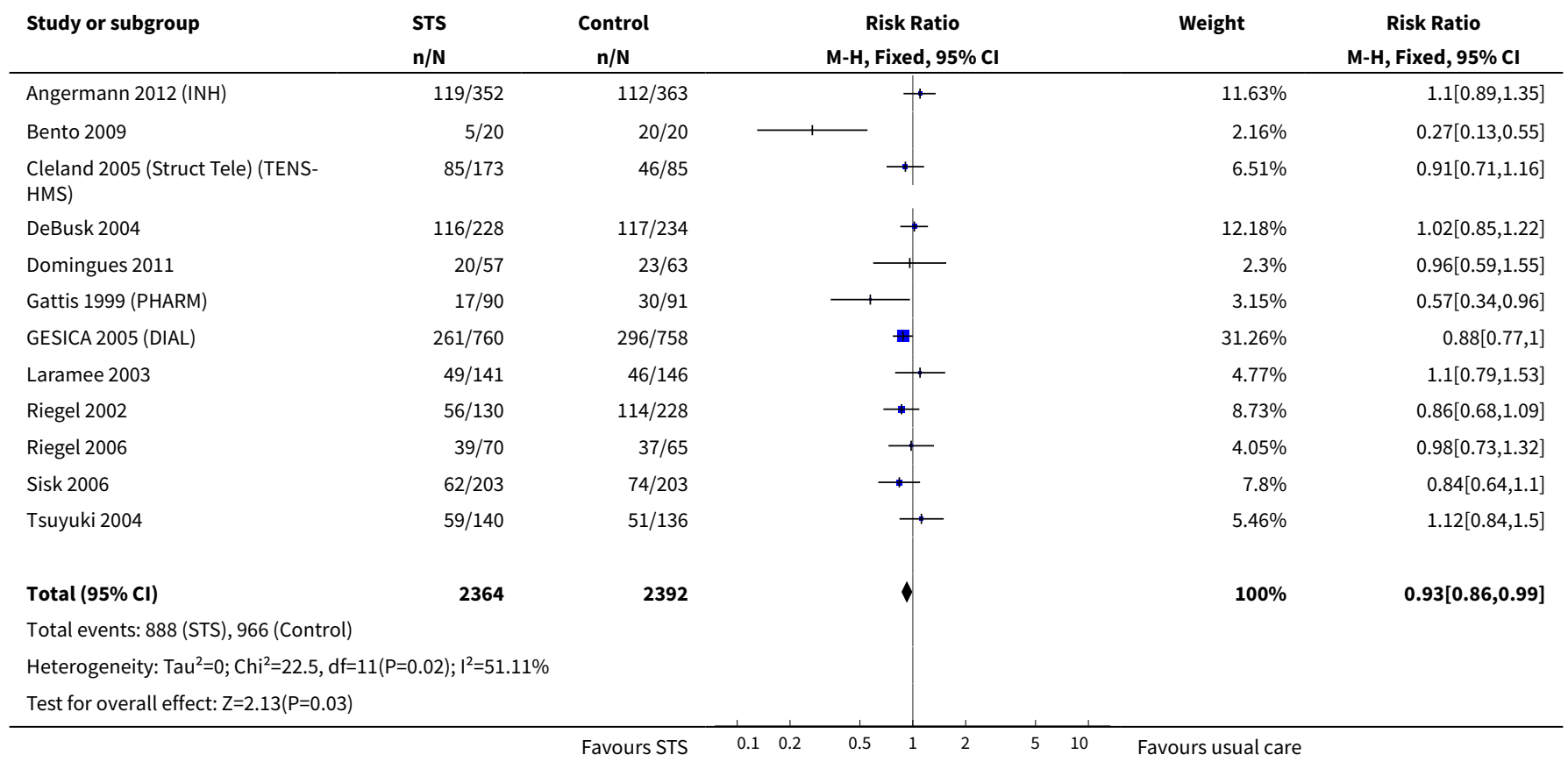


Analysis 2.4. Comparison 2 Impact of structured telephone or telemonitoring in heart failure on risk of all-cause hospitalisation, Outcome 4 Subgroup technology: all-cause hospitalisation Mobile/PDA vs UC.

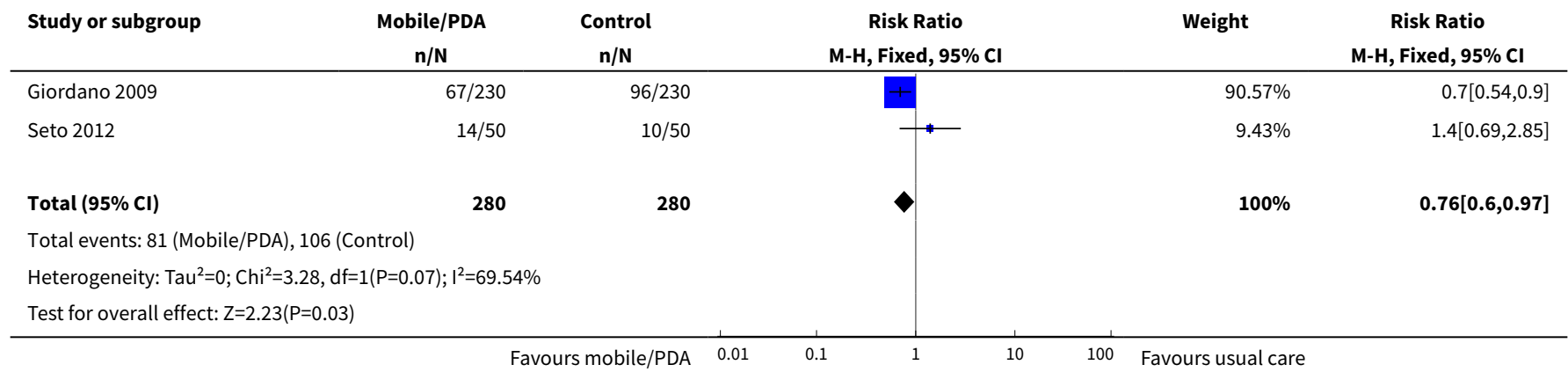

Analysis 2.5. Comparison 2 Impact of structured telephone or telemonitoring in heart failure on risk of all-cause hospitalisation, Outcome 5 Subgroup technology: all-cause hospitalisation Videophone vs UC.

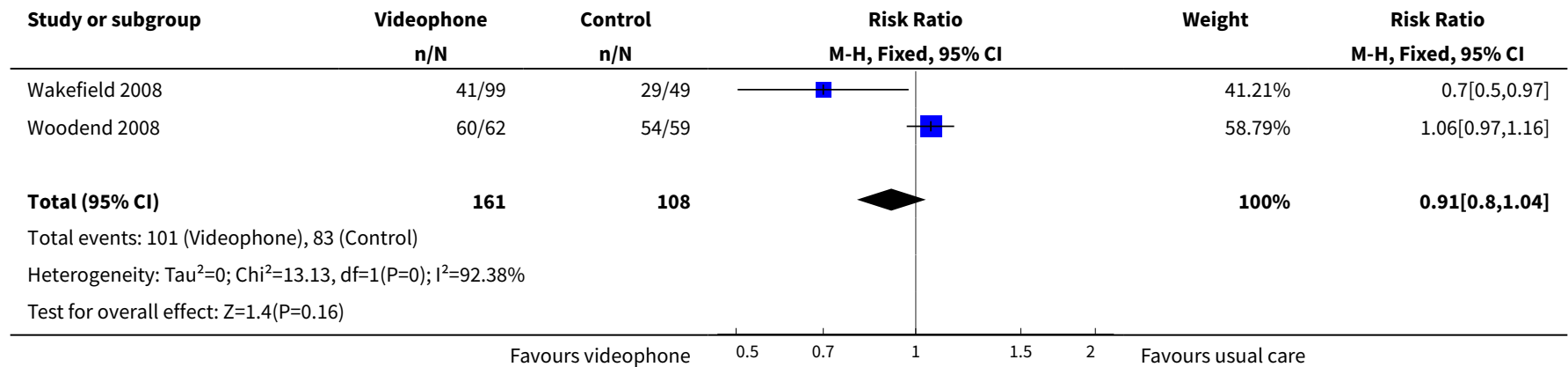

Analysis 2.6. Comparison 2 Impact of structured telephone or telemonitoring in heart failure on risk of all-cause hospitalisation, Outcome 6 Subgroup technology: all-cause hospitalisation IVR vs UC.

\begin{tabular}{|c|c|c|c|c|c|}
\hline Study or subgroup & $\begin{array}{l}\text { IVR } \\
\mathrm{n} / \mathrm{N}\end{array}$ & $\begin{array}{c}\text { Control } \\
\mathrm{n} / \mathrm{N}\end{array}$ & $\begin{array}{c}\text { Risk Ratio } \\
\text { M-H, Fixed, 95\% Cl }\end{array}$ & Weight & $\begin{array}{c}\text { Risk Ratio } \\
\text { M-H, Fixed, 95\% Cl }\end{array}$ \\
\hline Chaudhry 2010 (Tele-HF) & $407 / 826$ & $392 / 827$ & - & $73.48 \%$ & $1.04[0.94,1.15]$ \\
\hline Krum 2013 (CHAT) & $74 / 188$ & $114 / 217$ & & $19.85 \%$ & $0.75[0.6,0.93]$ \\
\hline Mortara 2009 (Struct Tele) (HHH) & $34 / 94$ & $48 / 160$ & + & $6.66 \%$ & $1.21[0.84,1.72]$ \\
\hline Total $(95 \% \mathrm{Cl})$ & 1108 & 1204 & & $100 \%$ & $0.99[0.91,1.08]$ \\
\hline \multicolumn{6}{|c|}{ Heterogeneity: $\mathrm{Tau}^{2}=0 ; \mathrm{Chi}^{2}=8.36, \mathrm{df}=2(\mathrm{P}=0.02) ; \mathrm{I}^{2}=76.07 \%$} \\
\hline \multicolumn{6}{|c|}{ Test for overall effect: $Z=0.16(P=0.87)$} \\
\hline
\end{tabular}


Analysis 2.7. Comparison 2 Impact of structured telephone or telemonitoring in heart failure on risk of all-cause hospitalisation, Outcome 7 Subgroup technology: all-cause hospitalisation Complex TM vs UC.

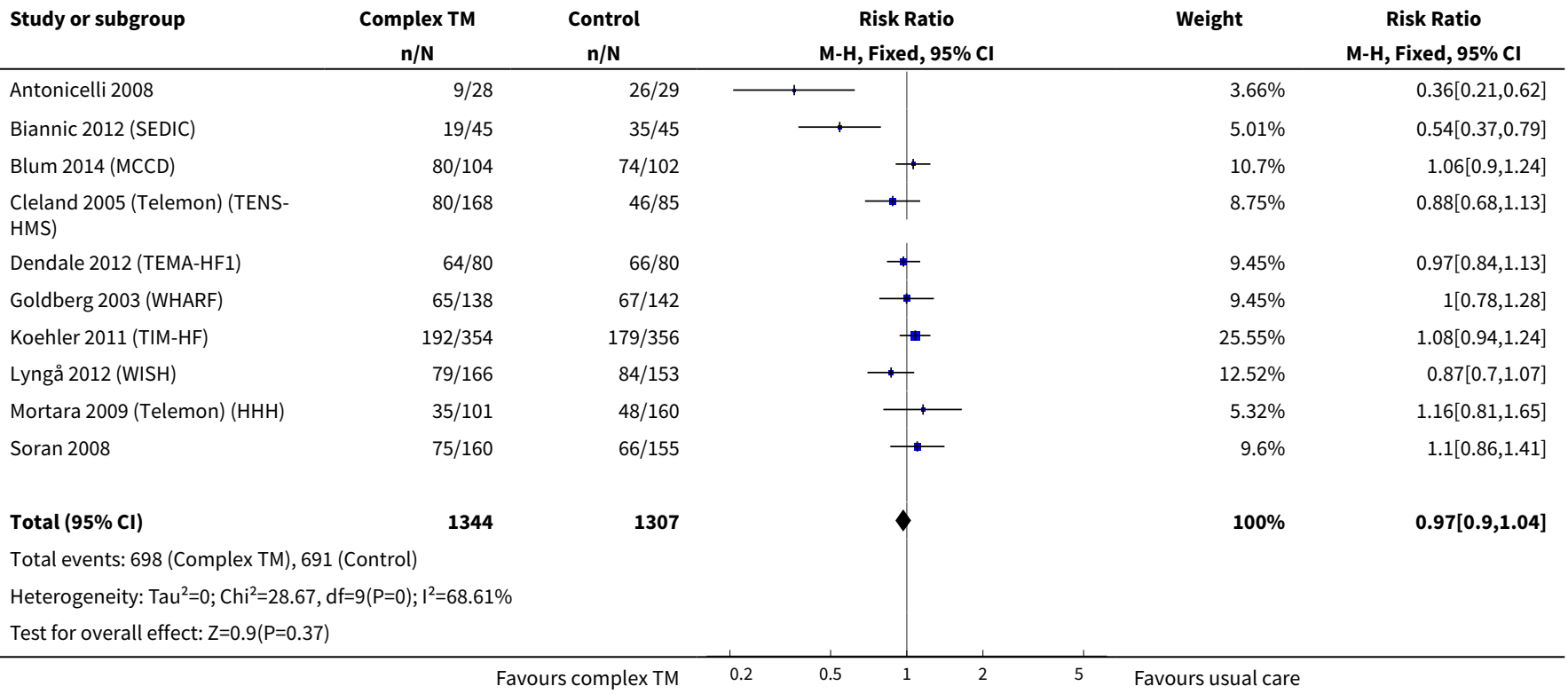

Analysis 2.8. Comparison $2 \mathrm{Impact}$ of structured telephone or telemonitoring in heart failure on risk of all-cause hospitalisation, Outcome 8 Subgroup TM intensity: all-cause hospitalisation Office hours vs UC.

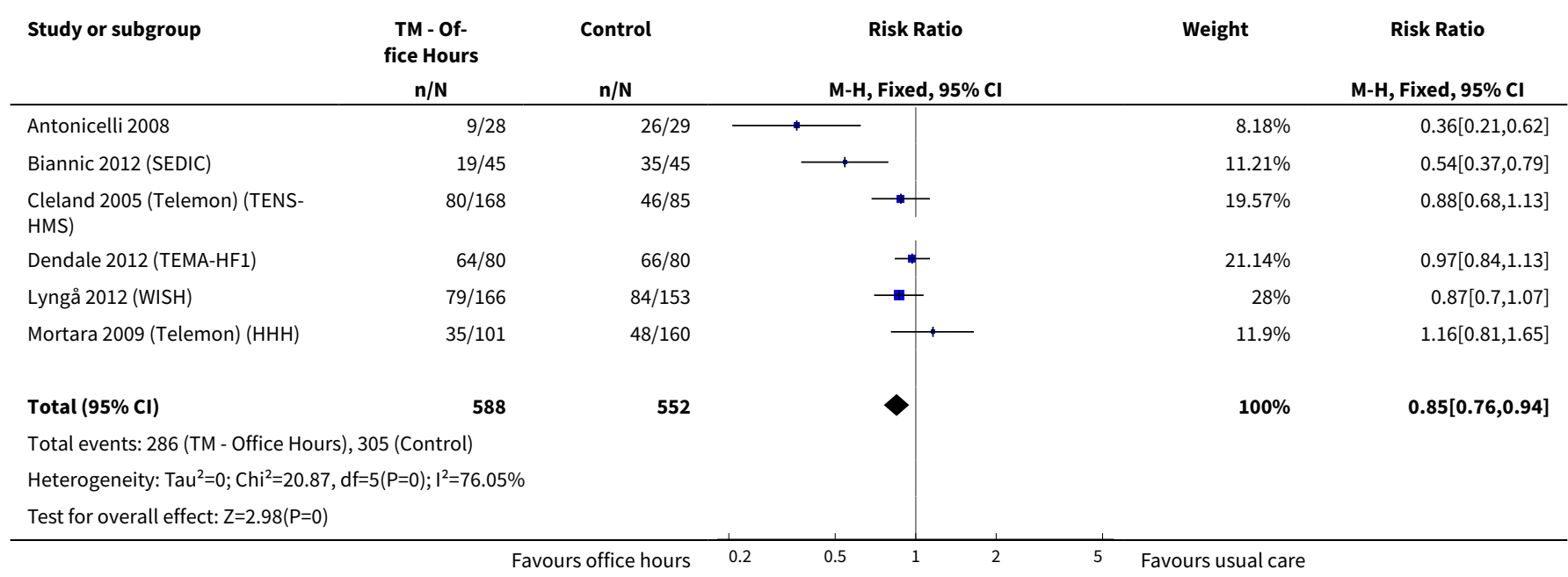

Analysis 2.9. Comparison 2 Impact of structured telephone or telemonitoring in heart failure on risk of allcause hospitalisation, Outcome 9 Subgroup TM intensity: all-cause hospitalisation 24/7 or 7 days vs UC.

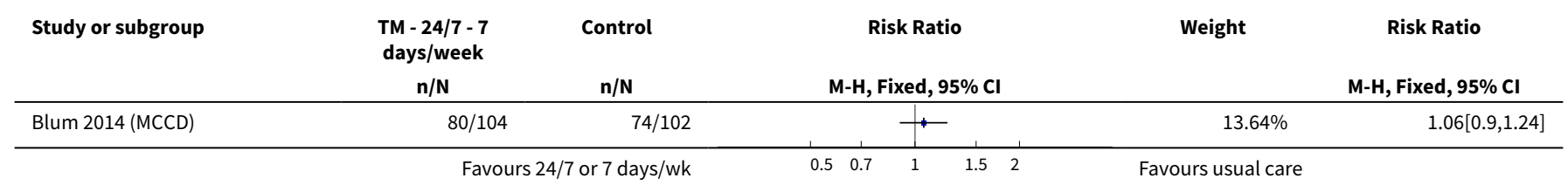




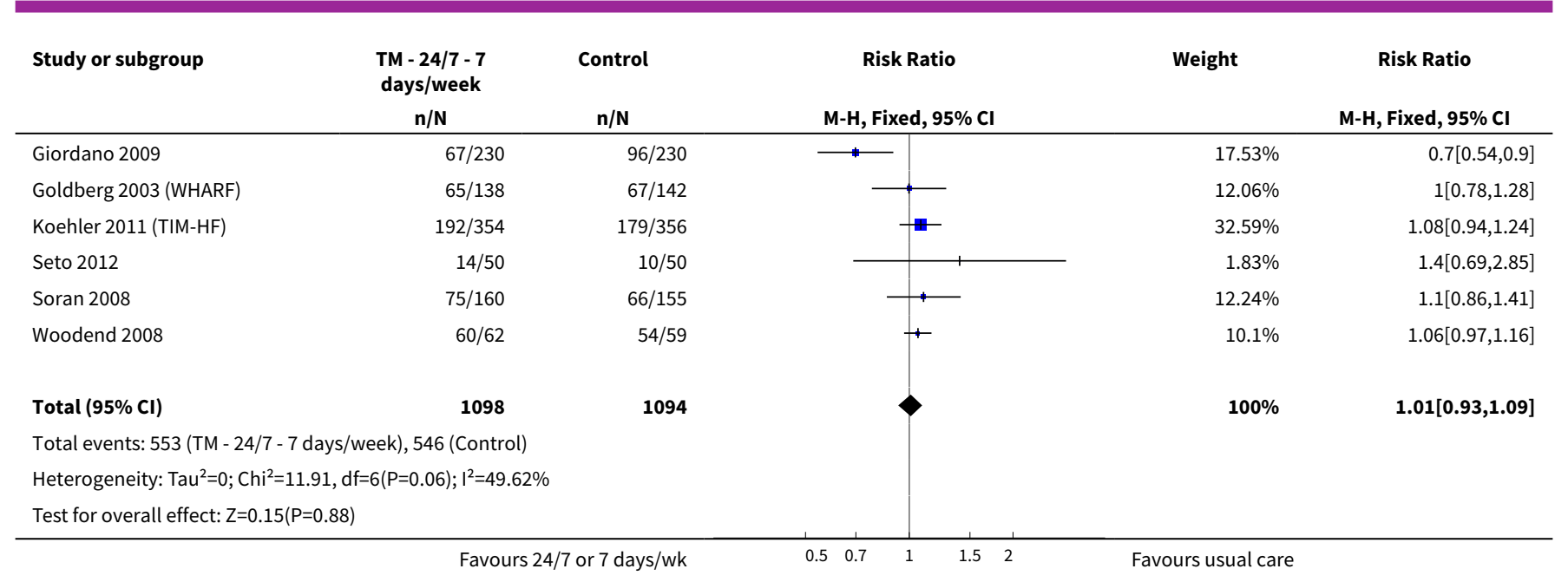

Analysis 2.10. Comparison 2 Impact of structured telephone or telemonitoring in heart failure on risk of allcause hospitalisation, Outcome 10 Subgroup Publication year: all-cause hospitalisation STS vs UC $<2000$.

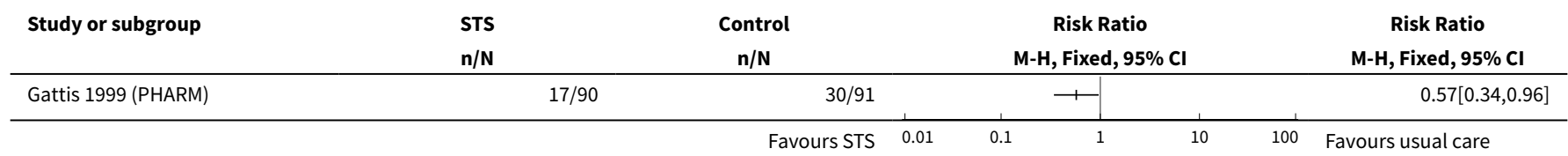

Analysis 2.11. Comparison 2 Impact of structured telephone or telemonitoring in heart failure on risk of allcause hospitalisation, Outcome 11 Subgroup Publication year: all-cause hospitalisation STS vs UC 2000 - 2007.

\begin{tabular}{|c|c|c|c|c|c|}
\hline Study or subgroup & $\begin{array}{l}\text { STS } \\
n / N\end{array}$ & $\begin{array}{c}\text { Control } \\
\mathrm{n} / \mathrm{N}\end{array}$ & $\begin{array}{c}\text { Risk Ratio } \\
\text { M-H, Fixed, } 95 \% \mathrm{CI}\end{array}$ & Weight & $\begin{array}{c}\text { Risk Ratio } \\
\text { M-H, Fixed, 95\% Cl }\end{array}$ \\
\hline $\begin{array}{l}\text { Cleland } 2005 \text { (Struct Tele) (TENS- } \\
\text { HMS) }\end{array}$ & $85 / 173$ & $46 / 85$ & \begin{tabular}{l|l}
$*$ &
\end{tabular} & $8.06 \%$ & $0.91[0.71,1.16]$ \\
\hline DeBusk 2004 & $116 / 228$ & $117 / 234$ & - & $15.08 \%$ & $1.02[0.85,1.22]$ \\
\hline GESICA 2005 (DIAL) & $261 / 760$ & $296 / 758$ & 田 & $38.71 \%$ & $0.88[0.77,1]$ \\
\hline Laramee 2003 & $49 / 141$ & $46 / 146$ & & $5.9 \%$ & $1.1[0.79,1.53]$ \\
\hline Riegel 2002 & $56 / 130$ & $114 / 228$ & + & $10.81 \%$ & $0.86[0.68,1.09]$ \\
\hline Riegel 2006 & $39 / 70$ & $37 / 65$ & & $5.01 \%$ & $0.98[0.73,1.32]$ \\
\hline Sisk 2006 & $62 / 203$ & $74 / 203$ & - & $9.66 \%$ & $0.84[0.64,1.1]$ \\
\hline Tsuyuki 2004 & $59 / 140$ & $51 / 136$ & & $6.76 \%$ & $1.12[0.84,1.5]$ \\
\hline Total $(95 \% \mathrm{Cl})$ & 1845 & 1855 & & $100 \%$ & $0.93[0.86,1.01]$ \\
\hline \multicolumn{6}{|l|}{ Total events: 727 (STS), 781 (Control) } \\
\hline Test for overall effect: $Z=1.79(P=0$. & & & & & \\
\hline
\end{tabular}


Analysis 2.12. Comparison 2 Impact of structured telephone or telemonitoring in heart failure on risk of allcause hospitalisation, Outcome 12 Subgroup Publication year: all-cause hospitalisation STS vs UC $\geq 2008$.

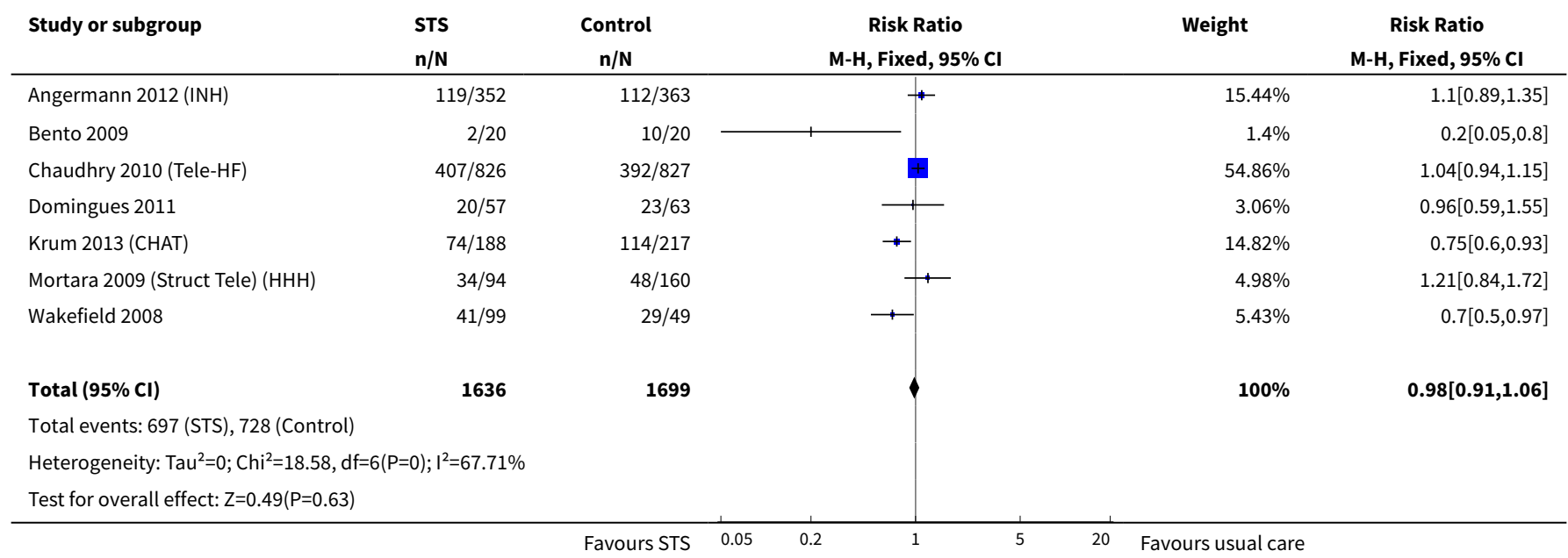

Analysis 2.13. Comparison 2 Impact of structured telephone or telemonitoring in heart failure on risk of allcause hospitalisation, Outcome 13 Subgroup Publication year: all-cause hospitalisation TM vs UC 2000 - 2007.

\begin{tabular}{|c|c|c|c|c|c|}
\hline Study or subgroup & $\begin{array}{c}\text { Telemonitoring } \\
\mathrm{n} / \mathrm{N}\end{array}$ & $\begin{array}{c}\text { Control } \\
\mathrm{n} / \mathrm{N}\end{array}$ & $\begin{array}{c}\text { Risk Ratio } \\
\text { M-H, Fixed, 95\% Cl }\end{array}$ & Weight & $\begin{array}{c}\text { Risk Ratio } \\
\text { M-H, Fixed, 95\% Cl }\end{array}$ \\
\hline $\begin{array}{l}\text { Cleland } 2005 \text { (Telemon) (TENS- } \\
\text { HMS) }\end{array}$ & $80 / 168$ & $46 / 85$ & -9 & $48.05 \%$ & $0.88[0.68,1.13]$ \\
\hline Goldberg 2003 (WHARF) & $65 / 138$ & $67 / 142$ & & $51.95 \%$ & $1[0.78,1.28]$ \\
\hline Total $(95 \% \mathrm{Cl})$ & 306 & 227 & & $100 \%$ & $0.94[0.79,1.12]$ \\
\hline \multicolumn{6}{|c|}{ Total events: 145 (Telemonitoring), 113 (Control) } \\
\hline \multicolumn{6}{|c|}{ Heterogeneity: $\operatorname{Tau}^{2}=0 ; \mathrm{Chi}^{2}=0.49, \mathrm{df}=1(\mathrm{P}=0.48) ; \mathrm{I}^{2}=0 \%$} \\
\hline Test for overall effect: $Z=0.67(P=$ & & & & & \\
\hline
\end{tabular}

Analysis 2.14. Comparison 2 Impact of structured telephone or telemonitoring in heart failure on risk of allcause hospitalisation, Outcome 14 Subgroup Publication year: all-cause hospitalisation TM vs UC $\geq 2008$.

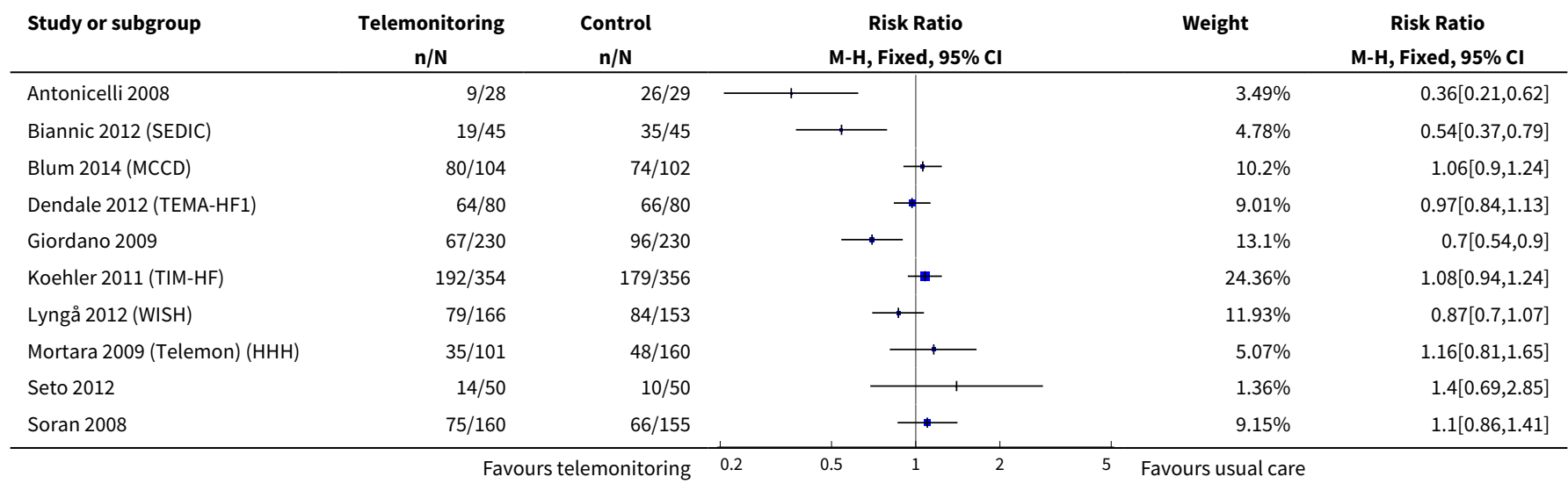




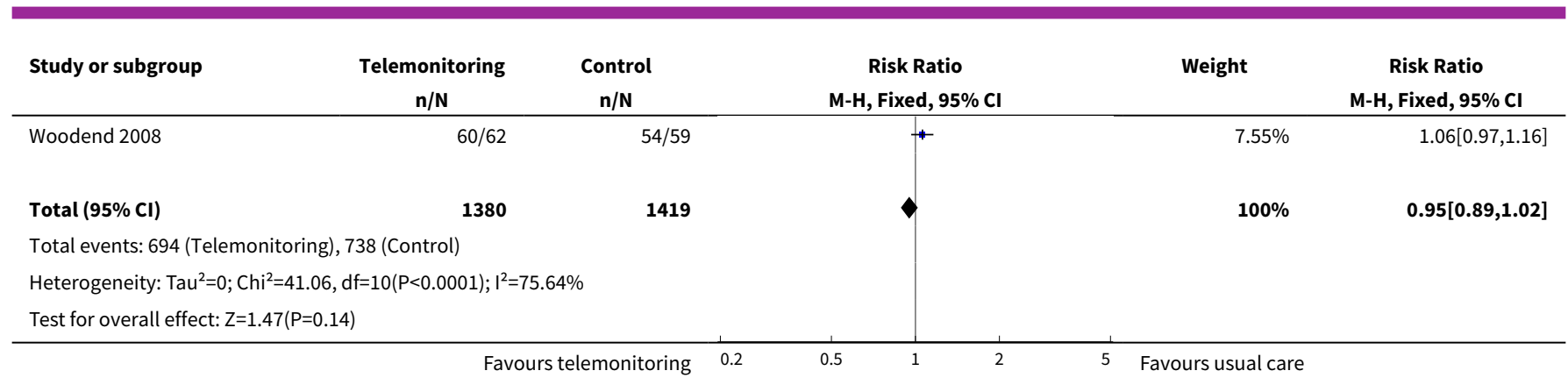

Analysis 2.15. Comparison 2 Impact of structured telephone or telemonitoring in heart failure on risk of allcause hospitalisation, Outcome 15 Subgroup Participant age: all-cause hospitalisation STS vs UC $\geq 70$ years of age.

\begin{tabular}{|c|c|c|c|c|c|}
\hline Study or subgroup & $\begin{array}{l}\text { STS } \\
n / N\end{array}$ & $\begin{array}{c}\text { Control } \\
n / N\end{array}$ & $\begin{array}{c}\text { Risk Ratio } \\
\text { M-H, Fixed, 95\% Cl }\end{array}$ & Weight & $\begin{array}{c}\text { Risk Ratio } \\
\text { M-H, Fixed, 95\% Cl }\end{array}$ \\
\hline DeBusk 2004 & $116 / 228$ & $117 / 234$ & - & $26.28 \%$ & $1.02[0.85,1.22]$ \\
\hline Krum 2013 (CHAT) & $74 / 188$ & $114 / 217$ & & $24.09 \%$ & $0.75[0.6,0.93]$ \\
\hline Laramee 2003 & $49 / 141$ & $46 / 146$ & & $10.29 \%$ & $1.1[0.79,1.53]$ \\
\hline Riegel 2002 & $56 / 130$ & $114 / 228$ & - & $18.84 \%$ & $0.86[0.68,1.09]$ \\
\hline Riegel 2006 & $39 / 70$ & $37 / 65$ & * & $8.73 \%$ & $0.98[0.73,1.32]$ \\
\hline Tsuyuki 2004 & $59 / 140$ & $51 / 136$ & 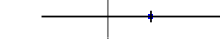 & $11.77 \%$ & $1.12[0.84,1.5]$ \\
\hline Total $(95 \% \mathrm{CI})$ & 897 & 1026 & & $100 \%$ & $0.94[0.85,1.04]$ \\
\hline \multicolumn{6}{|c|}{ Total events: 393 (STS), 479 (Control) } \\
\hline \multicolumn{6}{|c|}{ Heterogeneity: $\mathrm{Tau}^{2}=0 ; \mathrm{Chi}^{2}=7.85, \mathrm{df}=5(\mathrm{P}=0.16) ; \mathrm{I}^{2}=36.27 \%$} \\
\hline Test for overall effect & & & & & \\
\hline
\end{tabular}

Analysis 2.16. Comparison 2 Impact of structured telephone or telemonitoring in heart failure on risk of allcause hospitalisation, Outcome 16 Subgroup Participant age: all-cause hospitalisation: STS vs UC $<70$ years of age.

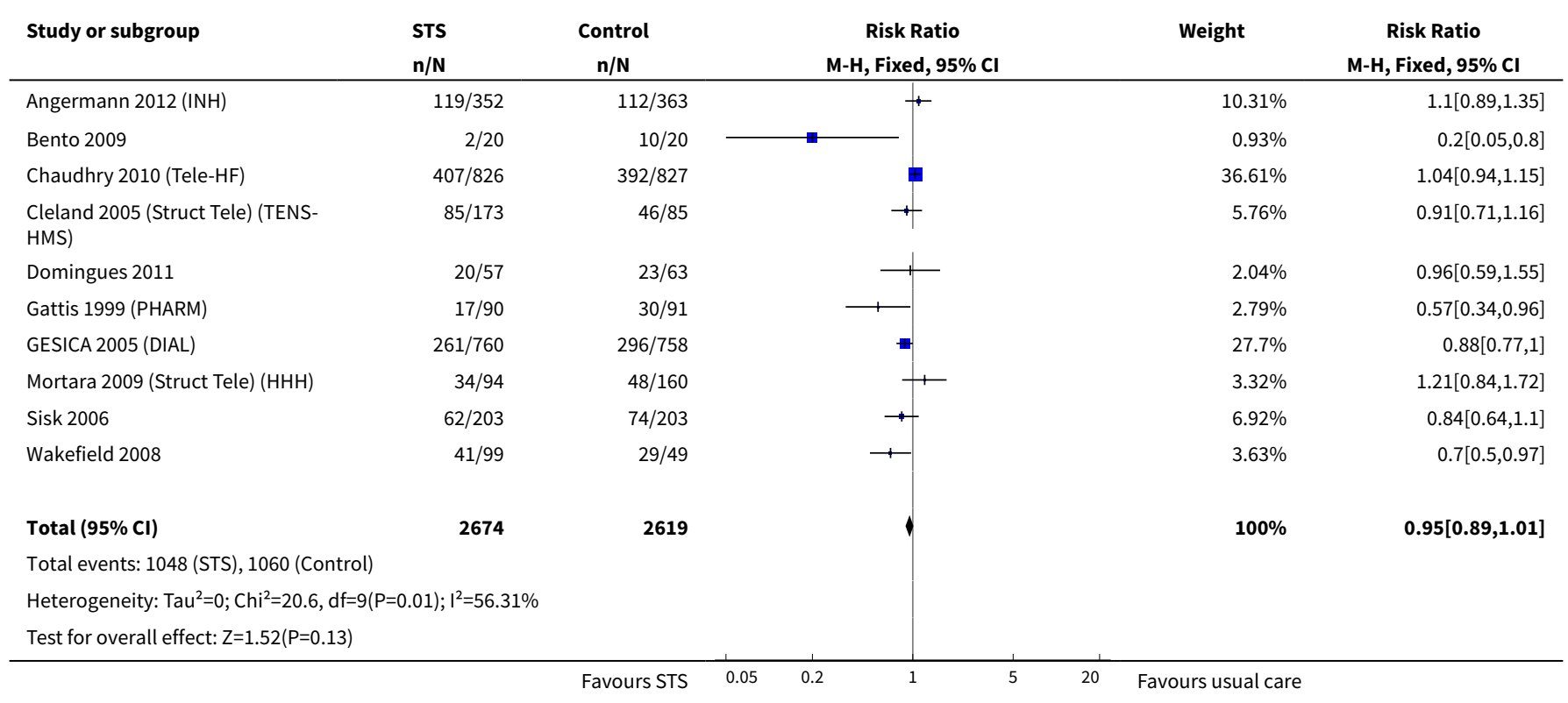


Analysis 2.17. Comparison 2 Impact of structured telephone or telemonitoring in heart failure on risk of allcause hospitalisation, Outcome 17 Subgroup Participant age: all-cause hospitalisation TM vs UC $\geq 70$ years of age.

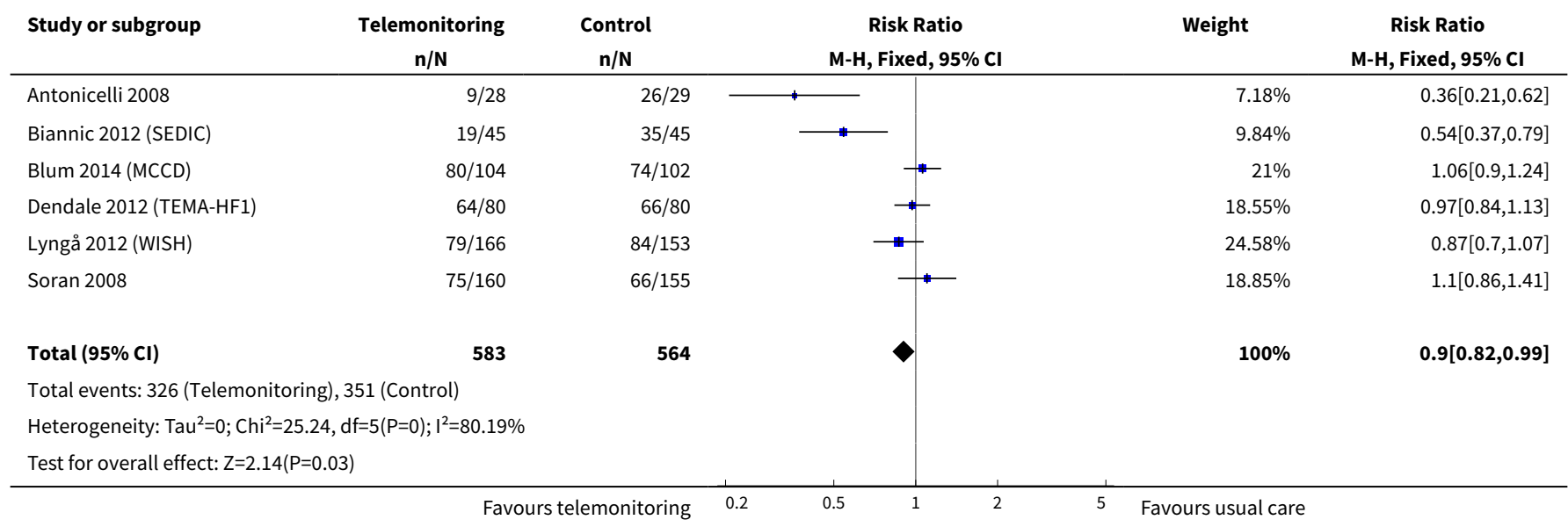

Analysis 2.18. Comparison 2 Impact of structured telephone or telemonitoring in heart failure on risk of allcause hospitalisation, Outcome 18 Subgroup Participant age: all-cause hospitalisation: TM vs UC < 70 years of age.

\begin{tabular}{|c|c|c|c|c|c|}
\hline Study or subgroup & $\begin{array}{c}\text { Telemonitoring } \\
\mathrm{n} / \mathrm{N} \\
\end{array}$ & $\begin{array}{c}\text { Control } \\
n / N\end{array}$ & $\begin{array}{c}\text { Risk Ratio } \\
\text { M-H, Fixed, } 95 \% \mathrm{CI}\end{array}$ & Weight & $\begin{array}{c}\text { Risk Ratio } \\
\text { M-H, Fixed, } 95 \% \mathrm{Cl}\end{array}$ \\
\hline $\begin{array}{l}\text { Cleland } 2005 \text { (Telemon) (TENS- } \\
\text { HMS) }\end{array}$ & $80 / 168$ & $46 / 85$ & $\longrightarrow$ & $12.12 \%$ & $0.88[0.68,1.13]$ \\
\hline Giordano 2009 & $67 / 230$ & $96 / 230$ & $\longrightarrow$ & $19.04 \%$ & $0.7[0.54,0.9]$ \\
\hline Goldberg 2003 (WHARF) & $65 / 138$ & $67 / 142$ & & $13.1 \%$ & $1[0.78,1.28]$ \\
\hline Koehler 2011 (TIM-HF) & $192 / 354$ & $179 / 356$ & $\rightarrow-$ & $35.41 \%$ & $1.08[0.94,1.24]$ \\
\hline Seto 2012 & $14 / 50$ & $10 / 50$ & & $1.98 \%$ & $1.4[0.69,2.85]$ \\
\hline Woodend 2008 & $60 / 62$ & $54 / 59$ & + & $10.98 \%$ & $1.06[0.97,1.16]$ \\
\hline Total $(95 \% \mathrm{Cl})$ & 1103 & 1082 & & $100 \%$ & $0.98[0.9,1.07]$ \\
\hline \multicolumn{6}{|c|}{ Total events: 513 (Telemonitoring), 500 (Control) } \\
\hline \multicolumn{6}{|c|}{ Heterogeneity: $\operatorname{Tau}^{2}=0 ; \mathrm{Chi}^{2}=13.86, \mathrm{df}=6(\mathrm{P}=0.03) ; \mathrm{I}^{2}=56.71 \%$} \\
\hline
\end{tabular}

Analysis 2.19. Comparison 2 Impact of structured telephone or telemonitoring in heart failure on risk of allcause hospitalisation, Outcome 19 Subgroup STS focus: all-cause hospitalisation: STS (clinical support) vs UC.

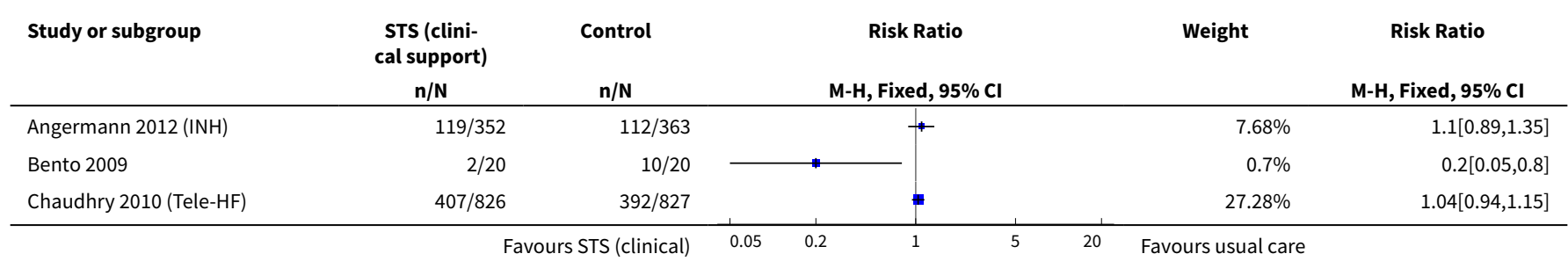




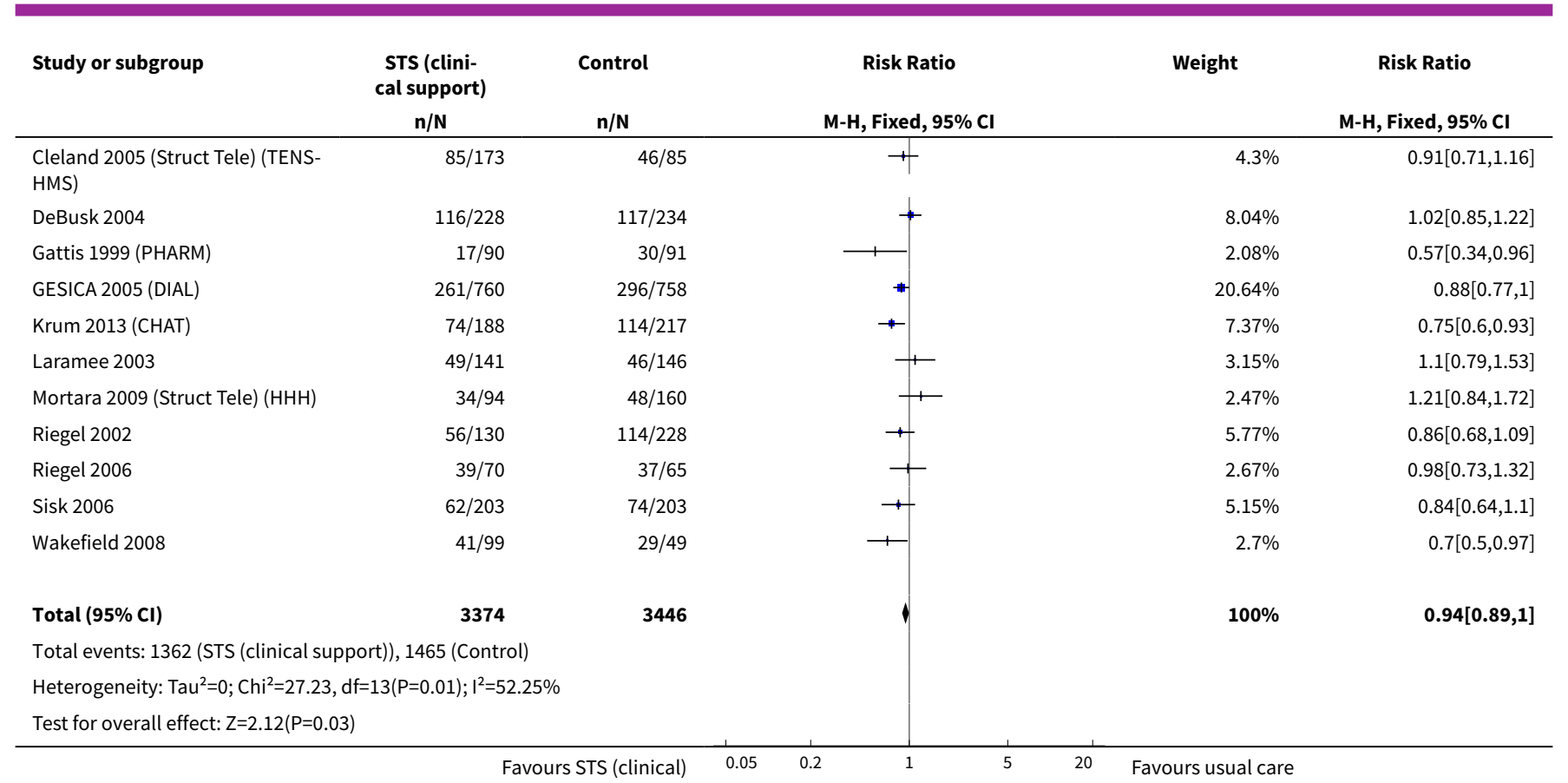

\section{Analysis 2.20. Comparison 2 Impact of structured telephone or telemonitoring in heart failure on risk of all- cause hospitalisation, Outcome 20 Subgroup STS focus: all-cause hospitalisation: STS (education) vs UC.}

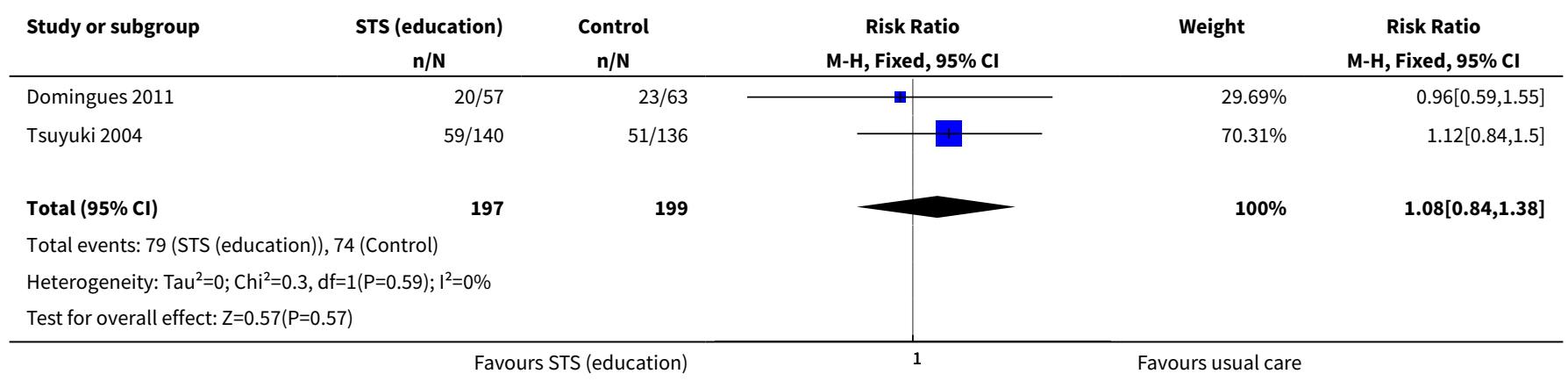

Analysis 2.21. Comparison 2 Impact of structured telephone or telemonitoring in heart failure on risk of all-cause hospitalisation, Outcome 21 Sensitivity analysis follow-up period (> 6 months), all-cause hospitalisation: STS vs UC.

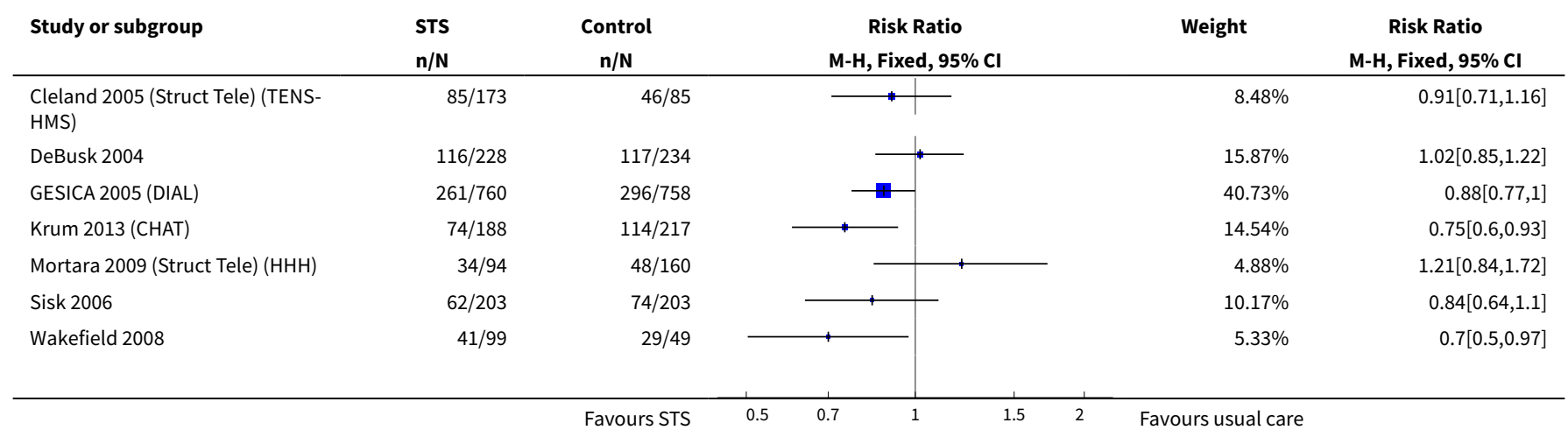




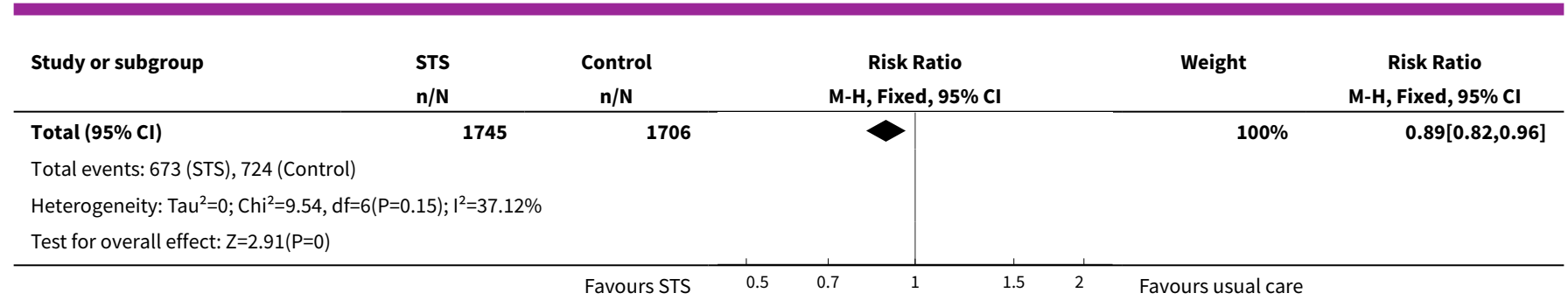

Analysis 2.22. Comparison 2 Impact of structured telephone or telemonitoring in heart failure on risk of all-cause hospitalisation, Outcome 22 Sensitivity analysis follow-up period (> 6 months), all-cause hospitalisation: TM vs UC.

\begin{tabular}{|c|c|c|c|c|c|}
\hline Study or subgroup & $\begin{array}{c}\text { Telemonitoring } \\
\mathbf{n} / \mathbf{N}\end{array}$ & $\begin{array}{c}\text { Control } \\
n / N\end{array}$ & $\begin{array}{c}\text { Risk Ratio } \\
\text { M-H, Fixed, 95\% Cl }\end{array}$ & Weight & $\begin{array}{c}\text { Risk Ratio } \\
\text { M-H, Fixed, } 95 \% \text { Cl }\end{array}$ \\
\hline Antonicelli 2008 & $9 / 28$ & $26 / 29$ & 1 & $4.15 \%$ & $0.36[0.21,0.62]$ \\
\hline Blum 2014 (MCCD) & $80 / 104$ & $74 / 102$ & + & $12.13 \%$ & $1.06[0.9,1.24]$ \\
\hline $\begin{array}{l}\text { Cleland } 2005 \text { (Telemon) (TENS- } \\
\text { HMS) }\end{array}$ & $80 / 168$ & $46 / 85$ & $\rightarrow$ & $9.92 \%$ & $0.88[0.68,1.13]$ \\
\hline Giordano 2009 & $67 / 230$ & $96 / 230$ & 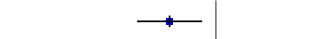 & $15.59 \%$ & $0.7[0.54,0.9]$ \\
\hline Lyngå 2012 (WISH) & $79 / 166$ & $84 / 153$ & $\rightarrow$ & $14.2 \%$ & $0.87[0.7,1.07]$ \\
\hline Mortara 2009 (Telemon) (HHH) & $35 / 101$ & $48 / 160$ & $\longrightarrow$ & $6.03 \%$ & $1.16[0.81,1.65]$ \\
\hline Woodend 2008 & $60 / 62$ & $54 / 59$ & *- & $8.99 \%$ & $1.06[0.97,1.16]$ \\
\hline Total $(95 \% \mathrm{Cl})$ & 1213 & 1174 & $\checkmark$ & $100 \%$ & $0.94[0.87,1.01]$ \\
\hline \multicolumn{6}{|c|}{ Total events: 602 (Telemonitoring), 607 (Control) } \\
\hline \multicolumn{6}{|c|}{ Test for overall effect: $Z=1.59(P=0.11)$} \\
\hline
\end{tabular}

Comparison 3. Impact of structured telephone support or telemonitoring in heart failure on risk of heart failurerelated hospitalisation

\begin{tabular}{|c|c|c|c|c|}
\hline Outcome or subgroup title & No. of studies & $\begin{array}{l}\text { No. of partici- } \\
\text { pants }\end{array}$ & Statistical method & Effect size \\
\hline 1 CHF-related hospitalisation: STS vs UC & 16 & 7030 & $\begin{array}{l}\text { Risk Ratio (M-H, Fixed, } \\
95 \% \mathrm{Cl})\end{array}$ & $0.85[0.77,0.93]$ \\
\hline 2 CHF-related hospitalisation: TM vs UC & 8 & 2148 & $\begin{array}{l}\text { Risk Ratio (M-H, Fixed, } \\
95 \% \mathrm{Cl})\end{array}$ & $0.71[0.60,0.83]$ \\
\hline $\begin{array}{l}3 \text { Subgroup technology: CHF-related hospital- } \\
\text { isation STS vs UC }\end{array}$ & 13 & 4718 & $\begin{array}{l}\text { Risk Ratio (M-H, Fixed, } \\
95 \% \mathrm{CI})\end{array}$ & $0.76[0.67,0.86]$ \\
\hline $\begin{array}{l}4 \text { Subgroup technology: CHF-related hospital- } \\
\text { isation Mobile/PDA vs UC }\end{array}$ & 3 & 674 & $\begin{array}{l}\text { Risk Ratio (M-H, Fixed, } \\
95 \% \mathrm{Cl})\end{array}$ & $0.58[0.44,0.77]$ \\
\hline $\begin{array}{l}5 \text { Subgroup technology CHF-related hospitali- } \\
\text { sation Complex TM vs UC }\end{array}$ & 5 & 1474 & $\begin{array}{l}\text { Risk Ratio (M-H, Fixed, } \\
95 \% \mathrm{Cl})\end{array}$ & $0.78[0.64,0.94]$ \\
\hline
\end{tabular}




\begin{tabular}{|c|c|c|c|c|}
\hline Outcome or subgroup title & No. of studies & $\begin{array}{l}\text { No. of partici- } \\
\text { pants }\end{array}$ & Statistical method & Effect size \\
\hline $\begin{array}{l}6 \text { Subgroup technology: CHF-related hospital- } \\
\text { isation IVR vs UC }\end{array}$ & 3 & 2312 & $\begin{array}{l}\text { Risk Ratio (M-H, Fixed, } \\
95 \% \mathrm{Cl})\end{array}$ & $0.99[0.86,1.14]$ \\
\hline $\begin{array}{l}7 \text { Subgroup TM intensity: CHF-related hospi- } \\
\text { talisation Office hours vs UC }\end{array}$ & 5 & 858 & $\begin{array}{l}\text { Risk Ratio (M-H, Fixed, } \\
95 \% \mathrm{Cl})\end{array}$ & $0.71[0.56,0.89]$ \\
\hline $\begin{array}{l}8 \text { Subgroup TM intensity: CHF-related hospi- } \\
\text { talisation } 24 / 7 \text { or } 7 \text { days vs UC }\end{array}$ & 3 & 1290 & $\begin{array}{l}\text { Risk Ratio (M-H, Fixed, } \\
95 \% \mathrm{Cl})\end{array}$ & $0.71[0.57,0.87]$ \\
\hline $\begin{array}{l}9 \text { Subgroup Publication year: CHF-related } \\
\text { hospitalisation STS vs UC }<2000\end{array}$ & 2 & 219 & $\begin{array}{l}\text { Risk Ratio (M-H, Fixed, } \\
95 \% \mathrm{Cl})\end{array}$ & $0.24[0.10,0.58]$ \\
\hline $\begin{array}{l}10 \text { Subgroup Publication year: CHF-related } \\
\text { hospitalisation STS vs UC } 2000-2007\end{array}$ & 10 & 3784 & $\begin{array}{l}\text { Risk Ratio (M-H, Fixed, } \\
95 \% \mathrm{Cl})\end{array}$ & $0.78[0.69,0.89]$ \\
\hline $\begin{array}{l}11 \text { Subgroup Publication year: CHF-related } \\
\text { hospitalisation STS vs UC } \geq 2008\end{array}$ & 4 & 3027 & $\begin{array}{l}\text { Risk Ratio (M-H, Fixed, } \\
95 \% \mathrm{Cl})\end{array}$ & $0.96[0.84,1.11]$ \\
\hline $\begin{array}{l}12 \text { Subgroup Publication year: CHF-related } \\
\text { hospitalisation TM vs UC } 2000 \text { - } 2007\end{array}$ & 1 & & $\begin{array}{l}\text { Risk Ratio (M-H, Fixed, } \\
95 \% \mathrm{Cl})\end{array}$ & $\begin{array}{l}\text { Totals not select- } \\
\text { ed }\end{array}$ \\
\hline $\begin{array}{l}13 \text { Subgroup Publication year: CHF-related } \\
\text { hospitalisation TM vs UC } \geq 2008\end{array}$ & 7 & 1895 & $\begin{array}{l}\text { Risk Ratio (M-H, Fixed, } \\
95 \% \mathrm{Cl})\end{array}$ & $0.69[0.58,0.82]$ \\
\hline $\begin{array}{l}14 \text { Subgroup Participant age: CHF-related } \\
\text { hospitalisation: STS vs UC }<70 \text { years of age }\end{array}$ & 8 & 5035 & $\begin{array}{l}\text { Risk Ratio (M-H, Fixed, } \\
95 \% \mathrm{Cl})\end{array}$ & $0.86[0.77,0.96]$ \\
\hline $\begin{array}{l}15 \text { Subgroup Participant age: CHF-related } \\
\text { hospitalisation: STS vs UC } \geq 70 \text { years of age }\end{array}$ & 8 & 1995 & $\begin{array}{l}\text { Risk Ratio }(\mathrm{M}-\mathrm{H}, \text { Fixed, } \\
95 \% \mathrm{Cl})\end{array}$ & $0.81[0.67,0.96]$ \\
\hline $\begin{array}{l}16 \text { Subgroup Participant age: CHF-related } \\
\text { hospitalisation: TM vs UC }<70 \text { years of age }\end{array}$ & 6 & 1898 & $\begin{array}{l}\text { Risk Ratio (M-H, Fixed, } \\
95 \% \mathrm{Cl})\end{array}$ & $0.75[0.63,0.89]$ \\
\hline $\begin{array}{l}17 \text { Subgroup Participant age: CHF-related } \\
\text { hospitalisation: TM vs UC } \geq 70 \text { years of age }\end{array}$ & 2 & 250 & $\begin{array}{l}\text { Risk Ratio (M-H, Fixed, } \\
95 \% \mathrm{Cl})\end{array}$ & $0.53[0.37,0.76]$ \\
\hline $\begin{array}{l}18 \text { Subgroup STS focus: CHF-related hospitali- } \\
\text { sation: STS (clinical support) vs UC }\end{array}$ & 15 & 6754 & $\begin{array}{l}\text { Risk Ratio (M-H, Fixed, } \\
95 \% \mathrm{Cl})\end{array}$ & $0.84[0.76,0.93]$ \\
\hline $\begin{array}{l}19 \text { Subgroup STS focus: CHF-related hospitali- } \\
\text { sation: STS (education) vs UC }\end{array}$ & 1 & & $\begin{array}{l}\text { Risk Ratio (M-H, Fixed, } \\
95 \% \mathrm{Cl})\end{array}$ & $\begin{array}{l}\text { Totals not select- } \\
\text { ed }\end{array}$ \\
\hline $\begin{array}{l}20 \text { Sensitivity analysis follow-up period (> } 6 \\
\text { months), CHF-related hospitalisation: STS vs } \\
\text { UC }\end{array}$ & 7 & 3341 & $\begin{array}{l}\text { Risk Ratio (M-H, Fixed, } \\
95 \% \mathrm{Cl})\end{array}$ & $0.76[0.66,0.88]$ \\
\hline $\begin{array}{l}21 \text { Sensitivity analysis follow-up period (> } 6 \\
\text { months), CHF-related hospitalisation: TM vs } \\
\text { UC }\end{array}$ & 4 & 1684 & $\begin{array}{l}\text { Risk Ratio (M-H, Fixed, } \\
95 \% \mathrm{Cl})\end{array}$ & $0.78[0.65,0.94]$ \\
\hline
\end{tabular}


Analysis 3.1. Comparison 3 Impact of structured telephone support or telemonitoring in heart failure on risk of heart failure-related hospitalisation, Outcome 1 CHF-related hospitalisation: STS vs UC.

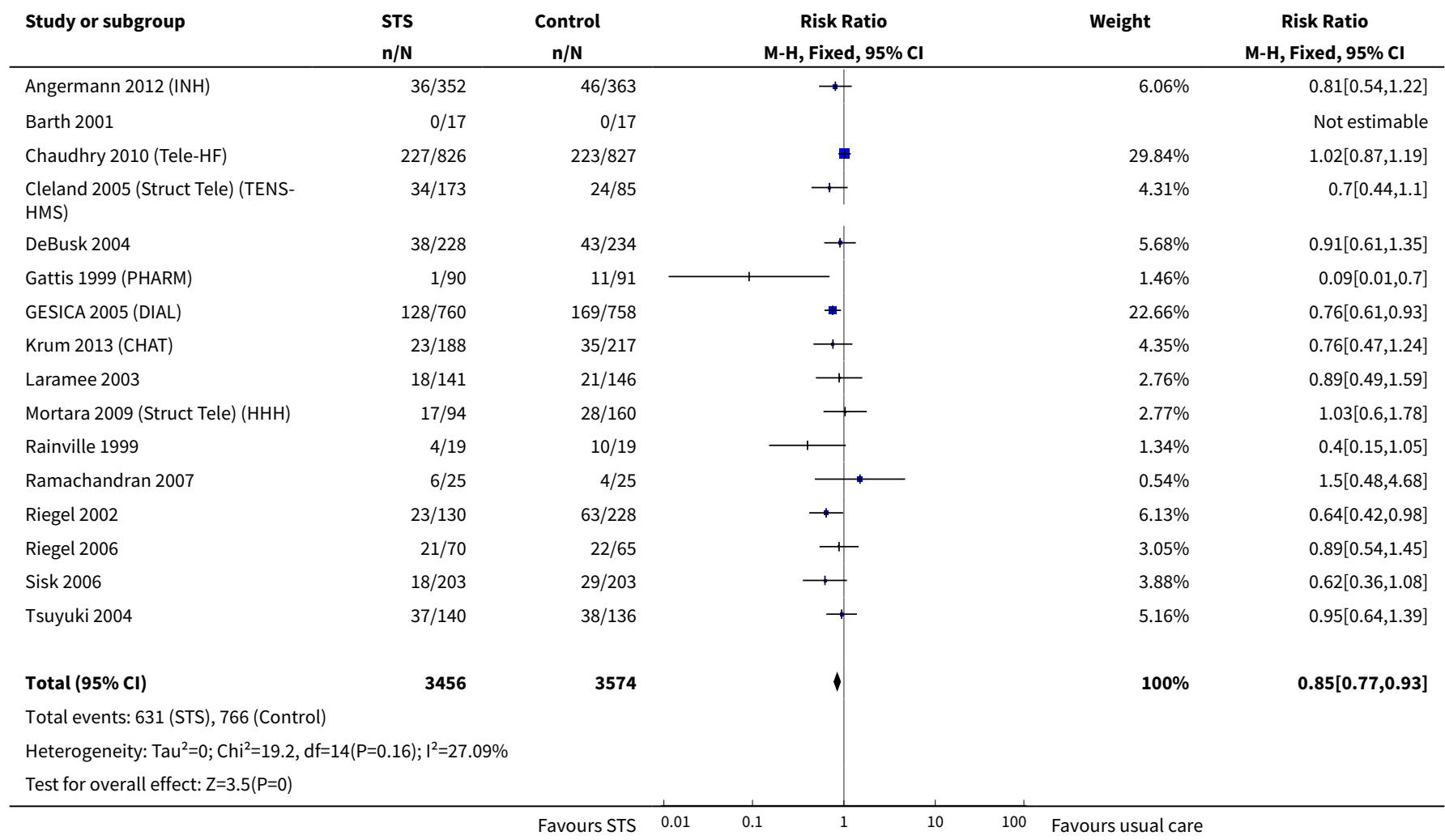

\section{Analysis 3.2. Comparison 3 Impact of structured telephone support or telemonitoring in heart failure on risk of heart failure-related hospitalisation, Outcome 2 CHF-related hospitalisation: TM vs UC.}

\begin{tabular}{|c|c|c|c|c|c|}
\hline Study or subgroup & $\begin{array}{c}\text { Telemonitoring } \\
\mathrm{n} / \mathrm{N} \\
\end{array}$ & $\begin{array}{c}\text { Control } \\
n / N\end{array}$ & $\begin{array}{c}\text { Risk Ratio } \\
\text { M-H, Fixed, 95\% Cl }\end{array}$ & Weight & $\begin{array}{c}\text { Risk Ratio } \\
\text { M-H, Fixed, } 95 \% \mathrm{Cl}\end{array}$ \\
\hline Biannic 2012 (SEDIC) & $12 / 45$ & $24 / 45$ & + & $8.27 \%$ & $0.5[0.29,0.87]$ \\
\hline $\begin{array}{l}\text { Cleland } 2005 \text { (Telemon) (TENS- } \\
\text { HMS) }\end{array}$ & $40 / 168$ & $24 / 85$ & -1 & $10.99 \%$ & $0.84[0.55,1.3]$ \\
\hline Dendale 2012 (TEMA-HF1) & $19 / 80$ & $34 / 80$ & - & $11.72 \%$ & $0.56[0.35,0.89]$ \\
\hline Giordano 2009 & $43 / 230$ & $73 / 230$ & & $25.17 \%$ & $0.59[0.42,0.82]$ \\
\hline Mortara 2009 (Telemon) (HHH) & $18 / 101$ & $28 / 160$ & & $7.47 \%$ & $1.02[0.6,1.74]$ \\
\hline Scherr 2009 (MOBITEL) & $11 / 66$ & $17 / 54$ & & $6.45 \%$ & $0.53[0.27,1.03]$ \\
\hline Vuorinen 2014 & $8 / 47$ & $13 / 47$ & 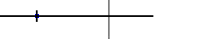 & $4.48 \%$ & $0.62[0.28,1.35]$ \\
\hline Total $(95 \% \mathrm{Cl})$ & 1091 & 1057 & & $100 \%$ & $0.71[0.6,0.83]$ \\
\hline \multicolumn{6}{|c|}{ Total events: 215 (Telemonitoring), 287 (Control) } \\
\hline Test for overall effect: $Z=4.36(P<$ & & & & & \\
\hline
\end{tabular}


Analysis 3.3. Comparison 3 Impact of structured telephone support or telemonitoring in heart failure on risk of heart failure-related hospitalisation, Outcome 3 Subgroup technology: CHF-related hospitalisation STS vs UC.

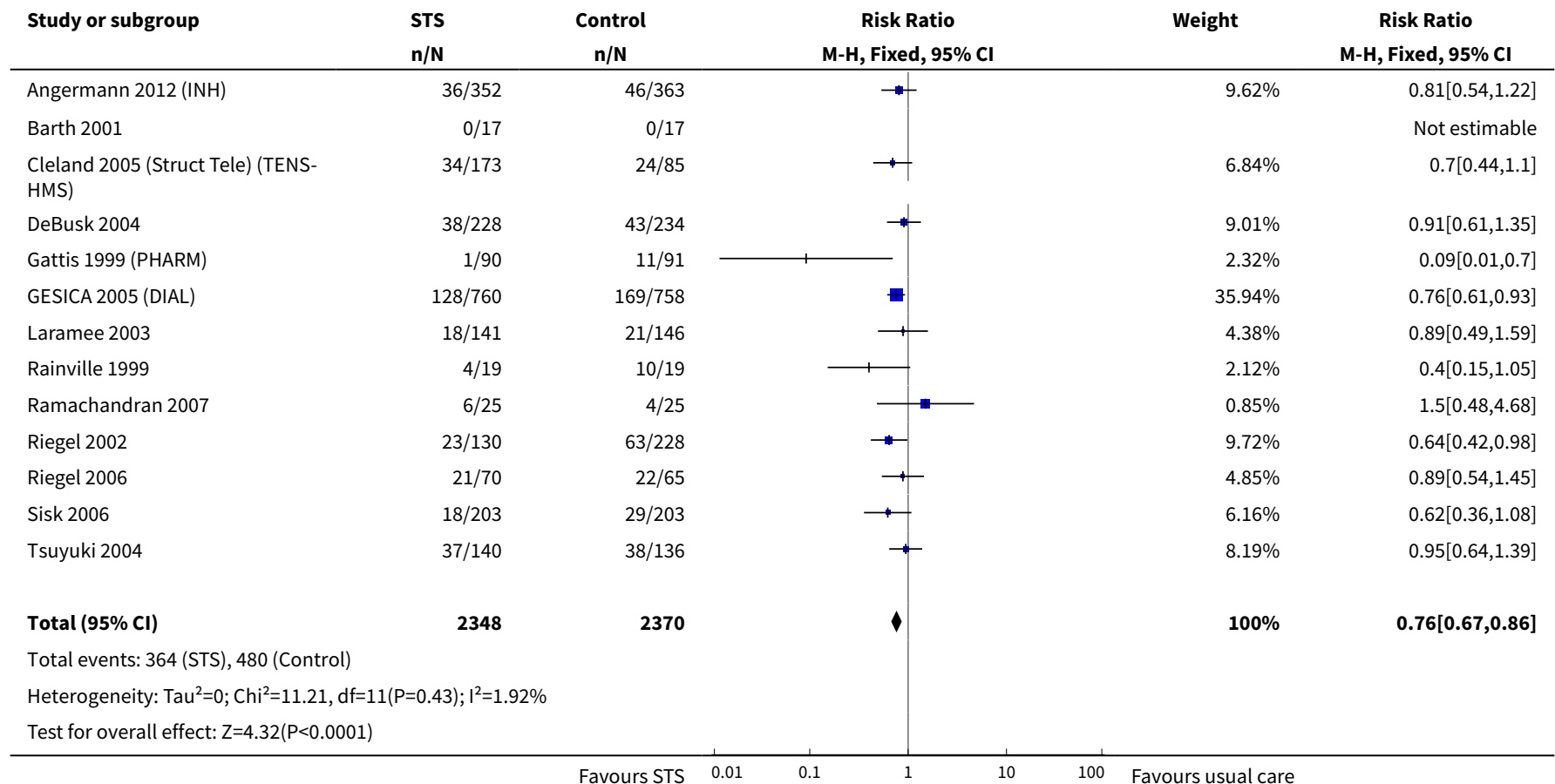

Analysis 3.4. Comparison 3 Impact of structured telephone support or telemonitoring in heart failure on risk of heart failure-related hospitalisation, Outcome 4 Subgroup technology: CHF-related hospitalisation Mobile/PDA vs UC.

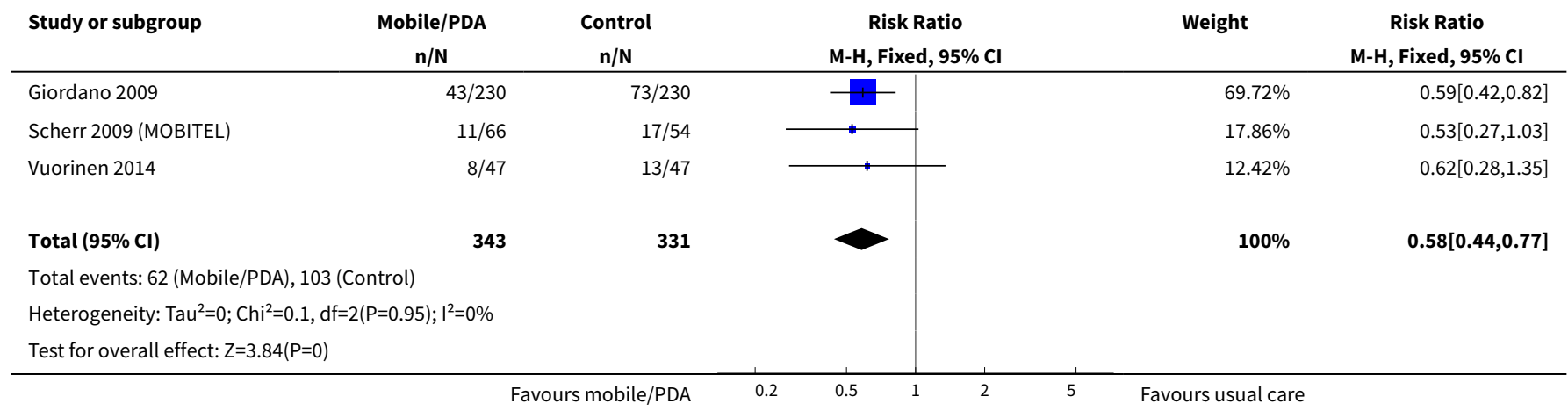

Analysis 3.5. Comparison 3 Impact of structured telephone support or telemonitoring in heart failure on risk of heart failure-related hospitalisation, Outcome 5 Subgroup technology CHF-related hospitalisation Complex TM vs UC.

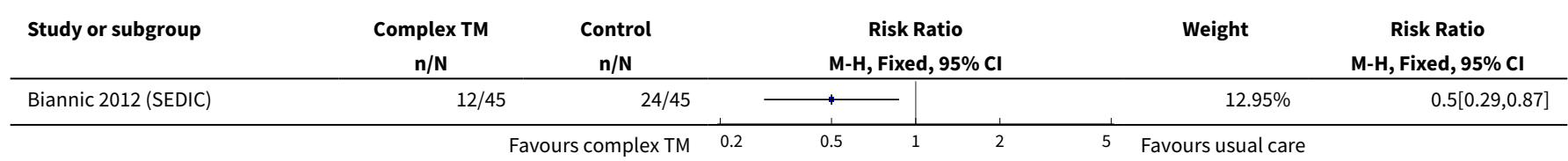




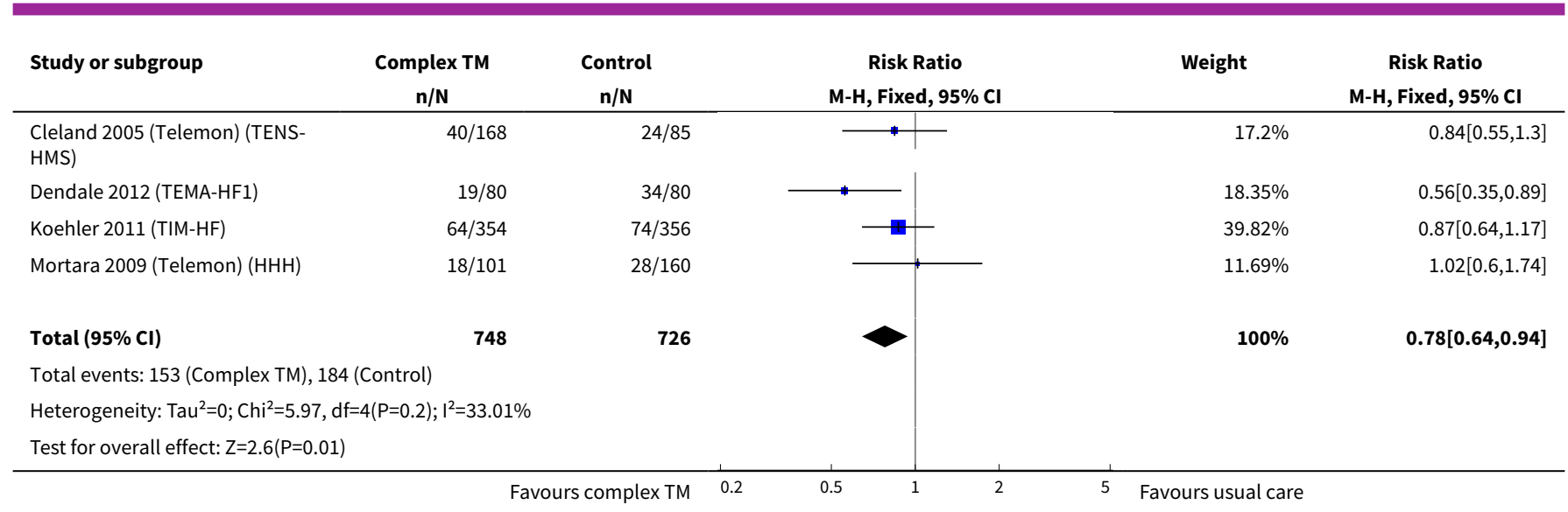

\section{Analysis 3.6. Comparison 3 Impact of structured telephone support or telemonitoring in heart failure on risk of heart failure-related hospitalisation, Outcome 6 Subgroup technology: CHF-related hospitalisation IVR vs UC.}

\begin{tabular}{|c|c|c|c|c|c|}
\hline Study or subgroup & $\begin{array}{l}\text { IVR } \\
\mathrm{n} / \mathrm{N}\end{array}$ & $\begin{array}{c}\text { Control } \\
n / N\end{array}$ & $\begin{array}{c}\text { Risk Ratio } \\
\text { M-H, Fixed, 95\% Cl }\end{array}$ & Weight & $\begin{array}{c}\text { Risk Ratio } \\
\text { M-H, Fixed, } 95 \% \mathrm{Cl}\end{array}$ \\
\hline Chaudhry 2010 (Tele-HF) & $227 / 826$ & $223 / 827$ & & $80.72 \%$ & $1.02[0.87,1.19]$ \\
\hline Krum 2013 (CHAT) & $23 / 188$ & $35 / 217$ & & $11.77 \%$ & $0.76[0.47,1.24]$ \\
\hline Mortara 2009 (Struct Tele) (HHH) & $17 / 94$ & $28 / 160$ & & $7.51 \%$ & $1.03[0.6,1.78]$ \\
\hline Total $(95 \% \mathrm{Cl})$ & 1108 & 1204 & & $100 \%$ & $0.99[0.86,1.14]$ \\
\hline \multicolumn{6}{|c|}{ Heterogeneity: Tau $^{2}=0 ; \mathrm{Chi}^{2}=1.3, \mathrm{df}=2(\mathrm{P}=0.52) ; \mathrm{I}^{2}=0 \%$} \\
\hline \multicolumn{6}{|c|}{ Test for overall effect: $Z=0.14(P=0.89)$} \\
\hline
\end{tabular}

Analysis 3.7. Comparison 3 Impact of structured telephone support or telemonitoring in heart failure on risk of heart failure-related hospitalisation, Outcome 7 Subgroup TM intensity: CHF-related hospitalisation Office hours vs UC.

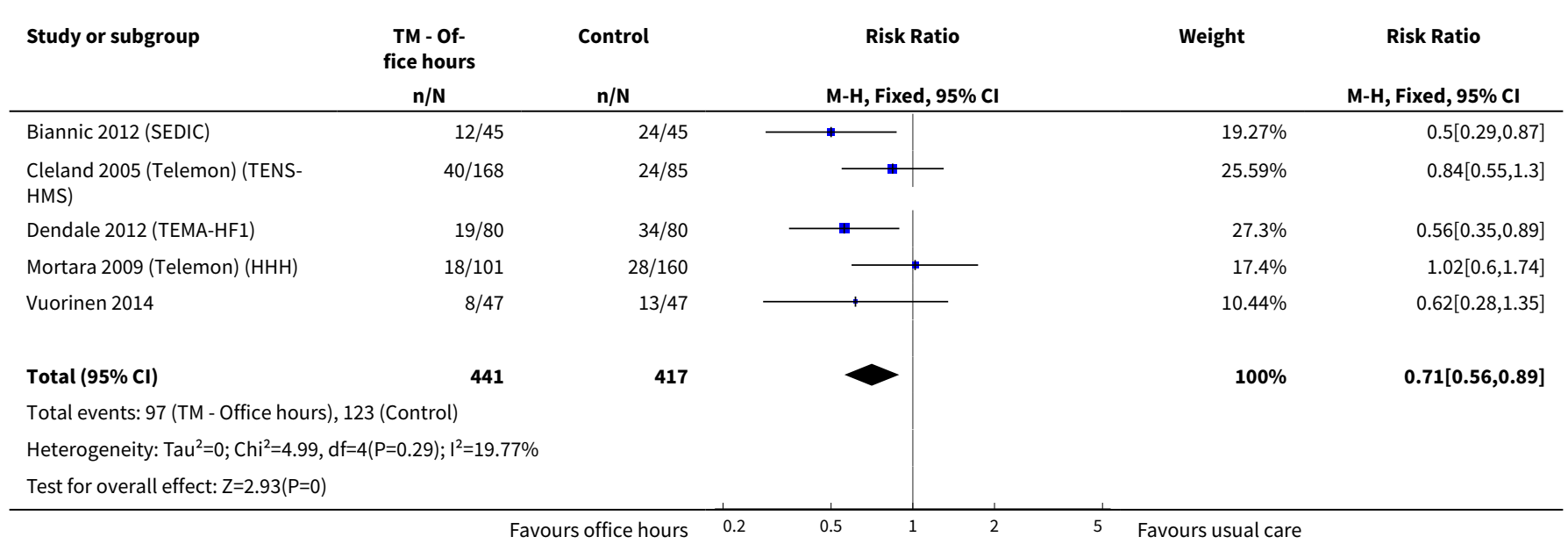


Analysis 3.8. Comparison 3 Impact of structured telephone support or

telemonitoring in heart failure on risk of heart failure-related hospitalisation,

Outcome 8 Subgroup TM intensity: CHF-related hospitalisation 24/7 or 7 days vs UC.

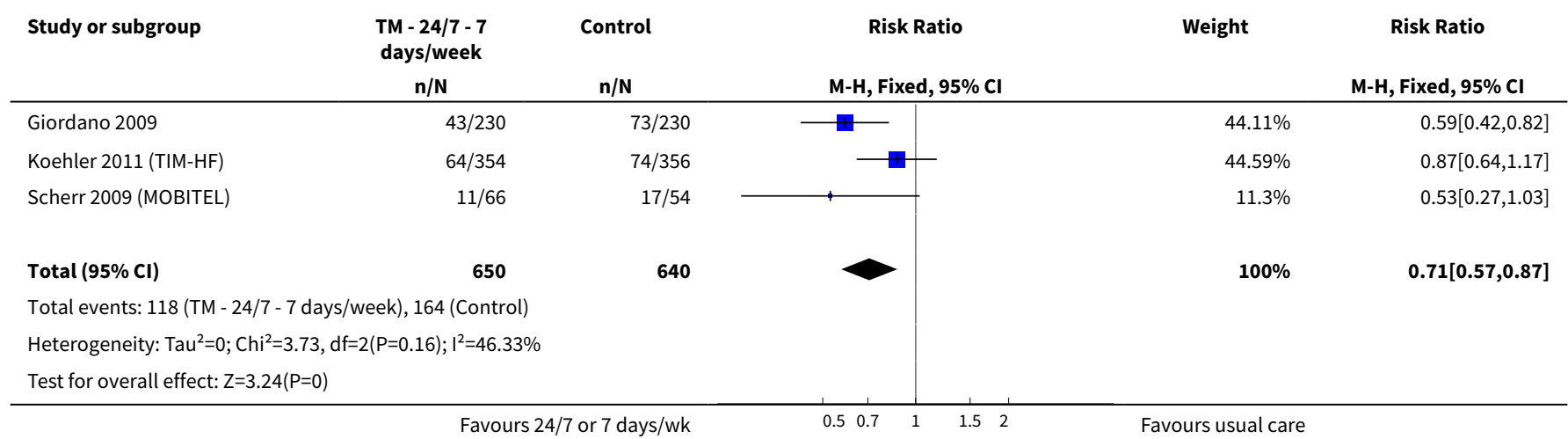

Analysis 3.9. Comparison 3 Impact of structured telephone support or telemonitoring in heart failure on risk of heart failure-related hospitalisation, Outcome 9 Subgroup Publication year: CHF-related hospitalisation STS vs UC $<2000$.

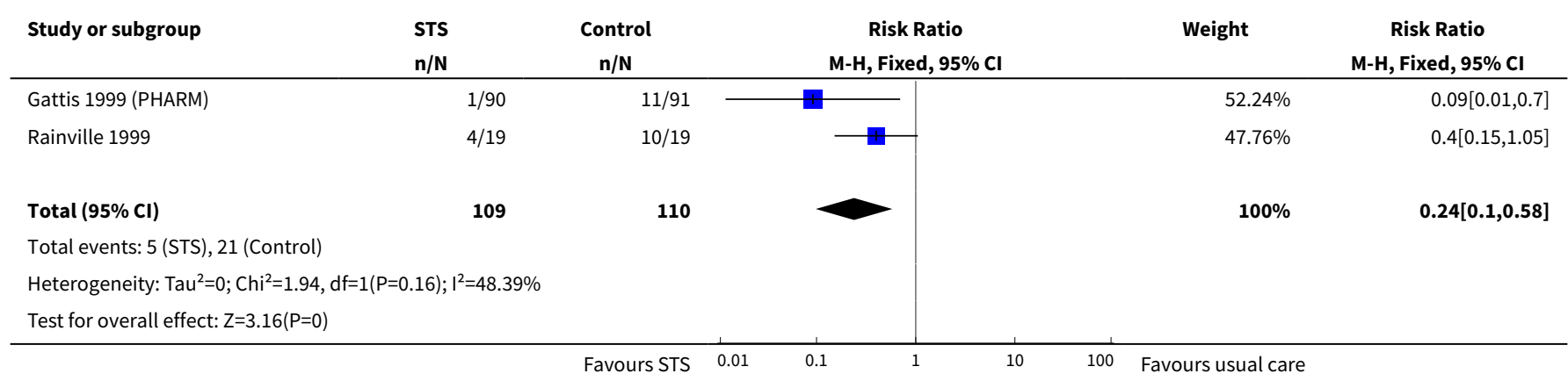

Analysis 3.10. Comparison 3 Impact of structured telephone support or telemonitoring

in heart failure on risk of heart failure-related hospitalisation, Outcome 10

Subgroup Publication year: CHF-related hospitalisation STS vs UC 2000 - 2007.

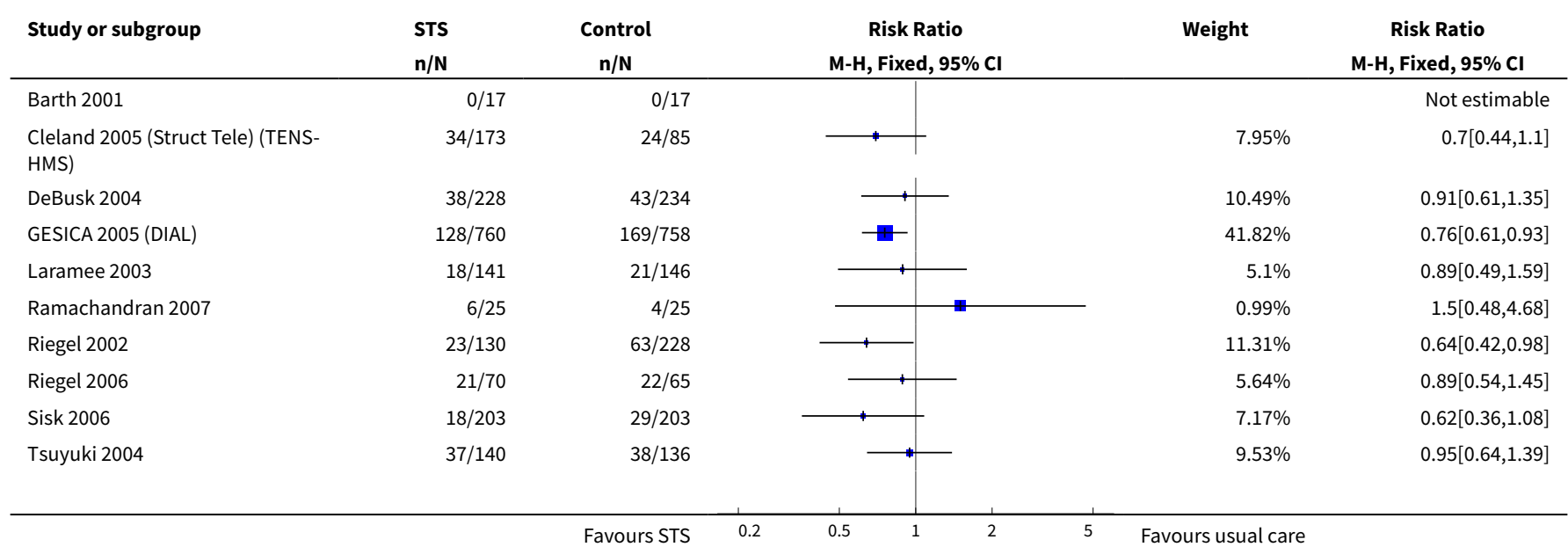




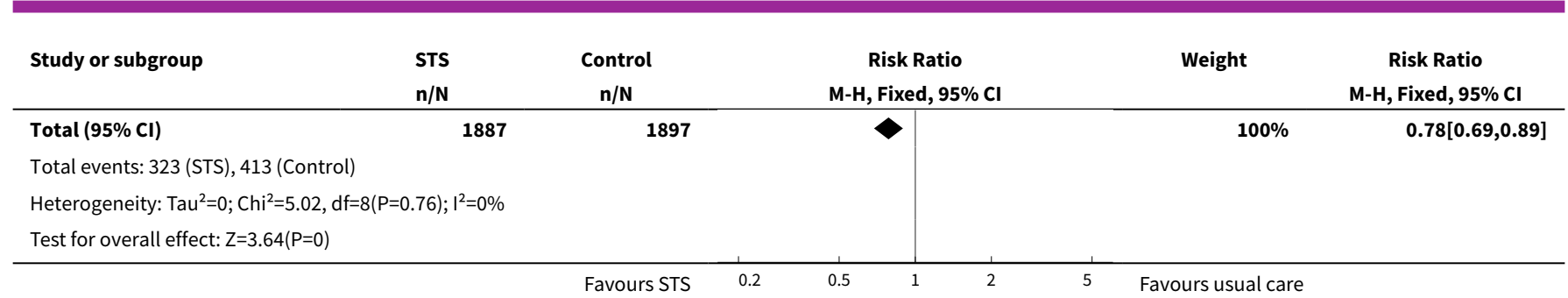

Analysis 3.11. Comparison 3 Impact of structured telephone support or telemonitoring in heart failure on risk of heart failure-related hospitalisation, Outcome 11 Subgroup Publication year: CHF-related hospitalisation STS vs UC $\geq 2008$.

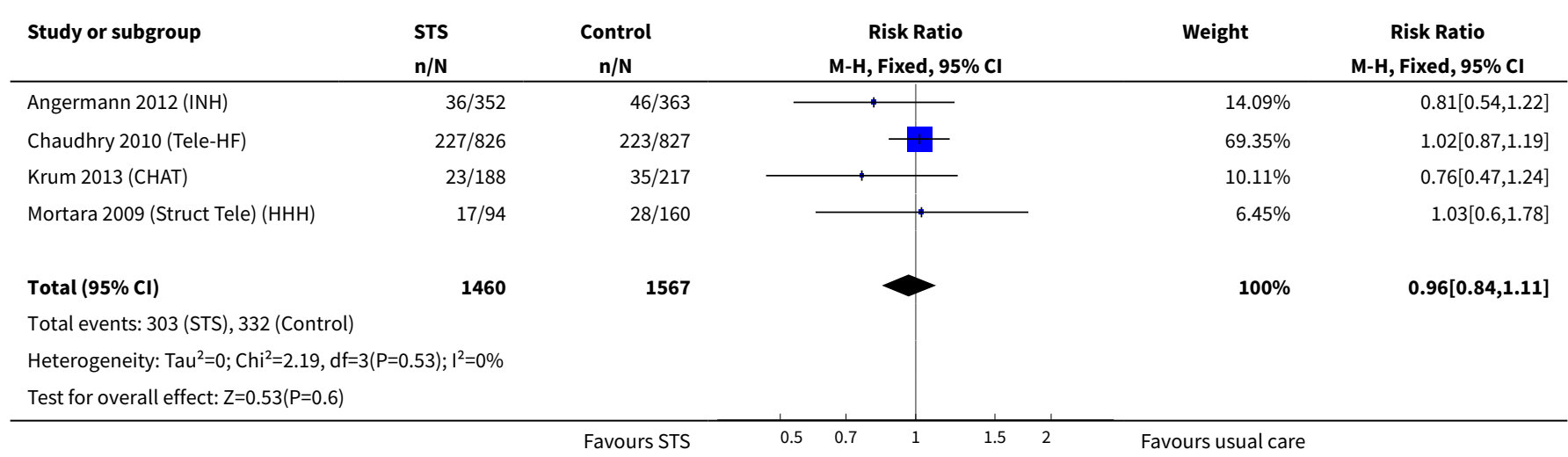

Analysis 3.12. Comparison 3 Impact of structured telephone support or telemonitoring

in heart failure on risk of heart failure-related hospitalisation, Outcome 12

Subgroup Publication year: CHF-related hospitalisation TM vs UC 2000 - 2007.

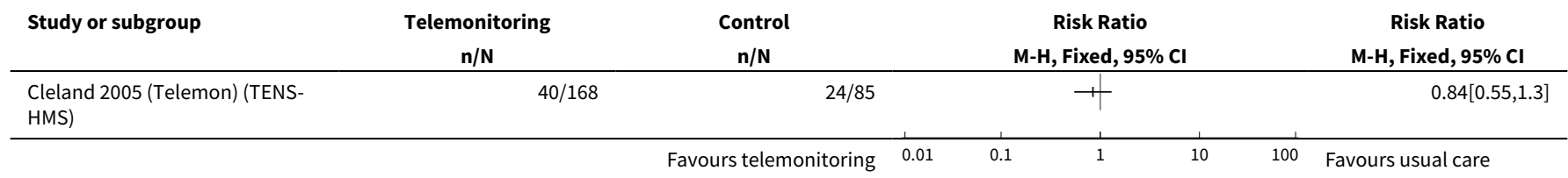

Analysis 3.13. Comparison 3 Impact of structured telephone support or telemonitoring in heart failure on risk of heart failure-related hospitalisation, Outcome 13 Subgroup Publication year: CHF-related hospitalisation TM vs UC $\geq 2008$.

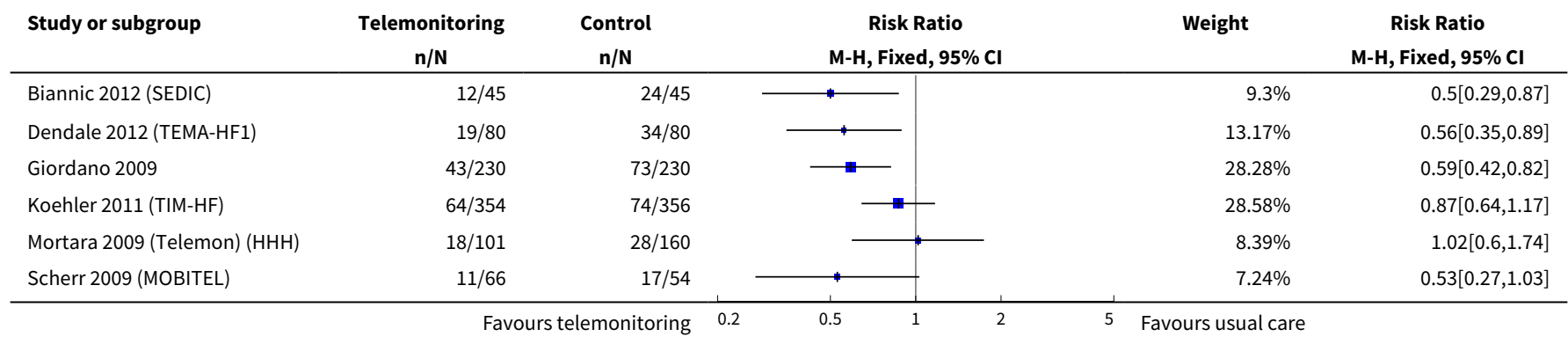




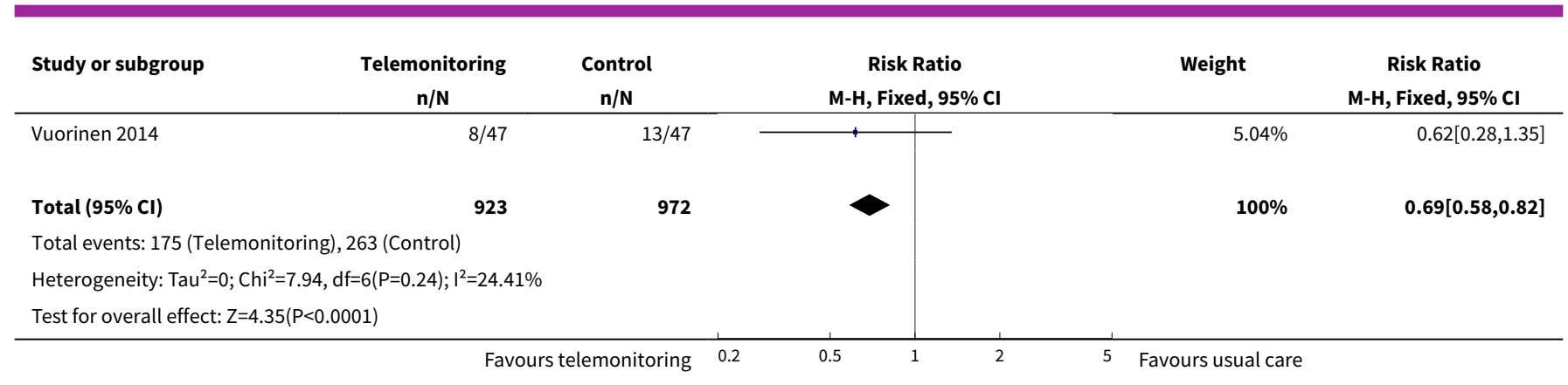

Analysis 3.14. Comparison 3 Impact of structured telephone support or telemonitoring in heart failure on risk of heart failure-related hospitalisation, Outcome 14 Subgroup Participant age: CHF-related hospitalisation: STS vs UC $<70$ years of age.

\begin{tabular}{|c|c|c|c|c|c|}
\hline Study or subgroup & $\begin{array}{l}\text { STS } \\
\mathrm{n} / \mathrm{N}\end{array}$ & $\begin{array}{c}\text { Control } \\
n / N\end{array}$ & $\begin{array}{c}\text { Risk Ratio } \\
\text { M-H, Fixed, } 95 \% \mathrm{CI}\end{array}$ & Weight & $\begin{array}{c}\text { Risk Ratio } \\
\text { M-H, Fixed, } 95 \% \mathrm{Cl}\end{array}$ \\
\hline Angermann 2012 (INH) & $36 / 352$ & $46 / 363$ & $\rightarrow$ & $8.48 \%$ & $0.81[0.54,1.22]$ \\
\hline Chaudhry 2010 (Tele-HF) & $227 / 826$ & $223 / 827$ & 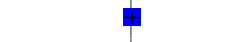 & $41.72 \%$ & $1.02[0.87,1.19]$ \\
\hline $\begin{array}{l}\text { Cleland } 2005 \text { (Struct Tele) (TENS- } \\
\text { HMS) }\end{array}$ & $34 / 173$ & $24 / 85$ & $\rightarrow$ & $6.02 \%$ & $0.7[0.44,1.1]$ \\
\hline Gattis 1999 (PHARM) & $1 / 90$ & $11 / 91$ & 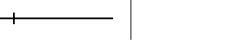 & $2.05 \%$ & $0.09[0.01,0.7]$ \\
\hline GESICA 2005 (DIAL) & $128 / 760$ & $169 / 758$ & - & $31.68 \%$ & $0.76[0.61,0.93]$ \\
\hline Mortara 2009 (Struct Tele) (HHH) & $17 / 94$ & $28 / 160$ & 1 & $3.88 \%$ & $1.03[0.6,1.78]$ \\
\hline Ramachandran 2007 & $6 / 25$ & $4 / 25$ & $\longrightarrow$ & $0.75 \%$ & $1.5[0.48,4.68]$ \\
\hline Sisk 2006 & $18 / 203$ & $29 / 203$ & $\rightarrow$ & $5.43 \%$ & $0.62[0.36,1.08]$ \\
\hline Total $(95 \% \mathrm{Cl})$ & 2523 & 2512 & ? & $100 \%$ & $0.86[0.77,0.96]$ \\
\hline \multicolumn{6}{|l|}{ Total events: 467 (STS), 534 (Control) } \\
\hline \multicolumn{6}{|c|}{ Heterogeneity: $\mathrm{Tau}^{2}=0 ; \mathrm{Chi}^{2}=14.23, \mathrm{df}=7(\mathrm{P}=0.05) ; \mathrm{I}^{2}=50.81 \%$} \\
\hline \multicolumn{6}{|l|}{ Test for overall effect: $Z=2.65(P=0.01)$} \\
\hline
\end{tabular}

Analysis 3.15. Comparison 3 Impact of structured telephone support or telemonitoring in heart failure on risk of heart failure-related hospitalisation, Outcome 15

Subgroup Participant age: CHF-related hospitalisation: STS vs UC $\geq 70$ years of age.

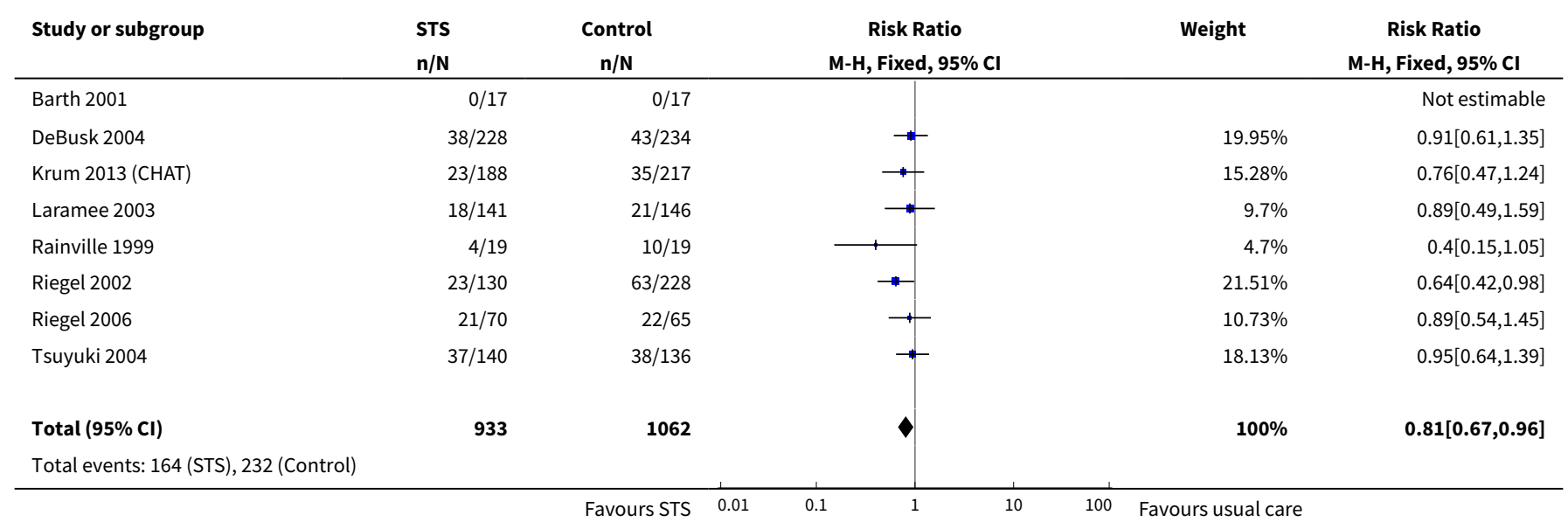




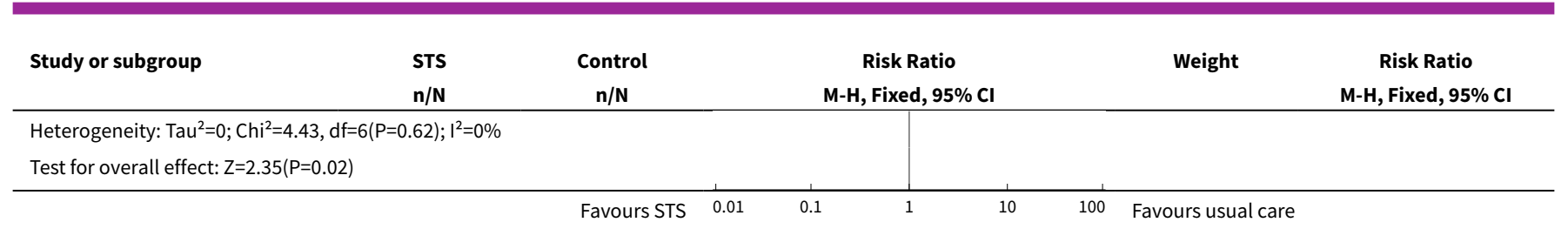

\section{Analysis 3.16. Comparison 3 Impact of structured telephone support or telemonitoring in heart failure on risk of heart failure-related hospitalisation, Outcome 16 Subgroup Participant age: CHF-related hospitalisation: TM vs UC $<70$ years of age.}

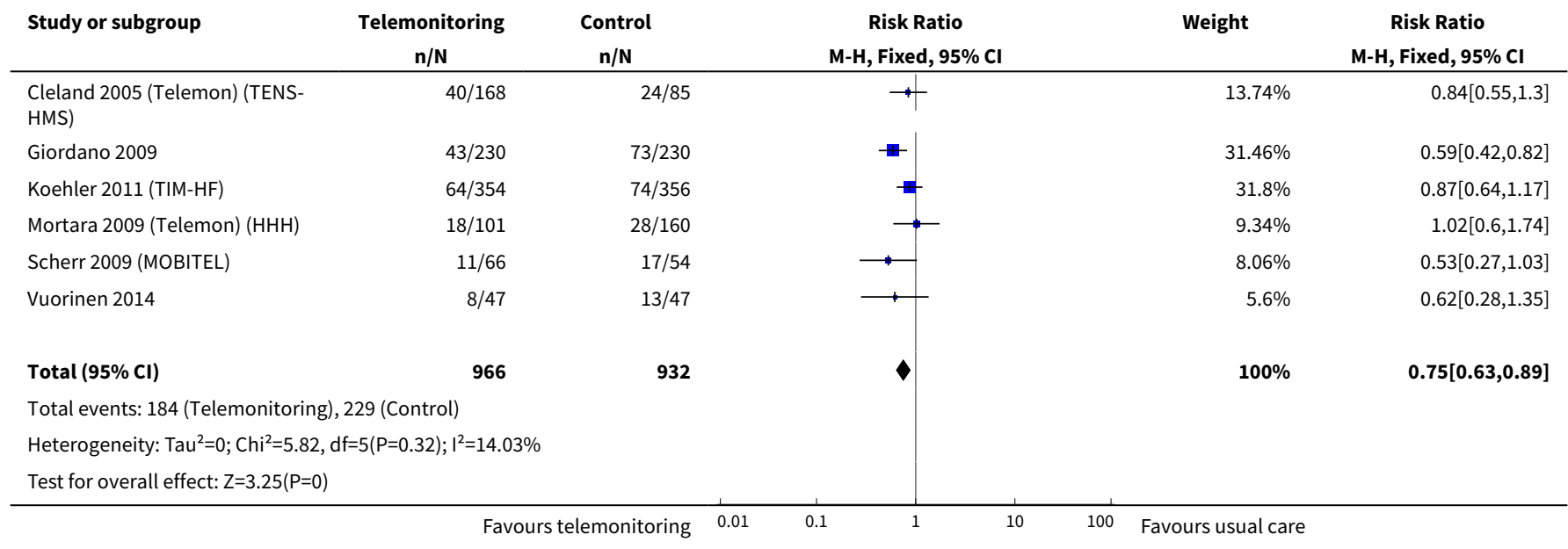

Analysis 3.17. Comparison 3 Impact of structured telephone support or telemonitoring in heart failure on risk of heart failure-related hospitalisation, Outcome 17 Subgroup Participant age: CHF-related hospitalisation: TM vs UC $\geq 70$ years of age.

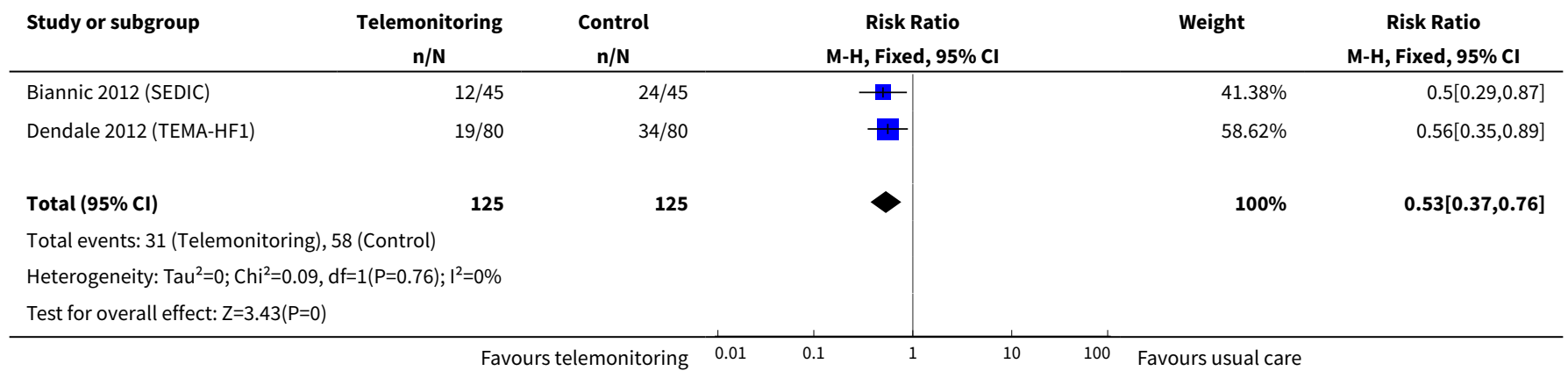


Analysis 3.18. Comparison 3 Impact of structured telephone support or telemonitoring

in heart failure on risk of heart failure-related hospitalisation, Outcome 18

Subgroup STS focus: CHF-related hospitalisation: STS (clinical support) vs UC.

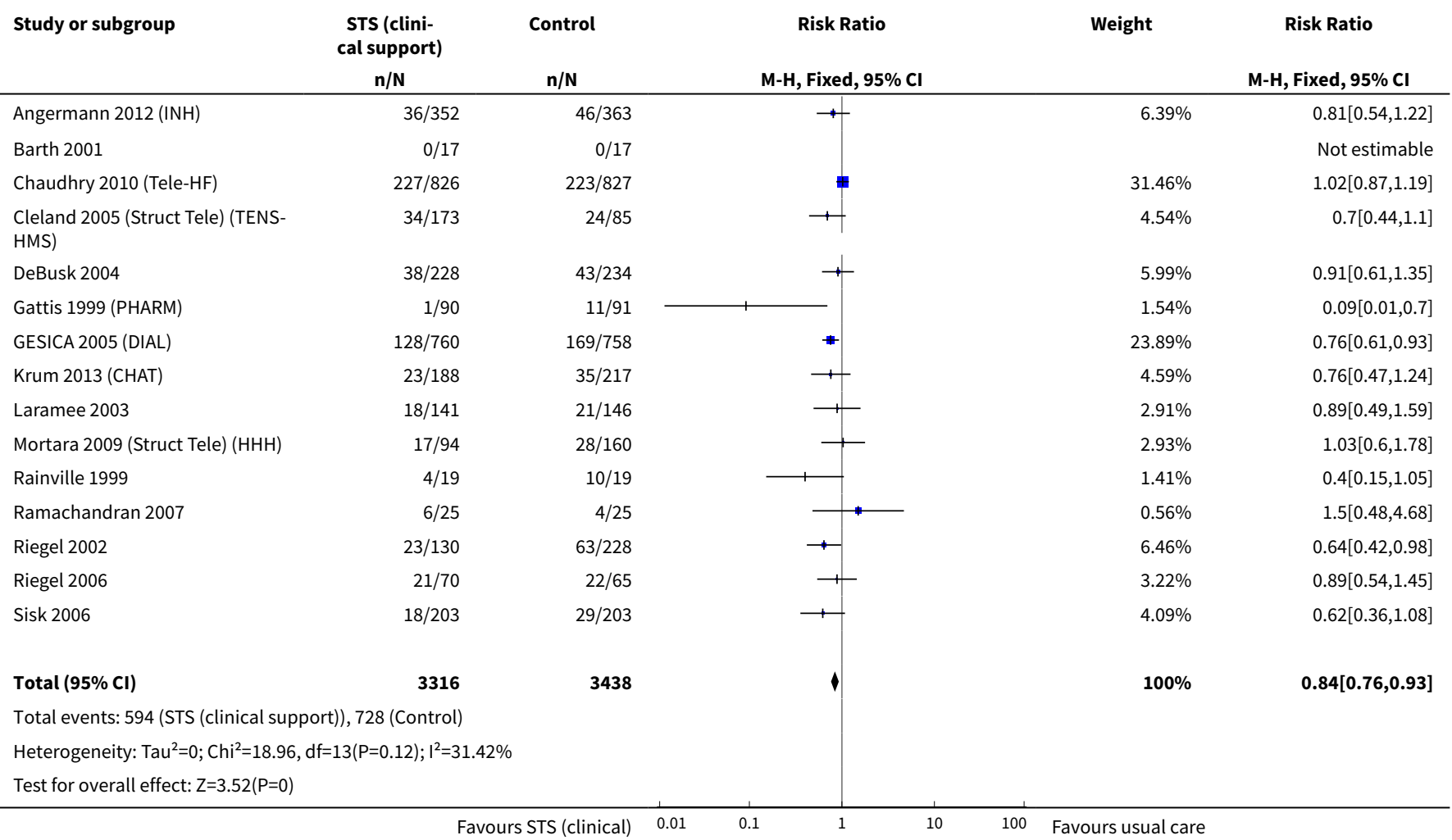

Analysis 3.19. Comparison 3 Impact of structured telephone support or telemonitoring in heart failure on risk of heart failure-related hospitalisation, Outcome 19 Subgroup STS focus: CHF-related hospitalisation: STS (education) vs UC.

\begin{tabular}{|c|c|c|c|c|c|}
\hline Study or subgroup & $\begin{array}{c}\text { STS (education) } \\
n / N\end{array}$ & $\begin{array}{c}\text { Control } \\
n / N\end{array}$ & & $\begin{array}{c}\text { Risk Ratio } \\
\text { M-H, Fixed, } 95 \% \mathrm{Cl}\end{array}$ & $\begin{array}{c}\text { Risk Ratio } \\
\text { M-H, Fixed, 95\% CI }\end{array}$ \\
\hline Tsuyuki 2004 & $37 / 140$ & $38 / 136$ & & + & $0.95[0.64,1.39]$ \\
\hline
\end{tabular}

Analysis 3.20. Comparison 3 Impact of structured telephone support or telemonitoring in heart failure on risk of heart failure-related hospitalisation, Outcome 20 Sensitivity analysis follow-up period ( $>6$ months), CHF-related hospitalisation: STS vs UC.

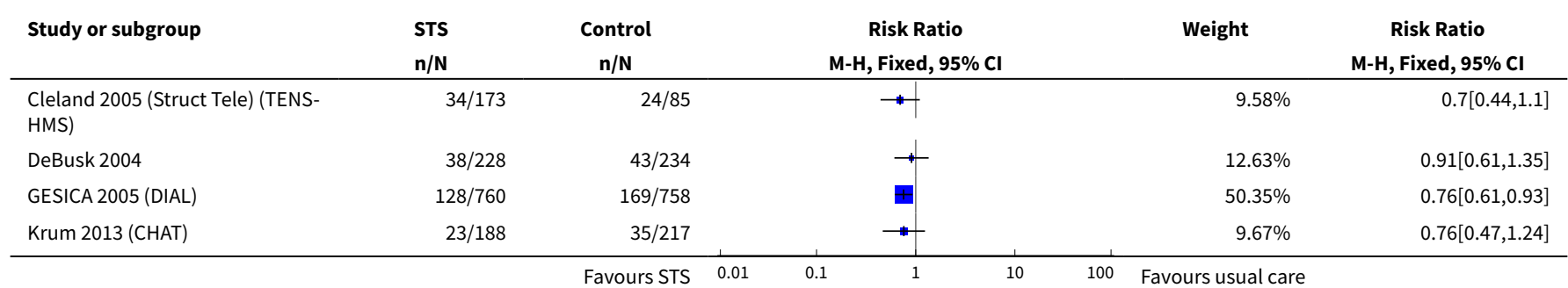




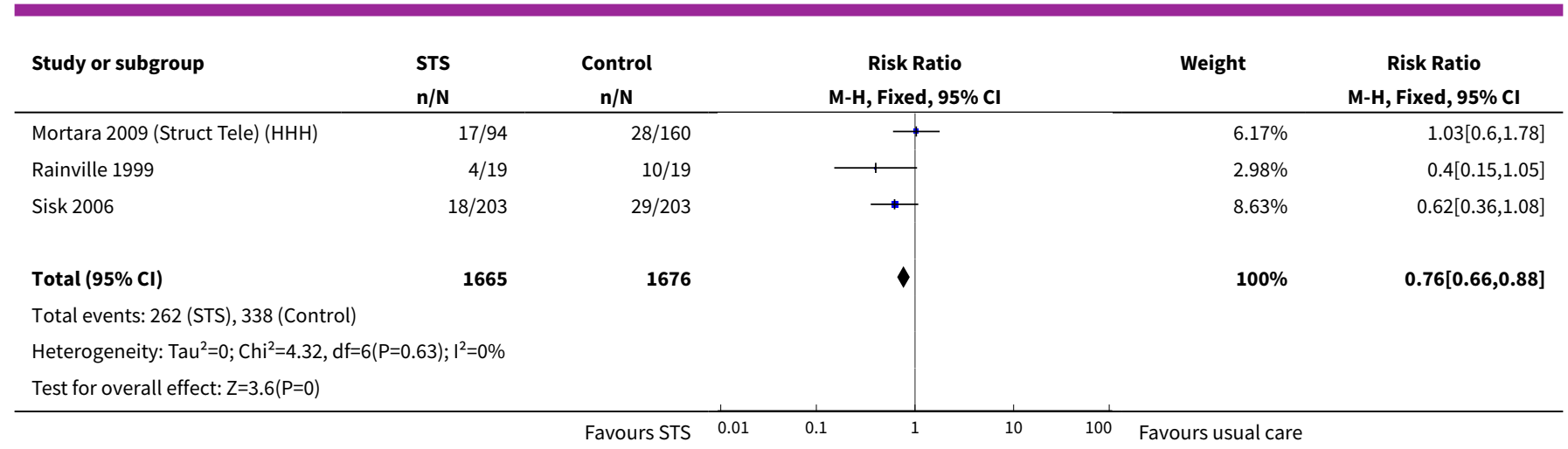

\section{Analysis 3.21. Comparison 3 Impact of structured telephone support or telemonitoring in heart failure on risk of heart failure-related hospitalisation, Outcome 21 Sensitivity analysis follow-up period (> 6 months), CHF-related hospitalisation: TM vs UC.}

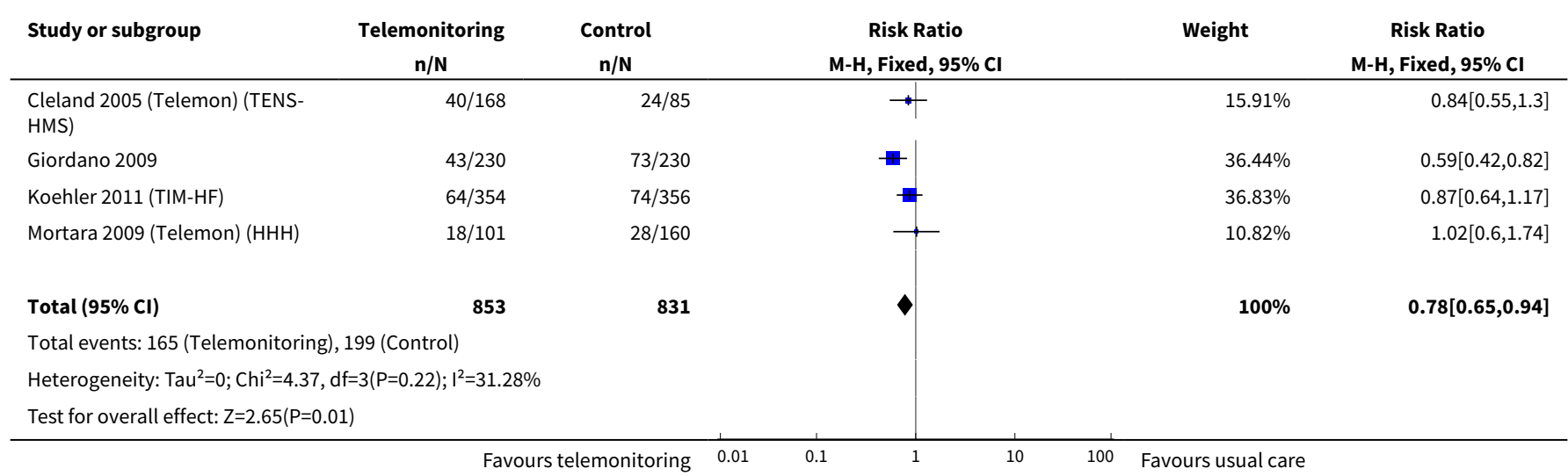

\section{ADDITIONAL TABLES}

Table 1. Secondary Outcomes - Length of Stay (LOS)

\begin{tabular}{lll}
\hline Study & Length of Stay & P value
\end{tabular}

\section{Structured telephone support}

Chaudhry 2010 (Tele- Mean total hospital days: Intervention vs Control: $7.2 \pm 14.6$ vs $7.0 \pm 14.9 \quad$ NS HF)

$P=0.27$

\begin{tabular}{ll}
\hline Galbreath 2004 & No statistical changes in participant bed days between groups \\
& $P=0.899$ (Group effect) \\
$P=0.117$ (Time effect) \\
\hline
\end{tabular}

Laramee 2003

Intervention vs Control: mean (SD) days of hospitalisation: $6.9(6.5)$ vs $9.5(9.8) \quad$ NS

$P=0.15$ 


\section{Table 1. Secondary Outcomes - Length of Stay (LOS) (Continued)}

Riegel $2002 \quad$ Heart failure hospital days (6 months): Intervention vs Control: mean (SD): $1.1 \quad$ NS

(3.1) vs $2.1(4.6)$

All-cause days (6 months): Intervention vs. Control: mean (SD): 3.5 (6.6) vs 4.8

$P=0.05$ (with covariate)

$\mathrm{P}=0.23$ (with covariate)

\begin{tabular}{lll}
\hline Riegel 2006 & Heart failure hospital days (6 months): Intervention vs Control: mean (SD, 95\% & NS \\
& Cl) & P value not reported
\end{tabular}

\begin{tabular}{|c|c|c|}
\hline Tsuyuki 2004 & $\begin{array}{l}\text { Cardiovascular hospital days ( } 6 \text { months): Intervention vs Usual care: mean (SD) } \\
6.4 \text { (6.0) vs } 11.6 \text { (10.3) } \\
\text { Total days } 341 \text { vs } 812 \\
\text { All-cause days ( } 6 \text { months): Intervention vs Usual care: Total days } 627 \text { vs } 1082 \\
\text { days }\end{array}$ & $\begin{array}{l}\downarrow \text { LOS } \\
P=0.003 \\
P=0.003 \\
P=0.001\end{array}$ \\
\hline Wakefield 2008 & $\begin{array}{l}\text { Quote: "No statistically significant differences in the mean number of hospital } \\
\text { days or the mean number of urgent care visits among the three groups, or be- } \\
\text { tween the combined intervention and control groups". }\end{array}$ & NS \\
\hline
\end{tabular}

\section{Telemonitoring}

\begin{tabular}{|c|c|c|}
\hline Balk 2008 & $\begin{array}{l}\text { Total days in hospital: Intervention vs Control } 759(0-116) \text { vs } 762(0-132) \text { : } \\
\text { Mean } 7.4 \text { vs } 7.9 \text { days }\end{array}$ & $\begin{array}{l}\text { NS } \\
\text { P value not reported }\end{array}$ \\
\hline Blum 2014 (MCCD) & $\begin{array}{l}\text { Total mean days in hospital (all-cause): Intervention vs Control } 40 \pm 74 \text { vs. } 24 \pm \\
35 \\
\text { Total mean days in hospital (heart failure-related): Intervention vs Control } 21 \pm \\
26 \text { vs. } 18 \pm 17\end{array}$ & $\begin{array}{l}\text { NS } \\
P=0.12 \\
P=0.75\end{array}$ \\
\hline $\begin{array}{l}\text { Dendale } 2012 \text { (TEMA- } \\
\text { HF1) }\end{array}$ & $\begin{array}{l}\text { Heart failure-related hospitalisation LOS: Intervention vs Control: } 2.5 \text { vs } 4.6 \\
\text { All-cause hospitalisation LOS: Intervention vs. Control: } 7.1 \text { vs } 8.0\end{array}$ & $\begin{array}{l}\text { NS } \\
P=0.10 \\
P=0.65\end{array}$ \\
\hline
\end{tabular}

Koehler 2011 (TIM-HF) Number of days in hospital (all-cause): Intervention vs Control: $16.7 \pm 32.3$ vs NS $13.7 \pm 22.7$

Number of days in hospital (heart failure-related): Intevention vs Control: $5.3 \pm$ 18.1 vs $4.9 \pm 13.2$

\begin{tabular}{|c|c|c|}
\hline Lyngå 2012 (WISH) & $\begin{array}{l}\text { Mean total days in hospital for cardiac disease: Intervention vs Control: } 7.5 \text { vs } \\
7.7\end{array}$ & $\begin{array}{l}\text { NS } \\
P=0.90\end{array}$ \\
\hline $\begin{array}{l}\text { Scherr } 2009 \text { (MOBI- } \\
\text { TEL) }\end{array}$ & $\begin{array}{l}\text { Length of stay: Intervention vs Control: median } 6.5 \text { days (IQR } 5.5-8.3 \text { ) vs } 10 \\
\text { days (IQR } 7.0 \text { - 13.0) }\end{array}$ & $\begin{array}{l}\downarrow \text { LOS with intervention } \\
P=0.04\end{array}$ \\
\hline Soran 2008 & $\begin{array}{l}\text { Heart failure hospital days ( } 6 \text { months): Intervention vs Control Mean (SD) } 9.3 \\
\text { (12.2) vs } 10.0(7.3)\end{array}$ & $\begin{array}{l}\text { NS } \\
P=0.22\end{array}$ \\
\hline Villani 2014 (ICAROS) & Hospitalisation > 3 days for heart failure: Intervention vs Control: 23 v 12 & $\begin{array}{l}\downarrow \text { number of hospitali- } \\
\text { sations }>3 \text { days }\end{array}$ \\
\hline
\end{tabular}


Table 1. Secondary Outcomes - Length of Stay (LOS)

(Continued)

$$
\mathrm{P}<0.03
$$

\begin{tabular}{|c|c|c|}
\hline Vuorinen 2014 & $\begin{array}{l}\text { Heart failure-related hospital days: Intervention vs Control: } 0.7 \text { (SD 2.4) vs } 1.4 \\
\text { (SD 3.5) } \\
\text { Quote: "No difference was found in the number of HF-related hospital days (in- } \\
\text { cidence rate ratio [IRR]=0.812)." }\end{array}$ & $\begin{array}{l}\text { NS } \\
P=0.351\end{array}$ \\
\hline Woodend 2008 & Mean total days in hospital: Intervention vs. Control ( 1 year): 7.13 vs 6.71 days & $\begin{array}{l}\text { NS } \\
\text { P value not reported }\end{array}$ \\
\hline Structured telephone & port and telemonitoring & \\
\hline $\begin{array}{l}\text { Cleland } 2005 \text { (Struct } \\
\text { Tele) (TENS-HMS) }\end{array}$ & $\begin{array}{l}\text { Heart failure hospitalisations ( } 240 \text { days) median (IQR) usual care }=11(6-20) \\
\text { nurse telephone support }=15(7-29) \text { home telemonitoring = } 11(6-19)\end{array}$ & $\begin{array}{l}\text { NS } \\
\text { P value not reported }\end{array}$ \\
\hline $\begin{array}{l}\text { Mortara } 2009 \text { (Struct } \\
\text { Tele) (HHH) }\end{array}$ & $\begin{array}{l}\text { No significant effect of home telemonitoring in reducing bed-days occupancy. } \\
\text { Quote: " There was no significant effect of HT in reducing bed-days occupancy } \\
\text { for HF". } \\
\text { Total days in hospital for heart failure: Usual care } 584 \text { days }(1.0 \%) \text { vs Home } \\
\text { telemonitoring } 1175 \text { Days (1.1\%), Strategy } 1: 477(1.2 \%) \text {, Strategy } 2: 374(1.2 \%) \text {, } \\
\text { Strategy 3: } 324(1.0 \%) \text {. }\end{array}$ & $\begin{array}{l}\text { NS } \\
\text { P value not reported }\end{array}$ \\
\hline
\end{tabular}

IQR: inter-quartile range; LOS: length of stay; NS: not significant; SD: standard deviation.

Table 2. Secondary Outcomes - Health-related quality of life (HRQOL)

\begin{tabular}{|c|c|c|}
\hline Study & HRQoL outcomes & P value \\
\hline \multicolumn{3}{|c|}{ Structured telephone support } \\
\hline \multirow[t]{2}{*}{ Angermann 2012 (INH) } & $\uparrow$ SF-36 (SF-36 Physical Health Component and Physical Functioning). & $\uparrow Q \circ L$ \\
\hline & & $\begin{array}{l}P=0.03 \text { (both compo- } \\
\text { nents) }\end{array}$ \\
\hline \multirow[t]{2}{*}{ Baker 2011} & $\begin{array}{l}\text { Improving Chronic Illness Care Evaluation (ICICE) Heart Failure Symptom Scale } \\
\text { (HFSS) }\end{array}$ & $\begin{array}{l}\uparrow Q O L \\
P=0.001\end{array}$ \\
\hline & $\begin{array}{l}\text { Quotes: "HFQOL improved from } 58.5 \text { to } 64.6 \text { for the TTG (intervention) group } \\
\text { but did not change for the BEI (control) group ( } 64.7 \text { to } 63.9 ; P<.001 \text { for the dif- } \\
\text { ference in change scores)." "The change was }-0.6 \text { versus } 6.7 \text {, respectively, for } \\
\text { the BEI and the TTG groups ( } P<0.001 \text { for comparison of the change between } \\
\text { the } 2 \text { groups". }\end{array}$ & \\
\hline \multirow[t]{2}{*}{ Barth 2001} & $\uparrow M L W H F Q$ pre and post-intervention for experimental group. & $\uparrow Q o L$ \\
\hline & $\begin{array}{l}\text { Quote: "Participants in the experimental group showed significant improve- } \\
\text { ment in the physical dimension }(\mathrm{t}=6.63, \mathrm{p} \leq .0005) \text {, the emotional dimension ( } \mathrm{t} \\
=4.55, \mathrm{p} \leq .0005) \text {, and the total LHFQ score }(\mathrm{t}=7.80, \mathrm{p} \leq .0005) \text { ". }\end{array}$ & $P=0.0005$ \\
\hline \multirow[t]{2}{*}{ Brandon 2009} & $\uparrow M L W H F Q$ & $\uparrow Q o L$ \\
\hline & $\begin{array}{l}\text { Quote: "Intervention group reported more improvement in overall QoL, and } \\
\text { the control group reported a decrease in overall quality of life". }\end{array}$ & $P=0.026$ \\
\hline
\end{tabular}


Table 2. Secondary Outcomes - Health-related quality of life (HRQOL) (Continued)

DeWalt 2006
Modified MLWHFQ

Quote: "In unadjusted analysis, the control group, on average, improved 5 points on the MLHF and the intervention group improved by 1 point. The difference was not statistically significant ( 3.5 points, $95 \% \mathrm{Cl} 11,-4, \mathrm{p}=0.36$ ). After adjusting for baseline differences between the groups, the difference was 2 points $(95 \% \mathrm{Cl} 9,-5, \mathrm{p}=0.59)$ suggesting no effect on heart failure-related quality of life".
NS

$P=0.36$ (unadjusted)

$\mathrm{P}=0.59$ (adjusted)

Quote: "Analysis of the SF-36 health transition measure showed a positive effect of the intervention on self-reported improvement in health at 6 months and at 12 months $(P=0.04$ and $P=0.004$, respectively). However, no effect of disease management was observed across any of the SF-36 components. Women and patients with diastolic heart failure had poorer HRQL scores."

$\uparrow Q O L$

$P=0.04$ (6 months)

$P=0.004$ (12 months) (reported in Smith 2005)

\section{Improved MLWHFQ}

Mean total score, intervention vs control 30.6 vs 35.0 (mean difference $=4.4$, $95 \% \mathrm{Cl} 1.8$ to 6.9$)$

\section{$\uparrow Q \circ L$}

$P=0.001$

\begin{tabular}{lll}
\hline GESICA 2005 (DIAL) & Improved MLWHFQ & $\uparrow Q 0 L$ \\
& Mean total score, intervention vs control 30.6 vs 35.0 (mean difference =4.4, & $\mathrm{P}=0.001$ \\
& $95 \% \mathrm{Cl} 1.8$ to 6.9$)$ &
\end{tabular}

Ramachandran 2007

\section{$\uparrow K C C Q$}

Quote: "There was no significant change in the mean HRQoL score of the control group during the study period (62.2 [22.6] to 63.4 [21.9]; $p=0.69$. However, there was a significant change in the intervention group $(0.60[23.6]$ to 76.3 $[17.3] ; p<0.05 "$

\section{$\uparrow Q o L$}

$\mathrm{P}<0.05$

\section{MLWHFQ}

NS

EQ-5D

Quote: "No beneficial effect was seen in the disease-specific or generic measures of HRQL".

\begin{tabular}{|c|c|c|}
\hline \multirow[t]{3}{*}{ Sisk 2006} & $\begin{array}{l}\uparrow M L W H F Q \\
\uparrow S F-12\end{array}$ & $\begin{array}{l}\text { Significant,(overtime } \\
\text { for the intervention) P } \\
\text { value not reported. }\end{array}$ \\
\hline & Physical component score (39.9 vs 36.3 , difference $3.6(\mathrm{Cl} 1.2$ to 6.1$)$ & \\
\hline & MLWHFQ (38.6 vs 47.3 , difference $-8.8(95 \% \mathrm{Cl}-15.3$ to -2.2$)$ & \\
\hline \multirow[t]{2}{*}{ Wakefield 2008} & $\uparrow M L W H F Q$ & $\uparrow Q o L$ \\
\hline & $\begin{array}{l}\text { Quote: "For all groups, quality of life scores significantly improved over time } \\
(\mathrm{F}=8.90, \mathrm{p}=0.0002) \text {. The magnitude of change was greatest in the telephone } \\
\text { group, followed by the videophone and control groups, although there were } \\
\text { no statistically significant differences among the three groups at baseline, } 3 \text {, } \\
\text { or } 6 \text { months. Post-hoc comparisons did not identify any significant differences } \\
\text { among the groups in pairwise comparisons." }\end{array}$ & $\begin{array}{l}P=0.0002 \text { (over time for } \\
\text { all groups) }\end{array}$ \\
\hline
\end{tabular}

\section{Telemonitoring}

\begin{tabular}{|c|c|c|}
\hline Antonicelli 2008 & $\begin{array}{l}\text { SF-36 } \\
\text { Quote: "...patients randomized to home telemonitoring were characterized } \\
\text { at follow-up by a significantly better reported health perception }(\mathrm{HP}) \text { score } \\
\text { as compared to baseline. This improvement was significantly greater as com- } \\
\text { pared to controls }(\mathrm{P}<0.046) . "\end{array}$ & $\begin{array}{l}\text { NS for component } \\
\text { scores. } P<0.046 \text { for } \\
\text { health perception over- } \\
\text { time with telemonitor- } \\
\text { ing. }\end{array}$ \\
\hline Balk 2008 & SF-36 & NS \\
\hline
\end{tabular}


Table 2. Secondary Outcomes - Health-related quality of life (HRQoL) (Continued)

MLWHFQ

Quote: "No differences in quality of life and self care behaviour were found between the Control group and the Intervention group either at the start or at the end of the study".

\begin{tabular}{ll}
\hline Biannic 2012 (SEDIC) & MLWHFQ \\
& $\begin{array}{l}\text { No difference between groups in quality of life (-10 in control and -11.9 in inter- } \\
\text { vention). }\end{array}$
\end{tabular}

\begin{tabular}{lll}
\hline Blum 2014 (MCCD) & Quote: "Scores for SF-36 and MLWHFQ improved ( $<<0.001)$ overtime, but there & 个QoL \\
& were no differences between groups". & Overtime: \\
& $\uparrow S F-36$ composite physical and mental scores & $P<.001$ \\
& $\uparrow M L W H F Q$ total, physical, and emotional scores improved statistically over & Between groups: NS \\
& the year with the 3 measurements & \\
& $\uparrow S F 36$ Mental composite and Physical composites improved & \\
& No difference between groups.
\end{tabular}

De Lusignan $2001 \quad$ CHFSQ NS

GHQ

Quote: "There is only a marginal difference between the telemedicine and control groups $\mathrm{CHF}$ questionnaire results there is no significant difference between the groups".

\section{Goldberg 2003}

(WHARF)

\section{MLWHFQ}

SF-12

HDS

All scores were improved but were not statistically significant.

Quote: "...patients in both groups experienced improvement between baseline and 6 months in their Minnesota Living with Heart Failure, SF-12 and Health Distress scores. Although no difference was statistically significant, the intervention group trended towards improvement in all quality of life measures".
Mean HRQoL at 12 months Intervention vs. Control: $54.3 \pm 1.2$ vs $49.9 \pm 1.2$

Mean HRQoL at 24 months Intervention vs. Control: $53.8 \pm 1.4$ vs. $51.7 \pm 1.4$

\section{$\begin{array}{ll}\text { Koehler } 2011 \text { (TIM-HF) } & \text { Quote: "Patients randomly allocated to the RTM (intervention) group com- } \\ & \text { pared with the usual care group showed an improved score for SF-36 physical }\end{array}$ \\ $\begin{array}{ll}\text { Koehler } 2011 \text { (TIM-HF) } & \begin{array}{l}\text { Quote: "Patients randomly allocated to the RTM (intervention) group com- } \\ \text { pared with the usual care group showed an improved score for SF-36 physical }\end{array}\end{array}$ functioning over the entire study period $(P<0.05)$." \\ $\uparrow Q O L$ \\ $\mathrm{P}<0.05$}

SF-12

Physical component score Intervention vs Control: $(31.3+9.0)$ vs $(31.6+8.0) \mathrm{P}$

$=0.76$

Mental component score Intervention vs Control: $(45.0+11.0)$ vs $(44.1+10.8) \mathrm{P}$

$=0.46$

Seto $2012 \quad$ 个MLHFQ

$\uparrow Q \circ L$

$\uparrow M L H F Q$ 
Table 2. Secondary Outcomes - Health-related quality of life (HRQoL) (Continued)

Quote: "The telemonitoring group had better post-study quality of life (emotional dimension)".

Physical $P=0.02$; Emotional $\mathrm{P}=0.03$

Quote: "Quality of life significantly improved for only the telemonitoring group $(P=0.02)$ including physical and emotional dimensions $(P=0.03)$ compared to overtime for telemonitoring only. standard care group $(\mathrm{P}=0.05)$ ".

Soran 2008 SF-12 NS

KCCQ

Quote: "Mean values for quality of life, as measured by the KCCQ and the SF-12, were also similar by treatment arm".

\section{个MLWHFQ}

Quote: "Patients randomized to receive telehome care had significantly better functional status on both the overall score (P .003) and the physical subscale $(P .001)$ of the LiHFe at 3 months than patients receiving usual care. There was no significant difference between randomized groups in the emotional subscale at 3 months."

\section{SF-36 improved}

Quote: "The only subscale on which telehome-monitored patients had significantly better quality of life at all three points in time was the vitality subscale,which reflects the subject's energy level and fatigue. Patients with HF in both randomized groups demonstrated significant improvements in quality of life over time in all of the SF-36 subscales"
NS

$\uparrow Q O L(M L W H F Q)$

Overall score $\mathrm{P}=0.003$

Physical $\mathrm{P}=0.001$ overtime with telemonitoring only.

SF-36 (all participants, overtime, all SF-36 subscales: $\mathrm{P}<0.05$

CHFSQ: Chronic Heart Failure Symptomatology Questionnaire; GHQ: General Health Questionnaire; ICICE: Improving chronic illness care evaluation; HDS: Health Distress Score; HRQoL: Health Related Quality of Life; HFSS: Heart Failure Symptom Scale (HFSS); ; KCCQ: Kansas City Cardiomyopathy Questionnaire; LiHFe: Minnesota Living with Heart Failure Questionnaire; MLWHFQ: Minnesota Living with Heart Failure Questionnaire; NS: not significant; SF-12: Short Form 12-Item; SF-36: Short Form 36-Item

Table 3. Secondary Outcomes - Cost of the intervention and cost effectiveness

Study Cost Outcomes

\section{Structured telephone support}

Barth 2001

Quote: "Data on unexpected office visits, emergency room visits, and readmis-

NS sions due to exacerbation of $\mathrm{CHF}$ plus costs were collected. No significant differences were found."

Cost of the intervention: USD 23.60 per participant.

Galbreath 2004
(reported in Smith
2008)

Quote: "Repeated-measures ANOVA for cost by group showed no significant NS differences, even in the higher NYHA class groups."

Quote: "Analyses of direct medical and intervention costs showed no cost savings associated with the intervention."

Cost of the intervention: Quote: "Based on the total cost of labor, benefits, and office and operating expenses, as well as the number of patient-months for which services were provided, the mean cost of DM services per patient per month was $\$ 246 "$. 
Table 3. Secondary Outcomes - Cost of the intervention and cost effectiveness (Continued)

Laramee 2003

Quote: "Total inpatient and outpatient median costs were slightly less for the intervention group ( $\$ 15979$ vs $\$ 18662, \mathrm{P}=0.14) "$

Cost of the intervention: not reported.

\begin{tabular}{|c|c|c|}
\hline Ramachandran 2007 & Cost of the intervention: INR 7680 per participant annually & \\
\hline Riegel 2002 & $\begin{array}{l}\text { USD } 443 \text { per participant. } \\
46 \% \text { reduction in inpatient costs. } \\
\text { Cost of the intervention: Quote: "The intervention was calculated to cost } \$ 443 \\
\text { per patient, if the cost of training is included". }\end{array}$ & $\begin{array}{l}\downarrow \text { Cost } \\
P=0.04\end{array}$ \\
\hline Riegel 2006 & $\begin{array}{l}\text { NS difference in HF cost of care or all-cause hospital costs. } \\
\text { Cost of the intervention: not reported. }\end{array}$ & NS \\
\hline $\begin{array}{l}\text { Sisk } 2006 \\
\text { (reported in Herbert } \\
\text { 2008) }\end{array}$ & $\begin{array}{l}\text { Quote" "Intervention cost } \$ 2177 \text { per patient were more than offset by reduced } \\
\text { hospital costs ( } \$ 2378 \text { per patient) but higher costs for outpatient procedures, } \\
\text { medications, and home health care but higher costs for outpatient proce- } \\
\text { dures, medications, and home health care prevented the intervention from be- } \\
\text { ing cost-saving over the } 12 \text {-month study." }\end{array}$ & NS \\
\hline Tsuyuki 2004 & $\begin{array}{l}\text { CAD } 2531 \text { per participant reduction with the intervention. P value not report- } \\
\text { ed. } \\
\text { Cost of the intervention: not reported. }\end{array}$ & $\downarrow$ Cost \\
\hline Wakefield 2008 & $\begin{array}{l}\text { Mean heart failure-related readmission costs were } 86 \% \text { and } 84 \% \text { lower in the } \\
\text { videophone and telephone groups, respectively, compared to usual care. } \\
\text { Cost of the intervention: not reported. }\end{array}$ & $\downarrow$ Cost \\
\hline
\end{tabular}

\section{Telemonitoring}

Balk 2008 Increase in costs in the Intervention group. $\quad \uparrow$ Cost

Quote: "With no decrease in hospital days or health care consumption and with the extra costs of the MOTIVA system and the Medical Service Centre, overall costs were higher in the Intervention group than the Control group."

Cost of the intervention: not reported.

Blum 2014 (MCCD) Total mean Medicare payment per participant (all-cause hospitalisation): Intervention vs Control: USD 64,788 \pm USD 100,452 vs USD 40,480 \pm USD 58,572 $(P=0.99)$

Total mean Medicare payment per participant (heart failure-related hospitalisations): Intervention vs Control: USD 33,944 \pm USD 29,804 vs USD 32,914 \pm USD 30,575 ( $P=0.83)$.

Cost of the intervention: not reported.

\begin{tabular}{lll}
$\begin{array}{l}\text { Dendale } 2012 \text { (TEMA- } \\
\text { HF1) }\end{array}$ & $\begin{array}{l}\text { Total cost of hospitalisation: Intervention vs Control: EUR 2557 (4094) vs EUR } \\
2643(4363) ; \mathrm{P}=0.90\end{array}$ & NS \\
& Cost of the intervention: not reported. \\
\hline Giordano 2009 & $\begin{array}{l}\text { Mean cost for hospital readmission was significantly lower in HBT group (EUR } \\
843 \pm 1733) \text { than in UC group (EUR 1298 } \pm 2322),(-35 \%, P<001) .\end{array}$
\end{tabular}


Table 3. Secondary Outcomes - Cost of the intervention and cost effectiveness (Continued)

Cost of the intervention: daily cost per participant of intervention was EUR 0.65 and mean annual cost per participant was EUR 185. 2010)

Soran 2008
(reported in Soran
2010)

Quote: "We found that both Medicare and total costs were significantly higher for patients who were randomized to the HFMS arm than they were for the patients randomized to the SC arm. The 6-month mean total costs were estimated to be $\$ 20,190$ and $\$ 15,966$ for the HFMS and the SC groups, respectively, whereas the 6-month mean Medicare costs were estimated to be $\$ 17,837$ and $\$ 13,886$, respectively. In fact, we found that costs were higher for most types of costs -inpatient costs, physician costs, home health costs, and outpatient costfor patients who were randomized to the HFMS than they were for patients randomized to SC. The difference in costs was much higher than the cost of the intervention."

Total cost to Medicare programme: Intervention vs Control: USD 17,838 (USD 20,559) vs USD 13, 886 (USD 17,556).

Total medical cost: Intervention vs. Control: USD 20,191 (USD 22,089) vs USD 15,967 (USD 18,878).

$\begin{array}{lll}\text { Villani } 2014 \text { (ICAROS) } & \text { Intervention costs (Intervention vs Control): USD 235,635 vs USD 81,275 } & \uparrow \text { Cost } \\ & \text { Total cost of management (Intervention vs. Control): USD 354,424 vs USD } & \text { P }<0.05 \\ & 284,464 & \end{array}$

NS: not significant

Table 4. Secondary Outcomes - Adherence to intervention

\begin{tabular}{ll}
\hline Study Adherence \\
\hline Structured telephone support
\end{tabular}

Angermann 2012 (INH) Participant compliance with self monitoring and drug adherence was high both at baseline and follow-up $(85 \pm 17 \%$ and $84 \pm 19 \%)$.

Chaudhry 2010 (Tele-HF) Quote: "...85.6\% of patients in the telemonitoring group made at least one call; among these patients, adherence to the intervention was highest, $90.2 \%$, during the first week of the study period and decreased to $55.1 \%$ by week 26 . A total of 29,163 variances were generated during the study period, with a median of 21 (interquartile range, 5 to 54 ) per patient."

Krum 2013 (CHAT) Adherence to intervention, 65.8\% $(P=0.001)$.

(reported in Clark 2007)

\begin{tabular}{ll}
\hline Laramee 2003 & Adherence to treatment plan (daily weighs) $(\mathrm{P}<0.01)$. \\
\hline Telemonitoring & $\begin{array}{l}\text { Adherence: mean of } 6 \text { days per week (95\% Cl } 5.93 \text { to } 6.03) \text { the } 1 \text { st year to a low of } 5 \text { days a week } \\
(95 \% \mathrm{Cl} 4.76 \text { to } 5.33) \text { in the 4th year. }\end{array}$ \\
\hline Blum 2014 (MCCD) & Compliance to telemonitoring was $82 \%$. \\
\hline De Lusignan 2001 & The telemedicine group was sufficiently motivated to record their weight $75 \%$ of the time. \\
& Blood pressure was measured 90\% of the time. \\
\hline
\end{tabular}


Table 4. Secondary Outcomes - Adherence to intervention (Continued)

\begin{tabular}{ll} 
Goldberg 2003 (WHARF) & Compliance with the monitoring system was $98.5 \%$. \\
\hline Koehler 2011 (TIM-HF) & $\begin{array}{l}\text { Quote: "RTM patients: } 81 \% \text { were } \geq 70 \% \text { compliant with daily data transfers and no break for }>30 \\
\text { days (except during hospitalisations)". }\end{array}$
\end{tabular}

\begin{tabular}{|c|c|}
\hline Lyngå 2012 (WISH) & Compliance with daily weighing: quote: "Mean compliance rate of $75 \% "$. \\
\hline Scherr 2009 (MOBITEL) & Adherence rate with the telemonitoring system: $95 \%$. \\
\hline Seto 2012 & $\begin{array}{l}\text { Quote: "...42, 33, and } 16 \text { out of the } 50 \text { telemonitoring group patients ( } 84 \%, 66 \% \text {, and } 32 \%) \text { complet- } \\
\text { ed at least } 91(50 \%), 146(80 \%) \text {, and } 173(95 \%) \text { of possible daily readings over the six months". }\end{array}$ \\
\hline & $\begin{array}{l}\text { Quote: "By the final week of our trial, } 89 \% \text { of our patients were taking their measurements at least } 3 \\
\text { times per week". }\end{array}$ \\
\hline
\end{tabular}

\begin{tabular}{ll}
\hline Soran 2008 & Compliance with the monitoring intervention: $97 \%$. \\
\hline Vuorinen 2014 & $\begin{array}{l}\text { Quote: "Patients adherence to the weekly reporting plan was close to } 90 \% " . \\
\text { Quote: "Adherence, calculated as a proportion of weekly submitted self-measurement, was } 86 \% \text { in } \\
\text { weight reporting and } 89 \% \text { in BP, HR and symptom reporting". }\end{array}$
\end{tabular}

\section{Structured telephone support and telemonitoring}

$\begin{aligned} & \text { Cleland } 2005 \text { (Struct Tele) } \\ & \text { (TENS-HMS) }\end{aligned} \quad \begin{aligned} & \text { Home telemonitoring had 80\% compliance with at least } 1 \text { daily measurement (weight or blood } \\ & \text { pressure). 55\% had } 80 \% \text { compliance with twice-daily measurements. }\end{aligned}$

$\begin{array}{ll}\begin{array}{l}\text { Mortara } 2009 \text { (Struct Tele) } \\ \text { (HHH) }\end{array} & \begin{array}{l}\text { Participants completed } 81 \% \text { of all practicable vital signs transmissions from home. Overall, 92\% of } \\ \text { practicable recordings were carried out by the participants. }\end{array}\end{array}$

$\begin{array}{ll}\begin{array}{l}\text { Mortara } 2009 \text { (Struct Tele) } \\ \text { (HHH) }\end{array} & \begin{array}{l}\text { Participants completed } 81 \% \text { of all practicable vital signs trans } \\ \text { practicable recordings were carried out by the participants. }\end{array}\end{array}$

Table 5. Secondary Outcomes - Acceptability (Satisfaction and Usability)

Study Acceptability of the intervention

\section{Structured telephone support}

\begin{tabular}{ll}
\hline $\begin{array}{l}\text { Krum } 2013 \text { (CHAT) } \\
\text { (reported in Clark 2007) }\end{array}$ & Participants assessed the CHAT project with a total acceptability rate of $76.45 \%$. \\
\hline Laramee 2003 & Satisfaction $(\mathrm{P}<0.01)$. \\
\hline Riegel 2002 & $\begin{array}{l}\text { Satisfaction was significantly higher among people assigned to the intervention group than among } \\
\text { those assigned to the usual-care group }(\mathrm{P}=0.01) .\end{array}$ \\
\hline Wakefield 2008 & Satisfaction between video and telephone (NS). \\
\hline Telemonitoring & $\begin{array}{l}\text { The ease of use was rated very good or good by } 80 \% . \\
70 \% \text { of the users mentioned that their access to doctors and nurses was better with remote patient } \\
\text { management compared to the service they had received before. }\end{array}$
\end{tabular}




\section{Table 5. Secondary Outcomes - Acceptability (Satisfaction and Usability) (Continued)}
Koehler 2011 (TIM-HF)
Usability of telemedical devices 224 (98.6\%), robustness (88.8\%), accessibility of the $24 / 7$ telemoni-
(reported in Prescher 2013) toring centre $193(84.6 \%)$

Vuorinen 2014

Quote: "The technology received excellent feedback from the patient and professional side with a high adherence rate throughout the study". "95\% of all patients found that making and reporting measurements with the mobile phone app was 'very useful' or 'quite useful'".

Woodend 2008

Overall participants found the equipment easy to use. 92 - $97 \%$ very highly satisfied.

\section{Structured telephone support and telemonitoring}

Cleland 2005 (Struct Tele) 96\% of participants were well satisfied with the system.

(TENS-HMS)

NS: not significant

Table 6. Secondary Outcomes - Heart failure knowledge and self care as assessed by validated questionnaires

$\begin{array}{lll}\text { Study Heart Failure knowledge and Self-care } & \text { Pvalue }\end{array}$

Structured telephone support

\begin{tabular}{|c|c|c|}
\hline Baker 2011 & $\begin{array}{l}\text { Adapted version of the ICICE telephone survey. } \\
\text { Quote: "The TTG (intervention) had greater improvements in } \\
\text { general and salt knowledge }(P<0.001 \text { ) and greater increases in } \\
\text { self-care behaviours (from mean } 4.8 \text { to } 7.6 \text { for TTG vs. } 5.2 \text { to } 6.7 \\
\text { for BEI (control) } P<0.001 \text { )." Adapted ICICE telephone survey. }\end{array}$ & $\begin{array}{l}\uparrow \text { Self care and knowledge } \\
P<0.001\end{array}$ \\
\hline Brandon 2009 & $\begin{array}{l}\text { Self-care behaviour scale. } \\
\text { Quote: "Improvement in the mean SCB score for the interven- } \\
\text { tion group (from } 95.9 \text { to } 128) \text {, whereas the control group's SCB } \\
\text { mean score did not change }(m=94)(P=0.009) " .\end{array}$ & $\begin{array}{l}\uparrow \text { Self care } \\
P=0.009\end{array}$ \\
\hline DeWalt 2006 & $\begin{array}{l}\text { S-TOFHLA } \\
\text { Quote: "Heart failure related knowledge improved more in the } \\
\text { intervention group than in the control group. Mean difference } \\
\text { in score improvement was } 12 \text { percentage points ( } 95 \% \mathrm{Cl} 6,18 ; \mathrm{p} \\
<0.001) . " \\
\text { Quote: "Heart failure self-efficacy improved more in the inter- } \\
\text { vention group than in the control group. Mean difference in } \\
\text { score improvement was } 2 \text { points ( } 95 \% \mathrm{Cl} 0.7,3.1 ; \mathrm{p}=0.0026) . " \\
\text { Quote: "Significantly more patients in the intervention group } \\
\text { than in the control group reported daily weight measurement } \\
\text { at } 12 \text { months ( } 79 \% \text { vs. } 29 \%, \mathrm{p}<0.001) . "\end{array}$ & $\begin{array}{l}\uparrow H \text { Heart failure knowledge improved } \\
(P=0.001) \\
\uparrow H \text { eart failure self efficacy improved } \\
(P=0.0026) \\
\uparrow H \text { Heart failure self-care behaviour im- } \\
\text { proved } \\
(P<0.001)\end{array}$ \\
\hline
\end{tabular}

Domingues 2011 Heart failure and self-care information questionnaire. Improvement in heart failure awareness and knowledge in both groups. Intervention vs Control from baseline $4.6 \pm 1.9$ to 3 months later $6.1 \pm 2.1$ vs from baseline $4.5 \pm 1.9$ to 3 months later $5.8 \pm 1.9$ (P

$\uparrow$ Heart failure knowledge improved over time for both groups $(P=0.001)$, no signifi$=0.001$ ). 
Table 6. Secondary Outcomes - Heart failure knowledge and self care as assessed by validated

questionnaires (Continu㭁ere were no statistically significant differences between the

two groups $(6.1 \pm 2.1$ versus $5.8 \pm 1.9, \mathrm{P}=0.41)$.

\begin{tabular}{|c|c|c|}
\hline Wakefield 2008 & Knowledge on medications improved. & NS \\
\hline \multirow[t]{2}{*}{ Zamanzadeh 2013} & $\mathrm{SCHFI}$ & $\uparrow$ Self-care behaviours $(P=0.003)$ \\
\hline & $\begin{array}{l}\text { Significant differences in self care between the control and in- } \\
\text { tervention groups and significant difference in self-care behav- } \\
\text { iours over the } 3 \text { months, such that as time progressed self-care } \\
\text { scores among participants in the intervention group continued } \\
\text { to increase. }\end{array}$ & \\
\hline
\end{tabular}

Telemonitoring

\begin{tabular}{|c|c|c|}
\hline Balk 2008 & $\begin{array}{l}\text { Dutch Heart Failure Knowledge Score. } \\
\text { Knowledge about heart failure increased significantly more in } \\
\text { the Intervention group. }\end{array}$ & $\begin{array}{l}\uparrow \text { Heart failure knowledge improved } \\
(P<0.001)\end{array}$ \\
\hline Seto 2012 & $\begin{array}{l}\text { SCHFI } \\
\text { Quote: "A comparison of the post-study data between groups } \\
\text { found only a statistically significant difference in SCHFI mainte- } \\
\text { nance scores, indicating the telemonitoring group had greater } \\
\text { self-care maintenance (ie, a higher SCHFI maintenance score) } \\
\text { (P=.03)." }\end{array}$ & $\begin{array}{l}\uparrow \text { Self-care behaviours } \\
(P=0.03)\end{array}$ \\
\hline Vuorinen 2014 & $\begin{array}{l}\text { European Heart Failure Self-care Behaviour Scale } \\
\text { Quote: "No statistically significant differences in patients clini- } \\
\text { cal status or their self-care behaviour". }\end{array}$ & NS \\
\hline
\end{tabular}

ICICE: Improving chronic illness care evaluation; NS: not significant; S-TOFHLA: Short Test of Functional Health Literacy in Adults; SCHFI: Self-Care Heart Failure Index

\section{APPENDICES}

\section{Appendix 1. Search strategies (2015 update)}

\section{CENTRAL}

\#1MeSH descriptor: [Heart Failure] explode all trees \#2 (heart or cardiac or myocard ${ }^{\star}$ ) near/2 (fail* or insufficien* or decomp*) \#3\#1 or \#2

\#4MeSH descriptor: [Telemedicine] explode all trees \#5MeSH descriptor: [Telecommunications] explode all trees \#6MeSH descriptor: [Case Management] this term only \#7MeSH descriptor: [Comprehensive Health Care] explode all trees \#8MeSH descriptor: [Disease Management] this term only \#9MeSH descriptor: [Home Care Services] this term only \#10MeSH descriptor: [Home Care Services, Hospital-Based] this term only \#11MeSH descriptor: [Nurse Clinicians] this term only $\# 12 \mathrm{MeSH}$ descriptor: [Nurse Practitioners] this term only \#13MeSH descriptor: [Monitoring, Ambulatory] this term only \#14MeSH descriptor: [Clinical Protocols] this term only \#15MeSH descriptor: [Patient Care Planning] this term only \#16tele* \#17(remote near/3 consult ${ }^{\star}$ ) 
\#18disease next management

\#19nurse next led

\#20phone*

\#21 (manage* near/3 program*)

\#22(nurse* near/3 manage*)

$\# 23$ case next management

\#24(home near/3 service*)

\#25nurse next practitioner*

\#26nurse next clinician*

\#27care next plan*

\#28ehealth

\#29mobile next health

$\# 30$ (remote* or distan*) near/2 (care or caring or monitor ${ }^{\star}$ or program ${ }^{\star}$ or help or support ${ }^{\star}$ )

$\# 31 \# 4$ or \#5 or \#6 or \#7 or \#8 or \#9 or \#10 or \#11 or \#12 or \#13 or \#14 or \#15 or \#16 or \#17 or \#18 or \#19 or \#20 or \#21 or \#22 or \#23 or \#24 or \#25 or \#26 or \#27 or \#28 or \#29 or \#30

$\# 32 \# 3$ and \#31

\section{MEDLINE OVID}

1. exp Heart Failure/

2. ((heart or cardiac or myocard $\left.{ }^{\star}\right)$ adj2 (fail ${ }^{\star}$ or insufficien* ${ }^{\star}$ decomp $\left.{ }^{\star}\right)$ ).tw.

3. 1 or 2

4. exp Telemedicine/

5. exp Telecommunications/

6. Case Management/

7. exp Comprehensive Health Care/

8. Disease Management/

9. tele med $^{\star} . t w$.

10. telecare ${ }^{\star}$. tw.

11. telecardiol ${ }^{\star}$.tw.

12. telemonitor ${ }^{\star}$. tw.

13. teleconsult ${ }^{\star}$. tw.

14. teleconferenc ${ }^{*}$.tw.

15. telecommunicat ${ }^{\star}$. .tw.

16. telephon*.tw.

17. telehealth*.tw.

18. telemetry.tw.

19. (remote $^{\star}$ adj3 consult $\left.{ }^{\star}\right)$.tw.

20. tele-med*.tw.

21. tele-consult ${ }^{*}$. tw.

22. tele-conferenc ${ }^{\star}$. tw.

23. tele-health ${ }^{*}$.tw.

24. Home Care Services/

25. Home Care Services, Hospital-Based/

26. disease management.tw.

27. Nurse Clinicians/

28. Nurse Practitioners/

29. nurse led.tw.

30. Monitoring, Ambulatory/

31. telehome.tw.

32. tele-home.tw.

33. phone ${ }^{\star}$.tw.

34. Clinical Protocols/

35. Patient Care Planning/

36. telefon ${ }^{\star}$.tw.

37. telemed ${ }^{\star}$. tw.

38. ehealth.tw.

39. mobile health.tw.

40. ((remote* or distan $\left.^{\star}\right)$ adj2 (care or caring or monitor ${ }^{\star}$ or program ${ }^{\star}$ or help or support $\left.\left.{ }^{\star}\right)\right) . t w$.

41. or/4-40

42. 3 and 41

43. randomized controlled trial.pt. 
44. controlled clinical trial.pt.

45. randomized.ab.

46. placebo.ab.

47. drug therapy.fs.

48. randomly.ab.

49. trial.ab.

50. groups.ab.

51.43 or 44 or 45 or 46 or 47 or 48 or 49 or 50

52. exp animals/ not humans.sh.

53.51 not 52

54. 42 and 53

$55 .\left(200811^{\star}\right.$ or $200812^{\star}$ or $2009^{\star}$ or $2010^{\star}$ or $2011^{\star}$ or $2012^{\star}$ or $2013^{\star}$ or $2014^{\star}$ or $\left.2015^{\star}\right)$.ed.

56.54 and 55

\section{EMBASE OVID}

1. exp heart failure/

2. ((heart or cardiac or myocard $\left.{ }^{\star}\right)$ adj2 (fail ${ }^{\star}$ or insufficien* ${ }^{\star}$ or decomp $\left.{ }^{\star}\right)$ ).tw.

3. 1 or 2

4. exp telemedicine/

5. exp telecommunication/

6. case management/

7. disease management/

8. telemed*.tw.

9. telecare ${ }^{\star} . \mathrm{tw}$.

10. telecardiol ${ }^{\star}$. tw.

11. telemonitor ${ }^{\star}$. tw.

12. teleconsult*.tw.

13. teleconferenc ${ }^{\star}$.tw.

14. telecommunicat ${ }^{\star}$.tw.

15. telephon*.tw.

16. telehealth ${ }^{\star}$. tw.

17. telemetry.tw.

18. (remote ${ }^{\star}$ adj3 consult $\left.{ }^{\star}\right)$.tw.

19. tele-med ${ }^{*}$.tw.

20. tele-consult ${ }^{\star}$. tw.

21. tele-conferenc ${ }^{\star}$.tw.

22. tele-health*.tw.

23. home care/

24. home monitoring/

25. disease management.tw.

26. nurse practitioner/

27. nurse led.tw.

28. ambulatory monitoring/

29. telehome.tw.

30. tele-home.tw.

31. phone*.tw.

32. patient care planning/

33. telefon ${ }^{\star}$. tw.

34. ehealth.tw.

35. mobile health.tw.

36. or/4-35

37.3 and 36

38. random\$.tw.

39. factorial\$.tw.

40. crossover\$.tw.

41. cross over\$.tw.

42. cross-over\$.tw.

43. placebo\$.tw.

44. (doubl\$ adj blind\$).tw.

45. (singl\$ adj blind\$).tw.

46. assign\$.tw. 
47. allocat\$.tw.

48. volunteer\$.tw.

49. crossover procedure/

50. double blind procedure/

51. randomized controlled trial/

52. single blind procedure/

53.38 or 39 or 40 or 41 or 42 or 43 or 44 or 45 or 46 or 47 or 48 or 49 or 50 or 51 or 52

54. (animal/ or nonhuman/) not human/

55.53 not 54

56.37 and 55

57. ("200845" or "200846" or "200847" or "200848" or "200849" or "200850" or "200851" or "200852" or 2009* or 2010* or 2011* or 2012* or $2013^{\star}$ or $2014^{\star}$ or $\left.2015^{\star}\right)$.em.

$58 .\left(2008112^{\star}\right.$ or $2008113^{\star}$ or $200812^{\star}$ or $2009^{\star}$ or $2010^{\star}$ or $2011^{\star}$ or $2012^{\star}$ or $2013^{\star}$ or $2014^{\star}$ or $\left.2015^{\star}\right)$.dd.

59.57 or 58

60.56 and 59

\section{Web of Science}

\#12 \#11 (Timespan=2008-2015)

\#11 \#10 AND \#9

\#10 TS=(random* or blind ${ }^{\star}$ or allocat ${ }^{\star}$ or assign ${ }^{\star}$ or trial ${ }^{\star}$ or placebo* or crossover $^{\star}$ or cross-over $\left.^{\star}\right)$

\#9 \#8 AND \#1

\#8 \#7 OR \#6 OR \#5 OR \#4 OR \#3 OR \#2

\#7 TS=("case management")

\#6 TS=("home care")

\#5 TS=("disease management")

\#4 TS=((nurse same led) or (nurse same practitioner $\left.{ }^{\star}\right)$ or (nurse same clinician*))

\#3 TS=(remote* same consult $\left.^{\star}\right)$

\#2 TS=(tele* or phone ${ }^{\star}$ )

\#1 TS=((heart or cardiac or myocard $\left.{ }^{\star}\right)$ near/2 (fail ${ }^{*}$ or insufficien ${ }^{\star}$ or decomp $\left.\left.{ }^{\star}\right)\right)$

\section{CINAHL}

S36 S34 AND S35

S35 EM 20081110-20150112

S34 S27 AND S33

S33 S28 OR S29 OR S30 OR S31 OR S32

S32 electronic* N6 communicat *

S31 remote $\mathrm{N} 6$ consult*

S30 (tele) N6 (communicat ${ }^{\star}$ or med $^{\star}$ or car $^{\star}$ or monitor $^{\star}$ or consult* or conferenc ${ }^{\star}$ or health or metr or nurs $^{\star}$ )

S29 telecommunicat ${ }^{\star}$ or telemed ${ }^{\star}$ or telecar ${ }^{\star}$ or telemonitor ${ }^{\star}$ or teleconsult* or teleconferenc $^{\star}$ or telehealth* $^{\star}$ or telephon* $^{\star}$ or telemetr $^{\star}$ or phon*

S28 (MH "Telecommunications") OR (MH "Interactive Voice Response Systems") OR (MH "Telecommuting") OR (MH "Teleconferencing") OR (MH "Telefacsimile") OR (MH "Telehealth+") OR (MH "Telephone") OR (MH "Wireless Communications")

S27 S3 AND S26

S26 S4 OR S5 OR S6 OR S7 OR S8 OR S9 OR S10 OR S11 OR S12 OR S13 OR S14 OR S15 OR S16 OR S17 OR S18 OR S19 OR S20 OR S21 OR S22 OR S23 OR S24 OR S25

S25 (MH "Patient Discharge+")

S24 discharge N6 plan*

S23 multidisciplin*

S22 (MH "Nurses") OR (MH "Advanced Practice Nurses+") OR (MH "Case Managers")

$\mathrm{S} 21$ nurs*

S20 (MH "Community Health Nursing") OR (MH "Home Nursing, Professional") OR (MH "Rehabilitation Nursing") OR (MH "Rural Health Nursing")

S19 (MH "Rehabilitation") OR (MH "Rehabilitation, Cardiac+") OR (MH "Rehabilitation, Community-Based")

S18 homecare or rehabilitat*

S17 disease $\mathrm{N} 6$ management

S16 secondary N6 prevent*

S15 home N6 intervention*

S14 (MH "Self Care") OR (MH "Self Administration") OR (MH "Self Medication")

S13 (MH "After Care") OR (MH "Cardiovascular Care") OR (MH "Home Nursing") OR (MH "Nursing Care")

S12 (MH "Patient Care")

S11 (MH "Home Health Care+") 
S10 (MH "Medical Practice") OR (MH "Nursing Practice+") OR (MH "Occupational Therapy Practice") OR (MH "Prescribing Patterns") OR (MH "Prescriptive Authority") OR (MH "Professional Practice, Theory-Based+")

S9 (MH "Health Maintenance Organizations")

S8 (MH "Managed Care Programs+")

S7 manag* N6 care

S6 (MH "Patient Care+")

S5 patient ${ }^{\star} \mathrm{N} 6$ care

S4 home 6 care

S3 S1 OR S2

S2 (heart or cardiac or myocard ${ }^{\star}$ ) N2 (fail ${ }^{\star}$ or insufficien* or decomp*)

S1 (MH "Heart Failure+")

\section{AMED}

1 exp Heart Failure Congestive/

2 heart failure.tw.

3 cardiac failure.tw.

4 or $1-3$

5 exp Telecommunications/

6 exp Comprehensive Health Care/

7 disease management/

8 telemed\$.tw.

9 telecare\$.tw.

10 telecardiol\$.tw.

11 telemonitor\$.tw.

12 teleconsult\$.tw.

13 teleconferenc $\$ . t w$.

14 telecommunicat\$.tw.

15 telephon\$.tw.

16 telehealth $\$$.tw.

17 telemetry.tw.

18 (remote $\$$ adj3 consult\$).tw.

19 tele-med\$.tw.

20 tele-consult $\$$.tw.

21 tele-conferenc\$.tw.

22 tele-health\$.tw.

23 Home Care Services/

24 disease management.tw.

25 nurse led.tw.

26 telehome.tw.

27 tele-home.tw.

28 phones.tw.

29 Clinical Protocols/

30 exp patient care management/

31 nurses/

32 Rural health services/

33 community health nursing/

34 or/5-33

354 and 34

$36(2008 \$$ or $2009 \$$ or $2010 \$$ or $2011 \$$ or $2012 \$$ or $2013 \$$ or $2014 \$)$.up.

3735 and 36

\section{Appendix 2. Search strategies (2010 review)}

Cochrane Central Register of Controlled Trials (CENTRAL), Database of Abstracts of Reviews of Effects (DARE) and Health Technology Assessment Database (HTA) on The Cochrane Library

\#1 MeSH descriptor Heart Failure explode all trees

\#2 heart next failure in All Text

\#3 cardiac next failure in All Text

\#4 (\#1 or \#2 or \#3)

\#5 MeSH descriptor telemedicine explode all trees

\#6 MeSH descriptor telecommunications explode all trees

Structured telephone support or non-invasive telemonitoring for patients with heart failure (Review) 
\#7 MeSH descriptor case management this term only \#8 MeSH descriptor comprehensive health care explode all trees

\#9 MeSH descriptor disease management this term only \#10 MeSH descriptor home care services this term only \#11 MeSH descriptor Home Care Services, Hospital-Based this term only \#12 MeSH descriptor Nurse Clinicians this term only \#13 MeSH descriptor nurse practitioners this term only \#14 MeSH descriptor monitoring, ambulatory this term only \#15 MeSH descriptor clinical protocols this term only \#16 MeSH descriptor patient care planning this term only \#17 tele* in All Text $\# 18$ (remote in All Text near/3 consult* in All Text) \#19 disease next management in All Text \#20 nurse next led in All Text \#21 phone* in All Text \#22 (manage* in All Text near/3 program* in All Text)

\#23 (nurse ${ }^{\star}$ in All Text near/3 manage* in All Text)

\#24 case next management in All Text

\#25 (home in All Text near/3 service* in All Text) 7

\#26 nurse next practitioner* in All Text

\#27 nurse next clinician* in All Text

\#28 care next plan* in All Text

$\# 29$ (\#5 or \#6 or \#7 or \#8 or \#9 or \#10 or \#11 or \#12 or \#13)

$\# 30$ (\#14 or \#15 or \#16 or \#17 or \#18 or \#19 or \#20 or \#21)

$\# 31$ (\#22 or \#23 or \#24 or \#25 or \#26 or \#27 or \#28)

\#32 (\#29 or \#30 or \#31)

\#33 (\#4 and \#32)

\section{Medline and Medline In Process on Ovid}

1 exp Heart Failure/

2 heart failure.tw.

3 cardiac failure.tw.

4 or $/ 1-3$

5 exp Telemedicine/

6 exp Telecommunications/

7 Case Management/

8 exp Comprehensive Health Care/

9 disease management/

10 tele med\$.tw.

11 telecare\$.tw.

12 telecardiol\$.tw.

13 telemonitor\$.tw.

14 teleconsult $\$$.tw.

15 teleconferenc $\$$.tw.

16 telecommunicat\$.tw.

17 telephon\$.tw.

18 telehealth $\$$.tw.

19 telemetry.tw.

20 (remote\$ adj3 consult\$).tw.

21 tele-med\$.tw.

22 tele-consult $\$$.tw.

23 tele-conferenc $\$$.tw.

24 tele-health\$.tw.

25 Home Care Services/

26 Home Care Services, Hospital-Based/

27 disease management.tw.

28 Nurse Clinicians/

29 Nurse Practitioners/

30 nurse led.tw.

31 Monitoring, Ambulatory/

32 telehome.tw. 
33 tele-home.tw.

34 phone\$.tw.

35 Clinical Protocols/

36 Patient Care Planning/

37 or/5-36

3837 and 4

39 randomized controlled trial.pt.

40 controlled clinical trial.pt.

41 Randomized controlled trials/

42 random allocation/

43 double blind method/

44 single-blind method/

45 or/39-44

46 exp animal/ not humans/

4745 not 46

48 clinical trial.pt.

49 exp Clinical Trials as Topic/

50 (clin\$ adj25 trial\$).ti,ab.

51 ((singl\$ or doubl\$ or trebl\$ or tripl\$) adj (blind\$ or mask\$)).ti,ab.

52 placebos/

53 placebo\$.ti,ab.

54 random\$.ti,ab.

55 research design/

56 or/48-55

5756 not 46

5857 or 47

5938 and 58

60 (2006\$ or $2007 \$$ or $2008 \$$ ).ed.

6159 and 60

\section{EMBASE (Ovid)}

1 exp Heart Failure/

2 heart failure.tw.

3 cardiac failure.tw.

4 or $/ 1-3$

5 exp Telemedicine/

6 exp Telecommunications/

7 Case Management/

8 disease management/

9 telemed\$.tw.

10 telecare $\$ . t w$.

11 telecardiol\$.tw.

12 telemonitor\$.tw.

13 teleconsult $\$$.tw.

14 teleconferenc $\$$.tw.

15 telecommunicat\$.tw.

16 telephon\$.tw.

17 telehealth\$.tw.

18 telemetry.tw.

19 (remote\$ adj3 consult\$).tw.

20 tele-med\$.tw.

21 tele-consult $\$$.tw.

22 tele-conferenc $\$$.tw.

23 tele-health\$.tw.

24 Home Care/

25 Home Monitoring/

26 disease management.tw.

27 Nurse Practitioners/

28 nurse led.tw.

29 Ambulatory Monitoring/

30 telehome.tw. 
31 tele-home.tw.

32 phone\$.tw.

33 Patient Care Planning/

34 or $/ 5-33$

354 and 34

36 controlled clinical trial/

37 random $\$$.tw.

38 randomized controlled trial/

39 follow-up.tw.

40 double blind procedure/

41 placebo\$.tw.

42 placebo/

43 factorial\$.ti,ab.

44 (crossover\$ or cross-over\$).ti,ab.

45 (double\$ adj blind\$).ti,ab.

46 (singl\$ adj blind\$).ti,ab.

47 assign $\$ . t i, a b$.

48 allocat\$.ti,ab.

49 volunteer\$.ti,ab.

50 Crossover Procedure/

51 Single Blind Procedure/

52 or/36-51

5352 and 35

54 ( $2006 \$$ or $2007 \$$ or $2008 \$$ ).em.

5553 and 54

\section{CINHAL (Ovid)}

1 cardiac output, decreased/ or heart failure, congestive/ or dyspnea, paroxysmal/ or ventricular dysfunction/ or ventricular dysfunction, left/ or ventricular dysfunction, right/

2 (heart adj failure).tw.

3 (cardiac adj failure).tw.

41 or 3 or 2

5 (home adj care).tw.

6 (patient adj care).tw.

7 patient care/ or case management/ or "continuity of patient care"/ or discharge planning/ or disease management/ or multidisciplinary care team/or nursing care/ or nursing care delivery systems/ or differentiated nursing practice/ or functional nursing/or modular nursing/ or primary nursing/ or progressive patient care/ or team nursing/ or total patient care nursing/ or nursing care studies/or nursing intensity/ or nursing process/ or nursing assessment/ or nursing care plans/ or nursing diagnosis/or nursing interventions/ or nursing outcomes/or nursing protocols/ or nursing care plans, computerized/ or nursing skills/ or patient care conferences/ or clinical conferences/ or patientfamily conferences/ or primary health care/ or "quality of health care"/ or accountability/ or guideline adherence/ or "outcomes (health care)"/ or medical futility/or outcome assessment/ or "outcomes of prematurity"/or treatment outcomes/or fatal outcome/ or treatment failure/ or practice guidelines/

8 (manag* adj care).tw.

9 managed care programs/ or health maintenance organizations/ or independent practice associations/ or preferred provider organizations/ or provider-sponsored organizations/

10 health maintenance organizations/or medical practice/ or nursing practice/ or advanced nursing practice/ or nursing practice, evidencebased/ or nursing practice, research-based/ or nursing practice, theory-based/ or "scope of nursing practice"/ or occupational therapy practice/ or prescribing patterns/ or prescriptive authority/ or professional practice, evidence-based/or medical practice, evidence-based/ or exp nursing practice, evidence-based/ or occupational therapy practice, evidence-based/ or physical therapy practice, evidence-based/ or exp professional practice, research-based/ or exp professional practice, theory-based/

11 (home adj care).tw.

12 home health care/ or home apnea monitoring/ or home intravenous therapy/ or home nursing, professional/

13 home care.mp. [mp=title, subject heading word, abstract, instrumentation]

14 patient care/ or after care/ or cardiovascular care/ or home nursing/ or nursing care/ or self care/ or self administration/ or self medication/

15 (home adj intervention*).tw.

16 (secondary adj prevent $\left.{ }^{\star}\right)$.tw.

17 (disease adj management).tw.

18 homecare.tw.

19 rehabilitat ${ }^{\star}$. tw.

20 rehabilitation/ or rehabilitation, cardiac/ or conditioning, cardiopulmonary/ or rehabilitation, community-based/ 
21 community health nursing/ or home nursing, professional/ or rehabilitation nursing/ or rural health nursing/

22 nurs*.tw.

23 nurses/ or advanced practice nurses/ or clinical nurse specialists/ or nurse practitioners/ or case managers/

24 multidisciplin ${ }^{\star}$. tw.

25 (discharge adj plan ${ }^{\star}$ ).tw.

26 patient discharge/ or discharge planning/ or early patient discharge/ or patient discharge education/ or transfer, discharge/

27 or/5-26

2827 and 4

29 telecommunications/ or interactive voice response systems/ or telecommuting/ or teleconferencing/ or telefacsimile/ or telehealth/ or telemedicine/ or remote consultation/ or telepathology/ or teleradiology/ or telenursing/ or telepsychiatry/ or telephone/ or wireless communications/

30 telecommunicat*.tw.

31 (tele adj communicat $\left.{ }^{\star}\right)$.tw.

32 telemed $^{*}$.tw.

33 (tele adj med $\left.^{\star}\right)$.tw.

34 telecar*.tw.

35 (tele adj $\left.\operatorname{car}^{\star}\right) \cdot$.tw.

36 telemonitor ${ }^{\star}$.tw.

37 (tele adj monitor ${ }^{\star}$ ).tw.

38 teleconsult ${ }^{\star}$. tw.

39 (tele adj consult*).tw.

40 teleconferenc ${ }^{\star}$.tw.

41 (tele adj conferenc ${ }^{\star}$ ).tw.

42 telehealth ${ }^{\star}$.tw.

43 (tele adj health $\left.{ }^{\star}\right)$.tw.

44 telephon $^{\star}$.tw.

45 telemetr $^{\star}$. tw.

46 (tele adj metr $\left.{ }^{\star}\right)$.tw.

47 (remote adj consult ${ }^{\star}$ ).tw.

48 phon ${ }^{\star}$.tw.

49 (electronic $^{\star}$ adj communicat ${ }^{\star}$ ).tw.

50 (tele adj nurs*).tw.

51 telehealth/ or telemedicine/ or remote consultation/ or telenursing/

52 or/29-51

5352 and 28

54 Experimental Studies/

55 exp Clinical trials/

56 ((control ${ }^{\star}$ or clinic $^{\star}$ or prospectiv $\left.{ }^{\star}\right)$ adj5 (trial ${ }^{\star}$ or study or studies)).tw.

57 ((allocat* or assign* or divid $\left.{ }^{\star}\right)$ adj5 (condition* or experiment* or treatment ${ }^{\star}$ or control* or group $\left.\left.{ }^{\star}\right)\right)$.tw.

58 ((singl ${ }^{\star}$ or doubl $\left.{ }^{\star}\right)$ adj $\left(\right.$ blind $^{\star}$ or mask $\left.\left.{ }^{\star}\right)\right)$.tw.

59 cross?over ${ }^{\star}$.tw.

60 placebo*.tw.

61 exp Clinical research/

62 Comparative studies/

63 exp Evaluation research/

64 exp "control (research)"/

65 Random assignment/

66 exp Prospective studies/

67 exp Evaluation research/

68 random ${ }^{\star}$.tw.

69 RCT.tw.

70 (compar ${ }^{\star}$ adj5 (trial* or study* or studies)).tw.

71 or $/ 54-70$

7253 and 71

77 limit 72 to $y r=" 2006-2008 "$

\section{AMED (Allied and Complementary Medicine)}

1 exp Heart Failure Congestive/

2 heart failure.tw.

3 cardiac failure.tw.

4 or/1-3 
5 exp Telecommunications/

6 exp Comprehensive Health Care/

7 disease management/

8 telemed\$.tw.

9 telecare $\$$.tw.

10 telecardiol\$.tw.

11 telemonitor\$.tw.

12 teleconsult\$.tw.

13 teleconferenc $\$$.tw.

14 telecommunicat $\$$.tw.

15 telephon\$.tw.

16 telehealth\$.tw.

17 telemetry.tw.

18 (remote adj3 consult\$).tw.

19 tele-med\$.tw.

20 tele-consult $\$$.tw.

21 tele-conferenc\$.tw.

22 tele-health\$.tw.

23 Home Care Services/

24 disease management.tw.

25 nurse led.tw.

26 telehome.tw.

27 tele-home.tw.

28 phone\$.tw.

29 Clinical Protocols/

30 exp patient care management/

31 nurses/

32 "Rural health services"/

33 community health nursing/

34 or/5-33

354 and 34

36 ( $2006 \$$ or $2007 \$$ or $2008 \$$ ).up.

3735 and 36

Science Citations Index and Conference Citations Index on ISI Web of Knowledge

\# 11 \#9 and \#10

Databases $=$ SCI-EXPANDED, CPCI-S Timespan $=2006-2008$

\# 10 ts=(random* or (clinical same trial) or rct or groups or (clinical same study)) and ts=("heart failure" or "cardiac failure")

$\# 9 \# 1$ and \#8

\# 8 \#2 or \#3 or \#4 or \#5 or \#6 or \#7

\# 7 ts=("case management")

\# 6 ts=("home care")

\# 5 ts=("disease management")

\# 4 ts $=\left(\left(\right.\right.$ nurse same led) or (nurse same practitioner $\left.{ }^{\star}\right)$ or (nurse same clinician $\left.\left.{ }^{\star}\right)\right)$

\# 3 ts $=\left(\right.$ remote $^{\star}$ same consult $\left.{ }^{\star}\right)$

\# 2 ts $=\left(\right.$ tele $^{\star}$ or phone ${ }^{\star}$ )

\# 1 ts=("heart failure" or "cardiac failure")

WHAT'S NEW

\begin{tabular}{lll}
\hline Date & Event & Description \\
\hline 20 July 2015 & $\begin{array}{l}\text { New citation required and conclusions } \\
\text { have changed }\end{array}$ & $\begin{array}{l}\text { We added 17 new studies of non-invasive telemonitoring or } \\
\text { structured telephone support in this updated review. }\end{array}$ \\
\hline 6 July 2015 & New search has been performed & $\begin{array}{l}\text { We have not included studies only available as conference ab- } \\
\text { stracts or theses and not a full-text peer-reviewed publication in } \\
\text { the review. }\end{array}$
\end{tabular}




\begin{tabular}{lll}
\hline Date Event Description & Den
\end{tabular}

We excluded studies if there was any documented previous exposure to telemonitoring or structured telephone support for the usual care or intervention arms prior to the start of the study.

We focused our searches on key databases and search engines.

We have added several new subgroup analyses, as well as a meta-regression examining subgroups.

We exclude Kielblock 2007 (included in 2010) from this version of the review.

Mortarra 2009 structured telephone support study arm revised to Strategy 2 (was Strategy 1 in 2010 review). Telemonitoring arm revised to Strategy 3 (was Strategy 2 and 3 in 2010 review).

We reclassified Capomolla 2004 as structured telephone support in this update.

\section{HIST O R Y}

Protocol first published: Issue 3, 2008

Review first published: Issue 8, 2010

\begin{tabular}{lll}
\hline Date & Event & Description \\
\hline 6 May 2011 & Amended & $\begin{array}{l}\text { Minor additions and corrections to Quality of life, Cost, Adher- } \\
\text { ence, adaptation, satisfaction and other outcomes section, in- } \\
\text { cluding Table 1. Additional references for included studies added } \\
\text { to 'References to studies' and study flowchart updated. Rela- } \\
\text { belled 'Parati 2007' as 'Villani 2007'. }\end{array}$ \\
& Amended & $\begin{array}{l}\text { As per correspondence with the author: The sensitivity anaylsis } \\
1.3 \text { has been corrected as the results for intervention and usual } \\
\text { care were interchanged. Text has been updated and Analysis } 1.3 \\
\text { renamed as correction. }\end{array}$ \\
\hline
\end{tabular}

\section{CONTRIBUTIONS OF AUTHORS}

Sally C Inglis: Responsible for conception and design of this study and the previous versions of this review (Clark 2007a; Inglis 2010). Responsible for co-ordinating and completing the review. Responsible for all data included in the review including designing, undertaking searches, retrieving search results, screening studies for inclusion, assessing risk of bias of studies, extracting data from papers, contacting study authors for information, entering data into Review Manager 5, analysis and interpretation of data, drafting of the review or revising it critically for important intellectual content and final approval of the version to be published. Performed previous work that was the foundation of this study.

Robyn A Clark: Responsible for conception and design of this study and the previous version of this review (Clark 2007a; Inglis 2010). Responsible for conceiving the initial idea for Clark 2007a. Responsible for all data included in the review including designing, undertaking searches, screening studies for inclusion, assessing risk of bias of studies, extracting data from papers, entering data into Review Manager 5 , analysis and interpretation of data, drafting of the review or revising it critically for important intellectual content and final approval of the version to be published. Performed previous work that was the foundation of this study.

Riet Dierckx: Responsible for all data included in the review including undertaking handsearching of conference abstracts, retrieving search results, screening studies for inclusion, assessing risk of bias of studies, extracting data from papers, entering data into Review Manager 5 , interpretation of data, drafting of the review or revising it critically for important intellectual content and final approval of the version to be published. 
David Prieto-Merino: Responsible for undertaking the heterogeneity tests between predefined subgroups and the meta-regression, review of statistical analyses undertaken in the review and drafting of sections of the review relevant to the heterogeneity analysis and metaregression findings and approval of the version to be published.

John GF Cleland: Consulted on design of review. Responsible for adjudicating study inclusion, interpretation of data (providing a methodological and clinical view), revising the review critically for important intellectual content and final approval of the version to be published. Guarantor for this review.

\section{DECLARATIONS OF INTEREST}

Sally C Inglis: no disclosures

Robyn A Clark was a PhD student involved in patient telemonitoring, data collection and tool development, analysis and publication of the CHAT Study which is included in this review (Krum 2013 (CHAT)). She was funded from NHMRC-funded grant for the CHAT study for one year then a Heart Foundation/NICS Scholarship.

Riet Dierckx: no disclosures.

David Prieto-Merino: no disclosures.

John GF Cleland has received research funds (within the last five years) from the European Union and Philips Health care for the conduct of TENS-HMS study (Cleland 2005 (Struct Tele) (TENS-HMS); Cleland 2005 (Telemon) (TENS-HMS)) and other research related to telemonitoring. He has received honoraria from Philips for speaking on the subject of telemonitoring (this funding is not connected with this review). As this author was a lead investigator of an included study in this review (Cleland 2005 (Struct Tele) (TENS-HMS); Cleland 2005 (Telemon) (TENS-HMS)) he did not undertake any risk of bias assessment or GRADE assessment of studies included in this review. Performed previous work that was the foundation of this study Clark 2007a and Inglis 2010.

\section{SOURCES OF SUPPORT}

\section{Internal sources}

- University of Technology Sydney Re-establishment Grant - A/Prof Sally Inglis, Australia.

\section{External sources}

- National Health and Medical Research Council of Australia, Australia.

(2010 version of review)

- National Heart Foundation of Australia, Australia.

(2010 version of review)

- National Institute of Clinical Studies, Australia.

(2010 version of review)

- Alberta Heritage Foundation for Medical Research, Canada.

(2010 version of review)

- NHS NIHR Cochrane Incentive Scheme, UK.

$£ 5,000$ to support completion of Cochrane Review (The views and opinions expressed therein are those of the authors and do not necessarily reflect those of the NIHR or the Department of Health)

- NSW Cardiovascular Research Network, supported by the Heart Foundation and the NSW Office for Science and Medical Research, Australia.

Cardiovascular Life Sciences Fellowship to A/Prof Sally Inglis (2013-2017)

\section{DIFFERENCES BETWEEN PROTOCOLANDREVIEW}

Changes from protocol to the 2010 version of review:

Since our protocol was published we decided to limit the studies included to randomised controlled trials only. We also decided to limit the inclusion of data in our meta-analysis to studies for which a full-text peer-reviewed publication was available. We also decided that the planned sensitivity analysis was no longer appropriate due to considerable advances in diagnosis and knowledge of chronic heart failure, and we performed sensitivity analyses examining the type of publication and length of intervention. We also included allcause hospitalisation and heart failure-related hospitalisations as primary outcomes, as it became evident that hospitalisations were 
an important measure of the effectiveness of these interventions, especially as over time survival from heart failure improves, but hospitalisations become frequent and costly. We also added adherence as a secondary outcome, as this was frequently reported in the individual studies and we realised it was important to consider whether or not participants were adhering to the intervention.

\section{Changes from protocol and 2010 version of review to current version of review:}

We have not included studies only available as conference abstracts or theses and not as a full-text peer-reviewed publication. This decision was based on research indicating inconsistency between trial findings presented as conference abstracts to those reported in a full, peerreviewed publication (Toma 2006). Another factor which was important in the decision to exclude studies only available as a conference abstract was the lack of detail provided regarding the intervention and usual care, thereby not providing confidence as to the classification of the study in terms of inclusion/exclusion in the review. Authors of studies available only as a conference abstract or study protocol were contacted in order to identify a full peer-reviewed publication for the study. A response was not received from several studies which are classified as ongoing or awaiting classification despite multiple attempts to contact authors.

We excluded studies if there was any documented previous exposure to telemonitoring or structured telephone support for the usual care or intervention arms prior to the start of the study.

Searches have focused on key databases and search engines, in particular, we excluded searching of trial registries as we were interested in studies published as full peer-reviewed publication only. We also excluded searching search engines such as Google Scholar as we found the enormous volume of hits which these types of search engines identify was unhelpful.

We have added several new subgroup analyses, as well as a meta-regression examining subgroups.

We have excluded Kielblock 2007 (included in 2010) from this version of the review.

Mortara 2009 (Struct Tele) (HHH) study arm revised to Strategy 2 (was Strategy 1 in 2010 review). Mortara 2009 (Telemon) (HHH) arm revised to Strategy 3 (was Strategy 2 and 3 in 2010 review).

We reclassified Capomolla 2004 as structured telephone support.

\section{N DEX TERMS}

\section{Medical Subject Headings (MeSH)}

*Telephone; Chronic Disease; Heart Failure [mortality] [*therapy]; Hospitalization [statistics \& numerical data]; Length of Stay [statistics \& numerical data]; Quality of Life; Randomized Controlled Trials as Topic; Telemetry [ ${ }^{*}$ methods]

\section{MeSH check words}

Aged; Humans 